Educational Governance Research 5

Michael Uljens

Rose M. Ylimaki Editors

\title{
Bridging
}

Educational

Leadership,

Curriculum Theory

and Didaktik

Non-affirmative Theory of Education

Springer Open 


\section{Educational Governance Research}

\section{Volume 5}

\section{Series Editors}

Lejf Moos, Aarhus University, Copenhagen, Denmark

Stephen Carney, Roskilde University, Roskilde, Denmark

\section{Editorial Advisory Board}

Herbert Altrichter, University of Linz, Austria

Stephen J. Ball, Institute of Education, London, England Y.C. Chen, Hong Kong Institute of Education, Hong Kong

Neil Dempster, Griffith University, Australia

Olof Johansson, Umeå University, Sweden

Gita Steiner Khamsi, Columbia University, USA

Klaus Kasper Kofod, Aarhus University, Denmark

Jan Merok Paulsen, Oslo and Akershus University College of Applied Science, Oslo, Norway

James P. Spillane, Northwest University, Chicago, USA

Michael Uljens, Åbo Akademi University, Finland 


\section{Educational Governance Research}

\section{Aims and Scope}

This series presents recent insights in educational governance gained from research that focuses on the interplay between educational institutions and societies and markets. Education is not an isolated sector. Educational institutions at all levels are embedded in and connected to international, national and local societies and markets. One needs to understand governance relations and the changes that occur if one is to understand the frameworks, expectations, practice, room for manoeuvre, and the relations between professionals, public, policy makers and market place actors.

The aim of this series is to address issues related to structures and discourses by which authority is exercised in an accessible manner. It will present findings on a variety of types of educational governance: public, political and administrative, as well as private, market place and self-governance. International and multidisciplinary in scope, the series will cover the subject area from both a worldwide and local perspective and will describe educational governance as it is practised in all parts of the world and in all sectors: state, market, and NGOs.

The series:

- Covers a broad range of topics and power domains

- Positions itself in a field between politics and management/leadership

- Provides a platform for the vivid field of educational governance research

- Looks into ways in which authority is transformed within chains of educational governance

- Uncovers relations between state, private sector and market place influences on education, professionals and students.

More information about this series at http://www.springer.com/series/13077 
Michael Uljens • Rose M. Ylimaki Editors

\section{Bridging Educational Leadership, Curriculum Theory and Didaktik}

Non-affirmative Theory of Education

照 Springer Open 


\section{Editors}

Michael Uljens

Åbo Akademi University

Vasa, Finland
Rose M. Ylimaki

University of South Carolina

Columbia, South Carolina, USA

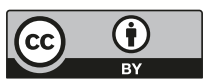

ISSN 2365-9548

Educational Governance Research

ISBN 978-3-319-58648-9

ISSN 2365-9556 (electronic)

DOI 10.1007/978-3-319-58650-2

ISBN 978-3-319-58650-2 (eBook)

Library of Congress Control Number: 2017951718

(C) The Editor(s) (if applicable) and The Author(s) 2017. This book is an open access publication.

Open Access This book is licensed under the terms of the Creative Commons Attribution 4.0 International License (http://creativecommons.org/licenses/by/4.0/), which permits use, sharing, adaptation, distribution and reproduction in any medium or format, as long as you give appropriate credit to the original author(s) and the source, provide a link to the Creative Commons license and indicate if changes were made.

The images or other third party material in this book are included in the book's Creative Commons license, unless indicated otherwise in a credit line to the material. If material is not included in the book's Creative Commons license and your intended use is not permitted by statutory regulation or exceeds the permitted use, you will need to obtain permission directly from the copyright holder.

The use of general descriptive names, registered names, trademarks, service marks, etc. in this publication does not imply, even in the absence of a specific statement, that such names are exempt from the relevant protective laws and regulations and therefore free for general use.

The publisher, the authors and the editors are safe to assume that the advice and information in this book are believed to be true and accurate at the date of publication. Neither the publisher nor the authors or the editors give a warranty, express or implied, with respect to the material contained herein or for any errors or omissions that may have been made. The publisher remains neutral with regard to jurisdictional claims in published maps and institutional affiliations.

Printed on acid-free paper

This Springer imprint is published by Springer Nature

The registered company is Springer International Publishing AG

The registered company address is: Gewerbestrasse 11, 6330 Cham, Switzerland 


\section{Foreword by William F. Pinar}

In this remarkable volume Michael Uljens and Rose Ylimaki juxtapose leadership and curriculum, scholarship from and about Europe and North America, a collection acknowledging the past's presence in the present, pointing to futures few in either curriculum or leadership studies have plotted. In recasting the relations between school and society, Uljens and Ylimaki contest the terms of the neoliberal present as they invoke them: yes, education is preparing students for the existing world, but it does so by problematizing that world. That world is of course a globalized one, but here globalization is defined as tendencies toward standardization, quantification, and homogenization but also as localization and cosmopolitanism, what Uljens and Ylimaki term "globopolitanism." Accordingly, they encourage us to attend to the interplay among curriculum, teaching, and leadership at various levels: the global, the national, the institutional. Educational leadership is also a curriculum work, and, given their reciprocal relationship, research becomes reconstructive.

While I concentrate on clarification rather than comparison as a methodology for studying across and within national cultures and histories - even the same curriculum concepts reverberate differently in different locales - the Uljens-Ylimaki conception of education as "summoning" the Other seems congruent: in being summoned the leader or educator or researcher is also being provoked to reflect over his or her positioning and relationship. Such a calling is clear when Uljens and Ylimaki cast education as "the cultivation of discernment with the help of reason." Like erudite and engaged teachers, educational leaders "summon" others - including the public, Uljens and Ylimaki suggest - "in reflective self-activity (Bildsamkeit) in order for them to transcend what is given." Transcendence can follow, even accompany, clarification of the given.

In a secular sense what is given is fundamentally historical; it requires historical scholarship to clarify, composing intellectual histories that intersect with present circumstances. Only from within nations and regions and locales - in complicated conversation with colleagues worldwide - can educators and leaders clarify together what "collective belonging and coherence" might mean. Even if democratically determined, such ongoing ever renegotiated internationalism requires, it seems to me, "becoming historical" so that the "outside" - including institutional, disciplinary, 
and (on the level of the state) legal arrangements - supports "a unified whole with spaces that allow for recognition of individual difference and freedom (autonomy)." In France that "unified whole" might be the state's enforcement of laicity, structurally not entirely different from Canada's enforcement of a multinational, multicultural state that, at least to some critics, threatens a tyrannical conformity.

In reaction, as Uljens and Ylimaki note, intensifying "pluralization" is also underway, a term referencing phenomena as varied as political polarization in the USA, in the UK, and across Europe, in the civil war in Syria. They also associate "pluralization" with "economic specialization" - they cite regulation and deregulation, so-called creative destruction - and these terms remind us that worldwide shifts in social formation and political movement are multileveled, often uncoordinated and unpredictable.

When academic study is undertaken with "double openness," Uljens and Ylimaki suggest, the researcher as well as the educator is engaged in dialogical encounter with oneself and others, with past and present, the local and the global. Due to the scale of such engagement, curriculum work and educational leadership are, as Uljens and Ylimaki remind, "human personal practices." So conceived, "curriculum making is educational leadership." Now often impersonalized and inhuman as scores substituting for actual children and educators, our professional calling as curriculum-leadership specialists calls upon us to study curriculum and leadership's enactment as it becomes (dis)embedded within locales, in its relations to larger social formations and political movements.

In such study, can Common Core be conceived as a compensatory contradiction of what sometimes seems intensifying social disintegration and political polarization in the USA? The very concept of Common Core - evocative of curriculum designs 100 years earlier - seems out of sync not only with the present but with the future of curriculum, at least as Williamson (2013) envisions it. In his chilling portrait, pluralization and economic specialization are elevated to somewhat superstructural elements of curriculum and leadership, Steve Jobs style.

In contrast to that soft authoritarianism decentralized as compulsory "collaboration," Uljens and Ylimaki affirm "comparative dialogue," supplemented by cosmopolitan ideals and realities, reconstructed by recursive returns to intellectual histories (and specifically classical theories of education) juxtaposed with present circumstances. As Aoki (2005 [1995]) appreciated, such sites of generative tension can encourage curriculum in a new key, what Uljens and Ylimaki foresee in "new research studies and fields" that could produce "a coherent language for policymakers, preparation programs, school development programs and practitioners." This is a vision at once theoretical and empirical, pedagogical and political, considering both policy and practice, a scale of aspiration one can only admire. That scale is shown too in their multiplying of the canonical curriculum question - what knowledge is of most worth? - into three: "Three questions rise above all: what are we going to live for (culture), what are we going to live of (economy), and how are we going to decide about these challenges (democracy)?" These questions specify the challenges humanity faces. 
As such, they might serve as organizing centers of the K-12 school curriculum, a curriculum focused less on academic vocationalism and standardized test-taking than on these fundamental questions of human life. It is a life, one hardly needs reminding, embedded in a biosphere being destroyed before our eyes by greed and power, themselves transhistorical, transcultural phenomena that could also serve as organizing centers for multidisciplinary academic study. From such study might emerge "conceptual and practical answers concerning citizenship" - answers that address, with cultural, political, and historical specificity, the fundamental questions concerning the future of life on earth. Such study could comprise a cosmopolitan education in our time.

By emphasizing intellectual histories as well as present circumstances, Uljens and Ylimaki remind us (in my terms) that the path forward is not in front of us, but in back. Grounded in history, they wonder to what extent fundamental concepts of education can be reconstructed in response to the world-historical situation today. In so doing, they aspire to "contribute with a reconstruction of fundamental tensions, issues and features of modern educational thought." This is the multileveled labor of internationalization and reconceptualization, labor that leads one to return to the relation between education and democracy, a move echoing the 100-year anniversary of Dewey's effort to do so.

The reconstruction of civil society - not only in the West - is just as crucial as it was in Dewey's time: social media and information technologies intensify those aforementioned movements of greed and profit-seeking. Feeling the emergency of "now," Uljens and Ylimaki assert that "curricula specify what education should be aiming at and they specify what cultural contents should be selected in order to reach these aims." Surely curricula can address "aims," but any promise to reach them risks reinscribing instrumental rationality, itself cause and consequence of the present crisis of sustainability. Becoming historical through academic study seems professional promise enough.

As did Huebner, Uljens and Ylimaki speak of "educational influence." For me the question of educational influence is necessarily a retrospective judgment: whether or not one was influenced, by whom and what, when, and to what effect. Emphasizing influence at the outset risks reinscribing instrumentalism encoded in objectives objectives to be assessed by quantitative outcomes. While there can be no predictable relation among curriculum, leadership, and democracy, surely the first two might mediate as they support the study of conflicting, even contradictory, currents within civil society: secularism and spiritualism, market economies and socialism, electronic media, and embodied educational experience.

Despite the confidence of the so-called learning sciences - and the neurology and pharmacology on which they sometimes rely - the relation between teaching and learning cannot be specified from the outset, only in retrospect, rendered as professional and personal judgments. The concept of "human personal practices" is here no warmed-over discredited humanism. Instead it invites, as Uljens and Ylimaki acknowledge, curriculum and leadership "pointed beyond the particularity of the 
nation-state - towards spatial universalism and temporal eternity," expansive sites of generative tension encouraging educational experience. You are entering one now.

University of British-Columbia

William F. Pinar

Vancouver, BC, Canada

\section{References}

Aoki, T. T. (2003 [1995]). In the midst of doubled imaginaries: The pacific community as diversity and as difference. In William F. Pinar \& Rita L. Irwin (Eds.), Curriculum in a new key (pp. 303-319). Mahwah: Lawrence Erlbaum.

Autio, T. (2006). Subjectivity, schooling, society: Between and beyond German Didaktik and Anglo-American curriculum studies. Mahwah: Lawrence Erlbaum.

Grumet, M. R. (1988). Bitter milk. Women and teaching. Amherst: University of Massachusetts Press.

Huebner, D. E. (1999). The lure of the transcendent. Mahwah: Lawrence Erlbaum.

Lilla, M. (2015, March 5). France on fire. The New York review of books, LXII(4), 14, 16.

Taubman, P. M. (2009). Teaching by numbers: Deconstructing the discourse of standards and accountability in education. New York: Routledge.

Toews, J. (2008). Becoming historical: Cultural reformation and public memory in early nineteenth-century Berlin. New York: Cambridge University Press.

Williamson, B. (2013). The future of the curriculum. School knowledge in the digital age. Cambridge, MA: The MIT Press. 


\section{Foreword by Carolyn M. Shields}

This book provides a stimulating opening for a thoughtful dialogue about the relationships among the concept of Didaktik, best known in Europe, the traditional North American understandings of curriculum theory, and thinking about educational leadership. It introduces important and foundational historical and philosophical concepts; it identifies tensions, disagreements, and false steps; and it profers some tentative solutions to some of the greatest challenges of our times. There is no doubt that the ideas espoused and the questions posed here offer ways to move the field of education away from the rational, technical, and scientific approaches that have framed much policy and discourse to date and have the potential to engage scholars and researchers for years to come.

Uljens and Ylimaki pose three organizing questions which, they believe, reflect the core tasks of education: what are we going to live for (culture), what are we going to live of (economy), and how are we going to decide about these challenges (democracy)? They indicate that the educator's role is to mediate between the culture and the individual's developing awareness of his or her potential and freedom as a cultural and political being. And they argue for a non-affirmative approach that does not violate the freedom of the individual learner.

To provide conceptual clarity, they clearly state that

For the purposes of our project, we begin with a classical approach and define curriculum practice as the process of formulating aims and selecting contents and defining methods, including the methods or pedagogical experiences through which content is enacted.

This debate is also reflective of the questions posed by Reyes-Guerra and Bogotch (2011) who argue that "the field of educational leadership needs to profoundly embrace the teachings of curriculum theorists" (p. 137) and assert:

Our argument is unequivocal: unless and until we can refocus on the learners' needs in context, we will remain captive inside the political apparatus of external state and institutional authorities [...]. Using curriculum theory/inquiry teachings to develop educational leadership programs, we can educate school leaders grounded in transformative leadership theory and practice, and affirm the values and processes of the American educational leadership profession on democracy. (p. 139) 
Uljens and Ylimaki have, of course, affirmed the need for a democratic focus as well, thus articulating one point of agreement among the authors, despite the many tensions and debates found in this volume. Uljens and Ylimaki maintain that they "defend a so-called non-affirmative position with respect to norms." They go on to explain that this means that existing knowledge, values, or ideals are definitely taken seriously but not affirmed. Affirmation in their view denies the possibility of critical reflection, confirming a present situation in a "rather uncritical fashion." Yet, one could argue that a non-affirmative position is one which simply does not explicitly recognize and identify implicit norms. Having a norm or end goal in mind whether democracy, individual freedom, or an open dialogue - opens up what Pinar calls "complicated conversations" still without advocating a specific path or determining a detailed set of steps to attain the goal.

Despite their previous assertion that democracy is an appropriate criterion to guide our responses to their three questions - how to decide about the challenges related to what we are going to live for, and of - Uljens and Ylimaki critique educational leadership theories as being "trapped either in an empiricist or descriptive approach or in a prescriptive and normative approach." Here, I think, is one of the challenges posed by their argument. Using the phrases "prescriptive and normative" or "normative prescription," as though the two were inextricably and permanently linked, seems to me to be erroneous.

Whether one is approaching education from the Kantian transcendental philosophy of freedom, from concept of collective nationhood, or from Heller's (1999) self-reflective consciousness of modernity itself, one's approach both arises from particular social and political contexts and is deeply embedded in the values (albeit often implicit) of those contexts. Thus, given that human existence is always embedded in a context, and given that contexts are deeply imbued with implicit or explicit values, it is difficult to overcome the fact that all theories or approaches are, in some way, normative. If one is identifying the "aims or content" of education, there will be at least an implicit sense of what is either "standard" or "ideal" in order to develop the student's understanding of his or her freedom (another value). If one believes that the goal of education is to enable a citizen "to participate in common tasks of the society, culture, politics, and economy," this undoubtedly comprises the standard for which content and pedagogy will be selected.

To be sure, there is no prescription in the above thinking, no sense of specific injunction, despite the fact that in recent years, under many regimes, authority and accountability have demanded conformity or prescription. Indeed, it might be argued that norms and prescriptions are of different natures and are both qualitatively and quantitatively distinct, in that norms relate to values and goals while prescriptions refer to rules and injunctions.

Moreover, not only might one argue that education is normative in the sense that, whether the valued end is freedom, equity, democracy, or something else, it expresses a desired norm, but that it should be normative. Giroux (2004), for example, when discussing critical pedagogy, stated that 
its normative nature does not offer guarantees as much as it recognizes that its own position is grounded in modes of authority, values, and ethical considerations that must be constantly debated in terms of the ways in which it both opens up and closes down democratic relations, values, and identities. (p. 36)

Being normative does not offer guarantees, but recognition of a starting position for a debate - the very activity in which Uljens and Ylimaki invite us to participate. Indeed, it seems to me that the central question is not whether the theory is normative, but whether, instead of being uncritically accepting of the status quo, it opens up debates about "relations, values, and identities" in such a way as to be nonprescriptive. Reyes-Guerra and Bogotch (2011) asked us to consider how we might "rewrite the field and profession of educational leadership as if curriculum theory/ inquiry were a leadership skill" (p. 138). In their response, they asserted that "both the purpose and process questions [of education] turn on the values and goals of democracy." Similarly Shields (2012) grounds her discussion of transformative leadership in concepts shared with curriculum inquiry - democracy, globalization, and dialogue. These and many other scholars thus come down on the side of a normative but not prescriptive argument, as they embed the concept of education itself in the norm of democracy, without identifying a list of rules or injunctions about how to attain it. Because Uljens and Ylimaki seem to be in fundamental agreement with Shields and Reyes-Guerra and Bogotch that education must be grounded in a concept of democracy, one might question whether they are really arguing for a "non-affirmative" approach. At the very least, one might wonder about the meaning and value of a non-affirmative approach.

At the same time, being non-prescriptive is an essential aspect of education if we are not to produce robots or widgets, but independent individuals, capable of innovative thinking and of challenging the status quo in order to build a better future (yes, another normative concept, but also without a clear prescriptive path for its attainment). This certainly requires, as Uljens and Ylimaki have done, opening up the dialogue and creating space in which various perspectives may be heard and paths taken to make sense of the selected curriculum materials on one's journey to individual freedom.

The European understanding of Didaktik is concerned with selection of the content to be taught as well as its "relation to the aims of teaching." Teaching and education, the editors state, "is about dealing with how to live out our responsibility to support the student's stepwise development toward an independent cultural being and citizen able to participate in common tasks of the society, culture, politics, and economy." When discussing mainstream leadership studies and trends, they assert that they find "little attention" to "education theory and its interests in schoolsociety relationships." Yet, when they describe the interpretive paradigm, they acknowledge that "an organization approach to organizations focuses on social life," and when they discuss educational leadership scholarship from a critical theory background, they describe it as "an approach to leadership grounded in a critical consciousness about power, privilege, and social inequities." These more recent approaches to leadership most certainly include discussions of pedagogy and curriculum especially as they pertain to the education of students who generally come 
from groups in society that tend to be marginalized. Hence, to bring together the concepts of Didaktik, curriculum inquiry, and educational leadership, it may be useful to pay more attention to recent and more critical theories that recognize the importance of the social and political context and that help to counteract the pervasive influence of the earlier more rational, technical, and scientific paradigms of educational leadership.

Some of the more technical educational leadership literature has sometimes attempted to draw sharp distinctions (falsely in my opinion) between leadership and management, with the latter representing the more routine functions that promote the efficient operation of an organization and the former requiring more proactivity and vision. Regardless of the emphasis, educational organizations must focus on functions of teaching and learning, and hence, educational leaders must be informed, at least in part, by conceptions of Didaktik and curriculum. And, as Uljens and Ylimaki argue, it is important to make the connections among policies, aims, curriculum, Didaktik, and leadership explicit in order for the theoretical grounding to be open to the dialogue and debate that is the foundation of a robust system of education.

Following their discussion of curriculum/Didaktik and educational leadership, Uljens and Ylimaki turn their attention to a universal and increasing cosmopolitanism, the third element of their discussion. Here they ask, "How do we explain curriculum making and educational leadership relations within and between nation states?" After describing some universal driving forces - a globalized economy, technology, and neoliberal politics - they go on to ask, "Do we, for example, imagine our theories being of universal validity over time and cultures or do we see them as regionally limited?" Is it possible, they ask, to have a universal theory of education or do we have to choose between the particular and the universal? These questions raise again the issue of whether a quest for a grand theory, responding to all times and contexts, is possible or even desirable. Perhaps a focus on guiding questions that may be answered in different ways in different contexts would be more appropriate. Yet regardless of the approach, the invitation to dialogue is central; the "dynamic" approach that emphasizes comparison of similarities and differences within and between levels from classrooms to transnational levels amidst the current globalization moment is necessary if we are to understand the ways in which education plays out, demanding not only individual freedom, but the freedom of local communities and nation states to choose their own way.

In their conclusion, Uljens and Ylimaki state that autonomy is "the highest objective of education." Indeed, it is the autonomy of the other authors in the book to forge their own paths, to agree or disagree with the editors, and to propose alternative responses to the questions of the relationships among educational leadership, Didaktik, and curriculum theory, which comprises the strength of this volume. Among the provocative ideas to be found here, Knapp and Hopmann suggest that increased attention to organizational perspectives of neo-institutionalism might contribute positively to an understanding of school leadership. Biesta posits that a language of education always needs to pay attention to questions of content, purpose, and relationship, hence arguing for a broader view of education than simply 
that of teaching and learning. Only then, he argues, can we overcome the current equation of learning with test scores and recognize that

education is always about the transmission and acquisition of some content (knowledge, skills, dispositions), but always also 'connects' students to particular traditions and ways of doing and being and, in addition, has an impact on their formation as a person.

His argument connects directly to that of Uljens and Ylimaki in that he asks for an integrative approach to thinking about education - one that does not separate curriculum from the aims and goals of educational leadership. Yet, neither does he call for a non-affirmative approach.

From different continents and different traditions, there is often agreement about some aspects of the conversation. Bogotch, Schoorman, and Reyes-Guerra situate the questions in a specific context, and provide a "tentative US framework," thus implicitly responding to the question of whether a theory may be universal in its application, given the various traditions from which education arises. They agree that "curriculum inquiry demands that participants have the freedom to be creative and innovative," and, at the same time, they argue the need for conscientization, for a recognition of injustice, and for dialogue. Huber, Tulowitzki, and Hameyer also emphasize the need for more attention to be paid to the interplay between educational leadership and curriculum theories, arguing that we need to see leadership "as a means to reach pedagogical goals and focus on education principles and not on bureaucratic ones." Sivesind and Wahlström take the argument farther, and while they argue the importance of taking both fields into consideration, they assert that it is also important to do so with "a reflexivity of how reform and research are intertwined."

As can be seen, as I refer to only a few snippets from the disparate chapters in the book, the dialogue here between leadership and curriculum theorists is rich and farranging. Yet, there is general agreement that the fields can benefit from a recognition of their mutual interdependence and from greater awareness of theories that help us to respond to the three questions Uljens and Ylimaki pose at the outset.

My intent here was to respond to the invitation and summons of Uljens and Ylimaki to reflect on possible responses to the dilemmas presented and the questions posed here, and to raise some questions and present some possible alternative ways of thinking, as a way of adding to the dialogue. If these reflections and the readers' careful considerations of the thoughtful chapters contained here prompt others to do the same, the book will have fulfilled the purposes of bringing the fields together in dialogue, if not in agreement. And this, after all, is the nature of freedom, autonomy, and inquiry. 


\section{References}

Giroux, H. A. (2004). Critical pedagogy and the postmodern/modern divide: Towards a pedagogy of democratization. Teacher Education Quarterly, 31(1), 31-47.

Reyes-Guerra, D., \& Bogotch, I. E. (2011). Curriculum inquiry as a transformative educational leadership skill. In C. M. Shields (Ed.), Transformative leadership: A reader (pp. 137-154). New York: Peter Lang.

Shields, C. M. (2012). Transformative leadership in education: Equitable change in an uncertain and complex world. New York: Routledge. 


\section{Foreword by Tomas Englund}

In their bold, rich, and very ambitious compilation of contributions trying to combine theories and studies on curriculum, Didaktik, and educational leadership into an integrated educational theory framework, Michael Uljens and Rose Ylimaki raise many fruitful questions and perspectives. Their explicit starting point is that "curriculum making is educational leadership" because, in the construction and implementation processes of curriculum, there is "educational leadership at multiple levels from classroom to transnational levels." Starting from a nonhierarchical and a non-affirmative position, they come close to a pragmatic tradition and give cosmopolitanism a central role.

The first chapter by the editors, Uljens and Ylimaki, presents a common general framework bringing the two disparate fields of curriculum theory and leadership studies together along with critical understandings from discursive institutionalism. Following an introductory framing of the book in Part I, Uljens and Ylimaki, then, expand the focus of the volume in four additional subsections: (1) curriculum theory vs Didaktik - USA and Europe, building partly upon the transatlantic project Didaktik meets Curriculum from the 1990s; (2) societal and policy context; (3) leadership, Didaktik, and curriculum; and (4) discursive and multilevel perspectives. The volume is then finalized in a conclusion by the editors.

This theory-building project to fuse curriculum theory and leadership studies is remarkable in its perspective and attempt, going beyond earlier theoretical developments made within curriculum theory - namely, the reconceptualist movement, the new sociology of education and studies presented in the Didaktik meets Curriculum project - in which leadership studies received very limited attention. This also means that the critical and conflictual perspectives of these theories and their follow-up theoreticians, analyzing curriculum as a political problem exposed for social struggles as in historical and educational policy studies of curriculum, have had no obvious place in leadership studies. Could these curriculum theorists and followers open up for leadership studies with critical and conflictual perspectives? It does not seem probable because the new accountability and testing environment of education may not give room for critical perspectives that challenge the narrow, organizational management language. 
Could leadership scholars engage in broader general education, critical education, curriculum, and policy studies in ways that challenge the dominant policy agenda? Jorunn Moller, a prominent Norwegian researcher on educational leadership, told us recently in her keynote at the ECER conference in Dublin in August 2016, based upon many years of experiences from the project ISSPP, known as the "International Successful School Principalship Project," that educational leadership in educational research needs to be complemented and informed by theory and research which focuses on recent changes in the political economy that have influenced education severely. She writes, "Although the reports briefly mention that education policies need to be aligned with other government policies, such as housing and welfare, to ensure student success, recommendations are mainly connected to improvement within organizations." She exemplifies saying that the discourse is mainly connected to the framework of increasing excellence in literacy and numeracy and is based on data from international large-scale student assessment. Moller argues that we, as researchers, have a responsibility to challenge this discourse in which this current policy agenda for equity is embedded.

She also stresses that the language we have adopted in education for the last decades may erode a broader discussion about education for citizenship and social justice over the long term: "One of the main tensions seems to be between discourses of competition and privatisation, which underpin new public management and discourses rooted in socially democratic ideologies, linked to notions of equity, participation and comprehensive education. We need to know more about the conditions which sustain education as public good, and it is urgent that we manage to initiate productive dialogues with practitioners and politicians about knowledge claims grounded in rigorous research". She concludes: "To lead education beyond the agenda of 'what works' we need different approaches to research, including critical studies addressing the power structures." But it is exactly this kind of perspective that studies of leadership are lacking.

How does this volume answer these kinds of challenges? In the next section, the one on historical societal-policy context, the four authors all stress the ongoing instrumentalization in this age of new public management and measurement with the implication of psychologization of education to learning.

A central part of the compilation of chapters aiming at bridging Didaktik/curriculum theory and leadership studies is the next section. We find here five chapters written by groups of authors, a total of 16 authors involved. Of course, there are, in this section, many different starting points for dealing with the relationship between curriculum theory and leadership studies, and the analyses also start from very different contexts.

The last two chapters of this section represent two different versions developed within a follow-up of the reconceptualization of curriculum movement, historically separated from each other for decades, one more macro and structurally oriented and the other more psychological. What we can see here, as in many of the other chapters and in educational research of today in general, is how the new educational policy language infiltrates the analyses and forces educational researchers to make 
use of a top-down perspective of goal achievement, learning outcomes, assessments, testing, and so on.

As formulated in one of the contributions in the last section, "educational leadership has in general focused on organizational conditions and expectations for managing and leading activities; in parallel, curriculum theories have offered insights into substantial societal problems that must be addressed in school and society." Is the future solution as developed in this chapter, to link curriculum theory to organizational theory, discursive institutionalism and educational leadership policy and research? Maybe, but the attempt to fuse curriculum theory and leadership studies will also have different implications in different contexts. Even though the possibilities of each nation-state are limited and profoundly changed by the globalization movement, there are still different preconditions in different countries.

To summarize, there is also a rather weak but anyway all-pervading and constant theme based in pragmatism (Dewey and Habermas) regarding the need to develop a deliberative stance in many of the contributions (e.g., the editors, Moos). There is also the recurring theme of cosmopolitanism, also presented in the introduction and referred to later in many of the chapters. These perspectives on pragmatism and cosmopolitanism could have been further developed. What might also have strengthened a book with this level of ambition would have been to go deeper into the ongoing changes that create new conditions for education, such as the increased parentocracy of schooling, the parental right to educational authority, legitimizing school choice, and the dissolution of public education.

Örebro University

Tomas Englund

Örebro, Sweden

Linnaeus University

Växjö, Sweden 


\section{Contents}

Part I Re-theorizing the Field: Foundations of a Research Program

1 Non-affirmative Theory of Education as a Foundation for Curriculum Studies, Didaktik and Educational Leadership .............................. 3 Michael Uljens and Rose M. Ylimaki

Part II Transnational Developments Challenging Leadership and Curriculum

2 Neo-liberal Governance Leads Education and Educational Leadership Astray . . . . . . . . . . . . . . . . . . . . . . . . . . . . 151 Lejf Moos

3 Lead Learner or Head Teacher? Exploring Connections Between Curriculum, Leadership and Evaluation in an 'Age of Measurement' Gert Biesta

4 Against the Epistemicide. Itinerant Curriculum Theory and the Reiteration of an Epistemology of Liberation João M. Paraskeva

Part III Curriculum Theory and Didaktik in US and Europe

5 The Didaktik/Curriculum Dialogue: What Did We Learn? . . . . . . . 219 Walter Doyle

6 School Leadership as Gap Management: Curriculum Traditions, Changing Evaluation Parameters, and School Leadership

Pathways

Mariella Knapp and Stefan Hopmann 
7 Curriculum Theory in Contestation? American Curriculum, European Didaktik, and Chinese Wisdom Traditions as Hybrid Platforms for Educational Leadership Tero Autio

\section{Part IV Leadership, Didaktik, and Curriculum Studies}

8 Forging the Needed Dialogue Between Educational Leadership and Curriculum Inquiry: Placing Social Justice, Democracy, and Multicultural Perspectives into Practice

Ira Bogotch, Dilys Schoorman, and Daniel Reyes-Guerra

9 Curriculum and School Leadership - Adjusting School Leadership to Curriculum

Stephan Huber, Pierre Tulowitzki, and Uwe Hameyer

10 Teachers and Administrators as Lead Professionals for Democratic Ethics: From Course Design to Collaborative Journeys of Becoming

Daniel J. Castner, Rosemary Gornik, James G. Henderson, and Wendy L. Samford

11 Codification of Present Swedish Curriculum Processes:

Linking Educational Activities over Time and Space

Eva Forsberg, Elisabet Nihlfors, Daniel Pettersson, and Pia Skott

12 Rethinking Authority in Educational Leadership William F. Pinar

Part V Discursive and Multi-level Perspectives

13 National Curriculum Development as Educational

Leadership: A Discursive and Non-affirmative Approach

Michael Uljens and Helena Rajakaltio

14 Curriculum and Leadership in Transnational Reform

Policy: A Discursive-Institutionalist Approach

Kirsten Sivesind and Ninni Wahlström

Part VI Conclusions and Implications

15 Curriculum Theory, Didaktik, and Educational Leadership:

Reflections on the Foundations of the Research Program

Rose M. Ylimaki and Michael Uljens 


\section{Part I \\ Re-theorizing the Field: Foundations of a Research Program}




\title{
Chapter 1 \\ Non-affirmative Theory of Education as a Foundation for Curriculum Studies, Didaktik and Educational Leadership
}

\author{
Michael Uljens and Rose M. Ylimaki
}

\begin{abstract}
This chapter presents non-affirmative theory of education as the foundation for a new research program in education, allowing us to bridge educational leadership, curriculum studies and Didaktik. We demonstrate the strengths of this framework by analyzing literature from educational leadership and curriculum theory/didaktik. In contrast to both socialization-oriented explanations locating curriculum and leadership within existing society, and transformation-oriented models viewing education as revolutionary or super-ordinate to society, non-affirmative theory explains the relation between education and politics, economy and culture, respectively, as non-hierarchical. Here critical deliberation and discursive practices mediate between politics, culture, economy and education, driven by individual agency in historically developed cultural and societal institutions. While transformative and socialization models typically result in instrumental notions of leadership and teaching, non-affirmative education theory, previously developed within German and Nordic education, instead views leadership and teaching as relational and hermeneutic, drawing on ontological core concepts of modern education: recognition; summoning to self-activity and Bildsamkeit. Understanding educational leadership, school development and teaching then requires a comparative multi-level approach informed by discursive institutionalism and organization theory, in addition to theorizing leadership and teaching as cultural-historical and critical-hermeneutic activity. Globalisation and contemporary challenges to deliberative democracy also call for rethinking modern nationstate based theorizing of education in a cosmopolitan light. Non-affirmative education theory allows us to understand and promote recognition based democratic citizenship (political, economical and cultural) that respects cultural, ethical
\end{abstract}

\author{
M. Uljens ( $\square)$ \\ Åbo Akademi University, Vasa, Finland \\ e-mail: michael.uljens@abo.fi \\ R.M. Ylimaki \\ University of South Carolina, Columbia, SC, USA \\ e-mail: YLIMAKIR@mailbox.sc.edu
}


and epistemological variations in a globopolitan era. We hope an AmericanEuropean-Asian comparative dialogue is enhanced by theorizing education with a non-affirmative approach.

\section{Challenges for Policy, Educational Leadership Research, Curriculum Theory and Didaktik}

The main argument driving this volume is that educational leadership and policy research, curriculum theory and Didaktik, despite their unquestionable contributions over the years, appear limited in their capacity to conceptually explain institutional education in increasingly complex environments and globalized nation-states. In addition, we will argue, many contributions in curriculum theory, educational leadership research and Didaktik are typically either remarkably functionalistinstrumentalist or prescriptive-normative in ways that make them appear, if not problematic, less fit for democratic education systems around the world.

We think, a mere dialogue between these strands of research does not suffice. What is needed is a conceptual system capable of coherently taking care of and relating these research specializations to each other. Without conceptual coordination, contributions in curriculum theory and Didaktik, as well as in educational leadership research, run the risk of being counterproductive or dysfunctional in terms of representing a too limited perspective on education, not only from the teachers' and principals' perspective but equally for policymakers and governance (Uljens 2015, 2016; Uljens and Ylimaki 2015; Ylimaki et al. 2016). Let us explain.

Nation-states and our schools of today find themselves operating in permanently renewed national and global contexts. We have witnessed new geopolitical repositionings and changes in the economic production. We see new energy systems evolve; we identify a need for sustainable development. Increasing multiculturalism, demographic movements, and aging population challenge us in new ways. Technological developments provide us new public spaces, and a global economy results in increasing interdependencies on all societal sectors. Many of these changes have been positive. Globally, poverty has decreased, more countries call themselves democracies, the number of military conflicts are, for the time being, less than in decades, and the education level is higher than ever. Yet, the developments mentioned all have created profound challenges for nation-states and their schools, for curriculum making, policy work, leadership and teaching. The political agenda in global, post-industrial, knowledge economies and information societies has thus changed the role of the nation- or federal state, the ethos of knowledge, education and research as well as the governance and leadership of the education sector.

Recent neoliberal educational and accountability policies have intensified a focus on school leadership, learning results, and national curriculum standards. The increased focus on leadership occurs as parts of new policies of curriculum making, leadership as enacted practice, and evaluation as a steering vehicle, all of which are occurring amidst increasing global interdependencies among all societal sectors as 
well as increasing multiculturalism and rapid developments in communication technology re-defining spaces for learning and teaching.

We can observe a redefinition of how power and influence is distributed anew between levels, e.g. transnational agencies, central administration and local schools, between state level administration and private (family) interests, but also within each level. These changes are far from only being functional or organizational but ideological. This is particularly well demonstrated by the move from a socialdemocratic welfare state (old public governance) to a neoliberal competition-based model (new public management/governance). This shift has had profound consequences for professional activity, identity and development. In a neoliberal, accountability paradigm, the customer is at the center, with various institutions organized around customer choice in the free market. In other words, neoliberal policies and trends have, in effect, shifted the focus of administration from the state as a provider of services to a buyer of services. This movement and related discourses are truly international, but they take different forms and stages in various countries (Paraskeva and Steinberg 2016; Gunter et al. 2016; Pinar 2004). We can observe, for example, an increase and changes in policy borrowing patterns, the ways in which curricula are harmonized, and new procedures of how we work with evaluation (SteinerKhamsi 2013).

Second, while a kind of cultural homogenization has been ongoing for decades transnationally and has become dramatically intensified through modern technology, the situation within states is the opposite. Here we see, simultaneous to global homogenization, an increasing cultural, ethnic, linguistic, professional, political pluralisation in most countries, as well as an economic differentiation and specialization (in the labourmarket). Recent neo-conservative movements both in Europe and in the USA have been interpreted as reactions to this pluralization and globalisation, which by definition, challenge established ways of sustaining unity and national identity or sense of belonging within states.

Third, we have been witnessing various types deregulation and decentralization since the 1980s as well as reregulation and recentralization of political power within nation-states (Gunter et al. 2016). However, an increasing number of counterproductive consequences emanating from deregulation of laws, decentralization of administration, a focus on cost-benefit and efficiency including idea of increased individual choice and reduced focus on egalitarianism to minimize disparities, initiated stepwise since 1980s, especially after 1989, have resulted in increasing mistrust, especially in Europe, as to whether a neoliberal model may provide sustainable solutions. Diane Ravitch (2010) and many others (e.g. Sennett 1998; Frontini 2009; Lingard and Rizvi 2009; Lindgard et al. 2015) have persuasively argued that neoliberal approaches have had unexpected results in application in various societal factors (e.g. healthcare, housing, education). For example, it seems obvious that large portions of citizens in the US and in Europe feel that recent developments regarding welfare, health-care, education and work have developed in a very unfavorable direction. It may be that the roots for expanding political populism in the USA and Europe partly are to be found here. These counterproductive consequences make it more important to see connections between economic neo-liberal globalization, 
national and transnational governance policies, educational ideals as well as curriculum and leadership practices.

Neoliberalism has many meanings and phases, with many references beginning in the 1980s with the Reagan and Thatcher administrations, the fall of the Berlin Wall, and the collapse of the Soviet Union. For some, neoliberalism equals globalization. Smith (2001) identifies three forms of globalization relevant for curriculum and leadership studies. Globalization One arises from the 1980s collapse of binary logics regarding cold war national borders and consonant revival of liberalism toward a borderless world vision of open markets guided not only by nations and states but also by newer transnational institutions with a global reach. Neoliberalism, then, redefined the rules of obligation between nation-state governments and people to privilege the free operation of a global market system over the state as the primary means for solving social problems, shifting the focus of administration from a provider of services to a buyer of services (Friedman, von Hayek).

In the education sector, the presence of the cosmopolitan dimension (Beck 2006; Kemp 2010) is practically visible both in terms of increasing global harmonization regarding core curricula but also through evaluation procedures (e.g. PISA) (Sahlberg 2015). Many countries have moved from an input oriented government policy driven by curricula towards an output centered governance policy driven by evaluations. An increasing number of transnational organizations influence, and are influenced by, national education systems. The globalization process inevitably leads to a restructuring of the role of the nation state and its governance practices in relation to the global scene. The nation state and its education must be thought anew in the light of new forms of, and previously unseen, versions of transnational and even global practices.

In a broad sense, then, the challenge for changing nation states involves organizational relations among education, politics, economy, and culture in order to continuously establish a sense of collective belonging and coherence to a unified whole with spaces that allow for recognition of individual difference and freedom (autonomy). Further, such societal and ideological changes, including the tension between the European social-democratic welfare state model (old public administration) and a neoliberal competition-based model (new public management and new public governance) and tensions between unity and plurality have consequences for professional activity, identity and development. This movement, and related discourses, are truly international, but they take different forms and stages in various countries (Roth and Burbules 2007; Paraskeva and Steinberg 2016).

These challenges have turned our attention towards understanding educational leadership as a much broader and complex undertaking than typical perceived of. It is a project with power redefined and redistributed anew between levels, interest and professional groups, e.g. central administration and local schools, between state level administration and private (family) interests, companies, between transnational organizations (e.g. European Union, OECD) and nation states, but also within each level. These developments make it crucial to understand the interplay among policies, societal trends/aims, methods, governance, social interactions of leadership-teaching-studying-learning within and between levels. We think that 
both curriculum research and educational leadership research would benefit from seeing how each connect sociocultural aims to curriculum contents and methods.

Our point of departure is that any successful accomplishment of educational practice, be it teaching, educational leadership or policy work on different levels, is partly guided by prevailing conceptual frameworks and theories, dominating policies, cultural and historical traditions. However, if the undertakings are informed by theoretical positions that conceptually highlight only disparate if important activities and processes of the educational system, and even in conflicting ways, their guiding power may be limited. While we recognize the value of debates within and between fields and disciplines, we argue that a more general and theoretically solid educational framework will inform new research studies and fields, as well as create a coherent language for policymakers, preparation programs, school development programs and practitioners. As we argue that curriculum theorists, Didaktik, leadership scholars, political scientists and policy scholars have yet to make these connections explicit this volume bring representatives from all these fields together for deliberation.

Deliberation has to be deliberation about something for some reason. The reasons were pointed at previously. Regarding the contents of this deliberation the leading question throughout this volume is: how should we understand the character and task of educational theory and research in order to handle the contemporary challenges? What should those conceptual answers concerning citizenship (e.g. cultural, economical and political) be and look like in order to understand and guide leadership and governance of our educational institutions in an era where all nations worldwide internally develop towards increasing plurality while being simultaneously framed by challenges that call for a cosmopolitan view? Where should we look for answers with the aim being a more profound and foundational approach to curriculum, educational leadership and teaching? How could a position look like and be constructed for these purposes? In the following, first, we outline the research strategy and how it was applied to meet the challenges described above. After this we describe how the volume as a whole is structured.

\section{Approach and Guiding Questions}

Given the existing multitude of theories on educational leadership, curriculum and Didaktik, are there any reasonable possibilities of developing a position that would allow us to approach these fields of research, i.e. curriculum, Didaktik and educational leadership, coherently? Because, if these theories are being developed with a limited awareness of an interest in each other they run the obvious risk of providing possibly distinct, yet only limited and disparate, answers. The strong twentieth century expansion of research has been welcome, but it has brought with it differentiation and specialization that is increasingly counterproductive in an ever more complex world. 
As a result, a fruitful strategy may be to move the starting point of theorizing educational leadership, curriculum and Didaktik to a level beyond each and all of them. Where and how can we find such a level? Our assumption is that such a metalevel does not need to be invented. It is provided to us by a long tradition of theory making in education, also called general education in Europe (Benner 1991; Uljens 2002). In this volume the German-Nordic tradition of general education will be made use of for these purposes, i.e. used as a meta-language and meta-perspective. Often general education theory starts off by asking foundational questions about the nature of education as such, as well as questions related to what role institutional education plays in and for societal development, politics economy and culture. Moving to a level beyond the specific theories and model of teaching, leadership and curriculum becomes our choice.

This chapter builds upon the non-affirmative theory of education as this position has been developed by the german education scholar, professor Dietrich Benner in Berlin. The non-affirmative theory of education is very much a German phenomenon. The approach was introduced in the Nordic countries in the mid 1990s at a education theory symposium on European identity in change in Finland (Uljens 1997b). More extensively the approach was discussed by Uljens (1998), Kivelä (1998), and Siljander (2000) in Finland and Sweden as well as by Oettingen (2001) in Denmark. This dialogue has stepwise expanded in all Nordic countries the past two decades (e.g. Bengtsson 2003; Kivelä 2004; Benner 2015; Damgaard Knudsen and Andersson 2008; Oettingen 2006, 2016; Saeverot and Werler 2017). The position has partly been made accessible to english speaking readers, primarily within philosophy and theory of education (e.g. Uljens 2002; Benner and English 2004; Friesen and Sævi 2010; Siljander et al. 2012; English 2013; Schaffar and Uljens 2015; Saeverot 2016; Uljens and Ylimaki 2015, 2016; Ylimaki and Uljens 2017). Benners works have been translated into Chinese, Japanese and some European langauges, but not yet into English. The specific contribution of the present volume and other publications by the co-authors the past 3 years relate to the application of this non-affirmative approach to bridging educational leadership, Didaktik and curriculum theory in a transnational perspective. Following this tradition we assume that following questions are central to answer in any theory of education, curriculum or educational leadership:

(a) How is education related to politics, economy and culture?

(b) How do we explain educational interaction?

(c) How do we deal with cosmopolitanism and the nation-state in a globalized world?

Next, the first two questions above are elaborated on and a preliminary answer on them is provided. We do not consider these questions as hierarchically ordered, but rather share the view that in conceptualizing education we need to understand how individual interaction and human agency is related with socioeconomic and political structures as well a historical developments and cultural patterns (Engeström et al. 1999). 
In order to understand institutional education, we must first (a) explain how a theory specifies the relation between education and other societal practices including e.g. culture, economy, politics and religion. To exemplify: how do we explain how political interests transforms into pedagogical action? Obviously national curricula, educational leadership and evaluation practices, teacher education and many other things mediate in complex networks between politics and schools' practices. In this respect, educational leadership is a mediating activity between different epistemic practices and value-spheres. But the question must also be put the other way around: How should education be organized, led and practiced to support and contribute to political democracy, if that is a system considered central? Naturally both questions are crucial from a curriculum and leadership perspective as practical tasks. For curriculum theory and educational leadership research, the problem is present as a theoretical dilemma and requires a position to be taken.

To indicate the idea of a non-affirmative position it can be contrasted with two normative-prescriptive positions frequent in contemporary curriculum theory: a reproduction-oriented model and a transformation oriented model.

From education history we know the conservative, socialization and reproduction oriented model in many versions since John Locke's book On Education from 1693 (see e.g. Durkheim 1893/1997; Parsons 1963; Bourdieau 1977). The radical, transformation oriented model is familiar since Rousseau's study Discourse on the Origin and Basis of Inequality Among Men from 1755. The first, reproduction oriented model subordinates education to politics by locating education within the existing society or culture. Here the task of education equals socialization into an existing society and culture wherein societal practices and norms function as the guiding principles. For example, according to Dewey, education in schools should be organized according to democratic principles as schools are expected to prepare for democracy. In contrast, transformation oriented models are guided by ideals for future. In its most radical form, revolutionary or transformation-oriented education allows itself to be positioned as super-ordinate with respect to societal interests (Freire 1970/2000; Giroux 1983; McLaren 1998). Educational efforts are made legitimate by their intentions - that education may liberate citizens from oppressive social values and practices aiming at a more socially just society. The ideals to be worked towards are not yet real, but can be made real.

According to non-affirmative theory of education both socialization/reproduction models as well as transformative curriculum models, are educationally problematic, regardless if they are ideologically conservative, radical, or counter-hegemonic. One of the fundamental issues is that these positions may be counterproductive with respect to political democracy. Strongly normativeprescriptive models run the risk of manipulation, indoctrination and of turning education, curriculum work, teaching and leadership, into a technological-instrumental profession.

In the mainstream literature these models are typically considered as critical of and opposite to technical-instrumental models, such as the Tyler rationale (1949). However, the normative-prescriptive character of both socialisation and transformative curriculum and leadership models make them congruent with technological 
instrumentalism: to the extent values and norms are prescribed, as they are in most models above, the task for education is to fulfill these pre-determined ideals as efficiently as possible. Yet, a non-affirmative position is not value neutral as it is assumed as a theory in and for a political democracy. Transformative theories are more explicitly suggesting what interests teaching and leadership should promote within democracies. Explicitly normative models often enter a dialogue with curricula as existing policy documents. Critical discussions of such documents belong to a public debate in democracies, but the question is if a curriculum or leadership theory is to be seen as a policy document among others?

The line to be drawn between critical-transformative and a non-affirmative position is fine. Both belong to a western family of critical theories. Yet we think this difference is worth pointing out. A non-affirmative theory is critical in that it reminds of that the task of education in and for a democracy is to recognize the existing individual, cultural and societal interests, yet not affirming them, but keeping these interests open as topics for reflection. As a theoretical construct non-affirmativity asks to what extent a given practice or policy allows for teachers and learners to cocreate spaces for critical reflection, not only to substitute one ideology with another. Although education is always political, the task of education is also to prepare for political participation the forms and aims of which are not determined in advance.

Coherent with the non-affirmative theory the position developed in this chapter, education stands in a non-hierarchical relation to politics, culture and economy (Benner 2015). Education is not solely placed either "outside" or "inside" society and is thus neither super- nor subordinated with respect to politics, economy and culture, but intends to mediate between these. In this non-hierarchical conceptualization, educational institutions are given relative independence with respect to societal and other interests. It is this space that both allows for and requires reflective, professional educational leaders on each level of the education system. Discursive institutionalism then offers a language for explicating these discursively identified spaces.

It should be observed that a non-hierarchical understanding accepts that hegemonic political interests frame and influence education, but this position recognizes that if the curricular policies and practices would be reduced in the service of some political ideology, it would be in conflict with principles for democratic education. Thus, political democracy requires a specific form of critical curriculum and educational leadership, including a relative independence for educational practitioners guaranteed by the political system itself. The same holds true regarding the relation between, for example, law and politics. In the modern, post-Kantian tradition laws are established by humans themselves. In a political democracy laws are established by an elected parliament. Simultaneously, however, laws regulate political activity. In the same sense, education is partly subordinate to political influences but simultaneously superordinate with respect to politics. Education, then, prepares the subject for active citizenship and political democracy as well as for a working life to be developed by the individual. Importantly, in this tradition, the individual is prepared to transform the very same society or culture into which that person is educated, but 
how this is or should be done cannot be decided upon in advance thus avoiding the normativity problems associated with the two dominant perspectives.

A problem with the closed models is that they do not leave room for developing the principal's, teacher's or learner's ability to decide upon what is to be considered valuable and meaningful. Pushed to an extreme, these approaches do not prepare the individual for self-reflective decision making about the future in a democratic society. Given that the future is thought of as open and undetermined and the question of morality something that cannot ultimately be decided upon in advance, the individual's cultural competence to critical reflection - autonomy, self-awareness and self-determination - is seen as something which must be developed.

The second question above (b) includes an expectation that any theory of educational relevance must explain human educational interaction. Although the individual can learn in and from all kinds of situations, all possible human interaction cannot reasonably be considered as educational or pedagogical in nature. Where, then, does educational interaction start and where does it end? Is there something we have to presuppose in order for education to be possible? For what reasons or ends would education be necessary? While the first question above (a) point at aims and contents of education, this second question (b) asks about the methods of education. How do we explain how teachers and learners' activities are related? How do we understand pedagogical leadership in contrast to other forms of leadership? How does educational leadership relate to teaching? Although educational leadership and teaching refer to different professions, what would the differences and similarities, given that both work with and relate to aims, contents and methods of education? Can educational leadership at a district or national level be seen as educational interaction of some kind?

In this chapter a non-affirmative position regarding pedagogical work is defended, regardless if we talk about teaching, educational leadership or curriculum work at the national level. The two previously described normative-prescriptive conceptions regarding the relation between education and societal development correspond to affirmative education. Affirmative education theory means that a theoretical position for curriculum, education or leadership is ideologically explicit regarding what interests and aims practitioners should affirm, regarding the present situation, or then regarding aims considered important for future needs of society. A dilemma with such an ideologically loaded position is that it runs the risk of ending up in manipulative education. Affirming a given reality, given values or future ideals can mean to uncritically relate one's professional practice to these. Affirmative teaching would be concerned with that learners really reach certain aims as given. An affirmative attitude ends up having a fundamental dilemma: to the extent that aims are given and accepted, educational leadership and teaching is expected to fulfill these resulting in a technical rationale for teaching and leadership.

In contrast, this chapter argues for the non-affirmative position. This means, for example, that existing knowledge, values or ideals are recognized but not affirmed. Non-affirmative education then means, in the pedagogical situation, to focus on the questions to which existing practices, experiences, norms or knowledge are seen as 
answers. By learning to understand how contemporary practices are seen as answers to given problems the learner is expected to develop a reflective relation not only to the answers provided (positive knowledge) in order to evaluate their relevance or accuracy, but also to develop an awareness regarding the questions behind the answers. This awareness includes reflection on in whose interest certain questions are. Of equal importance is the ability to learn to formulate new answers to old questions as well as to produce totally new questions to be answered. This means that irrespective of what values or norms education confess to, education cannot be about socializing the learners into these norms and values. The norms themselves must be brought into question for educational reasons. They are to be recognized, but not affirmed in order to create a pedagogical space for the learner to step back and see how one-self relate to these. In this respect non-affirmative education theory is clearly a critical theory. Some critical theories again are of course critical to existing hegemony. A counter-hegemonical discourse is crucial for any democracy. The question we raise is how valid such a position is as a foundation for education?

Our support of non-affirmative education theory is also grounded in that this approach lies, as will be demonstrated, at the heart of the western, modern tradition of education theory developed by "the classics". In our argumentation we rely on Dietrich Benners and others' reconstruction of this modern tradition, that for the most part has become forgotten. In order not to fall behind a theoretical level that is already attained such a historical awareness is necessary. We clearly understand that this such a position has developed within a given cultural, political and economic tradition. As will be shown this position was a response when leaving the dominating religious cosmology as societal or cultural "root metaphor".

The reason to why non-affirmative pedagogy reminds of pragmatism (Dewey), neo-pragmatism and deliberative democracy (Habermas) consist of their common roots. Both positions argue that there is an interdependent relation between education and politics. Also deliberative democracy requires individuals capable of participation in such a democracy. However, from a non-affirmative education theory perspective, a theory of how a deliberative democracy works is something else than a theory of educational preparation for participation in such a democracy. If this distinction is not identified there is a risk of ending up in normative-socialization oriented pedagogy again, now with deliberative democracy as the directing norm. Education "theory" would then reduced, once more, to drawing implications and developing prescriptive recommendations from e.g. sociology, psychology, cultural studies, ethics or the like, for how teaching should be organized to best prepare the learner.

We think that non-affirmative education theory is reflected in Bill Pinar's understanding of curriculum as complicated conversation. Pinar et al. (1995) observes that "Curriculum, then, is a provocation to reflect on and to think critically about ourselves, our families, our society" (p. 267). As we will see this kind of theory about educational activity, i.e. provocation to think critically, falls back on a long tradition of being aware of the difference between theory of Bildung and theory of education and how they are related. 


\section{The Modern Roots of Education: Cultural-Historical Grounding of Theory}

There is another reason to why we think it is not only valuable but also fruitful to turn to the German-Nordic tradition of general education to find a platform for coherently approaching educational leadership, curriculum and Didaktik. The reason is simply that the present day education dilemmas pointed out in the beginning of this chapter are not entirely new. The dilemmas we face today have a history, but not any history but a very specific one. It is the history of dealing with the question not only of how to educate in and for a pluralist society, but also to deal with the question of how education can, in principle, prepare the growing generation for a future that is not known by us? This move towards education for a future that is not known is one of the core dilemmas of modern education and schooling. How to support the development of the individual's cultural, economic and political citizenship, and educate for a society featured by mutual recognition and respect for differences, simultaneously creating societal cohesion without violating individual rights? The modern education theory was, in the end, a response to how to organize education in and for a pluralist society that left both religious frame of reference and a society built on given social classes.

These are questions we need answers to also today. Luckily these questions and answers to them are known to us as historical insights in the discipline of education. As principled dilemmas they point at those questions and answers that were identified in moving from a pre-modern to a modern societal order, i.e. moving from a pre-determined (teleological) cosmology to a view of the future as radically open where man no longer was only seen as law-abiding but also as law-making and where education became structured by a stepwise evolving nation state and new political order. The American revolution (1765-1783) and the European revolution (1789) came to be not only symbolic but historical turning points in this respect. The long history leading up to these occurrences included rethinking education. Is it too far-reaching to look back 200 years in order to find solutions for today's dilemmas? Our answer is a no. We argue that the modern classics of education theory like Rousseau, Fichte, Herbart, Schleiermacher, and also Dewey later on, formulated educational theory positions offering answers for the new postrevolutionary era. We think these philosophers of modernity and modern education developed conceptual categories and structures by which we still live, but have sometimes forgotten (Mollenhauer 2014). It is fascinating that these contributions were introduced as philosophical theories 100 years before any democratic societies were established, but they were not abandoned but rather built upon during the whole twentieth century until this day. These early contributions were not limited to teaching, curriculum or leadership alone but presented broader approaches to how education was to be understood. We argue for that a reconstructive visit to the core ideas in this tradition may revitalize and guide us in defining and specifying questions and answers to be provided. 
The first two questions (a) and (b) above constitute the core challenges in any education theory as we know of since ancient times. These questions were answered differently by the modern or classic education theory, as developed since Rousseau onwards including Kant, Fichte, Hegel, Herbart and Schleiermacher, i.e. as developed between 1760s and 1830s (Benner 2015; Uljens 2002; Schaffar and Uljens 2015). Our intention is to ask and answer to what extent this modern mode of theorizing education, as developed by these classics, is still relevant? And why should we bother about in asking if these classics make sense for us? There are mainly two reasons for this.

The first is connected to the fact that the modern, or today classic, education theory was developed parallel to the establishment of the modern concept of the pluralist, independent and liberal nation-state and a corresponding concept of the subject, not determined by origin, heritage or anything else This modern view of man, in contrast to a pre-modern conception thus recognized the subject as free or indetermined. The establishment of freedom of speech, religion and thought, as well as abandoning the religious idea of a predetermined cosmological development required a dramatic reform of educational thinking and theory. Previous religious cosmopolitanism as a regulating idea for explaining what it meant to be a human being and develop as such, was stepwise, by and large, replaced by the concept of the autonomous citizen in the independent nation-state. Consequently, the modern society could no longer manage with a pre-modern theory of education. The task of a pre-modern theory of education was about socialization the subject into a given order and future. Despite the original sin the individual was seen as free to make up her mind regarding the predetermined options available.

When religion stepwise was replaced by humanism and later with language as the constitutive dimension for nation-state, new building blocks were established framing education. Now education was supporting the birth of a man expected to make herself. Modern education was to prepare for a society that was in a stage of continuous development. Norms and values could be renegotiated. Westerners learned to live with the question of good life as an open one. Language was upheaved not only to a functional role but was made a constitutive question of personal and collective identity by e.g. Herder. An education for a pluralist society was created. We see that the challenges regarding unity and plurality have remained but that they have received new shapes. Today societies are more plural than ever, as societies have become increasingly multicultural, while at the same time increasingly interdependent both locally and globally. Modern education thus corresponded to a moving from an agricultural to an industrial society. In the agricultural society education as reproductive socialization was good enough, in the modern industrial society the individual's role and position was no longer given. In essence modern theory of education originally was an answer to the same principled dilemmas we experience today. Yet, educational theory as well as the discipline of education has in many instances lost the connections to its own roots as a discipline. This is why historical engagement with seminal ideas may be fruitful (Uljens 2002). 
Modern, or classic, European theory of education was developed along with the establishment of the ideas for the modern nation-state, abandoning previous forms of teleological views of societal development (Benner 2015). Through versions of enlightenment, human reason and rationality were elevated as to replace faith (Autio 2009). Education turned out as a crucial project for the nation-state from the beginning of the nineteenth century. How education was envisioned as a solution was evident in Fichte's Addresses to the German Nation (1808). This project was built upon the idea of establishing a connection between language, culture and state formation. In this concept religion was no longer considered crucial as the moral laws were to be established by man himself. While religious cosmology point beyond the particularity of the nation-state - towards spatial universality and temporal eternity, the modern project connects the concept of people as 'demos' (political citizenship) and 'ethnos' (cultural citizenship). In the beginning of this nation-state era, citizenship as cultural identity and religion was promoted over citizenship as political participation. Today the idea of education is, therefore, connected to a political-democratic citizenship idea, both in terms of that education was to be equally offered to each and everyone, but also that education was to prepare individuals for political participation, economic life and culture. The recent policy, education for the globalized competition state, is redefining concept of citizenship emphasizing the subject, not as a cultural or political citizen but as an economic one.

The seminal ideas developed in modern education theory were a response to an evolving new political, economical and cultural order. But these ideas were also partly utopian in that they presented ideas of how something could be, rather than they described as reality that was present. In a sense, the ideas regarding the task of education presented by of the classics were visionary as they went beyond their own contemporary societal practices and cultural contexts. In other words, these ideas were developed before any political democracies were established more broadly. Yet contemporary educational theory, curriculum and leadership research has to a large extent lost their connections to the seminal ideas of modern, western education theory. The loss of these roots make true progression difficult.

The point made here is that the demonstrated need to conceptually and practically rethink nation-state education reminds of what process the modern nation states lived through when the prevailing educational systems and philosophies were established (Oelkers 2000). There is a question of continuity and discontinuity. To what extent are we able to keep to seminal concepts of education developed as a response to the modern nation state? And to what extent are we forced to rethink citizenship as well as educational research, philosophy, policy and practice in the light of globalization? In order to accomplish this task, we will contribute to a reconstruction of fundamental tensions, issues and features of modern educational thought with a focus on post-Kantian educational thought as developed by Fichte, Schleiermacher and Herbart.

We do not make this argument in order to defend any classic philosopher or position in education. Further, this argument does not indicate a naïve relation to modernity. On the contrary, we emphasize a 'self-reflective consciousness of modernity 
itself' (Heller 1999). We see 'modernity' as one among many ways to organize and understand the individual, society and the world. But in order to move beyond contemporary positions and develop traditions, an insight in fundamental assumptions is necessary (Paraskeva 2016).

Another reason why we claim it is important to engage in the seminal ideas of modern education theory is that we today experience increasingly new forms of transnational organizational, national, corporeal, etc. aggregations of various kinds, new interdependencies and common challenges which require us to redefine what an "autonomous subject in an independent nation-state" is.

\section{The Return of Cosmopolitanism and Nationalism}

The third of the questions (c) pointed out above to be answered by a contemporary theory of education is that of cosmopolitanism. As a topic, cosmopolitanism has re-occurred many times in European history but always in new constellations and with new motives (e.g. Papasthephanou 2016). For example, both Kant and Herbart proposed cosmopolitanism as an ideal. "Das Weltbeste" (Kant 1915), meaning the best for the world, rather than private or national interests, was to be the aim of education (Perander 1883). What is needed today is a renewed and extended discussion on cosmopolitanism and the modern, nation-state centered heritage (e.g. Brincat 2009; Moland 2011). With the most recent 'globalization' movement, cosmopolitanism is back on the agenda after about 200 years of establishing independent, legal states (Rechtsstaat) based on some concept of collective nationhood, often invented around language, formal equity of citizens and history (Fichte 2009; Lewellen 2002). And while we recognize there have been perennial dominance and oppression issues with regards to language and distinctions among dialects, race/ethnicity, and class, the contemporary situation of globopolitanism is also radically different in many regards.

Whereas already Immanuel Kant replaced cosmopolitanism based on religion with universal principles of ethics, we today experience new, previously unseen practical cosmopolitanism or, rather, as we choose to call it, globopolitanism. In addition, with globalization, i.e. an ever increasing political, economical and technological process and policy of increasing interconnectedness, we witness a need for a better understanding of not how such processes are related to those processes within nation states. Many different transnational agreements and organizations today frame intentions and initiatives on the nation state level. Understanding educational leadership and curriculum today also must include the understanding of how nation states operate in relation to each other and in transnational aggregations. Educational leadership and curriculum theory, therefore, cannot be limited to the above two classical, questions but must be reconsidered in light of the global dimension. We argue this is a direction where curriculum theory, educational leadership and policy research should be moving, and, with this volume, we begin this direction. 
Globalization involve crucial developments with implications for curriculum work and policy as well as educational leadership and teaching. Globalization has many faces and is not least connected to technological developments as well as increased economic interdependencies. At other times globalization presents itself as cosmopolitanism. In an educational context cosmopolitanism can, and is to be seen as a question of educational ideals, contents and methods. But globalization as cosmopolitanism is also visible in the form of new interstate, international and transnational governance practices, policies, and procedures, including curriculum work and educational evaluation. Cosmopolitanism as a discourse on educational ideals reflected in curricula, and cosmopolitanism as interrelated governing and governance practices, are interrelated in complex ways. While the dilemma of cosmopolitanism as an educational ideal connect strongly to curriculum as a policy document, conceptually analyzed by Didaktik and curriculum theory, we see new transnational governance practices appearing as new empirical and conceptual challenges for educational leadership. We argue that these two forms regarding how education has developed with respect to global dimensions (cosmopolitanism as ideals and cosmopolitanism as policy practices) are to be treated in connection to, and not disparate, from each other. We perceive of educational governance and leadership as discursive practices at school, regional, nation-state and transnational levels, as well as in interactions between these (Uljens 2016; Uljens and Ylimaki 2015). These practices involve the making and practicing of politically agreed curriculum. The question is, then, how should a theory be constituted as to frame and deconstruct these dimensions?

It was previously concluded that the global or transnational level has complicated educational leadership, curriculum and evaluation. In our approach, we identify several levels of educational leadership as it relates to curriculum work with the intention to answer how the dynamics between, within and across these levels may be approached. It is not like this development has been hidden, on the contrary, empirically it is in front of everybody's eyes. Consequently, we have seen many languages and approaches developing around how the multi-level, multi-centered, and multi-professional processes should be approached. We see functionalist translation theory, systems theory and Luhmann inspired theory of policy borrowing (Steiner-Khamsi), network theories, Marxist inspired world systems theory (Wallerstein) and various versions of institutionalism (Frontini 2009). Few of these approaches have been developed with the core dilemmas of education as the ones to be solved. Most often the approaches advocated would be equally valid for understanding social work or health care. There is nothing wrong with that, except that education as a phenomenon (with curriculum, teaching and leadership as key dimensions) is treated as something what it may not be and as remaining blind for the complexity involved. In contrast, we navigate and position ourselves in this landscape by the help of education theory, not as our roadmap but rather as our guiding question aimed at reconstructing dominant positions. 


\section{A Comparative Perspective}

From a nation-state perspective these shifts in governance, commodification of education and pluralizing of identities reconstitutes the role of education for building social cohesion and national identity (Robertson 2006; Uljens et al. 2013). Especially in Europe with a tradition of "thick" states compared to a model of "thin" state in the USA, the policy shift is foundational. The Nordic welfare state has been built on the assumption of mutual positive effects between economic growth, welfare, educational equality as well as cultural and political citizenship. Movements towards entrepreneuralization of the subject have been received differently in the USA with a tradition of education more as a private rather than public good. More recent developments towards strengthening of nationalism all over the world, not the least in Europe, USA and Russia may be a reaction to economic liberalisation. In light of the complexity of the contemporary situation described above, we propose the need for a new approach to comparative methodology as well.

\section{Aim of This Chapter}

This chapter and the contributions included in this volume are, in different ways, part of a larger project and movement initiated and led by the co-editors with the expressed aim to develop a coherent non-affirmative theoretical framing (Benner 1991, 2005, 2015) for educational leadership and curriculum theorizing and Didaktik (Uljens 2015, 2016; Uljens and Ylimaki 2015; Ylimaki et al. 2016; Ylimaki and Uljens 2017; Uljens and Ylimaki 2017). Over the past 4-5 years (2013-2017) the co-editors have contributed to and engaged a wide range of prominent European and American scholars from the traditionally disparate fields of educational leadership and curriculum theory/Didaktik in a series of sessions, symposia and presentations at in Europe and USA (Uljens and Ylimaki 2017).

This initiative connects to and expands beyond the transatlantic dialogue on Didaktik and curriculum theory started in the beginning of 1990s (Hopmann and Riquarts 1995). Both projects share the idea to "investigate Didaktik and curriculum theory as historically evolved forms of reflection within the social system" (Hopmann 1992, 2015). The present project expands the focus in two ways.

Methodologically a meta-theoretical level is made use of with three guiding core questions: (a) how to specify the task of education in a democratic society; (b) how do we explain the nature of educational interaction; and (c) how can or should the above questions be treated at different levels within a nation-state (national, district, and school level) and beyond a nation state level. On the first question (a) a nonhierarchical understanding regarding the relations between education, politics, culture and economy is defended. Such a position is fundamental for democratic education as it avoids the pitfalls of functionalist and strong normative approaches to leadership and curriculum. The second question (b) argues for a non-affirmative 
interpretation of teaching, educational leadership and curriculum work. This question is opened up is handled by three classic education concepts - recognition, summoning to self-activity and Bildsamkeit as these are constitutive for a non-affirmative view for democratic education. The third question relates to cosmopolitanism and its necessary counterpart, i.e. individual and national identity, and citizenship. Moving from being an abstract, theoretical idea, cosmopolitanism has developed into an empirical reality; however, educational theory, leadership and curriculum research has only recently and still to a very limited extent developed a reflected position on cosmopolitanism and globalization. The nation-state perspective has dominated. We conclude that this theoretical framework allows us to more coherently conceptualize and study curriculum as policy and practice, educational leadership and teaching. In addition, a non-hierarchical position offers a foundation for discursive institutionalism valuable for understanding school work from a policy perspective.

Consonant with this purpose, this chapter is organized into four main sections. First, we analyze curriculum theory/Didaktik and educational leadership studies, including a discussion of strengths and limitations. Second, we present our general framework that considers core concepts and literature on non-hierarchical and nonaffirmative relations from general education theory, organizational theory, and discursive institutionalism that address the shortcomings from curriculum theory/ Didaktik and leadership studies, as well as cosmopolitanism as an educational ideal, and cosmopolitanism as empirical transnational relations. Fourth, our conclusions open the following chapters in the volume.

The first two sections analyses literature from two distinct fields, educational leadership and curriculum theory/Didaktik. Specifically, we examine strengths and weaknesses and then identify a shortcoming common to literature in both fields, a blind spot that, in our view, points toward the need for a new general framework that merges and extends educational leadership and curriculum theory/Didaktik. As it is not possible or meaningful within this frame to describe all varieties, we aim to identify core issues and distinctions.

\section{Theorizing in Didaktik and Curriculum Studies}

As the aim is to contribute a theoretical framework not only bridging European Didaktik and the Anglo-American curriculum studies but also to find out how these may be related to educational leadership studies in a broad understanding of the concept, we are engaged in a complex comparative dialogue. It is a dialogue moving across disciplines and fields of research, between epistemological schools and research methodologies, and between traditions in different countries and continents. Needless to say, such an attempt is a challenging undertaking. Within both fields of research considerable variation may be found. In addition, between European countries there is a variation in how education and curriculum is theorized. The same holds true for the US, although writing the US tradition equals 
writing the history of one European country. Despite a long and still ongoing dialogue on Didaktik and curriculum between US and Europe (Hopmann and Riquarts 1995; Hamilton 2000; Hamilton and Gudmundsdottir 1994) insight in each others traditions is weak. Some approach may attain significant interest in a country or two, but are hardly known in neighbouring countries. Also contemporary developments in one of the continents can pass relatively unnoticed in the other. For example, the remarkable Dewey interest in the 1990s remained for a long time mainly a movement within anglo-saxon discourse, including Scandinavian research, but hardly noticed in continental Europe (Oelkers 2000). As English has become the dominating 'lingua franqua' of Western academic discourse, parallelled by globalisation this has put non-english speaking countries in a very different position regarding their voices on education. Furthermore, in Didaktik we are talking about a historically seen very long tradition of different ways of structuring teaching, curriculum and education in general.

Although partly representing different approaches, curriculum studies and Didaktik have over time been mutually influential between US and Europe (Gundem 1995; Kansanen 1995, 2009; Kliebard 2004; Hamilton 2000). Joseph Dolch (1965) reminds us that the word 'curriculum' has occurred both in English and German but that while the notion survived in English, it was first replaced in the german tradition by 'Lehrplan' (instructional/teaching plan) by the end of eighteenth century. The curriculum term made its way back to Europe in the end of 1960s by Saul Robinsohn who, in turn, was influenced by Dewey's pragmatism (Robinsohn 1972). However, Dolch (1965, p. 359) observed that the distinction between a subjectcentered and a child-centered curriculum in U.S. corresponds to a movement from a 'Lehrplan' to a 'Bildungsplan' in Germany at the end of the nineteenth century, both acknowledging, on the one hand, structuring the contents and subject matter and, on the other, organization of teaching according to the learner's varying needs. In the U.S., the child centered, learning psychological approach to curriculum, originally based on Thorndike's idea of content neutral, psychological principles, grew strong early on.

Sometimes the differences are larger within Europe than between Europe and the US. Hudson and Meyer (2011) has argued that the increasing differentiation of Didaktik in Europe has resulted in a need to seek for common grounds within Didaktik. It is not clear how Didaktik should be understood as the research on teaching and learning has become very differentiated with various influences (Terhart 2009). In addition, European Didaktik has gained renewed interest in China (Bu et al. 2016; ChiKin Lee and Kennedy 2017), although European and German Didaktik was introduced in China via Japan already in the early 1900s (Deng 2016; Kennedy and Chi-Kin Lee 2016) when Herbartianism spreading also to the USA and Finland.

Most approaches to Didaktik, like theories of (general) education (Allgemeine Pädagogik), are directed by the ambition to cover both theory of Bildung (aims and contents) and theory of teaching (educational influence) in a systematic manner. Theory of Bildung traditionally then includes reflection on the aims of education and how selected cultural contents may support reaching these aims, while theory of education explicates those educative interactions involved in treating the contents for reaching given aims. Making use of these distinctions a first step must be a delin- 
eation structuring of the fields of Didaktik and curriculum studies with respect to the two questions posed as necessary for any theory of education to answer, i.e. (a) how educational interaction is explained, and (b) how the relation between education and other societal practices like politics, education and culture is defined. From a Didaktik perspective, however, questions of aims, contents and methods, or the why, what, and how of teaching are valid for both questions.

\section{Historical Developments: From Pre-modern to Modern Didaktik}

Historical studies have played an important role in Didaktik. Primarily this research has focused on the curriculum as a policy document and how it has developed (e.g. Sivesind 2008) or the history of ideas have dominated (Schaller 1995; Benner 1993, 1995; Gundem 2010). In order to understand the field both perspectives are required.

Although the contemporary western theory of education and teaching often relate to both ancient greeks and to rennaisance (fourteenth to seventeenth century) thinking the literature on Didaktik does not always point out features of these traditions compared with our contemporary, modern thinking. For this reason it is useful to discern between premodern and modern education theory (Benner 1991; Schaffar and Uljens 2015). The difference between these are related to the paradox of learning and teaching. We are familiar with the pre-modern paradox of learning as present in Plato's writings about the young Menon's learning of mathematics. Plato asks us how we should explain learning given that we cannot search for knowledge if we don't know what to look for. And, on the other hand, if we had knowledge it would obviously no longer be necessary to look for it. Plato's answer to this dilemma was the nativist one: paradoxically he claims that the condition for reaching knowledge is to already possess that knowledge, which obviously is paradoxical. How does he construct his case? According to his form of nativism a soul is connected to the individual by birth. This soul contains all eternal knowledge but the individual is unaware of that. Given this, the individual's knowledge cannot come from outside, e.g. from a teacher. Instead, the learner, according to Plato, had to learn to strive at reaching "in-sight", search for something that she already possessed. In other words, in this paradox the learner has to reach something she already has. The teacher's role is to direct the learner's attention by questions so that the learner may reach what she already is in possession of.

The Christian theological version of the paradox is also pre-modern. According to this version the paradox emanated from the view of man being created in God's image (1 Moses 26-27) and yet standing before the task of fulfilling this "likeness". The concept of Bildung (Ge. Bild meaning picture, image) has these roots. According to the Judeo-Christian doctrine, human beings are in need of maturing and becoming worthy of His image, while at the same time it is forbidden to make any image of Him (cf. the Ten Commandments). This dynamics, to strive for something that one cannot picture in advance, and of which one already is an image of is paradoxical (Meister Eckhart), and has since been a crucial dilemma of Bildung (Schaffar and Uljens 2015). For the major seventeenth century developers of 
Didaktik (e.g. Comenius) Christian cosmology was self-evident, a given. All education had the preparation for eternal life as its ultimate aim.

The paradox of learning changes in leaving the above described predetermined, pre-modern cosmology. By moving from a premodern to a modern ateleological view of individual and cosmological change, i.e. to viewing the future as indeterminate and not moving towards a given end, and the individual as no longer as predetermined, also the paradox of learning changes. Freedom and autonomy becomes key-concepts around which the modern paradox evolves.

Modern educational thought, from Rousseau and onwards, reformulated the premodern pedagogical paradox or paradox of learning. One of the cornerstones of modern pedagogy is the notion that autonomy (Mündigkeit) as the highest objective of education - discerning thought and action regarding issues of both knowledge and values. According to Herbart, moral freedom means following the reflected will, not acting conventionally from impulse or emotion. Consequently, education consists in the summoning of the Other to reflect over, for instance, the reasonableness of one's own will in relation to others and to the interests of others. Educating the will is then about the cultivation of discernment with the help of reason.

In modern theories of Didaktik knowledge is not considered to exist within the individual before experience nor that knowledge is transferred to the individual. Rather, education is often viewed as provocation to self-reflection. However, in order for the individual to transcend her present state, to reach autonomy, become self-directed, culturally free and reach competence to reflect, she must, according to this line of reasoning, already be conceived of as being autonomous, free and selfreflecting. This modern paradox of education is that education thus seem to presuppose the very existence of that which coming to existence education is considered necessary for. Expressed differently, in order for education to be possible there must be a somebody who's reflection is provoked, but simultaneously it is thought that the individual becomes a somebody, i.e. through the process starting with a provocation. Still differently stated, the pedagogical paradox concerns the following dilemma: in order for education to be possible the individual must be free and selfactive, and simultaneously, in order for the individual to become free and self-active education is necessary. Again we face the problem of how the individual can become something that she already is. The modern version of paradox is to consider the learner as already being able to do what she may become able to (Benner 1991, p. 71; Uljens 2002). Using this argument for understanding teaching it is assumed that the learner can reach cultural, productive freedom (ability to act), only by being recognized and treated as if she already is free (or reflective, capable, trustworthy). Teaching is therefore understood as an invitation, intervention or provocation, a violation, disturbance or expectation concerning the Others relation to herself, world and others. Teaching is to recognize somebody as if she already is capable of doing what she is supposed to become capable of - and act accordingly (Benner 1991). When discussing Didaktik, first, in terms of a relation between school and society and, second, as teaching-studying-learning process (Uljens 1997b) the above mentioned distinctions are interwoven in the following two paragraphs. 


\section{Didaktik as Theory About Educational Interaction}

It is quite obvious that Didaktik is widely considered a field of research and theorizing focusing on primarily educational interaction, especially from the perspective of initial teacher preparation (Jank and Meyer 2002). This is evident also in the etymology. 'Didaktik' originates from the Greek 'didáskō', which meant to demonstrate, show or teach. 'Didaskalos' ( $\delta 1 \delta \alpha ́ \alpha \kappa \alpha \lambda$ os) referred to the teacher. This term was also used in the Old and New Testament for a teacher of religion (Gundem 2010).

Historically, much research in both curriculum and Didaktik has focused either on epistemological, ethical, ontological, political, cultural, organisational, institutional and psychological foundations of substantial dimensions (aims, contents, methods, media) of teaching, or on the process aspect of curriculum as a plan for teaching, i.e. how curricula are constructed (initiated), communicated (implemented) and practiced (institutionalized) (Hopmann 2003). Following Hamilton's (2000) conclusions, modern didactics and curriculum arose "from a crucial distinction: the separation between the activity of 'teaching' from the activity of defining 'that which is taught' (p. 62). Before, this distinction between methods and contents was not made.

According to a widely accepted understanding, the German-Nordic Didaktiktradition of today would connect the mastery of subject matter as a necessary prerequisite for teaching activity, while the Anglo-American tradition (Tyler 1949) to a larger degree have treated principles of teaching as separate from any specific content. Instead reflection of methods would be grounded in psychological learning theory. Yet, these different positions have merged the past two decades. 'Didactics' may be contrasted with 'Mathetics' "for which the focus was the learner and the process of learning, as attested by the Socratic method of eliciting knowledge from the mind of a person through interrogation and logical reasoning (Maieutics)" (Sousa 2015, p. 282). This antique tradition of dialectic or dialogue centered reflection on education was focused on engaging the learner or the student in an reflective process. Dialectics rather than rhetorics made up the core of the teaching-studyinglearning process (Myhre 1976; Uljens 1997a). Later, for Descartes 'mathesis universalis' referred to general theory of quantities and proportions (Mittelstrass 1979).

Pierre de la Ramée (1515-1572) is often recognized as one of the first to use the word curriculum to signify goals and content for teaching while St. Victor in Paris in his Didascalicon from 1120 emphasized dialectics instead of rhetorics. Nordkvelle (2003) demonstrates how this dialectic tradition, where teaching was strongly connected to stepwise verbal commentary of the contents of a book in order to reveal its idea and structure for the student (lectio, meditatio), transformed into Didaktik. In essence, it was the rising number of universities and students attending classes that led to more structured presentations and monologic dictating of the contents (Nordkvelle 2003). However, with fifteenth century Italian humanism dialectics, classical rhetorics and dialogue were rediscovered from Antiquity, e.g. Quintilianus' (35-96) Institutio Oratoria consisting of 12 volumes describing the development 
from childhood to the adult speaker able to participate in public life. Hamilton $(1995,2000)$ argues that the mass schooling towards the end of the sixteenth century went through an "instructional turn" regarding how an existing body of knowledge, later a syllabus, could be organised to more effectively enhance learning (Hamilton 1995, 2000).

In this context the dynamic and vivid developments of Didaktik starting from the dialectic and rhetorical traditions of Antiquity, as well as transformations during the Middle Ages, Renaissance and Humanism can, however, not be explored. For extensive studies in history of Didaktik and curriculum research we refer to e.g. Diederich (1988), Schaller (1995), Nordkvelle (2003), Gundem (2010), and Hamilton (2000).

The intention of one of the classics in Didaktik, Johan Amos Comenius' (15921670) Didactica Magna (Comenius 1907/1633), aimed at presenting general principles valid for teaching of any contents. Comenius' idea was that the teacher reflects the contents (what), methods (how), when and in what order as well as with what kind of aids (Kroksmark 1989; Sadler 2007). Teaching principles transcending the contents have been starting from the familiar and moving to the unfamiliar, moving from the simple to complex, starting from sensory experiences in order to reach understanding through reason and pointing out the relation between principles and examples. Thus, despite continuous tensions, subject matter Didaktik (Ge. Fachdidaktik) and general Didaktik describe the same process but from different angles (Hultman et al. 2012). General Didaktik focuses on general principles.

In teaching, the teacher's task was to extrapolate the educative qualities (Bildungsgehalt) of the selected contents (Bildungsinhalt). To this end Didaktik aims at being a vehicle or a tool for thinking about or for reflecting education. Didaktik turns the teacher's attention towards the idea and character of the educational activity itself as it is related to aims and contents, as well as to the teacher's knowledge of the learners. For Klafki (1963) this type of pedagogical analysis (Ge. Didaktische Analyse), occurs before the actual planning of instructional and study activities.

For Wolfgang Klafki (1963), then, the idea with selecting content to be worked on had to represent or exemplify something general. It had to be exemplary and at the same time be experienced as meaningful by the student's given their present life-world or life-situation but also relevant in a future perspective. This Bildungtheoretical (Eruditioncentered) Didaktik continued the tradition of considering the task of teaching to develop the learner as a whole person, not just the individual's competencies.

We can now identify a difference between the Didaktik tradition in general and for example Shulman's pedagogical content analysis (Gudmundsdottir and Shulman 1987; Gudmundsdottir 1988). Bromme (1995) and Menck (1995) argues that Shulman's motive to focus pedagogical content knowledge stems from empirical studies on teacher effectiveness that identified a relationship among teachers' knowledge, treatment of contents, and successful student learning. However, because the Didaktik tradition as a whole puts the main focus on the teacher-student interaction as a reflective practice around selected cultural contents in essence as 
something non-predictable, this tradition stands in a radical opposition to contemporary output-centered approaches to learning, instruction and curriculum.

For the most part Didaktik has been developed as a doctrine, i.e. as principles for teaching directing planning of instruction. Didaktik is considered a field of knowledge central to the teaching profession and has a central position in teacher education programs (Jank and Meyer 2002). Given the practice oriented, methods centered and doctrinal status of the literature in Didaktik it has been a core element in teacher education over the centuries. Since its birth Didaktik has focused the structuring, sequencing, simplifying, organizing and communication of and around the teaching contents in order to facilitate learning, or rather pupils' studying hopefully resulting in learning what was intended (Uljens 1997b).

As a result, Didaktik, since Johann Amos Comenius' (i.e. Jan Koménsky) 'Didactica Magna' (originally the Czech Didaktik) has functioned both as "theory" or principles for teaching and a tool for lesson preparation, thereby receiving a similar role in teacher education as curriculum work did in the US (Hopmann and Riquarts 1995; Jank and Meyer 2002; Sousa 2015). Contributions to Didaktik with teacher education in mind (e.g. Wolfgang Klafki) typically emphasizes instructional planning with regards to aims, contents and especially the methods of teaching with the learner as well as the cultural context in mind, while contributions do Didaktik as curriculum theory (theory of the syllabus) looks at aims, contents and methods from a macro perspective focusing on what interests influence the making of a national curriculum so as to support transformation and reproduction of culture. However, teachers' professional development regarding their teaching competence is often not dealt within Didaktik and curriculum theory literature (e.g. Dreyfus and Dreyfus 1986; Berliner 1989; Dall'Alba and Sandberg 2006; Wermke 2013).

Despite the strong emphasis on Bildung in European Didaktik it should also be observed that much European empirical research on teaching and learning is not explicitly framed by a Didaktik theory. Rather, learning theory developed from the 1960s to a major frame of reference for research on teaching, especially various version of cognitivism.

However, in Scandinavian curriculum research and Didaktik in the 1970 and 1980s, the contents of teaching started to receive a more central position in research on teaching and learning. Curriculum research increasingly emphasized the differences between curriculum as intended, practiced and perceived. Instead of explaining differences in learning outcomes by the help of cognitive strategies applied by the learners studying the same contents, it was no longer taken for granted that learners perceived the content of teaching similarly or in the way the textbooks or teachers did. As a result, some scholars developed different approaches to studying how the contents was perceived and understood by the learners (Marton 1981) and teachers (Doyle and Carter 1984; Shulman 1986).

The turn towards individuals' experiences naturally required new research methods to be developed. Qualitative methods in educational research building on principles from hermeneutics and phenomenology (Bengtsson 2003), represented a significant shift in empirical research on Didaktik. European-Nordic research on Didaktik combined successfully empirical phenomenological research and the 
growing focus on teaching contents. Rather than focusing the learner's learning strategies, learners' ways of understanding the contents described. The phenomenographic approach developed by Marton and numerous colleagues (Marton 2014) has been influential since. Phenomenology and hermeneutics have most often been applied as epistemic positions (Claesson et al. 2011) but also as ontologically oriented theory of human experience and Bildung (Gustavsson 1996; Bengtsson 2003; Kristensson-Uggla 1999, 2010). This phenomenological-hermeneutic turn in Scandinavian Didaktik significantly contributed to expand the previously dominating interest in curriculum as primarily a governance issue. In Germany MeyerDrawe (1984) is a good exponent of this turn. However, these approaches were critiqued for disregarding sociocultural and institutional dimensions of education thereby remaining limited for understanding regarding the relation between politics and education pointed out as one of the two core questions to be answered by a theory of education.

Also cognitivist learning psychology as a foundation for research on teaching and Didaktik was criticized for disregarding the cultural and institutional context of learning. Which led to a support for a variety of sociocultural, situated and distributed approaches to learning inspired mainly by Vygotsky (e.g. Engeström 1987; Cole and Engeström 1993; Hutchins and Klausen 1990; Rogoff 1990; Lave 1988; Brown et al. 1989; Säljö 1994; Matusov 2011). These studies were mostly carried out in non-institutional learning, thus paying little attention to how organized teaching intervened in the learning (Bildung) process. Engeström (1987) developed an approach that was more relevant for Didaktik and institutional education by establishing the Scandinavian school of Vygotskian cultural-historical learning and activity theory (Engeström 2000). Further, this approach combined a contentcentered view of learning and teaching by locating this process in a larger social, cultural, historical and institutional activity system.

\section{Didaktik as Theory About the Relation Between Education and Society}

Didaktik grew into a more systematized field of reflection as a response to the sixteenth to seventeenth century developments in Europe with the embryonal construction of the modern state although teaching of reading was taken care of by the church. Naturally the printing press was a crucial technological invention allowing for a movement from an oral to a written culture (Ong 2002) that prepared for mass schooling and especially distribution of printed versions of the holy book. As the cultural institutions like the church and school were so crucial for the needs of the state it is easy to see why Didaktik covers both curriculum as the national or state based policy document (Lehrplan, syllabus, curriculum) and the practice of pedagogical or curriculum work within schools. With schools questions of teaching as 
separate from upbringing evolved. Also, as Hamilton (2000) reminds, the 'world of knowledge' began to be mapped pointing out the contents of teaching raising questions of how this contents should be organised to support learning (the course of schooling, curriculum). The questions has often been answered by describing how different levels decide on, act around and evaluate the selection of aims, content and method, i.e. curriculum work on a societal, institutional, interactional and personal level (Lundgren 2015; Gundem 2010; Reid 1994; Uljens 1997b).

In European practice of curriculum policy making the decisive selection, formulations and decisions regarding aims and contents of teaching is typically made on the nation-state level, far above each single school. Methods, in turn, have regarded primarily a topic to be decided by the teacher on the school or classroom level (methods freedom of teachers). A longstanding topic occurring in both curriculum studies and Didaktik is how the macro and micro levels connect (Goodlad 1966, Klafki 1995). While Didaktik as a field of research traditionally tries to keep together questions of aims, contents and methods of teaching as they occur different levels, in anglo-american research curriculum theory more often is discerned from instructional theory and its base in psychology or teaching contents.

While the curriculum as a public policy document, syllabus, and Lehrplan (since the seventeenth century in Europe) are seen as vehicles for governing the school as a state-driven public institution (Dolch 1965; Tenorth 1988) and for providing schools with the general aims of education and subject matter, such an understanding of a state or federal core curriculum is very recent in the US. In England and Wales national curricula were introduced 1988. In U.S., with a tradition of more state independent schools, the 'curriculum' has instead more often been considered a tool for local school leadership and organization of teaching (Robinsohn 1972; Hopmann and Riquarts 1995; McKernan 2008; Ylimaki 2011). While most European countries have lived through various stages of decentralisation of curriculum work the past three decades, the opposite movement have been visible in the anglophone world, thus reflecting a harmonisation across traditions.

For the Didaktik tradition, curriculum making involves an authoritative selection of contents from culture or tradition that must become embedded in the forms of teacher thinking (Hopmann 1997; Hopmann et al. 2000). This selection of contents is naturally done on a policy level as well as on a school or a teacher level. It is not surprising, then, that for one prominent twentieth century german researcher in Didaktik, Erich Weniger, Didaktik did not primarily refer to teaching methods or planning of teaching but mainly to the study of selection of contents for a curriculum as Lehrplan or syllabus (policy document) on a societal level (Weniger 1965). Today some might perceive this as the task of education policy research. As a consequence, for Weniger, Didaktik in narrower sense referred to the study of curriculum as a policy document while Didaktik in a wider sense also captures methods of teaching. Very often the opposite interpretation is found in the literature.

Throughout history, in Didaktik teaching in cognitive contents (qualification) has, in principle, been considered subordinated to and as a vehicle aiming at cultivation of more general capacities (reflected will, moral and rational reasoning, identity or character). That is, the selection and treatment of contents should serve 
educative purposes beyond learning the subject matter itself (e.g. Herbart's 'educative teaching', C. H. Judd's view of transfer; Somr and Hrušková 2014; Benner 1993). When saying that Didaktik also reflects contents in relation to the aims of teaching means that the core of education or Didaktik as an academic discipline is, in the end, about reflecting upon the ethical responsibility of teaching (Menck 1995). For what ends is education organized? What should the learner become?

The modern tradition, developed by Rousseau and Kant still today is a point of reference for the educational field as a whole, typically assuming that teaching and education is about dealing with how to live out our responsibility to support the student's stepwise development toward an independent cultural being and citizen able to participate in common tasks of the society, culture, politics and economy (labor market). This means that a theory of Didaktik is expected to answer how the question of pedagogical responsibility (Blankertz 1982). The ideal of the autonomous individual in the autonomous state was the regulative ideal that was transformed into a guiding point of reference for teaching.

Didaktik - A Theory for Educative Teaching In the German-Nordic tradition the discipline of education (Erziehungswissenschaft, Pädagogik), including Didaktik, identifies a difference between activity of teaching (Ge. Unterricht, Fi. opetus, Swe. undervisning) and activity of education (Ge. Erziehung, Fi. kasvatus, Swe. fostran). Some equate 'education' with primary socialisation typically occurring in the homes, while 'teaching' is seen as secondary socialisation that primarily would occur in schools. Others see education (Ge. Erziehung) and teaching (Ge. Unterricht) as two dimensions of one and the same process. Irrespective of position both notions refer to intentional activity to pedagogically influence the learner or student. 'Education' is focused on formation (Bildung) of the personality, i.e. supporting the individual's development towards autonomy and ability to self-directed responsible or ethically reflected action. 'Teaching', in turn, is not limited to the mere distribution of information or about training in a limited sense, but aiming at the development of learners' conceptual or theoretical understanding of a phenomenon, which also may guide future activity (Jank and Meyer 2002).

It is useful to be aware of the two different ways in which education and teaching may be considered as connected. Empirically seen schools always "educate" in the above sense of the word, intentionally or not (hidden curriculum): just by participating in social interaction and the practices of educational institutions values are always transferred and identities are being formed. Theoretically seen we may identify a more or less prescriptive relation between the two (teaching and education), i.e. that teaching always should educate (Jank and Meyer 2002). Through his concept of 'educative teaching' (Erziehender Unterricht) J. F. Herbart (1776-1841) pointed out that the primary idea or meaning of education was to support Bildung, i.e. the learner's development towards becoming autonomous (self-directed and responsible) and independent subject (Herbart 1908). Teaching that did not serve this educative purpose did not deserve to be called teaching but was rather about indoctrination or manipulation, according to Herbart. 
As we can see Didaktik primarily focus on the why-, what- and how-questions. Especially in broader conceptions of Didaktik like the one by Wolfgang Klafki the school's responsibility is clearly to be aware and reflect upon forces framing the school. Klafki's famous 'five questions' from the 1950s express the ambition to see Didaktik as a unifying theory regarding intellectual, social, historical and psychological justification inclusion of educational contents in the curriculum: (1). What wider sense of reality is prefigured in course content?, (2). What significance does course content already have in the learner's mind?, (3). What significance does course content have for the learner's future?, (4). How does course content relate to the other elements of a school programme?, (5). How does course content stimulate the learner's own inquiries?

Since 1960s, Didaktik has often been structured according to the research paradigm specific approaches represented. A paradigmatic division was made between an empirical-analytical approach, critical theory and hermeneutics reflecting a theory of science perspective, focusing on general ontological and epistemological positions. Both erudition centered Didaktik and progressivism during the twentieth century continued in many ways the longstanding tension between so called 'formal' theories and 'material' theories of Bildung. While the 'material' theories were emphasizing the importance of learning the contents in order to be socialized into the culture, 'formal' approaches focused on education as being about the development of general capacities to reflect, to solve problems and to deliberate. One of Wolfgang Klafki's contributions to german Didaktik was to connect the two by the concept of 'categorial Bildung' (Klafki 1995). The idea was that while the content in terms of categories for understanding opens up the culture for the learner, learning to e.g. reflect, argue and communicate 'opens up' the learner for the culture.

In Scandinavian Didaktik Swedish curriculum theory research by Dahllöf (1967) and Lundgren (1972) continued the approach initiated by Erich Weniger, viewing curriculum from a nation-state perspective. Curriculum theory was here not understood as theory or principles of teaching practice but rather as a framework for understanding how the school system was governed and "how knowledge transmitted in an educational context is selected, valued and organised and how such processes can be understood" (Linné 2015). This research developed later into studying curriculum codes inspired by Basil Bernstein.

Critical theory from the 1970s pointed out that traditional Erudition- or Bildung centered Didaktik was ideologically conservative contributing to preservation of the class society. The empirical turn in educational research in the 1960s in considered Bildung centered Didaktik as too philosophical and distanced from empirical practice of schools. As a result of this critique Wolfgang Klafki $(1994,1995)$ developed his 'critical-constructive' approach. As in critical curriculum and pedagogy in North American literature (e.g. Freire 2000; Apple 2005), this critical dimension is value laden in the sense that his critical-constructive Didaktik accepts self-determination, co-determination and solidarity as aims for education. Klafki's (1995) criticalconstructivist approach to education considers objective, institutional dimensions of schooling as well as the possibility of critical consciousness for eliminating barriers and changing society. The 'constructive' task of Didaktik then would initiate and 
develop new concepts, program, models for future educational practice moving beyond what is. Critical-constructive Didaktik thus is very close to a political program for democratisation of education (Jank and Meyer 2002).

\section{Sociocultural Reproduction and Transformation Perspectives in Curriculum and Didaktik}

More broadly, curriculum/Didaktik theorists also tend to fall between one of two dominant perspectives on relations between society and education but with more explicit ways of understanding education as subordinated to or located within existing society or superordinate to or located above existing society, socializing students into the values and norms of the organization and its goals to prepare students for an existing society or preparing them to change or transform society itself.

Curriculum theorizing and Didaktik scholars whose work is grounded in a sociocultural reproduction perspective explain curriculum in terms of a control function or paradigm, meaning that curriculum functions to prepare all students with existing important societal knowledge, values, and norms (Molnar and Zahorik 1977). Here, often drawing on Tyler (1949) and his followers, curriculum is a technical, rational system for planning and management. Dewey's (1916/2008) philosophy regarding relations among individuals, education, and society were not as widely utilized for curriculum development and planning in the US; however, his influence continues for many other contemporary curriculum theorists, including Henderson and Gornik (2007), Kesson and Henderson (2010), and Castner et al. (this volume).

Dewey (1916/2008) emphasized the idea of educational and social connections throughout his theories. As he described,

\footnotetext{
In directing the activities of the young, society determines its own future in determining that of the young. Since the young at a given time will at some later date compose the society of that period, the latter's nature will largely turn upon the direction children's activities were given at an earlier period. The cumulative movement of action toward a later result is what is meant by growth. (pp. 29-30)
}

In other words, education has no greater end than to create the capacity for further education in students; a democratic way of life is not a means to some larger end or outcome. It is in itself the realization of political, social, and educational ends supportive of growth (Ylimaki 2012). According to Dewey (1916/2008), such a democratic society must have a type of education that gives individuals a personal interest in social relationships and control, and the habits of mind that secure social change without introducing disorder. From this more or less sociocultural reproduction perspective along with a Darwin influence, Dewey (1916/2008) writes about curriculum subjects, arguing that the subject matter of education consists primarily of the meanings that supply content to existing social life. Here the continuity of social life means that many of these meanings are contributed to present activity by past collective experience. Dewey's rich pragmatic philosophy had a major impact 
on education in the early part of the twentieth century with many schools including his own Chicago lab school applying his ideas (results of which were reported in the Eight Year Study with Ralph Tyler as the primary evaluator).

Decades later, following in a neo-pragmatist and reconceptualist tradition of curriculum theorizing, Englund (1997, 2006), considered curriculum work as deliberative practice and communication. This deliberational and sociopolitical take on curricular dialogues in schools acknowledged how meaning was negotiated and constructed around subject matter in institutional settings, preparing the learner for a self-determined participatory and deliberative democratic citizenship. In the U.S., between Dewey and later reconceptualist theories (Pinar, Macdonald), Schwab (1969a, b) similarly drew on pragmatism to conceptualize curriculum making as deliberation in the "arts of the practical". Schwab considered the "practical' as a mode of inquiry or a way of dealing with the kinds of problems philosophers called uncertain (Gauthier 1963). To deliberate, according to Schwab (1969a, b), is to examine within a specific context, the complex interplay of means and ends in order to choose actions wisely and responsibly. While there are similarities with regard to a focus on environment or context and authority, these curriculum theorists did not explicitly consider organizational ontology, epistemology, or the role of leadership in these contexts.

Dewey's and later neo-pragmatist work (e.g. Englund, Schwab) notwithstanding, according to Westbury (2000), authority and management were central to anglo curriculum theory in the US with its decentralized system; authoritative agencies for public school systems, including district and school leaders were necessary to direct and institutionalize curriculum work and teaching in documents specifying objectives, experiences, and evaluation processes, such as in the system popularized by Tyler (1949). And while the role of district and school leaders is not explicitly considered here, classical anglo american curriculum theory was heavily influenced by behavioral psychology as well as the same rational perspectives and instrumentalism grounded in an underlying sociocultural reproduction perspectives that informed classic educational leadership approaches reviewed earlier. In one illustrative curriculum example, Bobbit's (1918) The Curriculum and its rational approach to curriculum development and planning were later popularized with Ralph Tyler's (1949) Rationale, identifying four broad open but fundamental questions concerning curriculum and instruction: (1) What educational purposes should the school seek to attain? (2) What educational experiences can be provided that are likely to attain these purposes? (3) How can these educational experiences be effectively organized? (4) How can we determine whether these purposes are being met? Tyler (1949) clearly disconnects ends and means; the question of objectives or aims must be identified first because they are considered "criteria by which materials are selected, instructional procedures are developed and evaluations are prepared" (p. 3). Values and morals are not an explicit part of curriculum planning as articulated in the Tyler Rationale (1949) and subsequent more instrumental models (e.g. Taba and Taba 1962).

In this control paradigm, the notion of human interactions around academic subjects (or the role of the student/subject) requires a rational approach to self-regulation, 
one that conflates morals and values with methods. From a classical curriculum theory standpoint, then, the success of rationalistic regulation of individual selves is dependent upon the behaviorist and cognitive measures tools of scientific psychology (i.e. curriculum). Subsequent scholarship regarding Tyler's Rationale tended to apply this system to very instrumental curriculum approaches grounded in behavioral psychology and learning theory, approaches that by design emptied the subject from consciousness. Pinar's critique of this grounding and underlying intent to empty the subject is explicit throughout his curriculum theorizing and that of his followers in the Reconceptualist Movement.

While U.S. educational leadership students and faculty members may not have read Ralph Tyler's (1949) Principles of Curriculum and Instruction, John Dewey's (1916/2008) pragmatic philosophy providing some counter arguments regarding the curriculum, the child, and democracy, or Pinar's (2004) contemporary curriculum theorizing as part of their leadership preparation courses, the above principles are very familiar in practical, mechanistic terms as the scientific procedures of Tyler's Rationale have become synonymous with U.S. curriculum work for decades. We can find a number of new practical models for curriculum planning and developed popularized in the wake of externalized evaluation policies connected to state and now more centralized (national Common Core) curriculum standards with Understanding by Design (Wiggins and McTighe 2005) probably among the most widely utilized example. Understanding by Design (UBD) is a curriculum planning approach to 'backwards mapping', looking at the outcome in order to design curriculum units with objectives (big ideas), performance assessments, and classroom instruction. Such curriculum planning also follows guidelines often promoted in practical workshops using divisional categories, such as the written curriculum, overt curriculum, recommended curriculum, and learned curriculum among others (Porter 2002). While Wiggins and McTighe clearly consider 'understanding' as a primary goal for curriculum work, we cannot consider this model as curriculum theorizing in the same way as Pinar's (2004) notion of curriculum as understanding and complicated conversation. In our view, these approaches to curriculum development and planning are control oriented in that the subject (both content and student) require a rational approach to regulation of learning from understanding self to the world. In other words, curriculum development models from Tyler (1949) and Taba and Taba (1962) through Wiggins and McTighe (2005) are also grounded in various learning theories. We do not go into all of that here but only to recognize that there is an underlying realist ontology and related epistemology at work, in for example, behaviorist theories that suggest knowledge is finite; learning is said to be overt, observable, and measurable, all of which aim for changes in behavior through some form of regulation and (implicitly) aim toward reproduction of existing society. We acknowledge that UBD and similar curriculum planning models now popular in the US context are not curriculum theories or theorizing as accepted in the curriculum field. During the same time, in the US and elsewhere, we also see a number of critical education scholars writing about the ideological conditions (e.g. neoliberalism) of social reproduction that influence schooling, including all kinds of curriculum work (e.g. Anyon 2006; Luke 2010; Purpel 1989). Across this work, we also find 
more attention to a critique of ideology and sociocultural reproduction than curriculum theorizing toward sociocultural transformation aims.

In contrast to sociocultural reproduction theories, sociocultural transformation oriented theories seek to critique the status quo and explain how radical change occurs in society. Much of the more recent curriculum theorizing in the North American context answers the question of relations among individuals, education and society in terms of societal change. In the US and elsewhere, this strand of curriculum literature takes its point of departure from either a subjective, radical humanist or psychoanalytic position radical structuralist position. Disputes between the more subjective, existential, even psychoanalytic perspective (exemplified by William F. Pinar), and a more objective structural perspective (exemplified by Michael Apple), have in many ways defined recent curriculum theorizing in North America and elsewhere. Regardless of epistemological and ontological differences, when applied to education, sociocultural transformation theorists argue that education is superordinate, to society (Uljens 2008; Uljens and Ylimaki 2015; Ylimaki et al. 2016). Curriculum functions in a superordinate position to society with planning, methods and content functioning to liberate citizens from existing, oppressive social norms and values. The aim of this scholarship is around liberation; however, according to Pinar and his followers, liberation begins with subjectivity Bildung and then moves outward for Apple, liberation is structural and overt from the beginning. Pinar et al. (1995) observe, "Curriculum is a provocation to reflect on and to think critically about ourselves, our families, our society" (p. 267). Specifically, Pinar et al. (1995) introduced eleven curricular subtexts: historical, political, racial, gender, phenomenological, poststructural, autobiographical/biographical, aesthetic, theological, institutional, and international. Here Pinar and colleagues draw on critical social theory as well as post-structural approaches to draw attention to a dual conception of the subject, content in texts as well as the individual subject and his/ her development through a journey of self-understanding (currere) and a complicated conversation with a range of texts. Reality is a social construction, meaning that becoming a person is a social act and that social meanings which sustain and organize a collectivity are created by the continuing pattern of interactions of increasingly diverse individuals in society.

Closely related, Freire (1970/2000) and his followers describe and critique what he calls 'banking' education as a term to describe the traditional education system. Similar models have followed, including funds of knowledge, defined as the historically accumulated and culturally developed bodies of knowledge and skills essential for households or individual functioning and well-being (Moll et al. 1992).

Other social change oriented curriculum scholars draw more explicit attention to connections among hegemony, ideology, power relations, official knowledge, and economic inequity (e.g. Whitty 1974; Apple 2004). Apple (2004) argues that curriculum must see as its task today the identification of moral, political, and ethical conflicts connected to social inequalities, including class structures, gender, race/ ethnicity, and intersections thereof. In Apple's (2004) view, the subjective, psychoanalytical principle of the social construction of reality does not explain why certain social and cultural meanings and not others are distributed through schools, 
nor does it explain how the control of knowledge preserving and producing institutions may be linked to power, particularly with regards to the ideological dominance of powerful groups in a social collectivity. Rather, Apple (2004) draws attention to the subtle connections among hegemony, ideological stability as raised by social reproductionist critics (e.g. Bowles and Gintis 1975; Bernstein 1975; Bourdieu 1977), curricular knowledge, and economic inequality. Across this critical education literature, curriculum decisions are political acts that contribute to social inequities, including class structures, gender, race/ethnicity and intersections thereof. Epistemological differences notwithstanding, pedagogical influences are not, however, explicitly discussed in much of this literature; however, others have applied the tenets of these critical education theories to various versions of critical pedagogy.

In the German Didaktik, Wolfgang Klafki $(1994,1995)$ would be the most wellknown European researcher representing a 'critical-constructive' approach. As in critical curriculum and pedagogy in North American literature (e.g. Freire 2000; Apple 2005), this critical dimension is value laden in the sense that his criticalconstructive Didaktik accepts self-determination, co-determination and solidarity as aims for education. Klafki's (1995) critical-constructivist approach to education considers objective, institutional dimensions of schooling as well as the possibility of critical consciousness for eliminating barriers and changing society.

\section{Post-Structuralism and Merging of Traditions: Postmodern Epistemology}

During 1990s post-structural developments in curriculum theory (e.g. Doll 1993b; Säfström and Östman 1999) were influenced by late modern philosophy (Lyotard, Foucault, Rorty, Cherryholmes) according to which social reality and its power relations are partly constituted by discourses, which were to be de- and reconstructed. The reception of this movement was received differently in arts, architecture, social and human sciences. In curriculum theory Doll's (1993a) interpretation is seen as a significative. In his reconstruction of historical developments in curriculum research he contrasted post-modern curriculum thinking as a departure from the modernist tradition. For Doll (1993a) modernism refers to Cartesianism which equals to realist ontology and representational epistemology as occurred in natural sciences. In this view learning is about aiming at reaching predetermined and true knowledge in a linear fashion assuming the "learner to be a receiver, not a creator, of knowledge, a spectator... who can only discover that which already is" (p. 283). The Credo of this modernist tradition is considered to be the Tyler rationale which "has led us to emphasize precisely defined, well-articulated, preset goals and a delivery system that matches the clarity of the goal statements" (ibid. p 284). This approach is, according to Doll (1993a), fundamentally challenged by postmodern paradoxical pedagogy viewing the future as unpredictable and where the "Self is understood only in relation to the other." (p. 285). This kind of curriculum thinking "shifts the 
focus of curriculum from receiving or developing to dialoguing, negotiating, interacting. These are not words found in either the Tyler rationale or in modernist curriculum thought." (p. 286). For Doll reaching back to issues Dewey dealt with, "a pedagogy based on Cartesian-modernist separation (of self and world) is either a copy-model pedagogy or, at its best, a discovery pedagogy". In developing his own answer inspired by post-structuralism, instead of replacing a causal view of teaching with a simple learner directed constructivism, Doll finds a role for the teacher: "The teacher's role is now to present the curriculum in just enough of challenging, controversial, "chaotic" manner so that self-organization will be encouraged" (p. 284). However, as will later be pointed out, in our reading of the history of education theory what Doll (1993a, b) describes as features of postmodern curriculum rather represents the very core ideas in modern education theory as developed from Rousseau and onwards, especially by Herbart and Schleiermacher. In Doll's reconstruction we cannot find any references to the classic core concepts of modern education theory as developed during romanticism, i.e. a period when education theory changed from representing a "closed" system and move towards an open one. Given that this tradition is invisible it can result in the conclusion that only post-modern education theory brought about something that was initiated almost two centuries before. We return later to what occurred between Doll's leap from Cartesianism to Bobbitt and Tyler.

In Autio's (2006) reconstruction, we find a comprehensive historical framing, both in terms of the reconstruction of the history of ideas as well as descriptions of the shifts in the political situation and the role of schooling in the nation state over time. Drawing on theological, sociological and philosophical sources, Autio (2006) identifies the roots of instrumentalist thinking and rationality operating in AngloAmerican curriculum theory and Didaktik today and then argues for a reconsideration of these traditions in the midst of contemporary globalization.

Autio (2013) argues that while this classical curriculum theorizing has been linked to the nation-state, the contemporary situation has also shifted with globalization. In his view, the traditional educational systems and models that have guided individuals towards the socialization process of becoming citizens of the nation state no longer apply. That is, an individual is no longer only a citizen of a nation but also has other concerns and responsibilities toward global citizenship. "Globalization is undermining the project of modernity by dis-embedding the political project of the state from the cultural project of nationhood" (Autio 2013, p. 31). For Autio, new conceptualizations of a nation-state force us to re-evaluate the curriculum as a project to build national identity through national ethos, and question the possibility of the curriculum or educational thinking to reflect and reinforce national belonging. Autio (2013) concludes by arguing that the post-national approaches to curriculum theorizing are needed in order to encompass the contemporary situation (p. 31). While Autio's reconstruction of ideas is comprehensive and considers intellectual shifts beyond Cartesianism, there are few references to the core concepts of modern education theory as developed during the later enlightenment and romantics, i.e. the seminal position in modern European theory. We will 
later consider the relevance of these core concepts to the contemporary situation. First, we must explicitly articulate what we mean by curriculum, its objects, processes, and underlying perspectives.

\section{Didaktik, Curriculum and Educational Leadership}

Classical North American curriculum theorizing and theories, when compared to Didaktik, featured a control managerial perspective, one that was formalized in the Tyler Rationale (Tyler 1949) and its psychology-based instrumental applications (Westbury 2000). In both classical American and European curriculum theory, answers to curriculum revolved around technical rationality connected to the idea of building systems of public schools with an authoritative agency directing teachers' curriculum work (i.e. implementation) with a written curriculum document (i.e. the syllabus) containing statements of aims, prescribed content (e.g. textbooks) and expected methods (Westbury 2000). Such control perspectives were not without early criticism, however, including most notably Dewey's (1938) charge that control theories masked value positions, giving the appearance of being value free when they clearly were not.

The managerial or control approach to curriculum (e.g. Tyler 1949) focused on practice, providing conceptual frameworks which were intended to increase the efficiency and effectiveness of the educational process, an intention that dominated much of curriculum theorizing until the 1970s. The reconceptualist Movement and parallel critical education movement in the New Sociology of Education. Since the 1970s, curriculum theorizing in North America has been focused around subjectivity Bildung and Currere in the Reconceptualist Movement (e.g. Pinar 1978, 2011, 2012; Macdonald 1971) and critical education studies in a parallel movement on the New Sociology of Education (Apple and Weis 1986; Bowles and Gintis 1976; Giroux 1980; Arnot and Whitty 1982), and, most recently in a post-reconceptualist era, cultural studies (e.g. Dimitriatis 2009; Helfenbein 2010; Mason and Helfenbein 2012). Across these strands, curriculum theorists have challenged the control or managerial perspectives of classical curriculum theory, such as the Tyler Rationale (1949).

Across these reconceptualist movements, the object of curriculum theorizing has been, on the one hand, critical studies of the marginalizing impact of policies or policy discourses and, on the other hand, a relation between identity construction (e.g. race, class, gender) and schooling. In the former, curriculum theorizing features the development of official knowledge emerging from societal structures, power, ideology, and schooling as exemplified in the work of Michael Apple (2004, 2014) and similarly articulated by others (e.g. Bowles and Gintis 1976; Giroux 1980). According to Apple (2014) schools preserve and distribute what is perceived to be "legitimate knowledge", meaning they confer cultural legitimacy on the knowledge of specific groups. Here the ability of a group to make its knowledge legitimate related to that group's power in the larger political and economic area. In a related strand, more recent cultural studies scholars remind us that such hegemonic 
processes create what Bourdieu termed symbolic violence, eradicating particular cultural identities from official curriculum documents and materials, all of which have a critical impact on identity construction. In other words, for critical education and cultural studies scholars, theorizing is cultural critique, engaging a counter hegemonic dialogue about the underlying ideological bases of curriculum as well as what happens with people's identities as a result of dominant curriculum/policy discourses and knowledge legitimation. Such critical education theorizing also dates back to Bernstein's theories of codes (1975) that heavily influenced the curriculum studies field in the Nordic countries/Europe, including Forsberg et al. (this volume) and is likely to increase with rise of neo-nationalism and authoritarian populism as well as increasing pluralism due to internal cosmopolitanism, population migrations, and refugees.

Pinar's complicated conversation or Bildung centered 'understanding curriculum' approach to curriculum theorizing took steps towards the German-Nordic tradition of understanding curriculum not only as a field of research among many, but as a discipline in its own right, comparable to psychology or sociology, aiming at theorizing curriculum (Uljens and Ylimaki 2017). Pinar gave little attention to institutionalized schooling in his curriculum theorizing, and in fact rejects the need to focus on it early on (Pinar 1978) while Apple has frequently critiqued the inequitable societal structures that schools as sociocultural institutions reproduce. During the same timeframe, Macdonald (1995) took steps toward a more hermeneutic approach to curriculum theorizing but did not explicitly consider organizations/ institutions or leadership therein. Grounded in hermeneutics, Macdonald's (1995) curriculum theorizing pushed beyond development frameworks to propose the importance of ongoing attempts to interpret curricular reality.

Across Pinar's $(1978,2004,2011)$ curriculum theorizing, critical education studies (Apple 1992, 2004, 2008) and various cultural studies literature (e.g. Dimitriatis 2009; Helfenbein 2010), we see important work dealing with contemporary political struggles, and we do not mean to question the importance of such identity issues as race, class, gender, sexual orientation and other -isms. Rather, we point out that theorizing identity in relation to sociopolitical struggles is not the same as theorizing education or curriculum/Didaktik as key aspects of it. At the same time, cultural studies oriented curriculum scholars also connect with those who write about inequitable power structures (e.g. Apple 2004, 2008) as well as related postcolonialism (Rizvi 2009) and critical race theories (Delgato and Stafanci 2000), opening the door for a consideration of internal and global cosmopolitanism as an empirical reality.

Perhaps as a result of these combined curriculum studies movements in the 1970s and 1980s as well as increasing emphasis on postmodern and poststructural approaches of the 1990s, we see fewer references to systems and institutional dimensions of education, and indeed curriculum as policy itself, in these later North American curriculum turns. Curriculum and related evaluation policy documents are studied in the US; however, this research is often carried out by political scientists or policy scholars, not curriculum scholars. Critical policy studies of US policy has been increasingly relevant with trends including policy appropriation (Sutton and 
Levinson 2001), policy enactment (Braun et al. 2010) and policy networks or assemblage (Koyama 2010).

Moreover, unlike recent educational leadership studies and the many empirically developed leadership approaches or 'theories' (e.g. instructional leadership; transformative leadership) in which curriculum is often an object of leadership influence on organizational or social interactions in schools, curriculum scholars, including those who emphasize cultural studies or critical education studies, are less interested in developing frameworks or approaches emerging from empirical realities of work in various educational systems. Moreover, in curriculum theorizing as cultural critique, we do not see explicit connections with educational ontologies and epistemologies.

In some connection to leadership early on, a few educational leadership scholars explained leadership as innate or internal to leaders and, thus, distinct from organizations. Here leadership is a function of a particular set of personal traits or characteristics, such as dependability, assertiveness, and adaptability (e.g. Stogdill 1948). Trait theories have made a resurgence in the wake of the need for so-called heroic leaders who have internal capability to turn failing schools around; however, such approaches do not theorize the leader as a subject in formation and/or one that invites or provocates others in ways that influence others pedagogically in the midst of their own self-formation. More recently, some scholars (Crow et al. 2016) have examined leadership identity, considering how leaders construct their identities as leaders in relation to how they see and are seen by others. While trait theories and more recent leadership identity scholarship could relate to the ontological dimensions of subjectivity in curriculum theorizing and Bildung, these literature bases never crossed, perhaps due to the differences in approaches to theory and theorizing describe here.

Earlier dialogues with regards to curriculum theory tradition (CT) and the European Didaktik tradition (e.g. Karseth et al. 1997; Gundem and Hopmann 1998; Hopmann et al. 2000) illustrated an implicit consideration of curriculum work as leadership, particularly in relation to issues of planning and forms of authority for content selection. In both classic American and European curriculum theory, answers to curriculum-work were connected to the idea of building systems of public schools with an authoritative agency directing teachers' curriculum work (i.e. implementation) with a written curriculum document (i.e. the syllabus) containing statements of aims, prescribed content (e.g. textbooks) and expected methods (Westbury 2000). The central construct is the school system as an agency for the institutionalized and intentional teaching of a 'content', seen in terms of selection from a subject matter. In terms of Schwab's (1978) curriculum theory triangle, transmission of knowledge occurs through an interaction of the system, student, and subject matter, all of which are contextualized in terms of culture and society. In Germany this approach was developed by Paul Heimann and was carried on in the Nordic countries as theory of school Didaktik (Uljens 1997b).

Educational leadership as system based governance was, however, more evident in Swedish curriculum theory research by Dahllöf (1967) and Lundgren (1972). Curriculum theory was here not understood as theory or principles of teaching 
practice but rather as a framework for understanding how the school system was governed and "how knowledge transmitted in an educational context is selected, valued and organised and how such processes can be understood" (Linné 2015). This research developed later into studying curriculum codes inspired by Basil Bernstein.

Ultimately, in Westbury's analysis, the American curriculum tradition and Didaktik differ substantially according to their focus in the core of teaching, the role of the teacher, and relationships between the individual (subject) and society. Specifically, for the American curriculum, the core (normative) foci of thinking centered, first on calibrating 'programs' to the 'needs' of different students and contexts, and second, on system effectiveness for achieving an appropriate and common learning. Due to the central position of the contents in Didaktik this naturally requires decisions concerning what contents should be chosen. In this process the question of the aims of education cannot be avoided. Therefore, the aims of education are also internally related to the content and methods. The classic didactic triangle focusing the aims, methods and content, describes this unity (Diedrich 1988; von Oettingen 2016).

Despite variations within and between European Didaktik and research on curriculum neither typically do not raise questions of educational leadership. Although the Didaktik tradition views the teaching-studying-learning process in a systems perspective acknowledging and embracing policy, governance and culture, the principal is an absent character. Curriculum theorists and scholars may consider and/or critique the role of leadership in social reproduction; however, they do not explicitly theorize leadership work with regards to curriculum and instruction or the social interactions around these in schools as societal institutions. As we see it, curriculum scholars propose strong intellectual arguments and analytical tools, but their views typically and still offer a normative view, promoting a particular set of values and ideals (Uljens 1997a, 2013; Ylimaki et al. 2016). Moreover, in large part, these strong intellectual arguments and debates have not reached educational leadership discourses, research traditions, and paradigms. That is, many curriculum debates establish the need and open the door for leadership and institutional/organizational analyses, but leadership has not been an area of focus in this literature. Most often, across this recent curriculum literature, leadership is only implicit in topics, such as "planning for new international subjects and curriculum homogenization" (Gough 2001), "accountability-based authority" (Palamidessi and Feldman 2003), and "requirements to mediate tensions between intellectual curriculum work and instrumental curriculum policies" (Green 2001).

For the purposes of our project, we begin with a classical approach and define curriculum practice as the process of formulating aims and selecting contents and reflecting methods, including pedagogical experiences through which content is enacted. Here we recognize an interplay among policies, societal trends/aims, methods, social interactions of leadership-teaching-studying-learning within and between levels, however, curriculum, Didaktik, leadership scholars, and political scientists/policy scholars have yet to make these connections explicit. A critical element is the dialogue between administration and politics/policy. Yet, identifying an 
object of research is not sufficient if the interest is to develop a conceptual language, a theory, through which these practices can be handled. In our view, we need a language to study how curriculum is constructed through mechanisms through initiation, implementation and institutionalization (Hopmann 2003; Lundgren 1972) and how teaching and leadership as activities may be treated coherently. We therefore argue that these processes are dialogical and discursively constructed in varying local contexts. And we argue that we must start with a non-hierarchical perspective. Our intention is to catch the process of curriculum; in the construction and implementation process, there is educational leadership at multiple levels from classrooms to transnational levels. In this sense, curriculum making is educational leadership (Uljens 2014; Uljens and Ylimaki 2015).

\section{Educational Leadership: Theoretical Roots and Empiricism}

In this section, we begin with a brief contextual discussion of the history of educational leadership research in the USA and Europe. We then analyze roots of educational leadership research in organizational theory, critical theories, and, in a few examples, hermeneutics. Here we distinguish between theory and theorizing (assumptions about ontology, epistemology, axiology and relations between individuals and society) as it has been utilized in educational leadership and the disciplines that inform these theoretical roots. This section is followed by a further examination of empirically developed leadership models or approaches that have dominated the educational leadership field with a focus on schools and districts within nation states since the 1970s. We conclude with a review of a growing number of studies that draw on institutional theories to posit leadership as a multi-level phenomenon, shifting the focus beyond schools and districts toward the national and transnational level.

\section{National Contexts for Educational Leadership Research in the USA and Europe}

Empirical leadership research has been predominantly carried out in a NorthAmerican context with its longer history of a stronger accountability orientation, decentralized governance systems, and the need for management of differentiated curriculum expectations. In European research, educational leadership studies (in the way they have been carried out in the US) have been far less prominent; however, scholarly interest has expanded partly along with the ongoing shift towards neoliberal accountability policies, emphasizing new ways of efficacy, excellence, competition and productivity but also due to new transnational evaluation and 
governance systems (Pedersen 2011; Moos 2013; Nordin and Sundberg 2014; Gunter et al. 2016).

In the North American context, educational leadership as a distinct field of research has a long history, from early studies reflecting organizational systems theories, scientific management perspectives on efficiency and productivity, and transactional leadership approaches from the twentieth century to more recent empirically developed models, such as instructional leadership that emerged from the effective schools research (e.g. Edmonds 1979; Hallinger and Murphy 1985), transformational leadership approaches developed in the wake of devolution policies and related site-based management mandates (Leithwood 1994), and transformative or social justice models developed as a reaction to classical systems theories and scientific management approaches as well as the needs of an increasingly diverse student population (Foster 1986; Shields 2010). Most recently, educational leadership scholars have also applied a multi-level perspective on schools as institutions, new (neoliberal) approaches to efficient and effective governance, critiques thereof, and alternative approaches oriented toward social justice.

All of these evolving approaches within the North American leadership tradition are also situated culturally, historically, and politically. For instance, the Coleman Report (1966) posited that student socioeconomic status made a larger contribution to student success that school practices, inspiring a host of studies on US effective "outlier" schools that were successful in educating all students regardless of socioeconomic backgrounds (e.g. Edmonds 1979). Such effective schools studies were also prevalent in the UK in the later 1980s and 1990s (Harris 1992). Similarly, the publication of A Nation at Risk (1983, p. 15) during Ronald Reagan's term in the U.S., accentuated a national perception that American schools were failing as well as a wave of local, state, and federal school reforms. A Nation at Risk foreshadowed a growing shift toward neoliberalism in the U.S. and a reformulation of the individual as responsible for his/her own life as well as societal progress. In that report, we clearly see the American ideology around education as a vehicle for equality of opportunity, individualism, and a collective effort toward an improved democratic society. The report saw that all "regardless of class or economic status are entitled to a fair chance and to the tools for developing their individual power of mind and spirit to the utmost. This promise means that all children by virtue of their own efforts, competently guided, can hope to attains the mature and informed judgement needed to secure gainful employment and to manage their own lives" (National Commission on Excellence in Education 1983, p. 15). In other words, the responsibility for students learning was moved towards the schools. This policy shift was echoing the responses to the earlier Coleman (1966) report that demonstrated that students sociocultural background was a strong predictor for success. However, in comparison, the same observation regarding everybody's right to access to a fair chance, "a school for all", was guiding the social-democratic initiatives in Europe in the 1960s. As we see it, this policy resulted in abandoning a differentiated system with parallel schools, and in the introduction of a comprehensive school equal for all regardless of region, language, religion social class or the like. In this context the 
conclusion was that the state took the responsibility developing schools with an all encompassing curriculum, accepting negative discrimination, i.e. that amount of support was increased to those in need (Blossing et al. 2014).

Effective schools and leadership research accepting local accountability has thus been strongly promoted by U.S. policy initiatives over the past five decades (Coleman Report, A nation at Risk, No Child Left Behind, Race to the Top). We also see the emergence of discourses around improving professional action on all levels through externalized evaluation and accountability ideology. This policy approach has become global (Sahlberg 2015). National reports in the U.K. (e.g. TIMMS) and policies signaled shifts in educational work toward a neoliberal agenda in other countries as noted in Leadership for Learning with members including England and Australia among others (MacBeath 2012).

In the European tradition, principals were to a larger extent civil servants taking care of the administration and management of schools but related to an idea of the school as a societal institution directed by a nation state curriculum. In Europe, with its tradition originating in the idea of education as a public good rather than a private good, schools have been less independent. Thus, the role of the principal as the single pedagogical leader for the school has been less prominent. Rather, we see a growing trend toward shared or collaborative approaches whereby leadership becomes a capacity for school improvement. Regardless of whether instructional/ pedagogical leadership is a single or collective construct, in much of the leadership research, curriculum is an object of leadership work, one that is under theorized.

Despite the variation between European countries in how leadership and governance is practiced and conceptualized, the recent change of the European policies moving from a social-democratic welfare state model towards a more social-liberal state model changed the nature of school governance and leadership. The movement towards performative accountability and educational managerialization consist partly in hopes expressed concerning the role education and innovation could play in post-industrial knowledge economies operating on increasingly globalized markets (Uljens et al. 2013; Gunter et al. 2016). Also, the past 25 years, after the collapse of Eastern Europe and the Berlin wall in 1989, the neo-liberal agenda emphasizing education has increasingly been received as a private rather than public good. New social technologies have become essential for new forms of public governance. These technologies in combination with neo-liberal policies transform complex and contextualized information to outcome indicators. At the heart of the new public governance is the idea of customer choice will boost quality of educational services in the 'competition state' (Cerny 1997; Genschel and Seelkopf 2015; Saari 2012). And while there is growing concern and resistance to the neoliberal agenda in many countries, the governance trends and leadership are evident on a global scale. With this historical and cultural framing in mind, we further consider approaches to educational leadership theories and theorizing that are important for understandings distinctions among educational leadership, curriculum, and didaktik fields. 


\section{Theory Versus Theorizing: Disconnected Roots of Leadership Studies}

Curriculum theorizing and Didaktik (described in the last section) and leadership theories have influenced each other over time but there are clear conceptual distinctions. More specifically, curriculum/Didaktik scholars frequently take a different approach to theorizing than we see in much of the educational leadership field, and these distinctions in theorizing traditions explain some of the differences between the educational leadership and curriculum fields as well as the difficulties in merging them.

Prior to the 1970s, there was an elaborated discourse about organizational theory that informed empirical educational leadership research; however our impression is that the connection has been weakened over the past three decades. Thus, our intention in the following is to return but expand on the theoretical base in order to retheorize educational leadership (Uljens and Ylimaki 2015; Ylimaki and Uljens 2017).

Social theories, including organizational theories and critical theories so prevalent in framing educational leadership studies, are based upon a philosophy of science and a theory of society, considering ontology, epistemology, and axiology, all of which are related to methodological assumptions as well as assumptions about human nature (relationships between the individual and his/her environment). In other words, social scientists approach their subject (e.g. organizations) via explicit or implicit assumptions about the nature of the social world and the way in which it may be investigated. To begin, assumptions of an ontological nature concern the very essence of the reality in general, such as whether the 'reality' to be explained is external to the individual, imposing itself on individual consciousness, or the product of individual consciousness (Burrell and Morgan 2003). Further, social theories are based upon a second set of assumptions of an epistemological nature, about how one might begin to understand the world and communicate this knowledge to others. As examples, we can distinguish between rational organizational theories as those theories that conceptualize organizations as material realities that can be observed objectively from the outside through a singular view of Truth and those that consider organizations as socially constructed based upon a vision of reality which holds no greater truth than alternate views. Here so-called natural organizational theorists seek to understand different individual realities in order to make generalized statements and provide a link between social experience and reality. In a third category, many organizational theories conceive of organizations as open systems that combine both rational and natural system assumptions about reality. Closely related, an axiological dimension of organizational theories whereby the 'organization' identifies its internal valuing systems and the ways in which values and ethics influence its decisions and actions.

The emphasis on how we view and come to know organizational reality and values is, of course, very relevant for leadership in schools and districts/municipalities as educational organizations/societal institutions. The poststructuralists remind 
us, however, that a dichotomy or even a continuum of what is true and what is false presumes a certain epistemological stance and indeed challenges the very notion of a paradigm. We further argue that organizational ontology, values, and assumptions about how we come to know these cannot be conflated with educational ontology, epistemology and axiology as in modern education theory and related assumptions about the Other, freedom, and the role of education. We will return to this point in more broadly in our presentation of non-affirmative education theory.

Closely related to all of these assumptions about human nature are the relationships between human beings and their environments and between schools/education and society. That is, all social theories are predicated upon a view of how humans individually and, in this case, within organizations like schools, respond to their environment (as an instrument and subordinate to society or as one with agency and superordinate to society). In one key relation within organizations, many theorists make references to 'authority' and 'management' with early descriptions of authority often informed by scientific management (Fayol 1949; Taylor 1947) practices aimed at productivity and formal hierarchical organizations of modern society in bureaucracy (Weber 1978). The word 'authority' is important here as it gave rise to early conceptions of transactional leadership in classical educational administration literature and management theory grounded in functionalist organizational theory and positivistic research approaches (Bass and Avolio 1990; Forsyth and Hoy 1978; Leithwood 1994) that have been applied to principals organization and management of teachers learning and effective classroom practices. Here authority can be given and taken away; managers and followers can make transactions or deals to accomplish organizational goals, and manager-worker relationships are connected to transactions. Here an organizational theory-based directs leadership focus to system elements (e.g. staff learning in relation to other system elements) rather than education as defined by education theory (Benner 1991). Further, we do not see the use of the term transforming leadership (Burns 1978) or transformational leadership; rather Leithwood (1994) and others (Avolio et al. 1999) developed these models from a combination of organizational theories and empirical generalizations or models. We will further describe these empirical generalizations as we explore the empirical turn in the educational leadership field in the next section. Although not always explicitly articulated in educational leadership studies, assumptions about knowledge, reality, and values have important consequences for the ways in which researchers attempt to study and obtain knowledge about the social world, including leaders and education (with curriculum/Didaktik as key aspects of it) in schools.

We also recognize that functionalist organizational theory, related systems theory, positivism, and their inherent assumptions are also grounded in a long history of functionalist sociology, and we do not go into full detail on the roots here. Briefly, elements of functionalist sociology can be traced to the Greeks and in many analyses to Comte, Spencer, Durkheim, Pareto, Simmel, Weber, and Mead. Because most references to organizational theory informing educational studies of schools/districts, and leadership therein date back to various systems theories (Mead 1967; Merton 1968; Follett 1918; Simon 1976; Weick 1976), we use examples from these latter theories frequently cited in leadership literature to illustrate our arguments. 
According to Merton (1968), organizational theory approximates interrelationships between the external environment and the organization; it is firmly rooted in the sociology of regulation and approaches ontology in terms of assumptions about reality of the system and its relations to organizations (educational and otherwise). Ontologically, then, from Merton's (1968) perspective, the organization exists as an observable reality with concrete boundaries within the larger societal/cultural/political environment, which corresponds to a version of representational epistemology. The environment influences organizational outcomes by imposing constraints and demanding adaptation at the price of survival, all of which affect the organizational behaviors, values and ethics, and the assumptions under which social scientists understand and communicate organizational behaviors.

In a related strand of organizational theory, drawing on Simmel and Mead, Follett (1918) theorized organizations in relation to understanding how social groups organize as interpreted by the social actors who are actually engaged in the experience; that is in terms of interpretation as the mode by which we live and interact with others in social organizations. Importantly, she acknowledges different individual uses of power operating within social interactions, gesturing closer toward an ontological dimensions of individual human relations. Follett's (1918) theorizing is important as she opens the door for the human relations approach and later scholarship. Here we also see some influence of movements such as phenomenology and hermeneutics; however, in the wake of Follett's (1918) theorizing, there is largely a white spot with regards to these perspectives in educational leadership studies. Further, we also argue that Follett does not consider ontology in terms of individuals' assumptions about the Other's freedom and intersubjective relations as educational problems. In the 1940s, other leadership scholars explained leadership work beyond organizational and societal functions; leadership is innate or internal to leaders. Here leadership is a function of a particular set of personal traits or characteristics, such as dependability, assertiveness, adaptability (e.g. Stogdill 1948), an approach that has regained popularity in recent studies of heroic leadership in turnaround schools (Drysdale et al. 2014).

Merton and Simmel among others also make distinctions among macro, disciplinary theory of societies (and organizations within them) and models of organizations developed from empiricism, even when these empirical models are framed by macro, disciplinary theory. Empirically developed forms or models are later subject to further empirical testing, but such testing does not make an empirical model a theory nor, according to Weick (1976), does such testing equate with the process of developing theory (i.e. theorizing). Here Weick (1976) echoes Merton and Simmel in arguing that theorizing is, of course, important but the ultimate goal in organizational theory is to produce a theory.

Critical social theorists, whose work heavily influenced contemporary educational leadership studies of social justice and culturally responsive leadership practices, have similar goals for theorizing (to produce a theory) but here the aim is to change society. Critical intellectual traditions and perspectives traced back to the tenets of German idealism and complex ontological views of the world as a product of individual consciousness or radical humanism as well as an objectification of the 
social world or radical structuralism (two views representing epistemological breaks of Marx).

More specifically, critical theorists' epistemological beliefs are largely based upon critique; the best way to know something is through criticizing it. Critical social theorists, thus, share a sociocultural transformation perspective on relations between the individual, systems (e.g. schools) and the environment with differences in ontological and epistemological positions. Critical theories, albeit through different epistemological perspectives, seek to explain social (dis)order and social change, differing largely in terms of whether the world is an objective reality (radical structuralism) or a subjective, constructed reality (radical humanism). Further, the axiology of critical theory stresses the importance of being upfront with values; values should guide research and theorists should strive to change the social construction that is the subject of their critique. From what has been referred to paradigmatically as radical humanism (Burrell and Morgan 2003), objectifications encountered in the social world are humanly created, pointing the way toward an emancipatory philosophy which stresses how individuals, through self-consciousness, could create and thus change the society in which he/she lives. In essence, the work of theorists located within the radical humanist paradigm (as it relates to education including curriculum/Didaktik and leadership), there is an aim toward setting human consciousness free and, thus, facilitating the growth and development of human potentialities.

In later works, beginning with The German Ideology, Marx (1946) moved away from the idealist perspective to one rooted in a more realist interpretation of the nature of the social world in what some paradigmatically refer to as the epistemological break toward radical structuralism (Althusser 1970/2006). Radical structuralism holds that modern social structures (and conflicts between them) exist in an external reality and can be investigated objectively. In other words, clear, observable superstructures legitimize power and domination such that radical social change can only be achieved through conflict (defined as crisis and revolution), placing much more emphasis on deep economic and political structures in their theories. Radical structuralism, then, is a view which focuses upon the essentially conflictual nature of social interactions and the fundamental process of social change this generates. It is a sociology of radical change, but in contrast to radical humanism, radical structuralism tends to place relatively little direct emphasis on 'man' as an individual human being. Importantly for some educational leadership scholars, in the context of radical structuralism, including the so-called radical Weberians (e.g. Rex, Dahrendorf), we see a focus on bureaucracy, power, conflict, and authority as points of concentration for theoretical analysis as a means of understanding important aspects of social life. Much of the most recent leadership literature, however, reflects empirically-developed theories more than theorizing associated with social theories that explain relations between organizations and society or the nature of societal change. 


\section{Social Reproduction and Social Transformation}

In the next two sub-sections, we further consider assumptions about relationships between the individual and his/her environment that have dominated macro social theories over time, including those that have informed organizational theories and critical theories that are often used to frame empirical leadership research: (a) social reproduction - schools should operate within society to transmit uniform knowledge and values to future generations who will inherit a largely unchanged society or (b) social transformation - schools should operate in a distinct role apart from society to prepare individuals to transform existing society and social values.

Social reproduction hinges on a certain set of shared fundamental ideals that are determined 'from above' in the form of ultimate values and also 'from below' in the form of more or less institutionalized objectives that individuals internalize as goals for their lives (Parsons 1963). Social reproduction perspectives often embrace a classic notion of subjectivity that is normatively tied to the idea of individual self-determination.

Bourdieu (2011) explains how reproduction occurs when individuals internalize the social world through habitus or a pattern of perceptions formed through mental structures, such as institutionalized values and objectives (Bourdieu 2011). More specifically, habitus is a type of socialization or "social conditioning" that predicates our class-based tastes, classifications, values, and choices. As such, "the habitus implies normative integration and a 'sense of one's place' but also a 'sense of the other's place, providing a "world of common sense, a social world which seems self-evident" (p. 132). Bourdieu (2011) further argues that education itself is an agent of cultural reproduction, which occurs primarily through the hidden curriculum or socialization; students acquire appropriate attitudes and values needed to further succeed within the confines of education. Thus education provides functional prerequisites whereby education provides individuals with necessary values and attitudes for future work. Regardless of the trade an individual participates, they will all need a similar set of social skills for their everyday interactions. In other words, education is an ideological state apparatus (Bowles and Gintis 1976), sorting individuals and assigning them the skills necessary to fulfill their destined occupation. Those students with high levels of accumulated social and cultural capital from parents or other sources are more easily able to excel within the system of education.

Social transformation perspectives date back to Rousseau (1762), who argued that education should not replicate problematic aspects of a less than ideal society; rather, education should promote a new ideal that does not yet exist. In other words, education would work toward an idealized more socially just future by educating a new generation of citizens entering society with social justice values. Social transformation theories critique the status quo and seek to explain, either through "subjective" (e.g., Lukacs; Frankfurt School) or "objective" (e.g., Althusser) ontologies, how radical change occurs in society. 
The above distinction (social reproduction versus social transformation) also resonates with the classical Aristotelian division between two modes of human action, techné and praxis in our time developed in Habermas' early works. The many versions of functional-instrumental approaches in educational leadership studies typically ask for efficiency - how should the work be rationally organized to reach intended aims? Overemphasizing rationality leads to a hiding of normative issues. Leadership is assumed to be adaptive to existing needs and ideals while ethics and politics dissolves. Today's educational governance initiatives operating over and across different levels operate along a logics of rational choice of means that work, both from the systems, organizational, and actor perspective. Yet few (empirical) leadership studies refer directly to these roots of theory and underlying assumptions (reproduction or transformation). regarding relations between individuals and societies.

\section{The Empirical Turn}

In the 1970s, the educational leadership field took an empirical turn in the North American context, resulting in a preponderance of empirically-developed models or approaches (e.g. instructional leadership, transformative leadership). Much of the empirical work was conducted at the nation state level; however, a growing number of international or transnational projects are evident. Across this literature, we argue that empirical leadership research is a bit free floating from theoretical roots. In our project, we seek to (re)theorize the educational leadership field with research closer to the roots.

In the 1970s, empirical approaches were primarily conducted from technologicalinstrumental or reformational perspective. Often a rationalist, functionalist and systems oriented thinking guided this research. Also the degree to which this research is empirically descriptive or guided by more or less explicit theoretical assumptions varies. Functionalist-empiricist research is often quantitative in nature, but equally often qualitative and interpretative. More recent empirical studies take a critical, transformation or social justice oriented position framed by various critical theories and earlier seminal research studies. Critical, transformation oriented research is often qualitative in nature.

\section{Systems-Theoretical and Functionalist Research into Educational Leadership}

Classical leadership literature has been firmly grounded in structural functionalism as it has informed classical organizational systems theories: (1) the rational systems theories and related scientific management principles of efficiency and effectiveness 
(e.g. Fayol 1949; Taylor 1911); (2) the human relations or natural systems theories (McGregor 1960) and (3) the open systems theories (e.g. Perrow 1970; Callahan 1962) merging both.

Such functionalist and reproduction oriented approaches may also include a larger perspective, looking at relations between organizations and broader social systems as is done in organizational theory (e.g. Fayol 1949; Simon 1976) interactions between levels of governance as well as interactions between different stakeholders as is done in institutionalist research. In using the term 'structural functionalism', we draw on Parsons and others who explain structural functionalism with regards to organizations in that organizational activities, including curriculum management and instructional supervision, are defined by social regulation and order at the micro level. In other words, behaviors within organizations (rational, natural, or open systems) are linked to the controlled functioning of social behavior and interactions within organizations. To promote labor efficiency and control behavior, rational systems utilize hierarchical structures that create clear divisions of labor and clear managerial spans of control (Taylor 1911); labor is expected to have specialized expertise in performing a function within the organization where all functions are broken down into their most basic components for effective and efficient completion (Gulick 1937). By contrast, the natural systems model views organizations as organisms emphasizing the perspectives of individuals as members of social groups with valuable input to the organization; relationships are valued above all other aspects of the organization (Metcalf 1942; Follett et al. 1942; Hoy and Miskel 2005). Systems perspectives have evolved over time in relation to historical, cultural and policy trends of particular times.

We spend a bit more time in a discussion of systems theories, including particularly open systems theory that represents a merger of scientific management/rational systems theory, bureaucratic theory, and natural systems theory. Taylor's scientific management theory proved successful in industrialized companies at the turn of the century but has not been utilized successfully in many modern companies as its philosophy of production over people has left a legacy of declining quality and work satisfaction as well as a loss of organizational pride. Weber (1947) expanded on Taylor's theories with a emphasis on the need to diverse ambiguity with clear lines of authority and a hierarchical structure of power in bureaucracies. Closely related, rational administrative theory (Fayol 1949) emphasized a universal set of management principles for all organizations. Such classical theories were rigid and mechanistic, shortcomings that quickly became apparent and gave rise to natural systems theory with its emphasis on relationships and human needs/motivations (Follett 1949). Natural systems theories were supported by several experiments of the time, including the now famous Hawthorne Studies in the Western Electric Plant (Mayo 1933) whereby the act of paying attention to employees in a nonthreatening way was more effective for productivity than manipulating the work environment. Simon (1991) made an important contribution to the study of organizations when he proposed a model of "limited rationality" to explain the Hawthorne experiments, arguing that workers could respond unpredictably to managerial attention and that external influences have a major effect on internal organizational 
behavior. Simon's insight was important to later articulations of open systems theory insisting on the importance of the environment and its impact on the organization (e.g. Perrow, Weick). Early open systems theories were subject to critique, including the lack of explicit attention to social interactions; however, these gaps were addressed by Rogers (2010) with a network analysis approach, Habermas (1984) with a communicative approach, and by Weick (1995) with a sensemaking approach that reminds of hermeneutics and phenomenology. We find Habermas (1984) and Weick (1995) particularly relevant as they argued that consensual meaning is created through iterative and adaptive communication (e.g. rational discourse, double interacts), referring to social norms and values. Across these open systems theories, Hoy and Miskel (2005) identified inputs, outputs, feedback and transformations of organizations in relation to schools as open social systems, giving more explicit attention to the importance of internal culture to social interactions, but their application gave little attention to educational activity.

For our purposes here, regardless of the particular approach (e.g. rational, natural, open), organizational theorists consider curriculum as an object of management; administrators must manage curriculum work in state, district and school systems, bringing disparate efforts toward a clear goal or outcome (Fayol 1949; Callahan 1962). While not explicit, organizational theorists (e.g. Callahan, Merton, Senge, Simon) understand education as located within existing society, socializing students into the values and norms of existing society, and thus, helping to maintain social regulation. For our purposes in making an argument for education theory, it is also important to note that organizational theorists (e.g. Fayol, Merton, Simon, Weick) and those drawing on organizational theory to study educational leadership (e.g. Bass and Avolio 1990; Hallinger and Murphy 1985; Leithwood 1994) have written in varying ways about the distinctions between "theory" and "theorizing". In the early North American educational leadership field as it developed in the 1940s-1960s, organizational theorists dominated this debate. According to Merton (1968), organizational theory approximates an explanation about interrelationships of a societal condition (i.e. organizations). He goes on to make a distinction between macro, disciplinary theory of societies and organizations and empirically developed forms or approaches to organizations, often distinguished by a particular aim or style. Empirically developed forms or approaches are later subject to further empirical testing, but even numerous empirical testing does not make for theorizing nor, according to Weick (1976), is the process of developing theory. And while rational (closed) organizational theory perspectives on educational leadership studies have been enhanced with open systems theory and, more recently, with institutional theory (Dimaggio and Powell 1991; Meyer and Rowan 1977, 2006) and critiqued with various critical/normative social theories (e.g. Apple 1992), the theoretical logics applied in empirical studies of educational leadership remain largely the same.

At the same time, we argue that much of the educational leadership theory development since the 1970s has been empirically-based, framed in seminal leadership studies that were explicitly grounded in structural functionalist perspectives and organizational theories. In two classical examples, Bass (1985) and then Leithwood 
(1994) drew on organizational theory, psychology, and as well as Burns' (1978) empirically developed theory of 'transforming' leaders to frame their empirical studies of school leaders' behaviors. Bass (1985) extended Burns' (1978) transforming leadership but used the term transformational, explaining the psychological mechanisms that underlie transformational leadership work in school organizations as open systems (Forsyth and Hoy 1978). Bass's (1985) aim was to measure transformational leadership in terms of influence on followers (we will come back to how influence is defined here in the next section). Bass' findings indicated that the followers of such a leader feel trust, admiration, loyalty and respect for the leader and because of the qualities, are willing to work harder than originally expected with leadership practices organized in four elements: individualized consideration, intellectual stimulation, inspirational motivation, and idealized influence. Later in the article, Bass (1985) clearly noted that the function of leaders and teachers revolved around school improvement in order to meet existing societal needs. Many others have followed Bass' example, drawing on functionalist organizational theory as well as Bass' empirical methods and tools to verify the use of transformational leadership with its four elements (e.g. Leithwood 1994).

In probably the most frequently cited example, Leithwood (1994) further studied Bass' (1985) transformational leadership elements with his own research of principals in effective schools. He later conducted a meta-analysis (with Riehl 2003) of additional effective leadership studies and a North American research project (with Seashore 2011), indicating that four leadership practices were necessary but not sufficient for effectiveness in any context: setting directions, developing people, redesigning the organization, and managing the instructional program. Leithwood and Seashore (2011) concluded, based upon a mixed methods study with a large North American sample, the effects of leadership influence on student outcomes is indirect. Teaching or instruction and related classroom practices have a direct causal effect. Further, while these and similar research studies have been widely cited, we argue that these transformational leadership approaches have become free floating from the original roots (organizational theory, political science, and psychology).

We can make similar examples from other empirically based leadership approaches or forms. A wide body of research examined principals' practices in socalled "effective" schools makes this point as these studies gave rise to instructional' leadership approaches. Effective schools were those that were successful with teaching all children regardless of SES, an important topic in the wake of the Coleman Report that concluded school-based poverty concentrations had far more impact on student achievement than school-based efforts (e.g. teaching, curriculum, culture). These effective schools studies most often considered schools as open systems with similar correlates of effectiveness, including safe and orderly environments, management of the curriculum, instructional leadership, and frequent monitoring of student progress (e.g. Edmonds 1979; Lezotte 1986; Purkey and Smith 1983). In conceptualizing effective schools as "open systems", Lezotte and others argued that the school organization always maintains effectiveness and efficiency through its formal structures, functions, and objects of these while concurrently acknowledging and serving the various aspects of the external circumstances 
(Hoy and Miskel 2005). Effective schools research led to the formulation of so-called instructional leadership approaches (e.g. Berman and McLaughlin 1976; Edmonds 1979; Hallinger and Murphy 1985; Lezotte 1979).

While recognizing the role of input from the external environment, according to Edmonds (1979) and other effective schools researchers, effective leaders influence followers' performance of various organizational tasks, including curriculum management and instructional supervision, all of which indirectly affect 'outputs'. Such leadership studies emphasize instructional leadership as the role of the principal in educational organizations (schools), including practices like supervision that affect classroom improvements, and indirectly affect student outcomes (e.g. Caldwell 1998; Edmonds 1979; Berman and McLaughlin 1976; Hallinger and Murphy 1985; Harris 1992). Across this literature, instructional leadership is an individual and often directive construct (Hallinger 2004).

In another frequently cited example specific to instructional practice, Hallinger and Murphy (1985) constructed an 'instructional' leadership model from earlier effective schools research (Edmonds 1979) and then tested that model with mixed methods on so-called outlier schools that exceeded expectations in neighborhoods with few resources. In this literature of the 1980s, it is important to note that relatively little reference was made to teachers, department heads or assistant principals as instructional leaders; there was little emphasis on instructional leadership as a distributed construct or a function to be shared. Instructional leaders were described as strong, directive leaders who had been successful in "turning their schools around" (Edmonds 1979; Hallinger and Murphy 1985); relatively few case examples of instructional leaders were conducted in schools with average or high performance. Drawing on results across this literature as well as their own empirical findings using survey methods, Hallinger and Murphy (1985) identified three dimensions of instructional leadership: (1) defining the school's mission, (2) managing the instructional program, and (3) promoting a positive school learning climate delineated into ten instructional leadership functions, including framing the school goals, supervising and evaluating instruction, coordinating curriculum, monitoring student progress, protecting instructional time, providing professional development, maintaining high visibility, providing incentives for teachers, and providing incentives for learning. While Hallinger and Murphy (1985) instructional leadership model has been widely tested and utilized worldwide since the 1980s, we argue that this model is grounded in empiricism and a language of the organization, and administrative functions therein, with little language of pedagogy, education or an educational ontology.

Another more collaborative leadership approach considers principals' instructional leadership in relation to other informal leaders and their social interactions within schools (e.g. Marks and Printy 2003; Jackson 2000; Hallinger 2004), defining instructional leadership as a shared capacity for school effectiveness improvement. Here the principal models appropriate leadership behaviors and invites teachers and others to join their efforts toward school improvement (e.g. Shephard 1996; Jackson 2000; Marks and Printy 2003; Hallinger 2004). Like earlier effective leadership studies that focused on individual principals, these scholars emphasized 
leadership roles in school changes/improvements but defined leadership more broadly (teachers as well as head teacher). Across these studies, we find quantitative methods, with regressions from findings from Hallinger's (2004) survey, mixed methods as in Marks and Printy's (2003) study of leadership in communities of practice or qualitative approaches with case studies of instructional leaders' collaborative approaches (e.g. Jackson 2000). With the growing pressures of external evaluation systems (e.g. PISA), North American and European scholars have renewed or focused more attention on instructional leadership approaches, leadership in professional learning communities, and teacher leadership (York-Barr and Duke 2004).

There is also a growing empirical literature in many countries on leadership in professional learning communities, learning organizations, or communities of practice (e.g. DuFour and Eaker 1998; Hord 2004; Silins and Mulford 2002). Although there is some variation, such approaches seek to understand how administrators, teachers, and others build capacity in schools to impact student achievement. While not explicitly connecting to natural systems approaches (Follett; MacGregor) much less Dewey's $(1916,1938)$ perspectives on growth and democracy in schools, DuFour and Eaker (1998) define a professional learning community as educators creating an environment that fosters mutual cooperation, emotional support, and personal growth as they work together to achieve what they cannot accomplish alone. Senge (1990) approach to learning organizations follows a similar conceptualization but adds an emphasis on organizational theory and adult learning in various disciplines. Across the above studies we see an empirical emphasis on knowledge constructed in professional learning communities. Lick and Murphy (2004) draw on Vygotsky and add an emphasis on staff development in collaborative teams and their use of study group plans focused on curriculum, instruction, and classroom assessment. Across this literature, argue that studies of professional learning organizations is useful as it connects leadership with curriculum and pedagogy; however, we also see much of this literature as free floating from Bildung and a theory of education.

More recently, still considering organizational elements of instructional leadership interactions but adding social capital theory, other scholars (e.g. Kelley and Halverson 2012; Klar 2012) have considered instructional leadership as a distributed construct, drawing on Spillane's (2002) research to consider how social networks affect such objects as curriculum and instruction. A distributive view of leadership recognizes that leading schools can involve multiple individuals in addition to the school principal_-"the leader-plus aspect"-and that leading a school is fundamentally concerned with social interactions around curriculum/instruction and other important objects of group leadership influence rather than about the actions of individual leaders- "the practice aspect" (Spillane and Healey 2010). According to Harris (2007), this view of distributed leadership also rests on an idea of leadership as cognition as well as an idea of leadership as mediating social networks and the capital needed to develop these. Thus, the democratic features are specifically taken into consideration, and they have also been the focus of several studies on educational leadership within the Nordic context (Moos et al. 2004; Moller 2009a). While perhaps not intentional, many of the recommendations from 
an organizational perspective (transformational, instructional, collaborative or distributed) on schools result in a technical or even instrumental approach, recommending leaders utilize a particular set of practices in order for educational organizations to develop effectively.

Essentially, research studies grounded in these organizational systems theories and more or less structural functionalist perspectives primarily focus on concrete relationships (rational, natural or open) among administrative functions, behaviors, social relationships or interactions, objects of these functions or behaviors and organizational effectiveness (e.g. Barnard 1968; Roethlisberger et al. 1943; Simon 1976). Such areas of focus are not surprising given the historical timeframe and the background of the seminal scholars (i.e. sociological perspectives on systems and later management practices associated with systems productivity).

\section{Critical, Transformative Oriented Approaches}

Educational administration scholars who conduct critical, transformative oriented empirical research draw support from Marx, Althusser, Freire, Gramsci, and Habermas as well as extant research studies devoted to explaining relationships between inequitable societal structures and education and/or the effects of various 'isms on leaders' work and the leaders themselves (e.g. feminist theories in women's leadership studies from Brunner 1998; Grogan 1996; Lumby 2008), racism from Gooden 2012; Tillman 2004). Drawing on radical humanist and radical structuralist versions of critical theory (e.g. Freire, Gramsci, Marx, Rex), Foster (1986) challenged classical educational leadership/management studies grounded in functionalist organizational theory. In Foster's (1986) argument, for instance, leadership must be critically 'educative', meaning leadership cannot only look at the conditions in which we live but it must also decide how to change them. Foster (1986) challenged the organizational ontology and values inherent in classical educational leadership literature, arguing that leaders needed to more explicitly understand the impact of macro policies and societal conditions on schools. Many leadership scholars have extended Foster's approach in various empirical studies with a transformation aim (e.g. McKenzie et al. 2008; Shields 2010; Theoharis 2009).

Although not always explicit, across this literature, leaders focus on education as a vehicle to improve the lives of all children, including the use of critical pedagogy to support development of a critical consciousness and social justice values (e.g. Brooks 2012; McKenzie et al. 2008; Shields 2014; Theoharis 2007). Scholars who focus on implementation of more revolutionary teaching methods aimed at radical social change through the creation of inclusive practices (e.g. Scheurich 1998; Shields 2014; Theoharis 2007) and critical pedagogy in schools (e.g. Boske 2014). Indeed, as Uljens and Ylimaki (2015) as well as Ylimaki and Henderson (2016) note, the educational leadership field has been recently dominated by various models and approaches to social justice leadership with a clear set of critical values and aims, such as the development of students who critique hegemonic ideologies, 
recognize market forces, work to eliminate injustices marked by various - isms (feminism, racism, classism among others). Yet as we argued earlier, teaching methods (revolutionary or otherwise) and student learning about critical perspectives are only parts of education. Closely related, some scholars devote more specific attention to the relevance of traditionally marginalized students' identities and home cultures for teaching, learning, and leadership (e.g. Capper 1993; Cooper 2009; Dillard 2000; Johnson 2006; Moller 2009a, b). We return to a discussion of culturally responsive leadership later in this chapter.

Taking a radical structuralist perspective on recent policy and related societal trends, other leadership scholars have examined the ways in which neoliberal discourses (e.g. modernizing the economy with educational competition in an open education market) and neoconservative discourses have the social life and interactions in schools (e.g. Anderson 2009; Apple 2008; Pedroni 2007; Ylimaki 2012). For example, Anderson (2009) takes a critical education perspective on schools as "sites of struggle over material and cultural resources and ideological commitments" (p. 38). Anderson (2009), thus, proposes advocacy leadership as a way for leaders to mediate between neoliberal and neoconservative ideologies and the pressures of these on what we teach and how we lead in an era of educational externalized accountability. Similarly, Ylimaki $(2011,2012)$ proposed critical curriculum leadership as a mediational approach between hegemonic social formations and progressive curriculum aims and values in schools.

Also transformative leadership forms stand in contrast to more functionalist views of transformational leadership, and transactional leadership approaches. Similarly, other scholars (e.g., Ryan 2003; Shields 2010) have indicated the importance of inclusive, transformative practices to effective educational leadership in diverse school contexts. Drawing on Freire (2000) and qualitative case study findings, Shields (2010), for instance, argued that transformative leadership begins with questions of justice and democracy, critiques inequitable practices, and offers the promise not only of greater individual achievement but of a better life lived in common with others. Distinguishing transformative leadership from transformational leadership, she argued that "transformative leadership holds the most promise and potential to meet both the academic and the social justice needs of complex, diverse, and beleaguered education systems" (p. 562). Shields also clearly acknowledged the challenges of transformative leadership work in diverse school contexts, including deficit thinking. Taking a similar approach $\mathrm{n}$ a specific 4-year examination of school leaders' perceptions of racism in diverse schools, Ryan (2003) explored the extent to which principals believed that racism existed in their schools and the ways in which they understood it. Specifically, Ryan (2003) found that many principals were reluctant to acknowledge that racism occurred in their schools. Those administrators who acknowledged the presence of racism in their schools identified situations of harassment, graffiti, and other similar forms of representation. In essence, then, the principals saw racism primarily in terms of individual actions or isolated incidents.

In such transformative or leadership for social justice models or theories, now dominant in the educational administration field, then, leaders are identified as an 
important counter hegemonic force in developing students through education, or critical pedagogy to critique and transform existing social structures and values, generally either through awakening an internal critical consciousness or recognizing the importance of conflict to radical social change. A number of scholars (e.g., Johnson 2007; McKenzie et al. 2008; Theoharis 2007) have examined leaders with a social justice orientation, identifying ideals for leaders, such as the importance of a critical perspective, self-awareness, ideological clarity, and inclusive pedagogical practices. Across this literature, scholars most often promote a future more equitable and ideal through school-based inclusive practices, attention to academic achievement, support for traditionally marginalized groups, and community activism with solutions grounded in previous empirical studies as well as various critical theories. In our analysis, we also note that transformative approaches to educational leadership or leadership for social justice so prevalent in the last three decades are increasingly grounded in previous empirically developed models and theoretical or conceptual frameworks more than the roots of critical theory.

Consider, for example, Capper et al.' (2000) study of principals' practices that influenced more inclusive democratic schooling for traditionally marginalized students. Capper drew on Foster's (1986) approach to educative leadership as well as Aronowitz and Giroux's (1985) notion of a transformative intellectual building on Gramsci's (1996) organic intellectuals. Drawing on her own empirical findings, Capper proposed an inclusive, transformative intellectual approach to leadership where leaders mediate between children's needs and structural inequities that affect schooling. Theoharis (2007) drew on Capper as well as Giroux and McLaren to frame his examination of principals' struggles and resistance practices to influence teachers, students, and parents to adopt an equity oriented agenda. Here influence is defined in critical terms as practices that develop teachers' and students' critical consciousness and agency aimed at social transformation. Other scholars (e.g. Bogotch 2002; Brooks et al. 2008) have similarly framed social justice leadership studies with a combination of previous transformative leadership models and their own empirical findings. Critical perspectives on educative influence notwithstanding, these social justice leadership approaches include principals' pedagogical influence on teachers' use of inclusive classroom practices or critical pedagogy as well as resistance strategies.

Most recently, a number of educational leadership scholars have studied leadership preparation programs with an explicit social justice mission to prepare leaders who can organize schools around inclusive principles and who can, if necessary, work through resistance to critically oriented changes, including critical pedagogy. Here scholars examine teaching methods in universities that have affected aspiring and seated principals to enact social justice ideals in their schools. In one example study with a clear expressed purpose for preparing social justice oriented leaders, Boske (2014) promoted the use of Freire's problem-posing cases and other related experiences to help leadership students develop a critical consciousness regarding various social injustices. Boske (2014) defined her course aim as social justice, identified relevant content from the literature on social justice leadership, and described educational experiences used to help students develop a critical 
consciousness about social injustice of all kinds. In other words, social justice is the educational purpose that the leadership program seeks to attain and educational experiences feature problem-posing cases and dialogue around social inequities. She used qualitative methods to assess the extent to which students' developed a critical consciousness and indicated intentions to seek social change. Here we can see Boske gesturing toward curriculum understandings that recognize the subject (leadership) formation process occurring through content and experiences. That is, Boske draws on Freire's work on critical consciousness development that students must read literature like Freire that help them become social justice leaders. She does not, however, specify an educational ontology in the importance of leaders' formation, being and becoming, which Dewey (2008) refers to as the heart of democratic living or that which Pinar (2004) maintains as essential to curriculum theorizing. Others have documented similar leadership preparation processes aimed at developing social justice oriented leaders who seek equity and excellence in schools (e.g. Brunner et al. 2006; Miller and Martin 2015). Recent syntheses of social justice leadership literature (e.g. Bogotch 2002; McKenzie et al. 2008; Ryan 2006), teaching practices focus on future opportunities for student success. It is never enough to merely transmit existing knowledge to students (Bogotch et al. in press). Rather, pedagogical practices must focus on creating new knowledge, new spaces for pedagogical practice, fighting and overcoming resistance and barriers (Ryan 2006; Theoharis 2007). Here social justice is an object to be realized after educators teach students how to attain social transformation by changing the material conditions of students, parents, colleagues, and community members (Bogotch et al. in press).

Regardless of underlying views of reality, epistemologies, and specific social justice applications, when applied to education and leadership thereof, we also argue that critical theory perspectives share a perspective that education is superordinate to society. In other words, education should promote a new generation of student or citizens (or educational leaders) who enter society (educational organizations/institutions) with critical theory informed aims and social justice values. Applied is a deliberate word choice here as our review of this literature indicated use of various critical theories combined with previous empirical studies that used the same theories. Thus, while critical leadership approaches contribute many understandings about how leaders can support radical social change and promote culturally diverse student identities in education/curriculum work, in our view, critically oriented scholars often take a position on a pre-determined vision of an ideal future society, one that is also sometimes a bit free floating from a critical theory base. Further, critical scholarship in educational leadership is often normative, promoting ideals, norms, and values that are decided upon in advance but is not explicitly theorizing in the ways we see in curriculum studies (critical education specifically) or education theory. Here we do not mean to minimize the importance of critical perspectives and an emphasis on identity. Rather we argue that the strong normative position taking on a future ideal solution does not support education in a democratic society. This is why a non-affirmative education theory perspective is so important. We see some scholars gesture toward a 
non-affirmative perspective in interpretive or hermeneutic approaches; however, these scholars do not focus on education theory as framed by Benner (1991) and precursors.

\section{Interpretive or Hermeneutic Approaches}

A third interpretative or hermeneutic approach may also be identified. We are then talking about research that is culturally and historically sensitive and oriented towards understanding the complexities involved in educational leadership practices. While functionalist-empiricist approaches are often guided by an instrumental knowledge interest, i.e. improving practices and collegial cooperation ultimately enhancing student learning, by utilizing research generating testable general explanations, this does not satisfy interpretative leadership research. Here interest is rather on studies accepting a multitude of dimensions operating in parallel.

Yet, interpretive leadership research may be equally strategic and instrumental, for example, by aiming at finding out the most efficient or successful practices. Such interpretative or hermeneutic, and sometimes phenomenological, approaches represent typically either an ideologically critical, a more neutral humanistic, or an ideologically unreflected position. At the same time, we see few leadership studies explicitly grounded in hermeneutics from philosophy. Rather, drawing on epistemological underpinnings of interpretivism from the social sciences, numerous educational leadership studies seek to understand leadership actions and their effects from the perspective of those actively engaged in that endeavor. Here methods are most often qualitative in nature.

In this strand, scholars often consider organizations, norms, and values as socially constructed and accessible through the subjective perceptions and experiences of people (Greenfield 1987). In other words, an interpretivist approach to organizations focuses on social life, interactions, and individually constructed meanings of these interactions as perceived by individuals, rather than on so-called objective reality (Salovaara 2011). While structural functionalist, classical organizational theorists are concerned mainly with how organizations operate, interpretivists are concerned mainly with how people experience them. In this paradigm, interpretivist perspectives (e.g. Fullan 2007) include suggestions that school group members ascribe different (even contradictory) meanings to educational work. School group members also assume particular roles in the development and support of a positive (or toxic) organizational culture, including those who are keepers of rituals and histories (Peterson and Deal 1998).

More specifically, in a number of empirical studies conducted from interpretivism or a social science version of hermeneutics, we see an increased emphasis on teacher interpretations or individual constructions of educational work (e.g. Peterson and Deal 1998; Fullan 2007). From a methodological point of view, such research studies proceed hermeneutically by depicted individual construction as accurately as possible in order to compare and contrast it dialectically with the aim of reaching 
and generating consensus with a saturation point of data. As examples, a number of empirical studies in schools (e.g. Day 2005; Ylimaki and Jacobson 2013) or districts (e.g. Brunner 1998) seek to understand leadership, from the perspective of teachers, students and parents as well as the formal leader. These empirical studies grounded in interpretivism focus more explicitly on study participants' interpretations of the phenomenon (e.g. leadership contributions to effective schools and districts with effectiveness defined in varying ways by individuals). Similarly, in some instances, scholars examine various interpretations of a particular leadership practice, such as curriculum work, with curriculum defined in varying ways by those involved in the process, from the content of instructional work in classrooms to the political and social intentions translated into content in K-12 schools (e.g. Norberg and Johansson 2010; Young et al. 2014; Ylimaki 2012). Here, on an epistemological level, these interpretivist studies reflect a contention that the inquirer takes on a subject-subject position whereas facts are values are inextricably linked. Hence, the knower and the known are inseparable; research is value-bound. Across these studies, the intention is to understand curriculum work, and in some studies, the macro influences on the work, as interpreted by those involved in that endeavor. The intention is not, however, to critique the broader macro influences (e.g. neoliberal policies) on the work. Ontologically speaking, there are multiple realities constructed by actors of leadership practice under study and by the researchers themselves. Thus, Guba and Lincoln (1994) argue that interpretive research is grounded on a relativist ontology which rejects the existence of any possible correct reality. And while we can identify a number of leadership studies categorized in an interpretive paradigm, there is largely a white spot in educational leadership studies with regards to an explicit use of hermeneutics from philosophy as described in this chapter. There are some studies that gesture toward hermeneutics grounded in the humanities to further consider multiple meanings of learning through participation. Taylor (1994) also argues that the more interpretive or hermeneutic version of psychology is best suited to the development of a theory of learning which explains how we make sense of, and act on, our worlds.

Across this educational leadership literature, we argue that functionalist research studies grounded in organizational systems theories, research studies grounded in critical theories and transformative perspectives, and various studies grounded in interpretive approaches give explicit attention to the micro level of schools and districts/municipalities shaped by aims and values of nation states as well as what we term globopolitanism. DiMaggio and Powell (1983) and Meyer (1997) have proposed new institutional theories, inspiring studies of leadership as a multi-level phenomenon with more explicit attention to relations between structure and agency beyond school and district levels. Going further, Schmidt posits an institution as a discourse in discursive institutionalism, providing an analytical framework to consider movements of discourses within and between levels. We will return to these points in our theoretical framing, but first we briefly examine some leadership studies that have moved toward considering leadership as a multi-level or transnational phenomenon. 


\section{Leadership as a Multi-level and Transnational Phenomenon}

Similar to curriculum theory, also leadership research has mostly been carried out within the frames of the nation-state. Today leadership is increasingly studied as distributed across levels and interest groups. Often versions of old, new, and neo institutionalism has informed this research, but also new approaches have developed. As education policies including comparative evaluation programmes today are increasingly driven by transnational organisations as OECD through PISA it is obvious that studies of educational leadership and governance expand beyond the nation-state level. However, these questions are typically rather taken care of by policy researchers. Yet, a growing emphasis on transnational organizations requires a corresponding consideration of organizational theory as well as institutional theories (Meyer 2010). Organisational theory and research relating to a transnational perspective has developed early on in the business sector. With an increasing pace after 1989 educational policy researchers have responded to the growing emphasis on transnational organizations in the educational sector.

More recently, also empiricist-functional or instrumental leadership studies have started to move beyond the individual or school level toward multilevel approaches, including both a nation-state perspective and sometimes transnational influences. There are many reasons to this. Investigations into success factors for school development have pointed out the need for a system wide perspective in understanding leadership for school development. Lateral and hierarchical cooperation and professional responsibility have been regarded as crucial for moving schools forward beyond high-stake testing (Hargreaves 2007).

Nation-State-Perspectives Functionalist and transformational understandings of leadership occur in a different fashion in studies adopting institutional theories. Some of these explain how societal and policy structures create homogenization in related organizations through isomorphism (Meyer and Rowan 1977), for example, Bennett et al. (2014) studied Southwest Arizona schools and district reforms embedded within state and federal policy pressures and changing demographics. Specifically, drawing on institutional theory, Meyer and Rowan (1977, p. 340) argued that organizations are driven to incorporate the practices and procedures defined by the prevailing rationalized concepts of organizational work to the point that they take on "rule-like status". According to these authors, new institutionalism explained how structures (policy and non-policy formulated or driven) replicate between related organizations through the mechanism of isomorphism and through leadership as a power dynamic. Pressures from the organizational field account for the homogeneity of organizations within them as a result of the press for the "legitimacy imperative," even though they may not result in increased technical efficiency (DiMaggio and Powell 1983, 1991). In other words, organizations experience change by adopting structures or routines that have been legitimized externally in organizational field in order to achieve similar status. Mimetic isomorphism explains the process by which organizations consciously or unconsciously borrow or mimic structures or processes that have been "legitimated" by their association with a 
high-status organization also considered to be an archetype (Meyer and Rowan 1977). Organizations seek to mimic archetypes in adopting various "templates for organizing" such as "organizational forms, structural components, and rules," which have emerged through interactions in the organizational field in response to scrutiny regarding technical outcomes (DiMaggio and Powell 1983, 1991a, b, p. 28). While institutional theory (Meyer and Rowan 1977; DiMaggio and Powell 1983; Greenwood and Hinings 1996) inspired a multi-level approach to understanding educational organizations (structures) within broader institutional fields, these scholars do not consider how power and ideas circulate within and between institutional levels. In other words, there is reason to observe that the focus in educational leadership research generally has broadened its scope of interest from an organizational to a wider systems oriented perspective.

In trying to understand educational leadership on a municipal level, which has been more or less disregarded in previous research, Moos et al. (2016), for example, suggest that using a filter of multi-level public governance logics would be beneficial for understanding how superintendents and local politicians are positioned in the system, how they develop their commitments and competencies for efficient work for democratic education. As educational leadership studies today acknowledge a multi-level perspective, these reflect a broadening of how educational leadership is conceptualized and points towards merging of curriculum research, organizational and institutional theory, leadership studies and policy research.

These approaches emphasizing the necessity of higher administrative levels expands traditional educational leadership research towards educational policy and governance research. Educational leadership as practised on a nation-state level by governments, central administration and municipalities is different to that of leadership in classrooms and schools - leadership is to govern within organizations on a system level. Moos (2013) and others examine educational leadership in new forms of governance and policies emerging from neo-liberal states. Moos (2013) argues that neo-liberal states have developed new forms and technologies of governance (Foucault 1991) that rely heavily on the market as the logical basis for policy, devolving management from the state to local institutional levels (e.g. self-managing or private schools), classrooms, and individuals. Here governmentality is a social technology of control or a set of strategic acts to act on others' actions as well as the self (e.g. leaders, teachers, students). Moos et al. (2016, p. 236) rely on Louis and van Velzen (2012) and Devos et al. (2012) who identify six key features aiming at understanding educational leadership a municipal level ((i). openness as stakeholders' access to political processes and structures, (ii). decentralization of power, (iii). egalitarianism as principles of justice, (iv). efficiency as scrutinizing public expenditures, (v). quality as a benchmark for effectiveness, (vi). choice as balancing between individual rights and social responsibilities).

Transnational Perspectives While functional and organizational approaches typically perceive their work on organizational change within a nation-state perspective, another way of approaching the multi-level perspective is to approach educational leadership including evaluation occurring at the nation-state level in a transnational 
perspective. We are thinking about the more or less globally occurring policy shift the global educational reform movement (Sahlberg 2015). The movement from a system of old public administration to new public governance and management has been based on focusing core subjects, competition, standardization, test-based accountability and control in ways where the transnational dimension cannot be overlooked. Although the effects of this shift vary, also within Europe. From a critical sociology perspective on educational leadership and policy Gunter and her colleagues (Gunther et al. 2016) have demonstrated that system-level and transnational modifications influence individual states', schools' and professionals' work. Nordin and Sundberg (2014) argue "that an increasing share of state policy formation is not bound to national boundaries but takes place in complex, dense and multi-directional transnational exchange."

In a European perspective, the development of the European Union (EU) quite obviously has contributed to the convergence of nation states toward a European knowledge discourse, identified as "Europeanization". As the European Union lacks coercive power over member states, Normand and Derouet (2017) note that soft governance in the form of expert knowledge and standardization has turned out as central governing strategy. They claim that benchmarks, targets, and recommendations are provided and can be voluntarily adopted to: "Common representation by standards specifies constraints for actors but also possibilities for action and cooperation". Nation-state policy systems featuring stronger regional autonomy demonstrate similar patterns of "governing at a distance" within the nation-state. Educational leadership as a policy and practice of governance from a distance through the use of standards makes it possible to bring together communicative activities across contexts and locations oriented towards simultaneously learning from each other and promoting a competition oriented mentality. This reflects a soft governance strategy identified as "competition oriented cooperation" (Grek 2008; Normand 2016) utilizing international evaluation data.

In explaining the dynamics of a multi-level regional school turnaround process in Finland, Uljens et al. (2016) demonstrated, applying Schmidt's discursive institutionalism and non-affirmative education theory, how Finland featuring a open coordinative policy discourse mediated transnational policies in a non-affirmative manner. Framed in this way regional school administrators acted in recognizing and inviting manner with respect to the schools rather than in coercive and accountability centered ways. Critical of accountability policies regional authorities were not required, by state authorities, to incorporate given evaluative practices, but were, however, invited to do so, allowing for various initiatives on a regional level without sanctions. This governing practice, recognized as historically developed, generated dynamic vertical relations between levels, horizontal relations between schools and diagonal relations between different institutions, each actor recognizing the other in a sustained developmental process over 6-7 years.

Including the transnational perspective turns attention to differences between states and nations but also connect to a sub-state level of analysis like districts and municipalities. It is not surprising that different countries and cultures have developed and operated with different policy cultures. Nor is it unexpected that regional, 
municipal or district level activities mediate between various epistemologies, values, and national policies in different ways. In curriculum theorizing and Didaktik, such a multi-level perspective including the school as organization, the district and the state-level, has been acknowledged for a very long time (e.g. Uljens 1997b).

Another approach receiving increasing attention in North American and European educational leadership research is actor network theory (Czarniawska and Sevón 1996, 2005; Czarniawska 2014; Latour 2005; Law 1986; Koyama 2011; Nyvaller 2015) inspired by Bruno Latour's sociology and Scandinavian institutionalism. Actor network theory (ANT) is considered fit for studying how socio-technological conglomerates operationalize and technologize educational policies into governable practises asking for new forms of professional leadership competencies. This approach represents a non-essential, or relational, thinking whereby the unit of analysis, actor, is not an isolated individual. Representing what is presented as a nondualist ontology the "volitional actant" can be a group or system that "that can associate or disassociate with other agents". ANT is seen as an initiative to integrate what is human and non-human (artifacts and organisational structures). Leadership operates as structured invitations including translational practices often based on new formats of data. Latour (2005) refers to Deleuze and Guattari's (1988) concept of network as a "rhizomatic" structure - a structure, pointing at various directions with no definite beginnings or ends. The dynamics of the rhizome makes it a structure at work, and in principle unpredictable, rather than a static. The power relations between nodes are reflected by the strength of the relations rather than by distances. An actor is seen as strong when it gets others to act according to intentions expressed. Or more precisely, as Latour (1986) argues, power is not something one can possess but rather something one can exert. Here the power of exerted actions are given by the follower. The paradox of exerting power is that mere having it is, in fact, powerless. In a translation model of power "a successful command results from the actions of a chain of agents each of whom 'translates' it in accordance with his/her own projects." As "the others perform the action" focus should be turned to "how people are associated together" according to Latour (1986). In contrast to traditional approaches to leadership, this theory acknowledges not only verbal activities but also coordinated material artefacts, procedures, technologies etc. Whereas transformational approaches in school leadership research have been focused on school internal dynamics in relation to external influences (e.g Leithwood 1994), a translation theory approach is more focused on identifying patterns for how 'master ideas' travel from one context to another by means of various translational processes. In their review, Wæraas and Agger Nielsen (2016) perceive translation theory as an approach with roots in actor network theory, Scandinavian institutionalism and knowledge management. In leadership as translation, different stages are identified - problematization, interessement, enrollment and mobilization (see Callon 1980). The translational approach in educational leadership is, according to Rövik et al. (2014), an instrumentalization of scandinavian-french organisational theory inspired by Latour (1986) and developed by Czarniawska and Sevon (2005). Following Latour's view of power, success of an educational reform is, thus, dependent of actors' competencies to translate these ideas into practice. The quality of 
these competencies consists in the actants' conceptual awareness, contextual knowledge, and knowledge of rules for translation. In this approach, we see a focus on translation of reform from one context to another as well as the social interaction with regards to reform among "actors" in particular settings. As in organizational theories, such approaches are grounded actions and behaviors in the micro level of educational changes or reforms in educational organizations. Again this is why we also see the relevance of institutional theories.

Based upon our review of strengths and limitations in educational leadership studies, we make several points. First, recent educational leadership theories or models have developed from an empiricist direction and second, while useful in many ways, theories of leadership are rather free floating from theory of education that drives our project. We further note that much of the empirical literature on leadership and in organizational theory, we see a focus on teacher/staff learning and other practices that indirectly affect student learning. By contrast, curriculum theory and didaktik focus explicitly on student learning. That is, in theories that frequently frame educational leadership studies (organizational theory, critical theories), general ontological and epistemological positionings only indirectly indicate what education should aim at, but do not explain in any great detail the nature of educational activity. In functionalist and reproductionist oriented leadership literature, there is an implicit acknowledgement that effective school leadership indirectly influences curriculum, teaching and student learning with schools serving a subordinate role preparing students for needs of an existing society. In transformative oriented educational leadership studies grounded in critical theory, we also see a strong focus on leadership as a counter-hegemonic force to overcome structural inequities. Here schools serve in a super-ordinate role, transforming society toward an ideal. As we shall demonstrate later, this is something that non-affirmative education theory handles differently.

\section{Conclusions Regarding Curriculum, Didaktik and Educational Leadership}

\section{Different Focus and Concepts on Different Levels}

The general picture emerging from the review of previous research is that various types of leadership may be identified at different administrative levels. Most often educational leadership studies are focused on the principal's work on a school level looking at how principals' or head teachers build up capacity, manage, lead and develop. Educational leadership focusing on leadership issues on higher administrative levels are closer to policy research. This research introduces different concepts and research questions compared with school leader research. These questions often have to do with the relation between politics, culture, legitimation on the one hand and educational activity on the other. Policy oriented leadership research no longer 
focus on single leaders' activities but on more general policies and principles featuring the governance or administrative system.

In Didaktik we can find a similar division of focus regarding different levels. As we have shown Didaktik theory focus on aims, contents and methods of teaching. Didaktik in a narrower sense has often been limited to questions of aims and contents of Bildung, while Didaktik in a wider sense also captures methods of teaching. The decisive selection, formulations and decisions regarding aims and contents of teaching in the European tradition of curriculum making is typically made on the nation-state level far above each single school. Methods in turn are regarded primarily a topic to be decided by the teacher on the school or classroom level. While Didaktik as a field of research traditionally tries to keep together questions of both aims, contents and methods of teaching as they occur different levels, in angloamerican research curriculum theory more often is discerned from instructional theory. Contemporary curriculum theory in the US is dealt as separate from theories of instruction and teaching as well as separate from educational leadership and policy.

The neoliberal shift toward new forms of governance within and across countries has demonstrated how strongly administrative initiatives, led by a new accountability ideology, influence professional educational activities on all levels. Moving from seeing the state as a provider of services to a buyer of services continues seeing the role of the state as collector of taxes but includes a privatization of public services previously organized by the state. In this respect USA with its privatized education governance sector and schools differ from Europe. Thus, we argue that curriculum theory/Didaktik and educational leadership studies would benefit from a closer dialogue as curriculum theory/Didaktik extends educational leadership studies' focus on local perspectives with an explicit consideration of how societal interests transform into educational content.

\section{Reproduction and Transformations Oriented Approaches Dominate}

As we have demonstrated a paradigmatic analysis regarding how leadership research and curriculum/Didaktik theory relate to the practice they are about, shows that either a functionalist instrumental-descriptive (sociocultural reproduction) or a critical-normative approach (sociocultural transformation) dominate the field. In addition hermeneutic oriented approaches may be identified within both.

From a sociocultural reproduction perspective, curriculum serves a preparatory function, preparing all students to become good citizens in existing society with its knowledge, values, and norms. Anglo American curriculum studies and European Didaktik focus explicitly on the interplay between the macro and micro levels. In particular, many scholars consider questions of how policy makers and educators decide on, enact, and evaluate the selection of aims, content, and method (Gundem 
2010; Reid 1994). Here, state curriculum is seen as a means for governing the general aims of education and academic subject content as well as the organization of teaching (Hopmann and Riquarts 1995; McKernan 2008; Uljens 1997b; Ylimaki and Brunner 2011).

Educational leadership studies, scholars tend to write about curriculum and pedagogy as objects of indirect leadership influence and productive social interactions that affect learning and student outcomes (e.g., Edmonds 1979; Hallinger 2003; Leithwood 1994). And while leadership scholars vary according to how they characterize leadership influence from an individual effort to a shared capacity, they frequently approach leadership through various forms and approaches with dispositions and practices that indirectly contribute to student learning. These educational leadership scholars do not explicitly conceptualize the school as subordinate to society. Indeed, there is little direct attention to societal aims and the role of schooling in this strand of literature; rather, scholars focus on social interactions among formal leaders, teachers, and sometimes students and parents that contribute to effective learning for all children.

Drawing on a tested transformational leadership model (Leithwood 1994; Leithwood and Riehl 2003), other scholars focused specifically on how individual administrators build organizational learning capacity in schools (e.g., Jackson 2000; Marks and Printy 2003). Findings from this later research indicated that by modeling appropriate instructional leadership behaviors, norms, and values, and by then inviting teachers to share leadership responsibilities, principals build instructional leadership capacity for systemic school changes that contribute to improved student outcomes. Instructional leadership research fits within social reproduction perspectives because it focuses on how school organizations and their leaders operate to create school conditions (normative school practices) necessary for schools to be productive and educate citizens in an existing, albeit a less than ideal inequitable society. In these leadership studies are implicitly grounded in normative views regarding the preparatory function of schools for society and its existing values (e.g., order, efficient work practices, high standards, strong academic performance) of the status quo. In these descriptions of instructional leadership and leadership for learning, there is also a clear lack of attention to the interplay between the broader society, culture(s), and school practices, including cultural responsiveness to increasing plurality, a gap that some scholars have attempted to reconcile in social transformation perspectives and social justice leadership.

Instructional and transformational leadership approaches are often contrasted with transformative leadership approaches whereby, as noted earlier, more revolutionary leadership practices are aimed at radical social change or transformation (e.g. Foster 1986; Scheurich 1998; Theoharis 2007). Shields (2014) calls for a distinction between transformative leadership for social justice education aimed at changing "the very nature of the school and its relationship with the wider community" (p. 325). Transformative leadership forms stand in contrast to more functionalist views of transformational leadership, and transactional leadership approaches. 
Here, curriculum functions in a superordinate position to society with plans, content, and methods functioning to liberate citizens from existing, oppressive social norms and values. The role of education, then, is to develop something more socially just, teaching students to work toward an ideal that does not yet exist. Apple (2004), for instance, draws attention to connections among hegemony, ideological stability as raised by social reproductionist critics (e.g., Bernstein 1975; Bourdieu 1977; Bowles and Gintis 1975), curricular knowledge, and economic inequality. In other words, it is in the interplay between curricular knowledge or what we teach, the "legitimate culture," and the social relations of classroom life that the reproductionist theorists describe, that we can begin to see some of the real relations schools have to an unequal economic structure. Apple identified four broad groups- neoliberals, neoconservatives, authoritarian populists, and the new middle class - that have sutured an uneasy compromise in a contemporary hegemonic alliance or the so-called conservative restoration. Thus, Apple (2004) argues that curriculum must see as its task today the identification of moral, political, and ethical conflicts connected to social inequities, including class structures, gender, race/ethnicity, and intersections thereof.

Critical curriculum scholarship has gained popularity in recent decades through various approaches to critical pedagogy (e.g., Giroux 1983; McLaren 1998) and Freire's (1970/2000) teachings aimed at developing critical consciousness about marginalizing conditions in society. Regardless of ontological differences, the role of curriculum is to prepare children in idealized, more socially just norms and values that they will apply as citizens who transform society. Notice, however, that the ideals, norms, and values for a transformed society are decided in advance, thus making social transformation perspectives also normative in nature (Uljens 2002). In our view, sociocultural transformation theories are also normative in that a predetermined set of idealized values guide practice.

Critically oriented curriculum scholars (e.g., Anyon 2008; Apple 2004, 2005) have written more explicitly and critically about formal leaders as agents of the system who direct teachers' work and, perhaps unconsciously, circulate a particular set of (neoliberal and neoconservative) policy discourses that institutionalize official knowledge and create a hidden curriculum.

In leadership for social justice theories, now dominant in the educational administration field, leaders are identified as an important counter-hegemonic force in developing students through education, or critical pedagogy to critique and transform existing social structures and values, generally either through awakening an internal critical consciousness or recognizing the importance of conflict to radical social change. According to McKenzie et al. (2008), a critical consciousness about power, privilege, and social inequities motivates leaders to ensure that schools are safe places for all children and that academic achievement is explicitly linked with activism. 


\section{Leadership and Didaktik/Curriculum: Mutual More and Less Blind Spots}

We observe that educational leadership research, curriculum theory and Didaktik all discuss values, identity, culture, social justice and learning but are often unable or perhaps even disinterested in relating to each other in a systematic way, remaining isolated although teachers' and administrators' work includes all. The practitioner is expected to combine disparate theories and perspectives or rely on "experts"often normative/instrumental recommendations - that research itself is not able to unify or correct.

A blind spot for both curriculum studies and Didaktik is the limited attention paid to leadership forms/structures and interactions (Gundem 2010). Perspectives developed within leadership studies, i.e. how professional groups (teachers, school leaders and superintendents) cooperate in order to work with school development together is an overlooked issue within curriculum studies and Didaktik research. In turn, educational leadership studies are typically less observant for the interplay between, on the one hand educational aims, contents and methods and social interactions within schools (e.g. teaching-studying-learning-leadership).

But, the degree to which Didaktik and curriculum theory has or has not paid attention to leadership really depends on which curriculum theories we discuss and how we define leadership. For example, taking Ulf P. Lundgren's curriculum theory as an example. This approach defines curriculum theory as the study of frame factors such as governance, legislation and national curriculum and their influences on teaching practice. A traditional educational leadership perspective in terms of principals' behavior is certainly not present. However, given that educational leadership research today expands its scope of interest from focusing intra- and interindividual dimensions of principals work in schools, as is done by Moos et al. (2016), then a conclusion would be that leadership in fact increasingly reminds of curriculum research carried out since almost five decades. In fact, Lundgrens systems- and governance-level oriented take on curriculum theory received critique for not paying enough attention even to the process of teaching.

Educational leadership as an organic part of the school system at different levels is a longstanding feature in the European tradition of "thick" states, i.e. a state formation with wide reaching responsibilities. This view of state responsibility include leadership in a broad sense as a question of how schools have been and should be financed, governed, evaluated and developed. This organic or broad approach to leadership, curriculum and teaching is well demonstrated in the first text-book in educational studies in Finland 'School Pedagogics' (Skolpedagogik), published 1884 authored by the hegelian educationalist in Finland Zachris Cleve (Cleve 1884). Here educational leadership (as principal's work) incorporated, first, an idea of the school's task in society, second, an interpretation of the curriculum as a policy document, third, a theory of teaching/didaktik including an elaborate view of the school's internal social climate, and fourth, an awareness of the state's regulations of a school's activities. Cleve's book, based on Hegelian philosophy, was aimed for 
teacher education. In it the task of the school is analyzed in relation to the family, culture and church, i.e. develops a theory of the school as a societal institution. Politics is not mentioned, but the book is written 32 years before all men and women won the right to vote in 1906. Further, Cleve identified different types of school institutions (primary school, comprehensive school, universities). The inner and outer organization, the tasks of the teachers, curriculum and laws and regulations are extensively discussed. The second and largest portion of the book deals with the process of teaching, studying and learning the curricular contents aimed at supporting the development of more general abilities among students. The third part of the Cleve's volume is then devoted to the teacher's profession and staff capacity and the role of the principal in these matters, i.e. how to best run a school given the task, the context, framing, contents and teachers. In essence, this description shows how educational leadership was from the beginning seen as an organic part of institutional education. In this contribution, understanding educational leadership is to see how curriculum and leadership are intertwined and connected to a wider understanding of the school as a societal institution. To connect leadership and curriculum and treat them as a whole is thus not a new phenomenon, although such a connection has got lost over the years, also in the European tradition of Didaktik.

\section{Beyond Prescription and Description?}

A frequent dilemma with reproduction oriented approaches is that they ontologically represent a form of naïve realism assuming that the reality may be described truthfully assuming scientific methods are used correctly. Empiricist-descriptive approaches often accept some version of representational epistemology. A classic dilemma with these approaches is that from an empirical description of how leadership or curriculum work is carried out in some given context, researchers draw conclusions concerning how one should teach or lead in the future. It may be that this research is not always aware of the functional role the results presented have. Despite that it is today widely accepted that empirical descriptions are never completely value neutral this is seldom a topic in this branch of leadership and curriculum research. This descriptive, functionalist research operating from a representative epistemology is a part of sociocultural reproduction. Bourdieu (1977) uses the term "symbolic violence" to explain how particular groups retain dominance in a society (without resorting to physical means). Symbolic violence is "the power to impose instruments of knowledge and expression, of social reality, which are arbitrary but not recognized as such" (Bourdieu 1977, p. 13). When most members of a society accept its norms as "natural," rather than arbitrary, then they also accept the outcomes of these norms as natural, even when the outcomes go against their own interests. To conclude, this reproduction oriented research, does not include any awareness of norms and values as being part of leadership as a societal activity. Yet, the results of this research can unintentionally operate as confirming status-quo. 
Socioculturally transformative approaches in turn accept that the task of counterhegemonic research is to reveal self-evident assumptions thereby revealing otherwise hidden interest. We perceive of this as a welcome approach but at the same time pay attention to that some approaches calling themselves critical instead document another type of naïve attitude. This has to do with the role academic research is assumed to have in democratic societies regarding value driven activities like education. The dilemma with strong normative positions may be that presenting solutions and ideals (aims) for education is maybe primarily a task for a public debate within democratic institutions and not something individual voices within universities should carry out. The dilemma is delicate. Assuming that educational research is never value neutral, one could claim that being silent regarding these values is to act deceptive with regards to users of research knowledge. On the other hand, does then every explication of these guiding values mean a negation of democratic processes? We support the view that neither educational research or educational practice can be value-neutral. Yet, in educational research it may be it necessary to differentiate between fundamental values guiding theory building, like assuming the research being aimed as valid for a democratic society, and more prescriptive-emancipatory aims.

We argue that regardless of whether leadership studies are grounded in structural functional, interpretive, or critical paradigms, the task for an educational leadership theory is to explain how this dilemma of normativity is handled. In line with questioning a too strict division between facts and norms in our view the step between what is (empirical description) and what ought to be (normative prescriptions) always include a discursive process, taking a position in value questions as related to a specific cultural context.

In our view, theories of education address school-society relationships, including the aims and character of teaching-studying-learning process. Regardless of underlying paradigm, conception of curriculum/instruction, or specific form or model, we argue that leadership studies do not really theorize education (with curriculum/ Didaktik as key aspects of it) or articulate an underlying educational theory. We will explain what we mean by an education theory in the next large section.

Although we appreciate that leadership studies, by adopting institutional theory instead of organizational theory, understanding schools as politically directed institutions, we also argue that organizational perspectives are still relevant and that leadership studies do not go far enough to consider education theory, curriculum theorizing, communication and cooperation across institutional levels, or broader questions of societal aims, content, and methods. We argue that educational theories and their core concepts from our framework will not compete with leadership studies but rather enhance empirical descriptions of these. By grounding empirical work in an underlying theory of education, we argue that educational leadership studies will more fully explain the current descriptive influence practices prevalent across much of the research.

To conclude, we see that the challenges in treating educational leadership and curriculum in the Anglo-American tradition is different from treating educational 
leadership and Didaktik in the European tradition. Yet, we have to remember there are common roots emanating from a common western philosophical tradition and many similarities regarding paradigmatic approaches. We argue that a simple merging of curriculum theory educational leadership studies is not possible. In the European tradition such a merging is not possible as governance, administration, curriculum and teaching is seen as a whole. In the American tradition a simple merging is not possible given the wide variety of isolated approaches. To address the interplay between schools and society, particularly in dealing with the normativity problem, leadership as a multi-level project in schools as societal institutions, or the paradoxical relationships inherent in notions of leadership influence requires something else.

\section{Non-affirmative Education Theory: Bridging Traditions and Grounding Comparative Research}

In this section, we aim at presenting a conceptual framework that will bring curriculum theory/Didaktik and leadership studies closer together to improve educational practice in the wake of a growing critique of neoliberalism. Our point of departure is, first, that any successful accomplishment of educational practice, be it or teaching or educational leadership on different levels and of different kinds, is partly guided by prevailing conceptual framework and theories, dominating policies, cultural and historical traditions. However, if the undertakings are informed by theoretical positions that conceptually highlight only disparate if important activities and processes of the educational system, and even in conflicting ways, their guiding power may be limited. Observing that the research traditions and paradigms approaching curriculum, evaluation and leadership, it is obvious that they are not developed in any closer connection to each other, rather quite disparate from each other.

Curriculum theory typically starts from a societal and philosophical perspective, operating nationally and on an interstate level, while leadership research has had a tendency to approach schooling on an individual, interactive, and a practitioner level, concentrating on the school (organizational) issues, effectiveness, and social dynamics between professionals. Didaktik in turn combines these two levels, the societal and the interactional eleve both philosophically and empirically, but lacks a conceptualisation of leadership. And while globalization and internationalization have been recognized in recent curriculum theory/didaktik (Pinar 2000, 2004) and educational leadership studies (e.g. Day 2005; Spillane 2002; Hallinger 2005), curriculum and leadership have not been explicitly connected in theoretical logics.

In the education sector the presence of an inter-state or the cosmopolitan dimension (Beck 2006) is visible both in terms of increasing global harmonization regarding core curricula but also through growth of neo-liberal transnational evaluation 
procedures (e.g. PISA) (Nordin and Sundberg 2014). Both contemporary European and US education policy reflects the neoliberal turn in public education, with an emphasis on accountability, standardization, and the comparative high-stakes testing of learning rather than on curriculum and pedagogy. Transnational institutions of different kinds have definitely challenged the nation-state perspective that has dominated curriculum research (Robertson 2006). The emergence of a 'European policy space' or the European Union as new 'transnational state' (Grek 2009; Lawn and Grek 2012) has naturally challenged the idea of the nation-state as the ultimate location for curriculum work or for any public policy making. Still, especially school leadership is mainly perceived as a 'within-state' dilemma (Clarke and Wildy 2009). Curriculum research and theorizing has partly turned into investigations into how policies travel horizontally between policy systems and how meaning translate between levels (Steiner-Khamsi 2004; Steiner-Khamsi and Waldow 2011). Curriculum and related evaluation policy documents are studied in the US and Europe; however, this research is often carried out by political scientists or policy scholars, not curriculum scholars. In particular, in critical policy studies on US policy, we can identify emerging trends, including policy appropriation (Sutton and Levinson 2001), policy enactment (Braun et al. 2010) and policy networks or assemblage (Koyama 2010). In Europe a transnational perspective on educational leadership is emerging within sociological policy research rather than within educational theory or curriculum theory (Gunter et al. 2016).

Given the above described developments regarding curriculum making and the renewed role of evaluation we can see that understanding how institutional education, including schools and universities, are governed and lead require we do not limit ourselves to those system-internal approaches dominating contemporary educational leadership research. This not to dismiss this research as unnecessary or the like. It is only a reminder that the object of research cannot be limited in this way if our aims is to understand how schools are led. In addition, to understand leadership as distinct or separate from eg. curriculum policies restrict our way of understanding leadership. School leadership and teaching are obviously not private activities but public ones. Leaders and teachers must adhere to curriculum policies regardless if these are aimed at social reproduction and cultural unity or social transformation, i.e. toward transformative (equitable) social justice norms and values that disrupt the status quo toward an idealized, predefined future that goes against existing societal norms and leadership traditions. In both cases leaders' professional identity is formed in the tension between on the one hand, their personal values and identity and, on the other, the institutional position they have accepted to be appointed to. Modern education systems typically operate along contradictory intentions - on the one hand schools are expected to adapt teaching to given needs and evaluate each student according individual progress, on the other hand schools are expected to evaluate each individual in relation to all other students. The principle of qualification stands in contrast to the principle of selection/stratification. Further, curricular policies often expect schools to fulfil both reproductive and transformative aims.

Viewing leadership as an object of study internal to the education system limits the attention to continuous reform irrespective of the aims of the education system 
are reproduction or transformation-oriented. In other words, a system-internal, reform- and betterment-oriented perspective on leadership tends to turn leadership initiatives primarily into questions of effectiveness. If research shares such a view of a system with internal leadership practice, then such research quickly turn into effectiveness research. In contrast, leadership as well as leadership research may also be aware and sensitive of historical, cultural, political, religious and societal dimension of education and break with a system-internal perspective. In this case a counter-hegemonic transformative view of leadership theory and practice is often adopted. When leadership scholars take a position on the role of leaders and schools aiming at creating a future more equitable this is guided by an interest in trying to understand how leadership activities influence and support pedagogical practice in revolutionary or reformatory ways, creating a new more socially just society through education. In leadership for social justice literature (e.g., Bogotch 2002; Ryan 2006), practices focus on future opportunities for student success. It is never enough to merely transmit existing knowledge to students. Exactly the same may be noted regarding to curriculum theorizing. Such a change-oriented interest constructs leadership and leadership research into a culturally critical voice. This may be perceived as problematic if such leadership research is presenting a competing or alternative ideal, compared with a politically agreed curriculum regarding the aims of education.

The dilemma of how educational theory may be related to educational practice goes back to double expectations on education theory. On the one hand educational research is expected to provide trustworthy or convincing descriptions of educational practice. On the other hand, this theory or research is expected to provide guidance for regarding how teaching should be practiced. The question is what role educational leadership theory and research should have and aim at? Should it present alternative views for the education system or should it be focused understanding the function of the system?

A second step in approaching these challenges will be the observation that although globalization/cosmopolitanism forces nation-states to restructure themselves regarding the ways in which political, cultural and economic unity and plurality is established and sustained, the dilemma of dealing with unity and plurality is not new. In fact, the stepwise movement from a premodern to a modern world corresponding to the establishment of modern nationstates (eighteenth to nineteenth century) in similar ways required a new approach to establish and sustain a relation between national and cultural collective unity (sense of belonging) and individual and regional plurality. The modern liberal nation state, framing previously unseen forms of liberalisms (freedom of speech, religion, politics, owning of property), required a corresponding educational philosophy, policy and organization able to meet up and conceptually make sense of the new situation. Although many differences may be identified, the solutions in USA and Europe were close.

In other words, the demonstrated need to conceptually and practically rethink nation-state education in a cosmopolitan light reminds of the process modern nation states lived through when establishing the prevailing educational systems and philosophies. There is a question of continuity and discontinuity. To what extent are we 
able to keep to fundamental concepts of education developed as a response to the modern nation state? And to what extent are we forced to rethink citizenship as well as educational research, philosophy, policy and practice in the light of globalization? In order to accomplish this task will contribute with a reconstruction of fundamental tensions, issues and features of modern educational thought, with a focus on post-Kantian educational thought as developed by e.g. Fichte, Schleiermacher and Herbart.

We ground our framework in a non-affirmative theory of education. This approach explicitly draws on Dietrich Benner's theory of education (Benner 1991, 2005, 2015). We elaborate on and use this non-affirmative approach because we consider this position to more broadly encompass and theoretically ground and thereby also frame a dialogue on curriculum theory, Didaktik and educational leadership. Representing general theory of education (Ge. Allgemeine Pädagogik) nonaffirmative education theory by definition is located on a more general and foundational, partly philosophical, level than various research specialisations. Typically a general education theory answers questions regarding the nature of education, what it means to be, become and grow as, a cultural being and citizen. Also how individual learning is related to societal change is at the fore as are questions around educational support, teaching and influence. Due to its foundational character this approach to education offers a meta-theoretical point of departure for understanding curriculum, Didaktik, and educational leadership amidst globalization processes.

\section{Theory of Bildung and Theory of Education}

A second preliminary observation may be helpful for some readers. In the GermanNordic tradition a distinction is made between Theory of Education and Theory of Bildung (Benner 1991). In German these two fields are often collectively referred to by the concepts of either Erziehungswissenschaft or Pädagogik. However, there is terminological confusion. In the literature, theory of Bildung refers at times to the content and aims of education, and sometimes Bildung refer more to the process of human growth in the broadest sense of the word, trying to explain what it means to become a person and a citizen (Bildung as educative formation). In its processual perspective Bildung is an enduring phenomenon - we learn continuously during our whole life. In this light one may see theories of Bildung as philosophical anthropology. For Herder (1744-1803) philosophical reflection was the core of Bildung. In this way, philosophical reflection and Bildung are both cultural enterprises asking what it is to be human, what it means to have knowledge and what is worthwhile pursuing in life. It is worth observing that the seminal works in modern education theory were clearly under influence of German romanticism indicating a reaction against french enlightenment emphasizing rationalism. A position balancing reason with passion (mind-body) endorsing an organic wholeness from the greek polis developed. According to this line of thought Bildung as the individual's 
self-formation is not limited to an individual vantage point but always completed with an understanding of how one's individuality connects to society in general. Socialisation and personalisation are seen as two sides of the same process.

In turn, Erziehung as an intentional activity on e.g. the teacher's part, is something we may experience at a younger and at an older age, but this is not an enduring process. Educational activity (Ge. Erziehung) has a beginning and an end, or, many beginnings and ends. This educative activity is sometimes perceived as influence, at other times rather as pedagogical interaction. As demonstrated earlier Erziehung sometimes refers to pedagogical interaction related to developing attitudes or values, while Unterricht (Teaching) would be more focused on the cognitive contents or competencies to be learned. Since Herbart we know the ambition of keeping these two together by talking about Erziehung as 'educative teaching' (German: 'Erziehende Unterricht', Swedish: 'fostrande undervisning', Finnish: 'kasvattava opetus'). Theory of Bildung is therefore not the same as theory of Education (Erziehung). Neither can be reduced to, deduced from or explained with the other. However, neither are each very meaningful without the other. The challenge for a theory of "education" (Pädagogik) is to explain how these two phenomena, the process of Bildung and the activity of Erziehung, are related to each other. In English 'theory pedagogical interaction' may cover all of the above. This is what we refer to in talking about 'theory of education'.

\section{Didaktik and Education}

Previously we have seen that a broader concept of Didaktik comprises typically both curriculum theory and instructional theory or theory of teaching. Despite this broad perspective Didaktik start from the school as a societal institution as a given. In turn, general education goes beyond activities within the school and rather sees the school as only one way how the relation between generations can be organized educationally in a society. While didaktik focuses the aims, methods and contents of education, general education often include the relations between politics and education as well as ethics and education to be solved. Both Didaktik and General Education theory typically confess to that the societal task carried out by schools is to bring forth reflective, mature and critically thinking citizens. The autonomy assumed is expressed by the german word 'Mündigkeit'. However, the dividing lines are not clear once for all: some approaches in Didaktik, like the one represented by Wolfgang Klafki are indeed broad, while some approaches to General Education are mainly focused around the school as an institution. Indeed, a general education theory always theorizes institutionalised education. This said, it should be made clear that although Didaktik as theory of school teaching is considered a subdiscipline reflecting crucial parts of the teaching profession, it does not theorize the development of professional teaching, or the leading thereof (see e.g. Berliner 1989; Sandberg and Dall'Alba 2006). Another way of putting it would be that while Didaktik assumes the existence of schools, general education starts by asking how 
the generational relations in a society are organized regarding culture, politics and economy. Given that general education by definition theorizes the fields of Didaktik, currículum and leadership on a more fundamental level it makes sense, from a Nordic-German perspective, to identify General Education as a level of analysis bridging Didaktik, Curriculum theory and educational leadership studies. However, as we have seen, neither the European (German-Nordic) tradition in Didaktik or in general education educational leadership has been debated as it is understood in most contemporary research. To our knowledge any such attempt has not been made anywhere before.

\section{Epistemology or Ontology First?}

Much research in education, including curriculum theory, Didaktik and educational leadership, can be structured according to which research paradigm they primarily represent epistemologically - a critical, functional, pragmatic or hermeneutic - or some combinations thereof. The paradigm debates are no longer as engaging as they were back in the 1970s or 1980s. A reason for this is that, first, the main paradigms have influenced each other. Second, prominent positions have lost their paradigmatic position, while new approaches like post-modernism, post-humanism and others have developed. 'The linguistic turn' during late-modern high-days brought new dimensions to interpretative, contextual or hermeneutic approaches through discourse analytic approaches. Recent posthumanist ideas have aimed at redefining relations between mind and matter, merging what is subjective, socially shared, manifest, represented, and material.

A paradigmatic division among positivism, critical theory and hermeneutics reflects a theory of science perspective, focusing on ontological and epistemological positions valid for any discipline. Such argumentations regarding the epistemological nature of human knowledge are also valid for educational theory, but, epistemological theory is not theory of education or theory of learning. The dilemma in structuring educational research following epistemological positions is that conceptually human growth as Bildung and Educational activity stay underdeveloped (Benner 2005).

This critique can also be directed regarding more recent epistemologies regardless if they are discursive, critical, phenomenological or post-humanist. Seldom do these argue for or against previous theories of curriculum, Didaktik or Education. In contrast, non-affirmative education theory does not start the journey from an epistemological perspective but from theorizing education as such, ontologically, asking questions like these: What is educational interaction? How can it be identified among all other human activities? As learning does occur all the time, as well as intentional activity aiming at learning, then what is teaching and leadership about? Teaching is to lead, and leadership can be about teaching. Of what nature are then these activities interfering with other individuals' relations to themselves, to others and the world? 


\section{Ethics}

Turning the attention from an epistemological way of structuring the field towards content oriented one immediately confront us with ethical and political questions. Educational leadership as policy and practice, teaching and curriculum work are never devoid of values. A first topic regarding educational ethics has to do with is the development of learners' (including professionals') moral reasoning. If educational theory is about individuals being, becoming, and development as human beings, and that being a cultural subject indeed includes morally reasoning, then a theory of education has to answer that question.

A second, related question, is what role intentional and reflective educative activity may play for this process to occur? How are individuals' to be approached in ethical issues? In moral professions and politically regulated activities practitioners make value decisions, and these are, in a sense, critical for many reasons. Critical perspectives are important to provide analytical tools (e.g. discourse, critique) in an era dominated by a particular set of political ideologies (Ylimaki 2011). Should education, for example, be about challenging learners' cognitive-reflective capacity to reflect one's own values in relation to others' values and interests (Herbart)? A third question regarding ethics in education has to do with how intentional education is legitimized.

How then does educational theory help us to reflect on the above mentioned questions? We have seen that sociocultural reproduction and transformation theories as well as ideologically critical, functional and hermeneutic theories all deal with these questions. A non-affirmative approach with its core concepts allows for critical discussion of the values lying at the foundation of democratic education and yet avoids the normativity problems of critical theory.

\section{Education as a Discipline of Its Own}

It is relevant to pay attention to that while 'education' typically is defined as a field of research in the US it is defined as a discipline of its own in the German-Nordic tradition (Uljens 2002). It is, however, not only American research that treats education as 'a field of research' where insights from other disciplines like sociology, psychology, subject matter knowledge or ethics, are applied. In such an applied view on educational research, education would be about applying insights from psychology or ethics. It is a widespread misinterpretation that even the founding fathers of education, like Johann Friedrich Herbart, would have understood ethics as providing the aims for education and psychology the methods. Especially, given that Herbart's idea indeed was to establish education as a discipline of its own during the first decades of the nineteenth century, it makes no sense that this initiative would start by drawing implications from psychology or ethics (Benner 1995). In fact, historically seen the relation between university chairs in education, sociology 
and psychology was the opposite. Psychology and sociology were established decades after the first chair in education was established in Finland (1852), but regarding the establishment of education as an academic discipline there are huge variations between countries. In any case, this instrumental view of educational knowledge emanating from seeing educational knowledge as implicational may be the reason to why so many still today treat models of teaching as falling back on sociological theory, psychological theories of learning or ethical-philosophical ideas (Uljens 1997b).

As education in the theory tradition framing this volume is seen as a discipline of its own there is naturally an expectation to develop theory of education, theories that are not applications of sociology, psychology or philosophy. Given the dramatic expansion and differentiation of education as an academic discipline the past 50 years there is an ongoing debate if education is one and only one discipline or if it can or should be divided in sub-disciplines based on the population, contents, institution or something else (e.g. special education, adult education, mathematics education, higher education). In any case general education (Allgemeine Pädagogik) is the most foundational or general field. Contrary to philosophy of education General Education aims at developing foundational and all-encompassing theory of education trying to keep many different questions and perspectives together in a coherent system. General education typically deals with an ontological level of analysis answering questions like: what is education, where does education start and where does it stop, what makes education possible, what makes education necessary, is a universal/regional theory even in principle possible, can or should a theory be prescriptive and normative or not? Dietrich Benner is one of the absolute leading researchers of this field in Germany.

Given the profound challenges of our own time with cosmopolitanism taking new shapes we find an approach in General Education that brings us into a dialogue with the modern western classics indeed as stimulating. We think that it is only by understanding how contemporary theory in curriculum, Didaktik and leadership reflects modern education thought that we may develop a more profound dialogue across different epistemic traditions in the in a global perspective.

At one level non-affirmative theory of education is much less familiar in a North American context than in Nordic/European contexts. In this respect the present project contributes to supporting a transcontinental dialogue on theory of Bildung and related concepts of education, now including educational leadership. But we would also like to remind of that more significant similarities may in fact be exist between the historical traditions in different countries than may be recognized. A first reason is that history of ideas at most universities receive a very limited space in curricula for education as an academic subject. Another may be that educational research is expected to mainly contribute to understanding contemporary empirical problems. A third reason is that education has developed very much as a regional science. Finally, we want to point out that earlier there was not the same culture of referencing to previous research as we see today. This holds true for European and North-American Scholars. For example, knowing the modern tradition one is able to see that the nineteenth century hegelian philosopher and educational theorist 
Johan Vilhelm Snellman in Finland brought his ideas from Herder, Hegel, Herbart, and Schleiermacher (Snellman 1861/1894). The same holds true for Dewey, who seldom makes references to these original sources in his theorizing of education. Yet it is obvious that his ideas, expressed for example in My Pedagogic Creed (1992), i.e. that "education must be conceived as a continuing reconstruction of experience" reflects a view of Bildung originally developed by e.g. Herder and Schleiermacher (Schlieiermacher 1826/1983). He also is very 'modern' in acknowledging the difficulty for education following from a non-teleological societal development, according to which we have to educate for a future we do not, and cannot, know. $\mathrm{He}$ thus concludes that the aim of education is personal autonomy, in german 'Mündigkeit': "To prepare him for the future life means to give him command of himself; it means so to train him that he will have the full and ready use of all his capacities..." (p. 445). Autonomy and self-determination as the aim of education developed early to the cornerstones of modern education thought. When Dewey concludes that "education, therefore, is a process of living and not a preparation for future living" this is here taken to mean that education cannot prepare for a future living as it is not known. Yet, here Dewey comes to equate life, process of learning and education. We think it is not unproblematic to say "the process and goal of education are the same thing" (Dewey 1992) but at this point of our project, it will suffice to say that it obviously may contribute to studying Dewey to read him through the conceptual apparatus outline here and elsewhere (English 2013).

\section{Three Questions as Core Topics}

In adopting non-affirmative education theory as a point of departure for understanding curriculum, educational leadership and Didaktik, then, we asked three questions as core topics. Methodologically, in order to bring together and merge objecttheoretical discourses on Didaktik, curriculum theory and leadership in (and of) schools as historically developed societal institutions, we see it necessary to move the analysis onto a meta-theoretical level. Therefore the point of departure is taken in core questions and concepts originally developed for a modern society within classical or modern continental education theory and philosophy.

By a systematic focus on foundational questions, we point out how a uniting discourse may be grounded in and merge the previously mentioned valuable but disparate approaches and research initiatives. Finally, in light of the contemporary situation, we move the meta-analysis beyond the nation state level to consider the pedagogical dynamics and relations between states as well as networks and states. Here we remind that organisational theory, discursive institutionalism and a globopolitan view complements this non-affirmative approach. These perspectives provide us with conceptual tools and analytical methods for understanding relations within and between levels. For empirical research on leadership, curriculum and Didaktik based on non-affirmative theory of education, critical hermeneutics is a corresponding epistemological school of thought. 
The questions we propose as starting point are:

(a) How does a theory of educational leadership, curriculum or Didaktik explain the relation between individuals in terms of pedagogical interaction and influence? That is, if leadership and teaching is to influence somebody else, then what kind of influence are we addressing?

(b) How do we define the relation between education and society, i.e. the relation between institutional education and other societal forms of practice (e.g. politics, economics, and culture)? How are the dynamics between e.g. education and politics explained?

(c) How do we explain cosmopolitanism and education, i.e. (i) cosmopolitanism as an educational ideal as related to individual identity, multiculturalism, the nation-state and social cohesion and (ii) cosmopolitanism as transnational network relations between nation-states and between nation-states and various kinds of conglomerates, institutions, etc.?

The first question refers to nothing less than to the classical issue of individual's freedom in education. What does this mean? Few would accept that education would not be about influencing the Other or intervening in somebody else's experience of the world. But, how do we explain theoretically the kind of influence education has, be in terms of leadership or teaching? Obviously few would consider leadership to have causal effects, for the reason that typically the individual being influenced is considered as being able to interpret impressions, i.e. free. However, if we accept that the subject is radically free, then in what sense is influencing really possible? A radical interpretation of freedom would mean that everything was in the hands of the individual so to speak. The individual alone would determine the extent to which leadership has an influence. In educational theory, this problem is not new. In fact it belongs to the fundamental core questions in any educational theory and many attempts have been made over the centuries to establish a position between external determination and internal freedom from influence (Oettingen 2001; Uljens 2002).

So, according to e.g. Kantian transcendental philosophy of freedom, the individual is free to establish her relation to the world alone. The theoretical problem which arises from such a position is that it does not seem possible to influence somebody from the outside, rendering leadership and teaching impossible in principle. Instead, the individual would be radically free to determine the meaning of her dynamic and open relation (Bildung) to others, to the world and to herself. In this subjectivist, or subject centered, or Cartesian tradition, it becomes theoretically difficult to explain why the presence and activity of the Other would be necessary for learning to occur, for example. The alternative approach has been to decenter a subjectivist approach and start from a pure intersubjective position, meaning that the individual, from the beginning so to speak, shares something (language, practices, values) before an empirical self is established, i.e. that the subject not only comes to share the world through the process of education, but that the subjects from the very beginning shares something (Masschelein 1991). The problem following from such an radical intersubjective position is that to the extent the world is intersubjectively shared in some sense of the word reaching a shared world cannot be something education aims at. For example, educational theories could not start 
from subjects being linguistic as this is something we learn to become (Kivelä 2004). Philosophical anthropology may explain that being human is to share a language and be linguistic, that thinking is not possible without language (Herder), but educational theory must explain how we come to share the world (Uljens 2016). To the extent the world is shared education is not any necessary activity.

The second question concerns how a theory explains the relation between education and politics, economy and culture, respectively (Benner 1991). Through what processes do societal interests transform themselves into practices of schooling? What are the mechanisms and degrees of freedom involved in these transformational processes at different levels? For curriculum theory questions such as the above ones are frequent. More generally, the question concerns the reasons and aims for which (liberal) education is promoted by the political system. But the converse perspective is also crucial: what kind of education is considered necessary or valuable in order for Western democracies to survive and develop? In essence, we focus here on how societal reproduction and transformation should be understood, organized and directed as a relation between generations. It is difficult to think of educational leadership theory neglecting such a question, especially when the previous levels are widely identified as relevant. In this respect, educational not only curriculum theory but also leadership theory must communicate how the role of the school as a societal institution is defined with respect to politics, economy and culture.

The third question stems from the ongoing crisis of the nation state, due to a conglomerate of issues often discussed in terms of 'globalisation'. The idea of 'the autonomous individual in the independent nation state', most often based on language, has been an explicit organizing principle for state construction for around two centuries in many parts of the world. The legal independence of nation states as such has not been discussed but instead a number of transnational institutions and organisations as well as a global economical mechanisms, corporate interests and non-governmental organisations and agreements have come to question the autonomy of the nation state. Regarding internal ethnic pluralisation we note that the pre-modern, multi-ethnic, absolutist european monarchies operated according to economic principles of mercantilism and saw religion playing a crucial role for establishing unity. The gradually evolving modern nation state instead coordinated the idea of people as etnos (e.g. language, race) with the idea of people as demos (citizenship). Obviously education received a paramount role in such a state construction. Today, cosmopolitanism presents itself as a practical educational challenge due to global interdependencies. As we answer question c, then, we consider transnationalism in terms of the crisis of the nation state and introduce our two interrelated approaches to thinking about transnational relations as a theoretical ideal and now as an empirical reality.

Answers to these questions are derived from modern education theory/philosophy, educational leadership studies, curriculum theory/Didaktik, and discursive institutionalism as well as theories and analytical frameworks used to understand transnational relations (e.g. on Luhmann's system theory, Wallerstein's world systems theory, and theories of intercultural relations from cultural anthropology and cultural studies). 


\section{Understanding Pedagogical Interaction}

The first question concerns what kind of interaction educational leadership and teaching is about and how these activities may be related to those involved. As pointed out before, the position outlined here draws on the seminal studies of modern education theory and later developments thereof. In this tradition the concepts (a) recognition, (b) summons to self-activity, and (c) Bildsamkeit occupy a central position (Benner 1991; Mollenhauer 2014). These concepts are considered fundamental for the post-Kantian pedagogy of which the main outlines were drawn by Fichte, Herbart, Hegel, and Schleiermacher among others. In the english speaking literature the tradition these concepts reflect, has received rather limited attention although the situation is gradually changing (Uljens 1997a, 2001, 2002, 2016, 2017; Kivelä 1998; Oettingen 2006, 2016; Schaffar 2009; Siljander et al. 2012; Friesen and Sævi 2010; Friesen and Hamelock 2012; English 2013; Schaffar and Uljens 2015; Horlacher 2015; Saeverot 2016).

The development of "modern" educational theory was indirectly prepared for by renaissance to humanism and enlightenment. But not before the latter half of the eighteenth century those crucial shifts that still frame our contemporary pattern of thought were starting to develop. The period between 1760 and 1830, i.e. from Rousseau's Emile, over Kant and Fichte, Hegel, Schleiermacher and Herbart, is thus to be seen as marking the period in European theory of education when true steps to what here is called modern education theory was taken. In his Didactica Magna Comenius was still a confessional Catholic who saw the ultimate aim of education connected to the preparation for eternal life. This pre-modern cosmology was teleological in two ways. First, human individual development was to large extent seen as determined by eternal sin and, second, the future of the world was also determined. Pre-modern education was tradition-based and reproduction-oriented, it was about socialisation into existing societal patterns and practices.

Modern education theory developed as a response to the movement from a religious teleological pre-modern cosmology. During the second half of the eighteenth and first third of the nineteenth century the pre-modern teleological view of the world as created by God and heading towards its own end was replaced by a nonteleological view of both history and individual development. Teleological reasoning was abandoned in two respects. In modern education theory the future was no longer considered determined but open, education as socialisation into an existing world was therefore no longer possible. The subject was no longer seen as determined by original sin, but an unwritten page. The shift had dramatic consequences for explaining educational aims and practices as it had from now on to prepare for a future which in principle was not knowable. The new liberties introduced (freedom of speech and religion and later political liberties) required a new education concept to which the Bildung tradition was a response.

In theories of Bildung framed by Christian theology the mundane process of Bildung was about developing an innate potentiality or image of God in order to be prepared for eternal life. J.A. Comenius is a well-known representative of this view 
in his Didactica Magna in 1657 (Schaller 1995; Sousa 2015). Later, when the future was seen as dependent on our own activities, and based on autonomous thinking and ethically reflected action, rather than dictated by given, external norms or directed by some innate developmental patterns, a new kind of Self and self-awareness was required. The modernist discourse on Bildung met this need when cosmological teleology and nativism was abandoned.

As educational theory and curriculum theory works with how the question of aims should be dealt with, the change from a pre-modern closed view of the future to a modern open view of the future changed everything. Modern education theory assumed a non-teleological view of history and future. The new intergenerational question that emerged as a continuous task was "what does the older generation want with the younger" (Schleiermacher)? No given answer to that question existed any longer, it had to be negotiated and had therefore to be the topic for an ongoing public discussion. As no objective or given values existed then the individual had, by education, to be prepared and learn to live with the question of good life as an open question that can never be definitely answered. To learn to live with a question mark as one's companion, rather than an exclamation mark, was necessary as the subject that was to participate in constituting new laws to be followed by all, i.e. normative ethical relativism. Moral education could no longer be a question of leading the child to a given morality. Rather the child had to be led to the question of morality itself.

Modern education theory, then, faced a new type of pedagogical question: if the individual was free and the future was open then a theory about the learning process (Bildung) was maybe possible to develop, but what about a theory of education telling us something about what it means to educationally influence another individual? Education could no longer be about nurturing a given seed, nor could it be about formation for something predetermined. The question remained - exactly how do we theoretically explain educational interaction if the individual was radically free?

The Non-affirmative Solution to the Paradox of Education Human interactive influence has been considered as a core question in all theory of human action and interaction. However, throughout history of education the answers to these questions have been paradoxical in nature. For example, in Plato's philosophy what makes teaching meaningful for learning is not that external and eternal knowledge is communicated or transferred to the learner but rather that the learner reaches insight that he or she already possesses (nativism). To learn is, thus, paradoxically, to attain something one already possesses. Also for Kant (1992) education was paradoxical: How may education, in the sense of external influence (coercion), support the development of autonomous thought and action? (see e.g. Roth and Suprenant 2012). Differently expressed, modern education theory typically views education as an invitation or a provocation to self-reflection and autonomy. However, in order for the individual to reach autonomy in self-reflection abilities, he or she must, according to this line of reasoning, be recognized as already autonomous, free and selfreflecting (Benner 1991; Uljens 2002; Schaffar and Uljens 2015). In other words, in order for education to be possible, there must be a free subject whose reflection is 
provoked, but simultaneously it is thought that the individual becomes a free subject through the process triggered by an educational provocation.

So, in order for education to be possible, the individual must be born free, indeterminate and self-active, and simultaneously, in order for the individual to become free in a cultural sense of the word, education seems to be necessary. Using this argument for developing an understanding of educational leadership, it is assumed that the individual can reach cultural, productive freedom (the ability to act), only by being recognized and treated as if she already is free (or reflective, capable, trustworthy). Educational leadership is, therefore, understood as an recognizing invitation, intervention or provocation, a disturbance or expectation concerning the Other's relation to himself/herself, the world and others. Educational leadership is, then, to recognize somebody as if they are already capable of doing what they are supposed to become capable of - and to act accordingly (Fichte, Herbart, Schleiermacher).

Intersubjectivity Replacing Subjectivity? In order to understand post-Kantian education theory primarily initiated by Fichte and Hegel which came to lay the foundation for all relational theories emphasizing intersubjectivity and recognition, a further analysis needs to be highlighted. From a curriculum theory perspective this analysis is relevant by pointing at recent and strong critique regarding the so called Cartesian subject (Doll 1993a, b) according to which isolated individual rationality has been considered as the metaphor for human reflection. It has also been argued that the pedagogical paradox as such emanates from a belief in a transcendentally free, logocentric rational subjectivity.

There have been many attempts to decenter the subject-centered position by introducing a philosophy where intersubjectivity is made the point of departure (Masschelein 1991; Crossley 1996). Despite differences between philosophers like Arendt, Buber, Goffman, Habermas, Honneth, Mead, Merleau-Ponty, Levinas, Ricoeur and Taylor, all argue in favour of intersubjectivity as a core concept. There is no room here for a detailed analysis of how intersubjectivity is interpreted differently by each representative mentioned above, i.e. as language, as shared values and culture, as something corporeal or as shared practices. If transcendental idealism (radical freedom philosophy) was considered educationally problematic, as it may be interpreted as making educational influence impossible, other problems appear with an intersubjective position. All intersubjective positions have in common that before subjectivity, there is something that is shared. This can be language, norms, practices or culture. This seems a very reasonable point of departure as long as we talk about encultured subjects who already are in possession of e.g. language. The dialogical, collective communicative system is indeed something framing or constituting individual experience. One of the ideas here is a separation between the individual's acts and the meaning of these acts. The meaning of the individual's acts are thus partly made dependent on somebody else's interpretation. Taking part in these interpretations then helps the subject to identify herself differently. The intersubjective linguistic relation would thus be something that cannot be treated in reductionist manner explained by each individual's contribution. Instead subjectivity would be constituted through this intersubjective relation. 
The intersubjective position is attractive yet problematic as a foundation for education theory (Uljens 1997a, 2002; Kivelä 1998). The problem arises from that an intersubjective position assumes that we already share something - language, culture practices or something else. To the extent we already share something we cannot, by pedagogical means or otherwise, move into this shared world; we are already there. Obviously language cannot be made such a foundation as language. The argument that 'we are with others before we are with ourselves' (Biesta 1994) is sympathetic but problematic. It appears that we have, in the end, to accept some fundamental or rudimentary version of subjective awareness to explain how a shared world is possible.

A solution to this dilemma is to discern between different notions of subjectivity and different notions of intersubjectivity. As argued in more detail elsewhere (Uljens 2002, 2008) we may discern between pre-linguistic subjectivity, cultural subjectivity (identity, me) and (self-)reflexive subjectivity. Correspondingly we may discern between corporeally constituted (pre-linguistic) intersubjectivity, linguistic or cultural intersubjectivity and (self-)reflexive intersubjectivity. While the first two forms are given by birth the following forms are empirically established first through socialisation while the last forms of subjectivity and intersubjectivity typically require educational support in order to develop and is also something that formal education aims at starting from pre-school to university education. In this educational process the empirical Me as well as shared practices, values or communicative patterns, ways of understanding the world are made the object of reflection. There is thus no reason to substitute a subjectcentered position with an intersubjectivism or relationalism. Rather these two perspectives complete each other, especially if we identify versions of these concepts as above. Instead of defending a reductionist approach pointing either at subjectivism or relationalism a complementarist position is regarded more fruitful. Accepting complementarism within and between notions of subjectivity and intersubjectivity helps us to identify those spaces where primary socialisation and education play a role (see Uljens 2015). In line with this, the individual would be recognised as being non-determined, free, but that reaching cultural freedom, to pursue one's aims would require an empirical other. In the hegelian tradition, the Other predates the birth of self-consciousness in that the self becomes an object for herself only mediated through the Other.

Following Benner (1991), Kivelä (1998), Oettingen (2001), and Uljens (1998, 2002) the move from Cartesian-Kantian transcendental concept of freedom to an idea of the subject based on mutual recognition and mediated freedom (focusing the ethical) was proposed by educational philosophers such as Fichte, Herbart, Schleiermacher and Hegel. These philosophers herald the end of the one-sided idealist philosophy of the subject, transforming it into a position understanding relationality or intersubjectivity as constitutive, in addition to transcendental freedom. It appears to be less known that the critique of logocentric rationality and that the decentering of the Cartesian subject is not anything recent but that occurred rather early in the educational theory (Benner 1991; Uljens 2002). In the following we return to how this topic was brought on the agenda by philosophers central to the development of modern education theory. The seminal discussion also helps us to 
see where the crucial demarcation lines are drawn. Philosophy combining subjectivity and intersubjectivity emerged historically partly through attempts to explain how education was a necessary intervention in the Other's process of Bildung for the subject's reaching of cultural freedom. This position was capable of explaining both the possibility and the necessity of pedagogical interventions without this being in conflict with individual freedom or the radical openness of the future. Fichte's innovation was to see consciousness of freedom as intersubjectively mediated by the Other as the self becomes aware of itself as free, experiences herself as free, only by being recognized by another (Williams 1997). The concept of recognition was developed further by Hegel and has influenced much later developments in education theory, for example, Mead and Dewey.

Recognition The origins of the concept recognition may partly be found in a modern, classical, theory of education, i.e. within a theoretical-philosophical context of the later enlightenment. It is often associated with Fichte's and, in particular, Hegel's philosophy. Although the concept has inspired many it has nevertheless long remained on the fringes of pedagogical discussions. The past two decades, however, the situation has changed through works by and inspired by Axel Honneth, Jürgen Habermas, Charles Taylor, Nancy Fraser, Judith Butler, Emanuel Levinas, Jaques Ranciere, and many more (e.g. Honneth 1992, 1995; Fraser and Honneth 2003; Taylor 1994; Levinas 1992; Williams 1997; Cobben 2010). Yet, the relation between recognition and some basic concepts in educational theory remain unclear. Also, what exactly is referred to by recognition varies. For instance, does recognition primarily refer to assumptions behind educational activity, e.g. that the subject has to be considered free or nondetermined, thus making pedagogical activity meaningful, or does it refer to some quality or dimension of the actual activity itself, i.e. that education would be about recognizing the present state of somebody, or, still, has the concept rather to do with pedagogical objectives? In this last case, recognition would be seen as a result of an educational process, for example that the subject experiences herself as being recognized. From the above we can see that the concept may refer to the premises, to the process, or to the results of pedagogical processes.

Recognition and Intersubjectivity in Seminal Education Studies In a historical perspective a crucial step for developing the notion of intersubjectivity in educational theory was taken by J. G. Fichte in 1796 who developed a severe critique of Kant's way of explaining self-consciousness of freedom. How does such a selfawareness develop? How do individuals come to understand themselves as free agents whose action is directed by themselves? Kant's explanation was that human beings are free to make choices. Thus he assumed that the individual can follow or not follow the so called moral law he thinks humans are aware of a priori, i.e. before experience. According to the categorical imperative the individual should only act according to principles that could be made universal laws. Further, the individual should never treat other human beings only as means to one's own ends. For Fichte the critical point Kant is referring to is a given awareness of the moral law (Fichte 1796/1992). In doing this, Fichte argued, Kant thereby, in fact, assumed an intersubjective life-world. 
Thus, Fichte's innovation was to realize that consciousness of freedom was in fact intersubjectively mediated by the empirical Other. Here the Self becomes aware of itself as free (experiences herself as free) by reference to the Other, i.e. by being recognized and treated by another as having the potentiality to move beyond her present state (Uljens 2002; Williams 1997; Pinkard 1994). In his Foundations of natural right Fiche $(2000, \S 10$, p. 102) defines the original right as follows: "The principle of any judgement of right is that each is to limit his freedom, the sphere of his free actions, through the concept of the freedom of the other (so that the other, as free in general, can exist as well". Here human rights, considered as universal (natural) rights, are made the foundation for the establishment of self-awareness. The individual has the right to be recognized as free. In addition, recognition of this original freedom and these rights does not suffice according to Fichte; in addition, the individual recognised as free has to be treated accordingly. What makes Fichte's argument interesting for educational and leadership theory is that he introduces the concept of Summoning to self-activity (Aufforderung zur freien Selbsttätigkeit) or the summons to engage in free self-activity. Based on a recognition of the individual's freedom and her rights the individual is invited to put her freedom in use. To be summoned to free self-activity means that the individual is expected to express her view and make decisions on her own part, based on one's own will. It is through this the individual comes to be aware of herself as having a will that is free. We can thus see that the Kantian self-awareness of the moral law is disregarded as the point of departure, rather an empirical other is introduced to explain self-awareness. Although summons to self-activity is considered necessary it never determines the outcomes: "rather, the rational being is to determine itself in consequence of the summons" (Fichte 2000, p. 35). However, in order for the individual to determine the consequences of the summons by herself, we must assume, as Fichte explains, that the individual at some level perceives the summons, which means that we have to assume some prior cognition. Fichte (2000, p. 35) writes: "Thus the external being that is posited as the cause of the summons must at the very least presuppose the possibility that the subject is capable of understanding and comprehending; otherwise the summons to the subject would have no purpose at all". In Dietrich Benner's (1991) formulation of this position education would, then, be about to treat the other as if he or she already is capable of doing what he or she is supposed to and may become capable of. Both recognition and a summons to self-activity are fundamental categories for understanding that the empirical freedom is intersubjectively mediated.

Even if J. G. Fichte, in his lectures from 1796, defines recognition as a mutual assumptional acceptance of each other's freedom, he simultaneously introduces the concept summons to self-activity as a fundamental category for understanding a human being's becoming human. This original freedom is not a gift given to the Other that we must recognize or refuse. Yet, through his critique of Kant's transcendental freedom Fichte introduces the idea that the empirical freedom is intersubjectively mediated through recognition and a summons to self-activity. The subject's empirical autonomy and experience of him or herself as a being of free will instead becomes dependent on the empirical Other, a position that Hegel develops further 
and which today constitutes the reference point for, for instance, Charles Taylor's and Axel Honneth's work. In this spirit, Axel Honneth, for instance, identifies three hierarchically related forms of needs of recognition that correspond to three "practical self-relations." Consequently, (i) love of home/parents/caretakers is necessary for the development of a fundamental self-confidence (Selbstvertrauen). The civil society (ii) recognizing the individual as a morally responsible legal subject, i.e., as having legal rights and responsibility for his or her actions as well as being a political citizen (respect for the freedom of will, freedom of expression, ownership), leads to self-respect (Selbstachtung). Finally, (iii) the individual would strive to be esteemed and recognized (self-esteem, Selbstschätzung) for the work he or she has performed or achievement in society.

In Charles Taylor's (1994) interpretation, to recognize another person with regard to a certain feature (e.g. race/ethnicity, gender), as a free or autonomous agent, you not only admit that he/she has this feature but you embrace a positive attitude toward him/her for having that particular feature. Such recognition implies that you bear an obligation to treat him/her in a certain way. In other words, you recognize the normative status of the other person as a free and equal person, but recognition transcends normativity. Misrecognition is the opposite, a relation that hinders or destroys persons' successful relationships to themselves (Fanon 1963). Fanon (1963), for instance, demonstrated the ways in which victims of racism and colonialism have suffered severe psychological harm by being demeaned as inferior human beings. Taylor and others have recently applied theories of recognition to struggles for particular identities (e.g. politics of difference around gay rights, ethnic or religious recognition, women's' rights, rights of differently abled individuals). Here justice is not primarily concerned with material goods but rather what kind of standing other persons deserve (Young 1998).

From the perspective of a non-affirmative theory of education it makes sense to clearly include the concept of 'summoning' (Aufforderung) in relation to recognition. Without a concept for pedagogical activity the concept of recognition runs the risk of becoming too wide and why the nature of the educative act remains unclear. Now, in order to identify pedagogical and educational (leadership) actions among all possible mediating actions (e.g. care), we draw on the concepts of summoning to self-activity and Bildsamkeit (Benner 1991; Mollenhauer 2014; Uljens 2002, 2015).

\section{How Pedagogical Interaction is Explained: Summoning to Self-Activity and Bildsamkeit}

The dramatic shift to modern philosophy of freedom presented itself as a difficult problem to be solved educationally as freedom could be interpreted in two opposite ways. We could, first, assume that leaving determinism for freedom philosophy results in an unrestricted possibility to influence the learner. But also the opposite interpretation is possible: this very freedom made education as external influence impossible, because how could anybody influence such a self-constitutive subject? 
How should one navigate, i.e. theoretically explain, what educational influence was about as determinism and radical freedom were educational unacceptable? Siljander (2008) draws attention to that Herbart's contribution was to introduce the idea of pedagogical causality to overcome the antinomy between freedom and coercion, between the causality of nature and the causality of freedom. The concepts Bildsamkeit and summons to self-activity have a bridging function for Herbart.

Summoning to Self-Activity The concept of summoning explains how a teacher or a principal has a mediating role with respect to the Other in the maintenance and development of the Other's self-relations. In order to identify pedagogical and educational leadership actions among all possible mediating instances, we also draw on the concept of summoning to self-activity. This concept explains how the school, teacher or a principal has a mediating role between the culture and the individual in the maintenance and development of the Other's self-relations. According to Fichte (1796), summons should be understood in a very specific meaning; we cannot summon somebody to gain an original state of consciousness. Rather, summoning assumes there is a somebody to be summoned before. Summoning should be understood only as an invitation to the Other's self-activity. An act of summons is the impulse to cultural self-reflection made on the part of a reasonable human being in developing into a culturally reasoning subject. In terms of school leadership, the act of invitation or summons is directed to toward a teacher or student's potentiality and invites the latter to become through own activity to become aware of his own freedom as a cultural and political being and ability to realize his own aims.

Bildsamkeit For Herbart Bildsamkeit was "ein Übergehen von der Unbestimmtheit zur Festigkeit", i.e. a movement from non-determinedness to firmness, a process through which the individual develops a personality. While Bildsamkeit often has been translated with referring to the learning ability or developmental capacity, also plasticity, it rather refers to that the individual is first recognized as being nondetermined (Bildsam), but second, being invited or summoned to make use of this freedom taking action during the pedagogical process (Benner 1991). Thus Bildsamkeit refers more to a process than to a state of mind (capacity). Bildsamkeit is about the learner's own intentional but relational activity rather than a process that happens to the individual. Studying, in terms of trying to move beyond one's present state, is an intentional activity, that the learner is invited to engage in, while 'learning' is something that 'happens' with us. Learning is something we typically experience when it has happened. Finally, Bildsamkeit is thought of as making possible that very activity of the learner that is occasioned by a corresponding act of teaching, leadership or education. Due to the close connection between educative activity and the learner's own activity Bildsamkeit has been conceived of as a relational concept that is connected to teaching (Mollenhauer 2014; Benner 1991; Uljens 1997a; Siljander et al. 2012).

While 'summoning' may be seen as a teacher's invitation of the learner to become engaged in a self-constituting and self-transcending process, Bildsamkeit refers to those latent capabilities made real through intentional efforts which individuals aim at making sense of the world and their experiences, given the summon- 
ing. The point is thus that this summons to free self-activity contributes to the establishment of a shared and mutual sphere, or a space wherein the subject may come to see the world in another light. The principle of Bildsamkeit, then, also means that the learner is recognized as a subject with a current potentiality for selftranscendence. But this potentiality is realized by the learner in and through the pedagogical space that is created through the summons to self-activity. The principle of Bildsamkeit refers to individual's own processing of one's experiences - ones relation to the world, others and oneself - through their own activity (Benner 1991, 2005; Uljens 2002). Pedagogical activity or summons to self-activity, then, means that the subject's potentiality for empirical freedom and world-relations are recognized (the principle of recognition, Anerkennung) and then intervened by a pedagogical act so that learners become aware of themselves as free to make use of their autonomy to become and develop as cultural beings.

This reasoning emphasizes that self-understanding in a wide sense is essentially dependent on social recognition, as for instance Honneth (1995) argues, while social recognition does not determine anything. If the individual's self-image is dependent on and is established through interaction with others, and if these dimensions of self are viewed as rights, it follows that pedagogical activity can be viewed as a response to the moral and political demands resulting from the recognition of these rights (Uljens 2016). The self is thus simultaneously both free and dependent, both as regards itself and others. Therefore, it is apt to say that the continuous selfforming (Bildung) process is about having learned to answer continuous questions of who I am, what I can do, know, and want.

It may be good to observe that pedagogues in their summons cannot assume or presuppose a shared life-world or some form of mutuality (symmetry) between the Self and the Other (intersubjectivity) as a starting point for education. Symmetry, or the negation of asymmetry, in the form of establishing a shared life-world, is rather something that is sought after to make real through the pedagogical process. But also the opposite is true. In their summons of the learner, teachers cannot exclusively take for granted there being a radical difference (asymmetry) between the Self and the Other, partly because corporeal intersubjectivity is accepted and partly because an asymmetry (individuation) is, in fact, something which is also sought through the pedagogical process. A "sought-for asymmetry," that is, the negation of symmetry, refers to the aim of the pedagogical process, namely that the individual develops uniqueness in a cultural sense, a uniqueness that did not originally exist.

Non-affirmative Summons A difficult question in all educational theory and practice is the question of norms and normativity. Few accept that educational practice and school development is value-neutral. On the other hand, if education would about socialisation of learners into given sets of values the freedom of the individual is violated and education would be counter-productive with respect to the aims of democratic society. The normativity dilemma is present and has to be solved both regarding constructing national curricula, in evaluation practices, in leadership and in teaching. The question is then how a theoretical position may look like in order to avoid turning education into technology or normative socialisation? As we have 
seen conservative theories tend to promote socialising into something existing for reproductional reasons while theories led by ideals for the future for transformational reasons both run the risk of turning education into a technology. Yet the challenge is there. Education must prepare for the world as it stands and for a future one. How can this be done without violating the learners' self-determination?

We defend a so-called non-affirmative position with respect to norms (see e.g. Benner 2005; Uljens 2002). This means that existing knowledge, values or ideals are definitely taken seriously but not necessarily affirmed. Non-affirmative education also means then, in the pedagogical situation, to focus on the questions to which existing practices, norms or knowledge are seen as answers. Through this the learner is thought to acquire a relation not only to given answers (positive knowledge), but also to the questions behind the answers. Of equal importance is the ability to learn to formulate new answers to old questions as well as new questions to be answered.

Affirmative education means that the practitioner confirms, in a rather unproblematic fashion, either the present situation or the learners' life-worlds, or the aims and content of schooling, reflecting the contemporary needs of society. Affirming a given reality, given values or future ideals can mean to uncritically relate one's professional practice to these. A version of affirmative teaching would have to be concerned with an expectation that learners absorb the given content of teaching, without teachers paying attention to the fact that curricular content in educational settings primarily serves as an example or means to support the development of principled insight, something for which the content is exemplary as such.

An affirmative attitude ends up having a fundamental dilemma: to the extent that aims are given and accepted, educational leadership and teaching is expected to follow a technical rationale. Such a (Tyler) rationale measures quality in terms of efficiency and efficacy. Non-affirmative summons involves a critique of both socializing and normative education. In accordance with this, in, for instance, emancipatory pedagogy, also called critical pedagogy, pedagogues tend to think they know and have the right to decide what students should be liberated for. A non-affirmative summons to self-activity, in contrast, highlights that the education process is dependent on an experiential address, but that when this provocation is a pedagogical the pedagogue consciously refrains from naively confirming either a prevailing or ideal future condition. With such a self-reflecting pedagogical discernment, the pedagogue is thought to be better able to create a space for an education process that recognizes the learner's self-activity and right to exercise conscious independence of thought so important in and for a democratic society and for developing oneself and one's culture in other respects. Also, in a non-affirmative view what "democracy" means, is or should be is an open question. Therefore, if democratic education aims to prepare the learners for democratic participation by mimics of such practices, one must understand the limitation of such methods.

Non-affirmative education that seeks to allow the learner to identify and deal with those problems to which existing knowledge is the answer (and also to assess the value of the existing problems) thus aim at preventing or restricting learners from unreflectively dedicate themselves to cultural content, practices, specific skills 
or concepts. In this limited sense, education is about hindering learning. A pedagogical activity which is educationally reflective presupposes that the school as a social institution is allowed enough free space for the establishment of necessary pedagogical fields of action in relation to other social interests.

The Paradoxical Nature of Non-affirmative Education With respect to the dichotomy between curriculum models emphasizing either learning of contents as such or curriculum models emphasizing learning of generic skills or concepts, a non-affirmative position would start from not affirming any of these ambitions as such. Instead, the educational activity would aim at working on the relation between the above curricular aims in connection to the learner's experiences. In nonaffirmative theory learning contents always also aims beyond itself. Working on the contents typically aims at a cultivation of more general capacities (self-concept, will, moral reasoning, identity, etc.) but also at identifying the principled meaning that a specific content exemplifies (Klafki 1994). That is, the modern tradition holds that selection and treatment of contents should also serve educative purposes beyond learning the subject matter itself (for Herbart's 'educative teaching' see Siljander 2000; Somr and Hrušková 2014). Learning generic skills or knowledge in turn occurs through working with contents, but focusing on generic knowledge can also work as a selection criterion for choosing curricular contents. Here the teacher's task is to extrapolate the educative power or qualities (Bildungsgehalt) of the selected contents (Bildungsinhalt).

The reason to why non-affirmative pedagogy reminds of pragmatism (Dewey), neo-pragmatism and deliberative democracy (Habermas) consist of their common roots. Both positions argue that there is an interdependent relation between education and politics. Also deliberative democracy requires individuals capable of participation in such a democracy. However, from a non-affirmative education theory perspective, a theory of how deliberative democracy works is something else than a theory of educational preparing for participation in such a democracy. If this distinction is not identified there is a risk of ending up in socialization pedagogy again, now with deliberative democracy as the directing norm. Education theory would then be about drawing implications and developing prescriptive recommendations for how teaching should be organized.

Non-affirmative approaches to educational leadership would be focused on creating a professional school culture where individual learners learn about what it means to find a voice of his/her own and what it means to develop towards democratic citizenship. Here the learner learns to make use of his/her own productive freedom. Insofar as district leaders and school principals act accordingly, they mediate between governance mechanisms, interpreting and translating them in dialogue with teachers. In such a process, the use of positive knowledge of e.g. new legislation or curricula may be focused towards not only understanding them as such but also towards reaching the questions or interests to which existing policies, norms, or practices are seen as answers or responses. An educational leader in this case invites (summons) colleagues or even the public to engage in reflective self-activity (Bildsamkeit) in order for them to transcend what is given. A non-affirmative sum- 
mons to self-activity highlights that the education process is dependent on an experiential address, but that when this provocation is a pedagogical the pedagogue consciously refrains from naively confirming either a prevailing or ideal future condition. With such a self-reflecting pedagogical discernment the pedagogue is thought to be better able to create a space for an education process that recognizes the learner's self-activity and right to exercise conscious independence of thought. Nonaffirmative education that seeks to allow the learner to identify and deal with those problems to which existing knowledge is the answer (and also to assess the value of the existing problems), thus aims at preventing or restricting learners from unreflectingly dedicate themselves to cultural content, practices, specific skills or concepts. In this limited sense education is about hindering learning. A pedagogical activity which is educationally reflective presupposes that the school as a social institution is allowed enough free space for the establishment of necessary pedagogical fields of action in relation to other social interests (Uljens 2016).

An educational leader who supports the identification of questions behind provided answers may result in the development of an ability to formulate alternative questions and agendas. Finally, it is to be observed that "summoning to self-activity" operates within (horizontally and between (diagonally and vertically) in institutional settings. Thus, we consider leadership as occurring within and across multiple institutional levels.

Using this argument for developing an understanding of educational leadership and curriculum work as they operate in educational/societal institutions, it is assumed that the individual can reach cultural, productive freedom (the ability to act), only by, paradoxically, being recognized and treated as if they are already free (or reflective, capable, trustworthy). Educational leadership is, therefore, understood as a recognizing invitation, intervention or provocation, a disturbance or expectation concerning the Other's relation to himself/herself, the world and others.

Following Dietrich Benner's (1991) theory of education, educational leadership would, then, be to recognize somebody as if they are already capable of doing what they are supposed to become capable of - and to act accordingly (Fichte, Herbart, Schleiermacher). This may result in learning on the part of the Other, but this is an open question. Teaching or educational leadership is not causal in nature. The nonaffirmative alternative argues that the growing individual is treated as though he or she is already capable of doing that to which he or she is summoned, and as someone who realizes his or her empirical freedom through his or her activity (Benner 1991). To say that pedagogical activity is to treat somebody if she already would be capable of that very thing one expects somebody to become capable of may at first sight be odd. A simple but strong example is when children learn the language or practical things like riding the bike. Parents and caretakers talk to the child as if she already would understand what he or she may start to realized through the process itself. The child only learns her being an individual with a name by being addressed as an individual having this name.

In this case, recognition is not only about recognizing a specific ability or competence, but about behaving toward the Other in an opportunity-identifying way. When Herbart refers to the concept of pedagogical tact, the intention is to demon- 
strate that summons not only falls back on the recognition of the freedom of the Other or the Other as worldly subject, but that summons must, in order to work, be experienced as reasonable by the Other. Through such a thought-provoking but sensitive activity, the educator recognizes the subject's empirical reality, life situation, as well as his or her self-expression and potentiality. However, nothing of this is affirmed, but rather challenged. Education more often aims at creating a headache, rather than at curing it.

The non-affirmative position (Benner 1991) outlined here must be further discussed but this theorizing shows that we in our educational theory tradition find ideas how to avoid the dichotomies between conservative reproduction oriented and normative transformation oriented curriculum theory, as well as descriptivetechnological approaches to teaching and curriculum work.

As we have seen from the above, curriculum theory and educational leadership cannot be limited to dealing with a theory of Bildung alone, but must explicitly and in parallel deal with a theory of education (pedagogical influence) leading us to different versions of the pedagogical paradox.

An act of summons is the impulse to cultural self-reflection made on the part of a reasonable human being in developing into a culturally reasoning subject. In terms of school leadership, the act of invitation or summons is directed to toward a teacher or student's potentiality and forces the latter to become aware of his own freedom as a cultural and political being and ability to realize his own aims. Education works with this double openness; the educator lives with the unanswerable question.

These concepts refer to a modern tradition of relational theory of education and form core concepts for educational acts in terms of summoning (inviting) the Other to self-activity (Bildsamkeit). 'Summoning' may be seen as the leader's or teacher's invitation of the Other to become engaged in a self-transcending process. In terms of school leadership, the act of invitation or summons is directed to toward a teacher or student's potentiality and forces the latter to become aware of his own freedom and ability to realize his own aims. In sum, the very same core concepts may be laid out as foundational for both teaching and educational leadership as human interpersonal practice. In fact, educational leadership as culture building or curriculum management, as example, is a process of intentionally creating professional working conditions and circumstances for colleagues to reconstruct their professional identities and capacities.

\section{Non-affirmative Theory and Critical Hermeneutics}

The non-affirmative education theory outlined here may also be clarified in relation to hermeneutics and phenomenology. Hermeneutics is relevant for non-affirmative general education theory for at least three reasons. First, the process of Bildung is traditionally perceived as the subject's dynamic relation to the world, to others and to herself. It includes an experiential (Ge. Erlebnis), a reflexive dimension and communicative dimension (cf. Ricoeur 1976; Kristensson Uggla 2010; Kemp 2010). 
In this process the subject develops both a personal and a cultural identity, i.e. a sense of self (Me) and sense of belonging (We). In English 'experience' refers to both the sensations one has and the experiences resulting from reflecting upon these sensations. In many other languages this difference is denoted by different concepts. The process of Bildung can now be seen as a change in one's experiences and identity. A change in experience may, with e.g. Gadamer (1975) be described as a loss, inasmuch it may be described as reaching of something. In the process of learning the negativity of experience may refer both to experiencing a dissonance between one's perception (Fi. elämys, Ge. Erlebnis) of the world and one's experiences (Fi. kokemus, Ge. Erfahrung), or to the actual loss of a previous way of understanding the world requiring a possible reorientation (Uljens 1992; Benner and English 2004; Marton 2014). Second, all of the above regarding Bildung as an interpretative process is equally valid for the leader and teacher. The leader and teacher stand in the very same position as any learner. Here the teacher/pedagogue, as an interpreting subject, also stands in an open relation to the world, others and herself requiring continuous interpretation of experiences. This is the teachers' or leaders' interpretative, meaning making process -process of Bildung. Third, the leaders and teachers are, however, expected not only to interpret the world for themselves, but also to explicate or communicate their understanding with the learners - the culture is mediated by the educationalist. When this mediation is carried out by the leader or teacher, it has to be done with the learners contemporary experiences and experiencing in mind, but also the learner's future. This explication is thus carried out and guided by pedagogical intentions. Educative leadership and teaching requires simultaneous awareness and recognition of many things, e.g. curricular expectations, the surrounding culture, and the learner's experiences - especially the learners' (phenomenologically seen) intentional object of awareness and her intentional activities aiming at reaching insight, i.e. the teaching-studying-learning process. Following the idea of a non-affirmative summoning the leader's and the teacher's intention would thus be to reconstruct or to open up for what is believed to be "educative" qualities of a selected learning contents in an invitational way from the learner's perspective. The truly educative qualities are therefore an emergent dimensions of the relations established, rather than something essential inherent in the contents as such (Klafki 1963). In a peculiar way the concept of Bildung aims at connecting the individual experience, the subjective, and the cultural contents, the objective, in ways that makes this contents accessible for the learner, yet in an interpretational way connected by the concept of meaning.

Non-affirmative leadership and teaching features not only the interpretative but also the mediating moment as developed in hermeneutics. The interpretative, sometimes mimetic, moment includes a reflective dimension, or rather is reflection, given in and through the non-affirmative act. Differently expressed, leaders and teachers experience external expectations, curricular interests and the like, but are not necessarily expected to affirm these interests. The 'tradition' exerts a normative force but in a non-affirmative light the individual interpreters are regarded able, not to stand out of the tradition but to making it an object for educative purposes. The tradition is recognized yet not affirmed. Would contemporary interests and the tradition be 
expected to be affirmed, the result might be manipulation and indoctrination rather than educative leadership and teaching. The explicative moment in hermeneutics, or, in other words, mediational, communicative re-contextualisation of understanding, corresponds to the concept of situational non-affirmative summoning to selfactivity reminiscent of Herbart's 'educative teaching' (Benner 1997; Uljens 2000). Educative leadership thus directs the followers attention and reflection to the problems at hand not only for seeking perhaps a practical solution for a problem at hand but also in order to support the development of discerning thought, which, in turn may orient the actor's future activity. Educational leadership that is educative in nature engages the learner in a process aiming at developing her professionality.

It is not much of an innovation to point out similarities between hermeneutics and Bildung, either as philosophical anthropology or as a language for interpretative processes in human learning or as an epistemological position for empirical research. Here the point is rather to observe that both theory of Bildung and hermeneutics typically starts from the perspective of the individual doing the interpretation or learning - there is always a somebody, a who. If the connection between hermeneutics and Bildung is more developed, a corresponding concept of hermeneutic teaching or leadership is much less studied. Therefore the contribution here is to remind of teaching as being congruent not only with the process of interpretation and understanding, which indeed is required, but the process of explication, which is also to be seen as a interpretative, but reconstructive initiative. Let us take one step back to see the logics of the above argument.

Important steps regarding hermeneutics were made since the eighteenth century in Germany as during the first half of the nineteenth by e.g. Friedrich Daniel Ernst Schleiermacher (1768-1834). His Hermeneutics and critique (Hermeneutik und Kritik) from 1838, published by his students 4 year after his death, significantly contributed to the development of hermeneutics as an empirical method, making hermeneutics a study of any texts, not only those connected to jurisprudence (law), linguistics and theology (Uljens and Mielityinen 2004). The view that hermeneutics is about studying texts is still widely supported (Palmer 1969). Being located between enlightenment and romanticism Schleiermacher was influenced by Hegel's dialectic, however without accepting Hegel's teleological view of historical development. It is vital to understand that Schleiermacher also was one of the major contributors to modern education theory, most famous for his Lectures in Education from 1826 (Schleiermacher 1826/1983). For him, as for Humboldt, Bildung was a concept referring to a dynamic relation between the individual and the world where the individual was by 'spontaneity' always directed to the world but simultaneously experiencing the world through 'receptivity'. The task of humanity, as Herder and Humboldt also argued, was to be involved in a continuous self-forming process. This tradition of theorizing beingness in the world constitutively including interpretation was later, in different ways, continued by e.g. Heidegger and also Gadamer.

Theory of Bildung and hermeneutics may include ideas about being from a philosophical anthropology perspective as well as theorizing what it means to interpret the world. From a Bildung perspective interpretation and understanding are then constitutive in defining human being and becoming, essentially connected to judg- 
ment, a view shared by also american philosophers like Charles Pierce, John Dewey and William James as well as Richard Rorty.

Theory of education includes the task of explaining how we think educational or pedagogical activity is related to a process of Bildung. Turning to hermeneutics is helpful for two reasons. First, obviously, also the leader is a subject interpreting the world. The process of Bildung, i.e. the unfinished or ongoing interpretative process in which the individual stands in an active position regarding herself, and the surrounding world ('spontaneity') as well as in an experiencing relation ('receptivity') is equal for all. However, in order to conceptualize educative activity we turn our attention towards our attention to the second moment of hermeneutics. This second moment or step of hermeneutics refers generally taken to explication, communication or the justification side of the interpretative process, methods and its results, understanding. In hermeneutics it has been disputed whether this second moment should be included in the act of hermeneutic interpretation or not. While Schleiermacher defined the task of hermeneutics primarily to be about the act and methods of understanding this corresponding to the process of Bildung, many hermeneutic theorists both before and after have, in addition, emphasized both moments of interpretation: understanding and clarification (Gallagher 1992).

From an educational perspective this second moment of hermeneutic interpretation, i.e. clarification, makes sense in that it does emphasize that e.g. a teacher or a school leader is not only carrying out an interpretative act in his or her own interest. Instead, the educational value of an (re)interpretation lies in what sense an this understanding arrived at may be helpful in supporting designing and carrying out a process aiming at enabling others in reaching beyond their experiences. This second moment of interpretation is closer to a pedagogical task despite the obvious differences between communicating one's results from empirical research within a "context of justification", which is driven by epistemological argumentation regarding validity and truth rather than by pedagogical motives.

Carrying out educative activities may be seen as a mediation between the learner and the world. The leader or the teacher stands in an interpretative relation to the learner, to the world and to herself. Within an already existing intersubjective space or shared world, the pedagogical leader reconstructs given cultural contents in a communicative interaction with the Other, aiming at establishing a second, "pedagogical space' (Uljens 1997a, 2002, 2016). Seeing pedagogical leadership in this way connects to earlier theorists in hermeneutics having emphasized the two moments of interpretation, namely understanding and explication. With some reservations, we can talk about a context of discovery for developing an understanding and a context of situated pedagogical justification where the contents is conveyed with educative intentions. Carrying out educational leadership or teaching the situation is radically open. As the responses and the outcome is not knowable in advance the leader and teachers are making continuous reinterpretations along the way, asking for the Other's intentions, experiencing, reflections, experiences (Uljens 1997a). In 'pedagogical explication', creating a space for Bildung, also continuous interpretative activity is present. Non-affirmative leadership is therefore both experiential and experimental. 
Teaching, according to Schleiermacher, was about protecting the child from outer less positive influences so that the inner good qualities could develop. 'Counteracting education' in turn was about working against individuals' less positive initiatives. In addition the teacher had to support reflection and development of competencies. Regarding ethics, Schleiermacher's idea was primarily about helping the subject to learn with the question of good life as an open one. Although education was an intentional act, it was not about being normatively closed, bringing the learner to a predefined understanding.

\section{The Other and Summons as Responsible Response}

As has been argued elsewhere this reconstruction of the modern education theory includes intersubjectivity and subjectivity as parallel non-excluding or complementary categories (Uljens 2001, 2015). Instead of making either intersubjectivity or subjectivity the ultimate point of departure it is possible and meaningful to accept both simultaneously. This requires, first, that different notions of both subjectivity and intersubjectivity are identified and, second, that we see how they are related to each other.

There is, however, a widespread understanding that this modern tradition represents a kind of subjective idealism which must be replaced by accepting intersubjectivity as the first philosophy or then replaced by some other way of decentering the so called Cartesian subject. Indeed a radical subjectivist epistemology is problematic in that the Self subordinates the Other, reducing the Other into being experienced by the Self. This is the fundamental problem of phenomenology. A decentering of such a view is represented by Emmanuel Levinas. Levinas turns around this subject-centered position in two ways by replacing epistemology with ethics and the primacy of the Self with that of the Other. As a result, by experiencing the Other the Self is awakened to awareness of one's responsibility. As formulated by Levinas, the face of the Other appeals to the Self to welcomingly open up for the Other, whereby the impregnable 'otherness of the Other' is simultaneously recognized. Here it is the Self that is summoned by the Other, through the Other's mere presence. The advantage with Levinas's position is that it draws attention to the Self as depending of the Other, but at the same time, this is interpreted as a reversed onesidedness in relation to the subject-centered tradition that Levinas critiqued. From a pedagogical perspective, Levinas's position is also limited.

Against this background, pedagogical activity, summons, can nevertheless be interpreted as the way in which the Self handles the responsibility to which we awaken through the presence or address of the Other. With Levinas, also the Self/ pedagogue is reminded of its/his or her "infinite" responsibility to respond to how the Other should be treated. Levinas's suggestion that the Other awakens the Self to responsibility simultaneously means that a reflective behavior is demanded of the pedagogue. How should I conduct myself? What does my responsibility involve? What constitutes a reasonable summons? 
It is possible here to discern a similarity or a point of convergence with the nonaffirmative summons: the non-affirmative summons is a reflected pedagogical act in the form of a full expression of a responsibility, and as such aware of its fundamental opportunity for affirmative or socializing teaching as well as prescriptive education, that is, freedom-depriving rather than freedom-developing pedagogy. The non-affirmative pedagogy that seeks to allow the Other to deal with the problems to which existing knowledge is the answer (and also to assess the value of the existing problems), and with issues about the good life, thus works to prevent the learner from unreflectingly dedicate to him or herself cultural content, and therefore represents a kind of restrictive pedagogy. A pedagogical activity which is ethically reflective presupposes that the school as a social institution is allowed enough free space for the establishment of necessary pedagogical fields of action in relation to other social interests.

Educational awareness could then be defined as the Self recognizing the unapproachable "Otherness" of the Other, but simultaneously viewing herself as an Other for the Other. The self-reflectivity of pedagogical thinking therefore means that the Self is forced to reflect on its I-ness and ask for its otherness from the perspective of the Other (who am I, what do I want, how does the other experience me?). In this way, the Self is and becomes both free and dependent on the Other.

According to this advocated perspective, there is also a pedagogical paradox, but in a new version. It implies that the growing individual is treated as though he or she is already capable of doing that to which he or she is summoned, and as someone who realizes his or her freedom through his or her activity (Benner 1991).

\section{A Non-hierarchical Relation Between Education and Politics, Economy and Culture}

The second question concerns how a theory explains the relation between education and politics, economy and culture, respectively, as non-hierarchical. How do we define the relation between education and society, i.e. the relation between institutional education and other societal forms of practice e.g. politics, economics, and culture? How are the dynamics between e.g. education and politics explained? In the attempt to understand how education (and leadership thereof) is related to politics, economy and culture, we must first negotiate a path through the various extant explanations in the history of education.

First, a pre-modern mode of thought understands education as being located within the existing society or culture (see Benner 1991; Uljens 2002, 2007; Uljens and Ylimaki 2015a; Ylimaki and Uljens 2017). This socialization-oriented model of education emphasizes the task of education as preparing the individual for an existing society and culture wherein societal practices and norms function as the guiding principles. In this model, educational leadership is subordinated to societal practices. Here education is not expected to have any particular developmental or trans- 
formative role with respect to the existing society, but rather education is preparatory in character. The power of societal transformation lies beyond education, and as a consequence, education is reduced to socialization.

Secondly, as described above, in contrast to the reproduction-oriented model, since Rousseau education theory has learned to conceptualize education as a revolutionary force with respect to societal practices (Uljens 2007; Rousseau 2002). In its most radical or critical form, revolutionary or transformation-oriented education is not only disconnected from society, but also allows itself to be positioned as superordinated with respect to societal interests (Benner 1991). According to Rousseau, there is not much point in educating individuals for an existing society, since education would then only reproduce unacceptable constellations (Rousseau 1979). Rather, the role of education would be to develop something new, something that does not yet exist. Education would work towards ideals, which may, in the future, become realities as a new generation enters society after having undergone education. In this model, education is superordinated with respect to societal interests. More specifically, social transformation theories critique the status quo and seek to explain, either through 'subjective', (e.g. Lukacs; Frankfurt School) or 'objective' (e.g. Althusser) ontologies, how radical change occurs is society. These "critical" theories do not place any critical distance between the values and norms they themselves represent. The similarity between these three positions is their normativity, meaning that a predetermined set of values guides educational practice. In addition, these values are defined irrespective of the educational leader's own interests.

Both the reproduction- and the transformation-oriented models are prescriptive while the first accepts existing norms of the society as guing for educating, the second starts from an ideal for the future, an idea that education should strive at. Then how might curriculum theory and educational leadership deal with value questions? How can they relate to ethics and politics? One of the founding fathers of modern education theory, was Johann Friedrich Herbart, Kant's follower on his philosophy chair. One of Herbart's ideas was that education should be conceptualised as an independent academic discipline of its own, not just a 'field of research'. Contrary to a widespread misinterpretation of Herbart's pedagogy he did not think that ethics could provide education the aims and psychology the methods of teaching as the individual is born neither good nor bad, nor was ethical realism taken seriously. If the modern human being is assumed to be the creator of laws as well as subjected to follow these laws, then moral principles are to be constantly negotiated. In addition, if humans are borne without eternal sin then moral thinking is rather something to be learned.

From a curriculum theory perspective the question is how such a (curriculum) theory may avoid being identical with the prevailing ideology or representing an ideologically opposite position to a politically agreed curriculum? In either case, curriculum theory would function as an instrument for either positive socialization or as a counter-hegemonic ideological discourse. Although educational theory is never value neutral there is reason to keep up the difference between politics and educational theory (Uljens 2016). Another problem with the previously described 
models is that they runs the risk of not leaving room for the principal's, teacher's or learner's to decide upon what is to be considered valuable and meaningful.

In the tradition of modern education theory education is therefore not subordinated to ethics, politics or psychology. If it were, the only remaining question would be from where and from whose ethics, politics and psychology the aims and methods are to be derived. Generally taken, throughout history we have seen two such normative positions: (a) in pre-modern thinking education is equated with normative socialization and the ideals for education stem from the values and practices of contemporary society, and (b) according to reformatory or transformative theories the ideals for education stem from how we envision the future of society (Uljens 2002 , 2007). The first position can be called sociocultural reproduction and the second sociocultural transformation.

A third line of reasoning opposes the above-mentioned ones by criticizing them for subordinating och super-ordinating education in relation to society and societal development (Benner 1991). Since the future is thought to be undetermined and the question of morality is something that cannot ultimately be decided in advance, the individual's reflective ability - self-awareness and self-determination - is seen as an ability that must be developed.

In this last model, education is seen in a non-hierarchical relation to politics, culture and economy. Education is not solely placed either "outside" or "inside" the society and is thus not either super or subordinated with respect to society, but attempts to mediate between the two. In this non-hierarchical conceptualization, educational institutions are given relative independence with respect to societal and other interests. It is this space that both allows for and requires reflective, professional educational leaders on each level of the education system.

It should be observed that a non-hierarchical understanding acknowledges that hegemonic political interests influence education, but recognizes that if educational leadership were to be reduced in the service of some political ideology, it would be in conflict with democratic principles. Thus, political democracy requires a certain form of critical educational leadership, that is, relative independence should be guaranteed by the political system itself. From a non-affirmative education theory perspective, a theory of how deliberative democracy works is something else than a theory of educational preparing for participation in such a democracy (Masschelein 2004). If this distinction is not identified there is a risk of ending up in socialization pedagogy again, now with deliberative democracy as the directing norm. Education theory would then be about drawing implications and developing prescriptive recommendations for how teaching should be organized (Uljens 2016).

From a non-hierarchical perspective, educational leadership is leadership that sustains democracy, related to an image of citizenship. From this point of view, education is allowed to critically examine the political system within which it operates, but it also leaves room for politics to be reflective and critical about contemporary education and educational leaders. The same relation occurs between education and economics: education must prepare individuals for an existing working life, but in such a way that the individual may transcend existing ways of working. In sum, the non-hierarchical position to the relation between school and society accepts 
that: (a) School prepares individuals for an existing world - though it does so in a problematizing, non-affirmative fashion, not confirming a present state of affairs; (b) Democratic ideals are defended: education prepares individuals for participation in societal political practices and change; (c) Human freedom is assumed - from provocation (intervention) to self-activity; (d) The question of the good life remains an open question; and (e) A relative degree of freedom is guaranteed for the state, district, principal, teacher, and ultimately, for the student. Valuable or ideal in society is decided upon in advance. Therefore, it is supposed that the previous models, taken seriously, in fact run the risk of indoctrination and of turning educational leadership into a technological profession.

\section{Organizational and Institutional Theories: Leaders and Actors}

For our theoretical framing, we put education theory in the foreground but also recognize that educational activity occurs within schools and other educational organizations as societal institutions. We appreciate the aspect of open systems theories that explains the interplay between schools/educational organizations and the broader environment, including neoliberal policies, centralization and decentralization trends, and related societal trends (e.g. globalization, cosmopolitanism) and internal dimensions of the system (e.g. school culture, technical requirements, structures, politics, formal and informal leadership). Yet we see the need to retheorize open systems organizational theory with its rational systems/mechanistic legacy combined with natural systems/human relations approaches. Further, we work from a non-hierarchical ideal of education (including organizations) in relation to the broader environment (e.g. politics, culture, economy) and a nonaffirmative education theory. Educational leadership scholars Hoy and Miskel (2005) remind us that open systems theories draw attention to the transformation of organizations through an interplay between the external environment and the schools, but their application give little attention to educational activity. In other words, open systems theories do not consider principles from educational theory or curriculum theorizing as we do here.

In our approach to educational organizations and institutions, we appreciate Greenfield's (1986) theory of organizations as the context of ideas by which we understand our experiences, Habermas' (1984) communicative approach, and Weick's (1995) sensemaking approach to systems that remind of hermeneutics and phenomenology. We find Greenfield (1986) Habermas (1984) and Weick (1995) particularly relevant as they argued that consensual meaning is created through iterative and adaptive communication (e.g. rational discourse, double interacts), referring to social norms and values as well as experiences and ideas. These theories of organizations grounded in hermeneutics and phenomenology are less prevalent in educational leadership literature than recent literature from business grounded in hermeneutics from philosophy and research. 
As we noted earlier, we recognize the challenges of integrating distinct theoretical traditions but suggest the relevance of an organizational context to educational activity. Here we recognize the contributions of various thoughts on how we organize in society to education but rather than a functionalist approach to systems theory, we conceptualize organizations through hermeneutics and phenomenology in relation to education theory whereby people form interpretations and develop understandings in a shared space for reflection and educational activity. Within organizations and institutions, Macdonald's (1995) perspectives are also important here as he advanced a broad understanding of curriculum to include hermeneutics, critical perspectives, and technical perspectives, such as the technical aspects of systems emphasized in much of the organizational theory literature. With Moos et al. (2016), Schmidt and others we also find a broader framework for understanding how the school system is governed and how knowledge is transmitted, selected, valued, and organized in an educational context. Closely related, taking a hermeneutic-phenomenological perspective, we appreciate Greenfield's (1986) educational organizations are the context of ideas, including an internal culture, by which we understand our experiences; however, like Lundgren (1972), we see goals and aims in terms of societal aim. That is, we also see organizations as societal institutions. Here institutional theory and actor network theory may fill a gap in open systems theory, arguing that much of that environment consists of social and cultural forces, not merely production resources and task-related information as some open systems theorists would suggest (e.g. McGregor). Actor network theory (e.g. Czarniawska and Sevón 1996, 2005; Czarniawska 2014; Latour 2005; Law 1986; Koyama 2011; Nyvaller 2015; Fenwick and Edwards 2010) has been utilized recently for studying how socio-technological conglomerates operationalize and technologize educational policies into governable practises asking for new forms of professional leadership competencies.

Along with actor network theory, new and neo-institutional perspectives from sociology extend our understandings of organizations and provide an opening for a multi-level perspective on educational leadership in relation to curriculum. As noted earlier, new institutionalism explains how structures like curriculum policy replicate between related organizations through isomorphism and leadership as a power dynamic. Institutional theories, thus, go beyond open systems theory in explaining the influence of policies and other dimensions of the external environment on organizational homogeneity. In particular, neo-institutionalism has influenced on scholars who seek to explain the transnational level and literature on global social change (Meyer 2010). Meyer and colleagues, for example, have developed world society theory to consider the relations among global institutions and culture and the structures and behaviors of individuals and organizations at nation state and transnational levels.

Organizational theories and related institutional theories, thus, apply to transnational organizations, including NGOs and organizations like OECD that oversee evaluations and other policies. Indeed, increasing complexity of multinational and transnational organizations has created the necessity of new structures, including federal decentralization (Drucker 1974). In federal decentralization, a company is 
organized so that there are a number of independent units operating simultaneously; each unit has its own management, which in effect runs its own autonomous unit. This structure has been utilized in large conglomerates with divisions in various countries and also in large school districts. There are many hybrid forms of transnational organizations, but classical organizational theories apply in established patterns of relationships among parts of these organizations (e.g. differentiation, structure of hierarchical relationships, formalized policies, organizational cultures and norms), but here the focus is on governance in various polities and the movements of ideas as discourses. This is where we need discursive institutionalism to analyze and understand policy discourses within and between levels.

\section{Discursive Institutionalism}

Above it was acknowledged that every theory of educational leadership must deal with three fundamental questions as well as their internal relations. These questions concern, first, how institutional education is related to politics, economy and culture in a democratic society and, second, how leadership as a professional, moral practice is explained in terms of power and influence, that is, as an ethical-political and epistemological relation between individuals in professional settings. On the first issue, this approach argues for a non-hierarchical view of the relation between societal forms of practice, thus holding to a western democratic tradition of citizenship and social transformation, allowing evolution and revolution. Thus a non-hierarchical view of leadership emphasizes the interdependent relation between modern democracy, dynamic culture and education that are each mutual preconditions. The claim that educational leadership is simultaneously a dynamic institutional, multilevel, diagonal, horizontal and vertical process, operating between different epistemologies and value-spheres, where the intersections between levels and interests may be described as negotiated discursive spaces, ultimately returns us to a theoretical definition according to which education stands in a non-hierarchical relation to politics, economy and culture. The very same structure explains how curriculum works, since educational leadership is meaningful as a dynamic relation between different levels.

So-called first order educational leadership refer here to teachers' leading the students' study activities in the teaching-studying-learning process. Second-order educational leadership shifts level and focus to principals' leadership of teachers' teaching activities and the school as a collective organization. Third order educational leadership again shifts level and focus to leaders who lead other leaders (e.g. municipal superintendents leading principals). Fourth order leadership involves developing and deciding about new national/state policies and curricula on a level authorized to establish laws - clearly a very different kind of educational leadership. As a result of recent political and economic aggregation processes, globalization, a transnational, fifth level of analysis as well as a cosmopolitan perspective has accentuated (Beck 2006). Typically contemporary Didaktik, varieties of curriculum stud- 
ies, leadership research and policy analysis and governance research have turned their main focus on typical one or two levels.

Accepting a multi-level approach to educational leadership and curriculum turns our attention not only to interaction between these levels but also interaction within them. Thus, the answer previously provided, i.e. the non-affirmative problem concerning the kind of influence educational leadership has, a non-affirmative approach was adopted. Educational leadership as non-affirmative summons to self-activity highlights that the education process is dependent on an experiential address, but that when leadership as summons is pedagogical the leader should consciously refrain from naively confirming either a prevailing or ideal future condition. With such a self-reflecting pedagogical discernment the leader is thought to be better able to create a space for an education process that recognizes the co-worker's selfactivity and right to exercise conscious independence of thought.

As we can see educational leadership as curriculum work, as evaluation, as school development etc. occurring within and between different levels this means that it is important to highlight mechanisms through which the political will formation and its results (ideas) transform or find their way, in cooperation with professional expertise, into a 'pedagogical agenda' by being communicated to practitioners. Curriculum/Didaktik typically identify several levels of activity, considered relevant for understanding the direct and indirect top-down/bottom-up dynamics between, within and across these levels. Leadership research has increasingly developed similar approaches since the 1970s (Meyer and Rowan 2006).

The question is now how to approach a multi-level, multi-centered and multiprofessional educational governance system given the fundamental theoretical positions developed above? Given the answers provided by a non-hierarchical view on the relation between politics and education as well as the non-affirmative approach of educational interaction we perceive of discursive institutionalism as offering a complementary and fruitful approach to understanding how educational policies, ideas and values (curriculum) relate to administrative processes on different levels beyond schools and districts/municipalities. As we see it, discursive institutionalism itself presupposes a non-hierarchical understanding of societal forms of practice.

According to Schmidt, discursive institutionalism aims at understanding how cognitive ideas (problems identification) and normative ideas (values that legitimize problems) are developed and communicated across societal, philosophical, policy, and program levels. This point is important for our framework in that it explains the interplay among societal values and aims, policies, and program interactions. The term 'discourse' refers not only to structure (what is said, or where or how) but also to agency (who said what to whom). Specifically, Schmidt argues that ideas operate as coordinative and communicative discourses. Coordinative discourses refer to policy construction among policy actors while communicative discourse refer to policy legitimization between policy actors and the general public.

These discourses operate within and between levels and in one of four directions. The first direction is top-down, meaning that policy elites generate and communicate ideas to the public, creating a master discourse, which inspires public debates and requires mediation. Second, discourses move bottom up, such as when social 
activists inspire mass protest against a policy or program, creating a counter discourse in mass media. Third, discursive interaction may remain solely at the level of civil society in the public sphere. Finally, there may be no "arrows" between coordinative and communicative discourses. Important questions are related to which way the arrows go-top-down, top-to-top, or bottom-up - and who is seen as the carrier of ideas (Schmidt). Schmidt's work on discursive institutionalism, thus, enhances our understandings about how curriculum as ideas and discourse may be communicated (and by whom) within and across macro (e.g. societal, policy) and micro (program, school) institutional levels.

Through vertical negotiations, political will transform into a 'pedagogical agenda' (policy) by being communicated to practitioners but agents' perceptions and enactments are also communicated back to the policy formulating process. The vertical discourses sometimes passes levels in between, like when transnational organizations like OECD communicate learning results directly to the schools without communicating these results to national authorities. The PISA process also demonstrates how result of evaluation is intended as influencing future curriculum work. Horizontal dialogues occur one all levels, including cooperative discourses within each level, but also between politicians and the administration.

Schmidt's discursive institutionalism provides a new point of departure to consider the "institutional" or "structural" dimensions, particularly those that are evidentinlaterdeliberative and discourse-oriented curriculum theory. Discourse-oriented curriculum theory provides a language for talking about the human interactive and interpersonal dimensions of any level, from classroom to transnational. Discursive institutionalism acknowledges the system-laws, policy documents, task descriptions, financing systems - that lies beyond individuals' everyday leadership practices. This position is coherent with the non-hierarchical view of the relation between societal practices in a democracy. The non-hierarchical point of departure offers us the principled position or rationale to approach both interactionalindividual and institutional structural levels from a discursive perspective. At the same time, with its grounding in public administration, Schmidt's discursive institutionalism does not have an underlying educational language or theory of education. Thus, we consider deliberative and discourse-oriented curriculum theory and classic concepts from educational philosophy described previously (recognition, summoning, Bildsamkeit,) as well as discursive institutionalism in our framework.

Coherent with discursive institutionalism, discourse-oriented curriculum theory, and the core concepts of modern educational theory described above, a curriculum includes the view of a curriculum as text as a systematic interruption in the practitioner's way of understanding herself and carrying out one's professional tasks. We see curriculum work as an interruption in the Other's relation to herself, other persons and the world (Honneth 1995).

This recognition-based Hegelian philosophy provides a general frame for understanding how the curriculum itself as well the construction process, works, and is used, as a pedagogical intervention in order to influence. Here leadership influence does not mean support for implementation of ready-made ideas but invitation to dialogue. In doing so, educational leadership as curriculum work recognizes the 
subject as radically free as this makes him/her able to transcend what is given. But the position also acknowledges the necessity of the subject's own agency as a necessary requirement for transcending a given state. The effects of a curriculum activity are, obviously, also in the hands of the receivers enacting these intentions. The curriculum making discourse as invitation to self-activity and self-formation creates spaces within and between institutional levels. By introducing these concepts, we create a more coherent language for school/district and curriculum work, educational leadership and school development activity, using the very same concepts that are relevant in describing the core object of curriculum theory and Didaktik, the teaching-studying-learning process. At the same time, we recognize that Schmidt's discursive institutionalism has been developed and applied to multiple levels of institutions and their structural dimensions within the nation state. Thus, we need to consider transnational organizations and relations through a combination of lenses, including organizational theory, new and neo-institutional theory, actor network theory, and world society theory.

\section{Cosmopolitanism as an Educational Ideal and as Transnational Relations in an Increasingly Plural World}

The third topic relates to cosmopolitanism and its necessary counterpart, i.e. individual and national identity, and citizenship. In the beginning of our chapter, we observed that we live through an era where the majority of nations worldwide internally have developed towards increasing plurality while being simultaneously framed by challenges that call for a cosmopolitan view. From being an abstract idea the relevance of a cosmopolitan perspective has developed into an everyday and self-evident issue through globalisation. However, educational theory, leadership and curriculum research has only recently and still to a very limited extent developed a reflected position in this matter. The nation-state perspective have dominated.

With this twofold challenge, how should we conceptualise education, leadership and curriculum in order to make sense of these phenomena in a contemporary cosmopolitan perspective including increasing local diversification? Further, how should we establish a notion of education, leadership and curriculum simultaneously relevant both for local, national and global citizenship in a post-national, neocosmopolitan era? In our understanding this dilemma mainly falls into two different parts: cosmopolitanism as an educational ideal and cosmopolitanism as transnationalism reflecting dynamics between states and between states and transnational aggregations or various kinds.

While the first has long historical tenets, the second presents itself as a rather recent topic. While cosmopolitanism as an educational ideal points at aims, contents and methods of education, i.e. curricular questions, cosmopolitanism as trans- 
nationalism points towards new features of contemporary educational leadership. Just to mention a too obvious example, transnational organisations like OECD influences and directs educational practices through the initiation and organization of international evaluations. New mechanisms and governance forms force traditional educational leadership research to develop new languages for dealing with the situation.

In the first part of the problem we meet the dilemma of cosmopolitanism as an educational ideal. A first dimension of this is what it means to have or accept cosmopolitanism, or world-citizenship, as an educational ideal? A second dimension of this problem is how cosmopolitanism relates to differences between nation states and the various educational aims they promote? Is it possible to unite different nation-state policies with a single cosmopolitan ideal? Do different versions of cosmopolitanism presuppose different versions of nation state education policies, or the other way around, is any version of nation state policy congruent with any version of cosmopolitanism? A third dimension related to increasing plurality within each nation state. How do national education systems approach increasing intrastate ethnic, religious, linguistic and ethnic/racial plurality - by differentiation/ diversification or unification and coherence making? As before religions unite peoples and individuals beyond nation state borders.

The second part of the problem concerning cosmopolitanism and education, curriculum and leadership is both practical and principled. It concerns how we conceptualize and empirically describe educational relations between and among nation states. Here we ask: How do we explain curriculum making and educational leadership relations within and between nation states, i.e. transnational, in an increasingly plural world? What kind of relation are we addressing?

Contemporary educational policy research has been active in approaching globalisation and the drivers in this process. This research has been successful pointing out globalized economy supported by technology and neo-liberal politics as driving forces in globalization. Often educational policy research has pointed out counterproductive consequences and other worrying effects of these developments. We highly appreciate this research although approaching the issue differently. The policy oriented literature is not always explicit concerning education theory. In curriculum theory, on the other hand, we see more clearly that the connections between globalization, cosmopolitanism and education tradition is much more present (Autio 2013; Hopmann 2015; Pinar 2011, 2013). Sociologists and philosophers of education were also early in bringing the cosmopolitanism anew (Beck 2006; Kemp 2010; Roth and Burbules 2007; Biesta 2009). In explaining educational leadership and curriculum work at a transnational level, our position again takes a non-affirmative position and draws upon the core concepts from Hegelian traditions, modern education theory, and later developments based upon subjectivity and intersubjectivity at the nation state level (i.e. recognition; summons to self-activity and Bildsamkeit) as well as discursive institutionalism. At the same time, we explicitly consider the contemporary globalized situation, looking at how these concepts apply in a drastically different context of relations between and among international organizations and increasingly pluralistic nation state. 
Cosmopolitanism as Universalism in Educational Research and Theory When saying that cosmopolitanism, as a version of universalism, always goes together with particularism, it is a proposition that is relevant for epistemology of education, i.e. the assumptions featuring our theories. Do we, for example, imagine our theories being of universal validity over time and cultures or do we see them as regionally limited? Now and then we still meet characterizations of the Western scientific tradition, obviously including social sciences, representing unreflected universalist knowledge ambitions as well as cultural insensitivity demonstrating versions of naïve ontological realism and representational epistemology. This view would have decentered recently, first in the 1960s with new insights developed in relation to the concept of paradigm (Kuhn, Lakatos, etc) and later with postmodern epistemology (Lyotard, etc.). However, in education, a non-foundationalist approach was developed early on by those classics being referred to here. In his classic lectures on education of 1826 Friedrich Daniel Ernst Schleiermacher asked the question concerning how far educational theory can be culturally universal. Being both one of the founders of modern hermeneutics, recently rediscovered, translated into english and today not only read through Dilthey, and a crucial contributor to culturalhumanistic pedagogical theory, it is not surprising that his own conclusion was negative: a theory of education is always relative to its own cultural context (Schleiermacher 1998), a position later supported by Dilthey (1883) and all subsequent anti-essentialist theories. A cultural space is thus dynamic, a map is a reconstruction of experiences given discourses and interests, rather that a representation (Saari 2012). In fact, according to Schleiermacher conceptual development and reflection takes off with life-world experiences. On the other hand, accepting the impossibility of a universally valid position does not have to mean we accept a view of theoretical pluralism where all theories are of equal value. Accepting epistemological pluralism rather changes the nature of the dialogue: it requires from participants even greater sensitivity to alternative views. With this must come an openness to different contemporary forms of discourse but also engagement in historical contributions in order not to loose the sight of historical roots. In this sense theorizing is reconstructive in nature.

Cosmopolitanism as an Educational Concept Cosmopolitanism as a theoretical idea has gone through many phases since it was introduced by the greek philosopher Diogenes (412-323 b.Chr). Diogenes conceived of himself not coming from any specific place but that his worth as human was not connected to from where he came. Before and after the Greeks cosmopolitanism and universalism has appeared in different shapes (Papastephanou 2016). Today being a cosmopolitan has an empirical, concrete meaning attached to it, intensified by the most recent globalization movement and related societal trends as well as technology (e.g. neoliberalism, population migrations, demographic shifts toward increasing plurality). In other words, we can, so much more easily than the greek Stoics for which cosmopolitanism was merely an abstract idea, think about the world as a real, common place in which we all are included. Kemp (2010) points at three core issues for today's cosmopolitanism: how does economic globalisation relate to democratic control of 
economy and technology, how to deal with conflicts between national or culturally related interests and challenges connected to sustainable development and, finally, how to deal with global responsibility?

Kant defended what he termed the universal cosmopolitan condition as comprised of two fundamental rights: the right of a state (Staatsrecht), which lays down the fundamental norms of rational political authority in the form of a rightful civil constitution and international right (Volksrecht), which sets out the basic principles that govern relations between states. The "right of peoples" (Volksrecht, international right) expressed a wish for peace, i.e. to avoid wars. Further, in addition to international right regulating conflicts between states Kant also defended the need of cosmopolitan right in terms of basic ethical existence. Cosmopolitan right defined an ideal equal for all. It was claiming that all rational beings, irrespective of their origin or race (some allusion to culture), should be regarded as ends in themselves and as also as lawgiving members of the universal kingdom. For Kant, cosmopolitanism was a moral orientation that provided the rationale for states to enter into a permanent peace treaty and confer rights of universal hospitality. In other words, all citizens would still belong to and reside in their own republics. Thus, while Kant sought to extend republican political philosophy into a wider framework beyond the relatively limited modern notion of the nation state, he did not completely abandon the importance of the nation state role. So recognized by a nation state any human being could therefore not be made use of by anybody else and their interests.

In his seminal work Cosmopolitanism and the Nation State, Meinecke (1970) captured the decline of the nineteenth-century cosmopolitan idea with the rise of the national idea. Meinecke captured Kantian cosmopolitanism and the so-called cultural cosmopolitanism of Enlightenment intellectuals and elites who did not succeed in translating their vision into a viable political order for the time. Further, Kantian cosmopolitanism did not explicitly address interculturality and other cultural dynamics (e.g. background, habitus) involved in relations between different cultures.

Education and the Establishment of the Nation-State As observed previously, the roots of European Didaktik are typically traced back to Comenius and his Didactica Magna from 1657. The establishment of modern Didaktik may be seen as a response to a need of the embryonal developments of a new school system providing the state with new competencies, but also resulting from new technology enabling mass schooling, i.e. the printing press. The format of the state before the modern nation state coming to existence was based upon an absolutist view of power demonstrated in monarchies. Mercantilism was the dominating economic paradigm primarily designed to securing the incomes for the state, military power being one of the main drivers. These earlier states were typically multi-ethnic although often one cultural group was the dominating. While territorial issue were central borders could change with marriage between monarchies. The teaching of reading cared for by the church started more systematically during mid seventeenth century in European countries, was very much connected to developing a capacity to perceive the meaning of (holy) texts. Teaching to write, i.e. productive communi- 
cative capacity around texts, was developed 200 years later, with the establishment of elementary schools. During the seventeenth century also significant expansion of universities occurred, with the aim to provide the state with workforce.

Herder was one of the first modern cultural theorists to use the plural version of cultures, considering particularly issues related to language (Herder 2002). In Herder's view, the movement toward the establishment of modern nation-states (eighteenth to nineteenth century) required a new approach to establish and sustain a relation among national, collective unity (sense of belonging) and individual and regional plurality. On the one hand, in the eighteenth and nineteenth centuries, this involved the task of building a single, new identity called the nation, encompassing several identities and affirmations. The relationship of new nations to pre-existing cultural identities was in part to be one of transcendence from cosmopolitanism to 'nation'. On the other hand, in agreement with Hegel, Herder argues that this very conception of encompassing diverse identities - social and regional, linguistic and cultural-meant giving a key role to the state through which the composite nation was to be created. These foundational social philosophers, then, lay the groundwork for understanding policy relations between nation states and basic human relations; however, they do not explicitly discuss the role of habitus or the ethical dilemmas that emerge from curriculum work across nation-state borders.

In a recent project exploring ethics in international curriculum work, Helfenbein (2010) explicitly considered ethical dilemmas in curriculum work occurring in various projects across borders defined by nation states, ethnicity, culture, and class. Helfenbein (2010) draws on Blackburn's (1996, p. 126) notion of ethics (i.e.the study of moral judgments and actions), Foucault's (1997) turn to ethics in his later writing, as well as critical geography to pose new questions revolving around ethical concerns about spacial relations, including power and identity. In our view, Helfenbein (2010) raises an important argument that while geographic borders have materiality associated with them, geographic places and spaces are socially constructed with real effects on lived experiences that are ethical in nature.

Hegel, in turn, defended the necessity of the nation state as the embodiment of ethical existence. For Hegel, the state is the last of three ethical components-the family, civil society, and the state, which together constitute the normative order known as ethical life. As the third ethical component, according to Hegel, the State should be seen as the pinnacle and as following logically from the other components. The state, then, is what allows human beings to take their place in the historical development of universal mind, and, hence, to make them capable of ethical living. Hegel also writes about the actual state where aims toward unity are viewed as a necessary evil. Hegel, thus, saw the state and its institutions, as part of the ongoing historical process of actualizing freedom, a kind of teleological philosophy of history.

Drawing on his Hegelian roots, John Dewey (1959) wrote about a similar harmony between people's particular and universal interests. For Dewey, as for Hegel, one must act in absolute cultural freedom; work, community, and the nation state must not confront an individual as alien but rather should be integrated with the self. Although not explicit, Hegel and Dewey use the term culture as singular for all 
humans, toward in Hegelian terms, the development of universal mind or the nation state. At the same time, Hegel argued that the nation state reconciles particularism (the individual) with the universal.

To conclude, today we do not have any global parliament or global state for which education would prepare. However, would some predefined idea of worldcitizenship be considered an ideal for education to strive towards this might result in an affirmative position, making contemporary education a tool for aiming at and reaching of a future state of art. Yet, what we do have is an increasingly strong awareness and understanding of sharing the planet or the globe as a place where we all live. Among other things, our increasing knowledge about the universe, cosmos or solar system has made us more aware of this than ever before in human history. While there is no global political state in which we would own or have citizenship the human population on earth form, to various degrees, a 'global community' that exceeds the borders of existing nation-states. Making use of Ferdinand Tönnies' (1855-1936) differentiation in 1887 between Gemeinschaft (community) and Gesellschaft (society), we may see Gemeinschaft as "a social group united by common beliefs" and Gesellschaft "a social group held together by practical concerns, formal and impersonal relationships". Given such definitions we can see that the experience of sharing the world as a common place for all known human life is certainly a concern of practical nature, but so is it about holding together with respect to common beliefs, let this be on a very general level. Now, given that contemporary institutional education aims at preparing the individual for a life in community and in society, i.e. for different "citizenships", this is valid also from a global or cosmopolitan perspective. Educating for globopolitan life is thus relevant although no global state exist. Educating for a life and living together on the earth exceeds traditional education for community and society framed by a notion of the nation-state. From an education theory perspective our general point of departure in this issue is not to make use any positive definition of globopolitanism as an ideal that narrows down how the future should look like. Rather, following e.g. Schleiermacher's definition of ethics, we have to learn to live with the question of globopolitanism as an open one.

Universalism and Particularism: Multiculturalism Philosophers and education scholars have long considered issues of identity in terms of the Bildung process occurring through 'experience', a conflict ridden process in the course of which a spiritual being discovers her own identity or selfhood while striving to actualize the selfhood. In many countries, scholars and educators have responded to the contemporary dilemma of increasingly culturally diverse student identities and universalism vs. particularism with multicultural education. Both US and European versions of multicultural education are frequently rooted in transformative sociological perspectives whereby schools play a role in overcoming social and political struggles against racism and other related 'isms'. By some contrast, European versions of multicultural education are often grounded in issues of nationality and language more than race and ethnicity; nonetheless, at a conceptual level, this literature is similar in that it is grounded in a social transformation perspective and a modernist 
critique of educational norms and values that privilege dominant groups and disadvantage traditionally marginalized groups (e.g. Gundara 2000; Portera 1998). Both North American and European scholars offer similar typologies and a range of pedagogical forms or approaches directed primarily toward either a particularist approach aimed at students from specific ethnic/cultural groups or a universalistic approach for all students irrespective of ethnic/cultural origin. The particularist approach is often supported by acceptance of positive discrimination, allowing for differentiation in favour of individuals or groups.

Drawing on critical theory notions of oppression, Sleeter and Grant (1985) posited four educational programs now popular in the United States and elsewhere (Human Relations, Teaching the Culturally Different, Single Group Studies, and Multicultural Education), all of which are grounded in an assumption that society will eventually change if we change the consciousness of individuals (children) through multicultural education and culturally responsive pedagogy. Within these approaches, then, there is also a range of emphasis on particularity over universality. For example, Single Group Studies support traditionally marginalized students as future citizen who will be educated to bring about structural and cultural changes in society for the benefit of "their historically oppressed group". This approach includes such programs as Black Studies, Chicano Studies and Women's Studies; it explicitly teaches students about the history of the group's oppression, how oppression works today, and how the culture the group developed within oppressive circumstances. Although embraced as particularized struggles within multicultural education, such single group studies can have a separatist emphasis and lead to dependency or tribalism.

In response to the separatist problem, Banks (2002) proposed multicultural education that teaches all children about cultural pluralism and diversity, as well as making sure all achieve well. Similarly, from a European perspective and responses to the needs of increasing immigration and population migrations, Gundara (2000) explained, multicultural education can be directed at all pupils (a universalistic approach). Across European multicultural education literature, scholars focus on the idea of nation and citizen as well as language diversity (e.g. Banks and Banks 2009; Gundara 2000; Portera 1998). In the wake of population shifts and more open immigration policies in many countries, the ideas of nation and citizen have to be revisited, in order to clarify not only rights, but also duties, and to develop social democratic norms for all citizens of a country (Schnapper 1994).

Multicultural education approaches have been recently tested or described empirically, examining various ways in which teachers respond to the needs of culturally diverse students in classrooms. For instance, Ladsen-Billings (1994) coined the term "culturally responsive pedagogy" in her now classic study of eight exemplary teachers of African American students. This 'pedagogy of opposition' drew on anthropological theories that noted a mismatch between students from culturally diverse backgrounds and their teachers, particularly in terms of language and verbal participation structures. Building on Ladson-Billings' study, Cooper (2003) investigated the practices of effective White teachers of African American students who had been nominated by the Black administrator and parents of their predominately 
African American schools. She found that they adopted many of the practices of effective African American teachers, including being "warm demanders" who held high expectations for academic achievement and became second mothers or "othermothers' to the children in their classrooms. In a similar project focused on Latino(a) children, Moll et al. (1992) referred to funds of knowledge as "the historically accumulated and culturally developed bodies of knowledge and skills essential for household or individual functioning and well-being" (p. 133).

While we noted differences in approaches to multicultural education from one country to another and from one area of focus to another, an ongoing harmonization of multicultural education may be identified. Across national contexts, discussions of multicultural education are frequently centered on questions of how far cultural diversity threatens the unity of society, and to what degree multicultural education programs for an increasingly pluralistic population of students hampers or encourages the realization of equality of opportunity for all students. However, these more universalistic approaches come to the same point of struggle: how to respond to increasing student plurality and yet retain a human relations approach that recognizes national and global unity for all students and perhaps dependency for some? As Helfenbein (2010) reminds us, we have to think simultaneously at the scale of the universal and the particular. While multicultural education research has provided important pedagogical understandings, these scholars have not used recognition and other modern educational concepts that may be helpful in the contemporary situation. At the same time we ask, what kind of postnational pedagogy might be important to educate for an aim of recognition? In our view, Benner's (1991) approach to recognition emphasizes initiative as well as need, an approach that may overcome concerns about inequities and dependency in particularist approaches.

In educational leadership studies, a growing number of scholars have applied multicultural education perspectives and culturally responsive pedagogy to leadership. (e.g. Johnson 2006; Scanlan and Lopez 2012). According to Johnson (2006), culturally responsive school leaders support academic achievement, work to affirm students' home cultures, empower parents in culturally and economically diverse neighborhoods, and act as social activists who advocate for societal change to make their communities a better place to live. Using a broader lens about culturally responsive leadership, Scanlan and Lopez (2012) conducted a narrative synthesis that examined 79 empirical studies related to best practices to promote equity and excellence for culturally and linguistically diverse students. Scanlan and Lopez (2012) concluded that principals serve a role as policy mediator; they need to "cultivate language proficiency, provide access to high-quality teaching and learning, and promote the socio-cultural integration of all students" (Scanlan and Lopez 2012, p. 149). Similarly, in a Nordic/European context, Moller (2009b) and Kalantzis and Cope (1999) write about leadership for democratic education arising from research at the intersection of educational leadership, critical theory, and critical multiculturalism. Such theories are rooted in social justice, equity, and the common good (e.g. Dewey 1916; Freire 2000). In Moller's (2009) view, most definitions of education for culturally responsive practice and democracy include themes such as recognizing the basic value and rights of each individual, taking the standpoint of 
others into consideration, deliberation in making decisions, embracing plurality and difference, and promoting equity and social justice. Multicultural education literature, culturally responsive pedagogy, and its application in culturally responsive leadership deal with social interactions and empirical realities around universalism versus particularism in schools. In the next section, we move back to empirical realities in literature on interstate relations in policy research.

Interstate Relations in Policy Research: Empirical Cosmopolitanism Current debates regarding international relations in policy research draw attention to the revival of a Kantian ideal as relevant in the present context of globalization, the socalled crisis of the nation state, and the need for a global, civil society (Bohman and Lutz-Buchmann 1997). The notion of cosmopolitanism put forward empirically in transnational relations is responsive to internal nation-state policy dynamics and responsive to a world system (Wallerstein), world society (Luhmann) or world polity (Meyer). Closely related, Rudd and Goodson (2016) developed a concept of 'refraction' as a holistic approach to understanding the empirical interconnectedness among structure, agency, ideology, history and individual beliefs. Here Rudd and Goodson (2016) highlight the areas of foci evident in particular historical moments of educational reforms across the UK.

Policy scholars have been studying recent globalizing curriculum policies through varying (and often Western) epistemologies and theoretical logics, frequently drawing on Luhmann's (1996) self-referential social systems theory and Wallerstein's (1974, 2006) world systems theory. According to Luhmann (1996), society can be interpreted as a functional integration of systems that follow an internally independent regulation that must accommodate the external requirements of other systems. Here society as a whole is defined by the medium of communication. Luhman extended his theory to the global level when he argued that communication had become globalized, meaning that society had to be analyzed on a global level within which states and other systems operate and regulate relations (Luhmann 1996, 2012). For Luhmann, then, educational theorizing is understood largely as a self-referential system of reflection rooted in distinct contextual conditions, intellectual traditions and value systems with respective systems of reference and contexts. Applying Luhmann (1996) to policy borrowing and lending, Steiner-Khamsi (2004) argues that borrowing (e.g. increasingly global core curricula) does not imply an exact duplication of reform; it draws our attention to processes of local adaptation and modification. Here contextual conditions are emphasized in policy communications, but there is little attention to critical geography and interculturality or communication between different cultures in these contexts.

While Luhmann based his theory of world society on Parsons, another seminal theorist Immanuel Wallerstein (1974) developed his world system theory out of a critical reading of Marx. For Wallerstein, the 'world system' is essentially the world economy emerging out of the rise of the west and the dominance of a single center over the periphery. Here cosmopolitanism concerns less the homogenizing capital world economy than the different combinations of periphery and centre. Wallerstein claimed that any social phenomenon of the present had to be studied in the context 
of the world system. However, instead of a functional theory, Wallerstein proposed a structural theory of power, extending Marx's distinction between capital and labor to the global level. Specifically, Wallerstein (1974) conceived of a world system as a multicultural territorial division of labor that leads to the existence of three interdependent regions: core, semi-periphery, and periphery. The core-periphery relationship is structural, one with a power hierarchy in which powerful and wealthy "core" societies dominate and exploit weak and poor (e.g. colonized) peripheral societies. The semi-periphery is supposed to mediate conflicts and to give the periphery hope for upward social mobility. Luhmann's functionalist systems theory and Wallerstein's world systems theory have both provided significant, if differing, theories and logics for understanding the structural dynamics between systems and their differential power structures, but these theories are not explicit regarding the discursive nature of relations, particularly as these may apply to education in a plural world.

Schmidt's (2016) discursive institutionalism as an analytical framework has largely been considered within nation states; however, her most recent conceptualization of DI focuses on the EU as a 'region-state', providing an important discursive strategy for understanding dynamics and relations between states as well as between states and networks. Again, discursive institutionalism aims at understanding how cognitive ideas (problems identification) and normative ideas (values that legitimize problems) are developed and communicated across societal, philosophical, policy, and program levels. Using discursive institutionalism, Schmidt argues that the substantive content of the idea 'region-state' serves as a lens to illuminate the EU in its current (emergent) development as a new international organizational form, one that has never been observed before as well as in relation to a line of development from existing supranational entities organized by and above the nationstate. Here discursive institutionalism frames Schmidt's conceptualization of the EU as a region-state in terms of the concept itself along with its discursive use of that concept to propose new ways of engaging in the EU based on the insights about the EU that emerge from the region-state concept" (p. 4). What the concept 'regionstate' offers for the EU is a new, readily understandable term capable of encompassing what it has become and what it is becoming" (Schmidt 2016, p. 2). Specifically, in the example of the EU emerging as a new international organization, Schmidt examines the substantive content of the EU as it is being and becoming a concept of 'region-state' characterized by shared sovereignty, variable boundaries, composite identity, highly compound governance, and fragmented democracy in contrast to sovereignty, boundaries, identity, governance, and democratic legitimacy as the main markers of the nation state. With Schmidt's approach in re-naming the EU as a 'region-state' we find a discursive strategy to understand the development of new ideas and global discourses within a new international organization and between nation states, a strategy that we argue is relevant to understand relations and dynamics regarding transnational curriculum policies. Still, we argue that Schmidt's discursive institutionalism does not have an underlying educational language or theory of education to explain pedagogical relations amidst increasing cultural plurality at all levels, including the transnational level. 
We see points in all of these theories that make a valuable contribution to understanding the current empirical realities as well as important missing dimensions. First, what is missing is a dialogue among these three perspective, all of which are valuable where the cosmopolitan moment may be conceived of as a creative combination of different forces-centre, periphery, and semiperiphery, the local and the global, and discursive institutionalism within and between institutions. While Luhmann's systems theory and Wallerstein's world system theory have contributed important, if differing, approaches to understanding relations within and between nation states and social systems generally, we also find a lack of attention to an educational language or the cultural dimensions of relations. This is where the nonaffirmative framework makes a valuable contribution. We recognize the structural and processual contributions of world society and world systems theories and position ourselves in relation to these. Further, we draw upon interculturality and discursive institutionalism especially as it emphasizes the cultural and ideational dimensions of discourses, respectively.

\section{Educational Core Concepts for Understanding Intercultural and International Relations}

We have discussed the core concepts of modern education theory at length in response to our first question regarding educational and pedagogical influence. In this section, we revisit these core concepts as well as interculturality concepts from cultural anthropology (e.g. Mead, Geertz, Wimmer) as these may provide an educational language for transnational and international relations beyond the language from policy studies. Again the core concepts from modern educational theory (Benner 1991) are:

1. Recognition - how the Self is aware of the Other as being free,

2. Summoning to self-activity - how a teacher or a principal has a mediating role with respect to the Other in the maintenance and development of the Other's self-relations;

3. Bildung (Bildsamkeit) - realization of the individual's possibilities through own conscious efforts aimed at making sense of the world and her experiences.

Recognition, summons to self-activity, and Bildsamkeit are fundamental categories for understanding intersubjectivity or what it means to become human (Fichte, Hegel). Interculturality, or communication between individuals (e.g. policymakers) from two distinctly different cultures, derives its role from the sphere of intersubjectivity According to Fichte, man should understand that, starting out from his own transcendental subjectivity, he discovers another self-consciousness in a process in which he is 'summoned' by 'the other' to jointly produce a third, a medium of the 'mind' in which both his own and his other's subjectivity are suspended. Hegel (1807) also theorized about intersubjectivity, arguing that 'self-con- 
sciousness exists because it is a self-consciousness in and by itself to another, meaning that it exists only if recognized by another.' In Fichte's view, adopted by Hegel, when attempting to view itself as an independent entity, the self must recognize the other as an equal subject so that, in turn, may be recognized by the other. Habermas (1981) expanded on Hegel's modern philosophy by drawing attention to the dialogic character of recognition and how communicative relations and actions can be distorted by force. Interculturality, grounded in cultural anthropology, furthers these theoretical propositions of intersubjectivity by considering the cultural dimensions of intersubjectivity. Specifically, interculturality focuses on relations among different cultural groups where the life-world is culturally different or not permanently shared (e.g. Mead et al.).

In a lecture connecting culture with intersubjectivity and recognition, Wimmer (2013) expanded upon Geertz' notion of culture as a system expressed through symbols that allow groups of people to communicate within and between cultures. Specifically, he conceptualized culture as an open and unstable process of negotiating meanings with three closely connected concepts: (1) the internalized culture of an individual, which forms the basis for processes of negotiation or habitus; (2) the common perceptions of the nature of the world as a result of those processes of negotiation (public-cultural-collective representation); and (3) cultural practices that represent the limits to social groups within which the processes of negotiation take place (cultural distinction). In other words, Wimmer (2013) argued that interculturality involves recognition; recognition is a foundational basis for multiculturalism and the coexistence of different ethnic or cultural groups in one country (nation state) as well as between individuals from two different countries with varying levels of cultural capital and power (p. 407). Here Wimmer's conceptualization reminds of Bourdieu's (1977) theories of cultural capital, habitus, and fields of power. Cultural capital and habitus affect intercultural relations as individuals use their own cultural capital as well as dominant societal norms to navigate policy relations and the education process. In our view, these perspectives on culture and interculturality have implications for educational relations at the transnational level as well as within nation states and their institutions.

At the same time, application to educational relations at the transnational level may be a hybrid version of both affirmative and non-affirmative perspectives. This means that, within a more mandated circumstance, educators/policymakers/international organization leaders' direct their own and others' (e.g. policy counterparts in other nation states or international organizations, state or region leaders, school/ district leaders, teachers) attention toward the relation between the experiential structure and dimensions of the policy ideas, requirements, and other content. In some similarity and contrast to the other four levels (classroom through nation state levels), educational leadership involves recognition and other human interpersonal practices. At a transnational level, recognition and interculturality are foundational to these relations. Again drawing on Benner's (1991) proposition, education would, then, be to recognize each leader or policy-maker in other countries as if he or she is already capable of doing what he or she is supposed to become capable of - and to act accordingly, Yet in the circumstance of transnational relations, there is also a 
potentially coercive relation or dynamic to these relations. From a non-affirmative approach, educational leadership and curriculum work may be focused on creating transnational policy cultures where international organization members/learners learn about what it means to position him or herself as both national and global citizens of democracy. Here the organizational member/learner learns to make use of his/her own productive freedom.

In some instances, one nation state organization and its results may "summon" another member to reflect its curriculum or evaluation system. Such relations, as a special form of curriculum (culture), are not directive or normative, but they contribute to global curriculum dialogue. Here discursive institutionalism also becomes important in, for instance, in international organization members' use of knowledge (ideas and content in transnational curriculum and evaluation policies) about curriculum and evaluation policies that may support mutual understandings or raise questions between nation states members with their differing cultural backgrounds, underlying values, and interests. Using the example of Schmidt's (2015) conceptual naming of the EU as a region-state, the very naming of transnational policy interests, knowledge and values (e.g. OECD), can illuminate concepts that support understanding transnational curriculum and evaluation policies, summoning activities grounded in affirmative and non-affirmative perspectives, and the development of international organizations around these. Such discursive relations may result in learning on the part of the Other as policymaker or international organizational leader or educational leaders at other levels within nation states, but this is an open question. At the same time, we recognize that transnational organizations in particular ways.

While we argue for the relevance of an educational language for transnational relations in a plural world, we must ask if these modern educational concepts are sufficient to understand the dynamics and relations at the transnational level. Such questions require a comparative study and, in our view, a new methodology. In a Hegelian tradition, the state guarantees that you are recognized so subjectivity is possible. In other words, the state expresses who people are. We ask: is this Hegelian tradition sufficient to understand human (e.g. educational) relations between nation states?

\section{Toward a Non-affirmative Hermeneutic Approach for Studying Educational Leadership, Curriculum and Didaktik}

In this section, we conclude our initiative by some reflections regarding epistemology and research methodology for studying educational leadership in a broad understanding of the concept. In this chapter we initially pointed out that large portions of research into leadership, curriculum and Didaktik have been framed by and grounded in various epistemological positions (critical theory, versions of positivism, and hermeneutics, postmodern positions). Often research is also profiled by 
identifying it representing certain methodology (discourse analysis, phenomenology, narrative research, etc.). It is indeed vital to establish a clear understanding regarding epistemological and methodological issues. However, as we have argued, research may also be guided by theory regarding its object. What do we understand by education in the first place? From our argumentation in this chapter it should be clear that we think we have been able to identify a very fundamental level of theorizing not only educational leadership, but also Didaktik and curriculum studies. We also think there has been several reasons to why re-theorizing these fields have been motivated.

The level at which we locate the non-affirmative theory of education is both foundational, and it is general, yet non-foundational in its epistemological attitude. The strength is obviously that we take our point of departure in the question of public and institutional schooling in our globalized world. We have also shown how we locate ourselves in the western, modern tradition without assuming any epistemological primacy of it (Paraskeva 2016). We have pointed out that the modern classics speak to us with convincing arguments, but that some of these connections have become forgotten. We also recognize the visionary qualities of these classics: while these theories were developed parallel or in relation to establishing modern nation states, these were years still far from anything like political democracies. The seminal ideas of modern education we have laid out were developed around a century before the introduction of the any early introduction of democratic popular vote (e.g. in 1906 in Finland) and it is still relevant more than 100 years after its introduction.

When saying that this position is epistemologically non-foundational it is not an acceptance of relativism nor that the theory advocated is a universal one. Universal theories might be necessary, but they are considered, in principle, impossible to achieve in education (Uljens 2002). This insight in the relative validity of education theory is not anything that has resulted from late-modern thinking (Rorty 1989), rather it has been understood within the discipline since long. Still we want to acknowledge that the late-modern period in philosophy helped us to clearly realize the limited validity of such a theory across cultures, partly representing other epistemologies. We see possibilities with critical hermeneutics for understanding Bildung and education in a globalized world (Kristensson Uggla 2010).

We think there is a value in engaging in an as-if foundational thinking in education theory given that foundational theorizing may be non-foundationalist regarding its epistemology stance. Maybe even more today than before. With the rapid expansion and differentiation of the pure quantity of research in addition to the urgent need for educational leadership research, curriculum studies, and policy research to support societal developmental work at different levels, combined with high pressure on publishing activity may have resulted in loss of theoretical groundwork and anchoring. We hope to some extent have met this need with this volume. Also we want to recognize the yet high quality of research on the various fields we reviewed. The task has not been easy and we hope we have succeeded in doing justice for work done. 
This project was initially driven by an European-American comparative interest (Uljens and Ylimaki 2015). We believe having pointed out that this theoretical framework recognizes common roots to American and European education theory and research, this hopefully for its part will enhance future dialogue. In a global perspective, for European and American research on curriculum, Didaktik and leadership to be able to communicate with e.g. Asian or Chinese traditions requires profound insights in one's own tradition.

We have also pointed out limitations we feel this foundational theory approach has for empirical research. The limitation does not consist in a conceptual weakness, although many aspects may be developed further. Rather, the position lacks conceptual structures for grasping the organisational dimension of institutionalized education. Second, we feel that discursive institutionalism offers us a policy language that does not emanate from education theory, but through which educational phenomena may be more easily understood. Third, increasing pluralisation of cultures within nation states across the world require us to develop a sensitivity which certainly was not present among philosophers of education 200 years ago, although the principles developed make sense as a foundation for this research. Fourth, as so much theory construction also education theory development has been carried out with the nation-state as the frame of reference. By our analysis of cosmopolitanism, and as we have called it, globopolitanism, we address questions and invite readers to rethink the relation between the global, the state and the local from an education theory perspective. Finally, we see educational leadership as truly a multi-level phenomenon where leaders mediate between and across levels.

We have also pointed out that an organizational perspective is required to grasp both educational leadership and teaching. In addition historical and cultural awareness and a policy perspective is considered necessary. In this respect we support a Habermasian awareness of both the systems-perspective and a life-world perspective. Throughout arguing for the non-affirmative position we have pointed out that at all levels of the education system there are individuals involved. Education cannot be understood without ethics, as supporting growth of ethical reasoning among learners is a topic of education but also as ethics is constitutive for educative activity itself. For these reasons we think that Ricoeur's (1989, pp. 114ff.) view of the relation between phenomenology and hermeneutics is valuable i.e. critical hermeneutic phenomenology. This position accepts the problem of meaning as the fundamental one both in interpretation theory or hermeneutics and phenomenology, but also in policy and education in general as well as in leadership. As Ricoeur (1989, p. 114) notes, in order for meaning to become a hermeneutic problem "the central question of phenomenology must be recognized as a question of meaning".

The problem of meaning in phenomenology refers to the nature of an experience, which again has a political, historical, cultural and a lingual aspect as discussed in Ricoeur (1989, p. 115). A second perspective on the relation between phenomenology and hermeneutics advocated by Ricoeur and conceived of as relevant here, is the distanciation from the "experience of belonging" (ibid., p. 116). That is, there is a connection between the hermeneutic concept of distanciation and the phenomenological epoché (bracketing), as long as the epoché is conceived of as "the intentional 
movement of consciousness towards meaning". In other words, to distance us from lived experience means to "interrupt lived experience in order to signify it" (ibid., p. 116). As previously pointed out we think this is close to what non-affirmative educational leadership and teaching is about, but in education the interruption in the Other's experiencing is done through the educative act. The relevance of this position for empirical research is first it helps us to define the relation between theory and practice as pedagogical practice and as empirical descriptions of this practice. Let us explain.

Sometimes it is claimed that pedagogical practice is primary in relation to educational theory, i.e. that practice is not dependent on theory. Schleiermacher's widely referred position from 1826 may exemplify this: "Still, it is nevertheless a fact that in every domain that goes under the name of Art, in a narrower sense, practice is much older than theory, so that it can simply not be said that practice gets its own definite character only with theory. The dignity of practice is independent of theory; practice only becomes more conscious with theory." Naturally the educational practice (Bildungswirklichkeit) is much older compared with a contemporary understanding of theory. Educational practice also continues to exist regardless of our description of it in the naive sense that it does not cease to exist if we stop talking about it. At least it would continue to exist as past "lived experience" (Van Manen 1991). However, in such past lived experience the meaning of the experience is not always evident. Therefore, precisely as Schleiermacher argues, a fundamental feature of theory is that it helps us to deepen our understanding of pedagogical reality. However, a deepening of our understanding must not be compared with a more detailed description of practice. To deepen our understanding is more; every description always has a constitutive function as well. Taken for granted that some kind of reflection is always connected with practice in a constitutive fashion, i.e. that practice gets its meaning only by virtue of this reflection, then practice is, exactly as Schleiermacher claims, not independent of theory (Bowie 1998). In this respect educational theory would now be primary in relation to practice; practitioners' theories contributes to defining the essence or the meaning of educational reality for the actor. In describing these core elements related to research, Max van Manen (1991) argued that phenomenological research is a lived experience for researchers as they relate with the ontological nature of the phenomenon while learning to "see" prereflective, taken-for-granted and essential understandings through the lens of their always already pre-understandings. van Manen (1991) posits principles for research activities: commitment to an abiding concern, oriented stance toward the question, investigating the experience as it is lived, describing the phenomenon through writing and rewriting, and consideration of parts and whole.

The conclusion defended here is that instead of claiming that theory is secondary to practice or that practice is secondary to theory, we should ask: "What kind of reflection is present in practice?" This position represents critical realism (or "epistemic" realism, Putnam 1988). In this view the world itself does not contain the limits for how it may be described. Only the describers themselves may decide upon which rules are to be followed, since the description is made in relation to previous 
knowledge and with certain interests in a given cultural and historical context. This means that models can be tested empirically, provided that the assumptions behind them are accepted.

One of the main roles non-affirmative theory has, with respect to empirical research, is that it offers us a framework for an empirical research programme regarding leadership and teaching practice including policy analysis, curriculum analysis and research supported school development. The non-affirmative theory also provide us with a thought model for teachers, principals, superintendents, officials at the national and transnational levels. The actual conceptual structure is a theory in the sense that it offers us a conceptual structure for our observations of the pedagogical reality. It is a constitutive theory defining what institutionalized education is about in the first place. Differently expressed, the analysis carried out is an ontological one as it asks about the fundamental nature of the institutionalized leadership-teaching-studying-learning process. Non-affirmative theory of education presents itself as a third position beyond traditional critical theory and functionaltechnological Tylerian models. The position is by no means valuefree or -neutral as it is a theory in and for a western democratic tradition as this has developed the past century. It criticality is visble in its non-affirmative stance demanding ethical, political, cultural and conceptual awareness from the practitioner. However, the practitioner must understand the difference between indoctrination and support for developing critical thinking among students but also professionals. At its core the position is a critical reminder that politics is not allowed to make use of education in its own interest, violating the possibility of contemporary and future democratic forms of social and societal life.

Comparative Inquiry Cross-national comparativists have long considered contextual differences within nation states, including cultures and language that affect categorical definitions; however, current challenges to the nation state raise new methodological dilemmas. Processes of transnationalization, such as the development of the European Union, have challenged the foundations of comparative social sciences methods as well as curriculum-leadership work itself with regards to the sovereign state and autonomous society. We agree with Charles Tilly (1984), who has written extensively about globalization movements, criticized the modern tendency for use of nation states as bounded units of comparative analysis because a strict focus on the nation state has run its course in a world characterized by vast population movements and vertical plurality which undermine the ideal of cultural solidarity. Further, neoliberalism, with its accompanying movements of capital, and the increasing relevance of transnational institutions may subvert formal procedures of inquiry with the nation-state as a strict, bounded analytical unit of comparison. In the contemporary situation, comparative inquiry approaches have also come under recent scrutiny because of the contradiction between different time-scales of different cultures and the linear conception of time (e.g. Abbott 1991; Sztompka 1990). As Sztompka (1990) has argued, globalization of the social world with its increasing plurality has reversed the cognitive and societal situation from one where a century ago heterogeneity and societal isolation was the reality. 
In our view, there will continue to be nation states with leadership and curriculum making occurring at school, district/municipal, state and nation state levels; however, in light of the contemporary situation, our methodological use of the nation state will be dynamic rather than static. We will compare similarities and differences within and between levels from classrooms to transnational levels amidst the current globalization moment. We will, thus, examine the ways in which globalization binds nation states/societies together along many dimensions, but simultaneously, illuminates diversity. Further, in our sampling and data analysis processes, we acknowledge the significance of diversity in terms of individual educators' and/or policymakers' habitus to interculturality or relations between individuals/subjects from distinct cultures. As noted earlier, in light of globopolitanism with its population migrations between nation states and increasing cultural plurality within nation states, interculturality is critically important to any human relations.

Thus, our research project seeks an alternative form of comparative inquiry for a multi-level examination of curriculum guides, leadership education standards or aims, policies, curriculum work-leadership within and between levels from classrooms to transnational levels. Specifically, we propose a formative comparative approach with an emphasis on contemporary moments that are informed by historical and cultural roots as well as the rapidly changing contemporary situation at the nation state and transnational levels, all of which are also shaped in certain ways by individuals with varying cultural backgrounds or habitus and epistemologies. This does not mean that we embrace plurality and diversity for its own sake. Rather, we address diversity, using comparison reflexively to situate diversity in the encounter of curriculum documents and inherent aims, policies, principal development documents, and educational relations amidst contemporary cosmopolitanism as an empirical reality and related dilemmas of universalism versus particularism.

In our view, a comparative research project examining curriculum workleadership must also entail a reflexive relation among fundamental social philosophy and its strength in pedagogical relations with more contemporary, postmodern philosophy (e.g. Deleuze) and its strength in identity. In our view, this reflexive relation involves simultaneously differentiating globalizing cultural times cognitively in policy and subject relations where the subject is considered, with some variation between the nation state curriculum/evaluation policy and leadership preparation level and the transnational policy level. At the nation state level (and states within these), we have curricula (e.g. content, methods) as well as policies. For our project, we ask: How do nation states initiate, develop, and mediate curriculum changes or reforms? If appropriate, we also ask how do states within nation states initiate, develop, and mediate curriculum changes or reforms? How are various nation states, with their differing cultural and historical traditions, responding to global, common changes and dilemmas? What are the conceptions of citizens' Bildung, skills, and learning of each nation state and how are these promoted through policies and the process of education? At the transnational level, we do not have curricula; rather, we have transnational curriculum and evaluation policies that could be compared. We ask: What curriculum and evaluation policies are promoted by transnational organizations as members of continuously evolving configurations 
of nation state systems and how is this promotion carried out? Here we may consider curriculum as a culture emerging in relations within and between new forms of governance. At school and district/municipality levels, we then ask: What notions of curriculum are promoted by transnational organizations, nations, and states, and how do they mediate among these and their students? How, if at all, did their principal and superintendent preparation support their curriculum work? At all levels, we acknowledge that categories are discursive, dynamic, and evolving within and between nation states.

A Note on Methods It is important to note that a refined version of critical discourse analysis or CDA (Fairclaugh 2005) is necessary because CDA begins with a normativity problem in clear social transformative ideal and an aim to correct a societal wrong. Our framework, rather, supports a non-hierarchical, non-affirmative perspective on criticality as well as an institutional perspective (Schmidt 2008a), and these combined perspectives necessitate a refined view of criticality to ground our discursive analyses. We also note, however, that some relations within and between levels may be affirmative and others may be non-affirmative, with for example, evaluation policies and legislations that are mandatory necessitating perhaps more affirmative perspectives and mediational approaches. Thus, we seek comparisons of connected segments that reveal emergent and, at times, contradictory discursive dynamics within classrooms, schools, districts, municipalities and/ or states, as well as in transnational relations between these nation states. Our framework will form the basis for data analysis of interview transcripts, including attention to discursive institutionalism (Schmidt) that may illuminate similarities, distinctions, old and new paradoxes, and emergent new constructions (Schmidt 2015). In so doing, we draw on discursive institutionalism for understanding how cognitive ideas (problems identification) and normative ideas (values that legitimize problems) are developed and communicated across societal, philosophical, policy, and program levels as well as how discursive constructions shape human educational/pedagogical relations. Going beyond Schmidt $(2009,2016)$, then, we analyze data, looking for a language of education, beginning with modern education theory and its core concepts (Benner 1991). From this foundational theoretical framing and methodological possibilities, we now introduce the remaining chapters in this volume.

\section{Overview of Book Structure}

This opening chapter presented a common general framework that brings two significant but yet unfortunately disparate fields (curriculum theory, leadership studies) closer together along with critical understandings from discursive institutionalism. Our framework moves beyond the limitations of curriculum theory/Didaktik (i.e. the lack of attention to the interplay between aims, contents, methods and leadership roles at various levels, the normative nature of dominant curriculum theories 
and their regulatory and transformational aims and underlying epistemologies and ontologies; limited language to talk about various systems). Discursive institutionalism, combined with classic educational philosophy concepts (recognition, summoning, Bildsamkeit), creates a language for curriculum work and other human-institutional interactions, including those concerning the broader system (e.g. law, policy documents). In our framework, then, curriculum theory/Didaktik (societal aims, content, methods, planning), leadership/institutional studies (structure) and policy (ideas) now complete each other conceptually. Educational leadership, including curriculum work is, thus, conceptualized as:

a non-affirmative, critical-interpretative and cultural-historical distributed multiprofessional practice carried out on different levels of the educational system, operating between different cultures, epistemologies (knowledge practices) and value-spheres (ethics and politics) where professional actors, through their roles (tasks) and persons, based on a recognition of the Other's potentiality, reality and possibility, aim at supporting teachers/ principals/students by summoning (inviting, intervening, demanding, supporting provocation) to engage in the transcendence of one's current pedagogical work.

As such, educational leadership may be accomplished through activities such as developing routines and cultures of change as well as post-national pedagogies, in order to create dynamic and reflected teaching opportunities, so that students become able to grow into an existing world while being prepared to change it according to their interests as related to others in a deliberative democracy. At the same time, we recognize that much of the theoretical and empirical work on educational leadership has been constructed for relations within the nation state.

Thus, we posed a third question derived from the ongoing crisis of the nation state, due to what we have termed globopolitanism. For over 200 years, in many parts of the world, independent nation states have developed education based upon the idea of culturally uniting 'the autonomous individual', most often through a common language. In the contemporary empirical reality, nation states remain legally and politically; however, new transnational and international institutions and organisations challenge the autonomy of nation states. Today, cosmopolitanism presents itself as a practical educational challenge due to global interdependencies as well as increasing internal, horizontal, ethnic plurality. Thus, while we see modern education theory and its core concepts essential for relations within and between levels of the nation state, we must consider the extent to which these core concepts are sufficient for understanding increasing transnational level of educational leadership and curriculum work relations. Moreover, in line with our non-affirmative position, we must continually live with the future as an open question, thus leaving our own framework a living, dynamic project open to question and revision. We hope our general framework may inform a new education field for theorizing curriculum/Didaktik and leadership according to which leadership and teaching professions may be conceptualized in a coherent way, not following different theoretical logics. Such a new research field requires a new comparative methodology. Further, we argue that our research agenda may also be productive for schools and new forms of governance at all levels as reflective professional learning communities being critically policy aware in their curriculum content and pedagogical develop- 
mental work amidst globopolitanism with perennial and contemporary tensions between unity and plurality.

\section{An Invitation to a Cross-National Dialogue}

The remainder of the book is organized into four additional parts. The first part provides a review of curriculum theory and Didaktik in the U.S. and Europe from authors who have been leaders in these traditions, including Stefan Hopmann and his colleague Mariella Knapp from Europe and Walter Doyle from the United States. In the second part, Lejf Moos, Gert Biesta, Tero Autio, and Joao Paraskeva further consider the societal and policy context for contemporary educational leadership and curriculum work. The third part brings leadership in closer dialogue with curriculum theory/Didaktik with chapters from Ira Bogotch, Dilys Schoorman, and Daniel Reyes-Guerra considering multicultural education and leadership, Daniel Castner, Rosemary Gornik, James Henderson and Wendy Samford with a constructive alternative to the Tyler Rationale in teacher leadership, Bill Pinar's chapter on rethinking authority in educational leadership, and Eva Forsberg, Elisbet Nihfors, Daniel Petterson and Pia Skott considering the commodification of the Swedish curriculum, leadership, and the need for a new code. Finally, in Part V, we explore discursive and multi-level perspectives with Michael Uljens and Helena Rajakaltio's chapter on national curriculum reform in Finland and Kirsten Sivisend and Ninni Wahlström's chapter on curriculum-leadership in transnational reform policy in Norway and Sweden.

One of the recurring themes across the chapters is a call for a renewed crossnational and cross-field/disciplinary dialogue on curriculum theorizing/Didaktik and leadership in an era of what we term globopolitanism. We hope that readers of this volume will approach the reading of this text as an opportunity to challenge their thinking and a space for dialogue as well, bringing their own theoretical backgrounds, experiences, thoughts, aims, and values to bear on the propositions and challenges that are raised and considered. We acknowledge that some authors and readers may be left with questions and ideas more than answers as cosmopolitanism and globalization challenge us to consider curriculum and leadership beyond the nation state level; however, we believe that the non-affirmative framework and this volume opens a space for a renewed and thoughtful dialogue on these issues. Future directions may include further cross-national dialogue toward new theoretical possibilities, a further consideration of cosmopolitanism as an empirical ideal and empirical reality, new comparative studies as well as new, less normative, approaches to educational leadership and curriculum work/leadership practice within and between levels. We hope that our framework and the kind of cross-field/disciplinary and cross-national dialogue initiated here will ultimately construct a foundation for the kind of curriculum-leadership work those of us who participated in this project seek to foster. This volume, thus, concludes with our own final thoughts about moving forward as well as commentaries from William Pinar, Carolyn Shields, and 
Thomas Englund, who contribute to this new cross-national dialogue from a North American context and a Nordic/European context as well as from curriculum and leadership perspectives.

\section{References}

Abbott, A. (1991). The order of professionalization: An empirical analysis. Work and Occupations, $18(4), 355-384$.

Althusser, L. (1970/2006). Ideology and ideological state apparatuses (notes towards an investigation). The Anthropology of the State: A Reader, 9, 86.

Anderson, G. L. (2009). Advocacy leadership: Toward a post-reform agenda in education. New York: Routledge.

Anyon, J. (2006). Social class and the hidden curriculum of work. Childhood Socialization, 162, 369.

Anyon, J. (2008). Social class and school knowledge. In The way class works: Readings on schools, family and the economy (pp. 189-209). London: Routledge

Apple, M. W. (1992). The text and cultural politics. Educational Researcher, 21(7), 4-19.

Apple, M. W. (2004). Ideology and curriculum. New York: Routledge.

Apple, M. W. (2005). Education, markets, and an audit culture. Critical Quarterly, 47(12), 11-29.

Apple, M. W. (2008). Knowledge, power, and education: The selected works of Michael Apple. Shanghai: East China Normal University Press.

Apple, M. W. (2014). Official knowledge: Democratic education in a conservative age. New York: Routledge.

Apple, M. W., \& Weis, L. (1986). Seeing education relationally: The stratification of culture and people in the sociology of school knowledge. Journal of Education, 168, 7-34.

Arnot, M., \& Whitty, G. (1982). From reproduction to transformation: Recent radical perspectives on the curriculum from the USA. British Journal of Sociology of Education, 3(1), 93-103.

Aronowitz, S., \& Giroux, H. (1985). Radical education and transformative intellectuals. CTheory, 9(3), 48-63.

Autio, T. (2006). Subjectivity, curriculum and society: Between and beyond the German Didaktik and Anglo-American Curriculum Studies. Mahwah: Lawrence Erlbaum Associates.

Autio, T. (2009). Globalisation, curriculum and new belongingness of subjectivity. Beyond the nexus between psychology (curriculum) and the nation-state (Didaktik). In E. Ropo \& T. Autio (Eds.), International conversations on curriculum studies (pp. 1-20). Dordrecht: Sense.

Autio, T. (2013). Subjectivity, curriculum, and society: Between and beyond the German Didaktik and Anglo-American Curriculum studies. Mahwah: Lawrence Erlbaum Associates.

Avolio, B. J., Bass, B. M., \& Jung, D. I. (1999). Re-examining the components of transformational and transactional leadership using the Multifactor Leadership. Journal of Occupational and Organizational Psychology, 72(4), 441-462.

Banks, J. A. (2002). Race, knowledge construction, and education in the USA: Lessons from history. Race, Ethnicity and Education, 5(1), 7-27.

Banks, J. A., \& Banks, C. A. M. (2009). Multicultural education: Issues and perspectives. New York: Wiley.

Barnard, C. I. (1968). Functions of an executive. Cambridge, MA: Harvard University Press.

Bass, B. M. (1985). Leadership and performance beyond expectations. London: Free Press/Collier Macmillan.

Bass, B. M., \& Avolio, B. J. (1990). Developing transformational leadership: 1992 and beyond. Journal of European Industrial Training, 14(5), 21-27.

Beck, U. (2006). Cosmopolitan vision. Cambridge: Polity Press.

Bengtsson, J. (2003). Possibilities and limits of self-reflection in the teaching profession. Studies in Philosophy and Education, 22(3/4), 295-316. 
Benner, D. (1991). Allgemeine Pädagogik. Weinheim: Juventa.

Benner, D. (1993). Die Pädagogik Herbarts. Weinheim: Juventa.

Benner, D. (1995). Wilhelm von Humboldts Bildungstheorie: Eine problemgeschichtliche Studie zum Begründungszusammenhang neuzeitlicher Bildungsreform. Weinheim: Juventa.

Benner, D. (1997). Johann Friedrich Herbart. Systematische Pädagogik Band 2: Interpretationen. Weinheim: Deutschen Studien Verlag.

Benner, D. (2005). Tekster til dannelsesfilosofi-mellem etik, paedagogik och politik. (Essays on philosophy of Bildung - between ethics, pedagogics and politics). Århus: Klim.

Benner, D. (2015). Allgemeine Pädagogik (8. upplagan). Weinheim: Beltz Juventa.

Benner, D., \& English, A. (2004). Critique and negativity: Towards the pluralisation of critique in educational practice, theory and research. Journal of Philosophy of Education, 38(3), 409-428.

Bennett, J. V., Ylimaki, R. M., Dugan, T. M., \& Brunderman, L. A. (2014). Developing the potential for sustainable improvement in underperforming schools: Capacity building in the sociocultural dimension. Journal of Educational Change, 15(4), 377-409.

Berliner, D. C. (1989). Implications of studies of expertise in pedagogy for teacher education and evaluation. In J. Pfleiderer (Ed.), New directions for teacher assessment: Proceedings of the 1988 ETS invitational conference (pp. 39-67). Princeton: Educational Testing Service.

Berman, P., \& McLaughlin, M. W. (1976). Implementation of educational innovation. The Educational Forum, 3(40), 345-370.

Bernstein, B. (1975). Class and pedagogies: Visible and invisible. Educational Studies, 1, 23-41.

Biesta, G. (1994). Education as practical intersubjectivity. Towards a critical- pragmatic understanding of education. Educational Theory, 44(3), 299-317.

Biesta, G. (2009). Good education in an age of measurement: On the need to reconnect with the question of purpose in education. Educational Assessment, Evaluation and Accountability, 21(1), 33-46.

Blackburn, S. (1996). Securing the nots: Moral epistemology for the quasirealist. In New readings in moral epistemology. Oxford: University Press.

Blankertz, H. (1982). Die Geschichte der Pädagogik. Von der Aufklärung bis zur Gegenwart. Wetzlar: Pandora.

Blossing, U., Imsen, G., \& Moos, L. (2014). The nordic education model: "A school for all" encounters neo-liberal policy. Dordrecht: Springer.

Bobbitt, J. F. (1918). The curriculum. Boston: Houghton Mifflin.

Bogotch, I. (2002). Educational leadership and social justice: Practice into theory. Journal of School Leadership, 12, 138-156.

Bohman, J., \& Lutz-Bachmann, M. (1997). Perpetual peace: Essays on Kant's cosmopolitan ideal. Cambridge: The MIT Press.

Boske, C. (2014). Critical reflective practices: Connecting to social justice. In International handbook of educational leadership and social (in) justice. Dordrecht: Springer.

Bourdieu, P. (1977). Outline of a theory of practice (Vol. 16). Boston: Cambridge University Press.

Bourdieu, P. (2011). The forms of capital (1986). Cultural Theory: An anthology, 1, 81-93.

Bourdieu, P., \& Passeron, J. C. (1990a). Reproduction in education, society and culture (Vol. 4). Newbury Park: Sage.

Bourdieu, P., \& Passeron, J. C. (1990b). Reproduction in education, society and culture (Vol. 4). London: Sage.

Bowie, A. (1998). Introduction. In F. D. E. Schleiermacher (Ed.), Hermeneutics and criticism. And other writings. Cambridge: Cambridge University Press.

Bowie, A. (2003). Aesthetics and subjectivity. Manchester: Manchester University Press.

Bowles, S., \& Gintis, H. (1975). Schooling in capitalist America (Vol. 57). New York: Basic Books.

Bowles, S., \& Gintis, H. (1976). Schooling in capitalist America: Educational reform and the contradictions of economic life. New York: Basic Books.

Braun, A., Maguire, M., \& Ball, S. J. (2010). Policy enactments in the UK secondary school: Examining policy, practice and school positioning. Journal of Education Policy, 25(4), $547-560$.

Brincat, S. (2009). Hegel's gesture to radical cosmopolitanism. Journal of Critical Globalisation Studies, 1, 47-65. 
Bromme, R. (1995). What exactly is 'pedagogical content knowledge'? Critical remarks regarding a fruitful research program. In S. Hopmann \& K. Riquarts (Eds.), Didaktik and/or curriculum (pp. 2015-2216., Report 147). Kiel: Institut für die Pädagogik der Naturwissenschaften an der Universität Kiel.

Brooks, J. S. (2012). Black school, White school: Racism and educational (mis)leadership. New York: Teachers College Press.

Brooks, J. S., Jean-Marie, G., Normore, A. H., \& Hodgins, D. W. (2008). Distributed leadership for social justice: Exploring how influence and equity are stretched over an urban high school. Journal of School Leadership, 17(4), 378-408.

Brown, A., Collins, A., \& Duguid, P. (1989). Situated cognition and the culture of learning. Educational Researcher, 18(19), 32-42.

Brunner, C. (1998). Women superintendents: Strategies for success. Journal of Educational Administration, 36(2), 160-182.

Brunner, C. C., Opsal, C., Oliva, M. (2006). Disrupting identity: Fertile soil for raising social consciousness in educational leaders. In Leading for social justice: Making revolutions in education (pp. 214-232). Boston: Allyn \& Bacon.

Bu, Y., Xu, J., \& Deng, Z. (2016). The new basic education and the development of human subjectivity. In G. Zhao \& Z. Deng (Eds.), Re-envisioning Chinese education: The meaning of person-making in a new age (pp. 201-2012). New York: Routledge.

Burns, J. M. (1978). Leadership. New York: Harper \& Row.

Burrell, G., \& Morgan, G. (2003). Sociological paradigms and organisational analysis (Vol. 248). London: Heinemann.

Caldwell, B. J. (1998). Strategic leadership, resource management and effective school reform. Journal of Educational Administration, 36(5), 445-461.

Callahan, R. E. (1962). Education and the cult of efficiency. Chicago: University of Chicago Press.

Callon, M. (1980). Struggles and negotiations to define what is problematic and what is not. In The social process of scientific investigation (pp. 197-219). Dordrecht: Springer.

Capper, C. A. (1989). Students with severe disabilities in the general education program: A conceptual and practical framework for rural school administrators. Journal of Rural and Small Schools, 41(1), 52-59.

Capper, C. A. (1993). Educational administration in a pluralistic society. New York: State University of New York Press.

Capper, C. A., Frattura, E. M., \& Keyes, M. W. (2000). Meeting the needs of students of all abilities: How leaders go beyond inclusion. Newbury Park: Corwin Press.

Cerny, P. G. (1997). Paradoxes of the competition state: The dynamics of political globalization. Government and Opposition, 32(02), 251-274.

Chi-Kin Lee, J., \& Kennedy, K. J. (2017). Theorizing teaching and learning in Asia and Europe: A conversation between Chinese curriculum and European didactics. London: Routledge.

Claesson, S., Hallström, H., Kardemark, W., \& Risenfors, S. (2011). Ricoeurs kritiska hermeneutik vid empiriska studier. Pedagogisk Forskning i Sverige, 1(16), 18-35.

Clarke, S., \& Wildy, H. (2009). The Europeanisation of educational leadership. European Educational Research Journal, 8(3), 352-475.

Cleve, Z. J. (1884). Grunddrag till skolpedagogik (Foundations of School Pedagogics). Helsingfors: Edlunds.

Cobben, P. (2010). Institutions of education, then and today: The legacy of German idealism. Leiden: Brill.

Cole, M., \& Engeström, Y. (1993). A cultural-historical approach to distributed cognition. In G. Salomon (Ed.), Distributed cognitions: Psychological and educational considerations. Cambridge: Cambridge University Press.

Coleman, J. S., \& National Center for Educational Statistics. (1966). Equality of education opportunity. Washington, DC: U.S. Department of Health, Education and Welfare.

Comenius, J. A. (1907). The great didactic. (M. W. Keatinge, Transl.). London: Adam and Charles Black. https://archive.org/stream/cu31924031053709\#page/n11/mode/2up 
Cooper, P. M. (2003). Effective White teachers of Black children teaching within a community. Journal of Teacher Education, 54(5), 413-427.

Cooper, C. W. (2009). Performing cultural work in demographically changing schools: Implications for expanding transformative leadership frameworks. Educational Administration Quarterly, $45,694-724$.

Crossley, N. (1996). Intersubjectivity. The fabric of social becoming. London: Sage.

Crow, G., Day, C., \& Møller, J. (2016). Framing research on school principals' identities. International Journal of Leadership in Education, 1-13.

Czarniawska, B. (2014). A theory of organizing (2nd ed.). Cheltenham: Edward Elgar.

Czarniawska, B., \& Sevón, G. (1996). Translating organizational change. Berlin: Walter de Gruyter.

Czarniawska, B., \& Sevón, G. (2005). Global ideas. How ideas, objects and practices travel in the global economy. Malmö: Liber.

Dahllöf, U. (1967). Skoldifferentiering och undervisningsförlopp. Stockholm: Almqvist \& Wiksell.

Dall'Alba, G., \& Sandberg, J. (2006). Unveiling professional development: A critical review of stage models. Review of Educational Research, 76(3), 383-412.

Damgaard Knudsen, L. E., \& Andersson, M. (2008). Skab dig! Paedagogisk filosofi. Köpenhamn: Forlaget Unge Pedagoger.

Devos, G., Ekholm, M., Kofod, K., Louis, K. S., Moos, L., Schratz, M., \& van Velzen, B. (2012). Political culture and educational reform. In Educational policy in an international context (pp. 5-27). Palgrave Macmillan US.

Day, C. (2005). Sustaining success in challenging contexts: Leadership in English schools. Journal of Educational Administration, 43(6), 573-583.

Deleuze, G., \& Guattari, F. (1988). A thousand plateaus: Capitalism and schizophrenia. London: Athlone.

Deng, Z. (2016). Rethinking and re-envisioning Chinese didactics. Implications from the German Didaktik tradition. In G. Zhao \& Z. Deng (Eds.), Re-envisioning Chinese education: The meaning of person-making in a new age (pp. 183-199). New York: Routledge.

Dewey, J. (1916/2008). Democracy and education. Boston: Seven Treasures (Original work published in 1916).

Dewey, J. (1938). Experience and education (The Kappa Delta Pi Lecture Series). New York: Collier Books.

Dewey, J. (1959). The child and the curriculum (No. 5). Chicago: University of Chicago Press.

Dewey, J. (1990/1915). The school and society and the child and the curriculum. Chicago: University of Chicago Press.

Dewey, J. (1992). The structure of experience. Chicago: Chicago University Press.

Dewey, J. (2008). Democracy and education. Boston: Seven Treasures. Original work published in 1916.

Dewey, J., \& Small, A. W. (1897). My pedagogic creed (No. 25). Chantilly: EL Kellogg \& Company.

Diederich, J. (1988). Didaktisches Denken. Weinheim: Juventa.

Dillard, C. B. (2000). The substance of things hoped for, the evidence of things not seen: Examining an endarkened feminist epistemology in educational research and leadership. International Journal of Qualitative Studies in Education, 13(6), 661-681.

Dilthey, W. (1883). Einleitung in die Geisteswissenschaften. Versuch einer Grundlegung für das Studium der Gesellschaft und der Geschichte. Leipzig: Duncker \& Humblot.

DiMaggio, P. J., \& Powell, W. W. (1983). And collective rationality in organizational fields. American Sociological Review, 48(2), 147-160.

DiMaggio, P. J., \& Powell, W. W. (1991). The new institutionalism in organizational analysis. Chicago: University of Chicago Press Books.

Dimitriadis, G. (2009). Performing identity/performing culture: Hip hop as text, pedagogy, and lived practice (Vol. 1). New York: Peter Lang.

Dolch, J. (1965). Lehrplan des Abendlandes (2nd ed., orig. 1959). Ratingen: Aloys Hann Verlag. 
Doll, W. E. (1993a). Curriculum possibilities in a "post-future". Journal of Curriculum and Supervision, 8(4), 277-292.

Doll, W. E. (1993b). A post-modern perspective on curriculum. New York: Teachers College Press. Doyle, W., \& Carter, K. (1984). Academic tasks in classrooms. Curriculum Inquiry, 14(2), 98.

Dreyfus, H. L., \& Dreyfus, S. E. (1986). Mind over machine: The power of human intuition and expertise in the era of the computer. New York: The Free Press.

Drucker, H. M. (1974). The political uses of ideology. Dordrecht: Springer.

Drysdale, L., Bennett, J. T., Murakami, E., Johansson, O., \& Gurr, D. (2014). Heroic leadership in Australia, Sweden, and the United States. International Journal of Educational Management, 28(7), 785-797.

DuFour, R., \& Eaker, R. (1998). Professional learning communities at work: Best practices for enhancing student achievement. Bloomington: Solution Tree.

Durkheim, E. (1997). Suicide. New York: Free Press. (Original work published 1893).

Edmonds, R. (1979). Effective schools for the urban poor. Educational Leadership, 37(1), 15-24.

Engeström, Y. (1987). Learning by expanding. Orienta: Helsinki.

Engeström, Y. (2000). Activity theory as a framework for analyzing and redesigning work. Ergonomics, 43(7), 960-974.

Engeström, Y., Miettinen, R., \& Punamäki, R.-L. (1999). Perspectives on activity theory. Cambridge: Cambridge University Press.

English, A. (2013). Discontinuity in learning. Dewey, Herbart, and education as transformation. Cambridge: Cambridge University Press.

English, A. (2014). Herbart, Johann F. In D. C. Phillips (Ed.), Encyclopedia of educational theory and philosophy (pp. 373-376). Thousand Oaks: Sage.

Englund, T. (1986). Curriculum as a political problem. Changing educational conceptions with special reference to citizenship education (p. 25). Uppsala: Uppsala Studies in Education.

Englund, T. (1997). Undervisning som meningserbjudande (Teaching as meaning construction). In M. Uljens (Ed.), Didaktik (pp. 120-145). Lund: Studentlitteratur.

Englund, T. (2006). Deliberative communication: A pragmatist proposal. Journal of Curriculum Studies, 38(5), 503-520.

Fairclough, N. (2005). Analysing discourse. Textual analysis for social research. London: Routledge.

Fanon, F. (1963). The wretched of the earth. New York: Grove Press.

Fayol, H. (1949). General and industrial management. London: Pitman.

Fenwick, T., \& Edwards, R. (2010). Actor-network. New York: Routledge.

Fichte, J. G. (1796/1992). Foundations of transcendental philosophy (D. Breazeale, Trans.). New York: Cornell University Press.

Fichte, J. G. (2000). Foundations of natural right (F. Neuhouser (Ed.), M. Bauer, Trans.). Cambridge: Cambridge University.

Fichte, J. G. (2009). Fichte: Addresses to the German Nation. Cambridge: Cambridge University Press.

Follett, M. P. (1918). The new state: Group organization the solution of popular government. University Park: Penn State Press.

Follett, M. P. (1949). The essentials of leadership. In L. Urwick (Ed.), Freedom and coordination, Lectures in business administration by Mary Parker Follett (pp. 47-60). London: Management Publications Trust.

Follett, M. P., Metcalf, H. C., \& Urwick, L. F. (1942). Dynamic administration. New York: Routledge.

Forsyth, P. B., \& Hoy, W. K. (1978). Isbridge University preolation and alienation in educational organizations. Educational Administration Quarterly, 14(1), 80-96.

Foster, W. (1986). Paradigms and promises: New approaches to educational administration. Prometheus books.

Foucault, M. (1991). Governmentalité. In G. Burcell, C. Gordon, \& P. Miller (Eds.), The Foucault effect: Studies in governmentality (pp. 87-104). Hempel Hempstead: Harvester Wheatsheaf. 
Foucault, M. (1997). Ethics. Subjectivity and truth. New York: The New Press.

Frank, M. (1977). Das individuelle Allgemeine. Textstrukturierung und-interpretation nach Schleiermacher. Frankfurt am Main: Suhrkamp.

Fraser, N., \& Honneth, A. (2003). Redistribution or recognition? A political-philosophical exchange. London: Verso.

Freire, P. (1993). Pedagogy of the oppressed. 1970. New York: Continuum.

Freire, P. (2000). Pedagogy of the oppressed. New York: Bloomsbury (Original work published 1979).

Freire, P. (2004). Pedagogy of indignation. Boulder: Paradigm.

Friesen, N., \& Hamelock, M. (2012). Augustine, Wittgenstein, and "the Call" in Mollenhauer's forgotten connections: On culture and upbringing. Phenomenology \& Practice, 6(2), 94-107.

Friesen, N., \& Sævi, T. (2010). Reviving forgotten connections in North American teacher education. Klaus Mollenhauer and the pedagogical relation. Journal of Curriculum Studies, 142(1), 123-147.

Frontini, S. (2009). Global influences and national peculiarities in education and training: The Finnish case. In I. H. B. Holmarsdottir \& M. O’Dowd (Eds.), Nordic voices. Teaching and researching comparative and international education in the Nordic countries. Rotterdam: Sense Publishers.

Fullan, M. (2007). The new meaning of educational change. London: Routledge.

Gadamer, H.-G. (1975). Truth and method. London: Sheed and Ward.

Gallagher, S. (1992). Hermeneutics and education. New York: SUNY Press.

Gauthier, D. P. (1963). Practical reasoning. Oxford: Clarendon Press.

Genschel, P., \& Seelkopf, L. (2015). The competition state. In: S. Leibfried, E. Huber, M. Lange, J. D. Levy, \& J. D. Stephens (Eds.), The oxford handbook of transformations of the state. Oxford Handbooks Online. doi:10.1093/oxfordhb/9780199691586.013.12.

Giroux, H. (1980). Critical theory and rationality in citizenship education. Curriculum Inquiry, 10(4), 329-366.

Giroux, H. A. (1983). Theory and resistance in education: A pedagogy for the opposition. South Hadley: Bergin \& Garvey.

Gooden, M. A. (2012). What does racism have to do with leadership? Countering the idea of colorblind leadership: a reflection on race and the growing pressures of the urban principalship. Educational Foundations, 26, 67-84.

Goodlad, J. (1966). The development of a conceptual system for dealing with problems of curriculum and instruction. U.S. Department of Health, Education, and Welfare, Office of Education Cooperative Research Project No. 454. Los Angeles: Institute for the Development of Educational Activities, UCLA.

Gough, I. (2001). Globalization and regional welfare regimes. The East Asian case. Global Social Policy, 1(2), 163-189.

Gramsci, A. (1996). Prison notebooks (Vol. 2). New York: Columbia University Press.

Greene, M. (2001). Educational purposes and teacher development. In Teachers caught in the action: Professional development that matters (pp. 3-11). New York: Teachers College Press.

Greenfield, T. B. (1986). The decline and fall of science in educational administration. Interchange, $17(2), 57-80$.

Greenfield, W. (1987). Instructional leadership: Concepts, issues, and controversies. Newton: Allyn and Bacon.

Greenwood, R., \& Hinings, C. R. (1996). Understanding radical organizational change: Bringing together the old and the new institutionalism. The Academy of Management Review, 21(4), $1022-1054$.

Grek, S. (2008). From symbols to numbers: the shifting technologies of education governance in Europe. European Educational Research Journal, 7(2), 2018-2218.

Grek, S. (2009). Governing by numbers: The Pisa "effect" in Europe. Journal of Education Policy, 1(24), 23-37.

Grogan, M. (1996). Voices of women aspiring to the superintendency. New York: SUNY Press. 
Gronn, P. (2000). Distributed properties a new architecture for leadership. Educational Management \& Administration, 28(3), 317-338.

Guba, E., \& Lincoln, Y. (1994). Competing paradigms in qualitative research. In N. Denzin \& Lincoln (Eds.), Handbook of qualitative research (pp. 105-177). SAGE: Thousand Oaks.

Gudmundsdottir, S. (1988). Knowledge use among experienced teachers: Four case studies of high school teaching. Unpublished Doctoral Dissertation. Stanford: School of Education.

Gudmundsdottir, S., \& Shulman, L. (1987). Pedagogical content knowledge in social studies. Scandinavian Journal of Educational Research, 31, 59-70.

Gulick, L. (1937). Notes on the theory of organization. Classics of organization theory, 3, 87-95.

Gundara, J. (2000). Interculturalism, education and inclusion. London: Paul Chapman.

Gundem, B. B. (1995). Historical roots and contemporary foundations. In S. Hopmann \& K. Riquarts (Eds.), Didaktik and/or curriculum (pp. 43-56). Kiel: IPN.

Gundem, B. B. (2010). European curriculum studies. Continental overview. In C. Kriedel (Ed.), Encyclopedia of curriculum studies (pp. 354-358). London: Sage.

Gundem, B., \& Hopmann, S. (1998). Didaktik and/or curriculum: An international dialogue. New York: Peter Lang.

Gunter, H. M., Grimaldi, E., Hall, D., \& Serpieri, R. (2016). New public management and the reform of education: European lessons for policy and practice. London: Routledge.

Gustavsson, B. (1996). Bildning $i$ vår tid. Om bildningens möjligheter och villkor $i$ det moderna samhället. Stockholm: Wahlström \& Widstrand.

Habermas, J. (1981). New social movements. Telos, 49, 33-37.

Habermas, J. (1984). Theory of communicative action (Vol. 1). Boston: Beacon Press.

Habermas, J. (1996a). The European nation state. Its achievements and its limitations. On the past and future of sovereignty and citizenship. Ratio Juris, 9(2), 125-137.

Habermas, J. (1996b). Between facts and norms: Contributions to a discourse theory of law and democracy (W. Rehg, Trans.). Cambridge: Polity Press.

Hallinger, P. (2003). Leading educational change: Reflections on the practice of instructional and transformational leadership. Cambridge Journal of Education, 33(3), 329-352.

Hallinger, P. (2004). Meeting the challenges of cultural leadership: The changing role of principals in Thailand. Discourse Studies in the Cultural Politics of Education, 25(1), 61-73.

Hallinger, P. (2005). Instructional leadership and the school principal: A passing fancy that refuses to fade away. Leadership and Policy in Schools, 4(3), 221-239.

Hallinger, P., \& Murphy, J. (1985). Assessing the instructional management behavior of principals. The Elementary School Journal, 86(2), 217-247.

Hamilton, D. (1995). Order and structure in Didaktik and curriculum. In S. Hopmann \& K. Riquarts (Eds.), Didaktik and/or curriculum (pp. 71-84., Report 147). Kiel: Institut für die Pädagogik der Naturwissenschaften an der Universität Kiel.

Hamilton, D. (2000). The pedagogic paradox (or why no didactics in England?). In M. Uljens (Ed.), Pedagogikens problem. Kulturella utvecklingslinjer och teoretiska spörsmål (pp. 57-76). Vasa: Åbo Akademi, Department of Education.

Hamilton, D., \& Gudmundsdottir, S. (1994). Didaktik and/or curriculum? Curriculum Studies, 2(3), 345-350.

Hargreaves, A., Moore, S., Fink, D., Brayman, C., \& White, R. (2003). Succeeding leaders? A study of principal succession and sustainability. Toronto: Ontario Principals' Council. Retrieved February, 20, 2008.

Hargreaves, A., Halász, G., \& Pont, B. (2007). School leadership for systemic improvement in Finland. A case study report for the OECD activity improving school leadership. Paris: OECD.

Harris, A. (1992). Effective leadership in schools facing challenging contexts. School Leadership and Management, 22(1), 15-26.

Harris, A. (2007). Distributed leadership: Conceptual confusion and empirical reticence 1. International Journal of Leadership in Education, 10(3), 315-325.

Hegel, G. W. F. (1807/1977). The phenomenology of spirit. Oxford: Oxford University Press.

Helfenbein, R. (2010). Thinking through scale: Critical geography and curriculum spaces. In Curriculum studies handbook: The next moment (pp. 304-317). New York/London: Routledge. 
Heller, A. (1999). A theory of modernity. New York: Columbia University Press.

Henderson, J. G., \& Gornik, R. (2007). Transformative curriculum leadership. Upper Saddle River: Prentice Hall.

Herbart, J. F. (1908). The science of education. Its general principles deduced from its Aim, and the aesthetic revelation of the world. Cambridge: D.C. Heath \& Co.

Herbart, J.-F. (1914). Umriss pädagogischer Vorlesungen [1841]. In O. Willman \& T. Fristch (Eds.), Johann Friedrich Herbarts pädagogische Schriften (Vol. 2). Oesterwieck: Verlag von U. W. Zickfeldt.

Herder, J. G. (2002). In M. N. Forster (Ed.), Philosophical writings. Cambridge: Cambridge University Press.

Honneth, A. (1992). Integrity and disrespect: principles of a conception of morality based on the theory of recognition. Political Theory, 20, 187-201.

Honneth, A. (1995). The struggle for recognition. The moral grammar of social conflicts. Cambridge: Polity.

Hopmann, S. (1992). Starting a dialogue. Roots and issues of the beginning conversation between European Didaktik and the American curriculum tradition. Paper presented at the annual meeting of the American Educational Research Association in San Francisco, April 1992.

Hopmann, S. (1997). Wolfgang Klafki och den tyska didaktiken (Wolfgang Klafki and the German Didaktik). In M. Uljens (Ed.), Didaktik: teori, reflektion, praktik (pp. 213-226). Lund: Studentlitteratur.

Hopmann, S. (2003). On the evaluation of curriculum reforms. Journal of Curriculum Studies, $4(35), 459-478$.

Hopmann, S. (2015). Didaktik meets Curriculum' revisited: Historical encounters, systematic experience, empirical limits. Nordic Journal of Studies in Educational Policy.http://dx.doi. org/10.3402/nstep.v1.27007.

Hopmann, S., \& Riquarts, K. (1995). Didaktik and/or curriculum: Basic problems of comparative Didaktik. In S. Hopmann \& K. Riquarts (Eds.), Didaktik and/or curriculum (pp. 9-40). Kiel: IPN.

Hopmann, S., Riquarts, K., \& Westbury, I. (2000). Teaching as a reflective practice: The German Didaktik tradition. Hove: Psychology Press.

Hord, S. M. (Ed.). (2004). Learning together, leading together: Changing schools through professional learning communities. New York: Teachers College Press.

Horlacher, R. (2015). The educated subject and the German concept of bildung. A comparative cultural history. London: Routledge.

Hoy, W. K., \& Miskel, C. G. (2005). Educational leadership and reform. Chicago: IAP.

Huber, S. G. (2004). School leadership and leadership development: Adjusting leadership theories and development programs to values and the core purpose of school. Journal of Educational Administration, 42(6), 669-684.

Hudson, B., \& Meyer, M. (Eds.). (2011). Beyond fragmentation: Didactics, learning and teaching in Europe. Opladen: Barbara Budrich Publisher.

Hultman, G., Löfgren, R., \& Schoultz, J. (2012). Subject didactics in practice - hidden in the process a study of teaching logics and classroom cultures. Education Inquiry, 3(1), 3-18.

Hutchins, E., \& Klausen, T. (1990). Distributed cognition in an airline cockpit. Paper presented at the 2nd international congress for activity theory, Lahti, Finland, May 21-25, 1990.

Jackson, S. (2000). A qualitative evaluation of shared leadership barriers, drivers and recommendations. Journal of Management in Medicine, 14(3/4), 166-178.

Jank, W., \& Meyer, H. (2002). Didaktische Modelle. Berlin: Cornelsen-Scriptor.

Johnson, L. (2006). "Making her community a better place to live": Culturally responsive urban school leadership in historical context. Leadership and Policy in Schools, 5(1), 19-36.

Johnson, L. (2007). Rethinking successful school leadership in challenging US schools: Culturally responsive practices in school-community relationships. International Studies in Educational Administration, 35(3), 49-57. 
Kalantzis, M., Cope, B. (1999). Multicultural education: Transforming the mainstream. In Critical multiculturalism: Rethinking multicultural and antiracist education (pp. 245-276). Milton Keynes: Lightning Source.

Kansanen, P. (1995) The Journal of Curriculum Studies, 27(4), 347-352.

Kansanen, P. (2009). Subject-matter didactics as a central knowledge base for teachers, or should it be called pedagogical content knowledge? Pedagogy, Culture \& Society, 17, 29-39.

Kant, I. (1915). Avhandlingar om fred och rätt. Stockholm: Albert Bonnier.

Kant, I. (1992). Kant on Education [Über Pädagogik]. Translated by Annette Churton. London: Kegan Paul, Trench, Trubner, 1899. Bristol: Thoemmes Press.

Kelley, C., \& Halverson, R. (2012). The comprehensive assessment of leadership for learning: A next generation formative evaluation and feedback system. Journal of Applied Research on Children: Informing Policy for Children at Risk, 3(2), 4.

Kemp, P. (2010). Citizen of the world: The cosmopolitan ideal for the twenty-first century. New York: Humanity Books.

Kennedy, K., \& Chi-Kin Lee, J. (Eds.). (2016). European didactics and Chinese curriculum: Curriculum thoughts in dialogue. London/New York: Routledge.

Kesson, K. R., \& Henderson, J. G. (2010). Reconceptualizing professional development for curriculum leadership: Inspired by John Dewey and informed by Alain Badiou. Educational Philosophy and Theory, 42(2), 213-229.

Kivelä, A. (1998). Gibt es noch eine Theorie pädagogischen Handelns? Zeitschrift für Pädagogik, 44(4), 603-616.

Kivelä, A. (2004). Subjektifilosofiasta pedagogisen toiminnan teoriaan. Oulu: Acta Universitatis Ouluensis.

Klafki, W. (1963). Studien zur Bildungstheorie und Didaktik. Weinheim: Beltz.

Klafki, W. (1994). Neue Studien zur Bildungstheorie und Didaktik. Weinheim: Beltz.

Klafki, W. (1995). On the problem of teaching and learning contents from the standpoint of critical-constructive Didaktik. In S. Hopmann \& K. Riquarts (Eds.), Didaktik and/or curriculum (pp. 187-200). Kiel: IPN.

Klar, H. W. (2012). Fostering department chair instructional leadership capacity: Laying the groundwork for distributed instructional leadership. International Journal of Leadership in Education, 15(2), 175-197.

Kliebard, H. M. (2004). The struggle for the American curriculum, 1893-1958. London: Routledge.

Koyama, J. P. (2010). Making failure pay: For-profit tutoring, high-stakes testing, and public schools. Chicago: University of Chicago Press.

Koyama, J. (2011). Principals, power, and policy: Enacting "supplemental educational services". Anthropology \& Education Quarterly, 42(1), 20-36.

Kristensson Uggla, B. (1999). Kommunikation på bristningsgränsen. En studie i Paul Ricœurs projekt. Stockholm: Symposion.

Kristensson Uggla, B. (2010). Ricoeur, hermeneutics, and globalization. New York: Continuum.

Kroksmark, T. (1989). Introduktion. In J. A. Comenius (Ed.), Didactica Magna. Göteborg: Daidalos.

Ladson-Billings, G. (1994). What we can learn from multicultural education research. Educational Leadership, 51(8), 22-26.

Ladson-Billings, G. (1995). Toward a theory of culturally relevant pedagogy. American Educational Research Journal, 32(3), 465-491.

Lahdes, E. (1977). Peruskoulun uusi opetusoppi. Otava: Helsinki.

Latour, B. (1986). The powers of association. In J. Law (Ed.), Power, action and belief. A new sociology of knowledge? (pp. 261-277). Keele: Sociological Review Monograph.

Latour, B. (2005). Reassembling the social: an introduction to actor-network-theory. Oxford: University Press.

Lave, J. (1988). Cognition in practice. Cambridge: Cambridge University Press.

Law, J. (1986). Power, action, and belief: A new sociology of knowledge. London: Routledge \& Kegan Paul. 
Lawn, M., \& Grek, S. (2012). Europeanizing education: Governing a new policy space. Oxford: Symposium Books.

Leithwood, K. (1994). Leadership for school restructuring. Educational Administration Quarterly, 30(4), 498-518.

Leithwood, K. A., \& Riehl, C. (2003). What we know about successful school leadership. Nottingham: National College for School Leadership.

Leithwood, K., \& Seashore-Louis, K. (2011). Linking leadership to student learning. San Francisco: Wiley.

Leithwood, K., Seashore, K., Anderson, S., \& Wahlstrom, K. (2004). Executive summary: Review of research: How leadership influences student learning.

Levinas, E. (1992). Jenseits des Seins oder anders als Sein geschieht. München: Verlag Karl Alber.

Lewellen, T. C. (2002). Anthropology of globalization, the: Cultural anthropology enters the 21st century. London: Bergin \& Garvey.

Lezotte, L. W. (1979). A policy prospectus for improving urban education. Hartford: Connecticut State Board of Education.

Lezotte, L. W. (1986). School effectiveness: Reflections and future directions.

Lick, D. W., \& Murphy, C. U. (2004). Whole faculty study groups (3rd ed.). Thousand Oaks: Corwin Press.

Lindgard, B., \& Rizvi, F. (2009). Globalizing education policy. London: Routledge.

Lindgard, B., Wayne, M., Rezai-Rashti, G., \& Sellar, S. (2015). Globalizing educational accountabilities. London: Routledge.

Linné, A. (2015). Curriculum theory and didactics towards a theoretical rethinking. NordSTEP 2015(1), 27002. http://dx.doi.org/10.3402/nstep.v1.27002.

Louis, K. S., \& van Velzen, B. (2012). Educational policy in an international context. Political culture and its effects. New York: Palgrave.

Luhmann, N. (1996). Social systems. Stanford: Stanford University Press.

Luhmann, N. (2012). Theory of society (Vol. 1). Stanford: Stanford University Press.

Luke, A. (2010). Defining critical literacy. In Moving critical literacies forward: A new look at praxis across contexts (pp. 19-31). New York: Routledge.

Lumby, J. (2008). Disappearing gender: Choices in identity. In H. Sobehart (Ed.), Women. Leading education across the continents: Sharing the spirit, fanning the flame (pp. 29-38). Lantham/ Toronto: Rowman \& Littlefield.

Lundgren, U. P. (1972). Frame factors and the teaching process. Stockholm: Almqvist \& Wiksell.

Lundgren, U. P. (2015). When curriculum theory came to Sweden. NordSTEP 2015, 1: 27000 http://dx.doi.org/10.3402/nstep.v1.27000. The postmodern condition. Manchester University Press.

Lyotard, J. F. (1979). Describing conceptions of the world around us. Instructional Science, 10, $177-200$.

MacBeath, J. (2006). School inspection \& self-evaluation: Working with the new relationship. London: Routledge.

MacBeath, J. (2012). Future of teaching profession. Cambridge: Educational International Research Institute and University of Cambridge.

MacDonald, M. J. (1971). Rigorous mortis: Allegory and the end of hermeneutics. Studies in the Literary Imagination, 28(2), 107.

MacDonald, M. J. (1995). Rigorous mortis: allegory and the end of hermeneutics. Studies in the literary imagination, 28(2), 107.

Marks, H., \& Printy, S. (2003). Principal leadership and school performance: An integration of transformation and instructional leadership. Educational Administration Quarterly, 4(4), 293-331.

Marton, F. (1981). Describing conceptions of the world around us. Instructional Science, 10, $177-200$.

Marton, F. (2014). Necessary conditions for learning. London: Routledge.

Marx, K. (1946). Kritik des Gothaer Programms. 
Mason, T. C., \& Helfenbein, R. J. (2012). Ethics and international curriculum work. The challenges of culture and context. Charlotte: IAP.

Masschelein, J. (1991). Kommunikatives Handeln und pädagogisches Handeln. Weinheim: Deutscher Studien Verlag.

Masschelein, J. (2004). How to conceive of critical educational theory today? Journal of Philosophy of Education, 38(3), 351-367.

Matusov, E. (2011). Authorial teaching and learning. In E. J. White \& M. Peters (Eds.), Bakhtinian pedagogy: Opportunities and challenges for research, policy and practice in education across the globe (pp. 21-46). New York: Peter Lang Publishers.

Mayo, E. (1933). The human problems of an industrial organization. New York: McMillan.

McGregor, D. (1960). The human side of enterprise. New York: McGraw-Hill.

McKenzie, K. B., Christman, D., Hernandez, F., Fierro, F., Capper, C., Dantley, D., ... Scheurich, J. (2008). From the field: A proposal for educating leaders for social justice. Educational Administration Quarterly, 44(1), 111-138.

McKernan, J. (2008). Curriculum and imagination. Process theory, pedagogy and action research. London: Routledge.

McLaren, P. (1998). Life in schools. An introduction to critical pedagogy in the foundations of education. Reading: Addison-Wesley.

McLaren, P. (2000). Che Guevara, Paulo Freire, and the pedagogy of revolution. Lanham: Rowman \& Littlefield Publishers.

Mead, G. H. (1967). Mind, self and society. Chicago: University of Chicago Press.

Meinecke F. (1907/2015). Cosmopolitanism and the national state. New Jersey:Princeton University Press.

Meinecke, F. (1970). Cosmopolitanism and the national state (trans: Robert B. Kimer) (Princeton, 1970), 10.

Menck, P. (1995). Anmerkung som Begriff der "Didaktik" in Deutschland. In S. Hopmann \& K. Riquarts (Eds.), Didaktik und/oder Curriculum. Grundprobleme einer international vergleichenden Didaktik. Zeitschrift für Pädagogik, 33. Beiheft. Weinheim: Beltz Verlag.

Merton, R. K. (Ed.). (1968). Social theory and social structure. New York: Simon \& Schuster.

Metcalf, H. C., \& Urwick, L. (1942). Dynamic administration: The collected papers of Mary Parker Follett. London: Routledge.

Meyer, H. (1997). Schulpädagogik (Vol. Bd. 1). Berlin: Cornelsen Scriptor.

Meyer, J. W. (2010). World society, institutional theories and the actor. Annual Review of Sociology, $36,1-20$.

Meyer, J. W., \& Rowan, B. (1977). Institutionalized organizations: Formal structure as myth and ceremony. American Journal of Sociology, 83(2), 340-363.

Meyer, H. D., \& Rowan, B. (Eds.). (2006). The new institutionalism in education. New York: SUNY Press.

Meyer-Drawe, K. (1984). Leiblichkeit und Sozialität: Phänomenologische Beiträge zu einer pädagogischen Theorie der Inter-Subjektivität. Munich: Fink.

Michelfelder, D. P., \& Palmer, R. E. (1989). Dialogue and deconstruction: The Gadamer-Derrida encounter. New York: SUNY.

Miller, C., \& Martin, B. (2015). Prinicipal preparedness for leading in demographically changing schools: Where is social justice training? Educational Management, Administration and Leadership, 43(1), 129-151.

Mittelstrass, J. (1979). The Philosopher's conception of Mathesis Universalis from Descartes to Leibniz. Journal of Annals of Science, 36(6), 593-610. http://dx.doi. org/10.1080/00033797900200401.

Moland, L. L. (2011). Hegel on political identity. Patriotism, nationality, cosmopolitanism. Evanston: Northwestern University Press.

Moll, L. C., Amanti, C., Neff, D., \& Gonzalez, N. (1992). Funds of knowledge for teaching: Using a qualitative approach to connect homes and classrooms. Theory Into Practice, 31(2), 132-141.

Mollenhauer, K. (2014). Forgotten connections. On culture and upbringing. (N. Friesen, Ed. \& Trans.). London: Routledge. 
Moller, J. (2009a). Approaches to school leadership in Scandinavia. Journal of Educational Administration and History, 41(2), 165-177.

Moller, J. (2009b). School leadership in an age of accountability: Tensions between managerial and professional accountability. Journal of Educational Change, 10(1), 37-46.

Moller, J., Eggen, A., Fuglestad, O. L., Langfeldt, G., Presthus, A. M., Skrøvset, S., \& Vedøy, G. (2005). Successful school leadership: The Norwegian case. Journal of Educational Administration, 43(6), 584-594.

Molnar, A., \& Zahorik, J. A. (1977). Curriculum theory. Selected Papers from the Milwaukee Curriculum Theory Conference (University of Wisconsin, Milwaukee, November 11-14, 1976).

Moos, L. (Ed.). (2013). Transnational influences on values and practices in Nordic educational leadership: Is there a Nordic model? Dordrecht: Springer.

Moos, L., Möller, J., \& Johansson, O. (2004). A Scandinavian perspective on educational leadership. The Educational Forum, 68(3), 200-210.

Moos, L., Johansson, O., Merok Paulsen, J., Strand, M., \& Risku, M. (2016). Democracy in complex networks: Political leaders and administrative professionals. In L. Moos, E. Nihlfors, \& J. Merok Paulsen (Eds.), Nordic superintendents: Agents in a broken chain (pp. 177-205). Dordrecht: Springer.

Muijs, D., \& Harris, A. (2006). Teacher led school improvement: Teacher leadership in the UK. Teaching and Teacher Education, 22(8), 961-972.

Myhre, R. (1976). Pedagogisk idéhistorie fra oldtiden till 1860. Oslo: Fabritius.

National Commission on Excellence in Education. (1983). A nation at risk: The imperative for educational reform. The Elementary School Journal, 84(2), 113-130.

Norberg, K., \& Johansson, O. (2010). The ethical dimensions of curriculum leadership in Scandinavian countries. Journal of Educational Administration, 48(3), 327-336.

Nordin, A., \& Sundberg, D. (Eds.). (2014). Transnational policy-flows in European education: The making and governing of knowledge in the education policy field. Oxford: Symposium.

Nordkvelle, Y. (2003). Didactics: From classical rhetoric to kitchen-Latin. Pedagogy, Culture \& Society, 11(3), 315-330.

Normand, R. (2016). The changing epistemic governance of European education. The fabrication of the Homo Academicus Europeanus? Dordrecht: Springer.

Normand, R., \& Derouet, J.-L. (2017). A European politics of education. Perspectives from sociology, policy studies and politics. London: Routledge.

Nyvaller, M. (2015). Pedagogisk utveckling genom kollegial granskning, Gothenburg studies in educational sciences 372 . Gothenburg: University of Gothenburg.

Oelkers, J. (2000). Democracy and education: About the future of a problem. In J. Oelkers \& H. Rhyn (Eds.), Dewey and european education: General problems and case studies. Dordrecht: Springer.

von Oettingen, A. (2001). Det pedagogiska paradoks. Et grundstudie i almen pedagogik. Århus: Klim.

von Oettingen, A. (2006). Pedagogisk filosofi som reflektert omgang med pedagogiska antinomier. Aarhus: Klim.

von Oettingen, A. (2016). Almen didaktik. Köpenhamn: Munksgaard.

Ong, W. J. (2002). Orality and literacy: The technologizing of the word (2nd ed.). New York: Routledge.

Orr, M. T., \& Pounder, D. (2011). Teaching and preparing school leaders. In Finding, preparing, and supporting school leaders: Critical issues, useful solutions (pp. 11-39). Lanham: Rowman \& Littlefield Education.

Palamidessi, M., \& Feldman, D. (2003). The development of curriculum thought in Argentina. In W. Pinar (Ed.), International handbook of curriculum research (pp. 109-122). Mahwah: Lawrence Erlbaum.

Palmer, R. E. (1969). Hermeneutics. Evanston: Northwestern University Press.

Papastephanou, M. (Ed.). (2016). Cosmopolitanism: Educational, philosophical and historical perspectives. Dordrecht: Springer. 
Paraskeva, J. M. (2016). Curriculum epistemicides. Towards an itinerant curriculum theory. New York: Routledge.

Paraskeva, J. M., \& Steinberg, S. (Eds.). (2016). Curriculum: Decanonizing the field. New York: Peter Lang.

Parsons, T. (1963). On the concept of political power. Proceedings of the American Philosophical Society, 107, 232-262.

Pedersen, K. O. (2011). Konkurrencestaten. Copenhagen: Hans Reitzels Forlag.

Pedroni, T. C. (2007). Market movements: African American involvement in school voucher reform. London: Routledge.

Perander, J. J. F. (1883). Herbartianismen i pedagogiken. Helsingfors: J. C. Frenckell \& Son.

Perrow, C. (1970). Organizational analysis: A sociological view. Belmont: Wadsworth Publishing.

Peterson, K. D., \& Deal, T. E. (1998). How leaders influence the culture of schools. Educational Leadership, 56, 28-31.

Pinar, W. (1978). The reconceptualization of curriculum studies. Journal of Curriculum Studies, 10(3), 205-214.

Pinar, W. F. (Ed.). (2000). Curriculum studies: The reconceptualization. Troy: Educator's International Press.

Pinar, W. F. (2004). What is curriculum theory? New York: Routledge.

Pinar, W. F. (2009). The worldliness of a cosmopolitan education: Passionate lives in public service. New York: Routledge.

Pinar, W. F. (2011). The character of curriculum studies: Bildung, currere, and the recurring question of the subject. New York: Palgrave Macmillan.

Pinar, W. F. (2012). What is curriculum theory? London: Routledge.

Pinar, W. F. (2013). International handbook of curriculum research. New York: Routledge.

Pinar, W. F., Reynolds, W. M., Slattery, P., \& Taubman, P. M. (1995). Understanding curriculum. An introduction to the study of historical and contemporary curriculum discourses (Vol. 17). New York: Peter Lang.

Pinkard, T. (1994). Hegel's phenomenology. The sociality of reason. Cambridge: Cambridge University Press.

Portera, A. (1998). L'educazione interculturale nella teoria e nella practica: Stereotipi pregiudizi e pedagogia interculturale nei libri di testo della scuola elementare. Padua: CEDAM.

Porter, A. C. (2002). Measuring the content of instruction: Uses in research and practice. Educational Researcher, 31(7), 3-14.

Powell, W. W., \& DiMaggio, P. J. (1991). The new institutionalism in organizational analysis. Chicago: Chicago University Press.

Purkey, S. C., \& Smith, M. S. (1983). Effective schools: A review. The Elementary School Journal, 83(4), 427-452.

Purpel, D. E. (1989). The moral \& spiritual crisis in education: A curriculum for justice and compassion in education. Westport: JF Bergin \& Garvey.

Putnam, H. (1988). Representation and reality. Cambridge, MA.: MIT Press.

Ravitch, D. (2010). Why I changed my mind about school reform: Federal testing has narrowed education and charter schools have failed to live up to their promise. Wall Street Journal. http:// online.wsj.com/news/articles/SB10001424052748704869304575109443305343962.

Reid, W. (1994). The pursuit of curriculum: schooling and public interest. Norwood: Ablex.

Ricœur, P. (1976). Interpretation theory: discourse and the surplus of meaning. Fort Worth: Texas Christian University Press.

Ricoeur, P. (1989). Phenomenology and hermeneutics. In P. Ricoeur (Ed.), Hermeneutics and the human sciences (pp. 101-128). Cambridge: Cambridge University Press.

Risk, A. N. A. (1983). National commission on excellence in education. Washington, DC: National Academy Press.

Rizvi, F. (2009). Towards cosmopolitan learning. Discourse: Studies in the cultural politics of education, 30(3), 253-268.

Robertson, S. L. (2006). The politics of constructing (a competitive) Europe(an) through internationalising higher education: Strategies, structures, subjects. Perspectives in Education, 24(4), 29-44. 
Robinsohn, S. B. (1972). Bildungsreform als Revision des Curriculum und eine Strukturkoncept für Curriculumentwicklung (4th ed.). Neuwied am Rhein: Luchterhand Vorlag.

Roethlisberger, R. C., Dickson, W. J., \& Wright, H. A. (1943). Management and the worker: An account of a research program conducted by the Western Electric Company, Hawthorne Works, Chicago. Cambridge, MA: Harvard University Press.

Rogers, E. M. (2010). Diffusion of innovations. New York: Simon and Schuster.

Rogoff, B. (1990). Apprenticeship in teaching. Oxford: Oxford University Press.

Rorty, R. (1989). Contingency, irony, and solidarity. Cambridge: Cambridge University Press.

Roth, K., \& Burbules, N. C. (2007). Changing notions of citizenship education in contemporary nation-states. Journal of Moral Education, 37(2), 257-270. doi:10.1080/03057240802009678.

Roth, K., \& Surprenant, C. W. (Eds.). (2012). Kant and education: Interpretations and commentary. London: Routledge.

Rousseau, J. J. (1979). Emile, or on education (A. Bloom, Trans.). New York: Basic Books, 54, $108-170$.

Rousseau, J. J. (1762/2002). The social contract and the first and second discourses. New Haven: Yale University Press.

Rövik, K. A., Eilertsen, T. V., \& Moksnes Furu, E. (2014). Reformideer i Norsk skola. Spredning, oversettelse, implementering. Oslo: Cappelen Damm.

Rudd, T., \& Goodson, I. F. (2016). Refraction as a tool for understanding action and educational orthodoxy and transgression. Revista Tempos e Espaços em Educação, Special Issue: Curriculum, Orthodoxy and Transgression, 9(18), 99-110.

Ryan, J. (2003). Leading diverse schools (Vol. 2). Berlin: Springer.

Ryan, J. (2006). Inclusive leadership and social justice for schools. Leadership and Policy in Schools, 5(1), 3-17.

Saari, A. (2012). The map is the territory: Educational evaluation and the topology of power. European Educational Research Journal, 11(4), 586-600.

Sadler, J. E. (2007). J. A. Comenius and the concept of universal education. New York: Routledge.

Saeverot, H. (2016). Revitalising Bildsamkeit? Studies in Philosophy and Education, 35(1), 1-16.

Saeverot, H., \& Werler, T. (2017). Pedagogikkens språk - Begreper i pedagogisk vitenskap (Language of education - Concepts in education as a science). Oslo.

Säfström, C. A., \& Östman, L., (1999). Textanalys: Introduktion till syftesrelaterad kritik. Lund: Studentlitteratur.

Sahlberg, P. (2015). Finnish lessons 2.0. New York: Teachers College Press.

Säljö, R. (1994). Minding action. Conceiving of the world versus participating in cultural practices. Nordisk Pedagogik, 14, 71-80.

Salovaara, P. (2011). From leadership-centricity toward leadership. A hermeneutic-narrative study. Tampere: University of Tampere.

Scanlan, M., \& López, F. (2012). ¡Vamos! How school leaders promote equity and excellence for bilingual students. Educational Administration Quarterly, 48(4), 583-625.

Schaffar, B. (2009). Allgemeine Pädagogik im Zwiespalt. Würzburg: Ergon.

Schaffar, B., \& Uljens, M. (2015). Paradoxical tensions between Bildung and Ausbildung in Academia. Moving within or beyond the modern continental tradition? In E. Westergaard \& J. Wiewiura (Eds.), On the facilitation of the academy (pp. 1-16). Rotterdam: Sense. https:// www.sensepublishers.com/media/2288-on-the-facilitation-of-the-academy.pdf.

Schaller, K. (1995). The Didactic of J. A. Comenius between instruction, technology and pansophy. In S. Hopmann \& K. Riquarts (Eds.), Didaktik and/or curriculum (pp. 57-71). Kiel: IPN.

Scheurich, J. J. (1998). The grave dangers in the discourse on democracy. International Journal of Leadership in Education Theory and Practice, 1(1), 55-60.

Schleiermacher, F. D. (1826/1983). Ausgewählte Pädagogische Schriften. Besorgt von Ernst Lichtenstein. Paderborn: Schöningh.

Schleiermacher, F. D. E. (1998). Hermeneutics and criticism. Cambridge: Cambridge University Press.

Schmidt, V. A. (2008). Discursive institutionalism: The explanatory power of ideas and discourse. Annual Review of Political Science, 11, 303-326. 
Schmidt, V. A. (2009). Re-envisioning the European union: Identity, democracy, economy. JCMS: Journal of Common Market Studies, 47(1), 17-42.

Schmidt, V. A. (2015). Conceptualizing Europe as a 'region-state'. In A. Spanakos \& F. Panizza (Eds.), Conceptualising comparative politics (pp. 17-45). New York: Routledge.

Schmidt, V. A. (2016). Conceptualizing Europe as a "Region-State". In T. Spanakos \& F. Panizza (Eds.), Conceptualizing comparative politics (pp. 17-45). New York: Routledge.

Schnapper, D. (1994). La communaute des citoyens: Sur L'idee modern de nation. Paris: Gallimard. Schwab, J. J. (1969). The practical: A language for curriculum. The School Review, 78(1), 1-23.

Schwab, J. J. (1978). Education and the structure of the disciplines. In Science, curriculum, and liberal education (pp. 229-272). Chicago: University of Chicago Press.

Senge, P. (1990). The fifth discipline: The art and science of the learning organization. New York: Currency Doubleday.

Sennett, R. (1998). The spaces of democracy. Ann Arbor: University of Michigan, College of Architecture+ Urban Planning.

Sheppard, B. (1996). Exploring the transformational nature of instructional leadership. Alberta Journal of Educational Research, 42(4), 325-344.

Shields, C. M. (2004). Dialogic leadership for social justice: Overcoming pathologies of silence. Educational Administration Quarterly, 40(1), 109-132.

Shields, C. M. (2010). Transformative leadership: Working for equity in diverse ontexts. Educational Administration Quarterly, 46(4), 558-589.

Shields, C. M. (2012). Transformative leadership in education: Equitable change in an uncertain and complex world. New York: Routledge.

Shields, C. M. (2014). Leadership for social justice education: A critical transformative approach. In International handbook of educational leadership and social (in) justice (pp. 323-339). Dordrecht: Springer.

Shulman, L. S. (1986). Those who understand: Knowledge growth in teaching. Educational Researcher, 15(2), 4-31.

Silins, H., \& Mulford, B. (2002). Schools as learning organisations: The case for system, teacher and student learning. Journal of Educational Administration, 40(5), 425-446.

Siljander, P. (2000). Kasvatus ja sivistys. Gaudeamus: Helsinki.

Siljander, P. (2008). Educability and Bildung in J.F. Herbart's Theory of Education. In P. Siljander, A. Kivelä, \& A. Sutinen (Eds.), Theories of Bildung and Growth. Connections and controversies between continental educational thinking and American Pragmatism. Rotterdam: Sense Publishers.

Siljander, P., Kivelä, A., \& Sutinen, A. (Eds.). (2012). Theories of bildung and growth. Rotterdam: Sense.

Simon, H. (1976). Administrative behavior: A study of decision making processes in administrative organization (3rd ed.). New York: MacMillan.

Simon, H. A. (1991). Bounded rationality and organizational learning. Organization Science, 2(1), $125-134$.

Sivesind, K. (2008). Reformulating reform: Curriculum theory revisited. Oslo: Faculty of Education, University of Oslo.

Sleeter, C. E., \& Grant, C. A. (1985). Race, class, and gender in an urban school a case study. Urban Education, 20(1), 37-60.

Sleeter, C., \& Grant, C. (1987). An analysis of multicultural education in the United States. Harvard Educational Review, 57(4), 421-445.

Smith, G. (2001). Curriculum and teaching face globalization. In W. F. Pinar (Ed.), International handbook of curriculum research (pp. 35-52). Mahwah: Lawrence Erlbaum.

Smylie, M. A., \& Denny, J. W. (1990). Teacher leadership: Tensions and ambiguities in organizational perspective. Educational Administration Quarterly, 26(3), 235-259.

Snellman, J. V. (1861/1893). Föreläsningar i pedagogik Lectures in Education (1861). In X. Band (Ed.), Samlade skrifter (pp. 193-309). Helsinki: Otava.

Somr, M., \& Hrušková, L. (2014). Herbart's philosophy of pedagogy and educational teaching. Studia Edukacyjne, 33, 413-429. Poznań: Adam Mickiewicz University Press. 
Sousa, J. M. (2015). Curriculum and Didactics: A matter of power. The case of the University of Madeira. European Journal of Curriculum Studies, 2(2), 282-296. http://pages.ie.uminho.pt/ ejcs/index.php/ejcs/article/view/92/46.

Spillane, J. P. (2002). Local theories of teacher change: The pedagogy of district policies and programs. Teachers College Record, 104(3), 377-420.

Spillane, J. P., \& Healey, K. (2010). Conceptualizing school leadership and management from a distributed perspective: An exploration of some study operations and measures. The Elementary School Journal, 111(2), 253-228.

Spillane, J. P., Halverson, R., \& Diamond, J. B. (2001). Investigating school leadership practice: A distributed perspective. Educational Researcher, 23-28.

Steiner-Khamsi, G. (Ed.). (2004). The global politics of educational borrowing and lending. New York: Teachers College Press.

Steiner-Khamsi, G. (2013). What is wrong with the "what-went-right" approach in educational policy? European Educational Research Journal, 12(1), 20-33.

Steiner-Khamsi, G., \& Waldow, F. (Eds.). (2011). Policy borrowing and lending in education. World yearbook of education. London: Routledge.

Stogdill, R. M. (1948). Personal factors associated with leadership: A survey of the literature. The Journal of Psychology, 25(1), 35-71.

Stoll, L., Fink, D., \& Earl, L. (2002). It's about learning. London: Routlege Falmer.

Sutton, M., \& Levinson, B. A. (2001). Policy as practice: Toward a comparative sociocultural analysis of educational policy (Vol. 1). Westport: Greenwood Publishing Group.

Sztompka, P. (1990). Agency and progress: the idea of progress and changing theories of change. In Rethinking progress. Movements, forces, and ideas at the end of the 20th century (pp. 247263). Boston: Unwin Hyman.

Taba, H., \& Taba, H. (1962). Curriculum development: Theory and practice. (No. 37.013 TAB). New York: Harcourt Brace.

Taylor, F. (1911). The principles of scientific management (pp. 5-29). New York: Harper \& Brothers Publishers.

Taylor, F. W. (1947). Scientific management. New York: Harper \& Brothers.

Taylor, F. W. (1949). Scientific management. New York: Harper.

Taylor, C. (1994). Multiculturalism. Princeton: Princeton University Press.

Tenorth, H.-E. (1988). Geschichte der Erziehung. Juventa: Weinheim.

Terhart, E. (2009). Allgemeine Didaktik: Traditionen, Neuanfänge, Herausforderungen. In M. Meyer, M. Prenzel, \& S. Hellekamps (Eds.), Perspektiven der Didaktik (pp. 13-34). Dordrecht: Springer.

Theoharis, G. (2007). Social justice educational leaders and resistance: Toward a theory of social justice leadership. Educational Administration Quarterly, 43(2), 221-258.

Theoharis, G. (2009). The school leaders our children deserve: Seven keys to equity, social justice, and school reform. New York: Teachers College Press.

Tillman, L. C. (2004). Chapter 4: African American principals and the legacy of Brown. Review of Research in Education, 28(1), 101-146.

Tilly, C. (1984). Retrieving European lives.

Tschannen-Moran, M. (2014). Trust matters: Leadership for successful schools. San Francisco: Wiley.

Tyler, R. (2013). Basic principles of curriculum and instruction. Chicago: University of Chicago Press.

Uljens, M. (1992). Phenomenological features of phenomenology. Gothenburg: University of Gothenburg.

Uljens, M. (1995). A model of school didactics and its role in academic teacher education. In S. Hopmann \& K. Riquarts (Eds.), Didaktik and/or curriculum (pp. 301-332., Report 147). Kiel: Institut für die Pädagogik der Naturwissenschaften an der Universität Kiel.

Uljens, M. (1997a). School didactics and learning. Hove: Psychology Press.

Uljens, M. (Ed.). (1997b). European identity in change - The meeting between German, Russian and Nordic Educational traditions. (Report 10). Vaasa: Åbo Akademi University. 
Uljens, M. (1998). School didactics and learning. Hove: Psychology Press.

Uljens, M. (2000). Allgemeine Pädagogik in Finland during the 20th century. In M. Uljens (Ed.), Pedagogikens problem. Kulturella utvecklingslinjer och teoretiska spörsmål (pp. 1-36. Rapport 13/2000). Vasa: Pedagogiska institutionen, Åbo Akademi University.

Uljens, M. (2001). On general education as a discipline. Studies in Philosophy and Education, 20(4), 291-301.

Uljens, M. (2002). The idea of a universal theory of education - An impossible but necessary project? Journal of Philosophy of Education, 36(3), 353-375.

Uljens, M. (2007). Education and societal change in the global age. In R. Jakku-Sihvonen \& H. Niemi (Eds.), Education as a societal contributor (pp. 23-51). New York: Peter Lang.

Uljens, M. (2015). Curriculum work as educational leadership: Paradoxes and theoretical foundations. Nordic Journal of Studies in Educational Policy, 1(1), 22-30. Retrieved from http:// nordstep.net/index.php/nstep/article/view/27010.

Uljens, M. (2016). Non-affirmative curriculum theory in a cosmopolitan Era? Revista Tempos e Espaços em Educação, 9(18), 121-132. Retrieved from http://www.seer.ufs.br/index.php/ revtee/article/view/4970.

Uljens, M. \& Mielityinen, M. (2004). Schleiermachers pedagogiska föreläsningar - en modern pedagogik för det moderna samhället. (Schleiermacher's Lectures on Education) I: L. Lövlie \& K. Steinsholt (Red.), Pedagogikkens mange ansikter. Pedagogisk idéhistorie fra antikken til det postmoderne (pp. 215-231). Oslo: Universitetsforlaget.

Uljens, M., \& Ylimaki, R. (2015). Towards a discursive and non-affirmative framework for curriculum studies, Didaktik and educational leadership. Nordic Journal of Studies in Educational Policy, 1(3). http://nordstep.net/index.php/nstep/article/view/30177

Uljens, M., \& Ylimaki, R. (2017). Implications and future directions for a New research agenda grounded on non-affirmative education theory. Leadership and Policy in Schools, 16(2), 389-396.

Uljens, M., Möller, J., Ärlestig, H., \& Fredriksen, L. F. (2013). The professionalization of Nordic school leadership. In L. Moos (Ed.), Transnational influences on values and practices in Nordic educational leadership: Is there a Nordic model? (pp. 133-157). Dordrecht: Springer.

Uljens, M., Sundqvist, R., \& Smeds-Nylund, A.-S. (2016). Educational leadership for sustained multi-level school development in Finland - A non-affirmative approach. Nordic Studies in Education, 36(2), 103-124.

van Manen, M. (1991). The tact of teaching. The meaning of pedagogical thoughtfulness. New York: Suny Press.

Wæraas, A., \& Agger Nielsen, J. (2016). Translation theory“ translated': Three perspectives on translation in organizational research. Working Papers No. 16/2015. Norwegian University of Life Sciences School of Economics and Business. https://www.nmbu.no/sites/default/files/ pdfattachments/hh_wp_16_2015.pdf

Waks, L. J., \& English, A. R. (Eds.). John Dewey's democracy and education: A centennial handbook. New York: Cambridge UP.

Wallerstein, I. (1974). The rise and future demise of the world capitalist system: Concepts for comparative analysis. Comparative Studies in Society and History, 16(04), 387-415.

Wallerstein, I. (2006). European universalism: The rhetoric of power. New York: The New Press.

Weber, M. (1947). Legitimate authority and bureaucracy. InThe theory of social and economic organisation (pp. 328-340). New York: Free Press.

Weber, M. (1978). Economy and society: An outline of interpretive sociology (2 volume set). Berkeley: University of California Press.

Weick, K. E. (1976). Educational organizations as loosely coupled systems. Administrative science quarterly, 21, 1-19.

Weick, K. E. (1995). Sensemaking in organizations (Vol. 3). Thousand Oaks: Sage.

Weniger, E. (1965). Didaktik als Bildungslehre. Band 2, Didaktische Voraussetzungen der Methode in der Schule. Weinheim: Beltz. 
Wermke, W. (2013). Development and Autonomy. Conceptualising teachers' continuing professional development in different national contexts (p. 16). Stockholm: Department of education.

Westbury, I. (2000). Teaching as a reflective practice: What might Didaktik teach curriculum. In I. Westbury, S. Hopmann, \& K. Riquarts (Eds.), Teaching as a reflective practice: The German Didaktik tradition (pp. 15-39). New York: Routledge.

Whitty, G. (1974). Sociology and the problem of radical educational change. In Educability, schools and ideology (pp. 112-137). London: Croom Helm.

Wiggins, G. P., \& McTighe, J. (2005). Understanding by design. Alexandria: Association for Supervision and Curriculum Development (ASCD).

Wimmer, F. M. (2013). Intercultural philosophy-problems and perspectives. Cirpit Review, 4, $115-24$.

Williams, R. R. (1997). Hegel's ethics of recognition. Berkeley/Los Angeles: University of California Press.

Young, M. F. (1998). The curriculum of the future: from the "new sociology of education" to a critical theory of learning. Hove: Psychology Press.

Ylimaki, R. M. (2011). Critical curriculum leadership. A framework for progressive education. New York: Routledge.

Ylimaki, R. M. (2012). Curriculum leadership in a conservative era. Educational Administration Quarterly, 48(2), 304-346.

Ylimaki, R., \& Brunner, C. C. (2011). Power and collaboration-consensus/conflict in curriculum leadership: Status-quo or change? American Educational Research Journal, 48, 1258-1285.

Ylimaki, R., \& Henderson, J. (2016). Curriculum in educational leadership preparation. In G. Crow \& M. Young (Eds.), Handbook of research on the education of school leaders (2nd ed., pp. 148-172). New York: Routledge.

Ylimaki, R., \& Jacobson, S. (Eds.). (2013). US and cross-national policies, practices and preparation: Implications for successful instructional leadership, organizational learning, and culturally responsive practices. Dordrecht: Springer-Kluwer.

Ylimaki, R., \& Uljens, M. (2017). Theorizing educational leadership studies, curriculum, and Didaktik - non-affirmative education theory in bridging disparate fields. Leadership and Policy in Schools, 17(2), 175-227.

Ylimaki, R., Fetman, L., Matyjasik, E., Brunderman, L., \& Uljens, M. (2016). Beyond normativity in sociocultural reproduction and sociocultural transformation: Curriculum work-leadership within an evolving context. Educational Administration Quarterly, 1-37. doi:10.1177/00131 $61 X 16669200$.

York-Barr, J., \& Duke, K. (2004). What do we know about teacher leadership? Findings from two decades of scholarship. Review of Educational Research, 74(3), 255-316.

Young, M., Lambert, D., Roberts, C., \& Roberts, M. (2014). Knowledge and the future school: Curriculum and social justice. London: Bloomsbury Publishing.

Open Access This chapter is licensed under the terms of the Creative Commons Attribution 4.0 International License (http://creativecommons.org/licenses/by/4.0/), which permits use, sharing, adaptation, distribution and reproduction in any medium or format, as long as you give appropriate credit to the original author(s) and the source, provide a link to the Creative Commons license and indicate if changes were made.

The images or other third party material in this chapter are included in the chapter's Creative Commons license, unless indicated otherwise in a credit line to the material. If material is not included in the chapter's Creative Commons license and your intended use is not permitted by statutory regulation or exceeds the permitted use, you will need to obtain permission directly from the copyright holder.

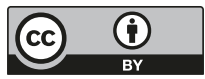




\section{Part II \\ Transnational Developments Challenging Leadership and Curriculum}

In Part II of this volume, we asked authors to explicitly consider the contemporary societal and policy changes arising from globalization. The reason is that both educational leadership and curriculum theory and Didaktik have typically been framed by and developed within a nation-state perspective. Although the modern origins to Didaktik, as represented by Johan Amos Comenius (Jan Komensky) and his Didactica Magna (1633), corresponds to the establishment of the modern school, Didaktik ultimately dealt with education as preparation for eternity in a cosmopolitan perspective. However in Didaktik and curriculum theory, for more than 200 years, curriculum aims, content, and methods have been theorized within and in relation to the morally autonomous subject in the autonomous nation state as the grand narrative. As we demonstrated in the Part I of this volume, however, today we face an ever increasing challenge of developing a conceptual language appropriate for understanding the global question. Increasing local and global interdependencies, neoliberalism, centralization of curriculum and externalized evaluation policies, changing demographics, and population migrations have all created profound challenges for nation states. Although the global perspective has been present as an educational ideal over centuries and we are familiar with policy borrowing since hundreds of years, the scale, nature, and practice of how curriculum development, educational leadership and policy are connected with transnational developments the globopolitan state - requires a new reconceptualisation.

More specifically, we argue that the most recent contemporary globalisation movement presents itself in terms of new international, transnational, and interstate governance practices, policies and procedures as well as in terms of cosmopolitanism. Thus, in our introduction, we argued for locating a theoretical starting point between traditional normative positions and functionalist positions. The authors in this part of the volume elaborate, in four different ways, the global perspective relevant for curriculum, Didaktik and leadership, expanding the argument that the renewed global developments require us to treat leadership and curriculum as related. 
Lejf Moos opens this part of the volume with a descriptive account of the empirical realities and relationships among new transnational governance structures and educational leadership in Europe. Specifically, Moos examines changes from leadership to new public management to new public governance and the consequences on what it means to educate citizens in nation states shaped by globalization and neoliberalism. Here Moos powerfully illustrates the ways in which new transnationally related governance practices appear as new challenges for educational leadership research. In the next chapter, Gert Biesta further highlights the ways in which neoliberalism and related externalized evaluation policies have instrumentalized administrative/leadership discourse, shifting from head teachers to lead learners.

Whereas the first two chapters empirically nail the contemporary neoliberal and transnational movements and their consequences for leadership and curriculum, Joao Paraskeva illustrate globalization as cosmopolitanism in terms of educational ideals in curriculum. In the next chapter, Paraskeva continues this line of argument with key decolonial thinkers (e.g. Santos 2007), demonstrating that bridging includes breaking. In so doing, Paraskeva argue that a Western eugenic curriculum of modernity has produced an abyssal thinking that constructs Western epistemologies as legitimate and all others as 'non-existent'. Paraskeva, thus, proposes a nonabyssal position that respects epistemological diversity in an era of globalization and cosmopolitanism. We see that although Paraskeva makes use of a different terminology, his position corresponds with the epistemological position we characterised in the non-affirmative position in Part I. Further, we must ask: can we imagine our theories or models being of universal validity over time and cultures or do we see them as regionally limited? Here Paraskeva joins a critique regarding positions that represent unreflected universalist knowledge ambitions and then points toward a cultural insensitivity, demonstrating versions of naïve ontological realism and representational epistemology. In the framework, we remind readers that the seminal ideas of a non-foundationalist epistemology were developed early by the modern classics of education. In his lectures on education, Friedrich Daniel Ernst Schleiermacher (1826) questioned how far educational theory can be culturally universal. His own answer was negative. But, accepting the impossibility of a universally valid position does not have to mean we accept a view of theoretical pluralism where all theories are of equal value. Accepting epistemological pluralism, rather, changes the nature of the dialogue: it requires from participants even greater sensitivity to alternative views. With this understanding must come an awareness about the positionality of one's own approach and an openness to different contemporary forms of discourse but also engagement in historical contributions in order not to loose the sight of historical roots. In this sense, theorizing is, by definition, reconstructive and evolutionary in nature; however, it can also be revolutionary.

In sum, these chapters both illustrate the dilemma of globopolitanism as new transnationally related governance practices that have created new challenges for educational leadership research, and globopolitanism as an educational ideal, which 
connect most strongly to curriculum theory/Didaktik. As we argued in part one, we think these two forms - regarding how education has developed with respect to global dimensions - are to be treated in connection to, and not disparate, from each other. In Part IV, we present chapters in which additional authors are attempting to connect curriculum/Didaktik and leadership in their work while Part V discuss nation-state education as a multi-level phenomenon.

\section{Reference}

de Sousa Santos, B. (2007). From an epistemology of blindness to an epistemology of seeing. In Cognitive justice in a global world (pp. 407-438). Lanham: Rowan \& Littlefield. 


\title{
Chapter 2 \\ Neo-liberal Governance Leads Education and Educational Leadership Astray
}

\author{
Lejf Moos
}

\begin{abstract}
The neo-liberal move to depoliticise and deregulate governance of public sectors by transferring them to the technocratic and administrative marketplace management endangers the political and democratic processes at all levels. It influences education and educational leadership at their very core: the purpose of education is shifting from intending to raise autonomous and critical participants in democracies, to aiming to produce an affirmative and employable workforce. This chapter analyses current developments in politics, administration and governance at state, municipal and institutional levels from a democratic perspective, and discusses concepts and practices of education and educational leadership with these same views. Two paradigms are described, in order to find the core of both kinds of theories: an outcomes-oriented, and a participation-oriented perspective. From a normative, democratic, viewpoint, it is argued that the core of each should be noncoercive relationships, developing deliberative practices in contemporary contexts is the main perspective in the chapter.
\end{abstract}

\section{Introduction}

It used to be self-evident that both the theory and practices of educational leadership and curriculum work are produced by societies, states and institutions. They are produced and developed in intricate networks by professional practitioners, policy makers and researchers with diverse backgrounds and with complex sets of interests. However, today we see that many governance ideas are borrowed from other systems. These borrowing industries want us to believe that 'best practices' and evidence of educational leadership and in education and teaching are of generic and global value. Two examples of this are the OECD understanding of 'autonomy' in governance and the PISA understanding of purposes and outcomes of education.

\footnotetext{
L. Moos (四)

Danish School of Education, Aarhus University, Tuborgvej 164, 2400 Copenhagen, NV, Denmark

e-mail: moos@edu.au.dk
} 
This chapter will insist that the contexts in which theories and practices are developed are closely connected to the forms and content of those applied theories and practices.

Especially in times with groundbreaking changes in societies, states and institutions, such as the current epoch, it becomes more important to try and find the connections and the dis-connections between context, practices and theories. It may be claimed that we are in the midst of one of those basic changes, as national states are being made less important, and transnational agencies and global structures and discourses emerge.

The theoretical filters and concepts used in this work will heavily influence the analyses. The governance and neo-institutional perspectives have been chosen because they create opportunities to look into the relationships between, and coupling of societies, states, organisations and agents. This is also at the core of theories of education and educational leadership: how do societies and states wish to educate the next generations, and to organise and lead the organisations and institutions in which this is supposed to take place? The filter for these analyses is the particular understanding and interests of the author, so it is also very Danish and Nordic.

The main intention of this chapter is to analyse policies and theories of educational leadership and curriculum work/didactics that are active in the current situation, moving from one paradigm to another, from a participatory, democratic paradigm, towards a management-by-objectives and outcomes paradigm, and it intends to help us to be clearer and more transparent in our arguments, intentions and descriptions.

The starting point for this chapter is a series of analyses and a critique of aspects of neo-liberal marketplace policies. They form the actual framework and context for analysing and discussing leadership and education from a deliberative, democratic perspective.

A New Global Order When nation states were the institutions that were the only agencies responsible for taking care of shielding and protecting their citizens, property, production and trade by means of the army, juridical system, policy, and export and import duty until somewhere around World War II - to put this extremely briefly - the war taught many politicians and business leaders that trade across borders in international or transnational companies boosted economy and profits. Collaboration could sustain peace, security, production and the economy. This is one of the reasons that states gradually agreed to form alliances, such as defence alliances, one of which was the North Atlantic Treaty Organisation (NATO), and economical alliances such as the World Bank, The International Monetary Fund (IMF), the European Union (EU), the Organisation for Economic Co-operation and Development (OECD), the General Agreement on Tariffs and Trade (GATT) and the World Trade Organisation (WTO).

Globalisation grew into an intricate pattern of changes in economics and the global division of labour (e.g. the emergence of more than 50,000 massive transnational companies loyal to their shareholders, and therefore able to force govern- 
ments to shape their financial policies according to market logic), changes in communication (especially the Internet and other forms of split-second, global mass media), changes in politics (with only one global political system remaining) and changes in culture (Martin and Schumann 1997). More recent areas of global interdependence are the financial market, and the climate and environmental problem. Thus, governments have tried to meet new challenges caused by transnational developments in forming transnational agencies or alliances, as mentioned before.

One global effect is the trend towards neo-liberal and marketplace politics in public governance (with a focus on decentralisation, output, competition, and strong leadership), as well as accountability politics (with a focus on recentralisation, centrally imposed standards and quality criteria, and on governing by numbers). This trend is known a neo-liberal New Public Management (NPM) (Hood 1991).

We know from research literature that is analysed in (Moos et al. 2015b) that the influence of transnational agencies, particularly of the Organisation for Economic Co-operation and Development (OECD), has been very visible in governance and education over the last 20 years (Hopman 2008). Therefore, it is interesting to investigate the ways in which these influences have been interpreted and translated into national political cultures and policies (Antunes 2006; Lawn and Lingard 2002). One transnational document seems to have been particularly influential: 'Governance in Transition: Public Management Reforms in OECD Countries' (OECD 1995). This was produced in accordance with the well-known OECD 'soft governance' strategy, the 'peer learning' method, by which member countries report trends in their public management to the organisation, where the complex picture is clarified and simplified, and trends and tendencies across countries are categorised into a smaller number of main categories:

1. Devolving authority, providing flexibility

2. Ensuring performance, control and accountability

3. Developing competition and choice

4. Providing responsive service

5. Improving the management of human resources

6. Optimising information technology

7. Improving the quality of regulation

8. Strengthening steering functions at the centre

9. Implementing reform

10. What next?

These themes were presented not as regulations or orders, but as recommendations from the OECD to member countries: national ministries could take, transform or ignore them (Moos 2009a). However, the language of the themes is extremely clear and informative, and reveals the OECD's neo-liberal political inclination (Moos and Paulsen 2014).

The recommendations meet country cultures, systems, traditions and politics, and are thereby transformed into new shapes and forms. The New Public Management 
approach had already been born (Hood 1991), but with this report it was baptised, blessed and registered as a fully developed child of the OECD. Since then, it has been adopted and transformed into many different incarnations. These tendencies form underlying the foundations for most transformations in society, governance and educational systems (Moos 2006a). Thus, special attention should be given to these categories: (1) devolving authority, (2) ensuring performance and (8) strengthening steering functions at the centre.

Barriers between nations in the areas of economics, industry and trade, and culture and communication are being torn down, and new relationships and new coalitions and liaisons are being formed. Some of these new relationships are ad hoc; some are more formal. Most of them have been established primarily in order to promote economic cooperation. Over the past decades, some of the alliances and agencies mentioned above have expanded their activities from economics into other spheres of life, such as governance and education. Governance and educational systems are also being subordinated to market thinking. The primary objectives and charters of most of these agencies exclude them from making decisions about educational policies. Nevertheless, they have begun to focus on education, as it is often seen as a cornerstone for national and global economic growth in the so-called 'global knowledge economy' (G8 2006).

\section{New State Model}

The economy frames contemporary states and transnational agencies. An example of this is when Commissioner J. M. Barosso, in the European Commission's strategy, Europe 2020, presented the Grand Challenges to the economies, and not to the societies (Barosso 2010). This was pivotal in its influence on public sector governance and thus on education and educational leadership. An effect of this is that many Western nations have developed from being primarily welfare states to being competitive states (Pedersen 2010), over the past 30-40 years.

In the years following World War II (WWII) we saw the emergence of welfare states, where areas of civil society were taken over by the state, which would protect citizens and thus further social justice, political equity and economical equality. Full employment was a major goal, and the public sector was seen primarily as delivering services to citizens: for example, citizens were supported in case of unemployment or sickness.

The transnational agencies mentioned above have been driving forces behind the opening of national economies to global competition since the 1970s, with increasing power since the mid-1990s. Their economic aims shifted from growth through full employment and increasing productivity through the labour force and technology, to growth through international trade and investment. Increasingly, national governments are members of international organisations on the regional markets. 
Moos (2014b) writes that beginning in the 1970s, governments started to turn economics in a neo-liberal direction built on rational choice ideology, increasing market influence and minimal state influence (e.g. deregulation, privatisation outsourcing). Citizens are seen less as citizens in a political democracy, and more as participants of the labour force with full responsibility for their situation, and as consumers. The public sector is seen primarily as serving production and trade in the national, innovative system. The state is influencing the availability and competence of the labour force and of the capital available. The competitive state is characterised (Pedersen 2010) as being regulating - by displaying best practices and budgets - by framing - the availability of a labour force, capital and raw materials and by being an active state - by encouraging individual citizens to enter the labour market. Pedersen argues that, based on a number of decisions with regard to the labour market and membership in the EU that were taken in 1993, this year was a turning point in the Danish development from a welfare state to a competitive state. The effects on the educational system and discourses will be discussed later on.

New Governance Neo-liberal states develop new forms of governance and new technologies of governance (Peters et al. 2000) that rely heavily on the market as the logical basis for public policy, and that involve a devolution of management from the state to the local level, to local institutions (in the case of education, to selfmanaging or private schools), to classrooms (classroom management techniques) and to individuals (self-managing students). Foucault calls this a process of neoliberal governmentalisation (Foucault 1991): governance presupposes agencies of management, but it also requires and gains the cooperation of the subjects involved. According to Foucault, this is the case in every modern society. What makes a difference is the logic or the rationale that seems to govern the fields. Governance and governmentality based on a management model are not legitimated by Weber's notion of legal-rational authority, but by a form of legitimacy or rationality that depends on market efficiency: 'No longer are citizens presumed to be members of a political community, which it is the business of a particular form of governance to express. The old and presumed shared political process of the social contract disappears in favour of a disaggregated and individualized relationship to governance' (Peters et al. 2000). People are transformed from autonomous citizens to choosers or consumers of services, as is the case in New Zealand and many other countries, where people are free to choose schools for their children (Marshall 1995). The fundamental principle at the heart of management ideology is 'freedom of choice', whereas a political perspective would stress the possibility of involvement, being a member of a community that could discuss and influence decisions.

The tendency towards more market and less state means that political logic is being replaced by capitalist logic, and that one logic is going to regulate all spheres of life.

Two Systems States, governance and leadership are, as mentioned, subject to influences from the transnational agencies, in this case, primarily the OECD and the 
European Commission (EC). However, the effects of the influences vary, because traditions, structures and discourses are different. In order to illustrate this, here is a short account of differences in selected features of the UK/US and Nordic countries (Denmark, Finland, Norway and Sweden) that are of immense importance to governance, education and the development of educational theories and practices. Here we will limit ourselves to a few pivotal values and discourses with consequences for the emergence of ideas and practices of schooling: the idea of state, schools and education. As mentioned, in the Nordic countries we see the development of the state from being first and foremost a welfare state to becoming a competitive state (Pedersen 2011), equipped to participate in the global competition for market shares in all areas of society. This development entails changes in the view of what is the optimal relations between state and individual, the governance; it brings changes in the dominant discourses on education and of the purposes of education, and it brings changes in the ideas of the best possible school organisation.

In the following sections we will sketch out some of the general ideas about important features in Nordic societies and in the UK/US, as they looked in the era from World War II until 1980s. This period has been chosen because many general ideas and practices were developed as part of 'a new beginning' following WWII, but also because they still live on in the discourses and practices today (Moos 2013).

We present indicators of core contemporary societal and educational values in Nordic education and compare them with core values in Anglo-American systems.

Relations among state, market and individuals

Proponents of the Nordic welfare states believed in a strong social democratic state and a well-regulated marketplace. The UK/US believed in a liberal state where the market was only minimally regulated by the state. We have chosen the following indicators of prevailing values to illustrate Nordic similarities and US/UK differences: GINI index, confidence in national institutions, trust, power distance and state funding of schools'.

The GINI index measures the extent to which the distribution of income or consumption expenditure among individuals deviates from a perfectly equal distribution. Thus a GINI index of 0 represents perfect equality, while an index of 100 implies perfect inequality: The Nordic GINI is 27 out of 100, indicating a high level of equality. The UK/US GINI is 38 to 41 of 100, meaning less equality (WorldBank 2015).

Confidence in national institutions is slightly higher in the Nordic countries: $65-75 \%$, whereas in the UK/US it is 65-68\% (OECD 2012b). The level of confidence is similar in Nordic countries and the UK/US.

Trust data are based on the question: 'Generally speaking would you say that most people can be trusted or that you need to be very careful in dealing with people?' Thus, trust reflects people's perception of others' reliability: in Nordic countries trust among most people was $84-89 \%$, and in the UK/US it was 49-69\% (OECD 2011).

The power distance in Denmark was 51 of 53; in the UK/US it was 40 of 53 (Hofstede 1980). 
School Organisation: The main school model in the Nordic countries was the comprehensive, non-streamed public school, while the UK/US school was more often privately funded, divided and streamed. One indicator of the importance attached to schools is the level of state funding: state funding of education is 98-100\% in the Nordic countries, while it is 79-92\% in the UK/US (OECD 2012a).

Dominant educational discourses: Nordic legislation and the dominant discourses have focused on a comprehensive school and an education for democratic Bildung, participation and equality, not only post-WWII, but for most of the twentieth'century. In the UK/US in the same era, we saw a very strong tendency to emphasise a scientific curriculum with a focus on national aims and measurable outcomes (Blossing et al. 2013).

\section{A compact overview}

\begin{tabular}{l|l|l}
\hline & Nordic indicators & UK/US indicators \\
\hline GINI & 27 of 100 & $38-41$ of 100 \\
\hline Confidence in national institutions & $65-75 \%$ & $65-68 \%$ \\
\hline Trust & $84-89 \%$ & $49-69 \%$ \\
\hline Power distance & 51 of 53 & 40 of 53 \\
\hline State funding of schools & $98-100 \%$ & $79-92 \%$ \\
\hline
\end{tabular}

It is reasonable to conclude that the UK/US had societal and political systems more inclined to build on rational choice theories, because of the belief in a liberal and weak state; on principal-agent theory, because of the greater power distance and GINI, and lower trust in other people; and on market thinking, because of the stronger belief in civil society and market. Thus, the UK/US seem better equipped to take in the transnational ideas of New Public Management.

\section{Influences from Supra- and Transnational Agencies}

The OECD and EU Commission (EC) are two powerful players in the global field of educational politics. Until now, they have not been positioned to make educational policy decisions on behalf of member governments. However, this may change with respect to the EU because of the Lisbon Agreement. National policies are influenced by supranational European Union policies 'that create, filter and convey the globalisation process' (Antunes 2006 p. 38). This influence is one of the purposes of the EC, but not the purpose for which it was originally intended. In the Lisbon Agreement, education is defined as an aspect of social services, and therefore falls within the range of Commission decisions and regulations (EC 2000).

Since both the OECD and EC - and their member governments - were interested in international collaboration and inspiration, they developed alternate methods to influence the thinking and regulation of education in member states. The EC devel- 
oped the 'open method of coordination' (Lange and Alexiadou 2007), and the OECD developed a method of 'peer pressure' (Moos 2006b; Schuller 2006).

The EC needed governance tools to influence public and private education within the member states. At the Lisbon EC meeting, participants agreed to develop a flexible method based on reflexivity and indicators. According to the meeting, this method had to include flexible governance tools that rely on 'soft law'. The divide between hard and soft law may be explained in this way: through hard law and directives, the $\mathrm{EC}$ is able to create regulations, legally binding obligations for states and individuals, whereas soft law may only be persuasive. The second feature of the open method is reflexivity: member states and institutions should inspire each other through 'peer reviews' and policy learning, such as best practices. An important tool is a set of indicators that was described in order to enable the identification of 'best practice' (Lange and Alexiadou 2007). Another very important tool is the Bologna Process of Higher Education, initially a project of homogenisation of higher education established by the European Ministers of Education in 1999, but later taken over by the EC.

CERI (Centre for Educational Research and Innovation) is the OECD bureau that manages education and educational research. The OECD does not see itself as, nor was it established to be, a federal or super-state with regulatory authority over its 30 sovereign member countries. Therefore, it has no formal power over member countries. However, the OECD/CERI was established as a powerful player in the globalisation of economies, and thereby, the restructuring of the nation states (Henry et al. 2001). Through this restructuring, it influences the policies and practices of member countries in ways other than regulatory means.

Both the EC and the OECD function in accord with the decision of the WTO's GATS agreement (WTO 1998) to include education services in the areas of free trade, thus transforming education into a commodity (Moos 2006b; Pitman 2008, 27-28 November). These influences on policy and practices, such as decisionmaking, are not linear and straightforward. Lingard (2000) describes them as 'mutually constitutive relations' between distinctive fields, or spaces. Lawn and Lingard claim that transnational organisations such as the OECD act as shapers of emerging discourses on educational policy as 'expressed in reports, key committees, funding streams and programmes' (Lawn and Lingard 2002). The main influence comes from the OECD setting the agenda (Schuller 2006), both within the whole organisation - for example, international comparisons such as PISA (Programme for International Student Assessment) (Hopman 2008) and TIMSS (Trends in International Mathematics and Science Study) - and within individual member nations. If a government wants to add an issue to the agenda, but lacks the ability to do so on its own, it can call on the OECD for help. The OECD then forms a team that reviews the state of affairs in the member state, based on a detailed and comprehensive framework designed by the OECD. The team's report often forms the basis for political action taken by the states. The review of educational research and development in Denmark is a relevant example (Moos 2006b). This strategy is explicated in the OECD publication, Education Catalogue (OECD 1998), as the 
strategy of 'peer pressure', which 'encourages countries to be transparent, to accept explanations and justification, and to become self-critical' (Ibid, p. 2).

Both the OECD and the European Commission distinguish between 'hard governance' and 'soft governance'. The choice of terms is interesting, because hard law stands for regulations that influence people's behaviour, while soft law/governance influences the way people perceive and think about themselves and their relationships with the outside world. Therefore, soft governance influences agents in much deeper ways. While these methods of influence may seem softer, in a sense, more educational, the effects of soft influence are harder and more profound. Governments and other authorities become 'leaders of leaders' through those indirect forms of power that produce 'conduct of conduct' - shorthand for Foucault's concept of governance (Foucault 1976/1994; Sørensen and Torfing 2005). These indirect forms are intended to influence the ways in which institutions and individuals perceive, interpret, understand and act. The values and norms are most important from a governmental point of view.

As mentioned above, analyses of governance in contemporary modernity show a general trend: Transnational agencies, government at the national and local levels, and agents at practical levels are increasingly attempting to use indirect forms of power, such as discourse, agenda-setting, sense-making and social technologies, instead of direct forms, such as prescriptions and instruction. Societies have become so complex that direct forms of power have become ineffective, because surveillance, control, and sanctions are impossible to implement, and because they are often not seen as legitimate forms of influence in democracies. Thus, there is a shift away from hard governance by regulation, towards soft governance by persuasion.

We see several examples of institutional isomorphism (DiMaggio and Powell 1983) among transnational, national and local institutions, such as agencies, governments and schools, based on coercion through political pressure, on mimicry of successful examples/practices, or on the transfer of norms through professional communication.

\section{Governance and Leadership}

Leadership and governance are at their very core about influencing institutions and agents through the use of forms of power. A working set of categories is: (a) regulatory leadership (e.g. regulations, legislation, direct use of power and sanctions), (b) discursive leadership and social technologies (e.g. societally constructed and naturalised as norms, discourses, strategies), and (c) reciprocal leadership (e.g. negotiations, dialogues, mediations and sense-making and enactment processes) (Moos 2009b; Moos et al. 2015a; Schmidt 2008).

Based on Foucault's post-structural perspective, influence and power may be described as a network of relationships where the poles (the agents) are defined by the relationships of which they are a part. For example, the special relationship 
between motherhood and childhood defines the mother and her child. The mother would not be a mother without a child, and vice versa. Another example is that of prisoners and their guards. The relationship, not the poles, defines the aspects of power and influence. Therefore, power is productive and relational. Influence is communication between a minimum of two poles/agents (Foucault 1983).

Pivotal situations, where influence and power are being used for leadership purposes are decision-making processes. As mentioned above, at the core of this concept of leadership is the notion that leadership does not consist of the actions of the leaders per se, but the interactions between leaders and other agents. Therefore, leadership is 'an influencing relation' between leaders and followers that takes place in situations (that may be described by their tools, routines and structures). Leadership is performed through interactions and communication that influence, and that are understood to influence other persons. This 'influence through communication' concept is parallel to Spillane et al.'s understanding of the interaction concept (Spillane et al. 2001, 2004), because both focus on the relations between leaders and teachers. The actions of the leader are only interesting if they are understood as leadership actions by the followers or co-leaders.

In principle, leadership decision-making may be understood as a three-phase process: there is (1) the production of premises for decision-making (sense-making or setting the scene); it is (2) decision making itself, and it is (3) the connections to decisions that are being made by followers (Moos 2009a).

In the first phase of decision-making - construction of premises - influence is present because of the way in which premises are defined or produced, and by whom: Who (individuals, groups, institutions) defines the situation or the problem at hand? How is the dominant discourse on which decisions and actions are based created, or how is 'the definition of reality' constructed? (DiMaggio and Powell 1983; March and Olsen 1976; Meyer and Scott 1983; Røvik 2007; Schmidt 2008; Sørensen and Tofting 2005).

It is important to distinguish between agent-driven and structural influences: There are a number of ways that individual agents or groups of agents can influence the minds and interpretations of other agents. They may set an agenda (Barach and Baratz 1962), influence sense-making and set the stage (Stacey 2001; Weick 2001), and enter into educational activities, negotiations, or other interactions (Spillane and Orlina 2005).

Secondly, decision-making is a complicated procedure involving the selection of accepted and sufficiently important premises that are influential enough to be taken into account. Individuals or collectives of agents may make decisions. Decisions may also result in a new agenda for discussing or making decisions about the field, or for the description and regulation of new behaviours. Decisions are often built into structures: legislation, societal, social and financial frameworks. Institutions are constructed because of political processes and power struggles that have sanctions attached to them. The agents' forms of direct power also have the possibility of sanctions being attached to them. However, none of these forms can guarantee results unless they are viewed - or even identified - as legitimate forms by the people and groups affected by them. On the other hand, decisions construct the prem- 
ises for new decisions. This construction is the case with leadership decisions that form the premises for employer decisions.

The third major phase of influence is the connection phase. Inspired by decisionmaking and communications theories (Thyssen 1997), a communication is only viewed as an effective communication if it 'irritates' the other pole to such a degree that it chooses to connect, to stop and reflect on, and possibly alter, their reflection processes and practices. Whether or not the other agent is connecting may be difficult to detect, since some reactions may occur long after the 'irritation' has taken place. On the other hand, there is no point in talking about influence without effects. If an act of law does not change anything concerning citizen behaviour, or if army privates do not follow a colonel's orders, then we cannot talk about real influence. The ways in which connections are made becomes an important feature of the construction of premises for future decisions.

An area of connections is constituted by evaluations and assessments. The broad field of evaluation and assessment is currently undergoing basic transformations. National as well as local systems and organisations need documentation for the use of resources in the organisations in their jurisdiction. An important aspect of the hunt for transparency involves finding out to whom agents and organisations should be accountable, and for which values they should be accountable. Schools must answer to a range of different forms of accountabilities including marketplace accountability that focuses on efficiency and competition, bureaucratic accountability that focuses on outcomes and indicators, political accountability that focuses on citizen satisfaction and negotiations, professional accountability that focuses on professional expertise, and ethical accountability that focuses on social justice (Firestone and Shipps 2005; Moos 2008). Schools must simultaneously answer to all these forms of accountability, consequently creating numerous dilemmas for schools and school leaders (Moos 2014b).

It is interesting to look at the deliberative and participative opportunities for leaders and teachers primarily because there are clear links and connections between the conditions that teachers have, and the conditions and frameworks that schools and teachers give students so they can develop a 'democratic Bildung'. This kind of Bildung is not only a matter of knowing about democracy, it is more a matter of acquiring democratic patterns of interpretation and democratic ways of life (Beane and Apple 1999; Dewey 1916/2005). Therefore, a 'democratic Bildung' must include the possibility of testing those interpretations and ways of living in real life (Moos 2011).

The concept of leadership as both influence and of decision-making is, as described here, built on the notion of relations and communication forming the very core of the understanding.

Acts of law and societal and discursive structures are institutionalised influences, some of which are called social technologies: routines, methods, work forms, and tools may all be used as social technologies, that is, technologies with a purpose or meaning (Dean 1999; Foucault 1991; Rose 1999/1989). These are used to influence people's norms and cognitive processes. They embody hidden decisions and influences from other places or other times, and form the premises for decision-making. 
Some of these technologies evolve from daily practices, while others are imposed or applied from outside actual practice. These methods might change over time, but at any given moment they are perceived as the 'natural way' of working. As they are not discussed, the power invested in them is concealed. Other social technologies are brought to the field of practice from the field of business life or from educational policy, often described as 'natural' or 'neutral' tools for practitioners to use. Here again, power is concealed, and therefore not discussed (Moos 2008). Therefore, in any setting, social technologies are powerful but silenced forms of power (Moos $2009 b$ ). The forms of power may also be compared to Scott's regulative, cognitive/ cultural and normative pillars of institutions (Scott 2001, p. 77).

\section{Social Technologies: Governing by Numbers}

The Programme for International Student Assessment (PISA) comparison has been imported into the European space as an important means of governing education (Moos et al. 2015b). It is in one package producing standards or indicators for learning, measurements for outcomes and tools for comparing students, schools and countries. This is not unexpected, as an OECD working paper shows (Wilkoszewski and Sundby 2014). This is a report on the use of a tool in the European Commission's 'open method of coordination' toolbox, the country-specific recommendations, presented in a comparison of three countries' cases of 'Steering from the centre - new modes of governance in multi-level education systems'.

Both the OECD and EC are working with the global trends to develop a new model of, and paradigm for governance of education. The central theme is that policymakers and practitioners should build on the quantitative sciences (of which psychometric comparisons are seen as a part), rather than the traditional, qualitative science of educational philosophy. These processes are called, 'The Political Work of Calculating Education' (Lawn and Grek 2012). Statistics becomes the science of the 'numerical study of social facts' and the foundation for the emergence of 'governing by numbers' (Nóvoa 2013). That means de-ideologisating and objectivising governance, leadership and education, making it possible to treat social facts as things (Desrosières 2000, page 122). Over the past century, this development has been the background for the emergence of a new group of experts in the educational field: experts in statistics and psychometrics. Politicians and policymakers are particularly interested in their work, as numbers are seen as the best and cheapest foundation for political and governance decisions. This trend is often called an 'evidence-based policy'.

When we take the foregoing observations together with the observation that the major tool, PISA, is actually measuring, what is not taught (Labaree 2014). National tests normally attempt to measure the outcomes of teaching in relation to national aims and standards. PISA was constructed as a tool that could facilitate a comparison of national outcomes across 20-30 different national educational systems. These national educational systems had their particular and very different sets of 
national aims and standards: therefore, a unified set of curricular aims was impossible. Thus, PISA constructed an independent, transnational set of aims: 'skills to meet real-life challenges'. Those aims are skills that productive workers anywhere in the advanced world would need. So the OECD reduced learning to the acquisition of economically useful skills - for employability. In order to be able to compare outcomes, a set of aims and skills was produced that are actually taught nowhere (Labaree 2014).

PISA is more economically focused than is usually acknowledged. This should be no surprise, as the OECD is the originator of the neo-liberal new public management system of thinking and governance (OECD 1995). Measuring outcomes, and in particular. outcomes along one global set of criteria, is a very powerful technology of soft governance. As time goes by, politicians, policymakers and professionals become accustomed to this, to thinking that this is the 'new normal'. As has already happened in so many ministries and local administrations, we will see a homogenisation of views on education, on the dominant discourses of education. This is like the old saying, 'You get what you measure - and only that'. That is basically economically defined and excellently calibrated to a technocratic and economic administration.

\section{Meta-Governance, Social Technologies and Self-Governance: New Public} Governance (NPG) In our study of Nordic superintendents (Moos et al. 2015b), we found that there are strong tendencies towards what we called 'governments bypassing the municipal level in matters of education'. The straight governance chain from government through municipal authorities to schools is rather unbroken, when it comes to finances and administration, but when it is about education - setting aims, developing means and measuring outcomes - the Nordic governments circumvent the municipalities (except for Finland): The aims for education and learning are being more detailed. The measurements follows through international and national tests and the means are developed on the basis of social technologies, presented by the ministry as advice to schools.

This situation fits nicely with the concept of a mixture of meta-governance and self-governance. Meta-governance involves implementing financial and legislative frameworks and initiating discursive governance. It is a form of governance that does not resemble governance: it imposes frameworks and attempts to influence discourse, yet delegates actual governance activities to various levels. Important tools of this kind of governance are social technologies such as standards and testing, quality reports and student plans, regular staff appraisals and budget models (Moos 2009a). Through various frameworks, as well as soft governance (Moos 2009b), the government encourages local authorities and individual institutions to produce and find their own identity as an institution (March and Olsen 1976) with their own specific aims, meanings and accountability. On the other hand, selfgovernance (Foucault 1983) means that institutions can - and are willing to - govern themselves in self-governing institutions and networks. Some decisions are made at state level, while others are delegated to lower levels, creating new relations between policymakers, and civil servants and various combinations of these members at all levels. 
In an overarching way, ministries and their agencies are still in control of purposes, aims, frameworks and organisation, since they make use of regulative forms of governance (legislation, regulations, economic frameworks, etc.) (Scott 2014). They set the goals and monitor the outcomes. However, for some areas of responsibility they have delegated decisions on how to achieve these goals and outcomes to lower-level agencies and to institutions. It is important to underscore the fact that the governments intend to keep a firm hand on issuing aims and measuring outcomes, 'steering from the centre' as the OECD called it in the 1995 report (OECD 1995). This produces a special form of self-governance: the neo-liberal, Panopticon form (Foucault 1961/1972; Foucault 1972), where the agents take over their own steering and monitoring, because they expect that they are being monitored, even if they cannot see a guard.

In all the Nordic countries, there are clear tendencies towards meta-governance when it comes to educational aims, accountability programmes and overarching financial frameworks for municipalities. Operations, human resource management and educational practices are, to some degree, left to self-governance by the practitioners in the workplace. However, the steering is left to practitioners to only a certain extent, because Ministries continuously attempt to influence reflection and practice through quality-assurance initiatives with clear national standards or indicators, and through monitoring and assessment of outcomes.

\section{Education Is Also About Content}

A general and ground-breaking analysis of education and student learning, write Moos et al. (2015b) with reference to Rømer et al. (2011), distinguishes between pure education, found in evidence-based and best practices, for example, and, on the other hand, impure education, described as follows:

The impure education is an education where methods of education cannot be separated from the content and the anchorage in cultural, ethical and political processes. (p. 7)

The argument is that in education one cannot separate form from content. It is an eternal and very old discussion in philosophy, dating back to Plato and to Kant. The proponents of impure education hold that one cannot separate the learning processes from the content, the object of learning. However, the separation of content from form is very common in contemporary educational policies, where learning has become the individual student's endeavour to lead and monitor her/his own learning processes. This is often labelled 'meta-learning': learning to learn, which may be supported through various methods of cognitive empowerment. In this understanding, students do not need a teacher or learning material, such as textbooks. They need to acquire only a set of cognitive learning strategies.

However, theories such as those of Dewey's and Brinkmann's (Brinkmann 2011; Dewey 1929/1960) hold that learning is not exclusively an academic, cognitive practice, but is also about establishing habits through non-verbal signals and con- 
crete manipulations with real objects and people. One learns in the interplay between student, teacher and content. Here, both academic and social learning take place, because all parties try to make sense of the information, the situation and the relations. Here, students also form their social identities, as aspiring members of the learning community of practice (Wenger 1999).

Making exclusive use of the social technologies mentioned above would exclude both the content and the relational aspects of learning. The social technologies describe procedures, in forms that are intended to be applicable in all similar situations. Therefore, they do not include the actual practice and situation, the actual people and learning objects involved in learning (Brinkmann 2011). The technologies make us forget that education, teaching and learning are, as also are all levels of leadership, very practical processes: students learn something when they manipulate objects and take part in communication, sense-making and enacting. School leaders and superintendents lead - they plan, they manage, they arrange, discuss, and negotiate real-life situations, challenges and problems. Budgets and strategies are not solely words on paper, but thoughts about actual schools, teachers and students. Therefore teachers as well as leaders need to be in close contact with the objects of their practices, both students and staff, so that they can interpret and act on both clear and weak signals about the practice processes.

The discussion looks very similar when we talk about educating students, leading schools and governing educational systems. Individualisation is spreading into more and more fields and levels. Both challenges and practices of course differ from level to level: what is meta-learning in the classroom is self-governance in schools and in municipal governance. But the basic understanding of what is needed is very much the same - or rather, should be the same - because all three levels of education work in pursuit of the general, overarching purpose of educating the next generation to take over, eventually. The superintendent strives to provide education in schools with the best of opportunities and frameworks. The school leader does the same at the school, as does the teacher with the class, groups of students and the individual students. However, the use of these internationally-inspired social technologies seems to determine the societal, cultural and political discussion of what they are there for. And the answer to that question is, for the purpose of schooling and upbringing. But the discussion of the upbringing and education of the kind of human beings to which society and schools want to contribute is often absent from discussion in the national contexts and obscure in the international context (Biesta 2009; Moos 2014a, b; Rømer 2011). The OECD has no public vision of a general Bildung with strong educational ties to history, ethics and culture; it issues directives related only to the question of skills required for the labour market.

School Leadership Functions In order to further explore concepts of school and educational leadership, we will look at the tasks and functions that school leaders are supposed to take care of. The first perspective will be that of the OECD, again, because it has had a great impact on the governments' thinking. In the Improving School Leadership Report (Pont et al. 2008), the functions are summarised into three categories, each with each three subsets. 
1. School autonomy with:

(a) 'Running a small business'

(b) Managing human and financial resources

(c) Adapting the teaching programme

2. Accountability for outcomes with:

(d) A new culture of evaluation

(e) Strategic planning, assessment, monitoring

(f) Use of data for improvement

\section{Learning-centred leadership with:}

(g) New approaches to teaching and learning

(h) Supporting collaborative teaching practice

(i) Raising achievement and dealing with diversity

The main concepts being used are clearly taken from areas of economy, (1a) and (1b), and governance, (2b) and (3c), with an emphasis on information technologies and number technologies, (2a), (2c) and (3c), and thus are very much in accord with the general governance trends, seen from the neo-liberal OECD kind of soft governances. Therefore, it does give advice to political and administrative managers, and not to school leaders. They are not given advice as to what to do, and how they may lead their schools.

Another approach would be to look at the findings of a big meta-study (Leithwood and Riehl 2005) of school leadership. Here, they found that four main categories of leadership functions were apparent in almost all contexts:

1. Setting the direction for the school with a focus on student learning

2. Seeing and empowering teachers

3. Structuring and culturing the organisation

4. Legitimising the school to the community

The first issue is very principal-agent driven: The principal sets the direction. In a participative, democratic setting one would phrase it differently: Leadership is setting and negotiating directions, making internal sense: even though schools in some systems are managed in some detail when it comes to outcomes (standards, inspections and tests), they have to find ways to achieve these outcomes themselves. They have to interpret demands and signals from the outer world and choose the means by which they want to respond to them. It is a major challenge to school leadership to interpret signals and make them into discourses and narratives, communications about differences, which form the premises for the next decisions in the community (Thyssen 2003a, b; Weick 1995, 2001).

The second issue acknowledges the fact that school leaders have no direct influence on student learning, as they do not participate in the daily interactions with students. Teachers do that, and therefore they need to be given the conditions in which to carry this out. They also need to be included in the school's educational community, and participate in maintaining and developing it. There are at least two 
reasons for that: they need to be part of, and sympathetic to the directions chosen in the school in order to contribute to a shared educational practice, and that is the second argument.

The third issue is acknowledging that learning, teaching and leading take place in organisations that need to be organised in ways that allow teachers and leaders to function in the best ways possible. One prerequisite for this is a focus on the integrity of the organisation: the ability for it to be both a convincing internal work- and life-framework, and to appear reliable in the eyes of all stakeholders. Inspiration for discussing community and membership may be drawn from Etienne Wenger's theory of how learning and identities are constructed within communities of practice (Wenger 1999). Identity construction is a dual process in a field of tension between our investment in various forms of belonging and our ability to negotiate the meanings that matter in those different contexts. The production is partly identification (investing the self in relations) and partly negotiability (negotiating meaning).

The fourth issue reminds us that schools are parts of larger systems, such as societies and communities, such as political and governance systems, and such as educational and cultural systems. It is important that schools acknowledge these systems in ways that may secure their support and acceptance. So, they can find them legitimised (March and Olsen 1976).

\section{Organisation or Organising}

Many theories are concerned with understanding and analysing organisations. Here, only one will be presented, because this new institutional understanding is in line with the general understanding of governance, leadership and education in this chapter: the core of all of them is communication and relations.

An organisation is a network of intersubjectively shared meanings that are sustained through the development and use of a common language and everyday interactions (Moos 2011s p. 38; Moos et al. 2015b; Walsh and Ungson 1991; Weick 1995).

Agents negotiate membership in a community as they share the meanings of relationships and tasks. Community and affiliations emerge in day-to-day interactions and communication.

The sense-making processes between school leaders and teachers are pivotal, because they can and should serve as models for the sense-making processes throughout the whole school. Sense-making takes place in many forms of communication, language and behaviour, interactions and communication. The sensemaking focus on language that has been at the forefront for some time should be supplemented. We need to focus more on what Weick (1988) describes 'enactment': the notion that when people act, they bring structures and events into existence, and put them into action. Weick uses this term in the context of sense-making by managers or employees. He also describes how they can enact 'limitations' on the system, to avoid unwanted issues or experiences. This too is seen as a form of social con- 
struction, focusing more on the actions we want to take in a given situation, and their materiality - for example, an agent's mimes, body language - as well as the purposes and organisational context of the interaction in which the communication takes place, and the content of the communication.

According to Weick et al. (2005), sense-making is communication through words and action that builds on the interactions that school leaders and teachers have experienced and undergone when 'the flow of action has become unintelligible' (2005, p. 409), and when external expectations seem strange and unintelligible, and there is a need for explanations and defence: What happened? What did I/we do? How can this be interpreted and understood? A similar argument is made by Vivien A. Schmidt (2008), when she discusses discursive institutionalism: Discourses are ideas in institutional contexts and interactions. Some of them are communicative discourses that emerge in the interactions between agents, when they are arguing for a given course of action. Therefore, deliberation is essential and at the core of discourse (Uljens and Ylimaki 2017).

The argument is similar to arguments about distributed leadership, made by Spillane and Woods et al. (Spillane and Orlina 2005; Woods 2004; Woods et al. 2004). They write that distributed leadership may take many forms. At the core of their concept of leadership is the notion that leadership does not lie in the actions of the leaders per se, but the interactions between leaders and other agents. Therefore, leadership is a relationship of influence between leaders and followers that takes place in situations (which may be described by their tools, routines and structures). Leadership is about interactions that influence and that are understood to influence other persons.

The basis for sense-making and for enactments is the life-world (Coburn 2004) of each participating group and individual. Life-worlds differ because of differences in background, experience, position and interests. This means that the positions, training and prior experiences of school leaders matter.

Weick's concepts of sense-making and enactment (Weick 2001) are often linked to face-to-face, real-time interactions and communications. As the distance between agents such as school leaders and teachers in contemporary Nordic schools grows so they seldom or never actually meet, they find it difficult to relate their understanding to other agents and communities, and correspondingly have a greater need to relate to regulations, norms, manuals and so on, that are transmitted in writing. This means that they have to operate at a more general level. They cannot, as in reciprocal, face-to-face encounters and deliberations, describe particular or specific situations, contexts and content. Therefore, we see the introduction of numerous social technologies that are intended to guide and lead agents to act and think along prescribed lines - models of classroom management; models of learning instruction that exclude teachers and enable individual students to learn at their own pace and in their own fashion; models of conflict resolution and peer support, such as supervision and mentoring schemes; comparisons made by translating learning outcomes to numbers (Moos et al. 2015b). 


\section{Diverse Visions of Education}

At present we see two dominant orientations or visions of education: one emerged from the welfare state model and may be called the 'deliberation orientation'. The other is attached to the competitive state, and we will call the 'outcomes orientation'. As has been already described, over the past two to three decades links have been constructed because of international competition in the global marketplace. Thus, education is intended to provide for a good position in the global race as constructed by international comparisons such as the PISA: In order to be competitive, education needs to produce students with high levels of attainment outcomes. Therefore, education is being constructed along 'management-by-objective' lines: The principal (the government) draws up the aims and measures the outcomes, while schools, teachers and students need to learn the correct answers to test questions. Very often, the curriculum that is developed in this situation is a scientific curriculum: experts know how to attain their politically administrative ends, and they describe every step in detail, for schools, teachers and students to follow. In this orientation there is a focus on 'back to basics' and skills, because those are what may be easily measured.

Core elements of the criticism of the foregoing orientation is, write (Blossing et al. 2013) firstly that the idea of curriculum objectives, originating from (among others) Franklin Bobbitt (1924); second, the conception of 'learning outcomes' as objectively measureable; and third, the technological means-end model formulated by Ralph Tyler (1949). These three elements have contributed to seeing education as an end rather than a process, as Dewey proposed; they serve as important tools in the neo-liberal governance systems that have been developed since the 1990s, across the world.

The first orientation, democratic Bildung for deliberation, has been an important vision in Nordic educational systems since WWII. Both education orientations have longer historical roots, but policies and practices have been located in the periods mentioned. The descriptions of the outcomes orientation is closely linked to the construction of the neo-liberal competitive state of the marketplace. The logic and theories governing this orientation are a good fit with the basic economy and management logic of the general governance. The description of the alternative, the democratic Bildung, it based on another kind of understanding of the needs of societies and agents: We need to develop democratic systems, thinking and practices, in order to develop sustainable societies (Moos 2011) that are able to survive the current economic and technocratic dominance. It is a normative choice, based on educational values and not on economic needs, but then, education is normative at its very core. 


\section{Democratic Bildung for Deliberation: The Core of Education and Schooling}

When we discuss education in a more educational/pedagogical way, we often use terms that are related to the intentions of education, rather than to the functions of schools (Moos 2003).

Educational functions are rarely explicit. Educational functions are effects of the educational system being one of the state institutions that is responsible for socializing (or forming) children to become well-functioning citizens in the society in which they are being brought up. Educational systems have this dual function: on the one hand, they further the optimal development of children's competence, and on the other hand, they teach them to be effective, well-functioning citizens. In this way, educational systems have always played a part in societal governance, which is about both building structures and institutions to maintain the dominant culture, and socializing citizens to willingly cooperate in this effort.

Educational intentions are reflected in a society's culture, in the formal objectives of educational institutions, and they are examined by educational theories. In Denmark, the intentions of the educational system are set out in Article 1 of the Act on the Folkeskole (1993, 2000), which states:

The school shall prepare the students for active participation, joint responsibility, rights and duties in a society based on freedom and democracy. The teaching of the school and its daily life must therefore build on intellectual freedom, equality and democracy. (authors translation)

In the Nordic countries, the foregoing means that teachers and principals must be aware of the socio-cultural environment and the learning conditions. Thus, students should not only be taught how a democratic society functions at a structural level (parliament, government, system of justice, police, and so on), they should themselves experience and live a democratic life, even at school. This means that not all methods of instruction or teacher behaviour are considered appropriate.

According to educational theory devised and discussed on the Continent in the historical epoch called Modernity (starting in the late eighteenth century), education is basically the responsibility of every generation to educate the next generation to be able to live in their society (Jean-Jacques Rousseau, Immanuel Kant, Friedrich Schleiermacher, Johann Friedrich Herbart and Dietrich Benner, to mention key French and German figures). In this discussion, I build on the work of Alexander von Oettingen (2001). Children depend on their parents to educate them, as they are born 'prematurely': they are born into a 'not-yet-condition'. In other words, they are not able to grow and survive without assistance from the older generations. Humanity depends on one generation of human beings educating the next generation of human beings (Uljens and Ylimaki 2017).

Education includes the acquisition of skills and proficiency, the assimilation and construction of knowledge, and the development of motives and values. It involves what is traditionally called subject content and liberal education, in German, 'Bildung'. Children must learn to become human beings, and therefore they must be 
educated so that they are able to function on their own in their culture and society. They cannot go on living with their parents forever, but must leave their childhood homes, and make a living and start families of their own. As these theories were devised in the 'Age of Enlightenment', they build on a concept of society - or rather, a vision of society - as enlightened and democratic. Therefore, the ideal human being, the goal of education, was the participating, democratically-minded citizen who was willing and able to be a qualified participant in the community and in society.

The debate on Democratic Bildung is still vivid in the Nordic and Danish contexts. In 1976 the Norwegian philosopher Jon Hellesnes formulated an often-quoted differentiation between conditioning and liberal education as two forms of socialization:

Affirmative education reduces humans to objects for political processes which they do not recognize as political; a conditioned human being is thus more an object for direction and control than a thinking and acting subject.

Non-affirmative education means that people are socialized into the problem complexes pertaining to the preconditions for what occurs around them and with them. Educational socialization emancipates humans to be political actors. (Fedotova 2014; Hellesnes 1976)

The ideal of liberal education, 'Bildung', is to educate human beings to be authoritative, competent and autonomous. In the Danish debate it has been called 'action competent': the individual is willing and able to be a qualified participant (Jensen and Schnack 1994). This ideal created a fundamental paradox, which has occupied theorists and practitioners ever since:

How is it possible - through external influence - to bring human beings to a state where they are not controlled by external influences?' (Leonard Nelson, 1970 in Oettingen 2001, p. 9)

This has been a fundamental question for all the educational theorists mentioned, and for many more. We know from experience that children are not able to take care of themselves; they must be educated. Parents educate children and they leave it to schools and other institutions to educate them on their behalf. Thus, education is an external influence that is somehow able to bring about a liberal education, a Bildung or educational socialisation. In principle, there are two agents in education: the child and the teacher. The questions are, what are the pre-existing conditions of the child, and what can the teacher do to further education for Bildung, for authority and autonomy?

According to Oettingen (2001), Rousseau, Kant, Schleiermacher, Herbart and Benner point to two fundamental principles in overcoming the paradox: the 'Bildsamkeit' of the child and the request for 'self-reflection'. 'Bildsamkeit' is difficult to translate into English, but it means the fundamental, innate ability (and willingness, I would add) to be open-minded and to participate in a shared praxis. The concept acknowledges the child's 'not-yet-condition' - it has not yet become what it is going to be - but it must participate in the educational interaction in order to become human. 
'Self-reflection' means that the self is able to focus its attention on something in the outer world and at the same time, on itself. This ability (and, again, willingness) enables the human being to act and to reflect on their actions, and thereafter initiate other actions. Therefore, educators should encourage children to engage in self-reflection.

When it comes to teacher activities Uljens and Ylimaki (2017) argue that two main actions are pivotal: recognition and summoning the child to self-activity. Recognition means respect, esteem, love and friendship (Honneth 1992), which are the basis for developing self-confidence and self-respect, and those are seen as the basis for learning. Following recognition is the quest for summoning to self-activity, inviting the student to become aware of her/his freedom as a cultural and political being with the option of realizing her/his aims. Thus, teachers may need to invite children to act and reflect in ways for which they are not yet ready, much like parents who invite children to walk, even when observation and experience indicate that they may not be able to walk yet. Focusing on these principles should facilitate the aim of all educational praxis, which is to ultimately render itself superfluous.

This outline of an educational introduction/discussion illustrates the ways in which educational problems and questions have a fundamental bearing on democracy: there is always a question of what kind of citizen a society or a culture wants to educate in the family, in the community and in institutional settings. Therefore, we cannot limit our discussions of education in schools to matters of subject content and curriculum. We must engage in research and debate the entirety of school life, the relations between students and teachers, the relations between teachers and principals and their relations to the local and national communities.

In bringing educational theories closer to practice, Dewey's writing has been a great inspiration. In Democracy and Educational Administration (Dewey 1937) he wrote:

What the argument for democracy implies is that the best way to produce initiative and constructive power is to exercise it. Power, as well as interest, come by useand practice ... The delicate and difficult task of developing character and good judgement in the young needs every stimulus and inspiration possible... I think that, unless democratic habits and thought and action are part of the fibre of a people, political democracy is insecure. It cannot stand in isolation. It must be buttressed by presence of democratic methods in all social relationships. (pp 345-346)

Participation Democratic education is (Moos 2014b) described by Biesta (2003) as 'creating opportunities for action, for being a subject, both in schools and other educational institutions, and in society as a whole' (Biesta 2003). Besides the opportunity for action or participation, the most important concepts related to democracy are the critique and diversity, because they give a more precise direction to the concept of participatory and deliberative democracy. In line with this understanding, Beane and Apple (1999), Furman and Starrat (2002), and Woods (2005) describe the central concerns of democratic schools as: (1) the open flow of ideas that enables people to be as fully informed as possible, (2) the use of critical reflec- 
tion and analysis to evaluate ideas, problems and policies, (3) the welfare of others and the common good and (4) concern for the dignity and rights of individuals and minorities.

This section is the brief interpretation of schools' ethical accountability. Pursuing goals of this kind has been a major concern for many educationalists over time. Besides the opportunity for action, participation and deliberation, the most important concept related to democracy is the critique, because it gives a more precise direction to the concept of deliberative democracy.

One cornerstone for learning is the capacity for self-reflection, enabling the self to simultaneously focus its attention on something in the outer world and on itself. This ability enables human beings to act and reflect on their actions, and then initiate other actions. So one of the primary tasks of teachers involves encouraging and helping children to engage in self-reflection (Moos 2003).

Deliberation If we again change the perspective from a micro- to a macrosociological and policy perspective on societies and states - a discussion of democracies - we may be able to shed new light on the micro-sociological analyses. The intention behind doing so is to try to develop links between the trends and intentions in democracies at a societal level, and the discussion of how leaders and teachers, the professionals in schools, can build the practices in schools in ways that are supportive of students' 'democratic Bildung'.

The theories mentioned in the previous section (Beane and Apple 1999; Bernstein 2000; Biesta 2003) demand that it is pivotal to give students voice and that is seen as the opportunity for deliberations in schools. This builds on a notion of a deliberative democracy that attempts to build a connection between liberal and communitarian democracy (Louis 2003).

The basis for liberal democracy is described as a special form of governance, where the free individual is capable of making his/her own choices and pursues his/ her own interests and so take care of his/her own life. Another dimension of this kind of democracy is the protection of the free individual, in that it is given certain rights or makes social contracts. In other words, individuals are seen as autonomous, even if they are part of a community and they have formed their opinions before entering the community. They are not bound by shared values, but majority votes are the preferred way of mediating opinions and reaching decisions.

In the communitarian democracy, individuals are seen as partners in social communities, bound by a set of shared moral and social values. Values are generated within the community, and may change over time. Members of a community are orientated towards a set of shared goals, and are conscious of the social bonds. These communities may be the state, or smaller parts of states.

The connection between those two forms of democracy thinking is the deliberative democracy: Both liberal and communitarian democracy concepts see the state as a central arena for all kinds of communities. The liberal concept sees politics as being formed through the complex interplay between agents in different arenas and networks, both within and outside the state. Society is seen as decentred, and political processes may take place in many arenas, within and outside elected bodies, 
such as parliaments and city councils. Deliberative democracies are seen as associations whose affairs are governed by public deliberation by its members (Englund 2006). A number of conditions must be met in this kind of democracy: The individual's rights that can be met in that the democracy is representative. The other condition is that the deliberations demand that individuals are able to a high degree of reflexivity and responsiveness towards other members of the community. A basic understanding in this concept is the concept of social identity.

The position Karen Seashore Louis takes is enlightening in this argument:

Many contemporary democratic theorists argue that the most essential element of democratic communities today is their ability to engage in civilized but semi-permanent disagreement. Articulating a humanist voice that calls for respecting and listening to all positions - but then being able to move forward in the absence of consensus - will be the critical skill that school leaders need to develop when the environment makes consensus impossible. (Louis 2003)

The theoretical or philosophical background for this chapter (Moos 2013) is a basic understanding of communication, the communicative rationale developed by the German philosopher Jürgen Habermas (1987). In his theory of universal pragmatism, a communication is seen as being legitimized if it strives for 'the strange unconstrained force of better argument'. This means that the relations in communication aim for mutual understanding with a minimum of domination it in what will always be asymmetric relations in bureaucratic organizations.

The potential for rationality in communication is inherent in communication itself. Thus, communicative rationality refers primarily to the use of knowledge in language and action, rather than to a property of knowledge.

This means that, on one hand, the person who produces the 'better argument' is the de facto leader in the situation. On the other hand, leadership in schools also consists of formal management delegated to formal positions in bureaucratic organisations: teachers over students, principals over teachers and so on.

The principal is of course the formal leader in a school, as teachers are in classrooms. Their position is designated as one involving the power to make decisions. According to the thinking presented here, anybody involved in the communication can influence the decision-making if they make the 'better argument', which refers to the argument that is being accepted as the better argument by persons who are involved in the communication, and who are affected by the associated decisions. This kind of influence is most often positioned in the 'construction of premises' phase or in the 'connecting phase', and these forms may be seen as deliberations or negotiations.

This ideal is often contested in real life, but according to Habermas (1987), this is still inherent in communication itself. Therefore there is a better chance for it to prevail if relations in schools involve communication at short range, where all participants have a chance of being heard, listened to and eventually given influence. Therefore, deliberation is the foundation on which schools may build their leadership, success and development, and thus for schools to become and stay 'self-renewing'. 


\section{Conclusion}

The vision of education for the competitive state is built on a set of core values or logic: management by objectives and outcomes-based accountability. Proponents of this paradigm often refer to scientific management and the scientific curriculum as core theories (Blossing et al. 2013; Moos et al. 2015b). It is a deeply inapproproiate that the adjectives of science are use in this way, as scientific management is fundamentally concerned with centralising the power at one centre, be it the owner, a manager or a government. Similarly, the scientific curriculum also hides the power to decide on the purpose, content, relations and methods of education behind the pretexts of expertise and value-free decisions.

This is only one interpretation of the shift in paradigm in educational governance and education itself. Both paradigms build on a set of a core logic and core purposes that are inseparable: The traditional governance paradigm, the welfare paradigm, advocates democratic equity and deliberation in society and its institutions, while the competitive paradigm builds on central management, managing by objectives and hierarchies. The traditional (Danish and European) educational paradigm builds on individual authority and democratic participation and deliberation for democratic Bildung, while the competitive paradigm builds on acquiring basic skills for employability.

It should be evident that the image drawn here takes an extreme view, but that may be legitimised by the argument that only by knowing the extreme can we see and understand the development and the moves.

In this chapter it was argued, partly on the basis of research, that competitiveand outcomes-orientated practices use more social technologies than we have seen prior to their appearance (Moos et al. 2015b). Social technologies can be seen as silenced carriers of power. They are made for a purpose - often hidden from the practitioners - and they specify ways of acting. Therefore, they point into a nondeliberative and top-down steered and managed practice.

School governance, finance and staff management, strategic development, liaising with external partners (parents, community, local authorities, politicians, national level) and educational leadership (of students as well as staff and organisations) are all parts of school leadership. These aspects of leadership cannot be separated, even if they are very different from one another. Ideally, they should be viewed from the same perspective: the education - the 'the democratic Bildung - of students and the leadership of professionals.

The leadership parallel to the Bildung of children should not be carried too far, but it may open our eyes to important aspects of educational leadership: if the intention of schooling is 'democratic Bildung', then the intentions of educational leadership must be to create a climate, a dominant discourse and a community that support the educational intentions. Thus, the community should not be governed by hidden structures and discourses of power, but should move towards transparency of relations, democracy and autonomy. If teachers and other staff are to support children in becoming democratic actors, then they themselves must be subject to transpar- 
ency, democracy, deliberation and autonomy. Staff must be treated as agents, not as subordinates.

We argued that teachers should focus on recognition, summoning to self-activity, and students' Bildsamkeit. In much the same way, school leaders should focus on recognition of the professionalism and the personalities of teachers, on encouraging to self-activity and a belief in teachers' ability and willingness to be autocratic, in sense-making and reciprocal interactions.

\section{References}

Antunes, F. (2006). Globalisation and Europeification of Education Policies: Routes, processes and metamorphoses. European Educational Research Journal, 5(1), 38-55.

Barach, P., \& Baratz, M. S. (1962). The two faces of power. American Political Science Review, 56(4), 947-952.

Barosso. (2010). Europe 2020. A European strategy for smart, sustainable and inclusive growth. Brussels, http://eur-lex.europa.eu/LexUriServ/LexUriServ.do?uri=COM:2010:2020:FIN:EN: PDF

Beane, J. A., \& Apple, M. W. (1999). The case for democratic schools. In M. W. Apple \& J. A. Beane (Eds.), Democratic schools, lessons from chalk face. Buckingham: Open University Press.

Bernstein, B. (2000). Pedagogy, symbolic control and identity: Theory, research and critique. Lanham: Rowman and Littlefield.

Biesta, G. (2003). Demokrati - ett problem för utbildning eller ett uttbildningsproblem? [Democracy - a problem for education or an educational problem?]. Utbildning \& Democracy, 12(1), 59-80.

Biesta, G. (2009). Good education in an age of measurement. Boulder: Paradigm Publishers.

Blossing, U., Imsen, G., \& Moos, L. (2013). Progressive education and new governance in Denmark, Norway and Sweden. In U. Blossing, G. Imsen, \& L. Moos (Eds.), The nordic education model: 'A School for All' encounters neo-liberal politics. Dordrecht: Springer.

Bobbitt, F. (1924). How to make a curriculum. Boston: Houghton Mifflin.

Brinkmann, S. (2011). Håndens epistemologi: Dewey som uren pædagog [The Epistemology of the hand: Dewey as an impure pedagouge]. In T. A. Rømer, L. Tanggaard, \& S. Brinkmann (Eds.), Uren padagogik. Aarhus: Klim.

Coburn, C. E. (2004). Beyond decoupling: Rethinking the relationship between the institutional environment and the classroom. Sociaology of Education, 77, 211-244.

Dean, M. (1999). Governmentality: Power and rule in modern society. London: Sage.

Desrosières, A. (2000). L'histoire de la statistique comme genre: style d'écriture et usasge sociaux. Genéses, 39, 121-137. Genéses, 39(http/dx.doi.org/10.3406/genes.2000.1626), 121-137.

Dewey, J. (1916/2005). Democracy in education. New York: Macmillan.

Dewey, J. (1929/1960). The quest for certainty. New York: Capricorn Books.

Dewey, J. (1937). Democracy and educational administration. In J. Ratner (Ed.), Education today. New York: G.P.Putman's sons.

DiMaggio, P. J., \& Powell, W. W. (1983). The iron cage revisited: Institutional isomorphism and collective rationality. American Sociological Review, 48(April), 147-160.

EC. (2000). Presidency conclusion. Lisbon, 23/24 March, DOC\&00\&8.

Englund, T. (2006). Deliberative communication: A pragmatis proposal. Journal of Curriculum Studies, 38(5), 503-520.

Fedotova, O. (2014). Modern education in the framework of affirmative and non-affirmative approaches. Procedia - Sociual and Behavioural Science, 2015(180), 55-60. 
Firestone, W. A., \& Shipps, D. (2005). How do leaders interpret conflicting accountabilities to improve student learning? In W. A. Firestone \& C. Riehl (Eds.), A new agenda for research in educational leadership (pp. 81-100). New York: Teachers College Press.

Foucault, M. (1961/1972). Galskabens historie [The history of insanity]. Frederiksberg: Det lille forlag.

Foucault, M. (1972). The archeology of knowledge. London: Tavistock.

Foucault, M. (1976/1994). Viljen til viden. Seksualitetens historie 1. København: Det Lille Forlag.

Foucault, M. (1983). The subject and power. In H. L. D. P. Rainbow (Ed.), Michel Foucault: Beyond structuralism and hermeneutics (pp. 208-226). Cambridge, MA: MIT Press.

Foucault, M. (1991). Governmentalité. In G. Burcell, C. Gordon, \& P. Miller (Eds.), The Foucault effect: Studies in governmentality (pp. 87-104). Hempel Hempstead: Harvester Wheatsheaf.

Furman, G. C., \& Starrat, R. J. (2002). Leadership for democratic community in schools. In J. Murphy (Ed.), The educational leadership challenge (pp. 105-133). Chicago: Chicago University Press.

G8. (2006). Retrieved from http://en-g8russia.ru/news/20060602/1151807.html.

Habermas, J. (1987). The theory of communicative action - lifeworld and system: A critique of functionalist reason. Boston: Beacon Press.

Hellesnes, J. (1976). Socialisering og teknokrati. København: Gyldendal.

Henry, M., Lindgard, B., Rizvi, F., \& Raylor, S. (2001). The OECD, globalisation and education policy. Amsterdam: Pergamon.

Hofstede, G. (1980). Culture's consequences: International differences in work-related values. Beverly Hills: Sage.

Honneth, A. (1992). Integrity and disrespect: priunciples of a conception of morality based on the theory of recognition. Political Theory, 20, 187-201.

Hood, C. (1991). A public management for all seasons. Public Administration, 69(Spring), 3-19.

Hopman, S. T. (2008). No child, no school, no state left behind: Schooling in the age of accountability. Journal of Curriculum Studies, 40(4), 417-456.

Jensen, B. B., \& Schnack, K. (1994). Action competence as an educational challenge. In B. B. Jensen \& K. Schnack (Eds.), Action and action competence. Danmarks Lærerhøjskole: København.

Labaree, D. (2014). Let's measure what no one teaches: PISA, NCLB, and the shrinking aims of education. Teachers College Record, http://www.tcrecord.org/Content.asp?ContentId=17533

Lange, B., \& Alexiadou, N. (2007). New forms of European governance in the education sector? A preliminary analysis of the open method of coordination. European Educationa Research Journal, 6(4), 321-335.

Lawn, M., \& Grek, S. (2012). Europeanizing education - governing a new policy space. Oxford: Symposium.

Lawn, M., \& Lingard, B. (2002). Constructing a European policy space in educational governance: The role of transnational policy actors. European Educational Research Journal, 1(2), 290-307.

Leithwood, K., \& Riehl, C. (2005). What we know about successful school leadership. In W. Firestone \& C. Riehl (Eds.), A new agenda: Directions for research on educational leadership. New York: Teacher College Press.

Lingard, B. (2000). It is and it isn't: Vernacular globalization, educationa. Policy, and restructuring. In N. C. Burbules \& C. A. Torres (Eds.), Globalization and education. Critical perspetives. New York: Routledge.

Louis, K. S. (2003). Democratic schools, democratic communities. Leadership and Policy in Schools, 2(2), 93-108.

March, J. G., \& Olsen, J.P. (1976). Ambiguity and choice in organizations. Oslo: Universitetsforlaget.

Marshall, J.D. (1995). Foucault and neo-liberalism: Biopower and Busno-power. Philosophy of education, Retrieved from http://www.ed.uiuc.edu/EPS/PES-Yearbook/95_docs/marshall.html

Martin, H.-P., \& Schumann, H. (1997). Globaliseringsfelden. Angrebet på demokrati og velstand Kobenhavn. Copenhagen: Borgen. 
Meyer, J. W., \& Scott, W. R. (1983). Organizational environments. Ritual and rationality. London: Sage.

Moos, L. (2003). Leadership for/as 'Dannelse'? In L. Moos (Ed.), Educational leadership. København: Danish University of Education Press.

Moos, L. (2006a). Fra politiske demokratidiskurser mod markedsorienterede effektivitetsdiskurser [From political discourses of democracy towards market oriented efficiency discourses]. Nordisk Pedagogik, 26(4), 322-332.

Moos, L. (2006b). A real change or a change in rhetoric? - comments to two OECD reviews on educational research: England in 2002 and Denmark in 2004. European Educational Research Journal, 5(1), 63-67.

Moos, L. (2008). School leadership for 'Democratic Bildung': fundamentalist beliefs or critical reflection? School Leadership and Management, 28(3), 229-246.

Moos, L. (2009a). A general context for new social technologies. Nordic Educational Research, 29(1), 79-92.

Moos, L. (2009b). Hard and soft governance: the journey from transnational agencies to school leadership. European Educational Research Journal, 8(3), 397-406.

Moos, L. (2011). Sustaining leadership through self-renewing communication. In L. Moos, O. Johansson, \& C. Day (Eds.), How school principals sustain success over time (pp. 127150). Dordrecht: Springer.

Moos, L. (2013). School leadership in a contradictory world. Revista de Investigacion Educativa, $31(1), 15-29$.

Moos, L. (2014b). Danish superintendent in a complex world. In A. E. Nir (Ed.), The educational superintendent: Between trust and regulation. New York: Nova Science Publisher.

Moos, L. (2014a). Leadership for creativity. International Journal of Leadership in Education, $18(2), 178-196$.

Moos, L., Nihlfors, E., \& Paulsen, J. M. (2015b). Directions for our investigation of the chain of governancd and the agents. In L. Moos, E. Nihlfors, \& J. M. Paulsen (Eds.), Nordic superintendents: agent in a broken chain (Vol. 2). Dordrecht: Springer.

Moos, L., Nihlfors, E., \& Paulsen, J. M. (2015a). Trends and tendencies. In L. Moos, E. Nihlfors, \& J. M. Paulsen (Eds.), Nordic superintendents: Agents in a broken chain (Vol. 2). Dordrecht: Springer.

Moos, L., \& Paulsen, J. M. (2014). Comparing educational governance. In L. Moos \& J. M. Paulsen (Eds.), School boards in the governance process. Dordrecht: Springer.

Nóvoa, A. (2013). Numbers do not replace thinking. European Educational Research Journal, 12(1), 139-148.

OECD. (1995). Governance in transition. Public management reforms in OECD countries. Retrieved from https://books.google.dk/books/about/Governance_in_Transition. html?id=TACcD2r0wDYC\&redir_esc=y.

OECD. (1998). Education catalogue. Paris: OECD.

OECD. (2011). Society at a glance 2011: OECD social indicators. http://www.oecd-ilibrary.org/sites/ soc_glance-2011-en/08/01/index.html;jsessionid=2a7fa4dh4ws3k.x-oecd-live-01 ?itemId=/ content/chapter/soc_glance-2011-26-en\&_cs.

OECD. (2012a). Education at a glance. Retrieved from.

OECD. (2012b). Society at a glance, trust in institutions. Retrieved from.

Oettingen, A. v. (2001). Det padagogiske paradoks [The pedagogical paradoxe]. Århus: Klim.

Pedersen, O. K. (2010). Konkurrencestaten [The competitive state]. Copenhagen: Hans Reitzels Forlag.

Pedersen, O. K. (2011). Konkurrencestaten. København: Hans Reitzels Forlag.

Peters, M., Marshall, J., \& Fitzsimons, P. (2000). Managerialism and educational policy in a global context: Foucault, neoliberalism and the doctrin of self-management. In N. C. Burbules \& C. A. Torres (Eds.), Globalization and education: Critical perspectives (pp. 109-132). New York: Routledge. 
Pitman, A. (2008). Capacity-building in South-East Asian Universities: International challenges. Paper presented at the Korean Education Research Association, Seul.

Pont, B., Nusche, D., \& Moorman, H. (2008). Improving school leadership. Policy and practice. Paris., http://www.oecd.org/edu/school/44374889.pdf: OECD.

Rømer, T. A. (2011). Dansk pædagogik i fald og bund [Danish pedagogy in fall and bottom]. In T. A. Rømer, L. Tanggaard, \& S. Brinkmann (Eds.), Uren paedagogik. Aarhus: Klim.

Rømer, T. A., Tanggaard, L., \& Brinkmann, S. (2011). Uren padagogik. Aarhus: Klim.

Rose, N. (1999/1989). Governing the soul: The shaping of the private self. London: Free Association Books.

Røvik, K. A. (2007). Trender og translasjoner. Ideer som former det 21. århundrets organisasjon. Oslo: Universitetsforlaget.

Schmidt, V. A. (2008). Discursive institutionalism: The explanatory power of ideas and discourse. Annual Review of Political Science, 11, 303-326.

Schuller, T. (2006). Reviewing OECD's educational research reviews. European Educational Research Journal, 5(1), 57-61.

Scott, W. R. (2001). Institutions and organizations. Thousand Oaks: Sage.

Scott, W. R. (2014). Institutions and Organizations. Ideas, interests, and identities. Los Angeles: Sage.

Sørensen, E., \& Tofting, J. (2005). Netvarksstyring - fra government til governance [Network governance - from government to governance]. Freederiksberg: Roskilde Universitetsforlag.

Sørensen, E., \& Torfing, J. (2005). Netvarksstyring - fra government til governance. Roskilde: Roskilde Universitetsforlag.

Spillane, J. P., Halverson, R., \& Diamond, J. B. (2001). Investigating school leadership practice: A distributed perspective. Educational Researcher, 30(3), 23-28.

Spillane, J. P., Halverson, R., \& Diamond, J. B. (2004). Towards a theory of leadership practice; a distributed perspective. Journal of Curriculum Studies, 36(1), 3-34.

Spillane, J.P., \& Orlina, E.C. (2005). Investigating leadership practice: exploring the entailments of taking a distributed persepctive. Paper presented at the AERA, Montreal.

Stacey, R. D. (2001). Complex responsive processes in organizations. New York: Routledge.

Thyssen, O. (1997). Vardiledelse. Om organisationer og etik /Valueleadership. On organizations and ethics]. København: Gyldendal.

Thyssen, O. (2003a). Luhman og ledelsen [Luhman and leadership]. In H. Højlund \& M. Knudsen (Eds.), Organiseret kommunikation - systemteoretiske analyser [Organized communication systemstheoretical analyses]. Samfundslitteratur: Frederiksberg.

Thyssen, O. (2003b). Organisationens usynlighed, Paper presented at the Professor tiltradelse. København: CBS, Handelshøjskolen.

Tyler, R. R. (1949). Basic principles of curriculum and instruction. Chicago: University of Chicago Press.

Uljens, M., \& Ylimaki, R. (2017). Non-affirmative theory of education as a foundation for curriculum studies, didaktik and educational leadership. In M. Uljens \& R. Ylimaki (Eds.), Bridging educational leadership, curriculum theory and didaktik. Non-affirmative theory of education. Educational governance research (Vol. 5). Dordrecht: Springer.

UVM (Ministry of Education). (1993, 2000). Consolidation Act on the Folkeskole. Copenhagen: Danish Ministry of Education.

Walsh, J. A., \& Ungson, G. A. (1991). Organizational memory. The Academy of Management Review, 16(1), 57-91.

Weick, K. E. (1988). Enacted sensemaking in crisis situations. Journal of Management Studies, 25(4), 305-317.

Weick, K. E. (1995). Sensemaking in organizations. Thousands Oaks: Sage.

Weick, K. E. (2001). Making sense of the organization. Malden: Blackwell.

Weick, K. E., Sutcliffe, K. M., \& Obstfeld, D. (2005). Organizing and the process of sensemaking. Organization Science, 16(4), 409-421. 
Wenger, E. (1999). Communities of practice. Learning, meaning and identity. Cambridge: Cambridge University Press.

Wilkoszewski, H., \& Sundby, E. (2014). Steering from the centre: New modes of governance in multi-level education ystems. Retrieved from Paris

Woods, P. A. (2004). Democratic leadership: Drawing distinctions with distributed leadership. International Journal of Leadership in Education, 7(1), 3-26.

Woods, P. A. (2005). Democratic leadership in education. London: Paul Chapman.

Woods, P. A., Bennett, N., Harvey, J. A., \& Wise, C. (2004). Variabilities and dualities in distributed leadership. Educational Managment Administration and Leadership, 32(4), 439-457.

WorldBank. (2015). GINI per capita. http://data.worldbank.org/indicator/SI.POV.GINI

WTO. (1998). Council for trade in services. Education Services. Background Note by the Secretariate S/C/W/49.

Open Access This chapter is licensed under the terms of the Creative Commons Attribution 4.0 International License (http://creativecommons.org/licenses/by/4.0/), which permits use, sharing, adaptation, distribution and reproduction in any medium or format, as long as you give appropriate credit to the original author(s) and the source, provide a link to the Creative Commons license and indicate if changes were made.

The images or other third party material in this chapter are included in the chapter's Creative Commons license, unless indicated otherwise in a credit line to the material. If material is not included in the chapter's Creative Commons license and your intended use is not permitted by statutory regulation or exceeds the permitted use, you will need to obtain permission directly from the copyright holder.

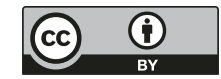




\title{
Chapter 3 \\ Lead Learner or Head Teacher? Exploring \\ Connections Between Curriculum, Leadership and Evaluation in an 'Age of Measurement'
}

\author{
Gert Biesta
}

\begin{abstract}
While 'head teacher' is still a prominent designation in many countries, there has been a tendency over the past two decades to refer to those 'in charge' of schools with a number of other words, most recently, that of lead learner. While one could say that these are 'only words' they nonetheless have a significant impact on how the position, role and responsibility of the head teacher is being understood. This chapter analyzes these conceptual shifts and the impact they have on perceptions, identities and relationship. For instance, the idea of lead learner fits within the ongoing 'learnification' (Biesta GJJ, Good education in an age of measurement: ethics, politics, democracy. Paradigm Publishers, Boulder, 2010a; Biesta GJJ, Stud Philos Educ 29(5), 491-503, Biesta 2010b) of educational thought and practice, that educational endeavours are increasingly being understood through a language of learning, learners - and now also lead learners. While the notion of the lead learner suggests democratic and empowering relationships, I will argue - mainly informed by a 'Continental' understanding of education (i.e. German 'kritische Pädagogik' and Philippe Meirieu's French educational thought) - for the need to reclaim the idea of the head teacher, in order to highlight that the responsibility of the head teacher is fundamentally an educational one, one that can only be accessed and conceptualized in terms of an updated understanding of curriculum/teaching.
\end{abstract}

\footnotetext{
G. Biesta $(\bowtie)$

Department of Education, Brunel University, London, UK

University of Humanistic Studies, Utrecht, The Netherlands

NLA University College, Bergen, Norway

e-mail: gert.biesta@brunel.ac.uk; http://www.gertbiesta.com
} 


\section{Introduction}

This volume of which this chapter is a part raises a number of important questions about the current climate under which schools in many countries function. Perhaps the most interesting observation concerns the ongoing divergence between three areas of scholarship: curriculum theory, leadership research, and evaluation research. These areas not only appear to have different foci and priorities but also seem to feed differently into educational policy and practice. Whereas curriculum theory on both sides of the Atlantic (albeit in very different modes and forms) focuses on the content of schooling in the widest possible sense, leadership research focuses more strongly on operational questions (again in the widest sense of the word), whereas much evaluation research continues to focus on what we might call the production of educational outcomes and the variables that impact on it - a central phenomenon in what I have elsewhere referred to as the 'age of measurement' (Biesta 2010a).

Whereas much evaluation research is closest positioned to neoliberal policies that continue to approach schooling in terms of the outcomes its produces - outcomes that are often exclusively valued with reference to the global economy leadership research is not entirely free from this either, not least because educational leaders are often seen as the first responsible persons for what it is that schools 'produce' in terms of statistics on student performance. From this angle curriculum theory seems to be operating a bit more on the sideline of where the policy heat is. This may partly be the result of the nature of curriculum scholarship itself. Here I do think that North American curriculum scholarship has moved itself much more away from the 'core business' of schooling than Continental traditions. If North American curriculum scholarship has morphed into a form of cultural studies which, although not without relevance for educational practice, approaches the question of the curriculum in a rather particular and distant way, much work on the Continent, but also in the UK, has been affected by the rise of national curricula which, at least in some countries, have stifled curriculum scholarship - a situation from which the field is only now beginning to recover (see, for example, Priestley and Biesta 2013).

If one way to understand the intention of this volume is to explore the interactions and possible connections between the three fields - curriculum, leadership and evaluation - then, in my contribution, I would like to explore two of these connections. One concerns the relationship between leadership and curriculum; the other the relationship between leadership and evaluation. I will look at both issues in a slightly more distant manner, as I do not wish to claim any specific competence in the field of educational leadership research. Nonetheless I hope that my observations provide some useful input for the discussion. 


\section{Leadership for What?}

The first point I wish to make has to do with the relationship between the field of leadership and the field of curriculum broadly conceived. (One question for discussion is indeed how broad or narrow we can conceive of this 'field.') That the observations I would like to make are important, was brought back to me by a recent experience and a more general observation. The recent experience concerned an invitation to contribute a theoretical perspective to a book with interview of educational leaders/managers in the Netherlands. I was happy to do so, particularly because the focus of the book was to gain an insight in what motivates leaders who work at a fairly 'high' level in educational organisations, that is, often with a responsibility for a number of schools or colleges. The interviews, to put it differently, tried to tap into the educational and professional values of educational leaders/ managers.

While most of the leaders/managers were able to speak about what drives them and about what they seek to achieve through their particular contributions to the education taking places in the schools and colleges they carry responsibility for, I was struck by the fact that the language they used to articulate this was predominantly a language of learning and development, including the popular/fashionable idea that education should allow children to develop their 'full potential.' Their orientations were generally positive and supportive but, in my view, vague and not really informed by what I would see as a distinctly educational outlook. This, as I will suggest below, partly has to do with the impact of the language of learning on contemporary educational research, policy and practice. But - and partly related to this - it also has to do with the role of particular traditions of thinking and doing. And perhaps here I was most surprised, as I had - until relatively recently - assumed that a country such as the Netherlands has a strong and long tradition of distinctive educational scholarship and practice, and I had expected to find at least sufficiently robust 'traces' of this. That this was not the case is, I think, also an important issue for this the discussions in this volume.

The impact of the language of learning on educational research, policy and practice is not only visible in the vocabulary I encountered with the educational leaders/ managers in the project above. It is also visible in the phrases that are being used to designate those who have responsibility for leadership and management in and across schools and colleges. The most striking change in this designation I have witnessed recently - particularly, if I am right, in the UK - is the use of the phrase 'lead learner' as the new name for what in the past was either called principal or head teacher. It is difficult to gauge how reliable Google still is in spotting trends, but when I checked "lead learner" it did generate 27.900 hits, not only from blogs and twitter accounts, but also from school websites - here is just a random quote: "My name is Matt Chappel and I am usually called the Lead Learner instead of Head Teacher because we want to show how important learning is for everyone at Thornhill, not just the children. (...) Thornhill's vision is that everyone at Thornhill should be a highly successful learner. Out motto is 'Leading and Learning Together.' 
We have one main school rule: "Help yourself and others to learn."' - and job descriptions for what formerly were principals' and head teachers' posts. This at least suggests at an anecdotal level that that language of learning has not only permeated the way in which many speak about the educational process, but now also on the way in which we speak about those who carry responsibility for it as managers or leaders. What is the problem here? Let me briefly try to make the point and then make some observations in relation to the theme of this volume.

The 'Learnification' of Education The issue at stake here is about what in other publications I have referred to as the 'learnification' of educational discourse and practice (see particularly Biesta 2009, 2010a). 'Learnification' refers to the relatively recent trend to express much if not all there is to say about education in terms of a language of learning. We can see, this for example, in the tendency to refer to students, pupils, children and adults as 'learners,' to refer to schools as 'learning environments or 'places for learning' and to see teachers as 'facilitators of learning.' Also the redesignation of the field of 'adult education' into that of 'lifelong learning' is an example of the rise of the 'new language of learning' (Biesta 2006). I would also say that the suggestion that the point of education is that students learn is part of this development, and there are indeed many examples - in national, local and school-level policies but also in descriptions of the task of teachers - that state that the task of schools is to make students learn and that teachers have a particular responsibility in facilitating the learning of their learners.

My point here is not to criticise the idea of learning in itself (although there are further issues that require discussion; see, for example, Biesta 2013), but to highlight the insufficiency of the language of learning as an educational language, that is a language of and for education and educators. In its shortest formula the issue is that the point of education is not that students learn, but that they learn something, that they learn it for particular reasons, and that they learn it from someone. The problem with the language of learning is that it is a language that refers to processes that are 'empty' with regard to content and purpose. So just to say that children should learn or that teachers should facilitate learning, or that we all should be lifelong learners, actually says very little - if it says anything at all. Unlike the language of learning, a language of education always needs to pay attention to questions of content, purpose and relationships. The danger with the rise of the language of learning in education is that these questions are no longer asked, or they are already taken to be answered (for example on the suggestion that the only relevant content is academic content, that the only relevant purpose is academic achievement, and the only relevant relationship is for teachers to train students so that they to generate the highest possible test scores, for themselves, their school, and their country).

The Question of Purpose in Education Of the three dimensions - content, purpose, and relationships - the question of purpose is the most important and fundamental question, because it is only once we have been able to indicate what it is that we seek to achieve through our educational activities and endeavours, that we can make decisions about the appropriate content students should engage with, and that we can decide how educational relationships can be used most productively and 
conducively. As I have suggested elsewhere (Biesta 2010a), what distinguishes education from many other human practices is the fact that it doesn't work in relation to only one purpose, but actually functions in relation to a number of 'domains of purpose.'

The argument is relatively simple and starts from the observation that in all instances of education - both at the 'big' level of national curricula or school systems and at the 'small' level of teachers working with their students - education is always about the transmission and acquisition of some content (knowledge, skills, dispositions), but always also 'connects' students to particular traditions and ways of doing and being and, in addition, has an impact on their formation as a person (either positively, for example by giving them knowledge, skills and connections to networks that empower them, or negatively when, for example, they are being told to 'know their place'). In more theoretical language I have therefore suggested that education always functions in relation to three domains: that of qualification, that of socialisation and that of what, with a technical term, I have referred to as subjectification, which is about the ways in which students can be(come) subjects in their own right and not just remain objects of the desires and directions of others.

If it is the case that all education always functions in relation to these three domains, then it is reasonable to ask from teachers and others who are involved in the design and execution of education to take explicit responsibility for the potential impact of their work in each of the three domains. This means that qualification, socialisation and subjectification not only appear as three functions of education, but also as three domains of educational purpose - three qualitatively different domains with regard to which we need to state and justify what it is we seek to achieve with our students, and what we seek our students to achieve.

Although qualification, socialisation and subjectification can be distinguished, it is important to see that they cannot be separated or singled out. This means, on the one hand, that even schools that claim only to focus on qualification are still impacting in the domains of socialisation and subjectification. It means, on the other hand, that teachers and others involved in the design and execution of education are always faced with finding a meaningful balance between the three domains, bearing in mind that what can be achieved in one domain often limits what can be achieve in the other domains. The latter can be seen, for example, in the negative impact an excessive focus on achievement in the domain of qualification can have on the formation of the personhood of the student (which has to do both with socialisation and with subjectification).

The Need for an Educational Perspective, And Where It Might Come From All this shows why it is so utterly unhelpful to say that the point of education is just to say that students should learn, just as it is utterly unhelpful to suggest that the sole task of teachers is to facilitate the learning of their students. Without specifying what it is that should be learned and, more importantly for what purpose it should be learned, the language of learning is unable to provide a sense of direction to the educational process, which is precisely where its deficiency as an educational language lies. 
I have been making this argument about the deficiency of the language of learning as an educational language for about a decade and although I do think that I have managed to raise some questions, it is remarkable how persistent the language of learning appears to be and, referring back to the anecdotes with which I opened this section, how it has also permeated the discourse and practice of educational leadership. There may, of course, be something attractive about the language of learning. It is generally (but mistakenly in my view; see Biesta in press) seen as an empowering and positive language that can provide an effective antidote against conceptions and practices of teaching as control - albeit that I would argue that learning has actually become a new mode or technology of control in contemporary societies (think of the strange imperative that we ought to keep learning throughout our lives), and also would like to highlight that learning is not just positive and easy, but can also be difficult and highly disturbing (for example when we learn our about inabilities and incapacities, or discover the darker sides of the histories that have formed us). But the problem with the language of learning, as I have shown, is that it provides us with very little direction for our educational efforts and that, in order to gain such direction, we need a much more robust educational language.

Here the point about different traditions becomes relevant, because - and again this is a topic I have explored in more detail in other writings (see Biesta 2011, 2012a) - whereas the field of educational scholarship in some countries and settings has developed a distinctive educational identity, in other countries and settings it has not done so. What I have particularly in mind here is the gap between what elsewhere I have referred to as the Anglo-American and the German-Continental construction of the field of educational scholarship. Whereas the latter has generated the idea of education as an academic discipline in its own right with its own forms of theory and theorising and, most importantly, with its own particular interest - an interest in the emancipation of the child, to put it briefly - the former has established education as an academic field of scholarship and research by conceiving of it as a multi-disciplinary effort to study education as an 'object.' The key characteristic of this effort is that education is not seen as a discipline in its own right, but just as an object or field of study, and that intellectual input needs to be 'borrowed' from 'real' disciplines, such as psychology, sociology, history and philosophy. Such disciplines do of course have interesting things to say about education but - as I have argued in more detail in my analyses - they do so in their own disciplinary terms, so that they ask psychological, sociological, philosophical and historical questions about education but fail, so we might say, to ask educational questions about education. The rise of the language of learning can, in my view, also partly be explained through the influence of the Anglo-American construction on educational research, policy and practice more world-wide, particularly through the influence of psychology (although I am inclined to think mixed psycho-sociological approaches such as socio-cultural theory have also contributed a lot to the popularity of the learning perspective on education).

From an analytical perspective these observations thus suggest that the 'field' of educational leadership and management may have turned away from its orientation on curriculum theory and has been pulled into a much more technicist and 
Anglo-American discourse of the evaluation of the 'operation' of education towards the achievement of learning and the generation of learning outcomes. The shift is both intellectual and political; it concerns both concepts, theories and vocabularies and social networks and alliances. From a more programmatic - or perhaps we should say: interested - perspective these observations suggest that there may be a need for the field of leadership and management to reconnect with curriculum theory (and I am not entirely sure whether I should add 'broadly conceived' as I have some doubts about the extent to which contemporary North American is able to generate the kind of educational questions and perspectives I have in mind; in my view what is happening in North America is mainly located in sociology and cultural studies) so as to be able to have a more robustly educational view about what leadership and management should be for, beyond the facilitation of learning or the promotion of development, such as the development of the child's full potential. The problem with the latter idea is that in such phrases it is assumed that everything we are potentially able to do is good and should therefore be allowed out. What is forgotten here is that the ability to do good or do evil are both within our potential which is why I have argued that the key task of education is actually to interrupt and question the child's development, and do so particularly with regard to the question whether what the child desires is actually desirable, both for the child and society at large. The task of education is, in other words, not to facilitate and to promote but to offer resistance - a point well made in the work of the French educational thinker Philippe Meirieu (e.g., Meirieu 2008).

If this sheds some light on the connection between leadership and curriculum theory, I now wish to turn to the other dimension, that is, the relationship between leadership and the field of evaluation (including evaluation research).

\section{Education, Measurement and the Professions}

One reason why educational leadership and management and education as a field more widely may have turned towards learning and its language, is because of changes in the policy environment in which education operates and functions. As many commentators have shown, education policy in many countries around the world - and increasingly also, we might say, 'between' many countries around the world - is caught in a culture (or cult) of measurement that seeks to express and assess the 'quality' of educational process, practices, institutions and systems through a measurement of the performance of such systems. Such performance is understood in terms of what the system 'produces' - hence the prominence of the notion of 'learning outcomes' - and is generally assessed in a comparative way, that is, that measurement is used to identify who performs better and who performs best on a particular standard or set of criteria. One problem in the age of measurement is that this has led to a culture of competition - competition between nations, but also competition between schools and school districts, between classes, between teachers and between students. 
The phrase 'race to the top' - the name of Obama's initiative to 'improve' American education - nicely captures what is going on here, that is, that education is caught in a race where there will be a winner but also many losers, and that the orientation of this race is that of teaching an alleged 'top.' The other problem with the culture of me asurement is that much is invested in the technicities of measurement - the reliability and the technical validity, that is, the question whether what we seek to measure is indeed being measured - but that very little attention is being paid to what I have coined the 'normative validity' of the measurement regime, that is the question whether what is being measured is indeed what we (and the democratic question is of course who is included in this 'we') value about education. Given the sheer force and size of the global measurement industry it looks like the tail is wagging the dog, that is, that we are in a situation where we are valuing what is being measured, and that we no longer take the time to ask whether we are measuring what it is we value about education. The means are defining the ends, in other words - which, in the definition of Habermas, is a clear cases of positivism or, in his term, of 'halved' rationality ('halbierte Rationalität').

The culture of measurement puts educational leaders in a difficult position because at first sight it looks like measurement contributes to accountability and democracy by giving all 'stakeholders' - itself not an unproblematic term; see below - transparent information about the performance of the education system. This is indeed how measurement is often defended, for example with reference to the social justice argument that everyone ought to have access to the same quality education, and hence we need to know how the system performs. (This argument already is spurious in at least one respect, in that it assumes that the 'outcomes' that can be measured are entirely the effect of - and hence the responsibility of - the school and the educational system. The shift towards 'value added' measuring was an attempt to address this issue, but remains caught in a rather limited input-output logic that does not really, in my view, engage with the complexities of educational purpose as indicated in the previous section.) But is it really the case that the culture of measurement improves accountability and democratisation of education? Is it therefore something that educational leaders and managers should embrace and full support - something they may feel they have to on the assumption that they should have nothing to hide? In recent work I have tried to raise some questions about this development, particularly with regard to the question whether the culture of measurement supports or distorts democratic professionality (see Biesta 2014). In this section I would like to share some of these insights in order to gain a better sense of the problematic side of what is happening in the domain of evaluation and measurement of the performance of education. I will do this through a discussion of three post-democratic distortions of professionality in education and related domains and will link this discussion back to the overall theme of this volume.

The Democratisation of the Professions The traditional case for professional autonomy, that is, for the idea that professionals should regulate and control their own work, relies on three assumptions (see, for example, Freidson 1994). The first is that the work of professionals distinguishes itself from many other areas of work 
in that it is concerned with the promotion of human wellbeing. This already indicates that professionality is not merely technical but always entails a normative dimension. Secondly, it is argued that unlike many other fields of work, professional work relies on highly specialist knowledge and skills, which is one of the reasons why the education of the traditional professions (doctors, lawyers and priests) has generally taken place in institutions of higher education. Thirdly, it is argued that the work of professionals distinguishes itself from other areas of work because professionals work in relationships of authority and trust. These three assumptions constitute, on the one hand, a definition of professionalism and therefore appear each time a new area of work seeks to elevate itself to the status of a profession. On the other hand the assumptions constitute a justification for the special status of the professions and for its system of self-regulation.

The traditional configuration of professionalism sees professions as closed and largely inward-looking entities that, although performing important functions for society, in a sense operate at a distance and even isolated from society. In their traditional set up professions thus largely operate beyond democratic control, either from clients or from society at large. This is most clearly visible in the fact that professions regulate their own functioning with regard to quality control, entrance to the profession - including the regulation of professional education - and, in case of professional failure and misconduct, also the 'exit' from the profession. This makes professions into powerful entities that exert power both over their own functioning and over important domains of human wellbeing. The power of professions also helps to understand why relations of authority and trust can easily turn into the unjustified exertion of power and even abuse of power.

The democratic deficit of the professions was fundamentally exposed and challenged in the 1960s and 1970s, partly as a result of client and patient emancipation (for example in the medical domain and psychiatry), and partly as a result of changing conceptions of health and mental well-being, for example in alternative medicine and anti-psychiatry (such as the work of R.D. Laing; see, for example, Laing 1960; Laing and Esterson 1964; for medicine see also Hellín 2002). These developments, which themselves were part of wider protest and emancipation movements at the time (including the student revolts of 1968 and the rise of the anti-education movement in Germany, known as anti-Pädagogik; see, for example, Von Braunmühl 1975), particularly exposed the abuse of power within professional relationships and, through this, were aimed at what we might call a democratic redefinition of the relationship between professionals and their clients. To the extent to which, after the Second World War, many professions became more central in the project of the welfare state (see, for example, Björkman 1982), a further shift occurred from a strict orientation on individual clients and their needs towards a wider concern for the common good. This can be seen as a second democratising impulse where professions increasingly established relationships of democratic accountability with their clients and society more generally.

This potted history of the development of professions and professionalism is first of all important in order to make visible how in the 1960s and 1970s a democratisation of the traditional configuration of the professions was set into motion, both as 
a result of a redefinition of the relationship between professionals and their clients, and as a result of the redefinition of the relationship between professions and professionals and their societal environment - something that was particularly connected to the role of the professions in emerging welfare states after the Second World War. Understanding these developments is also important, however, in order to grasp the significance of more recent shifts and changes in professional fields such as health care and education which, at first sight, may appear as furthering the case for the democratisation of professions but which, on closer inspection, turn out to be undermining the democratic configuration of professional work. In this section I focus on three 'post-democratic distortions': (1) the transformation of clients, patients and also students into customers; (2) the transformation of a democratic conception of accountability into a technical-managerial conception; and (3) the transformation of professional knowledge into 'evidence,' linked to the idea of evidence-based practice.

These developments should be understood against the background of the transformation of the welfare state and the rise of neo-liberal forms of governance and governing. The transformation of the welfare state - which was partly the result of economic crises such as the oil crisis in the 1970s, and partly of ideological interventions such as the conservative idea of the small state ('Thatcherism') (see Faulks 1998) - resulted in a shift from an orientations towards social justice and solidarity (the idea of 'the common good') towards a view of the state as a provider of a limited set of public services. What neo-liberalism added to this was the redefinition of the state as a regulator of the market of public services, no longer concerned with a substantive and hence political definition of the common good, but with formal notions such as 'quality,' 'choice,' and 'the customer always comes first.' As a result neo-liberal governments no longer see themselves as a key actor in the political debate about the definition of the common good, but increasingly understand themselves as process managers who, through a regime of standards, measurement and inspection, try to secure the quality of the products on offer. 'Quality' itself is understood in strictly formal terms, that is as the situation where a particular provision or service meets certain standards, without - as I have already hinted at in the introduction to this chapter - any concern for the question how meaningful those standards actually are. In what way, then, have professional fields been caught up in these developments and how has this distorted their democratic potential?

A First Distortion: From Client/Patient/Student to Customer I have indicated above that the emancipation of clients, patients and students in the 1960s and 1970s not only exposed the democratic deficit of many professions but also resulted in a transformation of professions and, more specifically, a transformation of professional relationships. Clients, patients and students literally made their voices heard in order to make clear that they were not just objects of the action and interventions of professionals, but subjects in their own right who therefore wanted to be treated as subjects of dialogue and not objects of intervention. From this angle it may seem that the recent trend to refer to clients, patients and students as customers and the tendency to emphasise that that in such domains as health care and education 
professionals must offer what their customers want, is the ultimate step in the democratisation of the professions - one where those at the receiving end, so to speak, are in total control.

But is this indeed the ultimate step in the democratisation of the professions? I have reasons to doubt that this is the case, and the main reason has to do with a fundamental difference between economic transactions and professional transactions (see Feinberg 2001). Whereas in economic transactions customers know what they want and would just look for a company that can provide them with what they want for the best possible combination of price and quality, a key aspect of professional relationships is that professional not just service the needs of their 'clients' but also play a key role in the definition of what it is that their clients need. Clients, patients and students, in other words, do not engage with professionals just to get what they already know that they want. Part of the process is precisely to figure what it is that clients actually need. As Feinberg (2001) explains: we go to the doctor because we have a headache, but we expect that the doctor figures out what the headache is an indication of and what can be done to get rid of it. This already suggested that the redefinition of clients, patients and students as customers is based on a fundamental misunderstanding of what professional practices are and what they are about.

A clear example of what is at stake here can be found in the domain of education and upbringing. If parents were only to give their children what they say they want, and never raise the question - for themselves but also in dialogue with their children - whether what their children say they want is actually good for them, it is quite likely that their children will turn into spoilt brats who remain slaves of their desires rather than that they are in a position where they can have a mature perspective on their desires in order to judge which of their desires are actually desirable. It is here that we can locate the specific responsibility of educators, and a similar argument can be made in relation to most if not all professions: just giving clients what they say they want may be utterly unprofessional. Doctors are not just there to give their patients just what they want, but have a key role in finding out what might be wrong with the patient in order then to propose possible treatments. Patients do have a voice in all this- for example with regard to questions concerning the risks and benefits of a certain treatment - but this is always to be understood as a dialogue between the experiential expertise of the patient and the professional expertise of the doctor. It is not a process where the doctor simply sells what the patient wants to have.

For precisely these reasons then, the redefinition of clients, patients and students as customers is not a further step in the democratisation of professional work and professional relationships, but rather a development that subverts the unique contribution professionals make. The reason for this lies in the fact that the redefinition of clients, patients and students as customers only puts the authoritarian relationship on its head by giving all the power to the customer. What it fails to see is that real emancipation and real democratisation requires a redefinition of the relationship between professionals and their clients where both play a distinctive role in a dialogical process of needs definition - it is not just a reversal of the traditional set up that would make the client/customer powerful and the professional powerless. 
A Second Distortion: From Democratic to Technical-Managerial Accountability A second distortion has to do with the way in which a democratic conception of accountability has been replaced by a technical-managerial one (on this see also Biesta 2004). In a democratic conception of accountability professionals are accountable for the quality of their professional action in a direct dialogical relationship with their stakeholders (clients, patients, students, and ultimately society as a whole). In a technical-managerial conception of accountability, however, the focus is no longer on the quality of professional action. Rather professionals are held accountable for the degree in which their actions meet certain standards. The role of the state in this set up, as already alluded to, is to guarantee the quality of the 'product' delivered by the professions. But it does not do so by engaging in a substantial political discussion about what, for example, good mental health care or good education ought to be, but by formulating standards and by initiating systems of inspection and control that need to make sure that professionals services meet the standards. The question of the normative validity of the standards is hardly ever discussed, or is brushed aside as 'ideological.' What happens as a result of this is the creation of a gap between professionals (redefined as providers) and their clients (redefined as customers). In this gap we find a whole machinery of often privatised quality controllers and inspectors, which means that the accountability relationships between professionals and their clients are no longer direct but have become indirect.

This is another example of what, at first sight, seems to further the democratisation of the professions but what, on closer inspection, turns out to be an erosion of the possibility for substantial democratic dialogue between professions/professionals and their clients. In her Reith lectures on accountability Onara O'Neill (2002) has shown in much detail what goes wrong here. She reveals two important shifts in the rise of the technical-managerial approach to accountability. The first has to do with a shift with regard to the different parties involved in accountability processes. She writes:

In theory the new culture of accountability and audit makes professionals and institutions more accountable to the public. This is supposedly done by publishing targets and levels of attainment in league tables, and by establishing complaint procedures by which members of the public can seek redress for any professional or institutional failures. But underlying this ostensible aim of accountability tothe public the real requirements are for accountability to regulators, to departments of government, to funders, to legal standards. The new forms of accountability impose forms of central control - quite often indeed a range of different and mutually inconsistent forms of central control. (O'Neill 2002)

A second shift has to do with definitions of quality. Here she writes:

In theory again the new culture of accountability and audit makes professionals and institutions more accountable for good performance. This is manifest in the rhetoric of improvement and raising standards, of efficiency gains and best practice, of respect for patients and pupils and employees. But beneath this admirable rhetoric the real focus is on performance indicators chosen for ease of measurement and control rather than because they measure accurately what the quality of performance is. (O'Neill 2002) 
O'Neill's observations thus clearly show the difference between a democratic and a technical-managerial approach to accountability and the slippery slope between the two.

A Third Distortion: From Professional Knowledge to Evidence-Based Practice The third arena in which the democratisation of professionalism has been distorted has to do with the way in which professional judgement in a range of different professional domains is increasingly being replaced or pushed out by a demand for an evidence-based approach (for more detail see Biesta 2007, 2010b). The idea here is that professional action can only become really professional if it is no longer based on the singular insights (or according to some: subjective opinions) of professionals, but when it becomes based upon secure scientific knowledge about 'what works,' And the claim is that the only way in which we can be certain that a professional intervention 'works' is by means of randomised controlled trials - in the literature known as 'golden standard' - which has even led to situations where professionals are prevented from doing anything unless there is positive evidence that their interventions will work.

While proponents of evidence-based approaches claim that professional fields such as education, social work and care can be improved dramatically if they opt for the evidence-based approach which, so it is claimed, has been the main driver of progress in such fields as agriculture and medicine (see for this particular argument Slavin 2002), there are a number of reasons why the idea of 'what works' is actually not that easily incorporated. One key issue is that in domains such as care and education - although this ultimately also holds for agriculture and medicine the question can never simply be about 'what works' but always needs to be phrases as the question 'What works for what?' The point is, that any idea of 'working' always needs to be understood in relation to the aim or aims of professional action in a particular field. This already shows that the question of 'what works' can, at most, be relevant with regard to the means of professional action, but not with regard to the ends.

The more important point with regard to the question whether the idea of an evidence-based approach makes sense in domains of professional action has to do with the fact that all professional action takes place in what Aristotle already distinguished as the domain of the 'variable' (see Aristotle 1980), the domain of actions and possible consequences, and not in the domain of the 'eternal,' that is, the domain of cause-effect relationships. One reason for this lies in the fact that professional action takes place between human beings who never appear just as objects of intervention which also shows that the language of intervention is actually quite misleading - but always also as subjects in their own right. In the domain of the variable research can at most provide us with information about possible relationships between actions and consequences. But research can never guarantee that relationships between actions and consequences that were found in the past will appear in exactly the same way in 
the present. While research therefore can tell us what in a concrete situation and under specific circumstances has worked in the past, it can never tell us what will work in the present or the future. Next to the need to make judgements about the ends of professional action, we therefore also always need judgement about how to act which is a judgement about the application of general and decontextualized knowledge to concrete situations and singular cases. Scientific evidence can neither replace judgements about how to act, nor can it replace judgements about the aims and ends of professional action - and where we find claims that it can or should, we have an example of positivism, where the means are defining the ends, rather than that we are in a position to define the ends of our actions ourselves.

The call, and in some cases even the blunt demand to work in an evidence-based way thus appears as an attempt to eradicate professional judgement with regard to the 'how' and the 'what for' of professional action from the domain of professionalism. It seeks to transform professions into abstract 'machines' in which reflection and judgement are seen as a weakness rather than as an essential part. This shows how the call for an evidence-based approach is not a deepening of the knowledge and judgement of professionals, but rather an attempt to overrule such knowledge and judgement. In precisely this sense the evidence-based approach is another erosion of the democratic dimension of professionalism and hence another post-democratic distortion.

The Role of Measurement If the above provides us with an insight into the ways in which recent developments in professional fields such as education are hindering rather than enhancing their democratic potential, there is the additional question how the culture of measurement is contributing to this. With regard to this question I wish to make two observations. Firstly I wish to argue that with regard to each distortion there is a need for data, information and measurement. After all, to give customers what they want and to give the choice and value for money they need data about the quality of the products on offer. Also: to hold professionals accountable for the quality of their performance we need data about the degree to which their work meets pre-set standards. And in order to make professional activity evidencebased there is a need for data about what works, particularly data that show correlations (if not causal connections) between 'interventions' and 'outcomes.'

We can say, therefore, that the culture of measurement has played and continues to play a key role in the post-democratic transformation of the professions. But it is not only that the transformations require data and measurement. At the very same time the availability of data, information and measurement reinforce these particular distortions rather than that they work against them. After all, once there are data available about the performance of individuals, groups or systems, it becomes increasingly difficult not to look at the data. Similarly, once there are data about the performance of individuals, groups or systems, it is difficult not to include them in any accountability exercise. And once some kind of apparent 'evidence' has been constructed about particular practices and ways of working, it becomes again difficult not to make use of it. The availability of data, information and measurement, to put it differently, is seductive and difficult to resist - which reveals another dimen- 
sion of the social psychology of the culture of measurement and provides a further explanation for its attractiveness and 'force.'

Reclaiming a Space? If my analysis of the distortions of professional fields such as education is sufficiently adequate, one question it raises is whether it is possible to regain a space for professional action - which is important for teachers but also for educational leaders and managers. There are (at least) three dimensions to this. The first is to challenge, interrupt and resist the redefinition of the professions, particular with regard to the three distortions I have discussed above: the redefinition of the client/patient/student as a customer; the replacement of democratic accountability with technical-managerial accountability; and the attempt to replace professional knowledge with evidence about 'what works.' In each case it is particularly important to show that these developments are based on a misunderstanding of what professional work is about.

Secondly it is important to expose the democratic deficit of these developments, that is, to show that in spite of what may seem to be the case at first sight, they are actually undermining and eroding the development of more democratic ways of working in professional fields such as health care and education. For this it is important to highlight that democratisation of the professions is not a matter of reversing the positions of the involved parties, that is, just turning authoritarian relationships on their head. It rather requires the establishment of new relationships between professionals and their clients - relationships of dialogue where both can contribute their particular experience and expertise, acknowledging that the experience and expertise of each of the parties involved (professionals and clients) is different and complementary, and that the differing contributions from all are needed in order transform authoritarian into democratic professional relationships.

To resist post-democratic transformations of dimensions of professional work, and to insist on the need to transform relationships rather than just reverse them, often means that one finds oneself defending ideas and positions that, at first sight, may look outdated. For example to argue against the 'learnification' of education, that is, against a conception of education that puts the learner at the centre and sidelines the teacher, and, in response, to make a case for the importance of teaching and the teacher (Biesta 2012b) - and also for the head teacher - is often perceived as a step back rather than a step forward. So it needs careful argumentation to show that the turn towards the learner and away from the teacher is actually an inadequate response to authoritarian forms of teaching as control, as it only reverses the position of the student and teacher, rather than that it seeks to transform the nature of their relationship. Similarly: to argue that education should be understood as valuebased rather than evidence-based (Biesta 2010b), is often perceived as a return to a pre-scientific age rather than as an attempt to show that science - in the form of evidence or otherwise - can never do away with the need for judgement in education. 
The Importance of an Educational Perspective At this point I come to a similar conclusion as the one I reached in the previous section because when we consider how educational leaders and managers are caught up these developments, it becomes again important on the one hand to understand - analytically - what has happened and why it has happened and, on the other hand - programmatically - to identify what kind of resources might be needed in order to regain a sense of professional control within these developments rather than to constantly being pushed in the position of being the servant or executor of other people's ideas - which is one way to think about how educational leaders and managers have become positioned in the culture of measurement. Perhaps to put it briefly - as a point for discussion - this analysis points again in the direction of the question whether those who play a key role in the design and enactment of education have access to educational ways of understanding what education is about (and I apologise for the multiple appearance of the word' education' in this sentence) or whether the only discursive resources they have are policy resources and a language of learning and development. To see how much effort is needed to make clear that teaching is a normative profession - a profession crucially built around the exercise of educational judgements - and not a technicist endeavour; to counter the influx of competence-based conceptions of teaching and teacher education that keep forgetting to engage with the question what all these competencies are for, that is, what they are supposed to bring about, indicates, even on anecdotal evidence, that the main stream of education - as practice, and as research - has lost contact with resources that have been central in (some) traditions of curriculum theory and wider educational scholarship. For a strong, self-confident educational profession the connection with these traditions remains important.

\section{In Conclusion}

Having put quite a lot 'on the table,' let me be brief in my conclusions. I have tried to analyse developments in relation to two aspects of the triangle proposed by the editors of this volume - the triangle being composed of curriculum, leadership and evaluation. On the one hand I have explored connections between leadership and curriculum directly, which I did through a critique of the language of learning and an argument in favour of the educational forms of theory and theorising. It may be that many leaders and managers have insufficient access to these - either because they have themselves been educated within traditions of thought and practice in which such perspectives were not present, or because they have lost touch with them as a result of the pressures of a more global culture of measurement. This is the other dimension of the triangle I have explored, that is, how the culture of measurement - which includes a particular way to 'evaluate' the 'quality' of education impacts on education and on the room for manoeuvre educational professionals, including leaders and managers have. Here again I suggested that one important precondition for countering the distortions that I think are still going on in education 
is to re-engage with vocabularies and discursive resources that keep education away from production metaphors and consumerist and technicist thinking and doing. Let's cautiously and provisionally conclude, therefore, that there may be a case to turn educational leadership and management - both as practice and as field of scholarship - 'back' or in the direction of curriculum theory, particularly those forms of theory that are informed by and built upon educational forms of theory and theorising. If my contribution reaches this conclusion in a more abstract sense - generalising rather quickly across a number of national contexts and settings - one interesting question is what might become visible in more detailed comparative studies, that some of the other contributions to this volume will provide us with.

\section{References}

Aristotle. (1980). The Nicomachean ethics. Oxford: Oxford University Press.

Biesta, G. J. J. (2004). Education, accountability and the ethical demand. Can the democratic potential of accountability be regained? Educational Theory, 54(3), 233-250.

Biesta, G. J. J. (2006). Beyond learning: Democratic education for a human future. Boulder: Paradigm Publishers.

Biesta, G. J. J. (2007). Why 'what works' won't work. Evidence-based practice and the democratic deficit of educational research. Educational Theory, 57(1), 1-22.

Biesta, G. J. J. (2009). Good education in an age of measurement: On the need to reconnect with the question of purpose in education. Educational Assessment, Evaluation and Accountability, 21(1), 33-46.

Biesta, G. J. J. (2010a). Good education in an age of measurement: Ethics, politics, democracy. Boulder: Paradigm Publishers.

Biesta, G. J. J. (2010b). Why 'what works' still won't work. From evidence-based education to value-based education. Studies in Philosophy and Education, 29(5), 491-503.

Biesta, G. J. J. (2011). Disciplines and theory in the academic study of education: A comparative analysis of the anglo-American and continental construction of the field. Pedagogy, Culture and Society, 19(2), 175-192.

Biesta, G. J. J. (2012a). Wanted, dead or alive: educationalists. On the need for academic bilingualism in education. In C. Aubry, M. Geiss, V. Magyar-Haas, \& D. Miller (Eds.), Positionierungen. Zum Verhältnis von Wissenschaft, Pädagogik und Politik (pp. 20-33). Weinheim: Beltz Verlag.

Biesta, G. J. J. (2012b). Giving teaching back to education. Phenomenology and Practice, 6(2), 35-49.

Biesta, G. J. J. (2013). Interrupting the politics of learning. Power and Education, 5(1), 4-15.

Biesta, G. J. J. (2014). De vorming van de democratische professional. Over professionaliteit, normativiteit en democratie. [The formation of the democratic professional: On professionality, normativity and democracy.] Waardenwerk: Journal of Humanistic Studies, 56(May 2014), 7-18.

Biesta, G.J.J. (in press). The rediscovery of teaching: On robot vacuum cleaners, the hermeneutical worldview, and non-egological education. Educational Philosophy and Theory.

Björkman, J. W. (1982). Professionalism in the welfare state: Sociological saviour or political pariah? European Journal of Political Research, 10(4), 407-428.

von Braunmühl, E. (1975). Antipädagogik. Weinheim: Juventa.

Faulks, K. (1998). Citizenship in modern Britain. Edinburgh: Edinburgh University Press.

Feinberg, W. (2001). Choice, autonomy, need-definition and educational reform. Studies in Philosophy and Education, 20(5), 402-409. 
Freidson, E. (1994). Professionalism reborn: Theory, prophecy, and policy. Chicago: University of Chicago Press.

Hellín, T. (2002). The physician-patient relationship: Recent developments and changes. Haemophilia, 8, 450-454. doi:10.1046/j.1365-2516.2002.00636.x.

Laing, R. D. (1960). The divided self: An existential study in sanity and madness. Harmondsworth: Penguin.

Laing, R. D., \& Esterson, A. (1964). Sanity, madness and the family. London: Penguin Books.

Meirieu, P. (2008). Pédagogie: le devoir de résister. In 2nd. Issy-les-Moulineaux: ESF.

O'Neill, O. (2002). BBC Reith lectures 2002. A question of trust. Retrieved from http://www.bbc. co.uk/radio4/reith2002

Priestley, M., \& Biesta, G. J. J. (2013). Reinventing the curriculum. New trends in curriculum policy and practice. London: Bloomsbury.

Slavin, R. (2002). Evidence-based educational policies: Transforming educational practice and research. Educational Researcher, 31(7), 15-21.

Open Access This chapter is licensed under the terms of the Creative Commons Attribution 4.0 International License (http://creativecommons.org/licenses/by/4.0/), which permits use, sharing, adaptation, distribution and reproduction in any medium or format, as long as you give appropriate credit to the original author(s) and the source, provide a link to the Creative Commons license and indicate if changes were made.

The images or other third party material in this chapter are included in the chapter's Creative Commons license, unless indicated otherwise in a credit line to the material. If material is not included in the chapter's Creative Commons license and your intended use is not permitted by statutory regulation or exceeds the permitted use, you will need to obtain permission directly from the copyright holder.

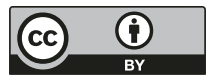




\title{
Chapter 4 \\ Against the Epistemicide. Itinerant \\ Curriculum Theory and the Reiteration of an Epistemology of Liberation
}

\author{
João M. Paraskeva
}

\begin{abstract}
The world of science has lost one of the most eminent figures and the race from which he sprang an outstanding member in the passing of Dr. George Washington Carver
\end{abstract}

Haber (1970, p. 104)

\begin{abstract}
Echoing Ettore Scola metaphor "Bruti, Sporchi \& Cativi", this chapter challenges how hegemonic and specific (or so called) counter hegemonic curriculum platforms - so connected with Western Eurocentric Modernity - have been able to colonize the field without any prudency to "fabricate" and impose a classed, raced and gendered philosophy of praxis, as unique, that drives the field to an ideological surrealism and collective suicide. Such collective suicide framed by a theoretical timesharing unleashed by both dominant and specific counter dominant platforms that tenaciously controlled the circuits of cultural production grooms the field as a ghetto, flooded with rudeness, and miserable ambitions, a theoretical caliphate that wipes out any episteme beyond the Western Eurocentric Modern terrain, insolently droving to sewage of society the needs and desires of students, teachers and the community.

Drawing from key decolonial thinkers, this chapter examines the way Western eugenic curriculum of modernity created an abyssal thinking in which 'this side' of the line is legitimate and 'the other side' has been produced as 'non-existent' (Sousa Santos B, Another knowledge is possible. Verso, London, 2007). The paper suggests the need to move a post-abyssal curriculum that challenges dominant and counter dominant traditions within 'this side' of the line, and respects 'the other' side of the line. The paper challenges curriculum studies to assume a non-abyssal position one that respects epistemological diversity. This requires an Itinerant Curriculum Theory
\end{abstract}

J.M. Paraskeva $(\bowtie)$

University of Massachusetts Dartmouth, North Dartmouth, MA 02747, USA

e-mail: jparaskeva@umassd.edu 
(Paraskeva JM, Conflicts in curriculum theory: Challenging hegemonic epistemologies. Palgrave Macmillan, London, 2011), which is a commitment and a ruthless epistemological critique of every existing epistemology.

Contrary to the nineteenth century in which, according to Marx and Engels (2012 [1848]) "a spectre [was] haunting [the Western civilization] - the spectre of communism [and that] all the powers of old Europe have entered into a holy alliance to exorcise this spectre - Pope and Tsar, Metternich and Guizot, French Radicals and German police-spies," at the end of the twentieth century beginning of the twenty first century, Western civilization is facing not just one a complex multiplicity of spectres. One of those is without a doubt the 'return of the repressed.' Although Eagleton (2011) and others are so right when they challenge the reductionist (and cheap) critic on Marx's framework, the truth of the matter is that 'the return of the repressed' cannot be explain historically as just a 'history of class struggles' since it is not just a political phenomenon. The field of curriculum studies here in the US and elsewhere cannot ignore these well-established conundrums.

Approaches, such as those of Mahbubani (2004), Sayyid (2015) or Haber (1970), testify to the importance of rethinking the way(s) internationalization debates have colonized the field - an issue that has caught the attention of many scholars in crucial settings, such as the American Association for the Advancement of Curriculum Studies. Sayyid's (2015) Fundamental Fear: Eurocentrism and the Emergence of Islam examines how modernity metamorphoses - anti, post, high, late, counter sweats full blast European nihilism in addressing the complex problems that were created by modernity. He (2015) claims:

Of the many spectres that have haunted western civilization from tine to time, perhaps none
is so perplexing or so irredeemably strange as the contemporary resurgence of Islam. The
spectral nature of this phenomenon arises not only from the way its emergence conveniently
coincides with the approach of the witching hour of the second Christian millennium, but
also from the way in which the Muslim presence for the West has tended to be grounded in
a 'hauntology' which finds it all too easy to conflate Muslims and ghosts. (p. 1)

While we are clearly facing a massive political issue, it is needless to add that such re-emergence "doesn't take only a political form" (Sayyid 2015, p. 4). The rise of such systematically repressed and in too many occasions - as history documents - butchered Islamic and other non-western civilizations goes hand in hand with the eugenic politics and praxis perpetrated by Western ideological apparatuses - such as 'the curriculum' - so well documented by Haber's (1970) Black Pioneers of Science and Invention.

Haber (1970) documents "significant contributions made by black scientists and inventors who were pioneers in various fields of science and the roles they played in the development of scientific progress in the United States" (p. vii). From inventors such as the genius Matzeliger (1852-1889) to whom "the modern shoe industry in [the US] and in the world is based upon his revolutionizing invention" (Haber 1970, p. 36) that was "patented on March 20, 1883" (Haber 1970, p. 46), to the "George 
Washington Carver Agricultural Chemist" (Haber 1970, p. 104), to "Garrett Morgan's Breathing helmet, patented on October 13, 1914" (Haber 1970, p. 93), 'another history' comes to the surface thus putting into question the knowledge that has been labeled as official bringing laudably to the fore the need to dismantled such curriculum epistemicide (Paraskeva 2014, 2016a).

Mahbubani's (2004) Can Asians Think? is a graphic example of the real color of what Mignolo (2013) and others frame as coloniality. He (2004) argues that "judging from the record of Asian societies over the past few centuries," Asians cannot think (p. 11); that is, societies "that take centuries to wake up cannot be said to think very well. It would be foolish for any Asian to deny [such] painful historical fact" (Mahbubani 2004, p. 11). Unfortunately, Mahbubani (2004) is not alone in such ideological statement. First, Mahbubani (2004) detached the history of colonialism and neocolonialism from a social constructed so-called darkness that drove nonWestern society to tough times in 'waking up' to use his own expression. In so doing, he also produces a specific eugenic historicity of history, one that denies irrefutable evidence of how inaccurate his claim is. Second, in so doing, Mahbubani (2004) not only strips such darkness from coloniality and its consequences. Such impossibility of wake up, according to him (2004), is due to the very Asian DNA that is devoid of any capacity to produce thinking and not a consequence of sanguinary centuries of imperial oppression. Such eugenic claim nulls any possibility of the oppressed to repel the oppressor and oppressed forms and to put themselves in a decolonial imaginary path (Perez 1999). Third, ignoring that right during the heyday of Enlightenment, "a number of prominent European political thinkers attacked imperialism not only defending non-European peoples against the injustices of European imperial rule, but also challenging the idea that Europeans had any right to subjugate, colonize, and 'civilize' the rest of the world" (Muthu 2003, p. 1).

As I have examined in other contexts (Paraskeva 2011, 2014, 2016a, b) Mahbubani (2004) and analyses such as his undermine that "to write about history is to write about of the lapses of history itself, of spaces blanked out by ruthless whiteness" (Young 2001, p. 1). Put it this way, "history is never is never for itself, it is always for someone" (Jenkins 1991, p. 21). Understanding Western Eurocentric history and its historicity as flooded by silences is perceiving not just the very color of coloniality, but also how such color was constructed right at the outset of colonialism. In 1670, Mbembe (2014) argues, "the big issue was how to create the conditions for a huge armada of labor to produce commodities from a far. The black ('o negro'; the other) addressed such challenge" (pp. 42-43). In fact, the black ('o negro'; the other) is the supreme invention of coloniality. Whereas on one hand, addressed the pressure for free labor, on the other raped Western democracies with an endemic issue; that is, "the fact that no black body arrived freely at the shores of the new world is a irresolvable problem for U.S. democracy" (Mbembe 2014, p. 147) - and one could add Western democracies.

When I was writing a new preface for the paperback edition of Conflicts in Curriculum Theory: Challenging Hegemonic Epistemologies (Paraskeva 2014), I remembered that a few years ago during a trip home, I came across an outstanding volume of Ezekiel Mphahlele's book in a shop in Hillbrow, Johannesburg. Mphahlele 
shares daily life stories of ordinary South Africans during the apartheid regime and discusses how such stories were crucial to understanding the complex struggle against oppression, poverty, and harsh inequality. There is a passage in the volume that I would like to highlight: "I want to write; I must write; I should write; I am going to write. This is what I said to myself one moonless night under an inky black sky ... [but] Write what? ... Why should I write?” (Mphahlele and Thuynsma 2011, p.7). With such words, South African intellectuals Ezekiel Mphahlele (known as Es'kia Mphahlele) and Peter N. Thuynsma (2011) begin “The Unfinished Story” in Corner B. Mphahlele and Thuynsma's questioning invokes for me so many other voices of intellectuals and countless horizons. Suddenly, I imagine a "trilogue" among Mphahlele, Steve Biko, and bell hooks. Biko (1978) would probably answer as follows: "You must write what you like." Quite rarely would hooks (1998) stress, "I write about work that does not move me deeply" (p. 137). However, Mphahlele would bring complexity to the arguments; one needs to say something to the world, and one needs to have something to say to the world. More to the point, one needs to have something to say to the world to be able to say something to the world. Mphahlele and Thuynsma insisted:

So much has been written on the Bantu, but I have always felt something seriously wanting in such literature. I told myself there must surely be much more to be said than the mere recounting of incident: about the loves and hates of my people; their desires; their property and affluence; their achievements and failures; their diligence and idleness; their cold indifference and enthusiasm; their sense of the comic; their full-throated laughter and their sense of the tragic with its attendant emotional sobs and ostentatious signs of pity. What could I say to the world?" (p. 14)

In writing Conflicts in Curriculum Theory: Challenging Hegemonic Epistemologies (Paraskeva 2011, 2014), I consciously faced the same challenges. Frustrated, like so many of us, with ambiguities and gaps within the vast and complex critical and poststructural terrains - despite the countless and crucial gains-I respectfully sought to go beyond such approaches and cautiously propose the need for an itinerant curriculum theory (ICT) to address the complex issues that we are all facing under the pressure of a liquid momentum (Bauman 1998), which characterize the current terrestrial globalization (Sloterdjik 2013). Arlene Croce (1998) argued that the critic has three options: "(1) to see and review, (2) to see and not review, and (3) not to see" (p. 16). She actually added a fourth option: "To write about what one has not seen; [that] becomes possible on strange occasions" (p. 16). ICT values not just the need to see and review; in fact, Conflicts in Curriculum Theory: Challenging Hegemonic Epistemologies does more than that. It reviews the field historically and addresses certain gains, strengths, and challenges of a particular radical/critical progressive river. By so doing, I claim a future-itinerary-path and justify why we need a critical itinerant approach, while being profoundly cautious about issuing any kind of recipe.

The field immediately reacted to ICT. Such reactions came from different Western and non-Western angles and epistemological axles, through varied informal and formal academic ways. Some were quite positive. Others raised justifiable concerns in particular cases, and others not only completely misrepresented ICT, but 
demonstrated by their objections precisely how important it is to challenge the epistemicide. It goes without saying that this is not an adequate space to address such reactions. But, for example, those who claim that I use ICT as an attack on JudeaChristian Western white male hegemonic epistemology-intentionally or nonintentionally-profoundly misinterpret the argument. ICT goes well beyond such notions. Other reactions, again some of them either welcoming and praising the merits of ICT or flagging understandable concerns, deserved attention, and I will likely address these concerns in the near future.

Needless to say, for so many liberals, epistemological differences are terribly inconvenient. Humanized capitalism, tempered with flamboyant forms of multiculturalism, are so dear to them and, in some cases, they are not even prepared to go that far. The problem is that "that far" is not enough. As Dwayne Huebner's words (personal communication, 2005) remind me repeatedly, "many educators are not necessarily magnanimous individuals — neither open to diverse ways of thought, nor to significant criticism. Welcome to the club."

ICT does try to say something to the field. It presents new terrains and theoretical situations. ICT participates in the complicated conversation (see Pinar 2000; Trueit 2000) - that cannot bend under the yoke of Western academicism-challenging Western curriculum epistemicides and alerting us to the need to respect and incorporate non-Western epistemes. William Pinar $(2012,2013)$ acknowledged the influential synopticality of ICT in his recent Curriculum Studies in the United States:

There are other discourses influential now, sustainability perhaps primary among them. Arts-based research is hardly peripheral ... One sign is the synoptic text composed by João M. Paraskeva. Hybridity is the order of the day. Pertinent to the discussion in that even Paraskeva's determination to contain in one "critical river" multiple currents of understanding curriculum politically floods its banks; he endorses an "itinerant curriculum theory" that asserts a "deliberate disrespect of the canon" $(2011,184)$. In Paraskeva's proclamation, this "river" has gone "south" $(2011,186)$. That South is Latin America, where we can avoid "any kind of Eurocentrism" $(2011,186)$ while not "romanticizing indigenous knowledge" $(2011,187)$. Addressing issues [such as hegemony, ideology, power, social emancipation, class, race, and gender] implies a new thinking, a new theory ... an itinerant curriculum theory. (Pinar 2013, p. 64)

Although Pinar's reading of ICT is crucial, I would clarify (maybe complexify) that "the" South is not just Latin America. Sousa Santos (2009) is vital here:

The South is metaphorically conceived as a field of epistemic challenges, which try to address and repair the damages and negative impacts historically created by capitalism in its colonial relation with the world. Such conception of South overlaps the geographical South, the group of nations and regions in the world that were subjugated to European colonialism and that, with the exception of Australia and New Zealand, never achieved levels of economic development similar to the Global North (i.e. Europe and the United States of America). (pp. 12-13)

Thus, we "designate the epistemological diversity of the world by South epistemologies" (Sousa Santos 2009, p. 12). In this way, ICT addresses Sousa Santos's (2006, p. xi) claim regarding the need for a new critical theory, a new emancipatory praxis that needs to be decolonized as well: 
Contrary to their predecessors, [such] theory and practices must start from the premise that the epistemological diversity of the world is immense, as its cultural diversity and that the recognition of such diversity must be at the core of global resistance against capitalism and of alternative forms of sociability. (p. xi)

ICT attempts to create an itinerant path to address a problem. In so doing, it faces undesirable yet unavoidable and needed black holes (Deleuze and Guattari 1987). ICT sees the confrontation with such holes as a reassembled set of processes toward a creative and desirable plan of consistency that is only possible by respecting a perpetual itinerancy. Such theory(ist) understands the structure and flows of a given social formation. Its itinerancy allows the theory(ist) to grasp the imposition, certification, and legitimization of particular un/re/coding metamorphoses, as well as the eclipse of so many others. ICT reads and challenges such codes that frame each social formation and fuel the wrangle of the oppressor-oppressed. This is crucial since it allows one to master the complex processes of axiomatization of specific codes within the capitalist society from slavery in the 1400 s to the current slavery constructions as de/re/coded flows of an economy and culture pumped by an epidemic of overproduction (Marx and Engels 2012).

ICT is an unblemished claim against dominant multiculturalist forms that are "Eurocentric, a prime expression of the cultural logic of national or global capitalism, descriptive, apolitical, suppressing power relations, exploitation, inequality and exclusion" (Sousa Santos 2007, pp. xxiii-xxiv) — that have been legitimizing a monoculture of scientific knowledge that needs to be defeated and replaced by an ecology of knowledges (Sousa Santos 2003a, b). ICT challenges the coloniality of power, being, knowledge, and labor (cf. Grosfoguel 2007; Mignolo 2000; Quijano 2000); it is sentient in that the "politics of cultural diversity and mutual intelligibility calls for a complex procedure of reciprocal and horizontal translation rather than a general theory" (Sousa Santos 2007, p. xxvi).

Formalizing ICT in my mind, through my writing, through dialogues with others and the wor(l)d, leads me to consider the intricacies of its conceptions and assertions. Yet, its conceptualization and creation comprise a natural complex interaction with the wor(l)d, as was perhaps the case for Michelangelo and Picasso with their art.

When one day Michelangelo was asked how a certain frame was painted, that is, where his idea came from, he answered, "I had no idea. The figure just stood there, looking at me. I just gave it life/birth.” Picasso had a similar dialogue with a Gestapo officer. In occupied Paris during World War II, a Gestapo officer who had barged into Picasso's apartment pointed at a photo of the mural, Guernica, asking, "Did you do that?" "No," Picasso replied, "you did." Writing is, Gilles Deleuze (1995) argued, "bringing something to life, to free life from where it's trapped, to trace lines of flight" (p. 141).

These words of Michelangelo and Picasso also highlight the theory of translation that works through art. Similarly, ICT is a theory of translation that attempts to prevent the "reconstruction of emancipatory discourse and practices from falling into the trap of reproducing, in a wider form, Eurocentric concepts and contents" 
(Sousa Santos 2007, p. xxvi). Translation is crucial to the processes of coding and decoding

between the diverse and specific intellectual and cognitive resources that are expressed through the various modes of producing knowledge about counter-hegemonic initiatives and experiences aimed at the redistribution and recognition and the construction of new configurations of knowledge anchored in local, situated forms of experience and struggle. (Sousa Santos 2007, p. xxvi)

In The Struggle for Meaning: Reflection on Philosophy, Culture and Democracy in Africa, Hountondji (2002, p. 26) confessed his search dilemmas under the supervision of Georges Canguilhem and later with Georges Balandier. Hountondji wanted to examine "all that history of ideas could teach us on the modes of existence of forms of knowledge and the conditions of the transition to science" (p. 26). Hountondji's ambition was to identify and delimit, within the existing corpus, something like an archeology of science and technology and apply it critically to Africa. Hountondji revealed the challenges in pursuing such object of study by engaging in a deep exegesis of Husserl's approach. Hountondji's approach is a vivid example of the inner challenges in examining Husserl without jeopardizing Africa as the focus of examination. Hountondji explained that his own strategy was his "struggle for meaning," which was to "work on the margins [and] to clear the field patiently, establish the legitimacy and the outlines of an intellectual project that was at once authentically African and authentically philosophical” (p. 78).

Examples such as Yacouba Sawadogo, an African farmer of Burkina Faso, who has been restoring the soil damaged by centuries of drought (and desertification) through traditional farming techniques, cannot be arrogantly minimized or eugenically produced as nonexistent or nonscience just because this work cannot be translated and framed within Western scientificity. Western intellectuals need to consciously acknowledge that the Western epistemological platform-both in its most sophisticated dominant and/or radical critical counterdominant perspectivesis insufficient and inadequate to explain and change its own effects (Seth 2011). A new system cannot emerge from the ashes of the old. It is pointless to think about the future solely with(in) the Cartesian modernity model. It is actually hopeless to frame the present within such a dated model.

Western counterdominant perspectives are crucial in the struggle for social and cognitive justice, yet they are not enough. As Sandra Corazza (2002) courageously argued, "we need to start taking seriously the task of a real theory of curriculum thought" (p. 131), one that opens the Western canon of knowledge and is responsive to the need for a new epistemological configuration. Such a journey of belligerent struggles - against dominant and within the counterdominant Western epistemological platform-aims to replace the so-called monoculture of scientific knowledge for an ecology of knowledges. Such ecology of knowledges is

an invitation to the promotion of non-relativistic dialogues among knowledges, grating equality of opportunities to the different kinds of knowledge engaged in ever broader epistemological disputes aimed both at maximizing their perspective contributions to build a more democratic and just society and at decolonizing knowledge and power. (Sousa Santos 2007, p. xx) 
As any other theoretical exercise aimed at understanding the educational world in order to transform it (see Pinar 2004), ICT certainly exhibits a latitude and longitude borderless space to deepen certain claims. For example, among many issues, ICT highlights the linguistic imperialism framed by the English language and culture as one part of the genocide. Conscious of this linguistic imperialism as a crucial part of the genocide, ICT allows one to respectfully understand, for example, how "camfrenglish"- "a language used in Cameron cities, invented [and] created daily by the Cameron's urban youth" (Ela 2013, p. 24)—a language that deliberately violates the linguistic rules of French and English, desacralizes such imperial languages Camfrenglish, in cities such as Yaonde, is the people's language.

Darder (2012), in her superb exegesis of the political economy of cultural theory and politics, brings language to the core of the battle against eugenics. As Darder (2012) claimed, "the complexity of language and how the students produce knowledge and how language shapes their world represent a major pedagogical concern for all educational settings" (p. 105). Language, Darder (2012) argued, is more than a tool that epitomizes a specific learning theory or the cult of a flamboyant method. The language question intersects other social nonepiphenomenons such as the question of authority, reframing equality and social and cognitive justice. Any critical theory that aims at cultural democracy cannot ignore the power of (noncelebratory forms of) biculturalism as a poesis that determines culture and power relations in the classrooms (Darder 2012).

ICT is a claim for a nonstop production of an epistemology of liberation, in the very best way promulgated by Sousa Santos, that rejects the perversity of colonial praxis of dominance based on "the ontic realization of Being" (Dussel 1995, pp. 44-45; see also Dussel 2013) and works based on and through a philosophy that liberates the very own liberatory philosophical posture-real philosophy of liberation that tries to

formulate a metaphysics - not an ontology demanded by revolutionary praxis and technodesign poesis against the background of peripheral social formations. To do this it is necessary to deprive Being of its alleged external and divine foundation; to negate fetishist religion in order to expose ontology as the ideology of ideologies; to unmask functionalisms - whether structuralist, logico-scientific, or mathematical (claiming that reason cannot criticize the whole dialectically, they affirm it more[;] they analytically criticize or operacionalize its parts); and to delineate the sense of liberation praxis. Only the praxis of oppressed peoples of the periphery, of woman violated by masculine ideology, of the subjugated child, can fully reveal it to us. (Dussel 1995, p. 15)

ICT consciously aligns with the need for an epistemology of liberation that requires the liberation of the epistemology itself. ICT also warns of the need to challenge any form of indigenitude or the romanticization of the indigenous cultures and knowledges, and it is not framed in any dichotic skeleton of West-rest. In fact, it challenges such functionalist forms. Its itinerant dynamic pushes the theorist to a pluri(nonnecessary) directional path.

More importantly, ICT confronts and throws the subject to a permanent unstable question of "what is there to think?" ICT pushes one to think in the light of the future as well as to question how "we" can actually claim to really know the things 
that "we" claim to know, if "we" are not ready specifically to think the unthinkable, to go beyond the unthinkable and master its infinitude. ICT is to be (or not to be) radically unthinkable. ICT is a metamorphosis between what is thought and nonthought and unthought, but it is fundamentally about the temerity of the colonization of the non/un/thought within the thought. ICT attempts to understand how big is infinite, the infinite of thought and action. If one challenges infinity, it is chaos because one is in chaos; that means that the question or questions (whatever they are) are inaccurately deterritorialized and fundamentally sedentary. The focus is to grasp that ICT implies an understanding of chaos as domestic, as public, as a punctum within the pure luxury of immanence. In such multitude of turfs, ICT needs to be understood as poesis. It plays in the plane of immanence. Being immanence, "a life," ICT is "a life.” Is it a life paced by a poesis or a revolution? Yes please, in a full Žižekian way. ICT is a poesis that itinerantly throws the subject against the infinite of representation to grasp the omnitude of the real(ity) and the rational(ity), thus mastering the transcendent. Being more poesis than just theory (and not because it is less theory), its itinerant position epitomizes a transcendent nomadography, which is not transcendental.

ICT challenges book worship (Tse Tung 2007, p. 45). In fact, ICT also encourages us to pay attention to the multiplicity of forms to read the wor(l)d. The verbalization of pain and oppression is quite visible in Africa, for example, in art forms, such as dance and painting. Dance, Ela (2013) argued, in a country financially and economically moribund, is not just a way to face inequality and oppression. It is, he stated, "the very best way to face discouragement" (p. 26). ICT is an attempt to help us to think in another form as a human being. Corazza's (2002) insightful framework is crucial here as well. As she claimed, and I honestly think ICT addresses her claim, the challenge is to fight against what she coined assentado curriculum toward a vagamundo curriculum; that is, "to create [or co-create] a vagamundo curriculum one needs to question how can one think about the unaddressable, the unthinkable, the non-thinkable of the curriculum thought, the exteriorities, the self different, the self other, the other self" (p. 140). Corazza added the following:

Such curriculum thought is meaningless, a real vacuum, without the effective forces acting upon such thought, as well as without the effective indeterminations that forces such thought [or forms of thought] to think otherwise, differently, through the creation of new concepts required by the real experience and not just by the possible experience, thus allowing new life experiences. [In fact,] the strength of (an)other knowledge, as well as a new philosophy, will be measured by the concepts that it is capable of creating, or its capacity to renew meanings which impose a new framework on things and to assentados actions, shuffle their syntax, and organizing its thought in a clumsy logic. (p. 140)

Corazza's sharp take equips intellectuals with the necessary extraordinary tools to understand why some African scholars, such as Axelle Kabou (2013), Jean-Marc Ela (2013), and others, justifiably counterargue the Western and non-Western hegemonic apparatuses with the following question: "What if Africa refuses development?"

The definition of development must be seen through other lenses beyond its Western monocultural conceptualization of the needed development for the global 
South. Whose purpose does this development serve? What is the cost to those beneath its grinding wheel of so-called progress? In such context, ICT is really a matter of human rights as well, due to its commitment to social and cognitive justice. This is a commitment that challenges dominant multicultural forms, creating the conditions for and intercultural reconstruction of human rights, toward intercultural postimperial human rights that respect, among other issues, (a) the right to knowledge, (b) the right to bring historical capitalism to trial in a world tribunal, (c) the right to democratic self-determination, and (d) the right to grant rights to entities incapable of bearing duties, namely nature and future generations (Sousa Santos 2007).

ICT is a clarion call to challenge curriculum epistemicides by engaging fully in the complex struggle for social and cognitive justice. This is an intergenerational matter of justice, as well. ICT is seeing to rely on Saramago's metaphor. In one of his best novels, Seeing, Nobel Prize winner and Portuguese intellectual, Saramago (2007) described pictorially how, with the vote, the citizens of one unidentified country (most likely Portugal) blocked the normal rhythms of daily life. On a typical gray, wet, winter day in Portugal, the huge majority of the population decided to not vote until late afternoon. The narrative explains the gradual panic of politicians who didn't know what to do before such a democratic scandal. Suddenly, almost at the end of the day, the citizens showed up and voted. Shockingly, after counting the votes, officials announced that the majority of the votes were blank. Such political embarrassment was examined and a lot of reasons came to the table, including the unpleasant weather conditions. The government scheduled another election the following week on a very pleasant and sunny day. To national consternation, the results were worse: More than $80 \%$ of the votes were blank. The government reacted immediately against such outcome as if a crime had been committed. A state of emergency was put in place; such state paved the way for a state of siege, with intelligentsia spying on citizens, taking them for interrogation, and administering liedetector tests. The story goes on with surreal examples narrated by Saramago.

Saramago's (2007) Seeing is crystal clear for those of us fully committed to the struggle against epistemicides. Seeing goes well beyond the understanding of how to use democracy to save democracy. It is a call for a blank vote for those of us committed to social and cognitive justice, not just against the modern Western dominant and specific counterdominant forms that colonize the very way we [can] think but also against the complex matrix of circuits of cultural production so well unmasked by Ahmad (2008), as well as our own very existence in our academic settings. In claiming a "seeing" position, ICT allows us to move on toward a world that we wish to see, a world that was proposed in the Bamako Appeal:

(1) a world based on solidarity among human beings and peoples, (2) a world based on the full and complete affirmation of citizenship and equality between the sexes, (3) a universal civilization that offers the greatest possibility for the creative development of the diversity in all areas, (4) a world that constructs civilization through real democracy, (5) a world based on the recognition of the non-commodity status of nature, the planet's resources and agricultural lands, (6) a world based on the recognition of the non-commodity status of cultural products, scientific knowledge, education and health, (7) a world that promotes policies that closely combine unlimited democracy, social progress, and the affirmation of the autonomy of all the nations and peoples, (8) a world that affirms the solidarity of the 
people of the north and the south in the construction of internationalism on an anti-imperialist foundation. (Amin 2008, pp. 108-111)

More to the point, and, as I mentioned in Conflicts in Curriculum Theory: Challenging Hegemonic Epistemologies (Paraskeva 2011), ICT will not please everyone, as I was able to observe in certain academic settings (to be honest, more so in the United States). While there are appeals for a copresence conversation to rub Sousa Santos's (2009) and Pinar's (2004) approaches against each other, it is not a cross-cultural conversation. We actually need to challenge the cult of cross-cultural conversations. Al-Azmeh (2009) helped a great deal here. One needs to radically question the notion of cross cultural conversation,

Not because [one] wishes there to be an eternal incomprehensibility between peoples, or because I wish to promote xenophobia, and encourage ethnic cleansing and correlative acts of barbarism. It is rather because I believe that the notion of cross cultural conversations rests upon an unreflected assumption of the fixity and finality of the interlocutors in this conversation which even at the ends of serious philosophical authors tends to cause reason to denigrate to the tritest statements on common maximums of etiquette. It is the very same assumption of fixity and irreducibility underlying the etiquette of interculturalism and multiculturalism as a form of conservatism etiquette, that [one] sees so apparently paradoxical correlative of the sorts of assumptions about others - other ethnoi, other religious groups-that prepare the grounds, in the realms of conceptions and imagination for the entire range of possibilitiesextending from the rapturous fascination with the exotic at one extremity, to bellicose dehumanization of the Other and genocidal dehumanization of the Other. (Azmeh 2009, p. 77)

As I discussed elsewhere (Paraskeva 2016b), this project attempts to address many of the important questions that have been raised regarding "internationalization." It attempts to bring to the fore voices/discourses that have been systematically produced as nonexistent. This project is part of a long itinerant, deterritorialized, decolonial walk, a mirror of a complex dialogue among many of us, a dialogue that keeps targeting major conceptual swamps, such as the following: What does one mean by "internationalization"? Whose "internationalization"? Which language dominates this "internationalization"? Whose voices have been silenced? Whose knowledge has been systematically dismissed, ignored, and produced as nonexistent? I reiterate that an emphasis should be placed on what Sousa Santos (2014) called epistemicides and that I championed in the field as "curriculum epistimicides."

By championing the commitment to a nonabyssal thinking and defying the eugenic cult of cross culturalism, ICT put forward, along with Mignolo (2000, 2013) and Escobar (2013), and others, un paradigma otro that "does not fit into a linear history of paradigms or epistemes [that] runs counter to the greatest modernist narratives [and] reaches towards the possibility of non-European modes of thinking" (Escobar 2013, p. 34).

Such paradigm otro frames and fuels the debate of Western modernity within the so-called modernity/coloniality research program (Escobar 2013, p. 33) that challenges dominant perspectives in the study of modernity that could well be framed as "intramodern perspectives" (Escobar 2013, p. 34). Eurocentered Western modernity cannot be dissociated from the quarrel of global-local, and, "as a particular local 
history-[it] lays in the fact that it has produced particular global designs in such a way that it has 'subalternized' other local histories and their corresponding designs" (Escobar 2013, p. 38; Mignolo 2013).

The modernity/coloniality research project (hereafter MC) conceptualizes such colonial-coloniality momentum "grounded in a series of events [social constructions] that distinguished it from established theories of modernity" (Escobar 2013, p. 38). That is,

(1) an emphasis on locating the origins of modernity with the Conquest of America and the control of the Atlantic after 1492, rather than in the most commonly accepted landmarks such as the Enlightenment of the end of the eighteen century; (2) a persistent attention to colonialism and the making of the capitalism world system as constitutive of modernity; (3) the adoption of a world perspective in the explanation of modernity, in lieu of a view of modernity as an intra-European phenomenon; (4) the identification of the domination of others outside the European core as a necessary dimension of modernity with the concomitant subalternization of knowledge and cultures of these other groups; (5) a conception of Eurocentrism as the knowledge form of modernity/coloniality - a hegemonic representation and mode of knowing that claims universality for itself. (Escobar 2013, p. 38)

Such MC frames its research agenda by emphasizing notions such as the following:

(a) modern colonial world system - as an assemble of processes and social formations that encompass modern colonialism and colonial modernities; (b) coloniality of power-a global hegemonic model of power in place since the conquest that articulates race and labor and peoples according to the needs of capital and to the benefit of white peoples; (c) colonial difference and global coloniality - which refer to the knowledge and cultural dimensions of the subalternization processes effected by the coloniality of power; the colonial difference brings to the fore persistent cultural differences within global power structures; (d) coloniality of being - as an ontological dimension of coloniality on both sides of the encounter; (e) Eurocentrism - as the knowledge model that represents the local European historical experience and which became globally hegemonic since the seventeenth century. (Escobar 2013, p. 39)

ICT needs to be seen in such a framework as well. It is sentient of MC, yet it is not exhausted by it. Its itinerant perpetual dynamic creates that incapacity of surrender to a concrete framework. However, ICT attempts to complexify MC. For instance, it does not necessarily "run counter the greatest modernist narratives" (Escobar 2013, p. 34). It definitely runs against dominant modernist great narratives and through some counterdominant modernist great narratives, such as Marxism, for example, and in so doing decolonizes it. However, even in the attempt to smash certain dominant Western modernist great narratives, ICT pays cautious attention to the wrangle of religion, that is, Christianity and spirituality and how such a yarn was/is crucial to the construction of the (non)existence of the "other" (see Ela 2013). In such a sense, ICT is a theory of liberation, a liberation from certain constraints of critical pedagogy, as well, without denying it. Critical pedagogy exhibits particular pedagogical forms

as part of an ongoing individual and collective struggle over knowledge, desire, values, social relations, and modes of political agency[; that is,] critical pedagogy is central in drawing the attention to questions regarding who has control over the conditions for the 
production of knowledge, values and classroom practices; [critical pedagogy] is a form of provocation and challenge [attempting] to take people beyond the world they are familiar with and makes clear how classroom knowledge [is] always implicated in power. (Giroux 2011, pp. 5-6)

ICT sees such a "collective struggle over knowledge" as a struggle that today needs to go well beyond the Western epistemological platform. We all stand respectfully in the shoulders of others, and Giroux's (2011) work helps a great deal. By insightfully framing critical theory and pedagogy as a language of critique and hope and possibility, a critical pedagogy "that addresses the democratic potential of engaging how experience, knowledge and power are shaped in the classroom in different and often unequal contexts" (Giroux 2011, p. 5), he built a foundational field that one can explore in the struggle against epistemicides. ICT is a clear call against the precariousness of any fixed theoretical position. Needless to say, this implies severe conflict, a conflict that was always a part of our daily lives. ICT is the people's theory, an epistemology of liberation quite sentient that there is no theoretical and/or political incompatibility between Marxist critical impulses and non-Western epistemes. For instance, if one pays close attention to Giroux's language of hope and possibility and the way that he frames critical theory and pedagogy, one does not see any incompatibility for an itinerant curriculum theorist to rub against other critical Marxist impulses and non-Western epistemes. This clearly implies decolonizing processes within the very core of the critical and Marxist matrix. Isn't this what Marx actually alerted us to when he claimed the need for a ruthless critique of everything that exists?

\section{References}

Ahmad, A. (2008). In theory. London: Verso.

Al-Azmeh, A. (2009). Islams and modernities. New York: Verso.

Amin, S. (2008). The world we wish to see: Revolutionary objectives in the twenty-first century (pp. 107-112). New York: Monthly Review Press.

Bauman, Z. (1998). Globalization: The human consequences. London: Blackwell.

Biko, S. (1978). I write what I like. Johannesburg: Heinemann.

Corazza, S. M. (2002). Noologia do currículo: Vagamundo, o problemático, e assentado, o resolvido. Educação e Realidade, 27(2), 131-142.

Croce, A. (1998). Discussing the undiscussable. In M. Berger (Ed.), Crisis of criticism (pp. 15-29). New York: The New Press.

Darder, A. (2012). Culture and power in the classrooms: Educational foundations for the schooling of bicultural studies. Boulder: Paradigm.

Deleuze, G. (1995). Negotiations 1972-1990. New York: Columbia University Press.

Deleuze, G., \& Guattari, F. (1987). A thousand plateaus: Capitalism and schizophrenia. Minneapolis: University of Minnesota Press.

Dussel, E. (1995). Philosophy of liberation. Eugene: Wipf and Stock.

Dussel, E. (2013). Ethics of liberation: In the age of globalization and exclusion. Durham: Duke University Press.

Eagleton, T. (2011). Why Marx was right. New Haven: Yale University Press.

Ela, J. M. (2013). Restituir a Historia as Sociedades Africanas. Lisbon: Edicoes Pedago. 
Escobar, A. (2013). Words and knowledges otherwise. In W. Mignolo \& A. Escobar (Eds.), Globalization and the decolonial turn (pp. 33-64). New York: Routledge.

Giroux, H. (2011). Zombie politics in the age of casino capitalism. New York: Peter Lang.

Grosfoguel, R. (2007). The epistemic decolonial turn: Beyond political economy paradigms. Cultural Studies, 21(2-3), 211-223.

Haber, L. (1970). Black pioneers of science and invention. Orlando: Odyssey Classic Hardcourt.

Hooks, B. (1998). Making movie magic. In M. Berger (Ed.), The crisis of criticism (pp. 132-146). New York: The New Press.

Hountondji, P. (2002). The struggle for meaning: Reflection on philosophy, culture and democracy in Africa. Athens: Ohio State University.

Jenkins, K. (1991). Re-thinking history. London: Routledge.

Kabou, A. (2013). E se a Africa se Recusar ao Desensvolvimento? Lisbon: Edicoes Pedago.

Mahbubani, K. (2004). Can Asians think? Singapore: Marshal Cavendish International.

Marx, K., \& Engels, F. (2012). The communist manifesto. New York: Verso.

Mbembe, A. (2014). Critica da Razao Negra. Lisboa: Antigona.

Mignolo, W. (2000). Local histories/global designs: Coloniality, subaltern knowledges and border thinking. Princeton: Princeton University Press.

Mignolo, W. (2013). Introduction. Coloniality of power and decolonial thinking. In W. Mignolo \& A. Escobar (Eds.), Globalization and the decolonial turn (pp. 1-21). New York: Routledge.

Mphahlele, E., \& Thuynsma, P. N. (2011). In corner B (pp. 15-29). New York: Penguin Classics.

Muthu, S. (2003). Enlightenment against the empire. Princeton: Princeton Univrsity Press.

Paraskeva, J. M. (2011). Conflicts in curriculum theory: Challenging hegemonic epistemologies. London: Palgrave Macmillan.

Paraskeva, J. M. (2014). Conflicts in curriculum theory: Challenging hegemonic epistemologies. London: Palgrave Macmillan.

Paraskeva, J. M. (2016a). Curriculum epistemicides. Towards an itinerant curriculum theory. New York: Routledge.

Paraskeva, J. M. (2016b). Introduction. In J. M. Paraskeva (Ed.), The curriculum: Whose internationalization? (p. XX). Peter Lang: New York.

Perez, E. (1999). Decolonial imaginary. writing Chicanas into history. Bloomington: Indiana University Press.

Pinar, W. (2000). Introduction: Toward the internationalization of curriculum studies. In D. Trueit, W. Doll Jr., H. Wang, \& W. Pinar (Eds.), The internationalization of curriculum studies (pp. 1-13). New York: Peter Lang.

Pinar, W. (2004). What is curriculum theory? Mahwah: Lawrence Erlbaum.

Pinar, W. (2012). Curriculum studies in the United States. New York: Palgrave Macmillan.

Pinar, W. (2013). Curriculum studies in the United States: Present circumstances, intellectual histories. New York: Palgrave Macmillan.

Quijano, A. (2000). Colonialidad del poder y classificacion Social. Journal of World Systems Research, 6(2), 342-386.

Saramago, J. (2007). Seeing (Reprint ed.). Washington: Harvest Books.

Sayyid, S. (2015). Fundamental fear. Eurocentrism and the emergence of Islam. London: ZED Books.

Seth, S. (2011). Travelling theory: Western knowledge and its Indian object. International Studies in Sociology of Education, 21(4), 263-282.

Sloterdjik, P. (2013). In the world interior of capital: Towards a philosophical theory of globalization. Cambridge: Polity Press.

Sousa Santos, B. (2003a). Prefácio. In B. Sousa Santos (Ed.), Democarizar a Democracia-Os caminhos da Democracia Participativa (pp. 25-33). Porto: Edições Afrontamento.

Sousa Santos, B. (2003b). Para uma sociologia das ausências e uma sociologia das emergências. In B. Sousa Santos (Ed.), Conhecimento prudente para um vida decente: Um Discurso sobre as ciencias revisitado (pp. 735-775). Porto: Afrontamento. 
Sousa Santos, B. (2006). The rise of the global left: The world social forum and beyond. London: Verso.

Sousa Santos, B. (2007). Another knowledge is possible. London: Verso.

Sousa Santos, B. (2009). Epistemologias do sul. Coimbra: Almedina.

Sousa Santos, B. (2014). Epistemologies from the South. Boulder: Paradigm.

Trueit, D. (2000). Democracy and conversation. In D. Trueit, W. Doll Jr., H. Wang, \& W. Pinar (Eds.), The internationalization of curriculum studies (pp. ix-xvii). New York: Peter Lang.

Tse Tung, M. (2007). Oppose book worship. In S. Žižek (Ed.), Slavoj Žižek presents Mao on practice and contradi ction (pp. 43-51). London: Verso.

Young, R. (2001). White mythologies. London: Routledge.

Open Access This chapter is licensed under the terms of the Creative Commons Attribution 4.0 International License (http://creativecommons.org/licenses/by/4.0/), which permits use, sharing, adaptation, distribution and reproduction in any medium or format, as long as you give appropriate credit to the original author(s) and the source, provide a link to the Creative Commons license and indicate if changes were made.

The images or other third party material in this chapter are included in the chapter's Creative Commons license, unless indicated otherwise in a credit line to the material. If material is not included in the chapter's Creative Commons license and your intended use is not permitted by statutory regulation or exceeds the permitted use, you will need to obtain permission directly from the copyright holder. 


\section{Part III \\ Curriculum Theory and Didaktik in US and Europe}

The present research program aims at bridging educational leadership research, curriculum theory and Didaktik. Further, this program connects to and moves beyond a previous large scale international dialogue, namely that between curriculum theory and Didaktik that was initiated in the end of 1980s. In Part III, chapter authors Walter Doyle, Mariella Knapp, Stefan Hopmann and Tero Autio, consider the impact of the classical Didaktik Meets Curriculum Project in which prominent U.S. and European scholars in curriculum and Didaktik, including one of the editors of this volume, met to consider relationships among these two dominant curriculum traditions. Stefan Hopmann and his colleagues led the project.

Walter Doyle belonged to the core group; thus, we asked him to give us perspectives on the impact of the earlier Didaktik/Curriculum dialogues, including any new relations among curriculum/Didaktik and leadership for the contemporary situation. From Doyle's perspective (this volume), the major contributions of Didaktik to the Anglo-American Curriculum tradition include a more explicit consideration of content as process endowed with educative significance, the transformation of content into pedagogical process, and the enactment of content in classrooms. Doyle, who had engaged with content related questions of teaching since the 1980s, further argues that the Didaktik/Curriculum dialogues changed his own scholarship and inspired many other US colleagues to conduct empirical studies of the relations among content, pedagogical processes, and the practicalities of classroom-level curriculum work. Lee Shulman, who also participated in the 1993 symposium in Kiel with Wolfgang Klafki, introduced pedagogical content knowledge (PCK) in the mid 1980s with, among others, Sigrun Gudmundsdottir, who participated in Shulman's project "The Knowledge Growth in a Profession" at Stanford. Both Doyle and Schulman have later considered how these perspectives relate to leadership.

The Knapp and Hopmann chapter provides a detailed analysis of similarities and distinctions between Didaktik and Curriculum Theory and then describes how this project inspired many empirical projects across Europe, including their own recent study of school leadership as gap management. Knapp and Hopmann remind us that at the time of the earlier dialogues, the American curriculum tradition and Didaktik 
differed substantially according to their focus in the core of teaching, the role of the teacher, and relationships between the individual (subject) and society. For the American curriculum, many scholars had focused on how educators align 'programs' to the 'needs' of different students and contexts as well as system effectiveness for achieving an appropriate and common learning. Rather, in Didaktik, central questions revolved around interrelations among content, process, and aims; the aims of education are, thus, internally related to the content and methods.

Tero Autio joined the Didaktik meets Curriculum meeting in 1995 and has since contributed to the field. His chapter is a well argued contribution regarding one of the principles advocated in Part 1: curriculum, Didaktik and educational leadership are cultural phenomena that have developed historically. A historical reconstruction is required in order to bridge these fields within different western traditions, the American and the European. Thus, Autio traces the history of ideas, traditions, and shifts in the social-policy context that have affected the role of schooling in the nation state. Specifically, Autio argues that a certain type of instrumentalist thinking and rationality may be identified through the history. He then argues for a reconsideration of this thinking in the contemporary situation, one that more clearly connects curriculum and leadership. In his final part of his chapter, Autio moves beyond the western tradition, and poses a view of educational leadership from a base of Chinese wisdom traditions as well as Western curriculum theorizing/Didaktik.

In a globopolitan perspective, we argue, it is crucial that the western tradition in curriculum studies, Didaktik and educational leadership also is aware of its modern roots and core concepts in order to establish and develop a Chinese-EuropeanAmerican dialogue.

We make two additional points regarding the Didaktik Meets Curriculum project as we introduce this part of the volume. First, in the wake of that critically important project, curriculum/Didaktik studies have also been affected by interrelated movements occurring around the same timeframe in North America, Europe, and elsewhere. Here we refer to the Reconceptualist Movement in the North American context and its later internationalization phase as well as the new sociology of education. In the Reconceptualist movement, by drawing heavily on subjectivity and Bildung thereby challenging scholars to think about curriculum as a complicated conversation, moving beyond traditional approaches to theorizing curriculum. Apple and colleagues theorizing curriculum as cultural reproduction, drawing heavily on transformative sociological perspectives and theoretical approaches which also influenced Scandinavian Didaktik (Englund). In addition cultural-historical psychology/activity theory significantly influenced European Didaktik and american research on teaching and reframed the US-European dialogue on teaching by offering a shared language including a cultural and an institutional perspective.

Second, leadership received little attention in the Didaktik Meets Curriculum project, Reconceptualist Movement, the New Sociology of Education or in Cultural Historical Activity Theory (CHAT), despite the orientation towards developmental work research. Knapp and Hopmann's chapter (this volume) clearly illustrates how the changing context clears the way to bridge curriculum/Didaktik and leadership in empirical work. More specifically, Knapp and Hopmann describe changing societal 
and policy (e.g. externalized evaluation) contexts in Europe that bring leadership into closer dialogue with Didaktik/Curriculum. Knapp and Hopmann also argue that Didaktik Meets Curriculum can offer a starting point for investigating how the construction of school leadership has changed in the new accountability and testing environment. We extend that argument with a further consideration of the contemporary policy and societal context for educational leadership and curriculum work in the next parts of this volume. 


\title{
Chapter 5 \\ The Didaktik/Curriculum Dialogue: What Did We Learn?
}

\author{
Walter Doyle
}

\begin{abstract}
In the late 1990s, scholars from the Anglo-American curriculum community began a conversation with scholars in the German didaktik tradition (see Westbury I, Hopmann S, Riquarts K, Teaching as a reflective practice: the German Didaktik Tradition. Erlbaum, Mahwah, 2000). One major difference between these traditions is the perspective on content. In the US tradition, content is often seen as (a) a given that does not need to be analyzed and (b) inert, i.e., unchanging as it passes from curricular documents through classrooms to pupils (and even to standardized tests). Within this frame, school leadership need not be centrally concerned with the content of curriculum. In didaktik, content is fundamental and regulation is ideally normative and intellectual, i.e., it provides tools for teachers to come to pedagogical terms with the contents they teach. What didaktik thinking potentially brought to life for US curriculum thought was a more fruitful understanding of content processes as (a) formation for community and society and (b) transformation to rich pedagogical potential. This chapter elaborates more fully the lines of similarity and difference between the didaktik and curriculum traditions and explores the reasons why didaktik has had only a modest impact on turning the Anglo-American curriculum tradition toward a more fully developed sense of content and content enactment.
\end{abstract}

In this chapter my task is to examine the nature and impact of the Didaktik and/or Curriculum project that took place in the late 1990s, the results of which are reflected in the volume on teaching as reflective practice edited by Westbury et al. (2000). At the core of the project, scholars from the Anglo-American curriculum community began a conversation with scholars in the German didaktik tradition. The US tradition of curriculum thought had its roots in the analyses of the graded school in the later half of the nineteenth century as a state system of universal education began to evolve. The perceived task was to decide which content should be covered when,

\footnotetext{
W. Doyle $(\bowtie)$

The University of Arizona, Tucson, AZ, USA

e-mail: wdoyle@email.arizona.edu
} 
where, and in what order so that documents could be created to synchronize and regulate the work of a largely female teaching force who were not trusted to have the capacity to decide or manage curriculum in their classrooms. The concern, in other words, was organizational and managerial. Although there were competing conceptions during this time of organizing principles for curriculum - what Kliebard (2004) called "ferment"- the traditional "subjects" of the academy were generally accepted as the organizing frame for curriculum. The German didaktik tradition traced its roots to Comenius' Didactica Magna-The Great Didactic - published from 1633 to 1638 . The central task of didaktik was the analysis of content from a social and a pedagogical frame. General didaktik focuses on bildung, i.e., the educative or formative potential of various contents within a social context. Fachdidaktik, or subject didaktik, associated with the gymnasium, concentrates on the elementarization and simplification of disciplinary content to create pedagogically functional representations.

The Didaktik and/or Curriculum project consisted of several activities:

1. Multiple conversations among a small group of scholars-Westbury, Gundem, Hopmann - on the likely points of similarity and difference between these traditions and on the difficulties of translating core terminology from the German didaktik corpus into English equivalents.

2. A major international conference at the IPN in Kiel attended by scholars from both traditions, including Lee Shulman, George Posner, Sigrun Gudmundsdottir, Tomas Englund, Michael Uljens, Peter Menck, David Hamilton, Ingrid Carlgren, and Wolfgang Klafki himself.

3. Articles in journals, including Bildung und Erzeihung and the Journal of Curriculum Studies.

4. Translations, for the first time, of major Didaktik writings into English.

5. Important summative commentaries from Hopmann, Riquarts, Westbury, Gundem, and others on the connections between the traditions.

This project and its attempt to connect the two great curriculum traditions was clearly a major event in the history of curriculum scholarship. But the impact is difficult to assess. As a participant in both the origins and the work of the project, I have had a continuing awareness of the value of didaktik thinking in my own work. Moreover, I have worked closely for the past 4 years with Fred Janssen and Hanna Westbroek in the Netherlands, and for them didaktik is part of their everyday thinking. So I am perhaps not representative of most US scholars on this topic.

There have been isolated references in the US curriculum literature over the years, but mostly by Europeans-e.g., Hopmann. Two other members of this panel have written on the topic. Autio (2006), writing from a Finish perspective, has produced a quite comprehensive analysis of the two traditions, seeing both as fundamentally complicit with neoliberalism and managerial instrumentality. Pinar (2011) has also authored a book that pays close attention to aspects of Didaktik. Finally, I would point to this session as a sign that the conversation opened in the 1990s is continuing, enlarged, of course, to include leadership studies. 
Rather than attempt some type of empirical estimate of the impact of the didaktik vs. curriculum project, I decided it would be most useful to map how my understanding of curriculum work has been shaped by my contact with the Didaktik and/ or Curriculum project. My contention is that this mapping can be useful in locating points at which the Didaktik tradition can potentially inform both Anglo-American curriculum thinking and leadership studies and practice.

\section{The Centrality of Content}

Let me start by noting that most of my scholarly work has been directed to understanding the culturally and historically situated forms and practices associated with teaching in classrooms. Such work is, of course, at the nexus of curriculum and pedagogy, a topic that I wrote about in the Jackson Handbook (Doyle 1992) and am preoccupied with even today. In the US curriculum tradition, content is bypassed, i.e., usually seen as (a) a given that does not need to be analyzed and (b) inert, i.e., unchanging as it passes through curricular documents and pedagogical material into classrooms to pupils and into standardized tests. Within this frame, school leadership need not be centrally concerned with the content of curriculum but rather with delivery systems and so-called professional development exercises to achieve implementation fidelity.

I might also point out that familiar, everyday pedagogical forms and arrangements are quite often objects of derision or at least disappointment among education academics throughout the world. As such, they are not items to be studied and understood but targets for removal and replacement with reputedly effective processes flowing from the superior minds of pedagogical scholars - to make the world a better place. Despite the premature reports of the impending death of conventional practice, these forms and arrangements are remarkably enduring and crop up, with few exceptions, across the world wherever schooling activity occurs. So while we wait for the end of schooling as we know it, I thought it might be useful to understand what existing practice is all about.

My contact with didaktik came about as a part of my effort to understanding teaching in classrooms in ways that went far beyond the content-free processproduct studies that grew out of the conventional educational psychology paradigm. As recounted in Westbury's origin story in the Preface to Teaching as Reflective Practice (2000), the Didaktik and/or Curriculum project began with an exchange Ian and I had around the Kirsch (1977) article on elementarization and simplification in mathematics teaching. But let me tell the rest of the story. For me it all began with an experience I had in a doctoral oral examination of one of our students who was majoring in what we called Teaching and Teacher Education and minoring in mathematics. The oral exam committee consisted of professors from the major and a minor professor from mathematics. After an hour or so of questions from the major committee, the mathematician began his turn with a comment that he found all the talk about teaching quite trivial. I, of course, bristled at this characterization of my 
career work and, when the mathematics professor finished his questions, I asked the student to go to the board and talk through the teaching of some topic in mathematics. The student selected "induction" as the topic and began to explain how he would teach it. The mathematician interrupted rather soon to question whether the student was actually teaching about induction. After a brief conversation, they agreed that induction was, in fact, the topic on the floor. Then as the student explained what he would do first, second, etc., the mathematician noted that the approach being taken would possibly result in confusion when the students reached a related topic, etc. This type of conversation when on for about 15 min and struck me as something I had never heard before-continuous talk about teaching that utilized content rather than psychological categories. When I relayed this experience to Westbury, he sent me the Kirsch article. Again, I had never seen such an analysis and asked him what this was. When he reported back that it was just an ordinary piece of facdidaktik, we began our quest to find out what this didaktik stuff was all about.

So in didaktik I saw a fundamental emphasis on content and on analysis and interpretation of content as essential to both curriculum work and teaching activity. From this perspective, my focus shifted from delivery and implementation fidelity to providing tools for teachers to come to pedagogical terms with the contents they teach.

Within this frame, curriculum is the process through which content is gathered from the world, brought into schools, transformed into pedagogical material, and enacted as classroom event. In other words, curriculum work is a process of interpreting content to connect the world to the classroom.

I see three major domains of content interpretation (i.e., curriculum discourse) in schooling. Although connected, the tasks and language differ, often substantially, across these domains.

\section{Levels of Curriculum Discourse}

\section{Societal Level Curriculum Discourse}

The first level of curriculum discourse is that which occurs at the juncture between society and the institution of schooling. It is usually quite difficult to locate where this discourse takes place and who the participants are, but this multi-voiced and multilocated conversation connects what Westbury (2000) called the "idea of curriculum" situated within the "the pervasive web of beliefs and understandings about what schooling is that is to be found embedded in any society or culture" (p. 106) and the institutional documents and arrangements that constitute the instantiation of that idea in schools. It is the discourse through which content in the world is curricularizedendowed with socially significant educative potential. Any particular curriculum, thus, is first a set of claims about the educative effects of certain contents (i.e., what outcomes can be expected of particular experiences) and the social significance of these effects (i.e., why such outcomes are important for children and youth to acquire). 
Let me quickly illustrate this process with one of my favorite quotations from Margaret Atwood's (2000) novel, The Blind Assassin:

Father had decided, correctly enough, that our education had been neglected. He wanted us taught French, but also Mathematics and Latin-brisk mental exercises that would act as a corrective for our excessive dreaminess. Geography too would be bracing.... He wanted the lacy, frilly, somewhat murky edges trimmed off us as if we were lettuces, leaving a plain, sound core (p. 161).

What we see here are working, and perhaps naive, theories of the educative potential of various contents as justification for their inclusion in a curriculum. Such theories of content are quite ubiquitous and easy to write. Their essence is twofold: (1) an interpretation of what experiences with the content achieve, and (2) an understanding of the fundamental educational consequences or importance of these experiences. What's more difficult to understand, of course, is how particular theories of content become hegemonic — why, for example, we generally accept that mathematics, history, literature, and science should be the backbone of curriculum. If curriculum is a complex conversation - and certainly it is, especially in very heterogeneous societies-where does that conversation take place and who listens to and accepts the claims of educative potential?

In contemporary curriculum theorizing, considerable discourse is directed to the connection between society and schooling - to what I am calling the curricularization of content. At a minimum, such discourse integrates theories of society, theories of the person, theories of knowledge, and theories of institutions. No wonder the curriculum conversation is complicated. Didaktik has certainly been seen as a resource for curriculum discourse about the connection between society and schooling and, thus, the fundamental role and purposes of education in a society (e.g., Autio 2006). This theme runs through the papers in this session and the argument is put forward that educational leaders can exercise influence in helping define how this connection should be interpreted, especially in light of the host of at best mis-educative standardizations and assessment practices being employed globally to manage schooling efficiencies while conveniently ignoring issues of race, poverty, gender, exploitation, and cultural funds of knowledge. Educational leaders, in other words, are being cast as the first, or perhaps the last, line of defense against neoliberal managerialism.

\section{Institutional Curriculum Discourse}

The second level of curriculum discourse occurs within the institutionalized enterprise of schooling and is focused on the transformation of content into pedagogical material. This transformation has several dimensions:

1. The writing of district curriculum documents that attempt to "resolve" issues of inclusion, scope, and sequence - a process that is currently being swamped by the Common Core Standards. 
2. The creation of textbooks and related materials (including assessments) by a variety of industries, nonprofits, and special interest groups.

3. The design and testing of curriculum approaches and materials by university professors, often with government sponsorship.

Whether consciously or not, these activities involve didaktik processes of elementarization, simplification, and representation. Moreover, they involve a theorizing of the content itself-what counts as mathematics, reading, science, history, geography, play, self-realization, etc. Arguments around these issues are especially inflamed in the fields of reading and mathematics. I would note that much of this kind of work is being done within disciplines, particularly mathematics education or science education rather than in traditional curriculum studies (e.g., Clements 2007). However, science and math educators in the US tend to view their work as pedagogical rather than curricular, which masks a bit the extent to which they are actually creating theories of content.

Didaktik has helped me identify and conceptualize these processes of content theorization and transformation that occur as content moves toward pedagogical material and classroom use. I would also see this as a rich arena for leadership studies. Educational administrators spend considerable resources to acquire and distribute pedagogical material, so enhancing their capacity to support, facilitate, and critically inform this process would seem to be essential.

\section{Classroom Enactment}

The third level of curriculum discourse occurs among teachers and especially within teachers (ultimately this is a private deliberation) as they engage in the practical task of enacting curriculum in classroom group settings. It is often true, of course, that curriculum designers often reach toward enactment by producing model lessons that illustrate how a program can be implemented in classrooms. Schoenfeld (2014), for example, in his TRU MATH project has given quite detailed and extensively tested lessons that exemplify his principles of teaching mathematics for rigorous understanding. Such model lessons are intended to serve as clarifications of what the basic principles of the approach are and as practical tools for teachers to learn how to implement the program in their own classrooms. But such lesson models, and the frameworks they represent, are intended necessarily for all classrooms and thus specific details of context and setting are stripped away. Teachers, however, deal with the particular so to do the practical work of teaching they must transform whatever curriculum they encounter into forms and practices they can use with specific students at a specific time and place.

I have written at length in other contexts about the myriad demands of the classroom setting and the complex tasks these demands pose for the practical work of teaching (Doyle 2006). For present purposes, I would like to underscore two major points. First, to enact a curriculum in a classroom, a teacher must design, bring to 
life, and sustain events that afford participation by as many students in the class group as possible. In other words, subtraction, poetry, or self-realization must become events to get on the floor in classrooms. At a minimum, such events (a) must not jeopardize student cooperation in future events - classes have a long historyand (b) must contain tasks that students accomplish with respect to the curriculum. Indeed, such tasks are, essentially, the curriculum on the floor in classrooms.

Second, practical designs, in teaching as elsewhere, must be procedural, suitable, and efficient. That is, to do practical work teachers face issues of:

1. Instrumentality — what procedures can be used to bring events to life and sustain them in a classroom

2. Congruence-how do these procedures fit existing circumstances in a classroom

3. Cost-how much of one's limited time and resources are required to bring the event to life in a classroom (Doyle and Ponder 1977; Janssen et al. 2013).

In contrast to what is often assumed in the Anglo-American curriculum tradition, curriculum processes do not stop at the classroom door as teaching begins. Rather, the interpretative processes - which I take to be the essence of curriculum-continue to occur as teachers engage in designing events, bringing them to life with groups of students, monitoring their progress, and judging student products. It is impossible, in other words, to obviate the teacher's perspective and essential theoretical commitments from the actual curriculum on the floor in classrooms. What teachers uniquely bring to the table is situated event knowledge of curriculum, which shapes in profound ways what occurs as curriculum in the real world.

School leaders and pedagogical reformers typically ignore the practicality demands of teachers' work and, in turn set teachers up to be seen as failures. Most professional development experiences either attempt to train teachers rather quickly in new pedagogies or place teachers in professional learning communities to deliberate about their practices. Since in most cases teachers are being asked to achieve goals they do not have with methods they do not know how to use, such efforts typically fail. However, since the pedagogies and/or learning community procedures are endowed with inherent "quality," leaders are off the hook. They have done all they can do, so it's the teachers who are to blame.

An emphasis on practicality leads to a quite different approach, one of seeking bridging tools that connect a world of possibility to a world of practicality, i.e., the general to the particular, and in this sense mirrors the very design processes of practicality that teachers face every day. I have had the privilege recently of working closely with Fred Janssen and Hanna Westbroek in the Netherlands (Janssen et al. 2015) and with Kristin Gunckel, Marcy Wood, and Erin Turner at Arizona (Doyle et al. 2013) on the design and use of such bridging tools. I do not have time here to go into this work in detail, but these tools have shown considerable promise in making innovations practical. In turn, I think this work in practicality studies can be the foundation for a reconstruction of how teachers are supported in accomplishing the design tasks they face in making the general particular. 
To return to the didaktik theme, I would underscore that an emphasis on the interpretation of content that exists at the core of curriculum processes in classrooms is an essential component of practical change in teaching. Reform is often seen largely as a matter of pedagogical practice. But different practices usually reflect fundamentally different conceptions of the content. If I think reading is primarily about pronouncing words correctly, then my reading pedagogy will differ markedly from someone who thinks reading is about updating personal knowledge. Or if I think mathematics is primarily about putting correct numerals into arithmetical sentences, then my math teaching will different from that of someone who thinks mathematics is about finding quantitative dimensions of real-world problems. In practicality terms, there is a problem of congruence. Asking me to change practices without exploring the congruence of my theory of the content with the intended pedagogy is quite likely to be unproductive.

\section{Summary}

To summarize, I think one of the major contributions of Didaktik to the AngloAmerican Curriculum tradition has been to make content process rather than managerial control central to the schooling enterprise. These basically interpretive processes include:

1. The curricularization of content, i.e., how content is endowed with educative significance

2. The transformation of content into pedagogical material

3. The enactment of content in the complex practical world of the classroom.

I want to emphasize that these processes do not disappear if one were to change the fundamental purposes of schooling. Whether schools seek reproduction, emancipation, or self-realization, there would still be the tasks of curricularizing specific contents, transforming them into pedagogical materials, and enacting them with group of children. An understanding of these processes empowers teachers and curriculum leaders to tackle the central educative issues of schooling. It also underscores the urgent need to the design sensible resources and support systems to sustain this practical conversation.

\section{References}

Atwood, M. (2000). The blind Assassin. Toronto: McClelland \& Stewart.

Autio, T. (2006). Subjectivity, curriculum, and society: Between and beyond the German Didaktik and Anglo-American Curriculum Studies. Mahwah: Erlbaum.

Clements, D. H. (2007). Curriculum research: Toward a framework for "research-based curricula". Journal of Research in Mathematics Education, 38, 35-70. 
Doyle, W. (1992). Curriculum and pedagogy. In P. W. Jackson (Ed.), Handbook of research on curriculum (pp. 486-516). New York: Macmillan.

Doyle, W. (2006). Ecological approaches to classroom management. In C. Evertson \& C. Weinstein (Eds.), Handbook of classroom management: Research, practice, and contemporary issues (pp. 97-125). New York: Erlbaum.

Doyle, W., \& Ponder, G. (1977). The practicality ethic in teacher decision making. Interchange, $8(3), 1-12$.

Doyle, W., Gunckel, K. L., Wood, M. B., Turner, E. (2013), Blending pedagogical theory and classroom practice in preservice science teacher education. Paper presented at the meeting of the European Science Education Research Association, Nicosia, Cyprus.

Janssen, F., Westbroek, H., Doyle, W., \& van Driel, J. (2013). How to make innovations practical. Teachers College Record, 115(7), 1-42.

Janssen, F., Westbroek, H., \& Doyle, W. (2015). Practicality studies: How to move from what works in principle to what works in practice. Journal of the Learning Sciences, 24, 176-186.

Kirsch, A. (1977). Aspects of simplification in mathematics teaching. In Proceedings of the Third International Conference on Mathematics Education (pp. 98-120). Karlsruhe.

Kliebard, H. (2004). Change in format made the struggle for the American Curriculum, 1893 1958. New York: Routledge.

Pinar, W. F. (2011). The character of curriculum studies. Bildung, currere and the recurring question of the subject. New York: Palgrave MacMillan.

Schoenfeld, A. H. (2014). What makes for powerful classrooms and how can we support teachers in creating them? Paper presented at the Annual Meeting of the American Educational Research. Philadelphia: Association.

Westbury, I. (2000). Toward an understanding of "aims" in music education. In R. Colwell \& C. Richardson (Eds.), The new handbook of research on music teaching and learning (pp. 105111). Oxford: Oxford University Press.

Westbury, I., Hopmann, S., \& Riquarts, K. (2000). Teaching as a reflective practice: The German Didaktik Tradition. Mahwah: Erlbaum.

Open Access This chapter is licensed under the terms of the Creative Commons Attribution 4.0 International License (http://creativecommons.org/licenses/by/4.0/), which permits use, sharing, adaptation, distribution and reproduction in any medium or format, as long as you give appropriate credit to the original author(s) and the source, provide a link to the Creative Commons license and indicate if changes were made.

The images or other third party material in this chapter are included in the chapter's Creative Commons license, unless indicated otherwise in a credit line to the material. If material is not included in the chapter's Creative Commons license and your intended use is not permitted by statutory regulation or exceeds the permitted use, you will need to obtain permission directly from the copyright holder.

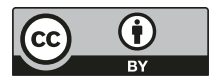




\title{
Chapter 6 \\ School Leadership as Gap Management: Curriculum Traditions, Changing Evaluation Parameters, and School Leadership Pathways
}

\author{
Mariella Knapp and Stefan Hopmann
}

\begin{abstract}
School leadership nowadays is confronted with ever-changing and fastgrowing expectations of what schools should be able to achieve. However, school leadership is an embedded activity, i.e. much depends on the underlying structure and culture of schooling. For instance, different traditions of defining schooling play a significant role in defining the role of school leaders. Therefore, it could be worthwhile to compare different traditions and current practices of defining school leadership with the traditions of conceptualizing the schooling within which they have evolved. Taking the well-known differences between the Didaktik and the Curriculum traditions as a starting point: Should one assume that these deeply rooted traditions have an impact on the leadership "pathways" which are determined by new expectations of the outcome of schooling? This becomes a fascinating empirical question the moment both traditions meet, e.g. by implementing in a Didaktik setting control patterns that historically have been developed within the curriculum tradition. For example, how do school leaders respond to the challenge of being measured by parameters that traditionally were none of their business? This chapter addresses conceptual issues of this question and empirical findings, based on a research project in Lower Austria.
\end{abstract}

\section{Introduction}

Different concepts of teaching, instruction and preparing lessons in different countries can be distinguishable when taking an Anglo-American Curriculum tradition together with a German Didaktik one (Hopmann and Riquarts 1995). An understanding of German, Scandinavian and Central-European schooling presupposes

M. Knapp $(\bowtie) \cdot S$. Hopmann

University of Vienna, Wien, Austria

e-mail: mariella.knapp@univie.ac.at; stefan.hopmann@univie.ac.at 
knowledge about the significance and role of Didaktik; such knowledge, however, has not been given the same importance in Anglo-American countries where the issues concerning Didaktik are expounded within the framework of "curriculum and methods" and "curriculum and instruction" (Hopmann and Riquarts 1995; Hopmann 2015). Linguistic and cultural differences also make it difficult to translate concepts and theories from one to the other. The difference between these two traditions cannot be regarded merely as a boundary, since it also offers an opportunity for each to learn from the other within their own possibilities and restrictions. "Didaktik meets Curriculum" is a topic on which researchers since the 1990s have been focusing, and its implications have become more important as extensive and large-scale changes in school systems, such as the introduction of Educational Standards and National Testing in Austria, continue to evolve.

Since curriculum development occurs on different levels of decision-making, educational leadership in the Anglo-Saxon tradition is also an important part of the discussion, selection and organization of the educational purposes of a school. The concept of school leadership became familiar in the 1990s and is connected to reforms towards a decentralization of the education system. While in the 1960s the activities of school principals were described as administrative tasks, emphasis has shifted to the discussion of effective school management (see Gunter 2014). In conjunction with the implementation of school-based management, the tasks of principals were increasingly seen as planning strategies, implementing proposals and motivating people. In the 1990s, mostly with the results and student outcomes of National Testing in mind that seemed to demonstrate a necessity for change in the education systems, this label changed again (see also Wissinger and Huber 2002). Research on school effectiveness indicated that leadership was an important factor for innovation and school turnaround since it would be able to create ideal conditions for school improvement (Gunter 2014). This marked a shift from the management label to an emphasis on leadership.

It is a different thing in the German-speaking context. A systematic confrontation with leadership matters has never occurred from the perspective of the Didaktik tradition. Nevertheless, the label of school leadership as a description of the tasks of principals here also is becoming increasingly common with the ongoing trend towards Standardization and National Testing. From this perspective, "Didaktik meets Curriculum" can offer a starting point for investigating how the construction of school leadership has changed in the new accountability and testing environment. The basic idea of this chapter is to define the dual tasks of school leaders as gap management: on the one hand, to ensure the requirements of school administration, but on the other, also to ensure local freedoms. In the following sections, the main elements of both traditions, Didaktik und Curriculum, will be described and their ramifications for leadership discussed. If and how school leaders deal with this kind of gap management in testing times will be investigated with recourse to a interview study from Austria.

"Didaktik meets Curriculum" is a project that started about 20 years ago (Hopmann and Riquarts 1995) to think about schooling using the differences and 
similarities of two different approaches. Both, Didaktik and Curriculum can be seen as different concepts associated with distinct traditions of dealing with the concept of schooling.

Both are historically evolved forms of reflection within distinct social systems (Hopmann 2015) and so are based on different understandings and images of schooling. These traditions have co-existed and from time to time have influenced each other in various ways, but until the late twentieth Century never in a way that changed the fundamentals of the other tradition. Due to developments stemming from social ones outside schooling, their current conjunction may be different in scope and consequences to any known hitherto. Besides the translation of important historical works of didactic and curriculum theory and making these accessible for both the English- and German-speaking worlds, another aim of the project is to discuss current developments and reforms in the field of education concerning the consequences of the ongoing mixing and transforming of both these traditions. The idea of the "Didaktik meets Curriculum" project can be seen as a background for the examination of actual trends in reforming education systems by implementing National Testing and modes of local accountability. Moreover, this new way of thinking about schooling also affects school leadership since reforms and changes in the context of implementing an accountability system often discuss school leadership as a key to the success of school effectiveness and related functions, but also new challenges, which were actually not part of the respective traditional activity set. Therefore, the different traditions of Didaktik Theory and Curriculum Research, but also current developments, are briefly characterized and serve as a background for the following description of the concept of school leadership as gap management.

\section{The German Tradition of Didaktik}

Whereas the German tradition of Didaktik and its central concept "Lehrplanung" (instruction planning) is typical for German, Scandinavian and Central-Europe schooling, Curriculum Theory was established in the Anglo-American area. Since the implementation of public mass schooling in the late eighteenth century, both have been established as distinguishable traditions. Historically, three different aspects are important for a characteristic of the traditional European Didaktik Theory: the term "Bildung", the pietistic understanding of schooling, and the implementation of a national curriculum regime.

A main feature of a pietistic understanding of schooling was not least the idea that teaching is more than acquiring knowledge, but that it renders teaching and learning as an unfolding of all the senses and powers (August Herman Francke). In this context the realization of teaching cannot be assumed but must be learned as a profession. Consequently, teacher seminaries as institutions for teacher education 
were established and used with the basic idea of teaching as an independent activity. Becoming a teacher was not about doing a job but following a vocation. This understanding of professionalism confers the teacher considerable "pedagogical freedom" in decision-making during lessons based on his or her "professional knowledge". In Curriculum Theory, this understanding is different and sees teaching more as an implementation and execution of curricular decisions (Westbury 2000).

Closely linked to the German understanding of teaching is also the implementation of a national curriculum regime or the so-called "Lehrplan", which is a product of the Prussian corporative state with its administrative structure, and was developed as a regulatory tool of education policy and school administration for controlling local schooling and classroom practice. It defines and specifies the social function of schooling, the objectives to be achieved and the content of teaching (Künzli et al. 1999). As a document, the "Lehrplan" describes the framework of teaching, but also grants teachers enough pedagogical freedom and professionalism (see Horlacher and De Vincenti 2014). Since the school administration is not in a position to compulsorily standardize the activity of teachers and student learning, it defines the achievement of students at best as expectations (Künzli 2006). In this sense, it reaches the classroom only indirectly (Künzli et al. 1999). The concrete realization of the intended goals needs transformation into a concrete methodical and didactical arrangement but also one in which teachers can appreciate the situation of their students. This traditional construct of state-based regulation has important consequences for teachers as it helps them legitimize pedagogical and administrative decisions for parents and students (Hericks and Kunze 2008). Teachers were not per se responsible for the performance of students or that something "works", but rather that something had been offered and done. Didaktik in this context was to close the gap between the regulations of the state and local teaching. For teachers this implies a scope of action that is manifested in a kind of "freedom of method" and a "pedagogical freedom". In a type of license principle, the teacher is seen as a legal person who can choose the methods of instruction and is responsible for conducting lessons (Hopmann and Künzli 1998). In this sense, Didaktik can be seen as important for the transformation from the national curriculum to lesson planning since teachers answer questions as to which specific content should be taught in a particular lesson and why. So, during teacher training the teacher has the license and the permission to act in class autonomously within the framework of the official guidelines, but still retaining full responsibility. Erich Weniger (1932) described the transformation of cultural heritage into the educational content of the "Lehrplan" as a struggle of powers between political agencies ("Kampf der geistigen Mächte").

The German tradition of Didaktik also established a close reference to the ideas of the Enlightenment of the eighteenth century and the German tradition of humanistic pedagogy ("geisteswissenschaftliche Pädagogik"). Most theories of Didaktik developed in the nineteenth century comprise as a constitutive element the category of "Bildung". In this context, the aim of teaching and schooling has nothing to do with transporting knowledge from society, science, or other domains to a learner, 
but uses knowledge for the transformation of the unfolding of a person's individuality and sociability. Bildung cannot be reached through Didaktik, but Didaktik makes it possible to "restrain teaching" in such a way as to allow for the individual development of the student to prosper (Hopmann 2007). Examples of this are the models of Wolfgang Klafki $(1958,1995)$, which until now are the most popular and bestknown references to school practice in German-speaking countries when it comes to planning lessons or evaluating the quality of schooling and instruction. In a survey of German teachers, in answer to the question as to which text best characterized the German Didaktik tradition, nearly all replied: Klafki's Didactic analysis as the core of preparation of instruction (Hopmann 1999). Here, one aspect of the "common core of Didaktik" becomes obvious, namely the difference between matter and meaning, which means the distinction between the content as such and its "educational substance". One and the same matter (Inhalt) can represent many different meanings (Gehalt), and one, and the same, meaning can be represented by different matters (Künzli 2002). Meaning is what emerges when content is enacted in a classroom based on the methodological decisions of a teacher (Hopmann 2007). In this sense, Didaktik becomes a tool for teachers to identify and transform curricular matters into local teaching (meanings). From the perspective of Wolfgang Klafki, this transformation from "matter" to "meaning" is only possible by analyzing and answering the basic questions of didactic analysis (this concerns the question of what relevance the content has for students present and past, what the content exemplifies, how it can be integrated into the overall structure of the lessons and how students can get access to this topic). Klafki's outstanding performance lies in the extraction and development of an argumentation structure for the planning of teacher lessons based on educational theory. As a student of Erich Weniger, he managed to reform and integrate the relationship between didactical and methodical problems (Primat pädagogischer und didaktischer Zielentscheidungen im Verhältnis zur Unterrichtsmethodik, Klafki 1976, S 81) and describes how methods, contents and aims are interrelated.

Connected with different traditions in philosophy and ideas about schooling, the concept of Didaktik as a systematic differentiation between curricular "matter" and local teaching "meaning" is also uncommon in the Anglo-Saxon world (Westbury 2000). While "Curriculum Studies" deal with the organization of curriculum and the processes of teaching and learning, classical questions of Didaktik, for example, how to structure schooling and school subjects, are discussed under the category of "classroom research" (Gundem and Hopmann 1998).

For curriculum as a scientific discipline in German-speaking countries, research on educational questions of the curriculum and syllabus discourse only existed marginally before the 1960s. So far, a research tradition or a research institution bearing the catchword "curriculum" has never existed (Tröhler 2014). Famous and wellknown Anglo-American curriculum literature, such as the Basic Principles of Curriculum and Instruction (Tyler, first published 1949), was first translated and only published in German 24 years after its first printing in the USA. In current German-speaking discourse on schooling such literature is either not mentioned or has been forgotten. Furthermore, other famous curriculum research works like 
Kliebard's "The Struggle for the American Curriculum 1893-1958" (first published in 1987) or Jacksons "Life in classroom" (1968) are only rarely addressed in German research on schools and education. German translations of these texts do not exist. There is also no German-speaking Educational Research Association with a division that focussing on curriculum studies (Tröhler 2014). As Tröhler (2014) mentions, this also seems interesting in the light of the 1961 OECD recommendation to found national institutions for the dissemination of the educational goals of the member states, which led to the initiation of the Max-Plank Institute in Germany with Saul Benjamin Robinsohn as Director. Robinsohn's publication "Bildungsreform als Revision des Curriculum [Educational Reform as Revision of the Curriculum 1971]" became popular in the German-speaking world and formed a basis for further curricular models (e.g. Frey 1971). The main idea was to build a scientific approach to curriculum planning by identifying through empirical investigation socially relevant qualifications and associated content, but also situations for achieving such qualifications. Like Tyler's "Basic Principles", Robinsohn's version of curriculum planning also focused on the importance of research, evaluation and expertise. Although it led to new models in Didaktik (e.g. Heimann et al. 1979) and to a new generation of "Lehrpläne" (Criblez 2009), this approach did not fit the German tradition of administrative curriculum work (Künzli Fries et al. 2013). Bearing in mind that learning goals were intended to be measurable and objective, it nevertheless can be seen as a precursor of the discussion on National Standards Testing, a discussion that is similar to the current discourse on Standardized Testing (Criblez 2009). During the sixties and seventies another American influence became very popular, namely, the concept of programmed learning and instruction. In hindsight, the traditional Didaktik was challenged by behaviourist ideas of learning, but also by more empirically based curriculum research (e.g. Heinrich Roth) and psychological testing (Terhart 2015). The interest in curriculum research in Germany finally waned during the 1980s, which is outlined in a "Renaissance of Didaktik" (Hopmann and Künzli 1992). Although the semantics were replaced, the rules and routines of state "teaching work as an administrative action" were reinforced (Hopmann 1988).

\section{Changing Times}

Amplified by international comparison studies like PISA or TIMSS a growing political interest in the direct regulation and effectiveness of schooling has shifted the traditional focus from central input control towards output control. Regulation through the formulation of expectations in the "Lehrplan" and the idea of work conforming to such expectations were queried by implementing a continuous evaluation and assessment testing of students and schools to control the realization of these formulated expectations. Over the last 30 years the trend in borrowing elements from the Anglo-Saxon curriculum tradition, where such product control and dealing with evaluation in the form of student test results is more common, could be 
observed. Whereas before, education planning was a promise without product viability, the implementation of standards as a new modus of regulation in education (which is also currently realized in Germany, Austria, Switzerland and in many other European countries) is oriented towards the idea of guiding learning processes through output control. This understanding emphasizes a strong rationalistic and deterministic view of teaching and learning and misjudges the fact that students do not automatically learn what they are taught. This is also called the "didactic difference" (Künzli 2006).

Although the Anglo-American "Curriculum tradition" is more oriented towards psychology models (Hericks and Kunze 2008), it, however, focused very early more on the learning process of students and how and what should be taught in school (Künzli et al. 2013). In contrast to the Didaktik approach, the term "Curriculum" is characterized by a culture of textbooks and learning materials (Künzli 2009). While ideally, and typically for curriculum theory, the preparation for everyday life is paramount, a central aspect of the Didaktik theory is the introduction into society through providing content or matter whose meaning should be learned (Westbury 2000). Furthermore, an output-oriented evaluation of learning results and of the public school system is more common in the Anglo-American discourse. Exemplary are Joseph Mayer Rice's 1912 claims for "Scientific management in Education", collecting data and developing common performance requirements in the form of educational standards (Kliebard 2004), but also as other American Educators like Leonard Porter Ayres, 1912, or Franklin Bobbitt, 1918, argued, reasons for regulating processes of teaching and learning through the results of tests. In this context, achievement testing and external evaluation for determining the quality of schooling increased and characterized the American school system in an important way. The College Admission Test (Scholastic Assessment Tests) was established already in 1901, but National Testing (National Assessment of Educational Progress $N A E P$ ) also was implemented before the seventies. As mentioned earlier, standardization was also a recurring topic in Western-European countries but never had a sustainable influence in the US context until recent years. Education Standards, implemented as an answer to "A Nation at Risk" have existed in the US since the nineties. Paradoxically, the discussion today on standardization is led in the context of the first implementation of a National Curriculum, the "Common Core State Standards", which defines in detail what K-12 students ought to know at the end of each grade. For England, the situation appears more moderate, but also there standards and attainment targets play an important role in describing the expected achievements of students, and being inspected and regulated by a government agency (OFSTED). Furthermore, national tests, teacher assessments and final examinations like GCSE are standard procedure. However, in contrast to the US, a National Curriculum as part of the "Education Reform Act" was implemented already in 1988. So what can be observed is an ongoing mixing of traditions in both directions, without at the same time neglecting the existing traditional form. Around the world, standards and tests are being implemented, which only intensifies the accountability problems of local teaching. 
Moreover, the political drive towards raising the bar produces serious and significant consequences, which Nichols and Berliner (2007) described as "collateral damage". There is considerable empirical evidence indicating that the more school systems focus on academic achievement as a key variable, the more they put pressure on disadvantaged students of all kinds (e.g. race, needs, migration) and promote social segregation (see e.g. Rustique-Forrester 2005; Braun et al. 2010; Ravitch 2011; Nichols et al. 2012). Another critical point is that standards also tend to draw attention and resources to certain subjects and therefore to knowledge and problems related thereto, which withdraws legitimacy from other subjects (Apple 1992). The more energy schools or students invest in achievement competition in key areas, the less they can really devote to other subject-matter areas such as civic education or the arts since fewer resources are left for other educational issues such as social activities or civic engagement (see e.g. Cuban 2007; Koretz 2008; Polikoff et al. 2011; Labaree 2010). Furthermore, curricular shrinking, also known as "Teaching to the test", is often described as a consequence of teachers and schools focusing so as not to be low down on the league table. Such rigid testing programs are associated with fostering educational inequality (Marzano 2000; Linn 2003). So, all in all, research on National Testing indicates the opposite effect to that which was expected and supposed to be established (for a summary see Hopmann 2013).

Concerning the topic, "Didaktik meets Curriculum" (Gundem and Hopmann 1998), it is interesting to see what happens when two different traditions of schooling come together. How do those involved handle the situation? How do they realize reforms, and how do they change their actions? Especially the introduction of National Education Standards is already a change from an input-control orientation in schooling to one of output control, which can be seen as a change from viewing the "location of schooling" to viewing the "measurement and assessment of schooling" (Hopmann 2006). School leadership is also affected by this mixing of cultures and the striving for enhanced accountability. During the eighties and nineties, through national and international discussions about reform, restructuring and improvement of the school system, the activity of school leaders evolved to itself become a subject of research. In German-speaking countries since the nineties the amount of literature on how to lead a school successfully, how school leaders should improve their schools and manage their staff in an effective way has been permanently increasing. The standard economic concept of leadership has become a common term for describing the duties and tasks of principals in the local improvement of schooling and teaching. Here, leadership concepts are often borrowed from the Anglo-American area, ignoring that the activities of school leaders differ according to the respective tradition. The approach of "Didaktik meets Curriculum" can therefore enable an understanding of these traditions of school leadership and facilitate the discussion of trends for further development and the related implications. 


\section{School Leadership as Gap Management}

Closely related to the discourse on "Didaktik and curriculum" is the idea of "School Leadership as gap management". Greatly simplified, the curriculum tradition was built around extremely high expectations of what local curriculum leadership meant. This was actually the basic notion of much of the curriculum work in the twentieth and mid-twentieth century. The institutional pattern of local curriculum leadership becomes obvious from Dewey (1902) to Tyler (1949) up to Schwab (1969), (1970). In Dewey's “Child and the Curriculum” (1902), learning is only possible by adapting the curricular subject matter to the local experiences and actual lives of students. The curriculum tradition directly addressed school leaders as curriculum makers. This already can be seen in Tyler's "Principles" (1949), which were developed in a University of Chicago course for school leaders. How should a school leader go about creating, defining, developing and controlling a curriculum at his or her school? By emphasizing the "rational" in curriculum planning, Tylers "Principles" matched the existing predominant paradigms of behaviourism, positivism and technical rationality and was thus often misinterpreted as a mechanistic understanding of curriculum (Pereira 1992). "The Practical" (Schwab 1970) criticized these principles and the idea of transforming scientific theories into pragmatic problems at school. But also for Schwab, curriculum was local, targeting a single school or small school districts and including a group of community members (and also the school leader) in the process of curriculum planning.

Up to the seventies and eighties, the idea that curriculum was publicly funded and locally decided was dominant in most of the Anglo-Saxon world. The basic idea of curriculum making was not only to locally define and determine its content, but also to regulate how and in what sequence, when and for whom, the content was to be considered. Since the late seventies, however, this tradition has been challenged not least as a consequence of reforms like "A Nation at Risk", the implementation of new core curricula (Common Core Standards) or the introduction of state-based standard testing. These changes can be interpreted as the consequence of the development of a phenomenon called "risk-sharing". The model of risk-sharing, which had been the basis for the development of the modern nation state, came under growing economic pressure (cf. Hopmann 2008). As with schooling, most societies met growing demands for health care, security provision, social services, etc. by simply expanding the institutions, professions and programs. There is an unavoidable limit to how much a society can spend on such risk-sharing without squeezing the tax-producing parts of society too much. Thus, since the eighties, almost all welfare states have had intensifying public struggles as to how much to spend on what, and most have had to adjust their risk programs to meet budget limitations. The people, as the other partner in the risk-sharing deal, do not simply accept that the State cannot deliver what was promised in exchange for loyalty and taxes; this has created an intensive search for ways of obtaining the same or even better services for less money. If more growth and expansion seemed not to be sustainable, the question instead was whether an "intensification" of public service delivery 
would do the trick. This gave rise to concepts like the one of "new public management" and accountability measures, with which those involved in public institutions should be forced towards a more effective and equitable use of public resources. This change can also be described as a switch from "management by placement" towards "management by expectations" (Hopmann 2008).

For schools, the introduction of National Testing and evaluation was intended to make teachers and schools accountable for the outcomes of schooling. This means a mixed transformation where local leadership diverges from curriculum leadership in the traditionally comprehensive sense and turns leaders into being accountable for executing curricula they did not themselves develop, or did not inspire or develop with their teachers. All in all, this means a reduction in locally based curriculum making, which today one can say has been destroyed in many places. The implementation of National Testing also has consequences for the relationship between inner- and extra-curricular activities. Only schools with very good conditions, such as a composition of students from a high SES background, or with an environment climate matching the requirements of the local school climate, allow leaders to be able to offer a program besides "teaching to the test". Under these conditions, reaching the standards of common core is just incidental. They can more or less act in the traditional way by planning and implementing their local curriculum in accordance with their students and situation. For schools with different conditions, the situation is different and National Testing has more relevance. These schools have to deal with the gap between local management and external accountability. So, what can be actually observed is that school leadership in times of accountability has to deal with new requirements. Gap management, in the sense of meeting local and statebased requirements, becomes an extended and transformed function.

In the midst of a transformation process towards school leadership as accountability management, issues like "fidelity", teacher control and evaluation outcomes also evolve. The interesting thing that is happening at the same time in the US context is that much of the curriculum studies field seems to be disappearing from the discourse and is being turned into a sort of cultural studies field not actually connected to what goes on in schools, or what goes on in leadership. This becomes clear, for instance, in discourse on curriculum and gender, race, class or multiculturalism. Nevertheless, some scholars in curriculum research are discussing ways of managing public schooling in testing times, and the plus of public schooling other than reaching good results in National Testing situations (for example DarlingHammond et al. 2014). There are many echoes of school not just being about testing (see Nussbaum 2011; Hansen 2011). In other words, with the continuance of highstake testing a new (didactical) pragmatism is also being discussed.

The Anglo-American perspective has now been described. The German, or Austrian, State-based system, however is a different matter. What many people do not know is that when State curriculum production was invented the recipient of this State curriculum was the school as a unit not the individual teacher. The original desire was that the State also control school plans to ensure that they were in line with State expectations. Although school leadership was meant to have a plan of its own, the national curriculum was simply a tool to see whether the local curriculum 
was covering enough of the material it indicated. Many of the very first curriculum documents in Europe in the first half of the nineteenth century actually dealt with which mathematics to teach. There were also all kinds of subjects available. In most European countries, the list of key subjects that we have nowadays was more or less finalized around 1850, and has not changed substantially since (Hopmann and Riquarts 1999). So the locally used curriculum became a matter for the individual teacher and not the school. The bridging gap here became Didaktik_Didaktik as a tool for teachers to define their work within the national frame.

However, leadership in this context was not to practice didactics on behalf of teachers, and so it became administrative. Each teacher was able to do as he or she saw fit, which reduced the leadership role in the course of the nineteenth century to a more administrative one. Indeed, leadership issues did not play a significant role either in prominent Didaktik theories (for example from Schleiermacher, 1810 to Weniger 1932; Klafki 1958) or in German Theories on Schooling. So, Didaktik generally addressed teachers and not school leadership, almost because leaders were not supposed to fill the gap. That was the teachers' view. Likewise, Didaktik had no key role in the history of leadership theories, which often focused on the administrative role of school leadership.

In this tradition, school leadership was primarily considered as an administrative task. A school leader represented the teachers and constituted the interface between school authorities and the matters of the local school. His or her central function in this model was the bureaucratic control and regulation of centrally based requirements of the school authorities. Legislation regulated the range of functions and duties of school leaders. This is still the situation today and school leaders fall under the responsibility of the local school and the proper implementation of rules, regulations and administrative provisions of the centralized school authorities. In this context school leaders have to deal with school authorities, teachers, and students and their parents. Schratz (1998) has summarized the traditional understanding of school leadership in German-speaking countries. In this sense, a "good" school leader is a person who is a good recipient and transmitter of orders in the interest of the smooth administration of schooling. In the traditional bureaucratic model the framework of the centrally regulated school is structured hierarchically and top-down.

In this sense, school leadership in State-based traditions also can be seen as gap management. This type of gap management has two distinctive sides. One is mainly located on the outside, and focuses on school leadership as an administrative and public task. School leaders have to show that their schools are firmly rooted in the institutional framework and perform the duties required of them by society and the State. But there is another side to the gap, which is located inside the school focusing on teachers and students. School leadership is also about defending the educative surplus of schooling (Bildung) as an outcome of the didactical use of teachers' pedagogical freedom. This State-based construction of school leadership remained basically unchanged until the late twentieth Century (see also Holtappels 1989) and was not discussed as a pedagogical issue (Wissinger and Huber 2002). Stimulated by Anglo-American school effectiveness research ("School Leadership matters") 
and international discussions on ensuring quality at schools, leadership started to become a topic in terms of school improvement. Connected with the idea of school leadership as a profession of its own, new intermediate programs and agencies (such as Landesinstitute), were created to offer training for school leaders. School leadership, however, did not become part of any didactical discussion and the programs did not really have an impact on everyday work in the schools. At best, they had an impact on the semantics of gap management.

\section{School Leaders as a Target for Educational Policies}

New inputs came from international developments like the results of large-scale assessments of PISA, TIMSS and PIRLS. The related recommendations of supranational policy organizations like the OECD led to pressure and stress concerning assessment. In Austria, for example, they were used as arguments for reforms such as the implementation of National Testing, a standardized school leaving examination, competence-based instruction or an inclusive and comprehensive school setting. By addressing the whole school as accountable for results, school leadership becomes a new issue. According to accountability, school leaders have to show that the students of their school fulfill the external State-based standards.

So over the last years, school leadership and leadership theory have become important issues for the output-oriented management of schools. This trend is driven by the school-improvement discourse. Guided by the argument that school systems need reforming, the importance of "successful school leadership" has also increased. Current discussion is led by school-effectiveness research, debate on strengthening the local responsibility of schools, and a series of empirical research studies on identifying factors and characteristics of effective and "good" school leadership styles. The success of school leadership is measured by student outcome variables. However, research on the effectiveness of school leadership has come to a different conclusion: for example, that targeted cooperation and innovation-oriented leadership has a positive effect on the actions and cooperation of teachers (e.g. Bonsen et al. 2002; Hallinger et al. 1996; Mackenzie 1983). This contribution also found a place in the concept of "Transformational Leadership" (e.g. Leithwood and Jantzi 1999; Dubs 1994) and "Contributed Leadership" (Mujis and Harris 2006), which are used internationally to describe and explain effective school leadership actions. Yet, findings concerning the relationship between school leadership and student achievement outcomes are inconsistent and very often without any theoretical substance. There is no evidence that a specific leadership style automatically leads to better achievement results. On the contrary, concrete leadership actions seem to be a response to the relationship between the contextual conditions of the individual school and the environment it serves as a moderating variable (Brauckmann 2012). Not personal factors, but the context as an interlock of institutional, system and personal factors, which cannot be investigated as separate, is important (ibid). In this sense, school leadership cannot be seen as the task of a single person, but as 
co-actions of a system. In terms of Spillane et al. (2004) this is called "Distributed Leadership". Charismatic and heroic school leaders who can perform all-important functions might be successful in reaching their goals, but there are only very few persons with this attitude. Over the last 10 years there also have been empirical references that focus on the interactions and active distribution of leadership functions which are helpful for identifying manners of organizational change (Harris 2008; Leithwood et al. 2007, Spillane et al. 2004).

Furthermore, school leadership is seen to influence student test results not directly and causally, but in indirect ways (Day et al. 2011). Effects seem small and often cognitive, and social- or organizational psychological models are borrowed to explain good leadership. Often these models take on an importance different to the context settings of schools. School context is discussed as a phenomenon of school culture, shared goals, trust and performance orientation, as well as cooperation among teachers, professional learning communities, capacity building, community partnerships and instructional settings. Often leadership theory is characterized by ideas of behavioural and personality theories, focusing on the person and the best leadership style, which imply normative ideas. Studies in this research context define and interpret very differently what a "good" school means and what successful leadership should look like.

So in the national and international discussion on school achievement, school improvement and school quality, often school leaders are seen as an important resource and dependent variable for influencing the development of their schools. New governance approaches and reforms, however, tend to extend the autonomy of the single school ("shift of powers") and lead to changes in the tasks and functions of school leaders ("shift of tasks"), and so school leadership is also discussed with stronger significance. The increased scope of actions and decisions in the pedagogical process should make it possible to better deal with the specific contexts and situations of the school and thereby use resources more effectively, which aims to improve the quality of both schooling and instruction. Coincident with the active use of these new scopes, school leaders are also increasingly responsible for processes concerning the management of quality. For individual school leaders this means new challenges and requirements, but also a new understanding of their own positions. On the one hand, they are more responsible for changes that happen under their leadership, on the other, they must have a deep insight into and local knowledge of what exactly is happening in their school. A decentralization of decisionmaking often coincides with greater responsibility for external standard setting and increased centralized output control.

There is now new research on how school leaders deal with these new scopes, how they interpret them and how the perception of more responsibility in more complex areas of activity can be successful in the social reality of individual schools (Brauckmann 2012). Furthermore, questions on how schools use the new open spaces and how and why some schools seem to act more intensively and innovatively than others are being addressed (Rolff 2009). In this sense, school leadership is about matching local and contextual demands with external requirements (Moos 2005). Surveys in the German and Austrian context show that school leaders still see 
their functions and duties as those of a steward and not as a developer and agent of school improvement (Bonsen 2010; Breit 2012). So the empirical questions now are: What happens to gap management in these contexts? How will an introduction of elements from the so-called "curriculum tradition" change the school leadership role? With the aid of examples of Austrian school leaders, we investigate how school leaders deal with these new challenges.

\section{The Implementation of Educational Standards and National Testing in Austria}

In the context of Austria, National Education Testing is a new form of accountability that was borrowed and adapted from Anglo-American school systems. As a reaction to the bad Pisa results in 2001 and 2006, reforms like the implementation of National Education Testing in Austria and a new school type, the "New Middle School" (NMS), were intended to help improve performance in international largescale assessments and achieve equity by reviewing and reconsidering traditional ways of teaching and learning. Evidence-based policy should thus help provide information about weaknesses and potential for improvement and increase quality not only in individual schools, but also in the whole school system (Haider et al. 2005). Unlike Germany and Switzerland, the Austrian education system is centralized, but similarly to the other two countries reforms are also intended to strengthen the responsibility and autonomy of individual schools. Discussion of National Testing is also embedded in this. While attention in the 90s focused on the improvement of the individual school, the results of international large-scale studies like TIMSS and PISA indicated a high variance between schools, which is considered problematic when it comes to the equity and efficiency of the education system (Freudenthaler and Specht 2006; Haider et al. 2005).

Conceptually, national testing in Austria is based on the construct of competence measurement and was tackled in 2003 after the publication in German of the socalled "Klieme Expertise", which contains detailed proposals for designing education standards (Zur Entwicklung nationaler Bildungsstandards, Klieme et al. 2003). Connected with the idea of being "objectively measureable", these standards, as in Germany, describe the normative expectations that schools should ensure (Lucyshyn 2006). Based on this definition, many more or less grounded competence models were introduced in the German-speaking world. Often these competences are criticized for being too focused on their measurability and not on their content (see e.g. Heid 2007; Scholl 2012), which may have far-reaching consequences for instruction and practical work in schools. In the Austrian school system, Schratz (2012), for example, observed that in discussions with their students, teachers focus more on the structure of learning processes than on examining more deeply the teaching content.

In Austria, National Testing takes place in the fourth and eighth grades. These are respectively the transitions from primary school to secondary I and from secondary I to secondary II. National Testing was first carried out in 2012 to measure compe- 
tences in English (as a foreign language), and in 2013 for Mathematics. Besides an analysis at the state level, school leaders also receive feedback from their own schools, teachers receive feedback from their classes and students receive feedback on their own achievements. School leaders are also bound by law to discuss the results and further implications with teachers and parents. In the framework of this context, the question arises as to how school leaders use this information for the improvement of their school and instruction. In contrast to other countries like the US or England, the results of National Testing are not connected to any consequences or incentives for schools, such as benefits, job positions, school closing, participation in improvement programs or financial disadvantages ("low stake"). Comparisons between schools are not explicitly intended and results of schools and classes are not made public.

Nevertheless, discussion seems important with respect to how school leaders and parents react to these changing contexts bearing in mind that educational standards and the competence-oriented curriculum cannot be introduced as a one-fits-all done template in a school. Schools and individual teachers do not have an executive function; their task at school is also to translate and contextualize the guidelines into the practices and conditions of their everyday lives. First, they have to make sense of the guidelines so as to embed them in a further step of their own instructional work. Here, the self-concept of teachers as professionals could be very important for how guidelines are followed. It is interesting to note that school leaders and parents react differently when bridging the gap between external demands and local situations of their school. The Ministry of Education also considers school leaders to be important for the successful implementation of reforms at school. Since 2004, the Ministry has been offering an official program under the name of "Leadership Academy" (LEA) to qualify school leaders in professional guidance for school improvement and the professional development of their teachers (Schratz et al. 2010). Furthermore, National Testing is on the agenda of this program and is intended to support school leaders in its strategic implementation.

But National Testing is not the only reform with which school leaders in Austria are confronted. In the school year 2008/2009 a new school type was established in secondary I, and will replace the hitherto lower secondary school by 2015/2016. Due to the fact that the school reform intends to be a school for all children, to intermix the social composition and reduce disparities by site-specific programs, school leaders have to engage with more possibilities and autonomy in order to cope with the requirements of the students in class. Both reforms tend to foster school improvement based on local awareness of the conditions of their school. Also here it would be interesting to gain a more differentiated insight and more information on how school leaders deal with the gap between school autonomy and standardization, and the bases of information they use in school improvement processes.

In international comparisons, school leaders in Austria more often cite that their assignments deal with administration and teaching than do school leaders from other countries (Suchari et al. 2010). School reforms, like the implementation of National Education Standards and the New Middle School, focus instead on school improvement and school management based on local circumstances, considering these the most important tasks for school leaders and emphasizing their responsibil- 
ity for them. So the question as to whether and how school leaders in their work approach this new aspect of school reforms seems to be very relevant. Do school leaders face more pressure or do they see no change at all? The answer to these questions would be of interest for the future development of schooling, school improvement and the schooling system.

\section{Mixed Messages: School Leaders Re-framing the Feedback from National Testing: Results from the Interview Study}

Interviews with ten school leaders of New Middle Schools in the State of Lower Austria were conducted to study in an adequate way the individual perspectives, perceptions and attitudes relating to school leadership and the tasks of school leaders concerning National Education Standards. This small-scale study is embedded in the government-funded evaluation project of the New Middle School in the state of Lower Austria "NOESIS" (for results see e.g. Feichter and Krainz 2015; Geppert et al. 2015; Geppert and Knapp 2015; Hörmann 2012, Kilian and Katschnig 2015; Knapp 2015; Retzl and Ernst 2012). In general, the interviews showed, that school leaders reported different reactions depending on which perspective they focused. If, in their function as a representative of the school, they were asked to describe the changes after feedback from National Testing, they reframed such testing as a useful evaluation tool for thinking and talking about school improvement. However, when asked to mention concrete activities, not much seemed to have changed in their everyday practice at school. From this within-school and didactical perspective, they seemed to reframe National Testing as a tool too narrow and reductionist to capture schooling and the work at their school. This also seems to reflect their ambivalent attitude concerning National Testing.

In the interviews and from the official perspective of administering and representing schooling, all school leaders reported that they discussed school-specific results from National Education Testing with the teachers. Changes after these discussions concerned in particular improvement in instruction and vocational development. Following feedback from National Education Testing school leaders also reported a focus on topics that needed improvement apparent through the introduction of observation by colleagues in the lessons, longer discussions during conference calls or a specific search for courses on vocational teacher training. The school principals also said that on the basis of the results they would place a new emphasis on learning and encouragement and that they would pay more attention to listening, writing and/or the corresponding verbal communication. Furthermore, exercises in tests, school- and homework seem to be progressively adapted to the ideas of competence models and multiple-choice tests. The following interview passage provides an example for these narratives.

In this school, before National Testing, I suppose, we didn't really deal with questions as we do now because we developed the exercises for the tests in the old-fashioned way. Since National Testing came in, we have been developing tests in a new format from first grade up. 
And I think we'll notice how the kids have become accustomed to these exercises in the next National Education Test. As I said before, the idea about what makes my colleagues special, well, we've also tried to bring this up at conferences and get it out there. (Interview 6)

What we actually see is that school leaders try to use the official language of the Ministry. From this perspective, they feel predominantly confident that National Testing offers a possibility for capturing and checking competences, but also for positioning their own school in an objective and fair comparison with other schools. They see it also as a way to evaluate and document the changes in their own instruction and to capture the effectiveness of that instruction. But we get another perspective if we look at the concrete changes at school. From this view, school leaders do not seem to be so sure anymore that National Testing can give them an orientation for further development at school. The ambivalent attitude towards National Testing would appear to be due to the perception that results of National Testing can be seen as snapshots of the current achievements of students that capture only a limited segment of schooling and what actually happens in the school system. This becomes obvious in the following statement of the interviews:

But they were certainly thought provoking, but not so much that, I wouldn't say we found a whole lot of information in it about what we can do differently. Some can, some areas always lag behind, okay? If I enhance reading, then some other area is lacking and that's the problem, okay? (Interview 10)

Whose fault is it? Attributing the Results from National Testing. We also see this ambivalence of different perspectives and in reframing National Testing when we look at how school leaders explain the results their school achieved. In the interviews school leaders mentioned three different ways of attributing the results from the National Education Standards. These possibilities can be arranged as in a triangle (see also Fig. 6.1).

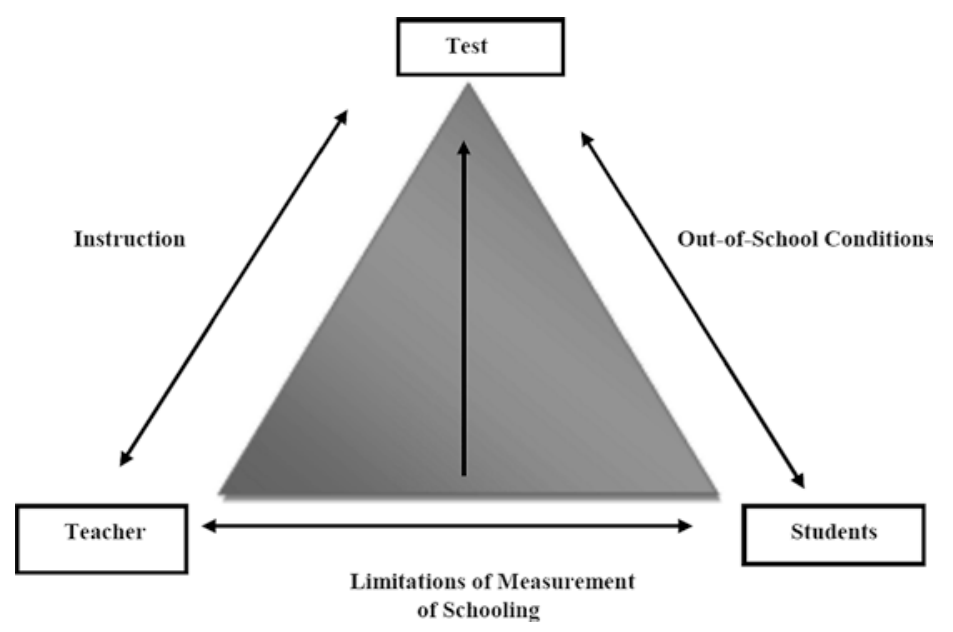

Fig. 6.1 Triangle to categorize the explanations for the scores achieved in National Testing of school leaders 
One way is that school leaders think that out-of-school conditions are relevant and important for outcomes. In this perspective they argue that the family's socioeconomic background and the missing commitment, but also the missing involvement of parents is responsible for bad outcomes. Because students do not bring the requirements necessary for schooling or because they have to deal with family problems, it is also hard for the school and teachers to prepare them adequately for the tests. Interestingly, results from National Education Testing mostly were attributed in the interviews to out-of-school conditions, such as the social background of the students. This perspective is illustrated in the following interview excerpt:

\footnotetext{
Last year we had some girls in the class where the families and the mothers had problems that were not really connected with the school, but we couldn't really get on with these people. Yes, definitely we had problems and I've seen the results of our school, because there were exactly three students who were hardly able to do anything properly in this class and there were these girls, who more or less failed themselves on purpose. So, umm, compared with the average, we didn't do so well in the English Test and were just below average for the overall test results. (Interview 3)
}

Another way to look at the results is to attribute the outcomes to the test itself, i.e. that the test can only measure what it measures and has some limitations. An example for this argumentation mentioned in the interviews is that, based on the construction of the test, it can only capture a snapshot of what schooling is about. So in this argumentation the inference that schooling is based only on National Education Testing is not a fair and good choice (for an example see the following interview passage).

Publicly, because in this form it's not possible to assess and evaluate schooling, and umm, this I wanted to add, if schooling is constructed only as an assessment of the National Education Test then Schooling and Education is interpreted very, very narrowly. (...) We're doing a good job, but also a job that can't be assessed in the form of a test. (Interview 1)

A possibility that was hardly noted was to explain the results as being due to the teacher's instruction, his or her personality and to what happened within a specific school. Although officially National Testing was intended to give feedback on learning processes and what had been learned at school, this argument rarely was used in the interviews. Here we can see the ambivalence in school leaders' views. While on the one hand school leaders reported that they had reflected on the results and used the testing for changes in instruction and school improvement, they, on the other hand, could not see the reasons for the results coming from themselves.

\section{Gap Management in Testing Times}

The present chapter defines school leadership as gap management, which can have different connotation depending on the tradition in which it is embedded, i.e. curriculum or Didaktik. In short, by gap management we mean that school leaders deal simultaneously with centralized and State-based regulations and demands but also with matters, needs and requirements of the local school. In the curriculum tradition, school leadership was a major part of local curriculum planning and making. 
By implementing State-based standards testing and a kind of national curriculum the local curriculum leadership became lost and school leaders were encouraged to execute an external curriculum.

In this context gap management deals with executing a centralized curriculum and being accountable for it, but also adapting this curriculum to local conditions. From a Didaktik-tradition, embedded in a centralized school system, school leadership has to deal with the gap between controlling and regulating the demands of centralized school authorities and defending the pedagogical freedom of teachers. This gap management becomes obvious as a "rhetorical shift" in the narratives of school leaders and seems to remain stable also after the implementation of (lowstake) National Testing. The interviews showed that school leaders also used this rhetorical shift when arguing for or against National Testing results. Whereas as part of their administrative duty they defended the results by explaining the changes they had initiated in their schools, they simultaneously defended the necessity of teachers' pedagogical freedom by emphasizing the education surplus of schooling beyond achieving good test results and the situatedness of teaching and learning.

This chapter also offers a background story on the current situation from the perspective of school leaders of the implementation of National Testing in New Middle Schools in Lower Austria. It emphasizes the importance of the contextual factors of the surrounding school area for school reforms, especially for reforms that deal with standardization in the context of achievement assessment and competence measurement. Often these reforms are constructed like a one-size-fits-all solution, which might not capture the real challenges and problems of the individual schools. For schools that face especially challenging circumstances, the results of National Education Testing might not be the first priority of their work. The same applies for schools that enjoy good conditions and have reached outcomes above the average. They often see the testing results as a way to acknowledge their good standing in comparison with other schools. The results of National Education Standards might be of greater interest for schools that due to their location find themselves in competition with academic secondary schools. Another important point is that changes because of the National Education Standards often do not go beyond the level of tests, tasks and exercise materials. Further interventions and reflection on instruction, the concept, preparation and course of the lessons, were not mentioned in the interviews in the context of the debate on National Education Testing. Furthermore, the idea of teaching and learning as a linear process and the deductive reasoning that excessive training in competences that had failed might lead to better results the next time round, seems to be an important point for future research. This information is not only of interest for policymakers, for implementers of reforms and for education researchers, but also for teachers and school leaders. Until now, it remains unclear which specific information is used by school leaders and how it is used for the determination and planning of interventions in their school. Thus, the question also arises as to which information resources seem to have the most relevance for school leaders.

As we know from the Anglo-American context, schools in future will have to deal with expectations constrained by resources. The quality of schooling is 
measured in terms of accountability for satisfying expectations within given resources. The interviews show clearly that school leaders are successful if they manage the gap and find ways and the capacity to react to the local problems of their school. Successful school leaders find it useful to take National Testing as a possibility and occasion to reflect on local circumstances. But that is not the only point. They also face the limits of National Testing and see the "added value" of schooling. In this sense, their aim is not only to achieve better test results, but also to find ways to deal with the problems facing them. This also necessitates allowing for local leeway in decision-making. The same applies for parents. Although National Testing also is intended to inform parents about the actual performance of their child and school, the results seem to hold only a part of parents' attention. In many schools parents do not seem to be surprised or affected by test results.

The results of the interview study indicate that school leaders try to deal with the challenges they face by managing the gap between the external expectations of National Testing and the local practices and demands of their school. In this sense, an interesting phenomenon can be observed and is described with the terms of "talk" and "action" of neo-institutionalism (Brunson and Olsen 1993). First introduced by Brunson and Olsen (1993) in research on reforms in public administration, they used this differentiation to explain dealing with contradictory or inconsistent institutionalized provisions of organizations. On the "talk" level, organizations master the proper and particular vocabulary of the reform, they present themselves as open-minded towards the reform and signal that the organization complies with the expectations and notions desired. However, the "action" level, which includes everyday behavioural patterns and interpretative patterns, is not affected. So the loose coupling of "talk" and "action" is seen as a possibility for creating a space of freedom for dealing with expectations at a distance (Schaefers 2002). Expectations, benchmarks and provisions that are not in line with the interests or conditions of the organizational actors are only symbolically realized at the "talk" level. NeoInstitutionalism Theory describes that this symbolic compliance helps to ensure the legitimacy of the organization (Meyer and Rowan 1977).

Based on these ideas, school leaders seem to be bridging the gap between the prescriptions of new reforms like National Testing or the implementation of a new school type (the New Middle School) and the local demands of their school by "talking the talk" they especially need. From the inside of schooling (or the organization) it is all about using teachers' "talk". In this context, school leaders are using the language of instruction, Didaktik, Curriculum, and professionalism when they discuss the results with teachers at their school. So, discussing the results of National Testing is all about a stronger representation of learning, exercises in tests, the adaptation of school and homework to the ideas of competence models, better receptiveness to the needs of students, observation by colleagues during lessons, teacher training, and so on. It is not very specific and it is not clear if much changes in practice, but it is about how these matters are discussed within the school. In this sense, school leadership and gap management are defined as turning official (reform) 
norms into building some kind of professional learning community and asserting the legal regulations symbolically. So school leaders "talk the talk" within the respective contexts they need.

In general, most of the school leaders seem to be positively disposed towards National Testing reform. However, it is questionable if this optimism is just an expression and reflection of the "talk" level to foster the legitimacy of their school. If you ask them what the results of National Testing tell them about their school, they answer: "not much". So what school leaders do is actually put the new challenge into the old gap management strategy. Outside school they talk the talk of accountability, but at the same time they see themselves as key figures taking care of the pedagogical freedom and the local autonomy of each and every teacher, and not interfering too much with their teaching. Here school leaders act as classical representatives of their school, so failure is of course attributed to the outside wherever possible, e.g. to characteristics of students, out-of-school conditions, school environment, parents, limitations of the test, but not to factors inside the school.

In summary, the interviews illustrated how school leaders try to translate reform demands into familiar activity and interpretative patterns for their school. They deal with these demands inside and outside the school differently and in a symbolic and ceremonial way. From the inside, School Leadership is about sending a signal about the legal regulations to teaching staff and transforming the demands into instructional and curriculum language. Outside the school, leadership is about ensuring legitimacy by using the vocabulary of the reform and showing that their school meets the norms that are demanded of a modern organization. Beside the "talk" level it remains unclear whether changes in activities and implementation of new activities are realized or if the routines and usual problem-solving processes remain stable. This is an important aspect for future research, but unfortunately would go beyond the scope of this chapter.

It should be noted that the presented results take place in a low-stake environment. Up to now, National Testing has had no real impact and it is not of much consequence in Austria to be low down in the national table. Nobody knows if this will change but the impression is that there are three conditions typical for many Western countries. The first perspective is that schools in Austria are not as different from each other as they are in the US. They are also very homogeneous and only a few schools really experience difficulties with National Testing. Another perspective is that schools in our system have little leadership to change the system. In the Austrian system, school leaders have to administer and not decide. Reforms like school-based management and movements of decentralization are only at their beginnings. Finally, school leaders are highly routinized in this gap management symbolism and are brilliant in changing their approach depending on to whom they are talking. So the treatment of keeping both approaches going is a key element of leadership development.

Nevertheless, in different contexts where National Testing is connected to important consequences, schools in a high-stake environment might react in different 
ways. Also, organizational theory describes a higher adoption of norms in an organization with a stronger dependency on the legitimacy of the norms. In the context of National Testing, a narrower adoption could mean a greater focus of curriculum on standards and "teaching to the test". In fact, school leaders in the interviews mentioned cases of cheating in other schools, like correcting the tests with students or studying the examples before the real test. Internationally, this is not a new phenomenon and Standardized Test cheating has already been observed in other countries (like the US or England). Nevertheless "talking about cheating" also emphasizes a strong feeling of competition and the fear of being compared with hardly controllable criteria and perhaps inconsistent goals, otherwise school leaders would not have mentioned this during the interviews. But on the other hand they only described the situation of "other schools" not their own schools and demonstrated they were ensuring legitimacy by their "talk" of the other schools (Standard Testing seems unfair, if other schools cheat) (e.g. see Berliner 2011; Petrilli 2012).

As already discussed, models and theories of school leadership have until now not paid much attention to organizational perspectives of neo-institutionalism that might afford a possibility for deepening the understanding of the actions and functions of school leadership. The results might also be connected to previous research on change and reform in the state-based "Lehrplan". Such research, too, indicates that the curriculum realized at a single school is only adapted to the new framework syllabus as far as necessary. This helps to ensure the established "curricular scripts" of teachers, which already secures their professional work at school (e.g. Vollstädt et al. 1999).

\section{References}

Apple, M. (1992). Do the standards go far enough? Power, policy, and practice in mathematics education. Journal for Research in Mathematics Education, 23(5), 412-431.

Berliner, D. (2011). Rational responses to high stakes testing: The case of curriculum narrowing and the harm that follows. Cambridge Journal of Education, 41(3), 287-302.

Bobbitt, F. (1918). The curriculum. Boston: Houghton Mifflin Company.

Bonsen, M. (2010). Schulleitungshandeln. [School leadership]. In H. Altrichter \& K. Maag-Merki (Eds.), Handbuch Neue Steuerung im Schulsystem (pp. 277-294). [Handbook of new governance in the school system]). Wiesbaden: VS.

Bonsen, M., von der Gathen, J., Iglhaut, C., \& Pfeiffer, H. (2002). Die Wirksamkeit von Schulleitung. Empirische Annäherungen an ein Gesamtmodell schulischen Leistungshandelns. [The effectiveness of school leadership. Empirical approaches of a complete model of achievement behaviour at school]. Weinheim: Juventa.

Brauckmann, S. (2012). Schulleitungshandeln zwischen deconcentration, devolution und delegation (3D) - empirische Annäherungen aus internationaler Perspektive. [School leadership between deconcentration, devolution and delegation (3D) - an empirical approach from an international perspective]. Empirische Pädagogik, 26, 78-102.

Braun, H., Chapman, L., \& Vezzu, S. (2010). The black-white achievement gap revisited. Education Policy Analysis Archives, 18(21), 1-99. 
Breit, S. (2012). Bildungsstandards und Qualitätsentwicklung an Schulen. Impulse für Schulleiter/ innen. Bildungsstandards - für höchste Qualität an Österreichs Schulen; Information für Schulleiter/innen. [Educational Standards and quality development at schools. Impulses for school leaders. Educational Standards - for highest quality of Austrian Schools: information's for school leaders].Graz: Leykam.

Brunson, N., \& Olsen, J. P. (1993). The reforming organization. Chichester: Wiley.

Criblez, L. (2009). Bildungsstandards. [Educational Standards]. Stuttgart: Klett und Balmer.

Cuban, L. (2007). Hugging the middle: Teaching in an era of testing and accountability, 19802005. Education Policy Analysis Archives, 15(1), 1-29.

Darling-Hammond, L., Wilhoit, G., \& Pittenger, L. (2014). Accountability for college and career readiness: Developing a new paradigm. Education Policy Analysis, Archives, 22(86), 1-38.

Day, C., Sammons, P., Leithwood, K., Hopkins, D., Gu, Q., Brown, E., \& Ahtaridou, E. (2011). Successful school leadership. Linking with learning and achievement. Berkshire: Mc Graw Hill.

Dewey, J. (1902). The child and the curriculum. In J. Dewey \& P. Jackson (Eds.)., (2004) The school and society and the child and the curriculum (pp. 181-208). Chicago: Univ. of Chicago Press.

Dubs, R. (1994). Die Führung einer Schule. Leadership und Management. [Managing schools. Leadership and Management]. Stuttgart: Franz Steiner Verlag.

Feichter, H. J., \& Krainz, U. (2015). Gemeinsames Nachdenken und Handeln: Über die Bedeutung partizipativer Evaluationsforschung für die Schulenwicklung. [Thinking and Acting together: About the relevance of participative evaluation for school improvement]. In NOESIS Project team (Ed.), Gute Schule bleibt verändert. Zur Evaluation der Niederösterreichischen Mittelschule (pp. 287-310). [Good schools stay changed. On the evaluation of the New Middle School in Lower Austria]). Graz: Leykam.

Freudenthaler, H. H., Specht, W. (2006). Bildungsstandards: der Implementationsprozess aus der Sicht der Praxis: Ergebnisse einer Fragebogen-Studie nach dem ersten Jahr Pilotphase II. [Educational Standards: the implementation process from the perspective of school practice: Results of a survey after the first year of pilot phase II]. Graz: Ministry of Education and Culture Austria.

Frey, K. (1971). Theorien des Curriculums. [Theories of the curriculum]. Beltz: Weinheim.

Geppert, C., Knapp, M., Bauer-Hofmann, S., Werkl, T. (2015a). "Das Rad muss sich drehen ... " - Zusammenfassende Ergebnisse der bisherigen Erhebungen im Rahmen der NOESISEvaluation. ["The wheel must turn..." - A summary of the results of the previous NOESIS Evaluation Study]. In NOESIS Project team (Ed.), Gute Schule bleibt verändert. Zur Evaluation der Niederösterreichischen Mittelschule. [Good schools stay changed. On the evaluation of the New Middle School in Lower Austria] (pp. 31-59). Graz: Leykam.

Geppert, C., \& Knapp, M. (2015b). Das verbale akademische Selbstkonzept als Capability Erweist es sich nach vier Jahren Neue Niederösterreichische Mittelschule tatsächlich als solche? [The verbal academic self-concept as a capability - Is that what it really is after four years of the New Middle School?]. In NOESIS Project team (Ed.), Gute Schule bleibt verändert. Zur Evaluation der Niederösterreichischen Mittelschule (pp. 93-120). [Good schools stay changed. On the evaluation of the New Middle School in Lower Austria]). Graz: Leykam.

Gundem, B. B., \& Hopmann, S. T. (Eds.). (1998). Didaktik and/or curriculum: An international dialogue. New York: Peter Lang.

Gunter, H. (2014). Educational leadership and management. In G. McCulloch (Ed.), The Routledge international encyclopedia of education. International encyclopaedia of education (pp. 202204). London: Routledge.

Haider, G., Eder, F., Specht, W., Spiel, C., \& Wimmer, M. (2005). Abschlussbericht der Zukunftskommission. [Final report by the committee on the future]. Wien: Ministry for Education, Science and culture. http://www.bmukk.gv.at/medienpool/12421/zk_endbericht_ neu.pdf. Accessed 12 Dec 2007.

Hallinger, P., Bickman, L., \& Davis, K. (1996). School context, principal leadership and student reading achievement. The Elementary School Journal, 96(5), 527-549. 
Hansen, D. (2011). The teacher and the world: A study of cosmopolitanism as education. Teacher quality and school development. New York: Routledge, Taylor \& Francis.

Harris, A. (2008). Distributed leadership: According to the evidence. Journal of Educational Administration, 46(2), 172-188.

Heid, H. (2007). Was vermag die Standardisierung wünschenswerter Lernoutputs zur Qualitätsverbesserung des Bildungswesens beizutragen? [What does the standardization of desirable learning outputs contribute to quality improvements of the education system?]. In D. Benner (Ed.), Bildungsstandards. Instrumente zur Qualitätssicherung im Bildungswesen; Chancen und Grenzen - Beispiele und Perspektiven (pp. 29-48). [Educational Standards. Instruments for quality development in education; chances and limitations - examples and perspectives]). Paderborn: Schöningh.

Heimann, P., Otto, G., \& Schulz, W. (1979). Unterricht: Analyse und Planung [Instruction: Analysis and Lesson Planning]. Hannover: Schroedel.

Hericks, U., \& Kunze, I. (2008). Forschung zu Didaktik und Curriculum. [Research on Didaktik and Curriculum]. In W. Helsper (Ed.), Handbuch Schulforschung (2nd ed., pp. 747-778.), [Handbook of school research]). Wiesbaden: VS.

Holtappels, H.-J. (1989). Der Schulleiter - zwischen Anspruch und Wirklichkeit. [The school leader - between ideal and reality]. Essen: Wingen.

Hopmann, S. T. (1988). Lehrplanarbeit als Verwaltungshandeln [Curriculum work as administrational action]. Kiel: IPN.

Hopmann, S. T. (1999). Wolfgang Klafki und die Tradition der Inhaltsorientierung in der deutschen Didaktik. [Wolfgang Klafki and the tradition of content orientation in German Didaktik]. In I. F. Goodson, S. T. Hopmann, \& K. Riquarts (Eds.), Das Schulfach als Handlungsrahmen Vergleichende Untersuchung zur Geschichte und Funktion der Schulfächer (pp. 75-92). [The school subject as a framework for action. Comparative Studies on the history and function of school subjects]). Köln: Böhlau.

Hopmann, S. (2006). Im Durchschnitt PISA oder Alles bleibt schlechter. [PISA on average or all stays worse]. In L. Criblez, P. Gautschi, P. H. Monico, \& H. Messner (Eds.), Lehrpläne und Bildungsstandards. Was SchülerInnen und Schüler lernen sollten (pp. 149-169). [Lehrpläne and Educational Standards. What students should learn]). Bern: h.e.p.-Verlag.

Hopmann, S. T. (2007). Restrained teaching: The common core of Didaktik. European Educational Journal, 6(2), 109-124.

Hopmann, S. T. (2008). No child, no school, no state left behind. Schooling in an age of accountability. Journal of Curriculum Studies, 40(4), 417-456.

Hopmann, S. T. (2013). The end of schooling as we know it? Journal of Curriculum Studies, 45(1), $1-3$.

Hopmann, S. T. (2015). 'Didaktik meets Curriculum' revisited: Historical encounters, systematic experience, empirical limits. NordSTEP, 1(1), 14-21.

Hopmann, S. T., \& Künzli, R. (1992). Didaktik-Renaissance - Zur Einführung [Didaktik Renaissance - Introduction]. Bildung und Erziehung (Cologne), 45(2), 117-135.

Hopmann, S. T., \& Künzli, R. (1998). Entscheidungsfelder der Lehrplanarbeit. Grundzüge einer Theorie der Lehrplanung. [Decision areas of curriculum-making. Main features of a theory of curriculum planning]. In R. Künzli \& S. T. Hopmann (Eds.), Lehrpläne: Wie sie entwickelt werden und was von ihnen erwartet wird (pp. 17-53). [Lehrpläne: How they are developed and what is expected of them]). Zürich: Chur.

Hopmann, S., \& Riquarts, K. (1995). Starting a dialogue: Issues in a beginning conversation between Didaktik and the curriculum traditions. Journal of Curriculum Studies, 27(3), 3-12.

Hopmann, S., \& Riquarts, K. (1999). Das Schulfach als Handlungsrahmen - Traditionen und Perspektiven der Forschung. [The school subject as a framework for action - traditions and research perspectives]. In I. F. Goodson, S. Hopmann, \& K. Riquarts (Eds.), Das Schulfach als Handlungsrahmen. Vergleichende Untersuchung zur Geschichte und Funktion der Schulfächer (pp. 7-28). [The school subject as a framework for action. Comparative Studies on the history and function of school subjects]). Köln: Böhlau. 
Horlacher, R., \& Vincenti, D. (2014). From rationalist autonomy to scientific empiricism. A history of curriculum in Switzerland. In W. F. Pinar (Ed.), International handbook of curriculum research (pp. 476-492). London: Routledge.

Hörmann, B. (2012). "Ja, also, das war nämlich so....”. Erzählungen von SchülerInnen über ihren schulischen Alltag als Mittel zur Evaluierung von Unterricht. ["So, it was like this ...". Narratives of students about their everyday experience in schools as a tool for the evaluation of instruction]. In NOESIS Project team (Ed.), Eine Schule für alle? Zur Evaluation der Niederösterreichischen Mittelschule. [A school for all? On the evaluation of the New Middle School in Lower Austria] (pp. 81-98). Graz: Leykam.

Kilian, M., \& Katschnig, T. (2015). Wohlbefinden als Komponente schulisches Lernens Veränderungen in der Wahrnehmung von ausgewählten Aspekten zum Wohlbefinden niederösterreichischen MittelschülerInnen im Verlauf der Sekundarstufe I unter Berücksichtigung des Schulstandortes. [Well-being as a component of learning in schools - changes in the perception of selected aspects of the well-being of students in the new middle school during secondary I, taking the school site into account.] In NOESIS Project team (Ed.), Gute Schule bleibt verändert. Zur Evaluation der Niederösterreichischen Mittelschule (pp. 121-148). [Good schools stay changed. On the evaluation of the New Middle School in Lower Austria]). Graz: Leykam.

Klafki, W. (1958). Didaktische Analyse als Kern der Unterrichtsvorbereitung [Didactic analysis as the core of lesson preparation]. Die Deutsche Schule, 50, 450-471.

Klafki, W. (1976). Aspekte kritisch-konstruktiver Erziehungswissenschaft: gesammelte Beiträge zur Theorie-Praxis Diskussion. [Aspects of critical-constructive educational science: collected contributions concerning the discussion about the relationship of theory and practice]. Weinheim: Beltz.

Klafki, W. (1995). Didactic analysis as the core of lesson preparation. Journal of Curriculum Studies, 27(1), 13-30.

Kliebard, H. M. (2004). The struggle for the American curriculum, 1893-1958 (3rd ed.). New York/ London: Routledge Farmer.

Klieme, E., Aventarius, H., Blum, W., Döbrich, P., Gruber, H., Prenzel, M., Reiss, K., Riquarts, K., Rost, J., Tenorth, H.-E., Vollmer, H. J. (2003). Zur Entwicklung nationaler Bildungsstandards. Eine Expertise. [The Development of National Educational Standards. An Expertise]. Berlin. https://www.bmbf.de/pub/zur_entwicklung_nationaler_bildungsstandards.pdf. Accessed 10 Nov 2015.

Knapp, M. (2015). “Der Standort macht den Unterschied!" - Schulische Aspirationen an Niederösterreichischen Mittelschulen im Passungsfeld von SchülerInnen-, LehrerInnenaspirationen und den Merkmalen des schulischen Umfeldes. ["School site matters!" - educational Aspirations in Lower Austrian New Middle Schools, the match between student and teacher aspirations and school environment.] In NOESIS Project team (Ed.), Gute Schule bleibt verändert. Zur Evaluation der Niederösterreichischen Mittelschule (pp. 195227). [Good schools stay changed. On the evaluation of the New Middle School in Lower Austria]). Graz: Leykam.

Koretz, D. (2008). Test-based Educational Accountability. Research Evidence and Implications. Zeitschrift für Pädagogik, 54, 777-790.

Künzli, R. (2002). The common frame and the places of Didaktik. In B. B. Gundem \& S. Hopmann (Eds.), Didaktik and/or curriculum. An international dialogue (pp. 29-46). New York: Peter Lang.

Künzli, R. (2006). Vermessene Bildung. TIMSS, WOV \& Consorten zugeeignet. [Measuring "bildung". Dedicated to TIMSS, WOV \& Consorts]. In L. Criblez (Ed.), Lehrpläne und Bildungsstandards : was Schülerinnen und Schüler lernen sollen (pp. 79-82). [What students should learn]). Bern: h.e.p.-Verlag.

Künzli, R. (2009). Curriculum und Lehrmittel. [Curriculum and teaching materials]. In S. Andresen, R. Casale, \& T. Gabriel (Eds.), Handwörterbuch Erziehungswissenschaft (pp. 134-148). [Dictionary of Educational Science]). Weinheim: Beltz. 
Künzli, R., Konstantin, B., Fries, A. V., Ghisla, G., Rosenmund, M., \& Seliner-Müller, G. (1999). Lehrplanarbeit. Über den Nutzen von Lehrplanung für die Schule und ihre Entwicklung. [Curriculum making. On the use of instruction planning for schools and their development]. Zürich: Rüegger.

Künzli, R., Fries, A. V., Hürlimann, W., \& Rosenmund, M. (2013). Der Lehrplan - Programm der Schule. [The "Lehrplan" - programme of the school]. Weinheim: Beltz Juventa.

Labaree, D. F. (2010). Someone has to fail. The zero-sum game of public schooling. Cambridge: Harvard College Press.

Leithwood, K., \& Jantzi, D. (1999). Transformational school leadership effects: A replication. School Effectiveness and School Improvement, 10(4), 451-479.

Leithwood, K., Mascall, B., Strauss, T., Sacks, R., Memon, N., \& Yashkina, A. (2007). Distributing leadership to make schools smarter: Taking the ego out of the system. Leadership and Policy in Schools, 6(1), 37-67.

Linn, R. L. (2003). Accountability: responsibility and reasonable expectations. Educational Researcher, 32(7), 3-13.

Lucyshyn, J. (2006). Implementation von Bildungsstandards in Österreich - Arbeitsbericht. [Implementation of the Educational Standards in Austria - a work report]. Salzburg: Bifie.

Mackenzie, D. E. (1983). Research for school improvement: An appraisal of some recent trends. Educational Researcher, 12(4), 5-17.

Marzano, R. J. (2000). Introduction to the special section implementing standards in schools updating the standards movement. NASSP Bulletin, 84(620), 2-4.

Meyer, J. W., \& Rowan, B. (1977). Institutionalized organizations: Formal structure as myth and ceremony. In W. W. Powell \& P. J. DiMaggio (Eds.), The new institutionalism in organizational analysis (pp. 41-62). Chicago: University Chicago Press.

Moos, L. (2005). How do schools bridge the gap between external demands for accountability and the need for internal trust? Journal of Educational Change, 6(4), 307-328.

Mujis, D., \& Harris, A. (2006). Teacher led school improvement: Teacher leadership in the UK. An International Journal of Research and Studies, 22(8), 961-972.

Nichols, S. L., \& Berliner, D. C. (2007). Collateral damage. How high-stakes testing corrupts America's schools. Cambridge: Harvard Education Press.

Nichols, S. L., Glass, G. V., Berliner, D. C. (2012). High-stakes testing and student achievement: Updated analyses with NAEP data. Education Policy Analysis Archives, 20(20), http://epaa. asu.edu/ojs/article/view/1048. Accessed 5 Sept 2015.

Nussbaum, M. (2011). Creating capabilities: The human development approach. Cambridge: Harvard University Press.

Pereira, P. (1992). Eine Einführung in Joseph J. Schwabs Theorie der curricularer Forschung. [An introduction to Joseph J. Schwab's Theory of Curriculum Research]. Bildung und Erziehung, 45(2), 159-174.

Petrilli, M. J. (2012). The newsroom's view of education reform. Surprise! The press paints a distorted picture. Education Next, 12(3), 77-78.

Polikoff, M. S., Porter, A. C., \& Smithson, J. (2011). How well aligned are state assessments of student achievement with state content standards? American Educational Research Journal, 48(4), 965-995.

Ravitch, D. (2011). The death and life of the great American school system: How testing and choice are undermining education. New York: Basic Books.

Retzl, M., \& Ernst, R. (2012). Schullandschaften: Schulen reformieren und entwickeln durch demokratische Einbindung von Schule, Familie und Gemeinde. [School settings: Reforming and developing schools through the involvement of schools, families and community]. In NOESIS Project team (Ed.), Eine Schule für alle? Zur Evaluation der Niederösterreichischen Mittelschule (pp. 81-98). [A school for all? On the evaluation of the New Middle School in Lower Austria]). Graz: Leykam. 
Robinsohn, S. B. (1971). Bildungsreform als Revision des Curriculums. [Educational Reform as curriculum revision]. Berlin: Neuwied.

Rolff, H.-G. (2009). Schulentwicklung, Schulprogramm und Steuergruppe. [School development, schoolprogram and steering group]. In H. Buchen (Ed.), Professionswissen Schulleitung (2nd ed., pp. 296-364). [The Profession of School Leaders]). Weinheim/Basel: Beltz.

Rustique-Forrester, E. (2005). Accountability and the pressures to exclude: A cautionary tale from England. Education Policy Analysis Archives, 13(26), http://epaa.asu.edu/epaa/v13n26/. Accessed 5 Sept 2015.

Schaefers, C. (2002). Der soziologische Neo-Institutionalismus. Eine organisationstheoretische Analyse- und Forschungsperspektive auf schulische Organisationen. [Sociological neoinstitutionalism. An theoretical analysis and research approach to schools as organizations]. Zeitschrift für Pädagogik, 48(6), 835-855.

Scholl, D. (2012). Are the traditional curricula dispensable? A feature pattern to compare different types of curriculum and a critical view of educational standards and essential curricula in Germany. European Educational Research Journal, 11(3), 328-341.

Schratz, M. (1998). Neue Rollen und Aufgaben für Schulleitung und Schulaufsicht. [New roles and tasks for school-leaders and school administration]. In A. Dobart (Ed.), Schulleitung und Schulaufsicht (pp. 93-116). [School Leadership and School Administration]). Innsbruck: Studien Verlag.

Schratz, M. (2012). Pädagogische Führung als Verantwortung für Bildungsprozesse wahrnehmen. [Appreciate pedagogical leadership as responsibility for educational processes.] In C. Nerowski, T. Hascher, M. Lunkenbein, \& D. Sauer (Eds.), Professionalität im Umgang mit Spannungsfeldern der Pädagogik (pp. 83-95). [Professionalism in dealing with educational tensions]). Bad Heilbrunn: Klinkhardt Verlag.

Schratz, M., Hartmann, M., \& Schley, W. (2010). Schule wirksam leiten: Analyse innovativer Führung in der Praxis. [Leading school effective: Analysis of innovative Leadership in Practice]. Münster: Waxmann.

Schwab, J. J. (1969). The practical: A language for curriculum. School Review, 78(1), 1-23.

Schwab, J. J. (1970). The practical: A language for curriculum. Washington, DC: National Education Association, Center for Study of Instruction.

Spillane, J. P., Halverson, R., \& Diamond, J. B. (2004). Towards a theory of leadership practice: a distributed perspective. Journal of Curriculum Studies, 36(1), 3-34.

Sucharí, B., Wallner-Paschon, C., \& Schreiner, C. (2010). TIMSS 2007. Mathematik \& Naturwissenschaft in der Grundschule. Österreichischer ExpertInnenbericht. [TIMSS 2007. Mathematics \& Science in Primary School]. Graz: Leykam. https://www.bifie.at/buch/1191. Accessed 22 July 2014.

Terhart, E. (2015). Drifting didactics. US-amerikanische Einflüsse auf die deutschsprachige Didaktik 1945-1975. [Drifting didactics. US Influences on German-speaking Didaktik 19451975]. In DGFE (Deutsche Gesellschaft für Erziehungswissenschaft) [German Association for historical Education Research] (Ed.), Jahrbuch historische Bildungsforschung 2014 (Vol. 20, pp. 285-306). [Yearbook of Historical Educational Research]). Bad Heilbrunn: Klinkhardt.

Tröhler, D. (2014). International curriculum research: Why and how? In W. F. Pinar (Ed.), International handbook of curriculum research (2nd ed., pp. 60-67). New York/London: Routledge.

Tyler, R. W. (1949). Basic principles of curriculum and instruction (29th ed.). Chicago: University of Chicago Press.

Vollstädt, W., Tillmann, U. R., Höhmann, K., \& Tebrügge, A. (1999). Lehrpläne im schulischen Alltag: Eine empirische Studie zur Akzeptanz und Wirkung von Lehrplänen in der Sekundarstufe I. ["Lehrpläne" in an everyday school setting: An empirical study on the acceptance and effect of "Lehrpläne" in Secondary I]. Opladen: Leske und Budrich.

Weniger, E. (1932/1952). Schulreform, Kulturkritik und pädagogische Bewegung [Schoolreforms, culture criticism and pedagogical movement]. E. Weniger (eds) Die Eigenständigkeit der 
Erziehung in Theorie und Praxis. Probleme der akademischen Lehrerbildung [The autonomy of education in theory and practice. Problems of academic teacher training] (pp. 59-70). Weinheim: Julius-Beltz Verlagsbuchhandlung.

Westbury, I. (2000). Teaching as a reflective practice: What might Didaktik teach curriculum. In I. Westbury (Ed.), Teaching as a reflective practice: The German didaktik tradition (pp. 15-40). Norwood: Ablex Publication.

Wissinger, J., \& Huber, S. G. (2002). Schulleitung - Forschung und Qualifizierung. [School leadership - research and qualification]. Opladen: Leske und Budrich.

Open Access This chapter is licensed under the terms of the Creative Commons Attribution 4.0 International License (http://creativecommons.org/licenses/by/4.0/), which permits use, sharing, adaptation, distribution and reproduction in any medium or format, as long as you give appropriate credit to the original author(s) and the source, provide a link to the Creative Commons license and indicate if changes were made.

The images or other third party material in this chapter are included in the chapter's Creative Commons license, unless indicated otherwise in a credit line to the material. If material is not included in the chapter's Creative Commons license and your intended use is not permitted by statutory regulation or exceeds the permitted use, you will need to obtain permission directly from the copyright holder. 


\title{
Chapter 7 \\ Curriculum Theory in Contestation? American Curriculum, European Didaktik, and Chinese Wisdom Traditions as Hybrid Platforms for Educational Leadership
}

\author{
Tero Autio
}

\begin{abstract}
In this chapter, I attempt to theorize and historize the current global education reform movement which the Finnish education policy analyst Pasi Sahlberg (Finnish lessons: What can the world learn from educational change in Finland. Teachers College Press, New York, 2011) has coined the GERM (Global Education Reform Movement), the "virus that is killing education." The key drivers of that global education movement adopted in Western countries with very few exceptions render the triad of accountability, standardization and privatization as a marker of the corporatization of educational provision. More specifically, I will analyze the intellectual history of neoliberal ideology, its complicit academic contributions in instrumental curriculum theory and educational psychology with its historical succession of theories from behaviourist psychology to cognitive and learning theories. In this sense, William Doll's (1993) recognition of the Tyler Rationale's (Basic principles of curriculum and instruction. Chicago University Press, Chicago, 1949) intellectual affinity to Descartes's Method as a core of modernization is not incidental. Descartes's curriculum theory overtly co-equalizes between knowledge and ethics, but actually subordinates ethics to instrumental science and knowledge. Descartes' s initiatives led to the modernist stratagem where ethics seeks its refuge in the self-referentiality of logocentric Reason and, by implication, seeks to legitimate the moral supremacy of instrumental mode of rationality in human activities: the good in terms of instrumentality is the moral interior of the logocentric reason; hence there is no proper reason to question the validity and legitimacy of instrumental rationality. Simultaneously and significantly, the logocentric subject provides the hidden place and source of colonialism and exploitation. Finally, this chapter considers non-Western perspectives on curriculum in China.
\end{abstract}

T. Autio $(\bowtie)$

University of Tallinn, Tallinn, Estonia

e-mail: tero.h.autio@gmail.com 


\section{Introduction}

The widely recognized crisis in education that has been nationally and internationally documented in many research findings (e.g. Apple 2006; Hargreaves et al. 2009; Pinar 2013, 2011; Sahlberg 2011; Terhart 2003; Autio 2014, 2006) is arguably a crisis in educational leadership as well. In this chapter I will make an effort to balance the managerial stress on educational leadership genre by incorporating elements from internationally vibrant field of curriculum theory studies drawing on two major schools of thought. Curriculum theory is arguably of great significance to registering intellectual coordinates of education policy adopted. Curriculum theory would also be instrumental in overcoming the genre of education policy writing often epitomized as chronicling without any noticeable account of theoretical and historical affiliations. Against David Berliner's claim, "education reform is the hardest science of all" (in Lather 2010, 93), the lack of elaborated intellectual coordinates of policies seems groundless indeed.

Another dimension ignored more often that not in scholarly reporting of education crises is world political affiliations of those reform oeuvres. The prime example of the intense link between changes in the world political arena and education reforms is the 1957 Sputnik shock in the US with significant detrimental consequences on education policies and curriculum practices. In order to position the current issues of educational leadership beyond managerial rearrangements, we need to provide a broader analysis of a history of the current crisis in education.

First, I make an effort to deploy European, Anglo-American or broadly Anglophone, and Asian/Chinese variants of curriculum theory, their differing intellectual affiliations and their possible implications in the respective education reform and leadership mindset. China has a longest known education history for thousands of years, but I start my brief excursion from Europe from where Modern Education in the sense we know education today was witnessing its birth particularly by Jean Jacques Rousseau: The beginning of modern education - if we follow the dominant historiographies and philosophies of education - can be precisely dated, (...) in the year of 1762, in the year of publication of Rousseau's Emile. ... There is an old and a new, and the line of demarcation is the publication of the educational novel Emile in 1762 (Tröhler 2011, 61-62).

Rousseau's groundbreaking impact precedes the political ideals of the French Revolution 1789: Liberty, Equality, and Solidarity ("Fraternity") as the guiding beacons for the modern nation state, ideals of modern citizenship and modern education. Already Rousseau's early reception - due to many factors not the least because of his polemics seeking traits of character - was quite problematic still ultimately indispensable: in Germany where his reception was most ambivalent, Karl Georg von Raumer, the author of the four-volume Geschichte der Pädagogik (1843-1847), after first demoting Rousseau as a simple critic of France, "'that civilization has gone to rot", in the conclusion he compares Rousseau to the one of the Seven 
Wonders of the World, the lighthouse of Alexandria, lighting the way for the French in politics and for the Germans in education" (In Tröhler 2011, 63-64, my italics).

In retrospect, Raumer's geographical division of Rousseau's reception in French notions of politics and German concepts of education appears illuminative and allegorical in our present context of education crises. The shared and critical core in both receptions is concepts of freedom and liberation in epistemic and psychological terms (liberation from ignorance and coping with "passions") and political liberation from societally produced inequalities. Rousseau emphasized the necessity to perceptively and constantly fight against the internal (passions) and external (societal) obstacles for freedom; he conceived of freedom as a desirable personal, social and civic virtue - and its lack as a vice, an index of weakness of character and subordination to institutions. "Freedom is found in no form of government; it is in the heart of the free man. He takes it with him everywhere. The wile man takes his servitude everywhere" (Rousseau, in Tröhler 2011, 36).

Rousseau's decisive impact on German concepts of education is reflected in the original ideas of Bildung that necessarily remained an unfinished project yet programmatic to this day.

The Bildung tradition is anything else but a coherent and unified school of thought. There are a myriad of internal debates, derivations and variants within the Bildung movement and it has also powerfully affected notions and practices of education outside Europe. I will choose, reconsider and reactivate some topics and issues that would, in my view, provide alternative intellectual resources and inform the ignored dialogue between educational leadership and curriculum theory amidst the worldwide crisis in education.

The first and inalienable still contested principle in Bildung theories with significant implications to all domains of education from education policy to teacher education is freedom. The principle of freedom was characterized in different aspects of modernity; in theological, political, philosophical-scientific, and educational redefinitions of respective realities. Modernism at large means to liberate, to get rid of theological, philosophical and scientific beliefs petrified as stable, unquestionable dogma in geographically distinctive realities postulated, initially and respectively, in ancient Jerusalem and Athens. The engine of modernity was a cognizance of possibility for dynamizing the assumedly stabile reality by introducing new concepts basically based on freedom, newly acclaimed liberties from traditional authorities. In theology, the principle of freedom embodied in an attempt to get free from the papal authority in the sixteenth-century movement for the reform of abuses in the Roman Church ending in the establishment of the Reformed and Protestant Churches. Theological discourses imply significant consequences to modern education, curriculum theories and practices, educational leadership as no exception. In fact, the two major paradigms of Western curriculum theory - Anglo-American Curriculum and North European Bildung - are reducible to secular embodiments of two variants of Protestantism - respectively Calvinism and Lutheranism - in their effort to discarding the papal authority appreciated as corrupt. In tandem with the 
huge impact on the birth of modern worldview by René Descartes (1596-1650), these two Protestant movements render the two distinctively different intellectual profiles for modern Western rationales of education.

The intellectual history of Bildung got a decisive impetus from Immanuel Kant (1724-1804). An anecdote of Kant's externally ordered life in Königsberg (the present Russian city of Kaliningrad) on the south eastern corner of the Baltic Sea anecdotally evidences the influence of Rousseau on the shape of German education. The story creates a stereotypical punctual picture of professor Kant who made his daily walk in the city with precision that inhabitants were able to check their clocks. The only exception from Kant's predictable routines took place - the story goes - when he started to read Emile and forgot the time.

The Rousseaun inspired concept of freedom featured prominently Kant's moral philosophy and reflected in Kant's own lectures on pedagogy (Kant 1991). Kant's moral philosophy had groundbreaking consequences not only in the domain of moral philosophy per se but also on theories of mind, subjectivity and education. The Kantian concept of freedom affected by Rousseau would be the first historically perceivable antecedent between the divide of Bildung and Anglophone psychologized Curriculum. The divide is predicated on methodological and educational implications of Kant's moral philosophy: is the human being capable of autonomous decisions or exclusively determined by natural forces?

Kant argued that conformity to the Categorical Imperative, the CI (a noninstrumental principle) and hence to moral requirements themselves, can nevertheless be shown to be essential to rational agency. This argument was based on his striking doctrine that a rational will must be regarded as autonomous, or free in the sense of being the author of the law that binds it. The fundamental principle of morality - the CI - is none other than the law of an autonomous will. Thus, at the heart of Kant's moral philosophy is a conception of reason whose reach in practical affairs goes well beyond that of a Humean 'slave' to the passions. Moreover, it is the presence of this self-governing reason in each person that Kant thought offered decisive grounds for viewing each as possessed of equal worth and deserving of equal respect. (http://plato.stanford.edu/entries/kant-moral/ Kant 1984).

Kant's perception of free will as a preconditon of morality is deeply predicated on the North European and particularly Scandinavian notions of education and curriculum: for instance, to position the teacher, ideally, as an autonomous and free professional as the center of an education system whose main mission is to advance the holistic development of her/his students: "scratch a good teacher and you will always find a moral purpose." The Moral in this broad sense is often misinterpreted and atrophied as moralistic but its historical core meaning is related to the holistic understanding of human condition where an individual with her/his developing capacity of personal judgment - as free moral agent - orchestrates the acquired content of education comprised in the curriculum (Autio 2014). More closely, in the Bildung concept, cognitive, aesthetic, and practical dimensions of curriculum are 
instrumental ones and what makes education educative is just moral judgment about the worthwhileness, value and relevance of studied and learned material. In Bildung inspired curriculum theories, cognitive, aesthetic and practical dimensions are related to instrumental rationality that focus on the pragmatic usability of acquired knowledge, skills and attitudes whereas the moral dimension of the Bildung curriculum represents the reflective modus of rationality that gives subjective meaning to the "content", the studied and learned in the sense what Max Weber called value rationality (Vernunft; Wertrationalität) beyond its direct pragmatic usability. Finally, the moral dimension of curriculum in the classical Bildung sense asks about conditions for possibilities and limits of instrumental rationality for meaningful, sustainable human existence (Klafki 1991, 31).

The disconnection of morality from intellectual agendas of education could be argued to have theoretically advocated the current crisis in education, curriculum and leadership. Without adopting a view on curriculum as "a complicated conversation" (Pinar) where contestation over goals, purposes and meanings necessarily are part and parcel of democratic and educational conversation, we suffice to witness the current simulation of education and educational leadership around managerialist "best practices", testing industry and test scores as goals and explicit business of education and education policy.

The eclipse of the comprehensive Vernunft rationality - embodied as a shortage of complicated conversation transcending means and methods - on the agenda of education, curriculum and educational leadership is an index of a larger historical phenomenon. Max Weber (1864-1920), in his classical but fragmentary studies on Western or "Occidental" rationalism (Weber 1978), made efforts to explain the peculiarly rationalized nature of "our European-American social and economic life," that is manifest specifically in the establishment of the capitalist economy and the modern state. Weber's treatment of the development of rationalization is pertinent and illuminative from educational viewpoint: how moral concerns give way to instrumental modes of rationality in curriculum theory and educational leadership. For Weber, rational action functions as two-way, reciprocal dynamics between single individuals and societal institutions; first, rational action by transcending individual interests advocates motivational anchoring of the individual in societal institutions and, second, posttraditional moral or psychological remakings of the self emerge as institutional embodiments. Instrumental rationality is deeply embedded in modern institutions; moral concerns are amenable to get reified as legal or other formal and regulative principles. An example would be the model of the Scandinavian welfare society where morality is instrumentally embedded in the mediating structures between self and society for the assumed and legally rationalized common good. The political ingenuity may not be related directly to solidarity - as it is often interpreted - but to sublimated and rationalized egoism, to moral and psychological remaking of the self by the state as a better deal to "me" (and indirectly to others as well) what would be the case without the mutual, instrumental social contract between egoistic "me" and society. 
Instrumental rationality stands for Weber as the ideal type of Western rationalism, as a yardstick against which other orientations of social action could be ordered and against which they could be assessed (Autio 2006, 114). "Ideal types" always guide education and curriculum thought. Western modernization is succinctly interpretable in terms of tight interrelatedness between instrumental rationality and knowledge subordinated to instrumental interests:

When we use the expression "rational" we suppose that there is a close relation rationality and knowledge. ... for rationality has less to do with the possession of knowledge than with how speaking and acting subjects acquire and use knowledge. (Habermas 1984, in Autio 2006, 114)

The methodical and pragmatic stress is characteristic for instrumental rationality, where knowledge is assessed by its assumed capacity for instrumental mastery of reality. Habermas (1984, 10; Autio 2006, 114) introduces the concept of cognitive-instrumental rationality that has, "through empiricism, deeply marked the self-understanding of the modern era". Instrumental rationality specified by Habermas leans on two basic premises. The first is the notion of truth conceived in empiricistic terms, and the second is the notion of effectiveness. This set of premises with its overall instrumental rationale render a major theme on educational agendas with minor still prominent variations (Dewey!) in the theme in the US since the turn of the twentieth century and in Europe more gradually since the end of WWII. The "icon" of modernist curriculum, the Tyler Rationale (Tyler 1949), would embody and deploy in an exemplary way the grand Western symbolic curriculum:

Empirical ("evidence-based"!) "truths" and pragmatic "effectiveness" stripped out of all metaphysical or moral considerations would form a kind of circular reasoning in curriculum planning, where educational goals are constantly revised in the light of "scientific findings" and "needs" of society, which, in turn, are to be tested against their effective applicability indicated as preferred behavior changes in students (Autio 2006, 114).

In such forms of knowledge, the answer to the basic curriculum question "what knowledge is of most worth" is obvious: instrumental knowledge - that still remains deeply problematic from sustainable education point of view:

Paradoxically, the greater the level of factual knowledge of the world the further the retreat of the possibility of discovering its meaning. Action based on scientistic knowledge tends to be instrumental, focusing on short-run calculators of self-interest rather than long-term commitment. (Crook et al. 1992)

There are countries that still are opposing the scientism of truth-effectiveness in education and its most recent, openly politicized form, neoliberalism, most notably Finland. More generally, while acknowledging some serious biases in the Bildung tradition (its gender-structured nature, elitism, idealistic aestheticism and apolitical propensity), some other vital elements of Bildung could still deserve resurrection to provide credible alternatives to narrow and biased scientist-empiricist concept of 
knowledge and curriculum. There are attempts to rephrase and reactivate comprehensive education discourse exemplified in international "complicated conversation" in US curriculum studies and Chinese education and curriculum reforms, but the international big picture of curriculum and educational leadership is the embodiment of presentist excesses of instrumentalism devoid of democratic dialogue and historical-theoretical reconsiderations.

\section{A Brief Intellectual History of Present Transnational Education and Curriculum Policy and Leadership Crisis}

I will engage closer with internal manifestations of instrumental rationality in education and their historical-theoretical conditionings. I will restrict my focus on some sets of those conditionings that would arguably play a complicit role in our present education and its leadership crisis. I will follow the European Protestant theological discourses and some Kantian-Herbartian educational ideas, their transatlantic travelling and their reception in the rapidly industrializing United States around the turn of twentieth century. That primary stage between the cross-continental academic studies of education, theological discourses and the economy would provide an allegory for the coming times in the twentieth and twenty-first century in Western education. I will start at the end by the characterization of the current crisis and then make efforts to make them more comprehensible by an appeal to those earlier intellectual developments.

As a beginning, I would provide a short diagnosis of the educational (policy) crisis of our times that has been named differently: in Andy Hargreaves et al. (2009) Bigger, Harder, Tighter, Flatter strategy, in William Pinar's (2006, 2011) several critiques of "The End of Public Education in The United States" to expose his worries about the deliberate destruction of public education. In Pinar's view

the end of education in America was indicated by the plundering of public budgets by private companies. And with the privatization of schooling teachers have devolved into bureaucrats, checking students' completion of online assignments. In universities, economists have replaced education professors as the experts in federally funded educational research. (http://www.ced.zju.edu.cn/english/redir.php?catalog_id=39270\&object_id=69658)

Diane Ravitch who worked for the President George W. Bush and initially introduced the triad accountability, standardization and privatization as the guidelines of neoliberal education and curriculum policy reforms changed completely her mind after the recognition of the detrimental effects of the Bush Regime's No Child Left Behind and President Obama's reform initiative Through Race to the Top (Ravitch 2010). The Finnish education policy analyst Pasi Sahlberg characterizes the current education and curriculum policy mainstream as the Global Education Reform Movement, "the GERM that is killing education" (Sahlberg 2011) and strictly contrasts it with the Finnish education reform strategy that will take an even further step 
away in the new 2016 Finnish National Curriculum Reform from the Anglophone driven accountability, standardization and privatization reforms.

\section{The Sputnik Shock and the "Educationalization of the Cold War" as a Precursor for the Current Neoliberal Education Policy}

The longstanding narrow Culture of Method (Autio 2014, 2006) in both education research and practical teacher education programs has often, paradoxically for methodological reasons (if we think of method as a way to comprehensive and truthful evidence), prevented from seeing education as affected by larger complex of political, historical, cultural and theoretical issues. The Cold War culminated in the Sputnik Shock 1957 when Russians seemed to win the first match in space race by sending the kerosene-driven Sputnik rocket on the earth-circulating orbit. The political consequences resulted in fundamental change in reform mindset in the U.S. education: “... the enemy was not only the Russians but also the progressive educational ideology that was dominant in the United States at that time, supported by philosophers of education and the powerful teachers' unions" (Tröhler 2013, 200, my emphasis). The establishment of the OECD in the aftermath of the Sputnik Crisis institutionalized the both efforts to "reform" (deform?) the then progressive U.S. education and combat the Soviet Union's assumed technological and educational superiority by the educationalization of the Cold War. Symptomatic of the educational paradigm that followed was the first founding meeting of the OECD that was occupied by the representatives of the military and economy with no education expert keynotes (Tröhler 2011, 205). The defensive political and economic agenda dictated new, radically narrowed guidelines for education and curriculum: mathematics, sciences, and foreign languages as the "core curriculum", almost identical to the PISA trilogy of today.

The founding event was a turning point when education policy and particularly assessment and evaluation as a natural part of pedagogic process and teachers' work are removed to external, quasi-authoritative sources of testing industry advocated and designed by educational psychologists. In academic terms, the Sputnik Shock prompted the shift from educational philosophy to psychology as an intellectual core of the curriculum and teacher education programs. The final impetus for assessment and testing as a core of education policy and educational leadership came some years later, in 1966, from the massive survey, "second largest social science survey in history", lead by the University of Chicago sociologist James S. Coleman: Equality of Educational Opportunity Study. What was striking and what made it "the most dangerous report in American education" (Moynihan, in Pinar 2006, 123) is that "After Coleman, ..., equal opportunity was to be measured by 'outputs,' among these (in Coleman's study) the test scores of 570,000 children. Only if students from differing groups (social background, race, color, religion, and national 
origin, my add.) scored roughly the same scores, Coleman insisted, could we conclude there was equal educational opportunity" (Pinar 2006, 124).

Central to those powerful standardizing efforts is the role of educational psychology, which meant a shift from pragmatic philosophy to schematic, radically simplified notions of human learning by behaviorism and cognitive theory:

The educationalization of the Cold War in the United States marked a transformation of the dominant reference discipline for education, for it switched from philosophy to psychology, more precisely from popular interpretation of Pragmatism to cognitive psychology, which was at its outset in the late 1950s - cognitive theory being the most important academic reference of PISA today, as the stakeholders admit themselves. (Tröhler 2013. 201)

The switch from philosophy to psychology also meant - paradoxically - the disappearance of the subject on the agenda of education for the abstract, reified and universal notions of "learning". The whole historical array of (educational) psychologies from behaviorism to cognitive theories to "Learning Sciences" is fundamentally a-psychological by nature without any substantive reference to human psyche as a distinctive, complex entity sui generis. Initially for behaviorism, consciousness was too complicated and messy phenomenon to be directly graspable and the study of consciousness was replaced by the observation of outer behavior with the methods already employed in natural sciences in accordance with the politics of positivist agendas: to see in order to control and predict.

The trend to a priori schematize human consciousness is closely related to the instrumentalism of modernization with the influential Cartesian emphasis on Method in the creation of new knowledge and the rise of natural sciences in the seventeenth century. One of the most pivotal figures in the history of education, Johann Friedrich Herbart (1776-1841), had ambivalent and contrasting alternatives for the notions of psychology (Blass 1978) with far-reaching implications for both European and Anglo-American developments of education and curriculum theory. Herbart, as the follower of Immanuel Kant (1724-1804) in Königsberg (today's Russian Kaliningrad), made efforts to combine freedom - necessary condition for Kant to design the moral sphere, free "judgmental reason" as the core of human rationality - with causal necessity. For Herbart, the intellectual "mission impossible" was to unite the moral end of education, the idea of inner freedom manifested ideally as knowledgeable and enlightened moral character, informed but not exclusively determined by external powers and authorities, with deterministic and exact ideals of scientific psychology developed in accordance with the methodology of the natural sciences (Autio 2006, 105).

The unbridgeable split remained to respectively live in two radically differing intellectual alternatives for Western curriculum theory: North European Bildung/ Didaktik and Anglo-American Curriculum. The European concept of curriculum initiated by Humboldt suggested that the cognitive, practical and aesthetic dimensions of curriculum are to be subordinated to the fourth dimension, the moral, (Klafki 1991), the decisive instance of human rationality and freedom (Vernunft) that would guarantee the educative and transformative nature of education beyond proceduralism. The practical and democratic implication would be that teachers and 
students alike are called to use their free judgmental faculties to richen the educative experience of all participants by subjectively scrutinizing the meaning(fullness) of the learned content and its context; in Pinar's current words: "curriculum as a complicated conversation" where "subjectivity is threaded through the curriculum" (Pinar 2013). Already Kant himself (Autio 2006, 102) warned in his pedagogic lectures - as if anticipating the present colonization and standardization of reason and educational experience by psychological, administrative and commercial instrumentalism:

Intelligence divorced from judgment produces nothing but foolishness. Understanding is the knowledge of the general. Judgment is the application of general to the particular. Reason is the power of understanding the connection between the general and the particular.

The moral, that is: the reflective, free faculty of human mind with its contextualized focus on "the primacy of the particular" (Pinar) is in strict contrast with the behaviorist tenet of inductive, non-subjective generalization of abstract "learning". Actually, this kind interpretation of the moral is one of the divisive intellectual factors between Bildung/Didaktik and the present of the neoliberal Anglo-American Curriculum and its global extension. The intellectual breakthrough in the US curriculum theory by Pinar and his colleagues (1995), the Reconceptualization, radically rephrased and opened new theoretical perspectives for curriculum thinking beyond procedural and abstract educational psychology. Simultaneously, like just the name of Pinar's et al. book, Understanding Curriculum, reveals, the Reconceptualization was intellectually affiliated with the Continental hermeneutic school of (educational) thought tentatively instigated by the German Movement around 1770-1830 but articulated more distinctively in the hermeneutic ("geisteswissenschaftliche") works of Friedrich Schleiermacher (1768-1834) and Wilhelm Dilthey (1833-1911). Dilthey's argument centered around the idea that in the natural sciences we seek to explain phenomena in terms of cause and effect; contrastively, in the human and social sciences, we seek to understand meanings in terms of relations between parts and a whole; "Die Natur erklären wir, das Seelenleben verstehen wir." (Dilthey 1894). In retrospect, the American Reconceptualization meant an advancement of hermeneutic understanding beyond the European tradition of the humanistic and nationalistic bound notion of the unitary subject with its postmodern fragmentation and explicit introduction of the interplay between language, power and knowledge to curriculum theory.

\section{Travelling Curriculum Discourses: From Herbart to American Psychologized Curriculum}

This intellectual division between hermeneutic Understanding and causal Explanation is already present in Herbart's blueprint for (inherently contradictory?) unified curriculum theory to combine the necessarily free will of the moral agent 
with the causative determination of behavior of the human creature not qualitatively different from other creatures or natural phenomena. Herbart was ambivalent - for good reasons - about the intellectual validity of his contradictory, pre-positivist suggestion and he proposed alternative, hermeneutic model for education and curriculum research that is recognizable today, for instance, in Kelly (2009) and Pinar et al. (1995). Herbart's second, hermeneutic model suggests the relative autonomy of curriculum studies apart from borrowing foreign concepts from other fields: anthropology, philosophy, and psychology. In Herbart's view, educational and curriculum concepts are educational more distinctively, einheimische Begriffe sui generis where education and curriculum should be conceived more autonomously in terms of how we define educative experience which arise from its own practice and its intellectual tradition rather than as conceptual borrowings from alienating and external realms of knowledge (Herbart 1804/1986, in Autio 2006, 105).

Yet, finally - likely due to the scholarly fashions in his day - Herbart's own intellectual ambition was to develop a universal model of a causal "mechanics of mind" in the spirit of deterministic Explanation - eine Mechanik des Geistes - and go down in history as "the Newton of Psychology" - als Newton der Psychologie in die Geschichte einzugehen (Autio 2006, 107).

Later in the Unites States, the short period of American Herbartianism around 1890-1900 was a decisive transition period to organize the assumedly chaotic and confused inner world of the child by organizing "the contents of knowledge in "well-organized"" textbooks and ensure that they are "stored in the mind in well arranged form". The standardization of learning and the child's psyche in the American Herbartianism still took place by reference to inner psychic life that behaviorism was coming to change for the favor of outer behavior. In the 1890s American Herbartianism, "the essence of the position was to produce an identity of outlook among the mass of population; the image of the industrial system demanding uniformity and interchangeability is dominant. The morality and character being sought was a conformity of wills and predictability of behavior; there was no intention of accepting individuality or personal autonomy (Bowen, in Autio 2006, 106 , my emphasis). The intellectual bridge for significant change from inner psychic life to outer behavior between Herbartianism and behaviorism was methodological and conceptual standardization of subjectivity in terms of "learning" as behavior that justified the discard of the assumedly redundant and messy conformity of wills for the assumedly law-like prediction of behavior.

Further, the political pressure by the industrial system for standardization in the late nineteenth century was instrumental to methodologically simplify the theory with displacement of any moral, metaphysical, or existential elements in education theory and remove the Kantian free will of the moral agent, the moral dimension, from the center of the curriculum and education.

As a consequence, there appears a split in the US between "a conformity of wills and predictability of behavior": the "conformity of wills" element remained out of the intellectual and methodological reach of behaviorist agendas. Behaviorism with its intent on the external determination and inductive generalizations of a behavior of an individual predicated on the agenda of positivism creates the powerful norma- 
tivity by the standardization of the subject; individuality and personal autonomy is to conceived not in their genuine idiosyncrasies but in universal, abstract, and collective terms of "learning".

\section{Neoliberal Cause as the Rule of Education}

These early historical, theoretical, and political incentives related particularly to instrumentalism in theoretical terms and industrialization and the economy in practical terms have arguably contributed to the shape of our present crisis of education where we can sense that something went wrong in the turn of the twentieth century in the intellectual design of education when moral and political aspects as goals of education was reduced to psychologized instrumentalism. The children's and peoples' capacity and talents are wasted, neglected or underused particularly by the external assessment obsessions of present education systems what the powerful national and transnational agencies EU, OECD, and the USA advocate. The excessively utilitarian thinking - "economic thought is coterminous with rationality" (Couldry 2011, 28) - sweepingly colonizes with intellectual and moral atrophy historical reminders, present circumstances and future imaginaries of education. Internally, the long tradition of the de-intellectualization of education render it complicit in the neoliberal reduction of the French Enlightenment rationality of liberty, equality and solidarity and its German Bildung equivalent moral, cognitive, aesthetic and practical dimensions of curriculum to instrumentalism of economic thought.

The adoption of the obsolete positivist image of science based on external observation and the ideal of exact measurement in social and education studies can further defy the complexity of education processes by preferring methodological reasons in the definition of educational reality. Neoliberalism policies purposefully but misguidedly advocate the ahistorical, a-theoretical, abstract system-driven and a kind of laboratory images of education research that still reflect the modernist, fundamentalist "quest for certainty"-posture in the numerically forced "evidencebased" interpretations of educational reality.

Paradoxically enough, the period of tumultuous change and instability of financial and economic systems since 2000 that reached the pinnacle in the 2007 financial crisis still going on has not prevented the forces of corporatization from losing their hold on social infrastructure (Goodson 2014, 14). Indeed, "economic thought is coterminous with rationality" (Couldry 2011) and in that all-eggs-in-the-samebasket spirit educational leadership promotes school-as-a-business model and respective business-like "profit projections" of standardized test score results through neoliberal education reforms. Despite the recognized failure of the new economy to create a sustainable new world order, transnational education and curriculum policy hold on the imitation of corporate logic as the educational rule: the "bottom line" in business is structurally and ideologically in congruence with the tested "learning outcomes" in education (Autio 2016, 113). 
These developments lead to the pervasive sense of inversion at many levels, for instance, the move from market economy to becoming market society - "everything is now saleable and available a site of profit making" (Sandel in Goodson 2014, 14). From curriculum and educational leadership perspective, if we are still able to think of education as a prime site for and of democracy, "the inversion of democracy" by neoliberalism would alarmingly mean the repudiation of "a system that was once set up to represent the people against vested power now seems to represent vested power (especially corporate power) against the people. Education policy and leadership can function like a tacit vehicle for these undemocratic ideals to creep into the socialization of future generations in advanced societies as, for instance, the US Through Race to the Top policy program would manifest by the absence of any explicit reference to democracy, education and personality ideals in any broader or holistic sense - except for competitiveness in the economy. The sense of national belonging is still there but subordinated to the assumedly more significant ideals of the market (Autio 2016, 113).

Indeed, "Neoliberalism has become a 'theory of everything' providing a pervasive account of self and identity, knowledge and information, economy and government" (Mirowski in Goodson 2014, 14). In terms of society and governance, "we would seem to be entering a period of 'corporate rule', where all criteria fit the prevailing neo-liberal dogma and where ... even alternative imaginary possibilities are clinically and forcefully expunged (Goodson 2014, 114).

In order to seek historical and theoretical composition and possible alternatives for neoliberalism as the hegemonic ideology of current educational leadership, we cannot evade the impact of Protestantism, especially Calvinism, on present educational landscape. In the context of neoliberalism, reactivation of Calvinist intellectual-theological heritage may illuminate the present in the long historical intertwinement prophetically recorded for first time in Max Weber's The Protestant Ethic and the Spirit of Capitalism (1930/1995). Within Protestantism, Calvinism and Lutheranism radically depart from each other in terms of their respective views on the interrelatedness between individuality and social organization. Both theologies stipulate individual responsibility for God as kernel of human existence with much less influence of the mediating instances of the Holy Scriptures - churchly authorities - that render the core of their "Protest" against Catholic belief and papal institutions. Lutheranism encourages for selfimprovement by advocating literacy, the translation of the Bible and other religious texts to mother tongue that make them available to personal study-motivated by enticing incentives like in the nineteenth and early twentieth century Finland as literacy was a precondition for marriage license. This episodic view on Lutheranism allegorically characterizes the relatively "free", educational nature of Scandinavian interpretation of Lutheranism and how education was connected to the building of the modern state by educationally conditioning the family formation as a basic cell of society. Yet, the mediations between self and society stamped by Luther's doctrine of Two Regiments that subordinate the Earthly to the Heavenly and particularly the Doctrine of Grace give to worldly social organization a kind of positive laissez-faire, less binding but also much more tradi- 
tional and conservative character than strongly interfering measures in Puritan Calvinism to arrange the relationship between the individual and social world (Weber 1995/1930; Autio 2006).

While Lutheranism's attitude toward the world remained to a certain degree indifferent without strictly bounding principles, Calvinism introduced a powerful curriculum to methodize the life of its supporters by interpreting devoted paid work as a sign of religious virtue. "Lutheranism, on account of its doctrine of Grace, lacked a psychological sanction of systematic conduct to compel the methodological rationalization of life. ... The Lutheran faith thus left the spontaneous vitality of impulsive action and naïve emotion more nearly unchanged. The motive to constant self-control and thus to a deliberate regulation of one's own life, which the gloomy doctrine of Calvinism gave, was lacking. ... The simple, sensitive, and peculiarly emotional form of piety, which is the ornament of many of the highest types of Lutherans, finds few parallels in genuine Puritanism (Weber 1995/1930 in Autio 2006, 67).

Calvinism's revolutionary secular impact on the new social order is psychologically based on its diabolically ingenious doctrine of Predestination. The constant uncertainty as to whether one is among the elect (due to the limited but secret number of the elect) creates the basic existential anxiety and the only way of alleviation is to exhibit one's spiritual worthwhileness through work. In the Calvinist view, calling is not a fate, but God's commandment to the individual to work for the divine glory ... with far reaching psychological consequences and, socially, work "became connected with a further development of the providential interpretation of the economic order which had begun in scholasticism (Weber, p. 160). The time horizon and the prime motive of Calvinism was in the future because only the fruits of labor were to reveal to mankind the providential purpose regarding the order of the world. The Calvinist-Puritan stress on the outcomes or the "fruits" of labor constantly challenged the present skills of its practitioners. Thus the human mind and human skills were in constant need of improvement, namely learning and education, in order to work better and better for the glory of God (Autio 2006, 66).

Theological scaffolding provided an early motivation for modern education and, paradoxically, its further, fully-fledged secularization and, most significantly, for the idea and concept of progress. These developments related to secularization and the pre-pragmatic notions by Francis Bacon (1561-1626): "Truth and Utility are ... the very same things" (Autio 2006, 20) can be read as a historical-theoretical prelude to our full-blown instrumentalist, neoliberal concepts of education, curriculum and leadership where the concept of "truth" as current anachronism is absorbed and removed by the guiding principle of education policies of today: high outputs at the lowest possible costs.

Initial theological incentives in educational thinking are buried in the tradition of Anglophone, especially in the Anglo-American Curriculum. The historical forging of American education has not of course any single origin, it is a long-term, com- 
plex process whose principles are assembled and connected to a myriad of different patterns that include religious, political, philosophical, social, and cultural discourses.

From the current perspective, the Bildung tradition is hardly present in any of present national educational and curriculum discourses, policies and practices. The only exception still may be Finland when the rest of the Scandinavian front (Denmark, Norway, Sweden) is fragmented to more or less neoliberal blocks with Anglophone accountability, standardization and privatization policy and leadership drivers. Finland's case is interesting in terms of the divide between psychologized Curriculum and Bildung that has not captured much attention in the PISA reception but what is highly significant in the current context of educational leadership. In a matter of fact, we can claim that the good Finnish results in PISA paradoxically and at least temporarily saved Finland from the Anglophone and OECD driven GERM, "virus that is killing education" (Sahlberg 2011). The success in the first PISA round in 2001 was a huge surprise in Finland; it was an unintended consequence and side product of the broad-based, holistic national curriculum, teachers' professional freedom and the democratic Finnish concept of comprehensive school (peruskoulu). The peruskoulu was constantly attacked since its creation from 1970s by the political right and the leaders of business sector but this critique was silenced overnight when the first PISA results were issued in 2001 (Saari et al. 2014). Without PISA surprise, Finland would most probably be engaged in transnational neoliberal policy drivers accountability, standardization and privatization. Characteristic to the Finnish peruskoulu is the academically qualified teachers, (master level requirement at all levels), implied in professional autonomy, freedom and high trust in teachers, the absence of external assessments and tests (practically all tests are teacherdriven), the not-stigmatizing support in cases of social and educational challenges, etc. Teaching is related to holistic education, in strict contrast with countries where teaching basically means teaching to the externally mandated tests. The decisive element in Finnish comprehensive school ideology is the interpretation of quality as equality; quality as equal educational opportunity regardless of social, economic or ethnic background. Again, that Finnish policy principle is in strict contrast with the Coleman report (1966, in Pinar 2006, 123-124) and its neoliberal offspring the NCLB and TRTT policy programs where educational (e)quality is linked to test scores by quasi-causal psychological argumentation used to advocate the intellectually dishonest and simplified conception of teaching as a "cause" and learning as an "effect". "Only if students from differing groups scored roughly the same scores, Coleman insisted, could we conclude there was equal educational opportunity" (Pinar 2006, 124).

Comparable to Sputnik shock in the 1950s USA, Germany experienced a PISA shock in 2001 that created a debate about the conflation between the concept of knowledge and competences, skills and performances. Apart from the shock of the PISA results as such, in the aftermath the debate in Germany created a conceptual shock that is very significant from the perspective of curriculum theory and educational leadership. To the proponents of traditional Bildung concept a shocking move 
by some German PISA experts was to suggest the concept of competence as a new Bildung concept: "Kompetenz - ein neuer Bildungsbegriff".

It is important to note, ..., that the merging of competencies and Bildung is not solely an act by historically blind empiricists ... Heinz-Elmar Tenorth, a genuine historian of education, did the very same thing: "Bildung and literacy, basic skills and modes of handling higher culture do not depict disjunctive classes of knowledge and behavioral patterns but specific developments of a single and identical dimension of human practice." (Tröhler 2011, 196)

Here, in an unexpected context of Bildung, we witness a single instance of neoliberalism as "a theory of everything", a discourse on education, knowledge and subjectivity, all conflated together as an image of the human subject reduced to a sheer aggregate of competences.

The example is a generalizable index of the current state of Bildung in its homeland Germany where it is increasingly colonized in the aftermath of the PISA shock by the instrumentalism of Anglo-American psychologized Curriculum.

PISA has led to the growing importance of principles such as outcome control, competence orientation and external assessment. The post-PISA academic discourse in Germany can be characterised by the re-orientation of educational studies towards a greater emphasis on the empirical research of pedagogic practice (empirische Unterrichtsforschung). (Ertl 2006, 619)

The reaction in Germany to PISA to get intellectually allied with transnational, narrow and detrimental psychologized agendas is just the opposite what is the case in Finland. PISA, paradoxically, has increased educational self-esteem to maintain and develop education, curriculum and leadership policies that preserve curriculum breadth and depth, academic teacher education with guided practice, teachers' untouchable professional autonomy and freedom and students' increasing involvement in school decisions that would affect them. In the 2016 new national curriculum reform students are invited from first grade on to actively participate with teachers in the assessment not only of learning and study process but also students' overall judgment over the quality of life at school. Despite the transnational terminological pressures to replace knowledge and education in favor of neoliberal and psychologized vocabulary of competencies, skills and performances, the atmosphere in Finnish comprehensive school still seems to adhering to the Deweyan conception of the school as a specific institution, confirming its status as a prime site of democracy: education is of and for democracy, strictly contrasting with antidemocratic, neoliberal drivers of external accountability, standardization and accountability based on rudimentary "evidence-based" scientism. 


\title{
Toward Genuine Theorization and Reactivation of the Past in Education: The Educational Landscape of Internationalization Between West and East (China) as a Reaction to Neoliberal Globalization
}

The German post-PISA case with all conceptual confusions like there were attempt to marry fire and water is to be conceived as an index of lack of theorization and lost sense of intellectual history of education and curriculum. Particularly in the Anglophone empiricist world, there is perceivable a long empiricist and pragmatist tradition where method is replacing comprehensive theorization in research and teacher education curricula. Not directly related to the aftermath of PISA shock, Ewald Terhart $(2003,25-26)$ registers the instigation of education paradigm shift in Germany toward ahistorical and a-theoretical Anglophone empiricism and psychologism:

\begin{abstract}
In Germany, it has become quiet around general didactics. The controversies of the late 1960s and early 1970s have died down; the theoretical situation has been basically stable for decades. ... this is surprising because one might perhaps expect, given the widespread talk about the crisis in instruction, in school, and the teaching profession, that the wheat of didactics would bloom on a theoretical level. Just the opposite is the case! In general didactics, there has been no theoretical discussion worth speaking of for around 2 decades ... genuine theoretical discussion has been largely replaced by the development and defense of certain teaching methods on a more practical level.
\end{abstract}

Increased awareness of the excessive instrumentalism and its detrimental effects on education in the United States that would urge genuine theoretical and historical reconsideration was embodying already in the 1970s in the scholarship of William Pinar. Reconsideration that was essentially drawing on critique of educational psychology, the Tyler Rationale as its icon, lead to re-conceptualizing of the ahistorical, psychologized concept of curriculum by behaviorism and cognitivism. The Reconceptualization Movement, "as an intellectual breakthrough" in the American context, materialized as a monumental magnum opus of American curriculum theory and history Understanding Curriculum coauthored by William Pinar et al. (1995), is sharing some intellectual affinities with German and north European Bildung but also critical reappraising and 'post-modernizing' some of Bildung tenets.

In terms of theory of science, the positivism of educational psychology as a vehicle of the psychologized Curriculum identify itself with the ideals of causal explanation, in turn, Bildung and the Reconceptualization share the common affiliation with hermeneutic concept of science with respective ideals of understanding of meanings, intentions and the interplay between the whole and the parts. Hermeneutics is a reaction to the methodological monism of positivism (Wright von 1971) and this reaction is specified respectively, in modernist terms, in Bildung and, in postmodernist sense, in Reconceptualization theories and concept. The American Reconceptualization meant a decisive advancement of hermeneutic understanding beyond the European tradition of the humanistic and nationalistic bound notion of 
the unitary subject with its postmodern fragmentation and explicit introduction of a interplay between language, power and knowledge to curriculum theory.

The hermeneutic critique directed to both Anglo-American abstract, instrumental, method-driven psychology and Bildung concepts of the unitary humanistic, nationally bound subject has transformed the educationally vital discourse on the subject from male dominated WASP discourse exemplified in the Tyler Rationale, the English gentleman ideal of education and the elitist, erudite, nationalistic male individual of the Bildung ideal toward gender-, culture- and internationally sensitive discourses on subjectivity. These intellectual shifts have radically transformed the landscape of education and curriculum through more nuanced dynamics of the subject facilitated by richer palettes of research methodology than is the case of routinized, unimaginative empiricism of surveys in current educational policies and leadership. For instance, the introduction of (auto)biographical research methods is essentially increasing knowledge and understanding what it is be a human being, teacher and student in the present world in more authentic, practical, and comprehensive ways than the mechanistic politics of behaviorist and cognitivist psychologies have provided or could in principle provide within their limited epistemic and methodological boundaries.

The interest to take individuality more comprehensively than traditional educational psychology by employing new methodologies implied in the intellectual legacies of Bildung and Reconceptualization has vital political implications. By scrutinizing internal and external circumstances of an individual, current curriculum theory/studies would reconsider and challenge the preconditions of democracy by amplifying and articulating more explicitly individual potentialities through narrative and biographical approaches. The urge to rethink individuality, subjectivity, agency, or self would denote a theoretical articulation of the current situation where individualization - biographical differences - has become "a structural characteristics of highly differentiated societies" (Beck and Beck-Gernsheim 2002, xxi; Autio 2006, 160).

The intensified individualism is tied to globalization, both of them marking the constitutive features of postmodernity, or in Ulrich Beck's terms, 'the second modernity'. Globalization has by the outsourcing of the functions of "the first modernity" effected a radical shift in the relationships between individuals and institutions (Autio 2006, 160). The neoliberal measures of external accountability, standardization and privatization are educational symptoms of globalization to which curriculum theory is reacting by inter-nationalization to effect the sense of historicity, locality, nuance, and fragmentation countering uniform standards of transnational reform and leadership mindset.

As Ulrich Beck and Elizabeth Beck-Gernsheim (Autio 2006, 161) point out, the move toward complexity has meant "a de-normalization of roles"; "the roles of the first modernity depended very much on what Kant called determinate judgment; on prescription, on determinate rules". 
Now, the individual must be much more the rule finder her/himself. Determinate judgment is replaced by "reflective judgment". Reflective judgment is not reflection because there is no universal to subsume the particular. In reflective judgment the individual must find the rule. Reflective judgment is always a question of uncertainty, of risk, but it always leaves the door open much more to innovation. (Lash, in Beck and Beck-Gernsheim, cited in Autio 2006, 162)

Mutatis mutandis, Scott Lash's description of a capable individual in the current world of "second modernity" or postmodernity could be a description what pedagogic practice has always been, "good teacherhood"(Goodson 2014) or "teaching as a reflective practice" (Westbury et al. 2000), in the spirit of Bildung - in sharp disagreement with pseudo-causal "evidence-based" and assessment-driven neoliberal education and leadership policies where - with glaring theoretical simplicity but political purposefulness - teaching is imagined as a "cause" and learning as an "effect".

the countries that have pursued neo-liberal reforms in the fastest and deepest manner, such as England, perform very poorly in educational standards. Meanwhile, those that have defended a social democratic vision and have explicitly valued professional autonomy, such as Finland, have produced top-rate educational standards. It would seem time to seriously scrutinise the neo-liberal orthodoxy in the field of education. (Goodson 2014, 43-44)

As "the curriculum provides a prism, a litmus test, through which to see and test societal health and character" (Goodson 2014, 14), likewise the positioning of the teacher within the curriculum is the litmus test of educational leadership. In terms of curriculum theory, there are basically two already described variants: psychologized Curriculum as managerial, transnational kernel of educational leadership or curriculum receptions motivated and reactivated by Bildung and Reconceptualization that stipulate the teacher as an academically educated, free professional rather than "the agent or the conduit of the system":

The managerial perspective of curriculum [as the embodiment of the dominant psychologized curriculum theory, my add.], teachers are always the invisible agents of the system, seen as "animated" and directed by the system, and not sources of animation for the system. This starting point leads to a view that existing teachers are a (if not the) major break on the innovation, change, and reform that the schools seem to require. ... it is this view of the teacher as a cipher for the formal curriculum that represents perhaps the major source of internal tension within contemporary, ... [psychologized, my add.] curriculum theory and practice. ... it is their respective views of the teacher, and the role the teacher is given within their theoretical and institutional systems, that represents the most dramatic difference in viewpoint between Didaktik and [psychologized, my add.] curriculum theory. (Westbury et al. 2000, 21)

The wider perception that there are theoretical alternatives and the recognition of respective impact of adopted curriculum theory on the work and professional identity of teachers is an international counter reaction to measures of neoliberal education policy and leadership. Yet, the reactions are uneven and vary paradoxically even in countries traditionally affiliated with Bildung theories like in post-Pisa Germany and in Sweden, Finland as an obvious exception. 
In the US, there are interesting efforts to overcome the Westburyan picture of the (American) teacher as a cipher for impersonal learning theories and the formal curriculum by the introduction of curriculum design and practice informed by Reconceptualized, autobiographical theories into the discourses of being a teacher and pedagogic artistry (Henderson et al. 2015) as guiding beacons for educational leadership. A kindred perception by Ivor Goodson $(2014,16)$ importantly extends the being a teacher and pedagogic artistry to education reform. The acid test here is the sustainability of change. The key lacuna in externally mandated change is the link to teachers' professional beliefs and teachers' own personal missions. New research findings in education reform patently show that personal and professional commitment must exist at the heart of any new changes or reforms. "Not only is it neutrally absent, it is in fact positively absent in the sense that there is a mixture of profound indifference and active hostility to so many changes and reforms" (ibid.).

One of the most interesting process in this sense of post-psychologism and poststandardization is taking place in China when the huge country is liberalizing and modernizing its education systems and developing curriculum theory and practice, internationally receptive and well-informed, still adjusted to the national, regional and local traditions, present circumstances and future imaginaries. China's education and curriculum strategy seems to be a hybrid one: the international comparisons like the PISA, TIMSS etc. keeps China, obviously for superpower reasons and its long tradition of externally mandated exams, alert to be competitive in the OECD and other organizations' tests and "racetracks". Simultaneously and apart from the standardizing global competition, China seems to make efforts to struggle against that 'global virus', Global Education Reform Movement, by seeking sources to rephrase and hybridize its "wisdom traditions" of Buddhism, Confucianism and Taoism together with Western theoretical novelties, like poststructuralism and postmodernism in curriculum theory.

China's modernization and its impact will not just be economic but cultural too. China's modernization may suggest in a longer run the way out - in a spirit of the Hegelian dialectic - both from the current fundamental and structural crises of Capitalism and obsolete and rigid Socialism. "The reason for China's transformation (...) has been the way it has succeeded in combining what it has learnt from the West, and also its East Asian neighbors, with its own history and culture, whereby tapping and releasing its native sources of dynamism. We have moved from the era of either/or to one characterized by hybridity" (Jacques 2012, 562).

China's hybrid modernization may signal a cultural feedback to Western notions of modernity and a future of an emergence of contested modernities. If we think about the age of the Enlightenment as the huge educational project, China's modernization and its global cultural impact would imply a need to reconsidering the European Bildung/Didaktik as well as Anglo-American Curriculum as two (Western) master narratives of curriculum theory. In the research project lead by William Pinar (2014): Curriculum Studies in China: Intellectual Histories, Present Circumstances, the chapters by Chinese curriculum scholars bear witness to the 
decisive turn away from the globally spread US reform model of accountability, standardization and teaching to the test - all based on superficial notions of human psyche, human activity and on absurdly narrow educational rationality. Intellectually and culturally profiled, emerging Chinese curriculum theory and practice seem to be affiliating with the North American post-reconceptualization Currere and older European Bildung thought reactivated, localized and hybridized by Chinese wisdom traditions. Chinese distinctive emphases on curriculum theory (Zhang 2014a) may as such work like antidote to schematic, routinized instrumentality and "teaching-by-numbers" mentality in education policy, leadership, and practice in most of Western countries. In Zhang's enthusiastic precondition for curriculum theory is echoed the hybrid resonances with the Eastern wisdom traditions and Western reappraisals of curriculum theory: "No Freedom, No Curriculum!" (Zhang 2014a).

Against the atrophy of economic and political liberalism and democracy to neoliberalism and neo-conservatism, educationally manifested in the totalitarian accountability and standardization, the Chinese opening might shed new light into the world of education by its contested modernity like the postmodern scholarship of Zhang Wenjun (2014b) signals in the Chinese context. Also the ongoing school reforms in China resonate in the reactivated Bildung-Currere spirit the marriage between agency and freedom, so vital to successful education system through the recognition of the significance of the broad-based teacher education curriculum and the positioning of the teacher beyond the sheer conduit of the system. Yuting Chen (2014) speaks powerfully against the grain of Western top-down reforms controlled by standardization and accountability by alternatively predicating on the necessary role of every single school as the "Reform Subject" when schools' role is transformed from the target of implementation, standardization and accountability, "From Follower to Creator", to the active agent of a reform.

China's monumental "liberalizing and modernizing education reforms" is informative in their attempts to overcome the intellectual limitations and exhaustion of presentist empirical social and educational sciences as resources for education reforms. Instead of the modernist four boxes model and division of labor in educational sciences - history, philosophy, psychology and sociology of education - academic study of education and teacher education in China is reorganized as Curriculum Studies.

Curriculum Studies, comprising curriculum theory, curriculum history and curriculum design create the intellectual center of educational sciences and teacher education curricula (Autio 2014) and provide an academic framework and intellectual support for education and curriculum reforms: curriculum becomes an organizational and intellectual center of education. While viewing curriculum as an intellectual and organizational centerpiece of education, Curriculum Studies in China can be seen as a reactivation of the double meaning of Bildung /Didaktik discourse in German-speaking and North European traditions where they can refer to both theory and practice. While reform in China is focused on questions of prac- 
tice, it is not exclusively organizational, "as quite an unproblematic syllabus or content to be taught/transmitted/delivered/tested" but strives "towards more intellectual, more complicated understanding of curriculum" (Pinar 2014, 182). And: "while definitely organizational, the current curriculum reform is profoundly political and intellectual, informed, ..., by culture and history" (Pinar 2014, 21).

\begin{abstract}
Rather than degrading public education, as US politicians have done since Sputnik, in China the Ministry of Education encourages reform through consultation with experts, including contributors to this volume. Rather than imposing a simplistic model of reform, as in the United States, in China the ministry demands complexity and local innovation, not in the service of standardization but to promote organizational diversity and studentcenteredness. In their intellectual courage, their ethical conviction, and their cosmopolitan incorporation of concepts, ancient and contemporary (East and West), curriculum researchers in China demonstrate that the future of education is not inevitably the tragic tale it too often is in the West today. (ibid., 1)
\end{abstract}

The lessons from internationally informed curriculum theory, one of the most vibrant fields of educational study, persuade us to believe that the world of education can be named differently. The fatal discard of moral, historical and democratic elements as vital preconditions for educational discourse by methodologies of positivism and presentist pragmatism has contributed to the neoliberal simulation of education. Separated divisions of labor in education research (history, philosophy, psychology, sociology) have been unable to provide a view comprehensive enough on the contested discourses of education so vital for sound educational leadership. Genuinely international curriculum theory could be a credible source for theoretical and practical uses of leadership by introducing educational concepts more sui generis which have hovered in the minds of prominent curriculum scholars throughout modern times from Herbart's einheimische Begriffe to Kelly's (2004) and Pinar's et al. (1995) views on curriculum as a study of its own right.

\title{
References
}

Apple, M. (2006). Educating the right way, markets, standards, God and inequality. New York: Routledge.

Autio, T. (2006). Subjectivity, curriculum and society. Beyond and between German Didaktik and Anglo-American Curriculum. New York: Routledge.

Autio, T. (2014). The internationalization of curriculum research. In W. F. Pinar (Ed.), International handbook of curriculum research (2nd ed.). New York: Routledge.

Autio, T. (2016). Contested educational spaces: Some tentative considerations inspired by curriculum theory and history. IJHE Bildungsgeschichte: International Journal for the Historiography of Education, Heft 1-2016.

Beck, U. \& Beck-Gernsheim, E. (2002). Individualization: Institutional individualism and its social and political consequences. London: Sage.

Blass, J. (1978). Modelle pädagogischer Theoriebildung. Band I. Von Kant bis Marx. Stuttgart: Verlag W. Kohlhammer.

Couldry, N. (2011). Why voice matters? Culture and politics after neoliberalism. London: Sage Publications. 
Crook, S., Pakulski, J., \& Waters, M. (1992). Postmodernization: Change in advanced society. London: Sage.

Dilthey, W. (1894). Ideen über eine beschreibende und zergliedernde Psychologie. In: W. Dilthey, Gesammelte Schriften, Bd. V. Stuttgart/Göttingen: B. G. Teubner Verlagsgesellschaft/ Vandenhoeck \& Ruprecht.

Doll, W. E. (1993). Post-modern perspective on curriculum. New York: Teachers College Press.

Ertl, H. (2006). Educational standards and the changing discourse on education: The reception and consequences of the PISA study in Germany. Oxford Review of Education, 32(5), 619-634.

Goodson, I. (2014). Curriculum, personal narrative and the social future. New York: Routledge.

Habermas, J. (1984). The theory of communicative action (Vol. 1). Boston: Beacon Press.

Hargreaves, A., Lieberman, A., Fullan, M., \& Hopkins, D. (Eds.). (2009). Second International handbook of educational change. Dordrecht: Springer.

Henderson, J., et al. (2015). Reconceptualizing curriculum development. New York: Routledge.

Jacques, M. (2012). When China rules the world: The end of the Western world and the birth of a new global order. [Greatly updated and expanded]. London: Penguin.

Kant, I. (1984). Kritik der praktischen Vernunft. Stuttgart: Philipp Reclam.

Kant, I. (1991). Education. Ann Arbor: University of Michigan Press.

Kelly, A. V. (2004). The curriculum: Theory and practice (5th ed.). London: Sage.

Kelly, A. V. (2009). The curriculum: Theory and practice (6th ed.). London: Sage.

Klafki, W. (1991). Neue Studien zur Bildungstheorie und Didaktik. Weinheim: Beltz Verlag.

Lather, P. (2010). Engaging science policy: From the side of the messy. New York: Peter Lang.

Pinar, W. F. (2006). The synoptic text today and other essays. New York: Peter Lang.

Pinar, W. F. (2011). The character of curriculum studies: Bildung, currere, and the recurring question of the subject. New York: Palgrave Macmillan.

Pinar, W. F. (2013). Curriculum studies in the United States: Present circumstances, intellectual histories. New York: Palgrave Macmillan.

Pinar, W. F. (2014). Curriculum studies in China: Present circumstances, intellectual histories. New York: Palgrave Macmillan.

Pinar, W., Reynolds, W., Slattery, P., \& Taubman, P. (1995). Understanding curriculum. Historical and contemporary discourses. New York: Routledge.

Ravitch, D. (2010, March 9). Why I changed my mind about school reform. Federal testing has narrowed education and charter schools have failed to live up to their promise. The Wall Street Journal.

Saari, A., Salmela, S., \& Vilkkila, J. (2014). Governing autonomy. Subjectivity, freedom, and knowledge in Finnish curriculum discourse. In W. F. Pinar (Ed.), International handbook of curriculum research (2nd ed., pp. 183-200). New York: Routledge.

Sahlberg, P. (2011). Finnish lessons: What can the world learn from educational change in Finland. New York: Teachers College Press.

Terhart, E. (2003). Constructivism and teaching: A new paradigm in general didactics. Journal of Curriculum Studies, 35(1), 25-44.

Tröhler, D. (2011). Languages of education. New York: Routledge.

Tröhler, D. (2013). Pestalozzi and the Educationalization of the World. Netherlands: Springer.

Tyler, R. (1949). Basic principles of curriculum and instruction. Chicago: Chicago University Press.

Weber, M. (1978). In G. Roth \& C. Wittich (Eds.), Economy and society. An outline of interpretive sociology (Two Vols). Berkeley: University of California Press.

Weber, M. (1995/1930). The protestant ethic and the spirit of capitalism. London: Routledge.

Westbury, I., Hopmann, S., \& Riquarts, K. (2000). Teaching as a reflective practice: The German Didaktik tradition. Mahwah: Lawrence Erlbaum.

Wright von, G. H. (1971). Explanation and understanding. New York: Cornell University press.

Yuting, C. (2014). From follower to creator: School as reform subject. In W. F. Pinar (Ed.), Curriculum studies in China: Intellectual histories, present circumstances. New York: Palgrave Macmillan. 
Zhang, H. (2014a). Curriculum studies and curriculum reform in China: 1922-2012. In W. F. Pinar (Ed.), Curriculum studies in China: Intellectual histories, present circumstances. New York: Palgrave Macmillan.

Zhang, W. (2014b). Growing with Postmodernism. In W. F. Pinar (Ed.), Curriculum studies in China: Intellectual histories, present circumstances. New York: Palgrave Macmillan.

Open Access This chapter is licensed under the terms of the Creative Commons Attribution 4.0 International License (http://creativecommons.org/licenses/by/4.0/), which permits use, sharing, adaptation, distribution and reproduction in any medium or format, as long as you give appropriate credit to the original author(s) and the source, provide a link to the Creative Commons license and indicate if changes were made.

The images or other third party material in this chapter are included in the chapter's Creative Commons license, unless indicated otherwise in a credit line to the material. If material is not included in the chapter's Creative Commons license and your intended use is not permitted by statutory regulation or exceeds the permitted use, you will need to obtain permission directly from the copyright holder. 


\section{Part IV \\ Leadership, Didaktik, and Curriculum Studies}

In this part, we invited prominent curriculum/Didaktik and leadership scholars from the United States and Europe to present their work bridging Didaktik/curriculum theory and leadership studies. We open this part of the volume with Bogotch, Schoorman, and Reyes-Guerra's chapter proposing multicultural perspectives on social justice as a platform for a dialogue between the two traditionally disparate fields of curriculum and leadership. Bogotch and colleagues review the history of curriculum studies and leadership in the United States, both of which have been subject to direct and indirect control by external authorities over time, most recently authorities emerging from neoliberalism. At the same time, Bogotch and colleagues argue that when curriculum and leadership literature has merged in the past, it has been less disparate with aims of multicultural education, democracy, and social justice. In so doing, these authors also provide powerful historical examples of vertical cosmopolitanism and the need for multicultural education as well as an analysis of the ways in which globalization, cosmopolitanism, and external accountability policies have profoundly affected educational leadership and curriculum work in the United States. In the next chapter, Huber, Tulowitzki, and Hameyer likewise consider tensions between recent accountability policy pressures on leaders in Germany. German speaking parts of Europe represents a strong and longstanding tradition in Didaktik and curriculum research. While Didaktik connects to the Bildung tradition the increasing focus on principal education and leadership research is recent and parallels the shift towards neoliberal policymaking. Drawing on empirical findings as well as curriculum and leadership literature, Huber and colleagues identify specific pressures on contemporary leaders in Germany, including changes in curriculum and authority at different levels. These two chapters, one focused on the United States and one focused on Germany, provide important examples of leadership literature benefits from a closer dialogue with Bildung, curriculum theorizing, and Didaktik.

One way in which leadership and curriculum research meet is a recent field called teacher leadership. James Henderson and his former students (Castner, Gornik, and Samford) were invited to provide a closer look at teacher leadership grounded in curriculum theorizing from the reconceptualist movement in North 
America (e.g. Pinar, McDonald). Here Castner et al. (this volume) draw on an action research project to exemplify a curriculum-based approach to teaching/leading in classrooms and schools. We also appreciate that Castner and colleagues offer a constructive alternative to the Tyler Rationale and a leadership approach grounded in understandings of teaching-learning relations. More broadly, for the next chapter, we asked internationally recognized curriculum scholar Bill Pinar to theorize leadership. Pinar begins by reminding us of Ted Aoki's insight that an educational leader (principal) once meant principal teacher whose authority could be exercised personally and pedagogically. He then engages readers in a complicated conversation regarding educational leadership, asking us to step back from leadership traditionally associated with institutional affiliations, and instead invoke leadership from traditions of study associated with psychoanalysis. Importantly, Pinar draws on his own curriculum theorizing and parrhesia ('frank speech') to posit a new lens for leadership authority, one that uses different language but relates to intersubjectivity.

The theoretical framing of this book apply a broad view on educational leadership as occurring on various levels, from the classrooms to transnational arenas. In our mind the curriculum theory tradition in Sweden (Dahhlöf, Lundgren, Englund, etc.) has for a long time developed a research perspective uniting educational governance, policy and curriculum research from a nationstate macro-perspective. In the final chapter of this Part, Forsberg, Nihlfors, Pettersson, and Skott draw on this tradition to consider leadership within new forms of authority and governance embedded in global movements and an increasingly multicultural society. Forsberg and colleagues illustrate the ways in which curriculum and leadership research have historically emanated from the same lines of inquiry and then make the case for a new code to explain the contemporary situation for curriculum-leadership. In this last chapter, Forsberg and colleagues, then, gesture toward the need for understanding leadership as a multi-level and discursive project. We take that argument further in Part V. 


\title{
Chapter 8 \\ Forging the Needed Dialogue Between \\ Educational Leadership and Curriculum \\ Inquiry: Placing Social Justice, Democracy, and Multicultural Perspectives into Practice
}

\author{
Ira Bogotch, Dilys Schoorman, and Daniel Reyes-Guerra
}

\begin{abstract}
History demonstrates that the relationship between curriculum studies and educational leadership is mediated by the conceptualization of curriculum adopted within specific community contexts. To this end, we identify four conceptualizations of curriculum that have been effected in the USA and explore the varied relationship between our two fields that each context portends. Our analysis demonstrates that only when both fields of study have come together for the benefit of society, the common good, will the emergent leadership and curriculum result in progressive attempts at multiculturalism, democracy and social justice. Towards that end, we offer a developmental dialogical framework transitioning from relations grounded in stratification, homogenization, transformation, and the stage we call leadership for social justice. This progressive dialogue is ever more problematic, as it has to navigate multiple obstacles of temporal policies and politics as well as ideological divides. Thus, the complicated conversations are among those who seek to maintain old values and norms (e.g., stratification and homogenization) with those who take a counterview (transformation), or critical perspective (social justice).
\end{abstract}

\section{Overview}

In this chapter, we situate the histories of school leadership (a subfield of educational leadership) together with curriculum inquiry within a larger USA political, cultural, and economic context. To be clear, our two disciplines have been subject to direct and indirect control by external authorities from colonial times to the present. Our analysis demonstrates that when both disciplines have come together

I. Bogotch $(\bowtie) \cdot$ D. Schoorman $\bullet$ D. Reyes-Guerra Florida Atlantic University, Boca Raton, FL, USA

e-mail: ibogotch@fau.edu 
progressively for the benefit of society, then the emergent leadership and curriculum result in real attempts at social justice, democracy, and multiculturalism. Yet, when we accept the historical dominance and subjugation of our fields as a permanent state of affairs, then it makes no difference whether or not we communicate across disciplines. We will explain.

History confirms that our struggles today have been confronted in the past. History also confirms that how the struggles are conceptualized, as stratification, homogenization, transformation or as social justice, are effected by politics and policies, locally and nationally. Such dynamic challenges occur in all national contexts as a complex conversation. Our narrative specifically captures the unique characteristics of the complex conversation within the USA context.

As such, we are obligated by our history to acknowledge the diversity of the nation's populations, highlighting the waves of voluntary and involuntary immigration as well as the horrific treatment of the original occupants of our land, that is, Native Americans. Therefore, our national focus has to be viewed through the lenses of (1) multiculturalism as context, (2) democracy as relationships and an experiment, and (3) social justice as purposes and the struggles to obtain equitable ends of education. The three co-exist; that is, it cannot and should not be otherwise in the USA context. Hence, the necessity underlying our historical and contemporary arguments. At the same time, within each era from the 1600s to the present, new and different circumstances have influenced the struggles for human rights, economic rights, and the quality of life, adding a dimension of contingent and temporal realities to the narrative.

Our purpose here is to provide context: that is, our perception of the current USA reality, how we got here, and how we hope to begin complex conversations. We identify four conceptualizations of curriculum, explore the relationship between curriculum and leadership that each would create, and then discuss how this all can come together into a framework of future dialogue.

\section{Where We Are}

We first ask, how did the recorded USA history bring us to a very different place than where other 'model' educational systems (i.e. Finland, Denmark, etc.) are today? Beyond the foundational cornerstones of democracy, individualism, and pragmatism upon which this nation and its public school system was built [even though it was built for some and not all], it is important to identify the major currents moving forward that prevented curriculum inquiry and school leadership from moving beyond their existing professional silos. Therefore, to better understand the USA context in the present, it is important to consider the rise of neoliberalism during the last quarter century, in particular how it stands in opposition to the conception of Didaktik in northern Europe.

Recent history demonstrates that the rise of neoliberalism marks an ascension to power that has institutionalized governance structures and policies including 
privatization, fiscal austerity, tax reform, deregulation, and free trade. This ideology and its accompanying policies have been embedded by governments on every continent, most notably in Chile under Augusto Pinchot, in the USA under Ronald Reagan, and in Great Britain under Margaret Thatcher. While the effects are worldwide, they have been most strongly felt in the United States in terms of their impact on public education moving further away from the ideals embedded in Didaktik.

Essentially, we see the way forward through a struggle in which the individual "fully enters into reality, so knowing it better, he or she can radically transform it" (Freire 2000, p. 39). We affirm that curriculum inquiry and educational leadership go hand in hand (Reyes-Guerra and Bogotch 2012). Pinar (2004) makes the argument that "As a distinct disciplinary field (rather than a subfield of a single academic discipline such as educational psychology or the sociology of education), curriculum studies may be the only academic discipline within the broad field of education" (p. 2). We believe that school leadership is also an academic discipline within the field because not only does it have an interdisciplinary structure, but it requires the understanding, knowledge, and critique of all the fields in education and society. What is important is the understanding that in northern European circles, Didaktik is still the strong and dominant approach to the foundation of teaching and learning, whereas school leadership and curriculum studies have been slowly neutralized and moved from "scholars and intellectuals to technicians in service to the state" (Pinar 2004 , p. 2). That is, school leaders were converted to managers unable to lead complicated conversations, and curriculum theorists were converted into curriculum designers allowed only to produce materials aligned to the dominant narrative.

Didaktik as a northern European tradition of thinking and studying teaching and learning is unfamiliar to most USA educators today. As Autio (2014) and Seel (1999) pointed out, Didaktik is based on the broader concept of Bildung, "the individual's competence to be able to lead public life: to participate in a knowledgeable way in cultural activities, public affairs, and politics, and to critique - ideally to reconstruct - society by transforming one's self through continuous study and different, idiosyncratic, activities" (Autio 2014, p. 18). Importantly, Didaktik is centered on four elements: moral, cognitive, aesthetic, and practical elements. Didaktik "[m]akes education educative in the real sense of the term: other elements are weighed by the moral and judgmental faculties of the teachers and students alike" (Autio 2014, p. 18). These elements are clearly central to the conceptualization of curriculum and school leadership disciplines, and necessary in building a framework to move the USA forward. Ironically, we will document historical eras, primarily within pragmatic and progressive periods in USA history when curriculum studies and school leadership have come close to a convergence with Didaktik in terms of process and purposes.

However, even when the processes and the purposes in this converging positionality of public education have occurred, it was, is, and will continue to be, as a uniquely "American" phenomenon. The Progressive Movement of the early 1900s marked the stance taken by John Dewey and others (e.g., Boyd Bode) as an educational bridge between "self realization and democratization" (Pinar 2004, p. 17). Therefore, it is this unique American Exceptionality - a term not without 
controversy - which guides our developmental framework by which we embrace the evolution of the social ideals surrounding multiculturalism, democracy and social justice. As a developmental framework in which we document transitions from stratification to homogenization to transformation and to social justice, our task has remained hopeful in spite of recent history and the rise of neoliberalism. We still believe the future is one of a progressive and pragmatic dialogue of mutual respect and interactions among equals, curriculum and leadership.

Nevertheless, dialogue is ever more problematic, as it has to navigate multiple obstacles of temporal policies and politics. Our two fields not only come together and separate contingently, but also are separated from constituents - i.e., practitioners within public education. We have professors of curriculum, professors of school leadership, teacher educators, public school teachers, and public school administrators, each operating independently within their distinct spheres of influence. As if these five constituencies were not complicated enough, there are within each group ideological divides, those who seek to maintain old values and norms (e.g., stratification and homogenization) and those who take a counterview (transformation), or critical perspective (social justice), asking different disciplinary questions. For example, Jerome Bruner (1960/2004) asked his two seminal questions: "What shall we teach and to what end?" (p. 1). Critical educators, such as Geneva Gay, James Bank, Joel Spring, Gloria Ladson-Billings and Michael Apple among others have asked why a curriculum is being developed (e.g. either for the perpetuation of an inequitable status quo; for transformation of the system); "Whose knowledge do we teach?" and "Who benefits from the curriculum?" In the USA, there have always been political struggles over who is included and excluded from public education. It is a perpetual motif in public education in the USA.

\section{Understanding USA Uniqueness: The USA Philosophy and Culture of Pragmatism}

Johann Gottfried Herder and Johann Gottlieb Fichte made strong arguments for the uniqueness of the language, traditions, and culture of a people as a whole that defines the limitations of the political state in dealing with the people that are its subjects or participants. There are strong debates by post-nationalists about US American exceptionalism, yet if there is one strong theme throughout USA history, it is the pragmatism found within the national culture. Pragmatism fits nicely into the "unfinished agenda" in which all USA ideas are debated and then re-interpreted to see which ones matter most in given situations. In William James' (1907/1963) own words, Pragmatism is a mediator/reconciler ... It " unstiffens' our theories" (p. 38, p. 71). "The world is full of partial stories that run parallel to one another, beginning and ending at odd times" (p. 64). It is by understanding pragmatism that we see how the meaning of the "totality of experiences" - a popular notion of curriculum - moved from being a Platonic Idea or Hegelian Consciousness to ongoing 
debates on what it means to be tolerant of queer ideas, on how important it is to listen, and why we need to reflect on alternative ideas of what might be true (Lippmann 1910, pp. 800-801). Pragmatism, according to Henry Steele Commager (1950), "was wonderfully adapted to the temperament of the average America" (p. 97). It translated abstractions into ideas comprehended by ordinary minds. The philosophy embodied a populist message.

William James argued that the disciplines of modern, academic psychology, traditional philosophy and the "science" of education were confusing to practitioners of education, deliberately, using a mystifying language of abstract ideas. His message instead (see his Talks to Teachers, 1899) was one of professional self-development.

You [the audience of teachers] make a great, a very great mistake, if you think that psychology, being the science of the minds' laws, is something from which you can deduce definite programs and schemes and methods of instruction for immediate school-room use. Psychology is a science, and teaching is an art; and sciences never generate arts directly out of themselves" (Psychology and the Teaching Art, Chapter 1 http://www.uky.edu/ eushe2/ Pajares/tt1.html)

James sought to "invent" a practical definition of how ordinary people can understand the meaning of truth or the meaning of any philosophical proposition; that is, he argued that truth comes down to particular consequences based on present and future experiences. There can be no meaningful difference in one's point of view unless it makes a real difference in concrete fact. Pragmatism was James' attempt to accommodate Idealists and pluralists by allowing for the co-existence of opposite points of view when their co-existence did not have any material consequences. It was a criterion for being tolerant, so necessary for any democratic society. By accounting for opposing views, he thought he had reconciled abstract philosophical debates as inconsequential to people's lives. "The true line of philosophic progress lies, in short, it seems to me, not so much through Kant as round him to the point where now we stand" (1907, p. 269)

Locke, Hume, Berkeley, Kant, Hegel, have all been utterly sterile, so far as shedding any light on the details of nature goes, and I can think of no invention or discovery that can be directly traced to anything in their peculiar thought, for neither with Berkeley's tar-water nor with Kant's nebular hypothesis had their respective philosophic tenets anything to do. The satisfactions they yield to their disciples are intellectual, not practical; (p. 83)

Thus, it was left to critical educational thinkers to bring curriculum and leadership to practitioners by engaging them in their lived realities (Boske and Osanloo 2015). This engagement is democratic at its core as it communicates with educators, not 'on behalf of' or 'to' or 'for'. Prepositions in language matter, for research and relationship building. But this in itself will not make any significant difference unless we actively listen to and learn from other educators, communities, and most of all, our students. The learning, listening and acting becomes the praxis or dialogue for our proposed framework. The primacy of practice purposes, processes, and outcomes are all contextual, thus requiring educators to continuously assess the consequences of leadership actions in terms of social justice outcomes (Ayers 2009; 
Bogotch 2002, 2008, 2014). Through James' public lectures to teachers, the meaning of education as negotiation and progressively moves from academics and social change.

As professors and researchers, it is one thing for us to diagnose the problems carefully and systematically and cautiously offer advice. But as educators, our responsibilities lie in our actions, in this case figuring out the next steps to a productive cross-disciplinary dialogue. But before we begin, we will next give a brief USA history to illustrate the twists and turns which have characterized our past relationships.

\section{Re-examining USA Histories as an Unfinished Agenda}

Because the forum for our ideas is international, we will follow an historical chronology so that readers familiar and unfamiliar with the uniqueness of the USA can follow the various twists and turns, continuities and discontinuities reflected in our history. Our illustrations in this narrative will unfortunately omit key personalities, facts, and events that some readers would have liked to learn more about. By necessity, our choices are arbitrary; that is a compromise all educational researchers face. More problematic is that historical figures do not always stand up well in today's light.

Two historical facts dominate any narrative of the USA context: first, we are a relatively young nation, and secondly, we are culturally diverse. As a young nation, the USA is and should continue to be an experiment in democracy, with principles of leadership based on checks and balances (what we call accountability) and the separation of powers (our national distrust of authoritarianism). This has been interpreted in many different pragmatic and progressive ways depending on which era from the 1600 s to the present is being highlighted. The Pulitzer Prize winning historian, Joseph Ellis (2007) refers to it as our history as an "unfinished agenda;" the "people's historian" Howard Zinn (1980/1988) called it the "possibilities of surprise (p. 435);"'and, the noted educational historian David Tyack (1974) saw history as a veritable kaleidoscope of competing interests.

In more practical terms, our history is a struggle between orthodoxy and tradition and modern day practices in all aspects of life. As E. J. Dionne (2012) puts it, "We are a nation of individualists who care passionately about community. We are a nation of communitarians who care passionately about individual freedom" (p. 5). We are a nation of culturally diverse peoples, even when the USA Constitution excluded Indians (Native Peoples) from citizenship (rectified in 1924), and for tax purposes and federal representation reduced Negro slaves to the fraction $3 / 5$ th ' $\mathrm{s}$ in terms of counting the population. Some of the noted architects of our republican form of government, such as Thomas Jefferson, were slave holders which speaks to the ongoing contradictions and tensions created by diversity. Nevertheless, as we will make clear in this chapter, USA public education was designed specifically and 
progressively for the "common man" (sic) as the great equalizer in society as essential to the great experiment in democracy.

Public Education Beginnings The origins of public schools was that they all functioned locally and autonomously for the first century and a half. When the church or orthodox educators controlled knowledge, then teaching the readings and moral lesson of the Bible was a complete answer to Bruner's (1960/1963) two questions: "What shall we teach and to what end?" (p. 1). Primary school students would not only be able to sing), but also have a sense of salvation, either in work, family or in an eternal afterlife. Replacing the Bible with secular readings, made spelling, literacy and calculating numbers not only basic skills, but depending on how many years of education a person received, provided access to secondary, colleges, and universities and a professional life - often a life inside the clergy. In rural settings, the curriculum was delivered by a single teacher in the proverbial one-room school house filled with a community's youth. In many instances, historical and contemporary, the curriculum was prepared outside the school setting and delivered, well or poorly by trained and untrained teachers.

One of the earliest secular - and urban - curriculums was set forth by Benjamin Franklin who synthesized many Western European ideas. In his Pamphlet.titled Proposals Relating to the Education of Youth in Pensilvania Philadelphia, (1749), he listed classical subjects and the reasons for their inclusion in the curriculum. The subjects were divided into two categories: "everything that is useful" and everything that is ornamental." Thus in this very early configuration of curriculum, we have both aesthetic and public ends of education.

Throughout the Pamplet are exhortations in defense of the term "public:"

- "Publick Disputes warm the Imagination, whet the Industry, and strengthen the natural Abilities;" "impressions of the Beauty and Usefulness of Virtue of all Kinds,

- Publick Spirt;" and, "the Necessity of a [CHRISTIAN]

- Publick Religion” (i.e., character education).

Yet, as an independent nation, circa 1776, and especially under the first governance structure, the Articles of Confederation, each of the original thirteen colonies maintained their autonomy, such that each printed its own currency, made its own domestic and foreign policies, each collected taxes, all based on own local, private interests. There was no central authority to coordinate both the business of America, the protection of America or the rights of citizens or establish a public identity (as Franklin's curriculum advocated) or a public good. But what was truly remarkable was that the very same group of well-educated men, landowners, who had conceived of the Articles of Confederation, understood that a new and different governance system was needed. The arguments were made in a series of New York Times essays under the title The Federalist Papers. The USA Constitution set forth a new direction based on the principles of minority rights, "checks and balances" and the "separation of powers," with central authorities focused on the rights of all citizens alongside local decision-making with respect to community and educational needs. 
What is most relevant to us here is the idea that when curriculum is transmitted rather than developed, the "what" or content has been determined externally, apart from the community or apart from the students' abilities and interests. When leadership and curriculum have worked in tandem to improve students' lives and uplift their communities, the first leadership decision is to create the conditions for engaging in curriculum inquiry in order to learn the values, beliefs, and customs of the community. When this leadership step has happened, the school becomes a part of a community with parental and community support. Unfortunately, educational history is replete with periodic examples of external authorities deciding from afar what all USA communities' need. The idea of school and community is as old as the nation. And yet, it remains problematic in terms of both leadership and curriculum inquiry and its development. But in order to understand the many examples of school and community throughout USA history and contemporary times, it is necessary to see how radical and dramatic governmental change happens.

\section{The Emergence of Public Education}

It was Horace Mann who insisted that public education be democratic in structure and practices. His design of normal schools in Massachusetts in the 1830s-1840sff were designed not to change the arduously difficult lives of immigrant parents, but rather through schooling to change their children who could rise up out of drudgery and poverty in one generation. Horace Mann himself personified the effective integration of leadership and curriculum. The design of normal schools was that teachers were educated in the mornings and then they taught students in the afternoon. And when it came to immigrants of Irish, Italian, Polish, and Jewish descents, his single generation goal became almost a two century mantra of those who enrolled in public schools. That said, for other non-white immigrant groups, they have never fared as well inside either separate but equal or integrated public schools (Greer 1972, Perlman 1988).

Although Mann himself championed integration and the end of slavery, it was not until the passage of the 13th, 14th and 15th Amendments to the USA Constitution after the bloody Civil War, in the 1860s, that a new transformational era in USA history was initiated by President Abraham Lincoln. Lincoln chose war in order to keep the nation whole and not let economic or political differences destroy the country's system of government. The above amendments abolished and prohibited slavery; conferred citizenship on persons born or naturalized in the US; prohibited denying voting right to citizens based on race, color and previous servitude.

By the turn of the twentieth century, public schools faced new challenges of immigration and poverty caused by urbanization, parental neglect and truancy. Schools were called upon to provide a new progressive curriculum and philosophy (i.e., Dewey). And thus were born schools in conjunction with settlement houses (Addams 1910/1961; Berger 1956/1980, Patri 1917). Quoting from Patri: 
We looked upon the settlement as a moving living force whose idea was one of service and not of power. Free from tradition, we felt that it would be the neighborhood social experimentation station, finding out, working out, and then beginning again, never stopping long enough to standardize. (p.134)

The settlement houses addressed economic issues while also instilling a meaning for democratic citizenship. The reform, like the origins of government were "experimental rather than permanent (Berger 1956/1980, p. 2) although the principles underlying this and many other educational reforms were meant to be permanently instilled in society. Even during the two World Wars and the Great Depression, public schools sought to become sanctuaries with "playgrounds and school gardens, shower baths and swimming tanks, manual training and domestic science, branches of the public library, vocation and evening schools, schools for deaf and blind children, auditoriums for use by pupils with free lecture courses and concerts, and in general the opening of schools after hours as neighborhood centers" (p. 90). For progressives, education was a social institution to promote community well-being and democracy (p. 105).

\section{An Historical Convergence}

The Progressive Movement in USA history had multiple philosophers, including James whom we have already presented. However, the premier philosopher in progressive education was John Dewey, who, inspired by James, then introduced instrumentalism as a philosophy and called for radical reform of curriculum and teaching. Always tempered by his primary ethical value of democracy, he countered the traditional approach to education with progressive education. His philosophy opened, on multiple levels, the doors to a strong convergence of leadership and curriculum that we are advocating. Dewey's emphasis on democracy as a way to protect popular interests, democracy as an expression of individuality, and democracy as a method of social inquiry were foundational as litmus tests of progressive education.

One of the most vivid examples of curriculum and leadership working in concert to build community was the planned community of Arthurdale, 1934-1936, the first subsidized project of the Roosevelt administration's New Deal. It was an experiment to educate the displaced coal mining families of West Virginia:

It is proposed that, just as the organization of this community represents an experiment seeking to discover means of needed adjustment in our social and economic life, likewise let this be a new school, providing for its citizens of all ages richer and more adequate educational opportunities (Stack 2004, p. 188).

The school principal, Elsie Clapp's leadership began with two non-negotiable demands: one, that she was able to bring her experienced staff with her to the new school community; and, two, was that she would not open the school until she and her teachers had acclimated and assimilated themselves into the community so that 
the curriculum would be based on the real needs of the students. The formerly city educators were now studying "farming, homesteading, village games" [because] "understanding cultural heritage was a central component of self-realization" (p. 196). The structure was to hold classes day and night for everyone in Arthurdalefrom ages 2 to 72 . Teachers became active members of the community, volunteering as firefighters and building greenhouses, recreation buildings, libraries, cafeteria and kitchens, home economics, a doctor's office, a bank and bookstore (p. 200).

But as history unfolded neither the settlement houses nor the Arthurdale experiments continued. What happened? For one thing, Dewey's progressive ideas were never embraced by traditionally trained educators whether in curriculum or leadership. As Dewey repeatedly noted, progressive education was too difficult conceptually for teachers and administrators who did not receive the kind of rigorous education advocated by Franklin, Jefferson or Mann. Instead teacher educators have participated in what William Doll (1993) referred to as the "methodologization" of curriculum. Progressivism required that the students' knowledge, interests and abilities inform the teacher who listens and learns from them, co-constructing the sequencing and pace of instruction within all subjects being taught. In contemporary terms, progressive practices have led to a more critical pedagogical approach which holds that "what works" for disadvantaged students, often students from minority cultures and races" actually works for all students, while culturally insensitive curriculum, that is, colorblind and objective practices, do not work for minoritized groups of students (Grant and Sleeter 2011). Moving from constructivism to critical constructivism has become one of today's curricular challenges inside the academy.

Resistance to progressive ideas had deeper roots in the USA society than just pedagogies and methods. And for this reason, Dewey's philosophy of education was grounded in his battle to combat the anti-intellectual state of affairs within education. He wrote essays on democratic relationships, educative habits and experiences, artistic appreciation, and engaging in the public's problems - all attempts to broadened progressive educational ideas beyond schooling.

\section{Wrong Turns: From Historical Injustices to the Neoliberal Assault on Public}

Education Throughout USA history, political, cultural and social ideals and ideologies have competed for power. It is always within a larger socio-cultural and political environment that we must see public education. But when education goes wrong, it goes wrong when curriculum and leadership come together to cause cultural and psychological damage to students and/or when the disparate impact of practices and policies keep minoritized groups from participating in the opportunities afforded to others in society.

The impact of scientific management imbued with the valuing of social efficiency has raised significant concerns for multiculturalism, democracy and social justice (Kliebard 2004). Bobbitt (1918/2013) made the case for the "technique of curriculum making along scientific lines" (p. 12) that prioritized the efficient use of resources. According to Flinders and Thornton (2013), 'For Bobbitt, 'scientific' suggested a systematic series of procedures, carried out by curriculum professionals, 
prior to implementation in a school district" (p. 4). Teachers and their supervisors were recipients of this externally derived curriculum. This methodolization of curriculum emphasized the "how to" of curriculum design, highlighting the interconnections among objectives, content, instruction and assessment, as exemplified by the work of Ralph Tyler (1949). This short text inspired scores of curriculum specialists in decades of discussions and debates on the development of objectives and varied models and frameworks for curriculum design. Although Tyler called for the purposes of curriculum grounded in broader concerns, preoccupation with efficient development and delivery became the focus of curriculum specialists. As Klein (2003) observed, instructional strategies such as "behavioral objectives, time on task, sequential learning, positive reinforcement, direct instruction, achievement testing, mastery in skills and content, and teacher accountability" (p. 21) became more valued as the domain of school-based educators.

The ability to "efficiently" deliver curriculum, rather than to question it, provided fertile ground for the proliferation of scholarship on curriculum design methodologies as well as for the growth of externally derived standardized curriculum. Teacher education programs honed the skills for practitioners' instructional development separate from the curriculum. Ensuring that the pre-determined learning outcomes are met and measured is a priority. Depending on the context teachers may have the license to achieve these outcomes in creative and personalized ways; in other contexts supervisors expect teachers to instruct in narrow and scripted ways. The convergence between curriculum and leadership within this paradigm is as seamless as it is sublime with the expression "aligned with fidelity" rolling off the tongues of today's teachers and principals as if their bonding over such reforms will in fact close the achievement gap and turnaround schools. We express this with as much sarcasm as we can muster because it reveals a larger political and economic agenda afoot.

To wit, the bogeyman that has extended the historical injustices into the present is often referred to as neoliberalism. According to Pinar (2004), it is complicit in schools and universities becoming a "skill-and-knowledge factory (or corporation); the education professoriate is reduced to supervisory personnel" (p. 3). Educators at all levels have lost the moral consideration so central to Bildung, Didaktik, and to Pinar's complicated conversation of currere in curriculum inquiry. There is even a bifurcation at the university level: at some institutions, students, teachers, and professors are not engaged in complicated conversations at any or all levels, while at others attempts are made, but they are isolated within the ivied walls of the institution and end when the teachers and leaders enter the school.

It must be noted that the overarching principle of neoliberalism is that all things have value because all things are commodities. Citizens are no longer citizens, as they are now global investors who can invest their capital for profit across the world, employers who look for the cheapest workers on the planet to produce goods, and consumers who purchase the production that then returns investment on the capital put out by the investors. There is no common good but that which is produced by the market forces of competition and the profit obtained by individual greed which then, somehow, allows the world to be a better place for all by meeting the 
self-indulgences of the consumers of these commodities. As Harvey (2005/2010) pointed out, the system works as long as there is a $3 \%$ average return on investment, but that 'system' is 'working' only for the investor and for those who are a part of the elite who manage those investments, be they corporate CEOs, stockbrokers, or those who have discovered a market where their labor is sufficiently valued to allow an income that places them in the investor class.

In this neoliberal setting, privatization, fiscal austerity, globalization, and free trade (competition) must become the focus of the government and policy in order to bring profit to the capitalists involved and cheaper commodities to the consumer. For public education, applying these principles results in two different trends. The first allows for education to be converted into a commodity, where students and parents are consumers. Privatization has meant turning public schools into independent, privately run schools or charter schools, whose purpose is to turn a profit as measured in the production of test scores or as measured in the efficiency of the private management organizations running and profiting from these schools. Education therefore is not about currere, it's about maximizing the 'profit' measures. Fiscal austerity, a means of getting more productivity out of less workers, has meant that schools and educational programs are given less resources (tax dollars) for funding in order to force greater productivity - consideration is not given to anything but those endeavors that will produce rising test scores, so moral, cognitive, aesthetic, and practical elements are ignored.

The second and more subtle trend is that by turning education into a commodity, the profession itself must change into a commodity producer and educators simply the corporate workers who develop and produce that commodity. And that commodity, learning, has to be packaged and delivered in uniform ways to allow for both an opening of the market and competition. This has meant that instead of curriculum or leadership becoming more contextual, more democratic and therefore by definition more focused on social justice and multiculturalism, these two disciplines have become commodified, specialized, and even further silo-ed by the policies enacted. Accountability, a word that plays on the sensibility of the general public, is actually a way for corporations to sell software, data tools, and testing materials to the over 13,500 school districts in the USA. But be clear, the USA is too small a market for the largest educational corporation Pearson Education, which is advancing globally and digitally into emerging markets, and taking full advantage of the newly created competitive market based on international standardized testing (e.g., PISA, TIMMS). Neither context nor currere exist. As Pinar (2004) states, "[by] tying the curriculum to student performance on test scores, teachers are forced to abandon the intellectual freedom to choose what they teach, how they teach, and how they assess student learning" (p. 164). 


\section{The Way Forward: A Tentative USA Framework}

Our reflection on USA history reveals the ongoing intergenerational struggles to realize the ideals of multiculturalism, democracy and social justice across curriculum theory and leadership. Just as the wrong turn was taken with respect to the infamous Native American Boarding schools, which turned out to be sites of cruel cultural genocide and militarism, and just as the enlightened turns of progressive and democratic community education were made within settlement schools, the two sides of the USA coin demonstrate that it is only when curriculum and leadership were inclusive of diverse participants coming together democratically and for socially just ends, that the dialogue was pragmatic and progressive, necessary and contingent.

The new conversation to be forged between leadership and curriculum is in our view best grounded in Freire's (2000) notion of dialogue between interlocutors engaged in a mutual struggle against injustice. In a way, dialogue serves both as metaphor as well as a process for re-framing the relationship between curriculum and leadership educators; it also signifies the desired relationship between curriculum/leadership professors and public schools. For Freire, dialogue is a transformative and humanizing process of mutual reflection and action, forged in love, humility, faith, hope and critical thinking, with a view to engaging in collective action in solidarity with oppressed groups.

Many public schools in the USA, particularly those that serve racially and economically marginalized students, offer appropriate yet urgent spaces within which such dialogue could occur. Currere holds, for us, the notion of curriculum as personal enactment - our lives as curriculum, as well as Freire's perspective signified by the dialogue: we make the road by walking. It is a notion of Freirean praxis forged through action and reflection in the dialogic encounter. The formal curriculum or conceptualization of the curriculum as a plan for instruction is only a limited facet of the definition of curriculum central to our discussion.

The context of curriculum will determine the nature and trajectory of the dialogue required between curriculum and leadership. Within the USA, the preservation of privilege has given rise to social efficiency undergirding the relationship between curriculum and leadership. The use of standardized curriculum in what has been variously referred to as the canon, a master-narrative, reflecting a culturally arbitrary (Bourdieu and Passaron 1977/1990) perspective, where assimilation of diversity towards a homogenized perspective was valued necessitates curriculum leadership that prioritizes monitoring, enforcement, and evaluation based on a priori, externally imposed standards. It is within this state of affairs today in contemporary USA that a significant amount of public funds are expended on the development of standardized tests, their administration and assessment, by private companies (e.g., Pearson), despite widespread criticisms by prominent USA educators such as Dianne Ravitch (2014) and David Berliner (2011).

Our path forward is necessarily informed by our critical reflection on our past. Our history reveals varied potentiality for the desired dialogue between our fields. 
On the one hand, we have spaces where limited dialogue in the context of common conceptualizations has been beneficial to maintaining an inequitable status quo; on the other hand, we recognize that curriculum as a complex, community based undertaking for social justice necessitates dialogue as counter-hegemonic praxis. Movement towards this latter notion of dialogue, is likely to require multiple steps. Gay (2001) wisely noted in the context of multiculturalism that an institution's development and readiness was a factor in the level of transformation to be expected. We reckon the same is true of transformative dialogue. History demonstrates that the relationship between curriculum and leadership is mediated by the conceptualization of curriculum adopted within specific community contexts. To this end, we identify four conceptualizations of curriculum that have been effected in the USA and explore the varied relationship between curriculum and leadership that each portends. For each, we look through the lens of multiculturalism, democracy, and social justice to examine the predicated outcomes.

Curriculum for Stratification Assimilationist and racial stratification policies have been endemic to US curricular practices in public education. The policies towards Native Americans, African Americans and subsequently towards generations of immigrants have included explicit plans for segmented assimilation (Portes et al. 2005; Portes and Zhou 1999). We see, on the one hand, the efforts to rid students of their native tongue, cultural values, in efforts to "Americanize" them; on the other, the denial of education either through explicit laws, or their lack of enforcement, ensured social control of different groups. (Spring 2013) quotes the advocacy of discrimination towards students of Mexican descent by a farmer, a school superintendent and a principal: "Educating the Mexicans is educating them away from the job, away from the dirt" ... It is up to the white population to keep the Mexican on his knees in an onion patch; this does not mix well with education .... Never try to enforce compulsory attendance laws with Mexicans. The banks and the company will swear that the labor is needed" (p. 95). Such overt racism also forced pragmatic educators, such as Booker T. Washington to press for "education before equality" in an effort to make the best of stratification policies.

Clearly, curriculum for stratification is a violation of any current conception of social justice. In fact, stratification is the opposite of inclusion, and inclusive schools as an overarching conceptualization "provides effective strategies school leaders operating from a social justice framework can implement to create more inclusive schools for all students" (Esposito and Normore 2015). Stratification parallels the curriculum of the status quo within the USA from which we wish to deviate. Alarming disparities in academic achievement due to the historic lack of resources and deliberate withholding of institutional support (Ladson Billings 2006) are further exacerbated through standardized testing regimes that link students' test scores to school funding and teacher salaries, reduce curriculum and leadership to a focus on test preparation through drills and instructional routines grounded in factory-like efficiencies. These contribute to the increasing racial re-segregation of public schools (Kozol 2005). Despite widespread scholarly condemnation of these practices, they remain well-entrenched within the public education system, supported in 
large measure by corporate entities who benefit financially from the increased testing, monitoring and data management that now characterize public education.

The widespread acceptance of this curriculum signifies a separate-but-equal compliance by curriculum and leadership practitioners in the perpetuation of narrowed, decontextualized and largely prescriptive curriculum. Educator preparation programs, swayed by test-based accountability and funding formulae, abandon any semblance of the principles linked to the notion of Didaktik to teach compliance with state standards for practitioners whose primary role is to uphold this system. The intensification of workload that comes with such de-professionalizing practices (Apple 1986) typically precludes opportunity for collaboration at any level, except for ensuring that standards are met across the system. Local control, democratic values that include social inquiry (Dewey 1916) are all violated in this form of curriculum.

Curriculum for Homogenization Curriculum for homogenization also draws on notions of standardized curriculum but with a distorted commitment to social justice and democracy that emerges from the concern about equal outcomes among different groups. Although framed also as the potential for access to knowledge variously described as cultural capital (Bourdieu 1986), cultural literacy (Hirsch 1996) and/or the culture of power (Delpit 1988), it often comes at the price of home language, cultural identity and curricular relevance. The curricular focus here typically privileges pre-determined content with emphasis on instructional methodology. Multicultural criticisms draw attention to largely monocultural (Banks 2001) and biased (Loewen 2008; Sadker and Sadker 2000) content that risks intellectual enslavement or colonization. As Woodson (1933) so eloquently reminded us, "If you can control a man's thinking, you do not have to worry about his action" (p. 84).

May and Sleeter (2010) report that standardization of curriculum towards homogeneity increased in the rhetoric against multiculturalism post 9/11; furthermore framing everyone as equal citizens detracted from the unequal power relationships that existed among groups. Goals of homogenization inevitably include deficit orientations where their "differences" are problems to be overcome, rather than assets to be built on. Thus, within the classroom, homogenization occurs through teachers' instructional strategies. At the level of the school, principals make professional development, budget, instructional support and teacher retention decisions that minimize the "gap," with little opportunity to question the received curriculum nor the policies that are foundational to such inequities. In the context of educator preparation programs, a curriculum as science perspective emphasizes lesson plan development aligned with state and local standards in teacher preparation (in plans that typically foreground procedures rather than concepts), scientific/rational curriculum design opportunities in the preparation of curriculum specialists and a managerial/administrative/supervisory focus in principal preparation.

Curriculum for Individual Transformation This conceptualization of curriculum more clearly identifies the potential for productive intersections between leadership and curriculum as the purposes and the intersections between the formal and the informal reveal a distinct departure from the previous two conceptualizations. 
It draws on a more polyphonic perspective of curriculum, rooted predominantly in the holistic development of the student as a human being. It integrates the multiple sources of curriculum to achieve a broader set of learning outcomes that extend beyond subject matter mastery per se, but where such knowledge becomes the basis of intellectual, social and civic development. Within this perspective, curriculum is broad-based, dynamic and multidisciplinary, drawing on the values of aesthetics as much as on science, social science, humanities, and languages and characterized by inquiry-based approaches to instruction. In this conceptualization, curriculum emerges as an art, not a science; it is a verb (Padgham 1988) not a noun. It is local and context-based, emerging from the specific needs and interests of the students, the community and their educators. Here tests and accountability do matter, but they do not drive the curriculum.

Historical manifestations of such an approach are evident in the work of Montessori (2013), whose pursuit of child study railed against the narrow conceptions of science noting, "The school must permit the free, natural manifestations of the child if in the school scientific pedagogy is to be born" (p. 25; italics in original). Dewey, whose prolific scholarship offered the balance between and among prescribed curriculum, student-centered learning and social responsibility in a democracy. Greene's $(1978,2000)$ exhortations for creativity and the imagination in the development of the consciousness of the individual, Eisner's (2002, 2003, 2005) challenge of the scientific notions of curriculum and pre-determined objectives, together with Robinson's (2006) observation that schools were both anachronistic and stifled growth and creativity support this conceptualization of curriculum, in strong criticism of the rational/scientific view of curriculum.

This democratization of curriculum demands vastly different responsibilities of teachers and leaders, including a shift in the responsibility of curriculum development. In the absence of the pre-packaged curriculum faithfully transmitted to students, school-based educators become responsible for facilitating the development of curriculum; what is worth knowing emerges from a process of collaborative investigation; lesson planning is conceptual rather than a technology. Outcomes such as students' self-actualization, identity development, cross-cultural competence and civic consciousness gain importance as knowledge is understood as dynamic and co-constructed. Inclusivity in this process will require comfort with divergent thinking and communication that fosters consensus building.

Consequently, how the fields of curriculum and leadership prepare their students for this reality becomes crucial. If curriculum studies programs adopt a curriculum as science perspective, their graduates will be ill-equipped for contexts requiring a more dynamic approach. Similarly, if educational leadership programs are solely focused on scientific approaches to ensure mastery of pre-determined and unquestioned curriculum, teachers prepared as curriculum inquirers will be stifled under such leadership. It thus becomes crucial for academics in these two fields to work closely as they prepare teachers, curriculum developers and leaders for conjoint practice in their communities. While such collaboration is central to the dialogue proposed at the next level of this typology, what is different here is a matter of focus. The focus on the individual lays the groundwork for transformative leadership 
(Shields 2014) and transformational curriculum (Banks 2001), each endorsing the value of divergent perspectives in a dynamic view of leadership and curricular implementation. This lays the requisite groundwork for transforming entire institutional structures. This is not a challenge to be undertaken by a single entity; it will require broad-based coalition building.

Curriculum for Social Justice Contemporary educational policy and practice in the USA, despite its rhetoric, has fallen prey to a technocratic managerial system, obsessed by measurement but with little meaningful learning going on in the 8 months leading up to the annual test (Rose 2011). Of particular concern is the impact of the current system on historically under-served students in the USA. As Rose (2011) notes:

This concern about the nature of a school's response to high-stakes pressure is especially pertinent for those students at the center of reform: poor children, immigrants, and racial and ethnic minority students. You can prep kids for a standardized test, get a bump in scores, yet not be providing a very good education. The end result is the replication of a troubling pattern in American schooling: poor kids get an education of skills and routine, a lower-tier education, while students in more affluent districts get a robust course of study. (pp. 34-35)

The ongoing struggle, especially in the current context of neoliberal politics that have gripped public education at the primary, secondary and tertiary levels, will require intentional coalition building between and among diverse constituents if a vision for social justice education is to be realized. Shields' (2014) call for transformative leadership for social justice education focuses on "the very nature of the school and its relationship with the wider community" (p. 325). This entails transformation in the formal, prescribed curriculum, as well as in its hidden curriculum of policies, practices, values, climate and community relationships. Apple and Beane (1995) observed similarly in their study of democratic schools in the USA:

Democratic schools, like democracy itself, do not happen by chance. They result from explicit attempts by educators to put in place arrangements and opportunities.that involve two lines of work: ... to create democratic structures and processes by which life in the school is carried out. The other is to create a curriculum that will give young people democratic experiences. (p. 9)

Curriculum for social justice builds on the legacy and vision of social reconstructionists who called for curriculum to be a catalyst in the transformation of inequitable social structures. It supports what Ladson-Billings (1995) calls culturally relevant pedagogy defined as a humanizing pedagogy of opposition that leads to collective empowerment, students' academic success, cultural competence and critical consciousness that facilitates their ability to question an inequitable status quo. This approach parallels the principles of critical pedagogy, inspired by the work of Freire (2000) and popularized in the USA by critical scholars (e.g. Apple 1986, 2006, 2012; Giroux 2011a, b, 2013; Kincheloe 2008). Critical pedagogy draws attention to the oppressive potentiality of curriculum, where students were treated as passive recipients of knowledge. Instead, Freire called for more emancipatory pedagogy that would facilitate active engagement through dialogue in a mutual process of reflection and action that constituted the praxis by which learners might act 
upon their world to transform it. The cases highlighted by Apple and Beane (1995), the (now banned) Mexican American Studies program of Tucson, AZ, and the Chicago Grassroots Curriculum Taskforce (CGCT) offers a prototype for community-based curriculum development integrating students, their families, community elders and school- and university-based educators to compile a collective knowledge of Chicago's communities (http://grassrootscurriculum.org/ about-us/).

\section{Curriculum Options for Democracy, Multiculturalism, and Social Justice}

As we mentioned earlier, there are four elements of Didaktik that provide a direction for bringing our two USA disciplines of leadership and curriculum together: the moral, cognitive, aesthetic, and practical elements. But the meanings of these terms are derived from history, national contexts and cultural perspectives. Thus the curriculum options begins with a four by three matrix in which we frame the four elements of Didaktik alongside democracy, multiculturalism, and social justice. Beyond social, political and economic stratification and beyond the "melting pot" metaphor of the Ss. Clearly, the two curriculum conceptualizations that begin to meet the criteria for effecting radical change are the Individual Transformation and Social Justice curricula. This means that curriculum and leadership programs must lead to the development of teachers and leaders who are able to effect needed changes in both individuals and communities.

For many scholar-practitioners who are working in pockets of isolation within the academy, where multicultural, democratic, and social justice concerns have been relegated to marginal status, this becomes an especially difficult challenge. Too few programs in education have made multiculturalism, democracy and social justice integral to their program philosophy in action, rather than merely in rhetoric. Too rarely does this philosophy extend to multiple programs such as its leadership preparation and its curriculum/teacher education. Instead, many programs still focus on the traditional curriculum developed with a certain dysconciousness (King 2000, 2015) of the structures of inequity that shape and are perpetuated by extant curriculum. Furthermore, in the high stakes context of assessment, where - in an egregious mismeasure of quality - educator preparation programs in public institutions will be measured and funded by the test scores of our graduates' students, educator preparation institutions appear more keen to gear their curriculum towards compliance with such policies, rather than interrogating them. 


\section{Conclusions for Complicated Conversations That Must Result in Action}

While in the broadest sense of leadership practices which does not delimit leadership to roles, functions, and structure), curriculum theorists are, in fact, educational leaders in that they interact with others. Our framework identifies these others not just in terms of the socio-cultural situation, historical eras, but also the key participants, which includes those held responsible for educational decisions and outcomes. Thus, the focus on the interactions between curriculum and educational leadership.

One of the very few curriculum theorists to have a clear view of these interactions with educational leadership was Alice Miel. In 1961, she delineated five prerequisites for leadership in schools: "(1) a professional atmosphere, (2) a climate of psychological safety with freedom, (3) staff learning opportunities, (4) opportunities for decision making, and (5) opportunities for cooperative evaluation and selfevaluation (p. 229). Following Dewey, Miel asserted that pedagogical content should never be limited to the transmission of already existing ideas, but rather engage with students in activities whereby new ideas emerged and were reconstructed. Thus, curriculum would never be static but always guided by a social construction through trial and error. For Miel, leadership was a matter of balancing safety and challenge, control and release with a freedom "to venture into uncharted territory where instructional material have not yet caught up with events" (p. 252). She called her leadership theory "ordered freedom" a liminal space between the status quo and agency for change - which for both administrators and teachers are a "never-ending challenge" (p. 252).

According to Reyes-Guerra and Bogotch (2012) "the field of curriculum theory and inquiry has been grounded in educational concepts, such as development, growth, discovery, progressivism, democracy, morality, and reconstruction" (p. 138). They stated that "While the formal definition of curriculum found inside catalogues, brochures, and Web pages describe it as what is taught and learned in single classrooms, curriculum [check the wording of this quote], as we learned in our undergraduate teacher-education years, is the totality of experiences - with multiple dimensions, including cognitive, social, political, economic, moral, aesthetic, cultural, and spiritual aspects of life - not just for living today, but also to be transmitted generatively from generation to generation. As such, curriculum inquiry demands that participants have the freedom to be creative and innovative." (p. 141).

It was the unintended consequence that two American pragmatists, William James and John Dewey, who were both trained as scientists, pushed open the door of tolerance, experimentation, and democracy only to witness how dominant groups in society walked through that door to impose their own selfish ideas or to propose a "new" science of psychometrics (e.g., Thorndike) and scientific management (e.g., Taylor, Bobbitt, Courtis) which has for a century now dominated USA education in policies and practices. Pragmatism and progressivism have struggled over the past century in the face of quantitative proofs and statistical methodologies. It is 
up to us to insist on the reconstituting a new pragmatic test: promoting dialogue focused on that which is good and eliminating that which causes psychological and bodily harm.

Dialogue, within the Freirean context arises from the identification (or "naming") of social injustice and is sustained through mutual engagement with the parties affected by the injustice in order to transform the conditions that give rise to it. The given name to that injustice will differ across the globe according to contexts from which opportunities arise. Contexts and opportunities differ nation by nation. In the case of the USA, we recognize that the inequities of contemporary education here are a legacy of the historical, economical, sociopolitical and moral debt owed to specific groups through educational policies and practices explicitly aimed at their annihilation (Grande 2004), stratification (Gonzales 1996) or miseducation (Woodson 1933). Thus it is incumbent on all educators who claim a stake in the equitable education of all students to identify ways that interrupt the current entrenched practices.

This interruption will require conscientization (i.e. critical awareness of the political dynamics that give rise to the unequal distribution of power) at multiple levels. First, is the recognition of the curricular problem itself, its causes, manifestations and results. Second, is the acknowledgement of our own professional culpability in sustaining this untenable curricular reality and our potential, individually and collectively in transforming the conditions that give rise to injustice. Third is our obligation to "wake" others up, - whether they are educators, students, administrators or community members - to the reality that injustices exist, and their own role in its perpetuation. Part of this awakening is to the institutional conditions in which all educators work.

The dialogue we seek, following Freire, must begin in the context of local manifestations of injustice. School-based or community-based injustices become the "laboratories" in which all educators (indeed all stake-holders) learn and work together. In these contexts all are learner-teachers; all are potential leaders (a role that has no connection with title or credentials); all are curriculum developers. In these roles we recognize that curriculum is not merely what we plan that students learn, but also the messages generated intentionally and unintentionally through our leadership actions in the community. Leadership, in the context of the dialogue entails active listening, soliciting the voices of those unheard and facilitating the collective critical consciousness and empowerment for action.

For university educators this engagement serves multiple purposes. It solidifies the recognition that, in much like the interdisciplinary projects undertaken by curriculum artists, the real problems of school and community cut across disciplinary boundaries. Stepping out of academic siloes to work collaboratively translating our theory into practice, provides us opportunity and rationale for moving beyond these boundaries, such as they exist, within the academy. Seeing the need for our collaboration and engagement in the sites of social injustice within our communities, would likely prove catalytic in our work, both separately and together within our own institutions. Separately, we engage in this work in our classes, our writing and 
institutional obligations but now ever mindful of our separate but equal paths in the collective undertaking towards social justice.

Yet none of this will lead towards an educational system that is led and engaged in curriculum inquiry undergirded by democracy, social justice, and multiculturalism if those three values are not embedded in the theoretical learning and practical application of our disciplines. What excuse is there for our disciplines if the educational leaders and teachers that we produce - both those dedicated to scholarship and those dedicated to its application in public schools - have not achieved conscientization and are not practicing to these ends. Moreover, if they are not engaged in questioning, understanding, and practicing these ends, then how will the students they teach be liberated? Clearly, in the USA our students of today have no conception of social justice, multiculturalism, or democracy as we understand them. They are, after all, like the educators that work with them, subsumed in a neoliberal system that rejects those values.

However, as we have discussed, the USA is pragmatic. Through compromise and practical actions our disciplines can effect change. The recognition of persistent injustices in education compels us to publicly ask of our programs, departments, colleagues and leaders about our institutional role in the interruption of or complicity with such policies and practices. When accrediting bodies or state certification regulations continue their historic practice of using education to marginalize diverse communities, or assume that homogenization of difference is a benevolent educational practice, what has been, is, and/or should be the role of professors in curriculum and leadership and how should they be confronted? Preparing future educators to recognize this injustice, and doing so in tandem, where future principals and teachers enter the field 'on the same page' with regard to diversity and injustices is an important step. But working in isolation in our isolating professional and institutional settings proves inefficient when the status quo of public education needs to be challenged.

Critical perspectives of our fields compel us to recognize our own complicity in setting up the conditions by which technocratic and marketizing forces have uprooted the democratizing aspirations of public education. The siloed existence has precluded dialogue as critical praxis within the academy to the extent that colleges of education as well as public universities themselves have fallen prey to neoliberal ideology and the logic of competition, profiteering and measurement. A naïve consciousness, as Freire described it, has engendered institutional complicity within systems and practices that disempower. While the specifics of how such dialogue occurs might be context-dependent, the rationale for it is clear. We must become "wide awake" rather than anaesthetized to the politics of neoliberalism and marketization that has de-professionalized our educators and that threatens the humanizing, democratizing and empowering potentiality of education, and we must become radical in our approaches to address them. 


\section{References}

Addams, J. (1910/1961). Twenty years at hull house. New York: Signet.

Apple, M. W. (1986). Teachers and texts: A political economy of class and gender relations in education. New York: Routledge.

Apple, M. (2006). Understanding and interrupting neoliberalism and neoconservatism in education. Pedagogies: An International Journal, 1(1), 21-26.

Apple, M. (2012). Foreword. In W. H. Watkins (Ed.), The assault on public education: Confronting the politics of corporate school reform (pp. ix-xiv). New York: Teachers College Press.

Apple, M., \& Beane, J. (Eds.). (1995). Democratic schools. Alexandria: ASCD.

Autio, T. (2014). The internationalization of curriculum research. In W. F. Pinar (Ed.), International handbook of curriculum research. Routledge. https://www.routledgehandbooks. com/doi/10.4324/9780203831694.ch1. Accessed on 27 June 2015.

Ayers, W. (2009). Handbook of social justice in education. Abingdon: Routledge.

Banks, J. A. (2001). Approaches to multicultural curriculum reform. In J. A. Banks \& C. M. Banks (Eds.), Multicultural education: Issues and perspectives (4th ed., pp. 225-246). New York: Wiley.

Berger, N. (1956/1980). The settlement, the immigrant and the public school. New York: Arno Press.

Berliner, D. (2011). The manufactured crisis revisited. http://schoolbriefing.com/1967/the-manufactured-crisis-revisited/ retrieved 8/14/2015

Bobbitt, F. (1918/2013). Scientific method in curriculum-making. In D.J. Flinders \& S.J. Thornton (Eds.), The curriculum studies reader (4th edition). (pp. 11-18). New York: Routledge.

Bogotch, I. (2002). Educational leadership and social justice: Practice into theory. Journal of School Leadership, 12, 138-156.

Bogotch, I. (2008). Social Justice as an educational construct. In I. Bogotch, F. Beachum, J. Blount, J. Brooks, \& F. English (Eds.), Radicalizing educational leadership: Dimensions of social justice (pp. 79-112). Rotterdam: Sense Publishers.

Bogotch, I. (2014). Educational theory: The specific case of social justice as an educational leadership construct. In I. Bogotch \& C. Shields (Eds.), International handbook of educational leadership and social (In)justice. Chapter 4. Dordrecht: Springer.

Boske, C., \& Osanloo, A. (2015). Living the work: Promoting social justice and equity work in schools around the world. Bingley: Emerald Group.

Bourdieu, P. (1986). The forms of capital. In J. Richardson (Ed.), Handbook of theory and research for the sociology of education (pp. 241-258). New York: Greenwood. Bordieu (1986) referred to at $\mathrm{p} 13$.

Bourdieu, P., Passaron, J-C. (1977/1990). Please fill in year/page/replace xx in the reference. Reproduction in education, society and culture.Thousand Oaks: Sage Publications.

Bruner, J. S. (1960/2004). The process of education. Boston: Harvard University Press.

Commager, H. S. (1950). The American mind: An interpretation of American thought and character since the 1880s. New Haven: Yale University Press.

Delpit, L. (1988). The silenced dialogue: power and pedagogy in educating other people's children. Harvard Educational Review, 58(3), 280ff.

Dewey, J. (1916). Democracy and education. New York: Macmillan Co.

Dionne, E. J. (2012). Our divided political heart: The battle for the American idea in an age of discontent. New York: Bloomsbury.

Doll, W. E. (1993). A post-modern perspective on curriculum. New York: Teachers College Press.

Eisner, E. (2002). What can education learn from the arts about the practice of education? John Dewey lecture the encyclopedia of informal education, www.infed.org/biblio/eisner_arts_and_ the_practice_or_education.htm

Eisner, E. (2003). What does it mean to say that a school is doing well? In A. C. Ornstein, BeharHoresnstein, \& E. F. Pajak (Eds.), Contemporary issues in curriculum (3rd ed., pp. 239-247). Boston: Allyn \& Bacon. 
Eisner, E. (2005). Re-imagining schools: The selected works of Elliot W. Eisner. World Library of Educationalists Series. New York: Routledge.

Ellis, J. (2007). American creation. New York: Vintage Books.

Esposito, \& Normore (2015) Race ethnicity and evolution of critically compassionate intellectualism.

Flinders, D. J., \& Thornson, S. J. (2013). The curriculum studies reader (4th ed.). New York: Routledge.

Franklin, B. (1749). Proposals relating to the education of youth in Pennsylvania. Philadelphia: University of Philadelphia.

Freire, P. (2000). Pedagogy of the oppressed (30th ed.). New York: Continuum.

Gay, G. (2001). A multicultural school curriculum. In C. Grant \& M. L. Gomez (Eds.), Campus and classroom: Making schooling multicultural (2nd ed., pp. 31-45). Upper Saddle River: Prentice Hall.

Giroux, H. (2011a). On critical pedagogy. New York: Bloomsbury.

Giroux, H. (2011b). Once more with conviction: Defending higher education as a public good. Qui Parle: Critical Humanities and the Social Sciences, 20(1), 117-135.

Giroux, H. (2013). Neoliberalism's war against teachers in dark times. Cultural Studies $<=>$ Critical Methodologies, 13(6), 458-468.

Gonzalez, G. (1996). Chicano educational history: A legacy of inequality. Humboldt Journal of Social Relations, 22(1), 43-56.

Grande, S. (2004). Red pedagogy: Native American social and political thought. Lanham: Rowman \& Littlefield.

Grant, C., \& Sleeter, C. (2011). Doing multicultural education for achievement and equity (2nd ed.). New York: Routledge.

Greene, M. (1978). Wide awakeness and the moral life. In M. Greene (Ed.), Landscapes of learning (pp. 42-52). New York: Teachers College Press.

Greene, M. (2000). Releasing the imagination: Essays on education, the arts and social change. San Francisco: Jossey Bass.

Greer, C. (1972). The great school legend: A revisionist interpretation of American public education. New York: Basic Books.

Harvey (2005/2010). A brief history of neoliberalism. Oxford: Oxford University Press.

Hirsch, E. D., Jr. (1996). The schools we want and why we don't have them. The Schools we want and why we don't have them. New York: Doubleday.

James, W. (1899). Talks to teachers on psychology and to students on some of life's ideals. New York: Henry Holt.

James, W. (1907/1963). Pragmatism and other essays. New York: Washington Square Press.

Kincheloe, J. L. (2008). Critical pedagogy primer (Vol. 1). New York: Peter Lang.

King, J. (2000). A moral choice. Teaching Tolerance Magazine, 18, 14-15.

King, J. (2015). Dysconscious racism, Afrocentric praxis and education for freedom: Through the years I keep on toiling. New York: Routledge.

Klein, M. (2003). Alternative curriculum conceptions and designs. In A. Orenstein, L. BeharHorenstein, \& F. Pajak (Eds.), Contemporary issues in curriculum (3rd ed., pp. 20-25). Boston: Allyn \& Bacon.

Kliebard, H. M. (2004). The struggle for the American curriculum, 1893-1958 (3rd ed.). New York: Taylor and Francis.

Kozol, J. (2005). The shame of the nation: The restoration of Apartheid schooling in America. New York: Random House.

Ladson-Billings, G. (1995). Toward a theory of culturally relevant pedagogy. American Educational Research Journal, 32(3), 465-491.

Ladson Billings, G. (2006). From the achievement gap to the educational debt: Understanding achievement in US schools. Educational Researcher, 35(7), 3-12.

Lippman, W. (1910). An open mind: William James remembered. http://www.nytimes.com/learning/general/onthisday/bday/0111.html 
Loewen, J. W. (2008). Lies my teacher told me: Everything your American history textbook got wrong. New York: New Press.

May, P., \& Sleeter, C. (2010). Introduction. In P. May \& C. Sleeter (Eds.), Critical multiculturalism: Theory and praxis (pp. 1-16). New York: Routledge.

McLaren, P. (1995). Critical pedagogy and predatory culture: Oppositional politics in a postmodern era. Hove: Psychology Press.

Miel, A. (1961). Creativity in teaching: Invitations and instances. Belmont: Wadsworth Publishing.

Montessori, M. (2013). A critical consideration of the new pedagogy in its relation to modern science. In D. J. Flinders \& S. J. Thornton (Eds.), The curriculum studies reader (4th ed., pp. 19-31). New York: Routledge.

Padgham, R. (1988). Correspondences: Contemporary curriculum theory and twentieth century art. In W. Pinar (Ed.), Contemporary curriculum discourses (pp. 359-379). Scottsdale: Gorsuch Scarisbick.

Patri, A. (1917). A schoolmaster of the great city. New York: Macmillan.

Perlmann, J. (1988). Ethnic differences: Schooling and social structure among the Irish, Italians, Jews and Blacks in an American City, 1880-1935. Cambridge: Cambridge University Press.

Pinar, W. (2004). What is curriculum theory? Mahwah: Lawrence Erlbaum.

Portes, A., \& Zhou, M. (1999). The new second generation: Segmented assimilation and its variants. In N. R. Yetman (Ed.), Majority and minority: The dynamics of race and ethnicity in American life (6th ed., pp. 348-362). Boston: Allyn \& Bacon.

Portes, A., Fernández-Kelly, A., \& Haller, W. (2005). Segmented assimilation on the ground: The new second generation in early adulthood. Ethnic and Racial Studies, 28(November), $1000-1040$.

Ravitch, D. (2014). Reign of error: The hoax of the privatization movement and the danger to America's public schools. New York: Vintage Books.

Reyes-Guerra, D., \& Bogotch, I. (2012). Curriculum inquiry as a transformative leadership skill. In C. Shields (Ed.) Transformative leadership: A reader (Chapter 10, pp. 137-154) New York: Peter Lang Publishers.

Robinson, K. (2006). Do schools kill creativity? TED 2006. Available at: http://www.ted.com/ talks/ken_robinson_says_schools_kill_creativity?language=en

Rose, M. (2011). The mismeasure of teaching and learning: How contemporary school reform fails the test. Dissent (Spring), 32-38.

Sadker, M. P., \& Sadker, D. (2000). Teachers, schools and society. Boston: McGraw Hill.

Seel, H. (1999). 'Allgemeine Didaktik' ('General Didactics') and 'Fachdidaktik' ('Subject Didactics'). Hudson, B., Buchberger F., Kansanen P., Seel H. Didaktik/Fachdidaktik as Science(-s) of the teaching profession? TNTEE Publications, Umea, 2(1), 85-93.

Shields, C. (2014). Leadership for social justice education: A critical transformative approach. In I. Bogotch \& C. Shields (Eds.), The international handbook of educational leadership and social (in)justice (pp. 323-340). Dordrecht: Springer.

Sleeter, C. (2015). Deepening social justice teaching. Journal of Language and Literacy Education [Online]. Retrieved from http://jolle.coe.uga.edu/.

Spring, J. (2010/2013). Deculturalization and the struggle for equality (6th ed.). Boston: McGraw-Hill.

Stack, S. (2004). Elsie Ripley Clapp (1879-1965): Her life and the community school. New York: Peter Lang. 
Tyack, D. (1974). The one best system: A history of American urban education. Cambridge: Harvard University Press.

Tyler, R. W. (1949). Basic principles of curriculum design. Chicago: The University of Chicago Press, 150, 6.

Woodson, C. G. (1933). The mis-education of the Negro. Trenton: Africa World Press.

Zinn, H. (1980/1988). http://zinnedproject.org/about/howard-zinn/

Open Access This chapter is licensed under the terms of the Creative Commons Attribution 4.0 International License (http://creativecommons.org/licenses/by/4.0/), which permits use, sharing, adaptation, distribution and reproduction in any medium or format, as long as you give appropriate credit to the original author(s) and the source, provide a link to the Creative Commons license and indicate if changes were made.

The images or other third party material in this chapter are included in the chapter's Creative Commons license, unless indicated otherwise in a credit line to the material. If material is not included in the chapter's Creative Commons license and your intended use is not permitted by statutory regulation or exceeds the permitted use, you will need to obtain permission directly from the copyright holder.

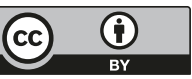




\title{
Chapter 9 \\ Curriculum and School Leadership - Adjusting School Leadership to Curriculum
}

\author{
Stephan Huber, Pierre Tulowitzki, and Uwe Hameyer
}

\begin{abstract}
This chapter looks at the role of school leadership vis a vis the curriculum. First, it offers a brief overview of school leadership in Germany, acknowledging the multitude of systems within the system as each German federal state has autonomy over educational matters. Next, curriculum development and research in Germany is briefly recapped, including historical aspects, and the curriculum work of school leaders on the school level is discussed. Then the discussion is linked to the international discourse on instructional leadership. Next, we conclude with the concept of organizational education ("Organisationspädagogik") as a perspective for viewing school leadership in conjunction with the curriculum. Finally, based on the material presented before, we take a reflective look ahead and ponder possibilities and desiderata of school leadership in the context of curriculum. The chapter shows that school leaders in Germany regard themselves as education professionals deriving from the teaching profession. Instruction and pedagogical tasks and developing a collaborative school improvement culture is what they prefer. Administrative tasks and certain controlling aspects of management are perceived as strain. It is argued that the concept of "educational leadership" is strongly - even if even implicitly - aligned with the knowledge base of instructional leadership as well as of the curriculum discussion in Germany.
\end{abstract}

This chapter is based on an article in LPS: Huber, S.G, Tulowitzki, P. \& Hameyer, U. (2017). School Leadership and Curriculum: German Perspectives. Leadership and Policy in Schools, 16(2), 272-302. The chapter is a shorter account of this article.

\author{
S. Huber $(\bowtie)$ \\ Institute for the Management and Economics of Education, \\ University of Teacher Education, Zug, Switzerland \\ e-mail: stephan.huber@phzg.ch \\ P. Tulowitzki \\ Ludwigsburg University of Education, Zug, Germany \\ e-mail: tulowitzki@ph-ludwigsburg.de \\ U. Hameyer \\ Kiel University \& Hameyer I Systemberatung, Nübbel, Germany \\ e-mail: uwe.hameyer@t-online.de
}




\section{Introduction}

The relationship between school leadership and curriculum in Germany is a complex one. The curriculum was long thought of as being tied to the individual state ("Land", plural "Länder") in the form of the "Lehrplan" (plan of learning). The school principal was and maybe still is often seen as of very limited authority when it comes to curriculum matters due to little decision making-power and due to the pedagogical freedom on the instructional methods level that teachers in Germany enjoy. And yet, the school principal being a former teacher, has a certain affiliation with curriculum matters as many school principals see themselves more as pedagogues and educational leaders than just as managers or administrators.

Curriculum, in turn, is related to the complex idea of Bildung. This concept is typically a normative concept with respect to the purpose and process of education, i.e. reflecting educational ideals as they occur in the curriculum thus guide teaching. According to a more or less common agreement on what Bildung is, the construct refers to a process of Selbstbildung, the individual's reflective acquisition of cultural knowledge, and personal growth and self-development. Especially in the tradition of Humanistische Bildung, i.e. humanistic Bildung, self-cultivation is essential in terms of being the path to cultural knowledge and to become a mature personality that can engage productively and critically in society. The task of education is to support this self-developmental process (Sorkin 1983). The connection between leadership and Bildung is thus established through the purpose of education.

It might be surprising to an international audience/readership that school leadership research is relatively young in Germany (it just started in the 1980s) and all the other German-speaking countries; Switzerland, e.g. even has established school leaders just the last 20 years (Huber 2016a, b, c). In other words, there are many facets and grey areas to this complex and not well elaborated and discussed is the relationship of school leadership and curriculum.

This chapter looks at the role of school leadership vis á vis the curriculum. First, it offers a brief overview of school leadership in Germany, acknowledging the multitude of systems within the system as each German federal state has autonomy over educational matters. Next, curriculum development and research in Germany is briefly recapped, including historical aspects, and the curriculum work of school leaders on the school level is discussed. We then link the discussion to the international discourse on instructional leadership. Next, we present the concept of organizational education (“Organisationspädagogik", see Rosenbusch 1997, 2005) as a perspective for viewing school leadership in conjunction with the curriculum. Finally, based on the material presented before, we take a reflective look ahead and ponder possibilities and desiderata of school leadership in the context of curriculum.

The Federal Republic of Germany is comprised of 16 federal states, known in German as "Länder". As a federal principle, matters of education and culture lie with each state. This means that each of the 16 states has its own school system framed by individual jurisdictional and administrative laws, encompassing its own 
educational-policy goals, school structures, school types, curricula, etc. Therefore, the 16 school systems in Germany feature different educational and governing traditions. Despite these differences, the governing of each state is organized according to a rather traditional bureaucratic governing model.

The Minister of a state usually represents the top of the governing structure (macro-level) with a succession of subordinate institutions (meso-level), with the schools themselves functioning as the lowest units (micro-level). In larger states like Bavaria, North Rhine-Westphalia, and Baden-Württemberg, there is a fourlevel administrative organization, which includes the ministry, a state office for education and/or school quality and the regional school supervisory administration, the school supervisory offices at the level of counties or county-independent cities, and finally school leadership at the school level (for further information see also Huber et al. 2016).

\section{School Leadership in Germany}

In comparison with their peers in many other countries, school leaders in Germany have limited authority, in part due to Germany's bureaucratic traditions. They have restricted authority over staff employment and dismissal, and they have limited control over financial resources. Nevertheless, school leaders are responsible for enforcing national and school's regulations and for the daily management of school life and lessons. Furthermore, they are responsible for representing the school, which includes maintaining contact with neighboring schools and institutions as well as the community. Historically speaking, they were in charge of the administrative tasks on organizational school level. Only over the last two decades further schoolbased responsibilities have emerged as a result of the decentralization of decisionmaking processes, usually shifting some of them from federal state system level or regional authority level towards the organizational school level. School-based management has been implemented in nearly all federal states over the last 10 years, known as "self-responsible school or autonomous school". However, the degree of decision making power as well as the resources allocated to the school varies from state to state. Generally speaking, and in particular when comparing Germany with other OECD countries, new public management is not implemented to the same extent. Hence, the influence of school leaders is still restricted while teachers are relatively free to make didactical and methodical decisions on the basis of their 'pedagogical freedom' as it comes to teaching and education. Furthermore, on organizational school level the school conference (or the school community conference), which consists of teachers and parents is the highest decision-making body and the school leader is obliged to implement and follow decisions made in this conference.

The school leader's teaching obligation depends on the kind of school, the number of classes and the number of pupils in her or his school. In a grammar school with more than 1000 students, the teaching obligation of a school leader is at least 
two lessons per week (the maximum is at $11 \mathrm{~h}$ a week in certain states); teachers at primary schools teach - depending on the state -23 to $27 \mathrm{~h}$ a week. Hence, school leaders in elementary schools have considerably more lessons to teach. School leaders are supported by vice-school leaders and by other staff who take over specific tasks, such as devising lesson plans, school career counseling and extra-curricular tutorship. In recent years all principals became real superior to the staff and took human resource management duties over from the local or regional authority like conducting the official assessments of teachers.

Vacant school leadership positions are announced publicly. Applicants' backgrounds are checked including an assessment of their past achievements and their teaching skills. A basic prerequisite for being appointed as a school leader is teacher training for, and teaching experience in the respective school type; additional qualifications are an advantage. These could be things like previous experience as a deputy school leader, experience on senior management teams or experience as an instructor in charge of the induction phase of teacher training. Mostly, however, the state examinations and in particular the official assessments of teaching competences by superiors as the deciding factors are taken into account. The candidates who are evaluated as most suitable are appointed school leader for life in a tenure track civil servant position.

\section{Curriculum in Germany}

Transforming the school curriculum and pedagogical leadership in Germany is not so much the result of a nationally orchestrated activity as it is an inside-the-state effort with partly growing local choices but also new systems of control by accountability measures, national education standards (Bildungsstandards), national standard testing, evaluation as well as systems monitoring agencies (Qualitätsagenturen) which are run by each of the majority of the 16 states. The degree of diversity between the schools have depended since decades, on how power and trust, theory and practical wisdom converge in common goals (Hameyer 2010; cp. Hameyer et al. 1983).

\section{A Brief Historical Look at Recent Curriculum History}

Curriculum development in Germany is embedded into an extended history of theorizing the syllabus (Dolch 1971; Meyer 1972; Paulsen 1892, 1896a, b; see also, Roth 1968; Weniger 1971, 1975) from the perspective of what "Bildung" is. The state does not always agree with what theorists proposed but German history provides examples of a powerful impact of theory on the syllabus (see the works of Humboldt, Dörpfeld, among other influential scholars of the nineteenth century). 
When curriculum research emerged in Germany particularly in the beginning of the seventies, the scientists studied approaches from abroad. Some of them combine German historical roots with what happens in the curriculum field in the US, in Switzerland, and Sweden (Elbers 1973; among many others cp. Frey 1971; Flechsig and Haller 1973 who investigate conditions for participative decision-making; Hameyer 1978; Hörner and Waterkamp 1981; comparative studies issued by the International Institute for Educational Research in Frankfurt).

In addition, syllabi and schoolbooks are analyzed and theoretically compared according to their impact on curriculum and instructional practice. Research projects are started, implementation issues more carefully integrated and curriculum process models developed. Curriculum research strives for scientific exchange and continuity both in terms of cross-national studies and with respect to what we learn from the "theory of the curriculum as syllabus" and various theories of Bildung (Oelkers 1983).

Within this scope of recent trends, there are attempts to redefine "Bildung". Redefining Bildung is a lasting process which is simultaneously given momentum particularly by the influential works of von Hentig $(1985,1993$, 1996), who conceives Bildung as a reflective effort of students ("sich bilden") to make up their own understanding of meaningfulness when they explore the world by reflective, experiential activities in schools seen as a place of democratic community and deliberate thought. A couple of years later, Tenorth $(1986,2001,2004)$ and other theorists redefine Bildung in association with modern views on the public curriculum.

Recently, schools are encouraged to develop local educational programs congruent with the syllabus. They do so by school-based curriculum renewal (often in intuitive ways). The respective state-run organization, the "Landesinstitute", that exist in each of the 16 states, is expected to help the schools in their own curriculum development by means of consulting the schools, providing materials, creating joint projects, qualifying teachers for this new demand, and networking schools within and beyond communities.

School development and syllabus work are considered major places to put conceptual ideas about Bildung into practice. This provides for an opportunity to combine and reground the domain of curriculum theory and the theory of Bildung in a more coherent, stimulating way. So far, the scientific communities of curriculum theory and school development, the theory of education, and the empirical stakeholders of instructional research are still separate worlds. There are still only a few attempts in Germany to unfreeze the separateness and establish a continued scientific dialogue between these worlds (Hameyer 2010).

Emergent Ways to Conceive the Curriculum Taba (1962) proposed the most simple definition of curriculum we are aware of: curriculum is a plan to learn. This is more than a headline, yet too vague to specify particular features of a curriculum so that the construct can be used in more precise ways. Defining curriculum is a task that has been the subjects of debates and shifts for several decades (Hameyer et al. 1983; Kelly 2009; Portelli 1987; Toombs and Tierney 1993; Wiles 2008). We therefore refer to Kerr's classic definition of curriculum being "all the learning which is 
planned and guided by the school, whether it is carried on in groups or individually, inside or outside of school" (1968, p. 16) as a humble point of departure although it does not include the hidden, tacit curriculum which got some attention in Germany. This simple definition implies what is taught to whom and by whom - questions that concern teachers, school leaders, but also policy makers. They are likewise influenced by the political as well as the socio-cultural sphere, but also the school culture and what happens on the informal stage in the school. The awareness towards the informal and hidden agenda in the instructional learning and teaching process is growing as to what has been published since two decades.

Like many other scholars, Pinar points to the intricate relationships between society, politics and education when laying out the educational aspect of the curriculum:

The educational point of the public school curriculum is understanding, understanding the relations among academic knowledge, the state of society, the processes of self-formation, and the character of the historical moment in which we live, in which others have lived, and in which our descendants will someday live. (Pinar 2004, p. 187)

Looking at the curriculum itself, even when it is framed by external parameters, it arguably has different faces. It can be codified or enacted (Hameyer 2007). Sometimes it is blended by a tacit set of personal or public norms. It can be specified in tests or standards, in approved textbooks or self-made teaching materials, in a guideline or it can grow out of local school programs and regional development plans.

Goodlad (1960) reflected the phenomenology of a curriculum, later referred to as the representative levels or modes of a curriculum as we mention later in this section (e.g. the hidden, the tested, the codified curriculum). The approach of Goodlad was later taken up by Jan van den Akker (2004) and Uwe Hameyer (2007). In this section, the idea is briefly discussed from a more systemic view, which reflects the representational levels in their interplay in various ways. This is needed if we want to understand the transformation of domain knowledge by curriculum reasoning, policy-making and its enactment in practice. The perspective of representation includes the invisible. An invisible curriculum is tacit or hidden. It is rooted in the minds of every teacher, policy-maker, parent, or student when they think about what should be learned at school. Tacit images shape not only what people think but also what they do and - at the same time - what they dislike (e.g. Morgan 2006). According to this view of the representational curriculum, we can discern the following 'faces' or levels:

- the codified curriculum

- the perceived curriculum

- the intended curriculum

- the enacted curriculum

- the experienced curriculum

- the tested curriculum

- the hidden curriculum 
The interplay of these levels helps us to understand what happens during the transformation process of domain knowledge on its way from outside into the school and its local enactment. A teacher who likes knowledge-based sequential learning within his subject perceives and interprets the curriculum probably differently from one who favors a daily-life-focused, exemplary design of instruction. Both will enact the compulsory curriculum in other ways according to their own aims which may be underpinned by tacit purposes.

A tested curriculum selects by nature something that is considered to be pars pro toto. Tests are norm-referenced; students are compared to others inside or outside the school. Test results are given institutional power which can be used for accountability goals and are individual placement decisions (see also Easley and Tulowitzki 2016).

In Germany, transforming the school curriculum is not the result of a national effort but rather that of efforts within individual states with a growing range of choices due to the federal autonomy described in the beginning of the chapter. A big impact stems from new systems of control by external evaluation, accountability demands, and changing patterns of leadership (Rolff et al. 2009).

Monitoring and evaluation systems, standards and national benchmarks have been put into place in many European countries (mostly initiated by central and regional authorities). For example, some cantons in Switzerland use quality and qualification plans (Q2E, for a brief presentation see Heidegger and Petersen 2005). This means that curriculum development is very much a matter of professional learning and continued development of competencies within and across schools, putting teachers in an expectation of constant learning but also putting school principals in a position where they need to have curriculum competencies. In Germany this can be seen in various ways where curriculum change is much more driven by the syllabus and by what the market offers than by developments from schools themselves.

At the same time, an argument can be made that teachers have not been sufficiently trained for systematic quality-based efforts and that school principals have not been sufficiently prepared for dealing with curriculum matters in depth. Many from inside and outside schools express concerns that teacher education, pre-service and in-service training only enables teachers to practice curriculum design and renewal on a limited scale (Handelzalts 2009). In addition, there is a lack of knowledge in schools when it comes to medium- and longer-term planning. In spite of this gap between the quality demands across schools and the given knowledge to implement goals inside schools in professional ways, schools have to master the challenges and choices that come with expanded freedom on a local level. We can specify some of the current challenges in terms of four major demands:

- The quality demand, i.e. to compare and compete with other schools, also to look at the quality of teaching and its impact on what the students learn; in addition, to improve the school curriculum quality according to internal and external standards. 
- The equality demand, i.e. to improve educational possibilities for all students, including the gifted, talented and the disadvantaged.

- The diversity demand, i.e. to take into account heterogeneous, sometimes diametrically opposed groups (e.g. poor vs. rich, employed vs. unemployed, social security vs. economic poverty, integration vs. segregation, minorities and migration background issues).

- The competency demand, i.e. a shift from the academic knowledge towards abilities (competencies) to use knowledge reflectively. This also includes mastering knowledge-based methods to solve key tasks in life and work. In addition, this demand entails a redefinition of how to learn and how to apply knowledge in practical situations. Thus, competency development comes into the forefront of syllabus work and curriculum renewal (Hameyer and Tulowitzki 2013).

For several years, one instrument above all has been increasingly affecting the curriculum practice in the schools: national education standards. These standards focus mostly on competencies to be achieved according to different levels of quality. This entails a shift away from the traditional German notion of Bildung towards the notion of literacy or competency, which is more prevalent in the US and the UK (Neumann et al. 2010). The standards are expected to stimulate schools to bring a certain level of cohesion across schools - even across the Länder boundaries. This marks an important development. Never after World War II has it occurred that all Länder agreed upon a common standard system for the school curriculum.

Another development is that schools in Germany must nowadays develop 1- or 2-year objective agreements ("Ziel- und Leistungsvereinbarungen") with governance authorities. These contracts focus on profile areas of the individual school. The curriculum is part of this contracting system. Additionally, individual schools are given various degrees of autonomy depending on the Land where they are located. Within this framework of relative autonomy, a school can attune its own curriculum to local demands and profile choices. Last but not least, schools have to work on getting and using data-driven feedback. This means that the curriculum work is framed by a mix of external parameters.

The scope of local or school-based curriculum development (Skilbeck 1998) has, to some extent, increased, i.e. schools have gained more autonomy and more duties, especially to create their own curriculum or adapt existing curricula in contextuallysensitive ways. These demands have to be (made) compatible both to the local and regional needs as well as to national standards and the syllabus. At least in Germany, there is still on-going irritation regarding how to handle this double-bind situation. Schools and teachers who are committed to these functions value this "tested curriculum approach" positively in contrast to those who think that tests are counterproductive with respect to local efforts to create own school-internal standards for student achievement and practice. With this in mind, these latter schools rely on the concept of autonomy as promised and granted by parliaments.

The current state of autonomy means that schools are facing a widening array of choices. This is also reflected in the growing number of documents relevant to the development and implementation of a curriculum. 'Public accountability and con- 
trol patterns' enter the school system through various measures: comparative achievement tests across all schools and all 16 'Länder, even though each 'Land' is, by law, independently responsible for its public schools. External evaluation schemes have taken root and new syllabi have been introduced. Monitoring systems have been designed and installed by state-level authorities. Internal quality management efforts are expected from each school. Such state-level measures are blended with external patterns to 'help' schools improve and aid them in using support systems.

Consequently, schools have to show themselves accountable of the results of their work, yet - at the same time - there is a need to individualize learning opportunities and instruction. The various, mostly state-driven forces behind the school curriculum give rise to a growing suspicion of many teachers and to a loss of confidence in the system (Hameyer 2006) because the politics of school autonomy, which started nearly two decades ago, are substantially contaminated by a growth of external forces bearing down on the school. This is also true for the domain knowledge as transformed in the curriculum.

Many professional schools cope with these developments akin to how they cope with external measures: in a more or less sovereign way. They master external requirements in strategic and creative ways, though some suffer for various reasons, such as bad working conditions. They are the losers and there are already programmes to work with these 'failing' schools. It is evident that the growth of the external pressure on schools increases the probability that the schools will differ much stronger in terms of quality than they did before; in other words: the 'accountability management pattern' can, paradoxically, lead to increasing the differences between schools. In Germany we call this divide a 'Schereneffekt' which does not only apply to the quality of schools but also to the discrepancies between the levels of curriculum representation shown above.

Leadership, especially shared leadership oriented towards improving student learning opportunities, can be seen as the lever activating productive work in the domain of the curriculum. However, as van den Akker has acutely pointed out, despite big investments in research, development and professionalization, "the target group of teachers often appears poorly informed about an intended innovation, while its practical application remains limited, and its impact on student learning is unclear. Simple explanations for innovation failures are inadequate, but a few gaps are often visible:

- weak connections between the various system levels (national, local, school and classroom)

- lack of internal consistency within the curriculum design

- insufficient cooperation between various actors in educational development" (van den Akker 2010, p. 178).

It can be argued that school principals are in a key position to strengthen the afore-mentioned weak connections and to ensure an internal consistency within the curriculum design as well as support cooperation between the various actors. This 
hinges on them being knowledgeable in the curriculum domain as well as them giving curriculum matters the attention and priority necessary.

As laid out before it is our contentation that teachers are not professionally educated for curriculum design and development roles and that school principals in Germany are equally ill-equipped in this regard. In domains such as project management, curriculum knowledge or teamwork, schools are more likely to improvise than to proceed systematically. Some competency requirements which would make the work at school more professional and effective are curriculum competencies, project competencies, team competencies, communication competencies, evaluation competencies and retrieval competencies. Table 9.1 shows detailed breakdowns of these competence groups (for further information about leadership development, see Huber 2013c).

To improve the odds of schools with curriculum-competent staff, it is important to not only instill these competencies in the teaching staff but also to ensure the school principals are proficient in them.

\section{Empirical Insights: Preferences and Strains in School Leadership Practices and the Importance of Curriculum}

In the following section, a study is presented which was conducted in the German speaking countries (Huber 2013a, b, 2016a; Huber and Schwander 2013; Huber and Wolfgramm 2013a, b; Huber et al. 2013a, b). It aimed to gain empirical insights into the work setting of school leaders. Its goal was to demonstrate which of their professional activities school leaders like to do (preferences) and which are a strain on them (strains). Moreover individual factors (e.g. aspects of one's professional biography) as well as institutional factors (e.g. conditions of the work setting) were tested as predictors of job strain. For operationalization purposes, Huber's (2012, 2013c, 2016b), Huber et al. (2012) model of school leadership practices was used and Böhm-Kasper's (2004) model of school-related strain was adapted to the contextual specifics of school leadership.

Altogether 5.394 school leaders participated in the general inquiry (representing a response rate of 49\%). The sample consisted of 3764 school leaders from Germany, 741 from Austria and 889 from Switzerland and Liechtenstein. The school leaders were between 25 and 66 years old $(\mathrm{M}=52.45 ; \mathrm{SD}=7.75)$ at the time of the study. For the analysis of quantitative data, structure equation modeling and path analysis were used.

By conducting exploratory and confirmative factor analysis, we can group activities to nine different fields of activities. Figure 9.1 illustrates the stress of and preferences for the nine different fields of activities on scale level differentiating for the three German speaking countries: Germany, Austria and Switzerland. The analysis of the specific strain experiences, which is the strain by specific activities, types of 
Table 9.1 NAME $\mathrm{x}$

\begin{tabular}{l}
\hline Curriculum competencies \\
\hline Defining a rationale \\
\hline Designing a curriculum \\
\hline Developing a coherent curriculum system \\
\hline $\begin{array}{l}\text { Setting up a process model for implementation and } \\
\text { feedback }\end{array}$ \\
\hline Structuring curriculum units and moduls \\
\hline Using key concepts and fundamental ideas \\
\hline Testing beyond one's own practice what works \\
\hline Evaluating a curriculum and its use \\
\hline Project competencies \\
\hline Planning curriculum work over longer time spans \\
\hline Linking different stages of projekt work \\
\hline Using project models for cross-case management \\
\hline Defining indicators of success \\
\hline Presenting results \\
\hline Team competencies \\
\hline Understanding the secrets of group dynamics \\
\hline Sharing work effectively \\
\hline Clarifying the starter aims \\
\hline Contracting team work \\
\hline Identifying and managing team conflict \\
\hline $\begin{array}{l}\text { Using methods of brainstorming and idea } \\
\text { production }\end{array}$ \\
\hline Sustaining team work over difficult times \\
\hline $\begin{array}{l}\text { Setting up different roles and commitments within } \\
\text { the team }\end{array}$ \\
\hline Comung \\
\hline
\end{tabular}

\section{Communication competencies}

Presenting clearly, also using advanced organizers

Giving and receiving feedback

Sharing rules of communication and feedback

Coaching others and being coached

Focusing complex stories down to a few major insights

Summarizing the easy and difficult points

Reflecting one's own patterns of communicating

Deliberating rather than stating

Using concepts from research (such as TZI or other)

Listening and paraphrasing

Clarifying a problem before valuing it

\section{Evaluation competencies}

Formulating indicators of success

Applying formative evaluation methods

Interpreting complex survey data (data-driven analysis)

Combining process and outcome data 
Table 9.1 (continued)

\begin{tabular}{l}
\hline $\begin{array}{l}\text { Writing a clearly structured report for } \\
\text { non-participants }\end{array}$ \\
\hline Exploring the impact of curriculum use \\
\hline Retrieval competencies \\
\hline Knowledge management \\
\hline Briefing and debriefing \\
\hline Knowing where to find important information \\
\hline Using expertise \\
\hline Retrieving knowledge from data baselines \\
\hline Simplifying complex information for practical use \\
\hline Supporting information work inside the school \\
\hline Reporting interim findings on demand in clear ways \\
\hline $\begin{array}{l}\text { Storing knowledge effectively over longer time } \\
\text { spans }\end{array}$ \\
\hline
\end{tabular}

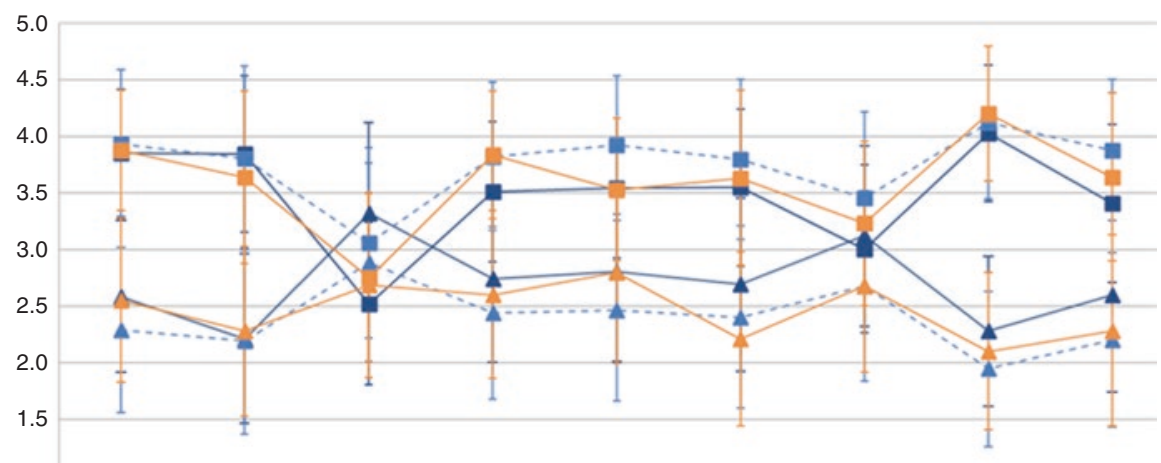

1.0

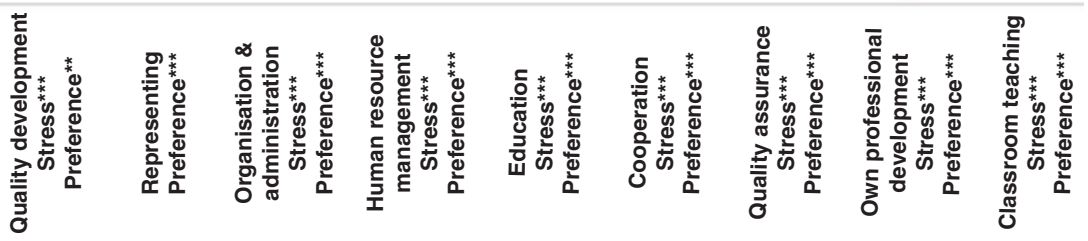

- Stress Germany (N=3515)

-2- Preferences Germany ( $\mathrm{N}=2831$ )

- Stress Austria ( $\mathrm{N}=473)$

- -1.1- Preferences Austria ( $\mathrm{N}=683$ )

- Stress Switzerland $(\mathrm{N}=721)$

-10- Preferences Switzerland $(\mathrm{N}=540)$

Fig. 9.1 Strain by and preferences for the different activity fields by German school leaders (compared to Austrian and Swiss school leaders)

activities and areas of practices clearly gives evidence that organizational and administrative activities are perceived as particularly stressful and most disliked. Activities closely connected with teaching and education (such as teaching in a class, talking with students, exchanging ideas with colleagues, and pursuing one's own professional development), proved to be very popular and were perceived as 
only slightly stressful. The same pattern can be found in the analysis of the types of activities: all school leaders experience activities that are close to education, close to classroom teaching and involving professional exchange with colleagues as less stressful than other types of activities.

In general it can be stated that school leaders who experience an activity as stressful do not like to perform this activity as much as activities perceived as not (or less) stressful, which, in turn, are more popular.

Moreover, the following tendency has become obvious: tasks that belong to the traditional range of tasks of teachers are more popular among school leaders and are experienced as less stressful than tasks that have been recently added to school leadership responsibilities through changes in the school system as a consequence of decentralization (new public management).

The fields of quality development, human resource management, education contain important singular activities associated with curriculum work. Quality development comprises activities such as contributing to the school's development plan, defining strategic aims for school development, initiating teamwork, stimulating interdisciplinary projects with teachers. Human resource management comprises activities such as evaluation of teacher lessons, encouraging teachers to try out new teaching methods, critically reflecting on teaching practice together with eteachers, advising teachers in their work, assessing teachers'performance. Education comprises activities such as developing teaching concepts with staff. All of these activities are associated with improving teaching and learning and hence are not seen as highly stressful compared to the other activity fields.

It could be argued and with further research easily demonstrated, that their original motives to choose the teaching and education profession and the professional socialization as well as the system of promotion contributes largely to this orientation of job preferences and the experience of strain.

Besides preferences, another question is, what school leaders actually do. The findings of the analysis of 4330 end-of-day logs of school leaders in Germany show that organizational and administrative activities take up most of a school leader's work day. Figure 9.2 shows that school leaders invest on average one-third of their time in these activities. About one-quarter of their time is used for activities concerning one's own classroom teaching, with huge variations seen according to the size and type of school (elementary/secondary, explanation see above). Education and guidance and personnel matters are in the mid-range. We can conclude, even if the preferences lay differently, the role as school leaders by just the analysis of daily practices is a more administrative one.

To sum up, the data suggests that school leaders in Germany (as well as in other German speaking countries) can be associated with the concept of primus inter pares. They are strongly rooted in the teaching profession. While the school principals' preference to teach classes cannot necessarily be identified with instructional leadership, it does at least indicate that their professional understanding is strongly rooted in teaching practices.

Besides their own teaching duties, leadership practices, which are associated with transformational and instructional leadership are preferred and perceived as 


\section{Percentage of work time for each practice on typical days}

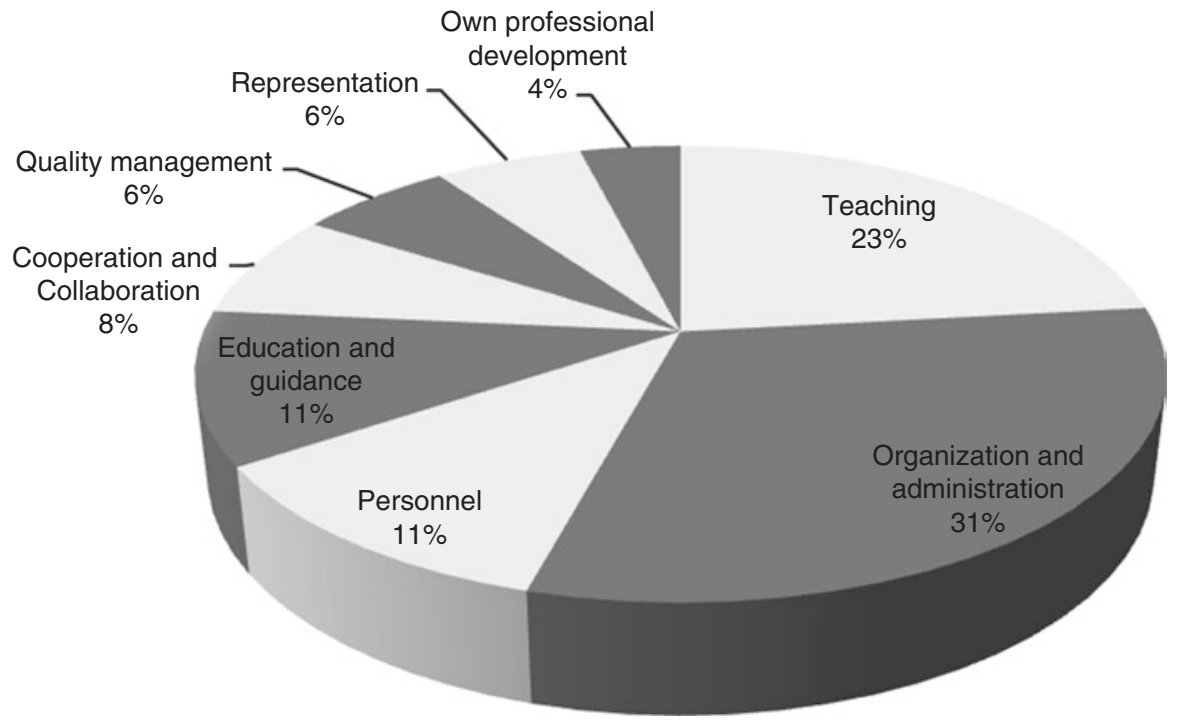

Fig. 9.2 Distribution of work time of school leaders (as percentage) in one German state

less stressful than other practices. Yet, school leaders mostly perform activities they do not prefer and they experience mostly as burden. Hence they do less work around curriculum in schools even if activities associated with curriculum work are experienced as preferred tasks and as tasks they do not experience as burden compared to administrative tasks. We therefore argue that this professional understanding is a positive prerequisite for curriculum work on the school level.

\section{Instructional Leadership}

In 1967 Bridges pointed out the fact that instructional leadership was an underdefined concept:

On the one hand, the principal has been exhorted to exert instructional leadership while on the other hand, he has been told flatly that such a role is beyond his or any other human being's capacity. The problem with these disputations is that the exponents of a given peosition have neither defined sharply what is signified by the concept of instructional leadership nor made their assumptions explicit. (Bridges 1967, p. 136)

In the US, the effective schools movement greatly spurred research in the domain of instructional leadership. Once the notion that schools did not matter (Coleman et al. 1966) had been refuted (Rutter 1979), attention quickly turned towards also looking at the school principals. Evidence suggested that in schools that were improving in challenging circumstances, the school principal was more likely to be 
an instructional leader (see for example Edmonds 1979). This led to increased research efforts in this area, characterising effective instructional principals also focused mostly on improving student outcomes (Hallinger and Murphy 1986; Leithwood et al. 1990). This was complemented by research on the work activity as well as the time-use of school principals (Kmetz and Willower 1982; Martin and Willower 1981), indicating that principals typically actually spend quite a scarce amount of time on instructional leadership due to a myriad of other activities and thus dampening the enthusiasm for the principal as omnipresent chief instructor (among many other things). Later studies from various contexts solidified these results, often finding that administrative duties overshadowed curriculum and instruction (Horng et al. 2010; Spillane and Hunt 2010; Tulowitzki 2013; Wildy and Dimmock 1993; Huber et al. 2013a, b).

The rise of transformational leadership triggered a discussion on the merits and characteristics of transformational leadership vs. instructional leadership. An oftenmade distinction in this regard is the more direct involvement of instructional school leaders in teaching and learning processes while transformational leaders typically seek to generate second-order effects (Hallinger 2003), trying to improve the capacity of staff who in turn produce first-order effects on learning.

Recapping its history and looking at its current state Hallinger and Wang conclude that "instructional leadership has become increasingly accepted globally as a normative expectation in the principalship" acknowledging that while other models have come and gone, "scholarly interest in instructional leadership has remained surprisingly consistent and strong" (Hallinger and Wang 2015, p. 15).

Despite or perhaps because of the fact that it has rarely been exhaustively defined, instructional leadership has maintained popularity in the leadership discourse (Hallinger 2005). Instructional leadership can be viewed as centered on the quality of teaching in classrooms. It "typically assumes that the critical focus for attention by leaders is the behaviours of teachers as they engage in activities directly affecting the growth of students" (Leithwood et al. 1999, p. 8). Emphasis is put - as the name suggests - on the principal having a succinct understanding of instruction in general, but also of the curriculum so as to be able to judge what is taught and how and to provide appropriate feedback. Thus, from an instructional leadership perspective, the principal is responsible for but also influential regarding the quality of teaching of her/his staff. Common areas of activity of instructional leadership include (Krug 1992, pp. 433-434):

- defining mission

- managing curriculum and instruction

- supervising teaching

- monitoring student progress

- promoting instructional climate

These areas are close to areas often associated with the tasks of teachers, highlighting how instructional leadership activities can often cross paths with typical teacher activities. 
Instructional leadership and matters of curriculum as well as curriculum research have been linked on several occasions. This even led to the rise of the term "curriculum leadership", often used similarly to "instructional leadership" (for example in Fidler 1997; Lee and Dimmock 1999) though never gaining the latter's predominance.

Data from the OECD PISA studies show that instructional leadership is being practiced by German school principals. Principals in the US, the UK and Australia (among other countries) tended to report they practice greater instructional leadership, while principals in Japan, Liechtenstein, France, Tunisia and Switzerland reported to practice this less than principals in other countries and economies with German principals reporting to practice instructional leadership slightly above OECD average (OECD 2013). The 2014 OECD Policy Outlook for Germany saw increasing autonomy and an above-OECD-average use of instructional leadership in Germany by German school leaders (Klumpp et al. 2014).

However, putting instructional leadership into practice is challenging to say the least. Echoing the curriculum competencies presented earlier in this chapter, Southworth (2002) found instructional leadership requiring school leaders to be competent in (among other things) the "knowledge of curricula, pedagogy, student and adult learning and skills in change management, group dynamics and interpersonal relations and communications" (pp. 85-86). Currently, these competencies are not systematically developed through pre-service or in-service training for German school principals (see also Tulowitzki 2015).

Echoing reservations regarding the actual feasibility in the German context Kuper (2008) deemed instructional leadership too complex, expressing skepticism that a principal might at the same time keep a good managerial overview and be deeply involved in the teaching operations, being able to give valuable feedback to teachers.

To conclude, we see that school leaders are grounded in education, we see the importance of school-based curriculum work, we also see the international discussion on instructional leadership and the emphasis given to the core purpose of school and schooling. However, the questions are, do German school systems, school leaders, teachers etc. tick the right way, do they have their perspectives and practices right, do they pick the right activities and do they carry them out rightly?

In our opinion, there is a long tradition in educational practices that allow an alignment of purpose and practices, also of school leaders as far as curriculum is concerned. However, there are bureocratic system traditions in Germany which interfer in school leadership practices of "doing the right rightly" as well as legal constraints and limitations of school leadership authority when it comes to pedagogical matters, including the curriculum. This can be viewed as a structural challenge. Finally, attempts to work on curriculum matters within a school need to be aligned with local as well as state and (when considering the national educational standards) possibly even national standards, making it challenging to achieve a coherence in curriculum agendas and settings. 


\section{Organizational Education}

In the German-speaking context, the notion of 'organisational education' as a field of research (see Rosenbusch 1997) focuses the mutual influence of the school as an organisation within an education system on the one hand and the educational processes on the other hand. The core question of organisational education raises a two-fold issue: which educational effects do the nature and conditions of school as an organisation have on individuals or groups within the organisation - and vice versa, which effects do the conditions in and the nature of individuals or groups within the school have on the school as an organisation. More to the point: how must a school be designed in order to guarantee favourable prerequisites for education and support educational work? Hence, organisational education would look at the influence of the school leadership on the teaching and learning process and would argue not only that learning should be the focal point of school leadership but also that leadership and the whole organisation should follow the purpose of school and schooling and be designed to best fullfil the core purpose.

Hence, organisational structures and process have to be brought in line with educational goals. This also implies a leadership approach. In the context of organizational education, school leadership practices become educational-organizational activities, and educational goals become super-ordinate premises of this action. This means that school leadership practices themselves must adhere to the four main principles of education in schools - that school leaders themselves assume or encourage maturity when dealing with pupils, teachers and parents, that they practise acceptance of themselves and of others, that they support autonomy, and that they realise collaboration. This adjustment of educational perspectives affects the school culture, the teachers' behaviour, and the individual pupils. Organizational conditions have to be modified accordingly, and be in compliance with educational principles. Thereby, the unbalanced relationship (which is historically conditioned in many countries) between education on the one hand and organisation and administration on the other hand can be clarified and aligned.

This implies, according to Rosenbusch (1997), that school leadership can be based upon certain constitutive educational principles:

- School leaders should adjust their educational perspective: educational goals dominate over administrative requirements, administration only serves an instrumental function.

- School leaders should take two levels of their educational work into consideration: first school leaders have to work with children and promote their learning, and second, as they also have to work with adults, they should promote their learning as well. Hence, conditions of adult education and adult learning have to be taken into account. This has to have an impact on their leadership and management style, particularly in professional dialogues, when knowledge is shared, expanded, and created. Therefore, school leaders have to integrate the two levels of child education and adult education in their educational perception and behaviour. 
- School leaders should be more resource-oriented than deficiency-oriented: a new orientation towards promoting strengths instead of counting weaknesses is needed. So far, in many countries bureaucratically determined school administration has concentrated on avoiding mistakes, on controlling, detecting, and eliminating weaknesses instead of - as would be desirable from an educational point of view - concentrating on the positive aspects, reinforcing strengths, and supporting cooperation; it should be about 'treasure hunting instead of uncovering defiencies'.

- School leaders should follow the 'logic of trusting oneself and others': it is necessary to have trust in one's own abilities and as well as in those of the staff and others so that empowerment, true delegation, and independent actions can be facilitated. Then, mistakes can be addressed more openly.

- School leaders should act according to the principle of 'collegiality in spite of hierarchy': individual and mutual responsibilities have to be respected and appreciated although special emphasis is placed on a shared collegial obligation regarding the shared goals.

In contrast to classical instructional leadership literature, the leadership concept of 'organisational-educational management' assumes a definition of 'educational' which not only incorporates teaching and education processes with pupils, but also with adults, as well as organisational learning. Organisational-educational management and leadership are committed to educational values, which are supposed to determine the interaction with pupils and the cooperation with staff as well. Administrative aspects fulfil a clearly defined function as instruments for reaching genuinely educational goals. These goals should determine the school as an organisation and thereby change it so that it becomes a deliberately designed, educationally significant reality for all. Leadership action also needs to be a model for what the school seeks to teach and preach, that is, it should shape a model-like social space for experiences for all the stakeholders by realising educational goals to the benefit of the organisation and the individual.

Consequently, the core principle of leadership action is to promote learning of all the members of the organization and in a democratic society to promote 'democracy', both as an aim and a method. Due to the complex hierarchy within the school, democracy represent an adequate rationale for actions concerning the intrinsic willingness and motivation of staff and the pupils for co-designing the individual school. However, democracy is not only valuable as a means for reaching goals, it is a decisive educational goal in itself. The same holds true for aspects of cooperation and collaboration. As far as 'cooperation' is concerned, following Wunderer and Grunwald (1980) and Liebel (1992) defines 'cooperative leadership' as (1) exerting goal-oriented social influence for performing shared tasks or duties (goal-achievement aspect) (2) in/with a structured working environment (organisational aspect) (3) in the context of mutual, symmetric exertion of influence (participative aspect) and (4) designing the work and social relationships in a way that enables a general consensus (pro-social aspect). Here, an organisational and a cooperative perspective are combined.

Developing these ideas would result in a broad distribution of leadership responsibility, that is in a 'community of leaders' within the school. This view is also taken 
by Jackson and West (1999), in their depiction of 'post-transformational leadership'. If the school is supposed to become a learning organisation, this implies the active, co-determining and collaborative participation of all (see also 'distributed leadership'). The old distinction between the position of the teachers on the one hand and the learners on the other cannot be sustained, nor can the separation between leaders and followers. Therefore, leadership is no longer statically connected to the hierarchical status of an individual person but allows for the participation in different fields by as many persons from staff as possible. This also extends to the active participation of the pupils in leadership tasks.

In the views of organizational education, we can argue that the delegation of decision-making power should not occur, however, in order to 'bribe' the stakeholders into showing motivation, but for the sake of a real democratisation of school. Therefore, cooperation or 'cooperative leadership' is not just a leadership style (like 'consultative leadership', 'delegative leadership' or 'participative leadership') but reflects a fundamental leadership conception as a general attitude. This can also be named 'democratic leadership'.

Overall, this has decisive consequences for teachers' actions and for school leadership actions; it also needs to be reflected in the preparation and qualification of those working in schools. Not only will the training of teachers benefit from this they also need to be trained for working within an organisation, whereas teacher training most often in many countries only focuses on how to teach the chosen subjects - but this will also affect the selection and development of the educational leadership personnel of the future.

\section{Conclusion}

We see that school leadership vis a vis curriculum is still an area that merits further research. School leaders in the German-speaking countries show a preference for activities from the domain of education and guidance. They enjoy teaching themselves. This could hint at school principals in these countries often still being closely tied to their identity as (former) teachers. Arguably, the long tradition of Bildung and didactics which has shaped the curriculum discourse in German-speaking countries (Hopmann 2015; Pinar 2011) has left its mark on the inherent professional identity of school leaders.

School leaders play an important role not only how the syllabus is implemented but also how curriculum work is planned, initiated, implemented and institutionalized in the school and how the school is embedded in the school environment (catchment area and community needs). At the same time, while school principals show an appreciation for teaching, education and guidance, they are not necessarily experts by tradition or training when it comes to curriculum matters. School leaders often lack the training necessary to make informed, appreciative assessments on curriculum matters. With national education standards taking root and the concept of pedagogical freedom still going strong, they also appear to have only restricted possibilities to influence curriculum matters. The interplay between leadership and 
curriculum is obvious but also still unfortunately underexplored and would well be worth of further analysis which would in turn require a bridging of leadership and curriculum theory as it has been done with the framework for curriculum studies, didactics and educational leadership by Uljens and Ylimaki (2015).

Looking ahead at possible developments in Germany difficulties are due to the state autonomy of the German Länder. However, one paradigm shift that was observed and that will likely continue is a shift towards national central standards of education. While these standards are mainly output-focused, they still have an impact on the curricula of the Länder. As the process of autonomy and accountability continues to evolve, it also stands to reason that more schools will try to stand out through their structure and curriculum.

The increase of accountability can also be seen in the testing process: more and more high school graduation exams are nowadays being developed and administered centrally by the state instead of the individual schools. Eventually, this might lead to a Germany-wide central procedure. This, in turn, would likely impact curricula in all states, which would then be likely to become more homogenous. Finally, while multiculturalism has long been a part of the German curriculum, it has so far been fairly centered on Europe (Faas 2011). The ongoing globalization process coupled with the continuing push of new media into the classrooms might entail a shift in curricula towards more global issues. In particular, the immigration of nearly one million people over the year of 2015 will also have and already has an impact on schools and schooling.

The concept of organizational education, as outlined above, can effectively support an adjustment of perspectives: To see leadership and management as a means to reach pedagogical goals and focus on education principles and not on bureaucratic ones. This would allow a shift of leadership practices, to what they prefer, away from what they experience as a burden, to what is desired from a curriculum perspective: focus on the core purpose of school and schooling, the learning of pupils, their development as persons within a community and the society, their Bildung. Yet, the basis for this change in perception is a higher range of autonomy and a higher degree of cooperative relationships across all hierarchical levels of the school system. It would result in a broad distribution of leadership responsibility and the networking of different systems: Distributed leadership for networked systems.

\section{References}

Böhm-Kasper, O. (2004). Schulische Belastung und Beanspruchung - Eine Untersuchung von Schülern und Lehrern am Gymnasium. Münster: Waxmann.

Bridges, E. M. (1967). Instructional leadership: A concept re-examined. Journal of Educational Administration, 5(2), 136-147. https://doi.org/10.1108/eb009614

Coleman, J. S., Campbell, E. Q., Hobson, C. J., McPartland, J., Mood, A. M., Weinfeld, F. D., \& York, R. L. (1966). Equality of educational opportunity. Washington, DC: US Department of Helath, Education, and Welfare, Office of Education/National Center for Education Statistics. Retrieved from http://files.eric.ed.gov/fulltext/ED012275.pdf 
Dolch, J. (1971). Lehrplan des Abendlandes. Zweieinhalb Jahrtausende seiner Geschichte (3rd ed.). Ratingen: Henn.

Easley, J., II, \& Tulowitzki, P. (Eds.). (2016). Educational accountability - International perspectives on challenges and possibilities for school leadership. London: Routledge.

Edmonds, R. (1979). Effective schools for the urban poor. Educational Leadership, 37(1), 15-18, 20.

Elbers, D. (1973). Curriculumreformen in den USA: ein Bericht über theoretische Ansätze und praktische Reformverfahren mit einer Dokumentation über Entwicklungsprojekte, M.P.I. für Bildungsforschung (Vol. 28). Berlin: Max-Planck-Institut für Bildungsforschung.

Faas, D. (2011). The Nation, Europe, and Migration: A comparison of geography, history, and citizenship education curricula in Greece, Germany, and England. Journal of Curriculum Studies, 43(4), 471-492. https://doi.org/10.1080/00220272.2011.584560

Fidler, B. (1997). School leadership: Some key ideas. School Leadership \& Management, 17(1), 23-38. https://doi.org/10.1080/13632439770140

Flechsig, K.-H., \& Haller, H.-D. (1973). Entscheidungsprozesse in der Curriculumentwicklung (Vol. 24). Stuttgart: Klett.

Frey, K. (1971). Theorien des Curriculums. Beltz: Weinheim.

Goodlad, J. I. (1960). Curriculum: The state of the field. Review of Educational Research, 30(3), 185. https://doi.org/10.2307/1169277

Hallinger, P. (2003). Leading educational change: Reflections on the practice of instructional and transformational leadership. Cambridge Journal of Education, 33(3), 329-351.

Hallinger, P. (2005). Instructional leadership and the school principal: A passing fancy that refuses to fade away. Leadership and Policy in Schools, 4(3), 221-239. https://doi. org/10.1080/15700760500244793

Hallinger, P., \& Murphy, J. F. (1986). The social context of effective schools. American Journal of Education, 94, 328-355.

Hallinger, P., \& Wang, W.-C. (2015). Assessing instructional leadership with the principal instructional management rating scale. London: Springer.

Hameyer, U. (1978). Innovationsprozesse: Analysemodell und Fallstudien zum sozialen Konflikt in der Curriculumrevision. Beltz: Weinheim.

Hameyer, U. (2006). School improvement in Germany - Current state and outlook. In J. Chi-kin Lee \& M. Williams (Eds.), School improvement: International perspectives (pp. 361-378). New York: Nova Science Publishers.

Hameyer, U. (2007). Transforming domain knowledge: A systemic view at the school curriculum. Curriculum Journal, 18(4), 411-427. https://doi.org/10.1080/09585170701688744

Hameyer, U. (2010). Transforming the school curriculum in Germany. In H. E. Law \& N. Nieveen (Eds.), Schools as curriculum agencies: Asian and European perspectives on school-based curriculum development (pp. 217-238). Rotterdam: Sense Publishers.

Hameyer, U., \& Tulowitzki, P. (2013). Reflecting curriculum trends in Germany - A conceptual framework for analysis. In W. Kuiper \& J. Berkvens (Eds.), Balancing curriculum regulation and freedom across Europe (pp. 81-98). Enschede: SLO.

Hameyer, U., Frey, K., \& Haft, H. (Eds.). (1983). Handbuch der Curriculumforschung. Weinheim: Beltz.

Handelzalts, A. (2009). Collaborative curriculum development in teacher design teams. Enschede: University of Twente [Host].

Heidegger, G., \& Petersen, W. (2005). Quality through self-evaluation and development. In W. Petersen, G. Heidegger, \& B. Niemeyer (Eds.), Re-integration (pp. 94-123). Norderstedt: GRIN Verlag.

Hentig, H. (1985). Die Menschen stärken, die Sachen klären : ein Plädoyer für die Wiederherstellung der Aufklärung. Stuttgart: Philipp Reclam jun.

Hentig, H. (1993). Die Schule neu denken : eine Übung in praktischer Vernunft: eine zornige, aber nicht eifernde, eine radikale, aber nicht utopische Antwort auf Hoyerswerda und Mölln, Rostock und Solingen. München: Hanser.

Hentig, H. (1996). Bildung: ein Essay (7. Auflage 2007). Weinheim: Beltz. 
Hopmann, S. (2015). "Didaktik meets Curriculum" revisited: Historical encounters, systematic experience, empirical limits. Nordic Journal of Studies in Educational Policy, 1(1):27007. https://doi.org/10.3402/nstep.v1.27007

Hörner, W., \& Waterkamp, D. (Eds.). (1981). Curriculumentwicklung im internationalen Vergleich. Weinheim: Beltz.

Horng, E. L., Klasik, D., \& Loeb, S. (2010). Principal's time use and school effectiveness. American Journal of Education, 116(4), 491-523.

Huber, S. G. (Ed.). (2012). Jahrbuch Schulleitung 2012. Befunde und Impulse zu den Handlungsfeldern des Schulmanagements. Köln: Carl Link.

Huber, S. G. (2013a). Forschung zu Belastung und Beanspruchung von Schulleitung. In S. G. Huber (Ed.), Jahrbuch Schulleitung 2013. Befunde und Impulse zu den Handlungsfeldern des Schulmanagements (pp. 222-240). Köln: Wolters Kluwer Deutschland.

Huber, S. G. (2013b). Schulleitung heute: Zwischen Begeisterung und Belastung? In Allgemeiner Schulleitungsverband Deutschlands (Eds.) b: sl Beruf Schulleitung. Berlin, Germany.

Huber, S. G. (2013c). Handbuch Führungskräfteentwicklung. Grundlagen und Handreichungen zur Qualifizierung und Personalentwicklung im Schulsystem. Köln: Carl Link.

Huber, S. G. (2016a). Switzerland: The school leadership research base in Switzerland. In H. Ärlestig, C. Day, \& O. Johansson (Eds.), A decade of research on school principals (pp. 421-442). Switzerland: Springer. Retrieved from http://link.springer.com/ chapter/10.1007/978-3-319-23027-6_18

Huber, S. G. (2016b). Schulleitung - Forschung in den deutschsprachigen Ländern: Belastungs und Beanspruchungserleben im Schulleitungshandeln. Münster: Waxmann.

Huber, S. G. (Ed.). (2016c). Steuergruppenhandbuch. Grundlagen für die Arbeit in zentralen Handlungsfeldern des Schulmanagements (4th ed.). Köln: Link-Luchterhand.

Huber, S. G. \& Muijs, D. (2010). School leadership effectiveness. The growing insight in the importance of school leadership for the quality and development of schools and their pupils. In S. G. Huber (Ed.), School leadership - International perspectives (S. 57-78).

Huber, S.G., \& Schwander, M.. (2013). Arbeitstagebuch: Wie verteilt sich die Arbeit von Schulleitungen?. b:sl, 03, 8-9.

Huber, S.G., \& Wolfgramm, C.. (2013a). Das Kollegium als soziale Ressource b:sl, 3, 7.

Huber, S.G., \& Wolfgramm, C.. (2013b). Was bedingt Unzufriedenheit und hohe Belastung?. b:s1, $3,17$.

Huber, S. G., Schneider, N., Gleibs, H. E., \& Schwander, M. (2012). Leadership in der Lehrerbildung: Entwicklung von Kompetenzen für pädagogische Führung. Stiftung der deutschen Wirtschaft.

Huber, S. G., Wolfgramm, C., \& Kilic, S. (2013a). Vorlieben und Belastungen im Schulleitungshandeln: Ausgewählte Ergebnisse aus der Schulleitungsstudie 2011/2012 in Deutschland, Österreich, Liechtenstein und der Schweiz. In S. G. Huber (Ed.), Jahrbuch Schulleitung 2013. Befunde und Impulse zu den Handlungsfeldern des Schulmanagements (pp. 259-271). Wolters Kluwer Deutschland: Köln.

Huber, S.G., Wolfgramm, C., \& Kilic, S.. (2013b). Tätigkeitsvorlieben. b:sl, 3, 10-11.

Huber, S. G., Gördel, B.-M., Kilic, S., \& Tulowitzki, P. (2016). Accountability in the German school system. In J. Easley II \& P. Tulowitzki (Eds.), Educational accountability - International perspectives on challenges and possibilities for school leadership (pp. 165-183). London: Routledge.

Jackson, D. \& West, M. (1999). Learning through leading: Leading through learning. Leadership for sustained school improvement. Paper presented at the International Congress for School Effectiveness and Improvement 1999, San Antonio, TX.

Kelly, A. V. (2009). The curriculum: Theory and practice (6th ed.). Thousand Oaks: Sage.

Kerr, J. F. (1968). Changing the curriculum. London: University of London Press.

Klumpp, L., Pont, B., Figueroa, D. T., Albiser, E., Wittenberg, D., Zapata, J., \& Fraccola, S. (2014). Education policy outlook Germany. Paris: OECD Publishing.

Kmetz, J. T., \& Willower, D. J. (1982). Elementary school principals' work behavior. Educational Administration Quarterly, 18(4), 62-78. https://doi.org/10.1177/0013161X82018004006 
Krug, S. E. (1992). Instructional leadership: A constructivist perspective. Educational Administration Quarterly, 28(3), 430-443. https://doi.org/10.1177/0013161X92028003012

Kuper, H.. (2008). Entscheiden und Kommunizieren Eine Skizze zum Wandel schulischer Leitungs-und Partizipationsstrukturen und den Konsequenzen für die Lehrerprofessionalität. In Pädagogische Professionalität in Organisationen (pp. 149-162). Springer. Retrieved from http://link.springer.com/chapter/10.1007/978-3-531-90777-2_8

Lee, J. C.-K., \& Dimmock, C. (1999). Curriculum leadership and management in secondary schools: A Hong Kong case study. School Leadership \& Management, 19(4), 455-481. https:// doi.org/10.1080/13632439968970

Leithwood, K., Begley, P. T., Cousins, J. B. (1990). The nature, causes and consequences of principals' practices: An agenda for future research. Journal of Educational Administration, 28(4). https://doi.org/10.1108/09578239010001014

Leithwood, K., Jantzi, D., \& Steinbach, R. (1999). Changing leadership for changing times. Philadelphia: Open University Press.

Liebel, H. (1992). Psychologie der Mitarbeiterführung. In E. Gabele, W. Oechsler, \& H. Liebel (Eds.), Führungsgrundsätze und Mitarbeiterführung: Probleme erkennen und lösen (pp. 109161). Wiesbaden: Gabler.

Martin, W. J., \& Willower, D. J. (1981). The managerial behavior of high school principals. Educational Administration Quarterly, 17(1), 69-90. https://doi.org/10.1177/00131 61X8101700105

Meyer, H. (1972). Einführung in die Curriculum-Methodologie. München: Kösel.

Morgan, G. (2006). Images of organization (4th ed.). Thousand Oaks: Sage.

Neumann, K., Fischer, H. E., \& Kauertz, A. (2010). From PISA to educational standards - the impact of large-scale assessments on science education in Germany. International Journal of Science and Mathematics Education, 8(3), 545-563. https://doi.org/10.1007/s10763-010-9206-7

OECD. (2013). PISA 2012 Results: What makes schools successful (Volume IV). Paris: OECD Publishing.

Oelkers, J. (1983). Kodifizierte Bestimmungsfaktoren curricularer Lernereignisse: Bildungskonzeptionen. In U. Hameyer, K. Frey, \& H. Haft (Eds.), Handbuch der Curriculumforschung (pp. 361-372). Weinheim: Beltz.

Paulsen, F. (1892). Einleitung in die Philosophie. Berlin: Hertz.

Paulsen, F. (1896a). Geschichte des gelehrten Unterrichts auf den deutschen Schulen und Universitäten vom Ausgang des Mittelalters bis zur Gegenwart. Mit besonderer Rücksicht auf den klassischen Unterricht. Band 2 (3rd ed.). Leipzig: Walter de Gruyter \& Co..

Paulsen, F. (1896b). Geschichte des gelehrten Unterrichts: Erster Band (3rd ed.). Leipzig: Veit \& Comp.

Pinar, W. F. (2004). What is curriculum theory? Mahwah: L. Erlbaum Associates.

Pinar, W. F. (2011). The character of curriculum studies: Bildung, currere, and the recurring question of the subject. New York: Palgrave Macmillan.

Portelli, J. P. (1987). On defining curriculum. Journal of Curriculum and Supervision, 2(4), 354-367.

Rolff, H.-G., Rhinow, E., \& Röhrich, T. (Eds.). (2009). Unterrichtsentwicklung - eine Kernaufgabe der Schule: die Rolle der Schulleitung für besseres Lernen. Köln: LinkLuchterhand.

Rosenbusch, H. S. (1997). Organisationspädagogische Perspektiven einer Reform der Schulorganisation. SchulVerwaltung, 10, 329-334.

Rosenbusch, H. S. (2005). Organisationspädagogik. München: Wolters Kluwer.

Roth, H. (1968). Stimmen die deutschen Lehrpläne noch? Die Deutsche Schule, 60(2), 69-76.

Rutter, M. (1979). Fifteen thousand hours: secondary schools and their effects on children. Cambridge, MA: Harvard University Press.

Skilbeck, M. (1998). School-based curriculum development. In A. Hargreaves, A. Lieberman, M. Fullan, \& D. Hopkins (Eds.), International handbook of educational change (pp. 121-144). Boston: Kluwer Academic Publishers.

Sorkin, D. (1983). Wilhelm Von Humboldt: The theory and practice of self-formation (Bildung), 1791-1810. Journal of the History of Ideas, 44(1), 55. https://doi.org/10.2307/2709304 
Southworth, G. (2002). Instructional leadership in schools: Reflections and empirical evidence. School Leadership \& Management, 22(1), 73-91. https://doi.org/10.1080/13632430220143042

Spillane, J. P., Hunt, B. (2010). Days of their lives: A mixed-methods, descriptive analysis of the men and women at work in the principal's office. Journal of Curriculum Studies, 42(3), 293-331. https://doi.org/10.1080/00220270903527623

Taba, H. (1962). Curriculum development: Theory and practice. New York: Harcourt, Brace \& World.

Tenorth, H.-E. (Ed.). (1986). Allgemeine Bildung. Analysen zu ihrer Wirklichkeit, Versuche über ihre Zukunft. München: Juventa.

Tenorth, H.-E. (2001). Kerncurriculum Oberstufe (2nd ed.). Weinheim: Beltz.

Tenorth, H.-E. (2004). Kerncurriculum Oberstufe II. Weinheim: Beltz.

Toombs, W. E., \& Tierney, W. G. (1993). Curriculum definitions and reference points. Journal of Curriculum and Supervision, 8(3), 175-195.

Tulowitzki, P. (2013). Leadership and school improvement in France. Journal of Educational Administration, 51(6), 812-835. https://doi.org/10.1108/JEA-03-2012-0026

Tulowitzki, P. (2015). The development of educational leadership and teaching professions in Germany. ECPS - Educational, Cultural and Psychological Studies, 11, 45-55. https://doi. org/10.7358/ecps-2015-011-tulo

Uljens, M., \& Ylimaki, R. (2015). Towards a discursive and non-affirmative framework for curriculum studies, Didaktik and educational leadership. Nordic Journal of Studies in Educational Policy, 13, 30177. https://doi.org/10.3402/nstep.v1.30177

van den Akker, J. (2004). Curriculum perspectives: An introduction. In Curriculum landscapes and trends (pp. 1-10). Netherlands: Springer. Retrieved from http://link.springer.com/ chapter/10.1007/978-94-017-1205-7_1

van den Akker, J. (2010). Building bridges: How research may improve curriculum policies and classroom practices. In S. M. Stoney (Ed.), Beyond lisbon 2010: Perspectives from research and development for education policy in Europe, CIDREE yearbook 2010 (pp. 175-196). Slough: NFER.

Weniger, E. (1971). Theorie der Bildungsinhalte (Didaktik als Bildungslehre. Teil 1) (9th ed.). Weinheim: Beltz.

Weniger, E. (1975). Theorie der Bildungsinhalte und des Lehrplans. In E. Weniger (Ed.), Ausgewählte Schriften zur geisteswissenschaftlichen Pädagogik (pp. 199-294). Weinheim: Beltz.

Wildy, H., Dimmock, C. (1993). Instructional leadership in primary and secondary schools in Western Australia. Journal of Educational Administration, 31(2). https://doi. org/10.1108/09578239310041873

Wiles, J. (2008). Leading curriculum development. Thousand Oaks: Corwin Press.

Wunderer, R., \& Grunwald, W. (1980). Führungslehre. Band II: Kooperative Führung. Berlin: De Gruyter.

Open Access This chapter is licensed under the terms of the Creative Commons Attribution 4.0 International License (http://creativecommons.org/licenses/by/4.0/), which permits use, sharing, adaptation, distribution and reproduction in any medium or format, as long as you give appropriate credit to the original author(s) and the source, provide a link to the Creative Commons license and indicate if changes were made.

The images or other third party material in this chapter are included in the chapter's Creative Commons license, unless indicated otherwise in a credit line to the material. If material is not included in the chapter's Creative Commons license and your intended use is not permitted by statutory regulation or exceeds the permitted use, you will need to obtain permission directly from the copyright holder.

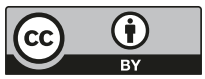




\title{
Chapter 10 \\ Teachers and Administrators as Lead \\ Professionals for Democratic Ethics: \\ From Course Design to Collaborative Journeys of Becoming
}

\author{
Daniel J. Castner, Rosemary Gornik, James G. Henderson, \\ and Wendy L. Samford
}

\begin{abstract}
The heightened level of attention being afforded to "teacher leadership" is palpable in the United States. At a national level, proprietary organizations are receiving funds from large philanthropic organizations (e.g., the Gates and the Wallace Foundations) to promote the development of teacher leaders. State departments of education are accommodating the federal push finding various ways to incentivize the efforts of teachers to lead from the classroom. Our institutions of higher education are also adjusting and accommodating by taking up the charge of preparing teacher leaders, theorizing, and researching the potential of teacher leadership through academic study. As professors of education in the United States, we are mindful of the contextualizing neoliberalism infused throughout our policy environment and are deeply concerned about the habits of competition, rigidness, bureaucratization, and overspecialization. Not surprisingly, such ways of thinking, acting, and being infiltrate our educational institutions and can have a dehumanizing effect on local teachers, their pedagogies, and their students (Noddings 2007; Nussbaum 2010). Such habits of mind and body can additionally reinforce a sense of isolation between teachers and their profession (Eisner 2001), perhaps even a loss of vocational calling (Hansen 1995; Palmer 2007). Along with this can come a sense of alienation from colleagues and administrators (Macdonald and Shirley 2009) as well as a loss of individual and collective voice and autonomy (Apple 2006; Ayers 2010; Miller 1990). This chapter reports on an action research project designed focused on teacher leadership and reconceptualist curriculum theorizing as an alternative to the Tyler Rationale.
\end{abstract}

\footnotetext{
D.J. Castner $(\bowtie)$

Bellarmine University, Louisville, KY, USA

e-mail: dcastner@bellarmine.edu

R. Gornik • J.G. Henderson • W.L. Samford

Kent State University, Kent, OH, USA

e-mail: rgornik@kent.edu
} 


\section{Introduction}

This collaboratively authored chapter is centered on the scholarly personal narratives of four educators, who maintain a faithful commitment to the integration of democracy and education a century after the publication of Dewey's (1916) seminal text. Their stories converge around a course at Kent State University that introduces fundamentals of curriculum to graduate students. Through the disciplined study of curriculum in this course, the taken-for-granted ideas that routinely circulate as common sense in many educators' habituated thoughts, discourse and daily practices are disrupted and personal pathways for new semiotic understandings are constructed. In specific terms, technical understandings of the fundamentals of curriculum, as symbolized by Tyler's (1949) "rationale," are critically challenged in light of a key normative question: what are educators' ethical responsibilities as lead professionals in societies with democratic aspirations, and how ought they to function in contemporary educational institutions that promulgate value-neutral decision-making?

This chapter begins with Jim Henderson's narrative of 25 years of action research in the curriculum fundamentals course, which has resulted in the creation of a constructive alternative to Tyler's rationale through a reconceptualization of curriculum development. Next, Dan Castner shares how his understanding of Jim's action research has informed his theory and practice of curriculum-based teacher leadership. Rosemary Gornik and Wendy Samford then discuss how their ongoing semiotic engagement with the fundamentals of curriculum wisdom has been applied to a grant project addressing the interplay of administrative and teacher leadership. Collectively, these narratives present a picture of educators engaged in collaborative journeys of becoming for the purpose of establishing an ethical community of democratic visionaries, colleagues and pedagogical artists.

\section{Jim Henderson: Course Design}

A Kent State University (KSU) graduate course, entitled "Fundamentals of Curriculum," (FoC) has been serving as my de facto research laboratory since 1991. It's been the place where I have been diligently refining a constructive alternative to Ralph Tyler's (1949) "rationale" which, despite the fact that it was published 67 years ago, is still considered the paradigmatic exemplar for conceptions of curriculum development (Null 2008; Walker and Soltis 2009). While teaching the FoC course for 25 years, I have examined and explored an open set of potentially fruitful curriculum concepts through informal action research activities, and I have been quite open and honest about my experimental approach. I tell students that I have had no other choice since what local school district would support such trial-anderror efforts? 
FoC is the core course in KSU's Curriculum and Instruction (C\&I) M.Ed. Program. This masters' degree program has seven subject-area concentrations and also serves as a key professional development course for non-C\&I students such as nurses and business people. In addition, $\mathrm{FoC}$ is a recommended course for new $\mathrm{C} \& \mathrm{I}$ Ph.D. students. As a result, the course is offered at least twice a year and draws on a diverse student population. I have taught FoC over 60 times during the past 25 years.

I have now reached the point in my ongoing FoC action research that I can confidently state that, with the help of my students in the course as well as many collaborating colleagues, I can now present a constructive alternative to Tyler's rationale. It's not surprising that it's taken me so long to get to this point, given the philosophical, personal, social and political challenges involved. In fact, when I started my experimental work in 1991, I didn't even fully understand these challenges. I do now; and as I have proceeded with my action research over the years, three broad ethical themes have emerged to guide my work: critical pragmatism, democratic hermeneutics, and holistic pedagogy. The themes are conceived as an open set of ethical end-in-views, not as a precise code of ethics, which allows for the play of diversified interpretations characteristic of democratic conversations and deliberations. As I introduce the three normative themes, I will be integrating nine concepts into my discussion. These nine concepts, which are presented in the FoC course as nine fundamentals of curriculum, provide scaffolding for the ethical mindfulness that I am advancing. In short, the FoC course is designed so that the three broad ethical orientations are grounded in a conceptual understanding agenda.

Critical Pragmatism The starting point for my years of FoC action research is the critical recognition that Tyler's rationale lacks a strong ethical basis. Tyler argues that educational workers need to systematically address the relationship between identifying clear curriculum purposes, designing learning experiences that address these purposes, organizing these experiences in accessible and coherent ways, and evaluating the learning results with the implication that curriculum development adjustments may need to be made. In effect, Tyler provides scaffolding for a seemingly thoughtful and recursive problem solving circuit. Herbert Kliebard-arguably the most influential and incisive North American critic of Tyler's rationale-notes that Tyler's purpose-experience-organization-evaluation circuit is, in fact, an "imperishable" feature of his curriculum development approach. Kliebard (1992) writes:

One reason for the success of the Tyler rationale is its very rationality. It is an eminently reasonable framework for developing a curriculum; it duly compromises between warring extremes and skirts the pitfalls to which the doctrinaire are subject. In one sense the Tyler rationale is imperishable. (p. 164)

It took me years to conceive of a constructive way to acknowledge the enduring relevance of Tyler's recursive problem solving while establishing critical distance from his inattentive ethics. I didn't want to throw the baby out with the bathwater. As Cherryholmes (1988) illustrates, Tyler's rationale is an example of "vulgar," not "critical," pragmatism. He writes: 
Tyler's proposal was attractive because it promised order, organization, rationality, error correction, political neutrality, expertise, and progress....[Unfortunately], the rationale is incomplete when it comes to making decisions about curriculum and instruction... There is no discussion of...politics, ethics, social criticism, social responsibility, or critical reflection (pp. 26, 40-41).

Tyler does not advance a deep critical and ethical awareness of curriculum purposes and practices. Any purpose can be plugged into his problem solving circuit: teaching dogmatic beliefs, teaching racism and sexism, teaching to standardized tests, etc. As the years progressed and I continued to think about this underlying normative-philosophical problem, I kept coming back to Dewey's (1897/2013) statement that teaching is "the supreme art" in a society.

Though space does not allow me to share my years of evolving critical and creative work on the organizing idea that teaching is a society's supreme art, I can state my current perspective on this matter. Educators become 'supreme' artists when they work on being lead professionals in their society for democratic ethics-when they cultivate a particular personal-professional journey of becoming. Here's how I present this perspective in my current FoC syllabus:

$* * * * * *$

In this course, "curriculum" will be defined as the deep-seated study and practice of the relationships between the what, the how, and the why of educational courses of action. Building on this definition, 'good' curriculum work will be interpreted as the study and practice of the relationship between educational courses of action and democratic living, particularly as informed by the writings of the great American educational philosopher, John Dewey. On the eve of World War II-in the context of America's upcoming fight with German Fascism, Japanese Imperialism, and Soviet Communism-Dewey wrote, "We [Americans] have advanced far enough to say that democracy is a way of life: What happened since...? We have yet to realize that it is a way of personal life and one which provides a moral standard for personal conduct" (Dewey 1939/1989, p. 101).

The eighteenth-century founders of the United States thought deeply about the quality of political and legal problem solving but gave insufficient attention to the quality of curriculum problem solving. If their constitutional work had been informed by disciplined curriculum studies (which did not formally emerge in the United States until 1918), they would have recognized the long-term societal implications of Dewey's (1897/2013) position that,

Education... marks the most perfect and intimate union of science and art conceivable in human experience. The art of thus giving shape to human powers and adapting them to social service is the supreme art; one calling into its service the best of artists; that no insight, sympathy, tact, executive power, is too great for such service. Every teacher should realize the dignity of his [or her] calling; that he [or she] is a social servant set apart for the maintenance of proper social order and the securing of the right social growth. (pp. 39-40)

In curriculum studies language, this historic limitation has created a profound "null curriculum" problem for the United States that has important implications for 
all societies with democratic aspirations, ideals, and/or social contracts. The "null curriculum" notion refers to educational topics that are ignored but should be taught. Eisner (1994) explains:

It is my thesis that what schools do not teach may be as important as what they do teach. Ignorance is not simply a neutral void; it has important effects on the kind of options one is able to consider, the alternatives that one can examine, and the perspectives from which one can view a situation or problems. The absence of a set of considerations or perspectives or the inability to use certain processes for appraising a context biases the evidence one is able to take into account. A parochial perspective or simplistic analysis is the inevitable progeny of ignorance. (p.158)

This course is designed to address the problem of underdeveloped ethical understanding and mindfulness in curriculum decision-making. You will be treated as having the potential to serve as one of your society's lead professionals for democratic ethics. In this course, you will not be treated as compliant, non-thinking semiprofessionals but as trustworthy professionals capable of visionary, informed ethical judgments based on disciplined study and practice. Acknowledging certain caveats, this is how Finnish educators are treated in their society (Sahlberg 2015). This is my relationship promise to you.

$* * * * * *$

Tero Autio's critique of the epistemological foundations of Tyler's rationale, as well as the many similar, modernist curriculum development processes, provides important pragmatic insights into the consequences of promoting value-free procedures. Autio (2006) notes that,

The aims and objectives pioneers [in the field of curriculum studies] gave to curriculum discourse....behavioral, scientific job analysis. The introduction of precise scientific methods in educational practice, drawing especially from industry...has been expanded to an ambitious, relatively autonomous and highly detailed classification of objectives.... At the center of this [work]...remains Ralph Tyler. His contribution was to capture the spirit and letter of the instrumental symbolic curriculum.... This aims-and-objectives movement, in which Tyler focused its scrutiny on the definition of curricular objectives, has been replaced by the neoliberal interest in the assessment of objectives by an intensified monitoring of individual performativity. (p. 13)

Autio (2006) then concludes his thorough, historical critique with a "dusting" metaphor, "The efforts to rescue education and educative experience from the stranglehold and seductions of vulgar instrumentalism is parallel to the act of dusting: There is a constant need for cleaning; dust never disappears completely" (p. 162). In the FoC course, I began working with the concept of curriculum criticism to refer to this continuous dusting.

The particular dusting that Autio (2015) has in mind is the establishment of critical distance from a "calculative instrumentality" embedded in a "culture of Method" in order to embrace and refine ethical values. He writes:

Scratch a good teacher and you will find a moral purpose. At its best, an emphasis on the moral shifts teaching from transmission to transformation, as the curriculum is no longer test preparation but a complicated conversation where all participants at every level think 
about the basic curriculum questions regarding the worth of and interconnections between the subject matter and the related subjective and social dynamics (pp. 180-181, 195).

This shift from transmission to transformation became a key focus in my FoC pedagogy as I began to think deeply about the concept of transformative curriculum leadership, which ultimately resulted in the creation of three editions of a coauthored text using the concept as the title of the book. The third edition of Transformative Curriculum Leadership provides the clearest definition of the concept:

Burns (1978) describes transformative leaders as individuals who encourage "principled levels of judgment" through dedicated effort (p.455). They inspire others to higher levels of performance. Because transformative curriculum leaders care so deeply about the facilitation of a personalized 3S [subject, self and social] understanding, they affirm the "best selves" of those who are around them (Noddings 1984). Burns (1978) contrasts transformative leadership with a more traditional understanding of leadership, with its focus on the efficient attainmentof organizational goals and not on "consciousness raising on a wide plane" (p.43) (Henderson and Gornik 2007, p.17).

In short, the concept of transformative curriculum leadership three key personalprofessional questions:

- Am I encouraging the establishment of critical distance from all personal and social factors that work against honest self-examination and visionary microand macro-cultural change?

- Am I inspiring best-self contemplations and deliberations?

- Am I fostering principled, deliberative judgments?

Because I recognized early on in the FoC course that the pursuit of these critical questions required the cultivation of a personal-professional journey of deepening understanding, I incorporated Pinar and Grumet's (1976) reframing of the Latin noun 'curriculum' as the Latin gerund, currere. In interpreting curriculum-ascurrere, Pinar and Grumet highlighted the relationship between an individual's personal past, present, and future when 'running' any particular educational course of action - a relationship that invited autobiographical narrative. In a later publication, Pinar (1994) summarizes the composition of currere narratives with a particular organizing question: "How is the future present in the past, the past in the future, and the present in both?" (p. 26). As Rosemary Gornik and I created the third edition of Transformative Curriculum Leadership, we decided that each of the nine chapters of the book would end with a specific currere narrative that she would compose from the perspective of a democratically-oriented educational practitioner who was committed to embodying and enacting inspired, deliberative judgments.

Over time, I came to realize that, with reference to educators becoming lead professionals for democratic ethics, my pedagogical focus was on journeys of understanding that positioned teachers to practice another key conceptual referent: $3 S$ pedagogy. $3 \mathrm{~S}$ pedagogy is an abbreviated shorthand way to refer to teaching for Subject understandings that are embedded in democratic Self and Social understandings. I first published this $3 S$ teaching notion in the third edition of my reflective teaching text (Henderson 2001a), and I was pleasantly surprised at how well it 
caught on in the FoC course. As a result, I have continued to refine the concept over the years; and in the first chapter of my latest collaborative text, I open with four snapshots of $3 \mathrm{~S}$ pedagogy composed by a kindergarten, a grade school, a high school and a college teacher. I introduce their four brief narratives as follows:

[Dan, Chris, Boni, and Beth] present four snapshots of their holistic teaching, which they see as personalized embodiments of their institutions' mission statements and their country's democratic aspirations. They hope that their individualized snapshots are instructive and, possibly, inspirational. Collectively, these four narratives are reminders that many educators feel they have a noble vocational calling which they understand in their own terms. There are many educators who don't see themselves as bureaucratic functionaries, corporate employees, or compliant technocrats. They view themselves as lead professionals with important visionary, progressive responsibilities. In broader cultural and policy terms, countries that do not recognize such educators' vital role in the dynamic health of their societies may be condemning their current and future generations to stagnant, regressive, and rigid lives (Henderson et al. 2015, pp. 1-2).

To summarize my pedagogical discussion to this point, the creation of a constructive alternative to Tyler's rationale required me to think through the vital academic, personal and social relationships between curriculum criticism, transformative curriculum leadership, currere narrative, and $3 \mathrm{~S}$ pedagogy. As I refined my thinking on this conceptual gestalt, I realized that this transformative work requires disciplined study; and hence, educators who are inclined to provide collegial leadership for this disciplined study should think of themselves as lead learners. Working with an international team of eighteen educators, I created a lead-learning text that would be incorporated into my FoC pedagogy. The book, entitled Reconceptualizing Curriculum Development (RCD), is based on Pinar's (2006) argument that disciplined studies, not technical protocols, should be the basis for rethinking curriculum development. Pinar writes:

Before the Reconceptualization, curriculum development was primarily procedural, epitomized in Tyler's (1949) four questions. ..[These many] years after the Reconceptuali-zation, I am proposing curriculum development that is simultaneously... [study-based]. ... As a curriculum developer, I compose synpotic texts to enable public school teachers to reoccupy a vacated public domain, not as "consumers" of knowledge, but as active participants in complicated conversations that they themselves will lead in their own classrooms. In drawing widely but critically from various academic disciplines, from interdisciplinary areas, from popular culture, the form of curriculum development I propose and demonstrate herein creates textbooks for teachers who can appreciate that our professional calling is the intellectual reconstruction of the public and private spheres (p. x).

The concept of lead learning is presented a certain way in the RCD text. Lead learning is defined as the formal and/or informal study of relevant curriculum and pedagogy topics that is initiated and modeled by one educator who then personally invites interested colleagues to join him or her. Lead learning does not require expertise; it only requires a willingness to engage in study relationships. Lead learning is decidedly non-hierarchical. It is highly collaborative and allows for diversified, idiosyncratic, and existential understandings. Barth (2008) writes that, "In our [education] profession, especially, one is a learner and THEREBY a leader. The moral authority of the educational leader comes first and foremost from being a 
learner" (p. x). Lieberman and Friedrich (2010) note that there are, increasingly, openings in the education profession for such collegial leadership. Stated in slightly different language, lead learning can be characterized as a type of humble "servant leadership" (Nichols 2011).

Democratic Hermeneutics Building educators' capacities for a lead learning grounded in critical pragmatism brings me to the second major ethical challenge that I faced in creating a constructive alternative to Tyler's rationale. In thinking through my understanding of democratic values, I realized early on that I needed to work with a hermeneutic rather than an ideological orientation. In other words, I needed to treat the topic of educators' democratic ethics through an open-minded, open-hearted frame of reference (Dewey 1910/1933), which Gadamer (1975) describes as the "dialogical playfulness" that cultivates a broadening of personal horizons. Such an existential, pluralistic commitment challenges rigid, dogmatic, close-minded, and ideologically-fused interchanges where options are limited to consensus or conflict.

Bernier and Williams (1973) define ideology as "an integrated pattern of ideas, system of beliefs, or a 'group consciousness' which characterizes a social group. Such a pattern or system may include doctrines, ideals, slogans, symbols, and directions for social and political actions" (p. 27). With reference to this definition, committed ideologues are not interested in the possibilities of achieving diverse, expansive empathy and interpersonal understanding through respectful dissensus. Their focus is on scripted agreement; and if such agreement is not achievable, conflict and, perhaps, even violent confrontation may be forthcoming. Committed ideologues eschew pluralism. By definition, passionate ideologues working out of their own specific scripts talk past one another. They make no effort to understand different ideas or beliefs; they are not interested in broadening their personal horizons; they are not interested in dialogical relationships. As Garrison (1997) writes, "Monism is dogmatism" p.15). I recognized early on in my FoC action research that democratic hermeneutics is based on a generative, creative dissensus (Rancière 2010).

Based on my educational experiences as an undergraduate history major at a private liberal arts college, I recognize the importance of curriculum liberalization in fostering communities of dissensus. Abraham Lincoln — who is arguably the best of all the USA presidents and, certainly, the pivotal lead professional for democratic ethics at a critical juncture in American history - created his cabinet as a community of dissensus. Goodwin (2005) characterizes Lincoln's cabinet as a "team of rivals." Concerning Lincoln's disposition toward his political rivals, she writes,

Though Lincoln desired success as fiercely as any of his rivals, he did not allow his quest for office to consume the kindness and openheartedness with which he treated supporters and rivals alike.... With his death, Abraham Lincoln had come to seem the embodiment of his own words-"With malice toward none; with charity for all"- - voiced in his second inaugural to lay out the visionary pathway to a reconstructed union (pp. 256, 749).

Kegan (1994) defines reconstructive postmodern leaders as individuals who "challenge the unacknowledged epistemological assumptions behind modernist 
conceptions of the unilateral leader" (p. 424). Lincoln was such a postmodern leader long before such terms as modernism and postmodernism had even been coined by academics. Kegan (1994) writes, "Postmodernism suggests a kind of "conflict resolution' in which the Palestinian discovers her own Israeli-ness, the rich man discovers his poverty, the woman discovers the man inside her" (pp. 320-321). Lincoln practiced such deeply empathetic conflict resolution at the precise historical moment that his country needed such presidential leadership. With reference to democratic ethics, he was not perfect; but for his era, he was an embodiment of the USA's Declaration of Independence assertion that all humans are created equal.

Over the years, I have experimented with the introduction of the concept of curriculum liberalization in a number of ways. My current approach is to ask students to think about the critical distinction between the neo-liberal/neo-conservative control of curriculum and curriculum liberalization through the study of selected work of three curriculum scholars: Michael Apple, Wesley Null, and Joseph Schwab. Apple's (2005) critique of the emergence of "audit cultures" in educational work around the world is based on his analysis of the interplay of neo-liberal and neoconservative ideologies:

Neo-liberalism requires the constant production of evidence that you are doingthings 'efficiently' and in the 'correct' way by examining the effects on the ground of the suturing together of the seemingly contradictory tendencies of neo-liberal and neo-conservative discourses and practices.... And this is occurring at the same time as the state itself becomes increasingly subject to commercialization. This situation has given rise to what might best be called an audit culture (p. 14).

Curriculum liberalization challenges the underlying ideological assumptions of audit cultures. It is based on the recognition that all humans can build their capacities to become "free thinkers who can draw upon many fields of knowledge, pursue truth, and solve problems" (Null 2011, p. 15). Humans can be more than mindless, manipulatable consumers of capitalistic products. Null (2011) writes that curriculum liberalization "pursues the goal of liberating minds so that they can become more fully human, make rational judgments, and provide civic leadership" (p. 15). He advances this goal by inviting his readers to think through the pros and cons of five curriculum traditions (systematic, existentialist, radical, pragmatic, and deliberative) in light of Schwab's (1973) five curricular commonplaces: teachers, learners, subject matter, context and curriculum making.

I tell students that, based on my own critical thinking, I don't completely agree with either Null's or Schwab's categorization schemes, and that they are equally invited to critically question the way Null and Schwab have constructed 'curriculum.' That's what free thinkers do! They recognize that 'curriculum' is an interpretive term. However, I want them to work with Null's book because it encourages synoptic thinking. His text fosters broad deliberations informed by many different, opposing curricular perspectives. He argues that good curriculum work is not locked into a single topic, whether it's standardized testing on the political right or social justice on the political left. He writes that, "Deliberators prefers a middle path that respects wisdom and tradition but also searches for new and creative ways to solve whatever problems arise in the world of practice" (p. 261). Curriculum liberation is 
about following this Aristotelian and Deweyan middle path. Kathleen Kesson and I created a curriculum wisdom book based on this premise (Henderson and Kesson 2004), which I used in the FoC course for a couple of years.

I recognized early on in my FoC pedagogy that curriculum liberalization can be powerfully fostered through the interplay of curriculum-based reflective inquiry and deliberative conversation. Collaborating with four colleagues, I created a book that addressed this interplay (Henderson 1992); and due to my own ongoing trailand-error learning in the FoC course, I continued to revise my conceptions of this interplay through two completely revised editions of the original 1992 text. As Dewey (1910/1933) argues and clearly illustrates in his seminal text on thinking, humans break dysfunctional and/or limiting habits and customs through reflective inquiry - through a continuous and recursive consideration of the consequences of their actions in a context of humble, open-minded questioning. In a similar vein, Schwab (1978) argues that curriculum work at its best requires a commitment to deliberation that is grounded in practical and eclectic arts; and parallel to both Dewey's and Schwab's arguments, Pinar et al. (1995) provide a comprehensive overview of understanding "curriculum as complicated conversation." As I refined my feel for democratic hermeneutics as a key ethical touchstone, I continued to delve into this interplay between curricular reflective inquiry (RI) and deliberative conversation (DC).

This conjunction of Dewey's, Schwab's, and Pinar's work—along with its vast related literature-became a key referent for my $\mathrm{FoC}$ action research. I was increasingly committed to explicating and clarifying curriculum-based RI/DC. My most recent collaborative text on the reconceptualization of curriculum development, which I have already introduced, advances a lead-learning study agenda of three RIs informed by four DCs. I introduce this agenda as follows:

\footnotetext{
Section I [of this book] is an open-ended arrangement of three interrelated reflective inquiries informed by four deliberative conversations. To quickly review, the reflective inquiries address the interrelated questions of how to teach for $3 \mathrm{~S}$ understanding, how to embody $3 \mathrm{~S}$ understanding, and how to build collegial and public trust for 3S pedagogy; while the four deliberative conversations are organized around the topics of management-to-wisdom critique/negotiation, social justice, democratic humanism, and mythopoetic inspiration. Over the course of six years of action research, this particular configuration of topics has emerged as a powerful way to introduce and sustain the collegial study and practice of $3 \mathrm{~S}$ pedagogy. In short, chapters two through eight are single-authored essays that function as an ensemble of lead-learning invitations... (Henderson et al. 2015, p. 35)
}

Holistic Pedagogy Through my continuing explorations of the ethics of democratic hermeneutics, I realized that the artistry of holistic pedagogy is another key ethical end-in-view. I've already introduced the notion of $3 \mathrm{~S}$ pedagogy. As I experimented with ways of introducing this pedagogical artistry, I became increasingly aware that this conception of teaching is informed by the European, particularly German Bildung/Didaktik heritage as summarized by Autio (2009), "Bildung can be understood as a kind of self-formation along the lines of a wider [societal] belonging and...Didaktik refers generally to the pedagogical techniques for intertwining if not spiraling subjectivity and society together" (p. 71). 
However, I approached this Bildung/Didaktik heritage with a certain Deweyan caution. Given my commitment to democratic hermeneutics, I didn't want to get caught up in Hegel's rational systemization, and I am indebted to Good's (2006) nuanced explanation of how Dewey established a constructive critical distance from Hegel's philosophy. Good's thesis is that Dewey advanced an American Bildung tradition that drew on Hegel's key concept of Aufheben, translated as sublation, without getting lost in Hegel's quasi-religious dogmatism-Hegel's pretense that, through his dialectical rationalism, he had achieved the proper overview of the mysteries of life. Good (2006) writes:

The American Bildung tradition is based upon an inherently expansive conception of philosophy because it requires its practitioners to be broadly educated, across academic disciplines, to better understand their society's ideals, practices, and institutions. Moreover, it demands that philosophers keep one foot firmly planted in their social and historical context and one in their study. More theoretically, the American Bildung tradition rejects mechanistic, static views of reality in favor of an organic and historical model according to which individual persons and objects are interrelated within a dynamic process. Rather than assume the Cartesian notion that knowledge is gained by reducing complex wholes to their constituent parts, the Bildung tradition maintains that knowledge of the part comes from attending to the ways it is related to other parts and the way it functions within the larger whole. ...The most significant Hegelian deposit in Dewey's mature thought is the Bildung model of philosophy. I hasten to concede, however, that Dewey rejected Hegel's systematic efforts... (pp. xx-xxi).

I quote Good at length for a particular reason. I wasn't interested in summarily rejecting Tyler's rationale, I simply wanted to sublate it. Good (2006) explains: "The dialectic, Hegel's perception of scientific method, always begins with a hypothesis in that it is always a position that is asserted provisionally, adapted, developed, and ultimately sublated (Aufheben), that is, incorporated without being eliminated, into a more inclusive understanding of the subject matter" (p.13).

Good's reference to an American Bildung tradition, initiated by Dewey and then refined in subsequent generations of educators, was articulated in the United States as the curriculum concept of democratic general education. Tanner and Tanner (2007) provide a thorough overview of the American heritage of this concept. They begin their book by noting that this democratic, holistic orientation can be traced back to Thomas Jefferson's views on the importance of education in a freedomloving society. They quote Jefferson, "If a nation expects to be ignorant and free in a state of civilization, it expects what never was and never will be" (Lee 1961, pp. 18-19). They then cite Lawrence Cremin's insight that "the entire course of American educational history is based on the gradual realization of the Jeffersonian ideal" (Cremin 1965, p. 40). In the context of a thorough historical analysis of the views of hundreds of American educational leaders in the nineteenth and twentieth centuries on the importance of a democratic, holistic approach to curriculum work, Tanner and Tanner (2007) write:

No document of the twentieth century was more influential in shaping the structure and function of the American educational system than the report of the NEA [National Education Association] Commission on the Reorganization of Secondary Education, Cardinal Principles of Secondary Education (1918)....Embracing Dewey's [1916] concept of social 
efficiency in a democracy..., in sharp contrast to social efficiency in undemocratic societies, the Cardinal Principles report called for the fullest release of human potential through the widest extension of educational opportunity (pp. 280-281).

In the FoC course, I point out to students that this historic commitment to democratic general education with its implications for practicing the artistry of holistic pedagogy is under attack from ideologues on the right and the left. With reference to the ideological right, I ask them to think about Ylimaki's (2011) critical analysis of the ways in which a hegemonic "conservative modernization"-involving the synergy between neoliberalism, neoconservatism, authoritarian populism, and public management policy and the social dynamics of an emerging managerial middle class - has resulted in the narrowing of the curriculum. With reference to the ideological left, I ask them to think about the fact that I am teaching them the fundamentals of curriculum as a holistic public intellectual, not as a narrow critical specialist (Henderson and Kesson 2001). Since academic cultures generally have a leftist orientation and since graduate students have a certain amount of curricular experience with such cultures, I ask them to ponder why more professors aren't working as public intellectuals? I also ask them if they feel they received a broad liberal arts education as an undergraduate. If not, why not? If they went through a preservice teacher education program, were they encouraged to think and act like holistic teachers committed to democratic general education; and if not, why not? Were they encouraged to think of themselves as lead professionals for democratic ethics?

I want to conclude my pedagogical narrative with a concise overview of the FoC design that has gradually emerged over 25 years of action research. The course is currently organized around the aim of building educators' capacities to work as lead professionals for democratic ethics, as guided by three thematic orientations that are advanced through the disciplined study of nine curriculum concepts: curriculum criticism, transformative curriculum leadership, curriculum-as-currere, $3 \mathrm{~S}$ pedagogy, curriculum liberalization, lead learning, curriculum-based reflective inquiry, curriculum-based deliberative conversation, and democratic general education. The focus of our collaborative chapter now turns to a discussion of the interrelated conceptions of teacher leadership and administrative leadership that emerge out of this approach to understanding the fundamentals of curriculum.

Teachers as Leaders Jim Henderson's narrative of theorizing curriculum leadership is one that unsettles polemic voices in the contemporary cultural milieu of educational study and practice. The fluidity of his 25-year narrative cuts diagonally across stable ideologies put forth both from the right and the left in the United States. From the right, a consistent and dominant commitment to technical rationality has maintained and promulgated key concepts of efficiency and development. Such trends were initiated at the very beginning of North American curriculum studies by Franklin Bobbitt, later refined by Ralph Tyler and are presently reified through the alignment of standards-based instruction scope and sequence charts and standardized test accountability. From the left, progressives have promulgated diverse epistemologies centering around two key concepts, social reproduction and political resistance (Pinar 2013). Following Schwab's declaration in 1969 that North 
American curriculum studies had become moribund, re-conceptualizers of curriculum have for decades turned their attention from development to understanding (Pinar et al. 1995). Henderson conceptualizes a middle way for curriculum workers and invites graduates students in FoC to consider in the possibilities for thinking, speaking and activating their own middle way ventures.

Though recently articulated as an alternative to the Tyler rationale, the nonideological have been a sustained feature of Henderson's scholarly trajectory. For example, when Wraga's (1999) and Pinar's (1999) contrasting ideas regarding the proper purposes and directions of contemporary curriculum scholarship were brought to bear on the pages of Educational Researcher, Henderson (2001b) interceded with an argument for an alternative that appreciated both tradition and the avant-garde. Understanding curriculum development as a circuit of problem-solving raises the very important and practical question of "Is this working?" However, understanding curriculum as an extraordinarily complicated conversation (Pinar et al. 1995) advances critical and useful questions regarding "what is implicated when one claims something is or isn't working?" The reconceptualization teaches us that matters of identity, power and language are always central and that curriculum is never apolitical or ahistorical. For Henderson (2001b), these are supplemental and not competing questions. His sustained dissatisfaction with either/or thinking was salient feature of the theorizing that eventually lead to Re-conceptualizing Curriculum Development (Henderson et al. 2015).

Limitations of space will not allow for a thorough examination of the impact of Jim's work as it has been experienced by diverse graduate students over the years. However, the pages that follow I (Dan) will write as a "teacher leader", who has been experiencing FoC, since 2008. I first engaged became engaged with Jim's work as a public school kindergarten teacher and doctoral student. Seized by both the content and method of the course, I remained interested in FoC as a teaching assistant, one of Jim's doctoral advisees and now as a teacher educator in another state. Therefore, my applications of the Henderson rationale are situated within my experiences as a teacher of young children, an emerging curriculum scholar and a teacher educator. In each of these capacities, I continue to draw on the ideas that Jim outlined above in a humble attempt to live out an Aristotelian telos of curriculum work and to keep going when reality makes it all too obvious that I have come up short. The two interrelated aspects of the Henderson rationale that I will emphasize in regards to how they apply to "curriculum-based teacher leadership" are: (1) the charitable listening and love of wisdom inherent to a commitment to finding a middle way; and, (2) the pedagogical roots of his curriculum theorizing that invokes one to walk their talk. Fusing these two attributes, I will argue constitutes something similar to what Kyla Ebels-Dugan's (2015) conceives of as "tenacious intellectual virtues", a quality that we must embody before we can ignite it in others. 


\section{Finding a Middle Way}

As mentioned above, I was introduced to curriculum theory in Jim's FoC course in 2008 working as a kindergarten teacher and beginning doctoral studies. I began my teaching career in 2000, shortly before the passage of the No Child Left Behind Act (NCLB). Therefore, my life as a teacher could be accurately described as taking place in the accountability era. Like so many teachers, I began my career with a vision of the teacher that I endeavored to be. It wasn't long until, I faced the common reality that my vision was at odds with the dominant culture of curriculum at my school. Enacting my ideals of being a caring and inclusive teacher of young children was a daunting enough challenge, which seemed to become increasingly thwarted the more dominant discourse-practices took hold. The rhetoric of neoliberal reformed seemed to at best trivialize and at worst distort the concrete realities of 5-year-olds with abstractions of preparing 'college and career readiness' that will prime them to 'compete in the 21 st century global marketplace.' Hence, I came to doctoral studies and to curriculum theory coping with a growing disconnect between the teacher I aspired to be and the contextualizing culture of curriculum that defined what was 'good.' This is a common, but nonetheless frustrating and even painful existential reality of many contemporary teachers. Speaking of her own experience, distinguished theorist, bell hooks (1994) powerfully testifies,

I came to theory because I was hurting- the pain within me was so intense that I could not go on living. I came to theory desperate, wanting to comprehend- to grasp what was happening around and within me. Most importantly, I wanted to make the hurt go away. I saw in theory then a location for healing (p.59).

The eloquent words of bell hooks continue to resonate with me, although in a very different way now than when I first read them. With an embarrassing amount of naiveté, I came to curriculum theory because I was morally outraged. The frustrations that I had experienced were so penetrating that I was ready for political resistance. I came to curriculum theory with a heightened sense of criticality wanting to accumulate knowledge for resistance- to understand the system and my capacity to disobey. Mostly, I wanted to win. I saw in theory the tools for formulating the right argument. For my first assignment in FoC, I wrote a scathing critique of a common system of instructional management. I chastised the hubris of scholars who either didn't understand or appreciate the value of inquiry-based pedagogy and the complexities of fostering a caring and cooperative classroom community. While honoring the sincerity of my concerns, Jim challenged me to reconsider the hubris in my own analysis, suggesting that I think about how I might engage in a collegial conversation by raising a good question, instead of asserting a strong opinion. A lead professional is a colleague who engages with peers to influence and be open to being influenced. I could no longer take solace in shutting my classroom door and comfortably conceding that my best work would be an uncover mission. In other words, Jim led me to search for a middle way. My options needn't be restricted to bureaucrat or dissident. A middle way of curriculum-based teacher leadership was indeed conceivable. 
The middle way of curriculum-based teacher leadership is methodologically speaking the enactment of critical bricolage enabling one to "make use of positive contributions of disciplines while avoiding disciplinary parochialism and domination" (Kincheloe 2001, p. 684). William Pinar (2006) suggests that disciplined study can indeed transform curriculum practice. Yet, Henderson and Gornik (2007) supplement such a commitment to study-based complicated conversation with an acknowledgement of the historical and systematic dominance of technical procedural rationality in curriculum practice. Underscoring the paradigmatic shifts, which are made evident in the complicated conversations among re-conceptualizers of curriculum, keeps theory grounded in pedagogical practice and away from tendencies to "become abstractions split-off from the concrete complexity of the historical moment" (Pinar 2013, p.7). Efforts to shed light upon matters of power, identity and discourse associated with the dominant paradigm, according to Pinar (2013), are intellectually exhausted. Moreover, when confined to "safe intellectual spaces for theorizing" complicated conversations remain detached from the public sphere, which continues on with the standardized management of curriculum. As a middle way, the Henderson rationale issues a provocative and inclusive invitation to all curriculum workers to consider the possibilities to think, speak and act as lead professionals for democratic ethics.

Many students in FoC spend the first several weeks of the course experiencing cognitive dissonance. This is to be expected, since apprehending curriculum as a complicated conversation represents a paradigm shift that requires many individuals to critically question taken for granted aspects of their daily practices. In other words, for many teachers finding a middle way involves fostering a critical relationship with inculcated assumptions. Such a paradigm shift includes a process of re-orienting oneself to habituated modes of understanding with a heightened level of critical awareness. Other students, such as myself, enter FoC already critical of the dominant discourse-practices. As indicated in the example above, the form of criticality that compelled me toward doctoral studies had its own, albeit very different, limitations. Overconfident in my own critical acumen, I lodged unforgiving negative critiques of authoritative structures without affirming any of their amenable qualities. Denouncing the status quo without advancing an alternative way of proceeding with educational problem solving, I was transmitting monolithic critical ideas of my own and failing to offer a transformative argument. Ebels-Dugan (2015) conceptualizes humility regarding one's own critical judgements and charity toward others' perspectives are the intellectual virtues central to cultivating autonomy. Hence, these intellectual virtues are key components to curriculum-based teacher leadership.

\section{Pedagogical Roots}

The second key component of curriculum-based teacher leadership to be highlighted in this chapter is that it is rooted in pedagogical practice. Neoliberal educational reform allied with accountability systems that are carried out by the audits of uncritical middle-managers who embrace "new professional identities" is a 
dominant force in P-20 educational institutions. An incredibly important aspect of Jim's evolving theorizing and scholarly voice narrated above, is that it is inseparable from the practical realities that comprised his 25 -year journey of teaching. Kincheloe et al. (2011) demand, "teachers must have more voice and more respect in the culture of education... [and] must join the culture of researchers if a new level of educational rigor and quality is ever to be achieved" (p.165). Conversely, to generate new possibilities of rigor and quality Henderson's narrative of action research and theorizing joins the culture of teachers. This is more than a matter of semantics. The eruditions of democratic curriculum-based pedagogy, as they are conceptualized in Jim's opening narrative and introduced to graduate students in FoC are more than theoretical abstractions. Rather, academic study was the means to inspire and inform a re-thinking, re-articulating and a re-working of one's curriculum practice. Furthermore, one's curriculum practice was the impetus for academic study. The Henderson rationale and the lead-learning invitations that have been extended over the years in $\mathrm{FoC}$ not only conceptualize but embody the normative ethical referents critical pragmatism, democratic hermeneutics and holistic pedagogy.

In my professional journey, the initial and immediate outcome of Jim's leadlearning invitation was that I was no longer content to shut my door and secretly do things my way. I encountered a new sense of responsibility to extend my sphere of influence by leaving my classroom door and an invitation for dialogue open. The particulars of my journey as a kindergarten teacher had the general implication of my following Jim's lead in thinking and talking about how curriculum studies can be the basis for the practice of a democratic ethic of teacher leadership. Alongside the theoretical eclecticism in the opening section of this chapter, I would like to bring attention to the concretized example of Jim's pedagogical practice. The process of replacing the Tyler rationale with a commitment to democratic, curriculumbased pedagogy, necessarily involved the careful and sustained narratives of a supreme artist, a reflective teacher engaged in disciplined academic study. This is the conceptual and operational definition of teacher leadership that guided my efforts as kindergarten teacher and emerging educational researcher.

Now I find myself working with graduate students working to earn Masters of Arts in education with teacher leadership endorsement. At the beginning of a course that I teach, my students read York-Barr and Duke's (2004) comprehensive review of two decades of research literature on the topic of teacher leadership. My students did not enjoy the reading assignment and offered two main criticisms. First, they found the technical procedures in the extant literature to be mostly comprised of strategies that are generally known among experienced educators. In this way, they complain, the authors' 62 pages offer them nothing new. Secondly, some students express exasperation with the forthright admission in the abstract that "the construct of teacher leadership is not well defined, conceptually or operationally" (York-Barr and Duke 2004, p. 255). They query how teacher leadership can be gaining political momentum nationally and among many state departments without a definition. Further, they wonder if this lack of a definition puts their program on a very unstable foundation. 
In search of clarity, we turn to our state department's website only to find wording that implicitly divides teachers and leaders into two separate groups. As the scrutiny intensifies, it is brought to bear that most of the rhetoric on innovation in schools is comprised of benign, commonplace catchphrases and slogans. Our state department provides a concise definition on a document titled the Kentucky Teacher Leadership Framework, "Teacher leaders transform their classrooms, schools, and profession, activating teacher growth and achieving equity and excellence for students" (Kentucky Teacher Leadership Work Team 2015). Eyes begin to roll as we unpack this simplistic sentence that vaguely charges my students with responsibilities to transform their context, galvanize imprecise growth among their colleagues, and achieve unprecedented equity for students. However, the faithful commitments of dedicated teachers persist in this conversation. After all, they do indeed endeavor to improve their schools, refine their teaching and do so to enhance student outcomes. They are not opposed to innovation. Yet, the surveillance of walk-through observations and the auditing of routine progress monitoring tests leave them little time and space to lead innovations.

Any mention of curriculum theory is absent from York-Barr and Duke's (2004) review of what is known about teacher leadership. Thus, at first glance, it seems as though using curriculum studies as the basis for apprehending and practicing teacher leadership is an idea the authors did not think to consider. Indeed, scholarship such as Ylimaki's (2011) fusing of the fields of educational administration and curriculum theory are unique and provide an imperative update to York-Barr and Duke's (2004) review. After all, if influential proprietary organizations, state departments and graduate programs are going to encourage teachers to lead it is reasonable to contemplate what is unique about the prospect of teachers leading as opposed to leadership from policy makers, school administrators or educational researchers. I challenge my students to consider how they are, as teachers, are uniquely situated so they can accomplish ends out of the reach of stakeholders with more traditional and formal authority. For these reasons alone as case is made for curriculum-based teacher leadership, since curriculum and pedagogy are the central facets of teachers' personalized and contextualized daily work.

However, I share with my students a second critique of the mainstream literature on teacher leadership. Reflecting upon the obvious absence of explicit reference to curriculum theory that is customary in mainstream literature, we take into account the conventions of curriculum theory implicitly infused throughout the review. In a laudable effort to make sense out of the extant literature, York-Barr and Duke (2004) begin with an apolitical, value-neutral conceptual definition that is too vague to strike controversy: "we suggest that teacher leadership is the process by which teachers, individually or collectively, influence their colleagues, principals, and other members of school communities to improve teaching and learning practices with the aim of increased student learning and achievement" (pp. 287-288). No reasonable person would object to teachers influencing improvements to bolster learning. However, my students cannot help but laugh how insights garnered from a comprehensive review of two decades of research can be deduced into the benign statement of "do and promote good things, so students will learn better". Is it 
possible that 20 years of research on teacher leadership has resulted in benign rhetoric without a substantive direction for prospective teacher leaders?

Perhaps this sort of conceptual emptiness did not sit well with the authors, because they persisted in operationalizing their loose conceptualization of teacher leadership. In doing so, they reveal how the predominance of technical rationale curriculum theory ensues as either a non-reflective or unacknowledged feature prevailing teacher leader discourse-practices. Tacitly utilizing each of Tyler's (1949) four fundamentals of curriculum development, York-Barr and Duke (2004) customarily begin the process of teacher leadership with "schools and districts must clearly articulate student learning and school improvement goals" (p. 290). Once these goals have defined the "purpose" of teacher leadership, the "possible ways in which teachers can lead efforts related to goal accomplishment must be generated" (p. 290). In Tyler's terms, this marks how teachers will experience opportunities to lead initiatives they may or may not have taken part in articulating. York-Barr and Duke (2004) suggest such experiences must be guided by the recognition that "unique and varied leadership capacities of individual teachers must be matched with unique and varied leadership functions..., [along with structures that] communicate purposes and expectations with staff..., [provide] school supports, [and] clear indicators of progress" (pp. 290-291). It is not insignificant to note that these purposes, experiential opportunities, organizational structures and evaluative systems of teacher leadership are developed at the systems level and may or may not include the personalized and contextualized perspectives of classroom teachers. Therefore, nowhere is it assumed that the development of teacher leadership is or ought to be led by classroom teachers.

Justifiably, my students are dissatisfied with the prospect of playing an ambiguous role in nebulously conceived teacher leadership. Why pursue teacher leadership if doesn't necessarily include an appreciation of teachers' intellectual autonomy and professional decision-making capacity? If curriculum and teaching are apprehended by these procedural mechanisms, then corresponding "new professional identities" will characterize dominant images of leadership (Ylimaki 2011). In plain terms, this puts teacher leadership on a trajectory of becoming an unappealing potential for teachers to be recruited as cost-efficient assistants to building principals in the carrying out middle-management accountability audits. The Henderson rationale offers an alternative and more complicated course. Recognizing the probable persistence of technical rationality, a democratic ethic of curriculum-based teacher leadership begins with an immanent break. Breaking from the taken for granted assumption of the dominant paradigm does not free a teacher leader from the practical constraints of instructional management. However, bringing curriculum theory into the reflective experience of teacher leadership sheds light on the habituated intellectual structures that are engrained in our dominant culture of curriculum. Curriculum-based teacher leadership is ignited with an event of becoming critically aware that "one of the most salient features of our culture is that there is so much bullshit" (Frankfurt 2005, p. 1).

Frankfurt's (2005) thesis is not that the bullshit is rooted in malicious deceptions or untruths. Rather, it is a product of lack of regard for the truth altogether. The truth is that curriculum and pedagogy are value-laden practices and value judgements are 
inscribed in all educational activities (Buzzelli and Johnston 2002; Hansen 2001). The imprecise rhetoric of "improving teaching" and "increasing learning" enable the neoliberal interests that drive educational policy, discourse and practice to be an elusive quality of the curriculum. To be certain, standardized systems of instructional management audit performativity without attending to the underlying ethics of curriculum practices. Therefore, one can make a career as a teacher, researcher or leader by way of bureaucratic functioning. Curriculum-based teacher leadership makes an alternative way available for educators who are committed to teaching and leading as a professional who "take an ethical stance in the world" (Block 2008, p. 416).

The chief distinctions between the technical way that teacher leadership is most prevalently being operationalized and Henderson's adaptive curriculum-based teacher leadership are the implicated ways for educators' being and becoming in the world. Dominant discourse-practices offer a way of deferring to intellectual authorities and complying with protocol to efficiently and effectively work toward the unacknowledged values of clandestine hegemonic sources. The Henderson rationale, on the other hand, challenges teacher leaders to think, speak and act in ways that work toward their becoming lead professionals for a democratic vision. Bringing a commitment to democratic ways of living with the normative referents of three broad themes to the fore, ventures of teacher leadership are more holistically conceptualized as encompassing personalized and contextualized journey of understanding. It might be said that the greatest irony of the accountability culture is that no one is held accountable for the value judgements inherent to any and all educational decisions.

Opting for a road less traveled, the efforts of teacher leaders who take an ethical stance in the world will often not be recognized or encouraged. Even more seldom will these efforts be commended. Hence, according to Kyla Ebels-Dugan (2015) intellectual virtues of humility and charity must be supplemented with tenacity. Her aims toward intellectual autonomy parallel Henderson's advancement of free thinking. What is more, her conceptualization of tenacity brings to bear the bureaucratic realities of P-20 teachers' situational contexts. Routinely expected and often directed to carry our bureaucratic functions, thinking as consumers and transmitters of knowledge pre-established by centralized authorities is indeed, for teachers, the path of least resistance. Hence, the research and policy on teacher leadership confine teachers' discourse-practices to the facilitation of technical conversations. Drawing upon his own experiential learning, as a free-thinking teacher, Henderson maps an alternate for teacher leaders. Imploring curriculum workers to think for themselves, as participants in an extraordinary complicated conversation, broad democratic ends-in-view emerging, powerful ethical communities can be affirmed on this road less taken. Therefore, the Henderson rationale invites teachers' discourse and practices to humbly inquire and charitably deliberate, while tenaciously engaging in transformative conversations.

Along the alternative route, democratic ethical norms fill the empty conceptual vessel within which veiled hegemonic interests operate through alleged value neutrality. In the spirit of Lincoln's "charity for all," as cited earlier in this chapter, it is important to recognize the contribution of Ralph Tyler. His linear circuit for 
curriculum problem solving has engrained habits of technical reflection into cultures of curriculum. This is his imperishable legacy of which bricoleurs make use, while circumventing the disciplinary narrowness that was likely not intended or even perceived in 1949. Benefitting from the reconceptualization studies, it is not too bold for us to supplement Tyler's technical rationale with a reflective "circuit of valuation" (Ryan 2011). In doing so, we are simply asserting that any set of beliefs, images and values are as good as any other. Though Tyler may have passively alluded to his progressive preferences, regarding ethics he only insisted upon an empty conceptual vessel, the creation of a philosophical screen. Since technical efficiency is equally well suited for any ethical or political orientation, curriculumbased teacher leaders do well to raise questions regarding how democratic virtues are made evident in educational enterprises.

Teaching is an innately moral endeavor (Buzzelli and Johnston 2002; Hansen 2001). How the value judgments that are inevitably infused in daily practice are incorporated into curriculum problem solving is where Tyler was flexible to the point of being relativistic and we are unwavering. Critical pragmatism, democratic hermeneutics and holistic pedagogy are the broad normative referents that constitute an open set of interpretations. However, they also demarcate an assertive boundary of what constitutes quality curriculum work in a democratic society. Not any philosophical screen is suitable for developing a curriculum in a free and just society. By merely stipulating that a philosophical screen be established, the Tyler rationale enjoys the same utility in an oppressive autocracy as a liberal democracy. While technical aspects of teacher leadership remain important, they are inept for ethically grounding teacher leadership.

Grounded in democratic ethics, I invite my students to think, discuss and act upon possibilities for curriculum-based teacher leadership that operate through adaptive, instead of technical fundamentals of curriculum. I ask them to consider transformative educational ends in view, instead of transmitting knowledge sanctioned by learning standards. Indeed, in my courses they are unsuspectingly invited to turn engage in an alternative discourse about their practices. They may discuss what is possible and what they find preferable, against the grains of data-driven standardization. They are encouraged to exercise and foster intellectual autonomy, while considering what is good for all. They are reminded that curriculum-based teacher leadership is deeply personal, historical, contextualized by the perspectives of diverse stakeholders and evolving through ongoing inquiry. We engage in holistic "aims talking" (Noddings 2013) that serve as ends-in-view for 3S pedagogy. Moreover, we proceed with a humble love of wisdom, and charity for all and a tenacious sense of commitment, as we know that leading this form professional artistry runs against the grain of dominant cultures of curriculum. This is how the adaptive fundamentals of curriculum highlighted in Jim's opening narrative are concretely operationalized in my classroom. Next, we will turn to how this work can be systemically supported. 


\section{The Interplay of Administrative and Teacher Leadership}

If we assume that schools will be organized within a refined and constructive alternative to Tyler's (1949) rationale; and if we assume that one aim will be to build educators' capacities to work as lead professionals for democratic ethics, as guided by three thematic orientations advanced through the disciplined study of nine curriculum concepts; and if we assume that teachers will flourish as democratic visionaries, colleagues, and artists through disciplined study and practice embodying the personal journeys that this work entails; then it stands to reason that the same three broad ethical themes that guide curriculum development and teacher leadership, must also guide the definition and operational aspects of administrative leadership and policy development. Just as Tyler's rationale is still considered the paradigmatic exemplar for conceptions of curriculum development (Null 2008; Walker and Soltis 2009), we critically recognize that the Standards for Advanced Programs in Educational Leadership for principals, superintendents, curriculum directors and supervisors, and policy "actions of government and the intentions that determine those actions" (Cochran et al. 1986, p. 2) do not advance a deep critical awareness of educational leadership and policy purposes.

Leadership Standards The common themes in the leadership standards represent what is known about current practice with an emphasis on the importance of a focus on student achievement; data-based decision making; communication and collaboration; shared leadership; instructional leadership and continuous professional development. One reason for the almost universal buy-in of this definition of leadership in schools, districts, communities, universities, and state and national government is its seductive rationality. Not unlike Tyler's rationale, these leadership standards do not advance a deep critical awareness of leadership purposes and therefore do not support critical pragmatism. Such a pattern or system is not interested in possibilities of achieving diverse, expansive understanding, but more focused on scripted agreement, and as such do not support educators' democratic hermeneutics through an open-minded, open-hearted frame of reference. Further, an educator operating out of holistic pedagogy would not find support in these standards to lead for the fullest release of human potential through the widest extension of educational opportunity.

\section{Public Policy}

Public policy is adopted by an agency of the government and is generally a principled guide for administrators to follow in carrying out their responsibilities" (Kowalski 2013, p. 163). In simple terms, the process of policy making is focused on establishing parameters about what should be done, and the constraints about what cannot be done in a school district. Schwartz and Sharpe (2010) remind us that we as human beings use two tools to manage organizational behavior: (1) rules and administrative oversight, and (2) incentives that encourage good performance by rewarding people for it. According to Kowalski (2013) properly constructed policy (rules) is a process that must consider four variables: legal, political, philosophical and professional. As such, we as lead professionals for democratic ethics find ourselves in a bind because while "good rules might be useful as guides as we 
try to manage multiple aims (legal, political, philosophical and professional), they will never be subtle enough and nuanced enough to apply in every situation (Schwartz and Sharpe 2010, p.7).

We posit for all societies with democratic ideals and aspirations, that at the policy level, educators be regarded as visionaries and trustworthy professionals capable of informed ethical judgments based on disciplined study and practice with a more hermeneutic rather than ideological orientation. More theoretically, as Jim Henderson asserts above, we reject the mechanistic, static views of reality in favor of an organic and historical model according to which individual persons and objects are interrelated within a dynamic process.

Administrative Leadership Defined With 41 years of experience as administrators in K-12, we contribute to this chapter with full empathy for the plight of administrators in this field today. We are not so far removed to pompously presume that by simply reading this section, full absorption of these theory-based suggestions will be incorporated into an already overloaded and demanding agenda. We are certainly aware of the many standardized requirements that administrators in the United States must perform on a regular basis, many of which leave us feeling disconnected from the very reason we chose this profession in the first place. We believe that 'good' curriculum work as indicated above, as the study and practice of the relationship between educational courses of action and democratic living, stirs and awakens a vital and missing link for educators living in societies with democratic ideals. As administrative leaders in education, we encourage you to imagine the moral possibilities that may be latent in your leadership repertoire and seek, as Jim Henderson refers to, a middle ground that begins with "reconceptualizing received standards and cultivating reflective inquiry" (Henderson and Gornik 2007, p. 93). We invite you to avoid meeting only the leadership requirements of neoliberal standardization (Watkins 2012) which causes our moral skills to be "chipped away by an over reliance on rules and procedures that deprive us of the opportunity to improvise" (Schwartz and Sharpe 2010, p. 111) and begin to imagine a cross-paradigm approach of standardized management and curriculum wisdom (Samford 2015). This work is invitational and hopeful. We invite you to join us in our quest for a better way by beginning the deliberative and complicated conversation this view of democratic administrative leadership embraces.

From a systems thinking point of view, if curriculum development is now being influenced by this open set of ethical themes, which allows for the play of diversified interpretations characteristic of democratic deliberations, then the culture needed to support this work will be central to its success. Within this culture, administrators committed to work as democratic visionaries, colleagues, and artists through disciplined study and practice while embodying a personal journey will simultaneously work to create a culture of support for teacher leaders working to do the same (Gornik and Samford, in press). These are two very distinct paths, which will be addressed in this section of the chapter. Administrative leaders are both lead learners and critical colleagues, supporting and nurturing while learning and 
growing all the while creating this same environment in their building or district for the teacher leaders to embark upon their own journey of understanding.

It is our contention that growth-minded leaders "start with a belief in human potential and development-both their own and other people's" (Dweck 2008, p. 125), so the focus becomes development and creation rather than answers and agreement. If we are seeking the kind of change, we must seek renewal (Sirotnik 1999) where every person is a contributor to improve our practice in a truly democratic forum (Nancy 2010); where all participants work toward a goal with a deep respect for the value that each person brings to the table. This culture of change is not possible in a district without the support of the administrative leader: "Conditions must be deliberately created to enable the mass of people to act on their power to choose" (Greene 1988).

Creating a Culture of Support Teacher leadership is not new. For over 25 years, Jim Henderson has been advancing democratically-inspired teaching and learning that uses deep subject matter understanding as a pathway to deeper understanding of the self and the society, or what he refers to as $3 \mathrm{~S}$ education. Teacher leaders can and will lead beyond the classroom using the $3 \mathrm{~S}$ design but without the support of the administration, efforts are stalled and energies frustrated. As Kegan so plainly states "a program can fail to provide the necessary evolutionary support by neglecting to build a bridge out of and beyond the old world" (1994, p. 46).

Our research is beginning to suggest that the prevailing conception of teacher leadership is not reaching beyond the individual classrooms. In other words, teachers are returning to their classrooms eager to utilize $3 \mathrm{~S}$ concepts in their classrooms but are unable to collaboratively share these practices with their colleagues because the basic infrastructure in most schools does not support this kind of ethical imaginings. If administrators are expected to provide this support for teacher leaders, they too need professional learning opportunities that empower them to embark upon their own journey of understanding. Due to the fact that current leadership standards do not define leaders as democratic visionaries, colleagues, and artists engaging with teachers in disciplined study, we propose working with administrative leaders to address these missing yet vital variables. Theoretically and pragmatically, we propose working with administrators to support a culture that "elicits administrative support to make time to develop trust thus promoting collegiality that expects sustained change grounded in democratic values to support curriculum development" (Samford 2016, p. 81).

Unfortunately, this is not the norm in administrative leadership in the United States today. The ideology that permeates our institutions of education not only discourages but prohibits a generative forum for multiple reasons, one of which is a lack of time dedicated to support such complicated conversation. There is barely enough time built into professional learning for maintenance of the banking concept of "container" to be "filled" (Freire 1997, p. 53) let alone the possibility that deep conversation may lead to questioning or even productive conflict. As a result, professional learning in an educational setting focuses primarily on "scripted agreement" painfully avoiding the "possibilities of achieving diverse, expansive empathy 
and understanding through respectful dissensus" as Jim Henderson suggests. We know that it takes time to develop the trust needed to support sustaining change in this level of curriculum development.

Taking Time to Develop Trust Apple notes there is blatant "loss of time to keep up with one's field" (Apple 2004, p. 189) and describes the "deskilling" of those in the field of education as "cut off from their own fields and again must rely even more heavily on ideas and processes provided by "experts" (p. 189). Time to develop trust so that all contributing educators have the opportunity to share curriculum-based deliberative conversations is imperative in creating a culture of support. As one teacher leader explains, "Trust is of the utmost significance in widening the possibilities of collegiality, of creative collaboration, and of expanding new horizons. The potential for growth is exponential when trust is established" (Griest et al. 2015, p. 161). In Finland, they know the value of taking time to cultivate trust. According to Sahlberg (2011), "Basic to this new culture has been the cultivation of trust between education authorities and schools. Such trust, as we have witnessed, makes reform that is not only sustainable but also owned by the teachers who implement it" (p. 2). Establishing this level of trust takes time, yet is imperative in sustaining change.

Sustaining Change to Support Curriculum Development We refer to Frank Ryan's (2011) interpretation of Dewey and Bentley' (1949) transactional "circuit of inquiry" to illustrate the change that both administrators and teacher leaders may undergo in sustaining a change in curriculum. In Ryan's interpretation, a nonreflective experience is interrupted by a problem. If the problem can be solved by habit, we return to a nonreflective state. However, if the problem is too big, we move forward to create a hypothesis and gather the tools and data to conduct an experiment. The object is to return to state of nonreflective experience, but not the same experience, we have changed. The nonreflective state is not the same but a new, changed nonreflective state. Interpreted, we must first realize that the problem of neoliberalism in education is too big to revert back to our habitual standardized management paradigm. We must move forward with a new hypothesis consisting of multiple theories and practical implementation to sustain a new nonreflective state. We look to Schwab's (1978) eclectic approach of curriculum theory by recognizing weaknesses of some theories while we seek to embrace that other theories may "provide some degree of repair of these weaknesses" (p. 295). Currently, we have embarked upon a journey to support administrators on their eclectic path of creating a culture to support their own journey and that of the teacher leaders.

The Research Award In spring of 2015, Rosemary Gornik received sizable award from the Ohio Department of Education to conduct research on improving teacher quality through teacher leadership. She formed a support team and together, we began to formulate the agenda for a 3-year journey to implement and support teacher leadership in two districts, one urban and one suburban. Our mission, to expose teachers and administrators to a definition of teacher leadership that embraces the three normative themes and nine concepts mentioned above for the purposes of 
advance a deep critical awareness of curriculum purposes in societies with democratic ideals. In addition to four graduate-level classes culminating in a Teacher Leader Endorsement for 40 lead teachers, all administrators in the two districts were to obligate $15 \mathrm{~h}$ of professional learning to gain insights about teacher leadership and create an action plan to support the teacher leaders when they returned to the building. Several suburban schools showed interest but one particular school volunteered immediately. We met with the district team of the suburban district and then with the entire administrative staff including all central office, all building principals, and assistant principals. They embraced the research goals with enthusiasm. Unfortunately, we did not have the same enthusiasm from urban districts. After spending weeks and months recruiting three different urban districts, the fourth one was fully on board. We learned that due to the accountability pressures urban districts experience, time spent on teacher leadership was considered a distraction from the real work of getting kids to pass the test. The superintendent of the fourth district we recruited was very eager to be a part of the grant as was their entire administrative team. Thus we moved forward with the commitment from two districts, one urban and one suburban, to implement a teacher leadership initiative including professional learning for administrators to support a culture of change.

We are in the process of creating the curriculum for four separate workshops over a 3-month time period for all administrators from both districts to meet and share ideas. The goals of these workshops are as follows:

- Create an action plan for teacher leadership in their building/district

- Define teacher leadership (with roles and responsibilities that support district goals that meet the learning needs of students).

- Understand and support 3S curriculum design principles

- Explore ways to establish a culture in the building/district to support teacher leaders

Prior to the meetings, a culture survey was electronically distributed to all employees in both districts. Interestingly, even with the diverse backgrounds of the two districts, the highest level of disagreement in both districts was with the statement "Our school has a trusting environment where teachers feel free to share opinions even if they may be controversial". This seemed to resonate with the administrators during the first administrative workshop and sparked deep discussion in both districts. Using the survey data, administrators will choose two specific areas of need in support of cultural change and create an action plan for teacher leadership in the district and then in the buildings. The administrators will be asked to define teacher leadership, brainstorm ways teacher leaders could support the goals and objectives of the district, and create a culture of support in the buildings. As we conclude this chapter, we are entering into the second professional learning workshop for these administrative leaders. 


\section{Conclusion}

This chapter presents interpretations of teacher and administrative leadership that emerge out of 25 years of action research in a graduate course introducing the fundamentals of curriculum - a topic that lies at the heart of educational practice. What if this FoC course never existed? What if the teachers and administrators who enrolled in the course were introduced instead to an uncritical appraisal of Tylerian fundamentals? In more general terms, what if societies with democratic aspirations didn't have critically-astute, ethically-aware educators to advance their ideals? What if these societies did not have educators who embodied and enacted critical pragmatism, democratic hermeneutics, and holistic pedagogy? What if these educators could not imagine becoming lead professionals for democratic ethics? What if these societies did not have educators who were engaged in personal journeys of ethical becoming? What if, instead, these educators practiced an ideologically rigid, faddishly narrow, vulgar pragmatism? What would be the future of these societies, and could they become "deep democracies" (Green 1999)? As rector of the University of St. Andrews, John Stuart Mill (1867) declares in an inaugural address at his school: "Bad men need nothing more to compass their ends, than that good men should look on and do nothing" (p. 36). As authors of this collaborative chapter, we passionately believe that those with undemocratic ends-in-view will not prevail in societies that are serviced by democratically committed educators. This is the vocational calling, the curricular platform, and the educational leadership we are advancing.

\section{References}

Apple, M. J. (2004). Controlling the work of teachers. In D. J. Flinders \& S. J. Thornton (Eds.), The curriculum studies reader (2nd ed., pp. 183-197). New York: Routledge Falmer. (Original work published 1986).

Apple, M. W. (2005). Education, markets, and an audit culture. Critical Quarterly, 47(1-2), 11-29.

Apple, M. W. (2006). Educating the "right" way: Markets, standards, God, and inequality (2nd ed.). New York: Routledge.

Autio, T. (2006). Subjectivity, curriculum, and society. New York: Routledge.

Autio, T. (2009). From Gnosticism to globalization: Rationality, trans-Atlantic curriculum discourse, and the problem of instrumentalism. In B. Baker (Ed.), New curriculum history (pp. 69-95). Rotterdam: Sense Publishers.

Autio, T. (2015). Tero's R2 and R3 contributions. In J. G. Henderson (Ed.), Reconceptualizing curriculum development: Inspiring and informing action (pp. 180-181, 194-195). New York: Routledge.

Ayers, W. C. (2010). The standards fraud. In A. S. Canestrari \& B. A. Marlowe (Eds.), Educational foundations: An anthology of critical readings (2nd ed., pp. 183-186). Los Angelos: Sage Publications. Original work published in 2000.

Barth, R. S. (2008). Foreword. In G. A. Donaldson (Ed.), How leaders learn: Cultivating capacities for school improvement (pp. ix-xi). New York: Teachers College Press.

Bernier, N. R., \& Williams, J. E. (1973). Beyond beliefs: Ideological foundations of American education. Englewood Cliffs: Prentice-Hall. 
Block, A. A. (2008). Why should I be a teacher? Journal of Teacher Education, 9(5), 416-427.

Burns, J. M. (1978). Leadership. New York: Harper and Row.

Buzzelli, C., \& Johnston, B. (2002). The moral dimensions of teaching: Language, power, and culture in classroom interactions. New York: Routledge Falmer.

Cherryholmes, C. H. (1988). Power and criticism: Poststructural investigations in education. New York: Teachers College Press.

Cochran, C. E., Mayer, L. C., Carr, T. R., \& Cayer, N. J. (1986). American public policy (2nd ed.). New York: St. Martin's Press.

Commission on the Reorganization of Secondary Education. (1918). Cardinal principles of secondary education. Washington, DC: National Education Association.

Cremin, L. A. (1965). The genius of American education. New York: Vintage.

Dewey, J. (1916). Democracy and education. New York: Macmillan.

Dewey, J. (1933). How we think: A restatement of the relation of reflective thinking to the educative process. Boston: D.C. Heath \& Company. (Original work published 1910).

Dewey, J. (1989). Freedom and culture. Buffalo: Prometheus. (Original work published 1939).

Dewey, J. (2013). My pedagogic creed. In D. J. Flinders \& S. J. Thornton (Eds.), The curriculum studies reader (4th ed., pp. 33-40). New York: Routledge. (Original work published 1897).

Dewey, J., \& Bentley, A. F. (1949). Knowing and the known. Boston: The Beacon Press.

Dweck, C. S. (2008). Mindset: The new psychology of success. New York: Ballantine Books.

Ebels-Dugan, K. (2015). Autonomy as intellectual virtue. In H. Brighouse \& M. McPherson (Eds.), The aims of higher education: Problems of morality and justice (pp. 74-90). Chicago: The University of Chicago Press.

Eisner, E. W. (1994). The educational imagination: On the design and evaluation of school programs (3rd ed.). New York: Macmillan.

Eisner, E. (2001). What does it mean to say a school is doing well? Phi Delta Kappa, 82(2), 367-372.

Frankfurt, H. G. (2005). On Bullshit. Princeton: Princeton University Press.

Freire, P. (1997). Pedagogy of the oppressed. New York: Continuum.

Gadamer, H. G. (1975). Truth and method (G. Barden \& J. Cumming, Eds. \& Trans.). New York: Seabury.

Garrison, J. (1997). Dewey and eros: Wisdom and desire in the art of teaching. New York: Teachers College Press.

Good, J. A. (2006). A search for unity in diversity: The "permanent Hegelian deposit" in the philosophy of John Dewey. Lanham: Lexington Books. Add pages referred to at p 18/replace xx.

Goodwin, D. K. (2005). Team of rivals: The political genius of Abraham Lincoln. New York: Simon \& Schuster.

Gornik, R., \& Samford, W. L. (In press). Into the light: Creating a culture of support for teacher leaders. Lanham: Rowman \& Littlefield.

Green, J. M. (1999). Deep democracy: Community, diversity, and transformation. Lanham: Rowman \& Littlefield.

Greene, M. (1988). The dialectic of freedom. New York: Teachers College Press.

Griest, J., Schneider, J. L., School, S., \& Stagliano, K. (2015). Lead learning stories: A narrative montage. In J. Henderson (Ed.), Reconceptualizing curriculum development: Inspiring and informing action (pp. 139-1168). New York: Routledge.

Hansen, D. T. (1995). The call to teach. New York: Teachers College Press.

Hansen, D. T. (2001). Teaching as a moral activity. In V. Richardson (Ed.), Handbook of research on teaching (4th ed., pp. 826-857). Washington, DC: American Educational Research Association.

Henderson, J. G. (1992). Reflective teaching: Becoming an inquiring educator. New York: Macmillan.

Henderson, J. G. (2001a). Reflective teaching: Professional artistry through inquiry (3rd ed.). Upper Saddle River: Merrill/Prentice-Hall.

Henderson, J. G. (2001b). Deepening democratic curriculum work. Educational Researcher, 30(9), 18-21. 
Henderson, J. G., \& Gornik, R. (2007). Transformative curriculum leadership (3rd ed.). Upper Saddle River: Merrill/Prentice-Hall.

Henderson, J. G., \& Kesson, K. R. (2001). Curriculum work as public intellectual leadership. In K. Sloan \& J. Sears (Eds.), Democratic curriculum theory and practice: Retrieving public spaces (pp. 1-23). Troy: Educator's International Press.

Henderson, J. G., \& Kesson, K. R. (2004). Curriculum wisdom: Educational decisions in democratic societies. Upper Saddle River: Merrill/Prentice-Hall.

Henderson, J. G., et al. (2015). Reconceptualizing curriculum development: Inspiring and informing action. New York: Routledge.

Hooks, B. (1994). Changes in format made Teaching to transgress: Education as the practice of freedom. New York: Routledge.

Kegan, R. (1994). In over our heads: The mental demands of modern life. Cambridge, MA: Harvard University Press.

Kentucky Department of Education [KDE]. (2015, December). Kentucky teacher leadership framework. Retrieved from http://education.ky.gov/teachers/Documents/Kentucky\%20Teacher\%20 Leadership\%20Framework.pdf

Kincheloe, J. L. (2001). Describing the bricolage: Conceptualizing a new rigor in qualitative research. Qualitative Inquiry, 7(6), 679-692.

Kincheloe, J. L., McLaren, P., \& Steinberg, S. R. (2011). Critical pedagogy and qualitative research. In Denzin \& Lincoln (Eds.), The Sage handbook of qualitative research (4th ed., pp. 163-178). Los Angeles: Sage.

Kliebard, H. M. (1992). Forging the American curriculum: Essays in curriculum history and theory. New York: Routledge.

Kowalski, T. J. (2013). The school superintendent: Theories, practice, and cases. Thousand Oaks: Sage Publications. Changes in format made.

Lee, G. C. (1961). Crusade against ignorance: Thomas Jefferson on education. New York: Teachers College Press.

Lieberman, A., \& Friedrich, L. D. (2010). How teachers become leaders: Learning from practice \& research. New York: Teachers College Press.

Macdonald, E., \& Shirley, D. (2009). The mindful teacher. New York: Teachers College Press.

Mill, J. S. (1867). Inaugural address: Delivered to the university of St. Andrews. London: Longmans, Green, Reader, and Dyer.

Miller, J. L. (1990). Creating spaces and finding voices: Teachers collaborating for empowerment. Albany: State University of New York Press.

Nancy, J. (2010). The truth of democracy (P. Brault, \& M. Naas, Trans.). New York: Fordham University Press.

Nichols, J. D. (2011). Teachers as servant leaders. Lanham: Rowman \& Littlefield Publishers.

Noddings, N. (1984). Caring: A feminine approach to ethics and moral education. Berkeley: University of California Press.

Noddings, N. (2007). Philosophy of education (2nd ed.). Boulder: Westview Press.

Noddings, N. (2013). Education and democracy in the 21 st century. New York: Teachers College Press.

Null, J. W. (2008). Curriculum development in historical perspective. In F. M. Connelly (Ed.), The Sage handbook of curriculum \& instruction (pp. 478-490). Los Angeles: Sage Publications.

Null, W. (2011). Curriculum: From theory to practice. Lanham: Rowman \& Littlefield Publishers.

Nussbaum, M. C. (2010). Not for profit: The human development approach. Cambridge, MA: The Belknap Press.

Palmer, P. J. (2007). The courage to teach: Exploring the inner landscape of a teacher's life. San Francisco: Jossey-Bass.

Pinar, W. F. (1994). Autobiography, politics, and sexuality: Essays on curriculum 1972-1992. New York: Peter Lang.

Pinar, W. F. (1999). Response: Gracious submission. Educational Researcher, 28(1), 14-15.

Pinar, W. F. (2006). The synoptic text today and other essays: Curriculum development after the reconceptualization. New York: Peter Lang. 
Pinar, W. F. (2013). Curriculum studies in the United States: Present circumstances, intellectual histories. New York: Palgrave Macmillian.

Pinar, W. F., \& Grumet, M. R. (1976). Toward a poor curriculum. Dubuque: Kendall-Hunt.

Pinar, W. F., Reynolds, W. M., Slattery, P., \& Taubman, P. M. (1995). Understanding curriculum: An introduction to the study of historical and contemporary curriculum discourses. New York: Peter Lang.

Rancière, J. (2010). Dissensus: On politics and aesthetics (S. Corcoran, Ed. \& Trans.). London: Continuum.

Ryan, F. X. (2011). Seeing together: Mind, matter and the experimental outlook of John Dewey and Arthur F. Bentley. Great Barrington: The American Institute for Economic Research.

Sahlberg, P. (2011). Finnish lessons: What can the world learn from educational change in Finland? New York: Teachers College Press.

Sahlberg, P. (2015). Finnish lessons: What can the world learn from educational change in Finland? (2nd ed.). New York: Teachers College Press.

Samford, W. (2015). Deliberative conversation: Cross-paradigm critique. In J. Henderson (Ed.), Reconceptualizing curriculum development: Inspiring and informing action (pp. 85-98). New York: Routledge.

Samford, W. (2016). Out of the dark: A direction for change in education. Eugene: Wipf and Stock.

Schwab, J. J. (1973). The practical 3: Translation into curriculum. School Review, 81, 501-522.

Schwab, J. J. (1978). In I. Westbury \& N. J. Wilkof (Eds.), Science, curriculum, and liberal education. Chicago: The University of Chicago Press.

Schwartz, B., \& Sharpe, K. (2010). Practical wisdom: The right way to do the right thing. New York: Penguin Group.

Sirotnik, K. A. (1999). Making sense of educational renewal. Phi Delta Kappan, 86(8), 606-610.

Tanner, D., \& Tanner, L. (2007). Curriculum development: Theory into practice (4th ed.). Upper Saddle River: Merrill/Prentice-Hall.

Tyler, R. W. (1949). Basic principles of curriculum and instruction. Chicago: University of Chicago Press.

Walker, D. F., \& Soltis, J. F. (2009). Curriculum and aims (5th ed.). New York: Teachers College Press.

Watkins, W. H. (2012). Re-imagining public education. In W. H. Watkins (Ed.), The assault on public education: Confronting the politics of corporate school reform (pp. 189-192). New York: Teachers College Press.

Wraga, W. G. (1999). Extracting sun-beams out of cucumbers: The retreat from practice in reconceptualized curriculum studies. Educational Researcher, 28(1), 4-13.

Ylimaki, R. M. (2011). Critical curriculum leadership: A framework for progressive education. New York: Routledge.

York-Barr, J., \& Duke, K. (2004). What do we know about teacher leadership? Findings from two decades of scholarship. Review of Educational Research, 74(3), 255-316.

Open Access This chapter is licensed under the terms of the Creative Commons Attribution 4.0 International License (http://creativecommons.org/licenses/by/4.0/), which permits use, sharing, adaptation, distribution and reproduction in any medium or format, as long as you give appropriate credit to the original author(s) and the source, provide a link to the Creative Commons license and indicate if changes were made.

The images or other third party material in this chapter are included in the chapter's Creative Commons license, unless indicated otherwise in a credit line to the material. If material is not included in the chapter's Creative Commons license and your intended use is not permitted by statutory regulation or exceeds the permitted use, you will need to obtain permission directly from the copyright holder.

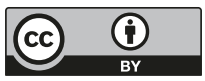




\title{
Chapter 11 \\ Codification of Present Swedish Curriculum \\ Processes: Linking Educational Activities over Time and Space
}

\author{
Eva Forsberg, Elisabet Nihlfors, Daniel Pettersson, and Pia Skott
}

\begin{abstract}
The aim of this chapter is to explore the relationship between curriculum and leadership research with examples of three recently completed mixed methods studies of assessment cultures and leadership as interlinked activities of governance and school management. We employ curriculum theoretical concepts like e.g. codes and arenas to illustrate their usefulness as a point of departure to further theorize a changing educational landscape. In our study, we illustrate how curriculum and leadership research are historically linked. We put forward some concepts to address the increased complexity of the governance system, and we stress the need to strengthen how different ways of forming the steering system interplay with key curriculum questions. Leadership researchers have, to a large extent, studied school development on a municipality- and organizational level asking questions on how to manage and guide school development. In contrast, curriculum researchers have studied school development from a reform- and governmental perspective more asking questions on how to steer educational development through law, curricula and evaluation. We suggest that these research traditions ought to be further united in order to develop both traditions in less normative, and more, critical ways, and to answer crucial educational questions in glocal times (Marginson and Rhoades. Conceptualising global relations at the glonacal levels. Paper presented at the annual international forum of the Conference of the Association for the Study of Higher Education, Richmond, VA, November 15-18, 2001). This chapter concludes with an argument for a new comparative curriculum code due to major shifts including curriculum practices, message systems, levels, arenas and number of curriculummakers engaged.
\end{abstract}

\footnotetext{
E. Forsberg $(\varangle) \cdot$ E. Nihlfors $\bullet$ P. Skott

Department of Education, Uppsala University, Uppsala, Sweden

e-mail: eva.forsberg@edu.uu.se; elisabet.nihlfors@edu.uu.se; pia.skott@edu.su.se

D. Pettersson

University of Gävle, Gävle, Sweden

Department of Education, Uppsala University, Uppsala, Sweden

e-mail: daniel.pettersson@hig.se
} 


\section{Introduction}

In Sweden and other Nordic countries the formation of educational systems has primarily been an issue for the national state in a rather homogenous society. Today, governance of education is embedded in global movements and a multicultural society influencing the role and function of the state. We will use two empirical cases, based in three recently completed research projects, to illuminate how curriculum and leadership research have worked in tandem to explain and develop both policy and practice. The cases focus on the assessment culture of the Swedish schools and implications of changes in governance for local educational leadership.

Ever since the establishment of the comprehensive school system in the 1950s and onward the interplay between educational research, policy and practice has been stressed and elaborated in various forms. Research focusing curricula, management, organization, evaluation and improvement was developed to support educational reforms. Curriculum and leadership research have often been labeled in different ways and partly they have been separately developed. However, in Sweden they emanate from the same line of studies with a tight connection between societal missions and research. The aim of this chapter is to explore assessment cultures and leadership as interlinked activities of governance and school management. We will employ curriculum theoretical concepts like e.g. codes and arenas to illustrate their usefulness as a point of departure to further theorize the changing Swedish educational landscape.

Different curricula, frames and regulations as well as curriculum-making constitute shifting educational experiences and paths and as a consequence also different ways to develop knowledge on education. With changing conditions follow both a window of opportunity and a need to scrutinize the way we conceptualize and research various educational phenomena. In the remainder of the chapter we first present changes in the Swedish educational system. This is followed by a short outline of some key features of research issues in the development of the Swedish comprehensive school system. We then turn to our two empirical cases on assessment cultures and local leadership. Finally, some conclusions are drawn about the development of curriculum and leadership research.

\section{Changes in the Swedish Educational System}

After World War II a comprehensive school system was developed in Sweden. It was unified and un-streamed enrolling all students - irrespectible of sex, social or geographical backgrounds and the students were put in the same age-based schools. In the case of compulsory and upper secondary education, the schools were almost exclusively run by municipalities. This set the agenda for constructing and negotiating the concept a school for all. Usually, students attended the nearest school and primarily tracking, if necessary, was meant to take place within schools. 


\section{The Swedish Democratic Welfare State}

For long, the Swedish Social Democrats formed governments by themselves having strong impact on policy, especially up until the mid-1970s. Education was regarded as an essential part of an all embracing welfare policy and the concept of equality was a guiding principle for reforms, and the method for achieving equality was a high degree of standardization with funding's and important decisions made at the national level. Sweden was characterized as a typical social democratic welfarestate regime (Esping-Andersen 1996). Hence, public education came to be both a part of the Swedish welfare project and a prominent example of it (Lindblad and Wallin 1993). During a period of expansion of the modern welfare systems in the 1950s, political governance and administration of reforms were relatively straightforward. Politicians made the priorities and decided upon the goals and resources that were supposed to guarantee attainment. Failures were usually attributed to shortcomings in the original plan or in the execution of the plan, and problems were expected to be solved at the next stage. Post-war expansion of education made central planning important and national educational administrators, politicians and educational researchers came to work rather closely together (Marklund 1985). Established within national educational planning were concepts like social engineering and a rational paradigm (Forsberg and Pettersson 2014; cf. Marklund 2008). The so called rolling reforms initiated in the 1960 s can serve as an example. Reforms based on findings in state-commissioned investigations were implemented and evaluated. Reformulations and further change were expected to take place on a regular basis (Johnsson Harrie 2009).

During the 1970s a number of factors, internal as well as external, challenged the perception of a welfare state. Globalization, new communication technologies, unstable political situation and the rise of discussions on a knowledge society all promoted changed relations between policy, labour market and economy (SOU 1990:44). In addition, the better-educated citizen called for enhanced influence. The criticism of the welfare state focused on the inadequacy of governance, increased costs, inefficiency and an overload of tasks (Held 1997). Despite educational reforms and resource allocations the system did not deliver what it promised. Even though reforms had changed the distribution of education socially and geographically, social background was still the best predictor of educational attainment (Härnqvist 1992). The Swedish model with a strong public sector was considered rather as a problem than an effective instrument for distribution of welfare and social change (cf. Forsberg and Lundgren 2004/2009).

\section{Changes in Governance on Both Vertical and Horizontal Axes}

In the last two decades the Swedish public sector and education system have been radically and extensively transformed (Englund 1995; Lundahl et al. 2014). Shifts in governance of Swedish education during the last decades include changes on 
both vertical and horizontal axes. These can be seen as expressions of changes in the relationship between the state, the society and the individual. On the vertical axes, globalization of education is manifested in transfer of policy and participation in programs initiated by international organizations like e.g. the EU and the OECD. At the same time, decentralization and deregulation have distributed responsibilities in new ways with municipalities as chief responsible for compulsory and upper secondary school. However, curricula, syllabuses and system for control and evaluation rest on the national level, even though there have been changes over time in the level of detail and degree of precision.

On the horizontal axes a number of suppliers of schools can be identified such as private companies or non-profit organizations running independent schools along municipality-driven schools. In addition, a third informal sector is growing, the so called shadow education sector, with offers on for example homework support (Forsberg 2015). Changes on both the vertical and the horizontal axes have together contributed to an increase of players involved in the governance of education; including governmental organizations (GO) as well as non-governmental organizations (NGO), international governmental organizations (IGO) and grey-zone actors (Lindblad et al. 2015). We will shortly describe two waves of reforms from the late 1980s up until today as a background for our cases on assessment cultures and leadership, respectively. These reforms have significantly altered the framing of schooling as well as the knowledge, values and norms codified in education.

\section{Two Waves of Reforms}

Two National Commissions paved the way for changes in the governing of education and introduced a move from centralism, universalism, social engineering and consensus to decentralisation, particularism and polarisation (Lindblad and Wallin 1993), as well as to marketization (Lindensjö and Lundgren 2000). The first wave of reforms was initiated in the late 1980 s with a clear policy shift illuminated in a series of activities that affected the structure, distribution and content of education but also its governance and control. The aim of these reforms was said to increase democracy, professionalism and efficiency (e.g. Lindblad and Popkewitz 2001). Up until the 1980s, the Swedish educational system was highly centralized. The Regional Boards of Education were responsible for enforcing educational policies and making decisions on the allocation of resources for each school. In 1991, municipalities were given full authority for primary and secondary education and granted responsibility for the organization, implementation, and operation of schools. This included staffing and in-service training for teachers and principals. Due to changes in the national regulations of independent schools they increased in numbers. Municipalities and independent schools became the principal organizers of schools, but it was still mandatory to hire a principal as an administrative and educational leader of the school. Many Swedish schools are small in comparison to large urban schools in e.g. America, and a single principal often leads a campus 
without additional administrative assistance. A result of these reforms of decentralization and deregulation was a more heterogeneous Swedish school system, opening up for a larger variation in organization, instruction and outcomes. In other respects the system was made more homogeneous in that upper-secondary school programs were made equivalent in enabling university studies and the pre-school system became more integrated with the comprehensive school (Skott et al. 2015b).

During the last years of the 1990s and the first decade in the twentieth century Sweden experienced a second wave of reforms. The line of action can be described in a two-way direction. The state is regarded as an important source of action, change and control. At the same time the municipalities, local schools and their actors are made accountable and responsible for the organization of education, allocation of resources, student achievements and school results. However, central means of control remained and became even more important e.g. the Education Act, objectives of the national curriculum and syllabi and the national system of testing and grading. These artefacts of hard governing have then been combined with soft governing tools introduced in the 1990s and reinforced in the 2000s, such as governmental development plans for education and a system of national quality assurance and assessments (Bergh 2010; Lundahl 2006; Nytell 2006). There was a shift into a more evaluation based discourse of governance with strong focus on assessment, monitoring and inspection on both the individual and system level. The curriculum and the assessment system also came to be more aligned and international comparative tests were included in the national system and discourse on assessment and evaluation (Pettersson 2008). The second wave has been described as reclamation of a more homogeneous and teacher-proof school system where school content, in particular subject knowledge, has once again been more nationally uniform (Forsberg and Román 2014). In all, changes in governance, curriculum, and assessments and their relations created a new frame for education. Questions may thus be raised on how assessment and educational leadership are discussed and analyzed within a societal discourse of education. This is particularly important when local actors are expected to take actions and the state monitor performances within an outspoken discourse of quality and equity in a regime of control. Not least, in relation to a society less homogenous and with students with increased variances in cultural background.

\section{From Frame Factor Theory to Code, Context and Curriculum Processes}

At an AERA meeting in 1979 professor Ulf P Lundgren stated that curriculum studies in Sweden are rooted in questions asked by national politics and answers given by research to the conflict between creating equal opportunities for all students and adjustments to a differentiated and technologically advanced society. In the following we will present how the early Swedish curriculum theory was developed. 


\section{Social Engineering: A Basis for Curriculum Studies}

An organized and agreed relationship between politics and science can in Sweden be dated back to at least the 1930s based on a specific collaboration between the state and organizations representing capital and labor interests. A key issue for the public sector was to simultaneously cope with demands on democratization and efficiency. The development involved various reforms that been summarized with concepts like - centralism, universalism, social engineering and consensus (Lindblad and Wallin 1993). In Sweden cultural homogeneity, secular modernity and socialdemocracy provided a basis for promoting a discourse on social engineering. But rather than an intermediary social engineer that runs in between politics and science, social science itself came to cooperate more closely with politics and administration (Marklund 2008).

\section{Educational Policy, School Development and Curriculum Research}

Swedish curriculum studies have been elaborated together and in close connection to politics and national agencies. The endeavor of launching a model for comprehensive education as well as the implementation of the model was closely followed by educational research. An early example is Torsten Husén, an internationally renowned Swedish researcher. Most of his research has been oriented towards policy issues closely related to school reforms in Sweden (e.g. Husén and Boalt 1968). Interested in comparisons of social and economic heritage and the development of a comprehensive school system Husén embodied two separate, but interrelated themes: the relation between education, social justice and meritocracy, and social efficiency and the allocation of individuals. However, Swedish curriculum research has also been closely connected to school policy and reforms (Vislie et al. 1997). Hence, Swedish researchers came to be engaged in the activity of providing politicians with empirically based knowledge for policy decisions. However, a sharp line was articulated between politicians asking questions and researchers giving answers (Säfström 1994). Within this tradition a number of empirical studies were conducted evaluating various educational reforms. In this way Swedish educational policy, practice and research to some extent submerged, creating specific educational researchers as state intellectuals giving answers to policy. 


\section{The Frame Factor Theory: A Step Towards Educational Sociology}

Within the paradigm of state intellectuals Urban Dahllöf in the 1960s formulated the frame factor theory giving special attention to what was conceived as a black box of educational research (e.g. Dahllöf 1967, 1971). The main question was if and how the un-streamed groups of students in the comprehensive school system had implications for classroom processes and school results. Dahllöf was especially interested in processes that were beyond control for teachers and students. The concept of frames, introduced by Dahllöf, came to be important in Swedish educational research and has later on been elaborated and discussed in a number of studies (e.g. Lundgren 1972; Gustafsson 1977; Skott 2009).

\section{Curriculum Codes: The Socio-economic and Historical Context of Education}

In the 1970s Ulf P Lundgren followed in Dahllöfs' footsteps, studying the framed classroom processes. Lundgren elaborated the concepts of steering group, codes and contexts. Codes relate to the purpose, content and method of a curriculum. He raised questions on how the frames were constituted, and identified historically developed curriculum codes manifested in the selection and organization of school knowledge (e.g. Lundgren 1977). Shifts in codes were related to changed relations between production and reproduction (Lundgren 1983); neither the purposes of education nor the subject content were taken for granted.

Since schools are institutions reproducing social values, the organization of teaching includes activities on three levels (e.g. Lundgren 1977, 1983): First, the selection and organization of knowledge, norms and values; second, the governing and organization of education including educational research and development and third, how the political curriculum document relates to classroom activities. The concept of code includes a multilevel way of thinking related to actions on different system levels, also expressed in terms of arenas for formulation, realization and mediation (Lindensjö and Lundgren 1986, 2000). This elaboration on linkage of levels includes thinking and reasoning on how different actors and educational activities are coordinated. As such, governance and leadership is integrated in the code concept. In the section Educational Leadership in a curriculum tradition we discuss this further. Curriculum research was presented as a pedagogical problem focused on the transformation of knowledge, values and norms from one generation to the next and the part played by education in reproduction (Kallos and Lundgren 1979). With an historical lens Lundgren identified four different curriculum codes: the classical, the moral, the rational, and the realistic. Even though they succeed each other there are also layers of the predecessors in later times. 
Curriculum research in Sweden came eventually to leave a positivistic behavioral tradition and entered research inspired by sociology and history due to the interest introduced by Lundgren concerning codes and context. As such, the work of Pierre Bourdieu (cf. Callewaert and Nilsson 1979, 1980; Broady 1990) and Basil Bernstein (cf. Bernstein and Lundgren 1983; Beach 1995) came to influence Swedish curriculum research and the further development of sociology of education. Through this influence reproduction theory became noticeable during the 1970s more focusing on the function of school subjects and school knowledge as sociocultural reproduction (e.g. Berner et al. 1977). The codification of educational knowledge was by Bernstein further differentiated in relation to three educational message system: curriculum, pedagogy and evaluation.

[...] curriculum defines what counts as valid knowledge, pedagogy defines what counts as valid transmission of knowledge, and evaluation defines what counts as valid realization of this knowledge on the part of the taught. (Bernstein 1975 p. 47)

This quote captures a complex reality especially relevant for our empirical cases and for analyzing the shifts and moves of contemporary education (cf. Forsberg 2011).

\section{Curriculum as a Political Problem and the Linguistic Turn}

The Swedish researcher Tomas Englund fits very well for explaining the shift towards sociology and history. He introduced curriculum as a political problem, as a site for different groups struggling over education and its content (Englund 1986). Englund analyzed the content of citizenship education in Swedish policy and textbooks concluding in an alternative curriculum code in relation to Lundgren - the civic curriculum code - including three conceptions: the patriarchal, the scientificrational and the democratic.

What became evident within studies influenced by sociology and history was the importance of language and consequently some of the curriculum research made a linguistic turn in the late 1980s and early 1990s focusing on meanings inherent in the curriculum and textbooks (e.g. Östman 1995; Selander 1992). Another prominent curriculum theorist employing historical and linguistic perspectives is Agneta Linné (1998, 2015), researching the formation of teacher education and knowledge traditions within higher education. The emphasis of language was also significant when Swedish curriculum research in the 2000s developed an interest for globalization and how this affected national curriculum and how different groups and organizations were struggling over education and its content (e.g. Pettersson 2008, 2014; Nordin 2012). 


\section{Curriculum as a Sociological Problem and Phenomenography}

Curriculum research in Sweden is normally discussed in terms of a bias towards systemic and structural issues, but Swedish curriculum research have also been influential in entering the classrooms to study curriculum as a practice and the role of agency. As a matter of fact the Swedish curriculum tradition was born within an empirical classroom research tradition (cf. Dahllöf 1967; Lundgren 1972, 1977; Gustafsson 1977). Researchers were interested in explaining the outcome of classroom processes, especially the underachievement of the educational system in creating a uniform, equivalent and democratic education, said to be a school for all. Within this specific focus several studies were conducted e.g. Callewaert and Nilsson (1979, 1980), Arnman and Jönsson (1983), Arfwedson (1985), and Arfwedson and Lundman (1984). The main analytical frame for catching these processes has been developed within the conceptualizations of what is perceived as the delivered and/or experienced curriculum. One scientific strand flourishing from these initial questionings is phenomenography with explicit claims on what and how students learn in school and higher education (Marton 1981). Within this trait of curriculum research students' conceptions of various phenomena are examined and findings show that educational phenomena and learning objects are experienced in qualitatively different ways.

\section{Curriculum as a Governance Problem}

Beside these inside and outside classroom studies in Swedish curriculum research there is also a profound tradition evident concerning governance, leadership, organization and development of the school. For instance, Erik Wallin and Gunnar Berg are inspired by a neo-rationalistic view on organizations, which they make relevant for the education sector by inscribing it in the main ideas and conceptualizations of the Swedish frame factor theory model. According to their view - the school-as-aninstitution is seen as established within society by dominating interest groups for promoting their goals and interests, which is formally codified and manifested in the curricula and other equivalent rule systems. Simultaneously, an organization is also under the pressure of informal control mechanisms, e.g. traditions, rituals, school codes, public opinion. The result of this is that the school-as-an-organization is considered as a result of interplay between the formal steering that emerges from the state and different kinds of informal influences rooted in the society in general, and particular in the local community. As such, the activities in schools are being shaped in the juncture between state legality and social legitimacy. Consequently, there is an essential established connection between an organization and the structure of society (Berg 1986; Berg and Wallin 1986). 


\section{Educational Leadership in a Curriculum Tradition}

In Handbook of Leadership for Learning (Townsend and MacBeath 2011) the editors argue that the use of the concept leadership became common only 20 years ago. Earlier the term was management but it became associated with conformity, uniformity and stasis. Leadership comes along as an alternative focused on change, development and movement (Townsend and MacBeath 2011). Educational leadership was used in the Nordic countries in the 1970s and 1980s but internationally:

[...] it was not until the 1990s that the interest in leadership really began to gather momentum. Chairs and centers were established in universities, new journals were created or renamed, development programs were introduced and governments began to pick up on the emerging trends. (Townsend an MacBeath 2011 p. 3)

Obviously, the concept of leadership is multifaceted and we need to clarify it. In this section, we elaborate three important concepts in Sweden - styrning, ledning and ledarskap (steering, leading and leadership) - for understanding the political and scientific discourse of leadership in Sweden. All three concepts are well rooted within the Swedish curriculum theory tradition and are often used for linguistic clarity of what in English most often is discussed in terms of governance and management of schools. The three terms steering, leading and leadership make sense from a Swedish context, but is maybe not that evident translated into English. The concepts are further discussed in case II.

\section{Steering, Leading and Leadership: Context Dependent Concepts?}

Steering, leading and leadership are elements of the steering system (Forsberg and Wallin 2006). Within the Swedish curriculum tradition it is looked upon as a system of rules, formally drawn up by the government to exert influence on processes and results in education (Skott 2009). The concept of leading captures the relational aspect where steering switches to local processes. Here individuals or defined groups of individuals (instead of the government) have the responsibility to organize and exercise influences (Nihlfors 2003).

Steering, leading and leadership create conditions for teaching and learning. We are especially focusing on leaders acting in-between groups of people, in and on different levels and in different networks (e.g. Robinson et al. 2009). We limit our discussion to individuals with formal functions and positions as educational leaders, well aware of that many other sources have influence (Seashore Louis et al. 2010). The formal functions and positions of leaders are manifold, from politicians and professionals on the national, regional and school owner (municipality and independent) levels to the school and preschool level (e.g. principals, preschool leaders, teachers). 
Within the Swedish curriculum theory tradition issues regarding the steering system have been analyzed in relation to school organization and school development as well as investigations directly addressing school leadership (cf. Svedberg 2014; Berg 1986). Using examples from our own and others research we will pay special attention to leading and leadership has in a curriculum perspective.

\section{Codes, Arenas, Curriculum Processes and Leading}

Following Dahllöf, it was above described how Lundgren raised questions on how frames are constituted. Discussing reasoning and concepts relating to leading and leadership the concepts of codes and arenas can be taken as starting points. Codes relate to the so called representation problem, emanating from the period when production and reproduction became separate processes. What is to be taught in schools is not up to the teachers to find out alone. Society and the state is strongly involved in what content is to be selected and how to organize schools and teaching in classrooms. Noteworthy is that one level of curriculum explicitly highlights the importance of organization. This means that the concept code can be used for analyzing linking of different levels at different times, but also to more narrowly study what happens at different system levels. As such the concept of codes can be most useful in discussing the activities of leading and leadership within a steering system.

Although the organizational level was recognized as important during the early days of curriculum theory in Sweden, it seems like the concepts were mostly developed either related to the national or the local level. However, during the early period of Swedish curriculum theory a profound tradition discussing leadership and organizational research was developed. This includes studies by researchers like Erik Wallin and Gunnar Berg, as mentioned above (e.g. Berg 1986; Berg and Wallin 1986). The curriculum theory concepts were however not elaborated further by them. This goes also for researchers studying school improvement (e.g. Ekholm 1976).

The concept of code thus includes a multilevel way of reasoning related to actions on different levels. This is where the analytical conception of different arenas for formulation, realization and mediation is important for understanding different activities (Lindensjö and Lundgren 2000). Today, the concept of curriculum processes is most used as an analytical tool for understanding variation in activities on different levels. These curriculum processes is understood as occurring at different levels but interlinked and related to each other in the form of textual activities. Some activities go on while text is formulated, while others are visible when texts are to be enacted (indicating that governing has an inbuilt direction, from the top to the bottom, but one can also identify curriculum cycles, when realization activities precedes formulation). During different times, and in different local settings, the complexity of the realization arena varies (Skott 2009). In this complex setting of curriculum processes on different arenas it is possible to identify various, what can 
be called, leading positions. These leading positions are not only recognized within the formal system, it is also possible to locate leading positions in an informal system of steering e.g. media actors, educational grey-zone actors (Lindblad et al. 2015), or national and international organizations involved in education, but they are crucial to uplift for discussing curriculum processes. All of these leading positions are important for the linking between different levels in the governing system, handling for one thing the curriculum as a governing means (Nihlfors 2003).

\section{Research on Curriculum Processes and Leading}

One position identified as important for curriculum processes is the superintendent (Moos et al. 2016). The educational superintendent is a research area that few researchers in Sweden and Europe until just recently have had in explicit focus for understanding curriculum processes. This can be contrasted against the American Association of School Administrators (AASA) that since 1923, nearly every tenth year has made a study of the American school superintendence (e.g. Glass et al. 2000; Kowalski et al. 2010). During the last years a series of symposiums (called In search of the educational superintendent) stressing research on educational leaders conclude that the term superintendent, frequently used in different settings, referred to different role expectations, duties and responsibilities, serving different purposes and needs (Nir 2014). A result of this cooperation became a survey instrument used in thirteen countries and the results of this survey are presented in the publication The Educational Superintendent; Between Trust and Regulation (Nir 2014).

Within this growing field of research various theoretical perspectives are used, and curriculum theory can be presented as one voice in a larger choir of perspectives. Scholars from different research areas have shown an interest in the black box of schooling where leading and leadership are important phenomenon. For one thing political scientists have shown an interest in the schools as a political organization (Pierre 2007) or in municipality ownership (Jarl 2012). One problem with the spreading, in terms of research areas, is that researchers on governing of schools represented by political scientists and researchers on leadership of schools represented by educationalists rarely meet at the same conferences. On educational conferences these studies have mostly been presented in leadership sessions and not in curriculum theory sessions, which might contribute to a low profile for this kind of research in some areas. This observation also goes for research studying the position of local politicians, which is surprisingly sparsely observed as an important actor for educational leadership. However, Wallin (2000) has evaluated projects with local politicians in focus. This is especially surprising for the Nordic context due to that the municipality level in many respects is an interface between the national and the local educational policies. The local is a level where national and local interests meet and political and professional standpoints on knowledge are interchanged and negotiated. This observation has been discussed by several Nordic researchers (e.g. Moos and Paulsen 2014) but in these discussions the results have primarily been 
highlighted, and seldom are the discussions placed within a context of curriculum and curriculum-making.

\section{Key Actors for Leading}

One actor that has been of interest is the principal, but more seldom pre-school leaders are in focus (Nihlfors et al. 2015). During the first 10 years of the twenty-first century the research on school principals have increased across Northern Europe. However, there is still a need for advanced research on these matters (Ärlestig et al. 2016). Noteworthy is that there is an emerging interest within leadership research to understand implications of context for principals' daily work. This, combined with a growing interest on theoretical elaborations for understanding aspects of leading and leadership, might indicate that the timing is right for a development of research on what can be called curriculum leadership. This can perhaps create a link between curriculum- and leadership research for a better understanding of the specific context of educational leadership.

\section{Case I: A Shifting National Assessment Culture}

Contemporary governance and discussions on school leadership in Sweden has enhanced the importance of quality and how to establish and develop it in a knowledge society. Assessment and evaluation take different forms, but during the last 25 years in Sweden there have been a strong emphasis on tests focusing on performance output, especially international large-scale assessments and activities that monitor, value and judge outcomes (cf. Forsberg and Wallin 2006; Lundahl 2006). We describe and analyze an assessment culture that has been on the move for some time now. We use data from research projects on assessment cultures, international large-scale assessments and educational control and quality regimes. Some have been funded by the Swedish research council (Forsberg 2006; Lindblad et al. 2015) and a number of studies (Forsberg and Wallin 2006; Nytell 2006; Pettersson 2008) were conducted within the research program of STEP at Uppsala University primarily inquiring educational changes from the 1990s and onward.

\section{Assessment Cultures and Key Actor Arenas}

With an elaboration on the concept of educational assessment culture we focus on conceptions of assessment and assessment practices that include different ways to measure, value, judge and document student achievement and school performances. Further it involves how outcome data is used as information or facts about how 
school performs. Assessment practices in Swedish schools can be categorized and to some extent divided into formal/informal and formative/summative assessments. Formal assessments are officially regulated whereas informal are the everyday assessments employed in the classroom and its context. Formative assessments are in many cases unrecorded and informal, while summative always are documented and formal. However, there have been examples of formative assessments being regulated in Sweden - e.g. the so called development dialogues (joint meetings between the teacher, the student and the parents) and individual development plans, IUP (recorded agreements between the teacher and student on goals to achieve). During the last 20 years of educational reforms in Sweden there has been an intensified formalization of assessment practices on three key actor arenas: politics/bureaucracy, school practice and educational sciences (cf. Forsberg and Román 2014).

\section{Assessment: A Multilevel and Multifunctional Phenomenon}

A variety of assessment activities on different levels co-exist in Sweden. They exist on different levels: international (large scale assessments and international cooperations on evaluations and inspections), national (assessment criteria related to syllabuses, grade system, national tests, guidelines for assessment, system for information and involvement of parents, follow-up, evaluation and inspection), local school board and agencies (follow up tasks and quality responsibilities), school/ classroom (teachers formative and summative assessment of student performances). In addition, requirements of teacher certificates and more differentiated teacher salaries increases demands on documentation of teacher performances and qualifications.

The assessment system with its different practices can be visualized as a very powerful multifunctional communication device that governs schools but also signals what school is all about. It provides information to teachers, students and parents on what knowledge and skills are to be given priority and puts up norms for how knowledge is to be observed, measured, valued and communicated. Assessments affect the relation between different actors. Assessments have a twofolded disciplinary functions; it corrects student achievement and behavior, the latter something that might be expressed in marks on conduct. Assessments also have a normalizing function when it is used for deciding whether a student is qualified for a certain school form or stage, whether a student should do an extra year or whether a student should be subject to special education. Educational assessments in such cases often interact with psychological and medical assessments. The selective function of assessment is related both to selective processes in society and the school system and to choices made by the student. Further, assessments have a prognostic function. These functions are not new to the educational system, but during the last decades they have been stressed on behalf of the curriculum. Finally, simple rather than complex assessment tools have been emphasized and are more frequently used. 


\section{Assessment: A Boundary Object Connecting Actors, Activities and Places}

Data on student performances are used to follow and determine the development of single students. Frequently, suppliers of schools also use surveys to map students, parents and teachers attitudes on different aspects of the school. Together data like these are used to inform on the effectiveness and efficiency of the school as well as to make up ranking lists of different kinds, nowadays often reported in media. For example, a research project on school management and performances showed that Swedish principals communicate by numbers to a rather large degree (Svedberg 2014). The findings pointed to a multi-faceted picture of principals' interaction with, meaning-making of and value attached to, educational outcomes. Four facets of the assessment culture of school leadership were identified: the narrative, the archive, the number, and the demand. The data also revealed two discourses of outcomes related to different objectives of education: the performance discourse and the care discourse (Forsberg et al. 2016). Data on individuals are accumulated and function as information about performances on other levels, and usually without being contextualized step by step. The other way around, policy and regulations on international and national levels as well as conceptions of assessment frame local schools and classroom practices.

An institutionalized, but in research often forgotten tool is homework and related feedback. Homework connects different actors and places. Yet another is related to the increase of players involved as suppliers of education. Evaluation and inspection are tools used to connect the public allocation of assignment and resources with accountability. Here public, private and non-profit organizations are connected. Today, with a changed system for funding, based on the thinking of a school for all, includes at least rhetorically the right to attend the nearest school or a private school, and reduced taxes for homework support and a proposal on mandatory provision of homework for municipalities can also be understood as a call for homework support for all (cf. Forsberg 2015). Assessments documented in written statements and marks are crucial at different stages of transitions and affect students' life chances. Assessment as a boundary object has a more elaborated and stronger position in the present education system. This is due to the emphasis put on student performance and outcomes and partly it depends on the increase in levels, arenas and actors involved in the communication with and about results - and not least, expectations on education in the so called knowledge society. 


\section{International Large-Scale Assessments as Practice and Discourse}

Sweden has been involved in international large-scale assessments first as one of the co-founders of the IEA and later on as an active part of the OECD and the EU. From the 1960s and onwards, international large-scale assessments were conceived as a technological/instrumental method for making the process of schooling more effective and as such were fitting with the dominant ideology of social engineering. The international tests primarily concern only a few subject matter areas and only a few age cohorts have been tested. In the international tests Sweden has normally been ranked above the average, and in many cases we have also held top, or close to the top, positions, but during the last 15 years there has been a falling trend for the Swedish student results. This has caused a lot of educational concern and dismay. It is not enough for Swedish education to be satisfied with medium or below medium results - Sweden is supposed to be at the top. In this discourse both researchers and policymakers have been deeply involved - discussing and referring to international tests - but more seldom teachers or principals participates. Today, the international assessments are an integral part of the national evaluation system of education and in the debate it stands out as an inevitable part of it. Nowadays Sweden also takes part in regional assessments.

The discourse about international tests in Sweden has been an ongoing debate around several issues, but the reliability of the tests is one of the more frequently raised. The discussion circles around methodological consistency with regards to national curriculum, objectives and content. However, commonly test outcomes are accepted as depicting the national school situation in a fair way, and the results emanating from the tests are mostly interpreted as evidence based results that in turn can be used for identifying reforms to be taken. In a study by Daniel Pettersson (2008) the reception of PISA on the Swedish politics/bureaucratic arena was studied. Conclusively, international large-scale assessments came to be important sources for information but different actors used them in different ways depending on individual purposes and goals. International assessments and the national discussions they lead to came, as such, to reflect actors view on education. The tests were given meaning in the context of politics, administration and media. They were also used as points of descriptions and departure for educational visions of the future. In addition, they were used to distribute shame, blame or glory (cf. Steiner-Khamsi 2004). In media debates the tests often served as a marker of legitimation, used as arguments for both stability and change (e.g. Forsberg and Román 2014).

In part the tests are used as a tool to evidence-base practice and politics. Today, schools by law, are expected to evidence-base their practice. However it is less common among practitioner than politicians, at least rhetorically. A much discussed Swedish example is the former Minister of Education Jan Björklund (2006-2014). He managed to successfully take the lead in the political and media debate on education. Björklund set the agenda in close connection to PISA and TIMSS, lending legitimacy from the research-like comparative tests. As often is the case in politics, 
there is already a solution at hand, searching for a problem to solve. While Björklund at first met some resistance, eventually his line of reasoning gained ground and several reforms during the last 10 years have been legitimated with reference to falling results in the tests and the urgency to act. In the Swedish case, the international assessments as such came to play an important part in the reforms of the curriculum, the syllabuses, the grading system and the organization of upper secondary schools.

\section{Grading, Leaving Certificates and Exams}

In Sweden, students have for several decades until recently not been introduced to marks and report cards until the eighth grade. There has been an intense and longlasting debate on marks and its didactical impact, which to some extent can be discussed as the perpetuum mobile of the assessment discourse in Sweden. When to start with marks and report cards seem to be the large gap in the assessment discourse today, with class-teachers, politicians to the left and education researchers generally more critical to marking compared to subject teachers, politicians to the right and researchers belonging to other disciplines than education. During the last 20 years we can observe a shift towards a more positive consensus. It is now more common that actors from all three arenas (politics/bureaucracy, research and practice) accept and advocates marking as a tool to promote justice, equality and quality. Instead of being questioned, marking is now more discussed in terms of mark inflation as well as mismatches between marking and national test results.

The previous focus on more complex assessment tools and a practice of mostly formative assessment are diminishing. One reason is that development dialogues have shown to be problematic in some aspects. Numerical marks seem to admit a reduction of complexity, which for many make them more clear-cut and paradoxically more informative compared to more elaborated verbal assessments (Forsberg and Lundahl 2006). Another reason why marking and other summative assessments have become more important has to do with the school choice reform of the 1990s. Schools now market themselves in order to attract students. Referring to good markings, i.e. a high ranking is a popular and successful marketing strategy for schools. The marking accordingly serve an economical function, since schools - especially independent schools - need students in order to get financial support (Lundahl et al. 2010).

Markings, leaving certificates and exams are meritocratic tools that regulate transitions within the educational system as well as between the system and the labor market. As such they are crucial, with an impact on students' life chances and opportunities to fulfill their individual goals. For long, all students leaving upper secondary schools with acceptable grades could enter higher education. This recently changed and now students can leave upper secondary education with one of three different exams - apprentice, vocational or academic, and it is only the academic exam that is directly qualifying for attending higher education. Today, 
grades, leaving certificates and exams are closing paths for students not able to produce expected results in various assessments.

\section{National Tests: Professional Guidance or Guardian of the Meritocratic Ideology}

In Sweden there was a previous system of testing the proficiencies of students with the help of national tests. From the middle of the 1940s until the middle of the 1990s the national tests were based on a system of norm-referenced grades. In the beginning there were norm-referenced tests in grade 2, 4 and 6 but later this changed to a compulsory achievement test in Mathematics only in school year 9 and in grade 3 for two of the programs in upper-secondary education. The main aim of the tests was to standardize teacher grading. In the first wave of reforms in the 1990s a new grading system was introduced to coincide with new curricula and syllabuses. At the same time national tests were introduced in three subjects in grade 9 to serve as professional guidance and to complement other kinds of assessments performed by an individual teacher. During the second wave of reforms the amount and extent of national tests came to gradually increase and as such laying a stronger foundation for evaluating students through summative assessments. Today there is a variety of tests in grade 3, 6 and 9. In the last years in Sweden national standards and tests have been formulated and implemented and in-service training materials have been produced and delivered to teachers. The purpose of the national tests has shifted from primarily a tool for professional guidance to more of a guardian of the meritocratic ideology of a justly system designed to measure the efficiency of Swedish education. (cf. Lundahl 2006).

\section{Reformed Tasks and Activities for National Agencies}

National school authorities have in Sweden had a crucial impact on evaluation regulations and teacher assessment. During the last decades the national agencies have gone through a number of make-overs. At the end, evaluation department and the agency responsible for school improvement were closed down. Instead, criteriabased control and inspection as well as the production of guidelines and evidencebased data and publications were reinforced and strengthened.

Starting in 2003, all schools in Sweden were inspected over a 6-year cycle. Control of output was introduced within an organizational tradition that previously foremost targeted input, processes and school development. Consequently, control is now seen as prerequisites for improvements. In 2008 the Inspectorate was moved away from the National Agency of Education (NAE) to an independent agency with a strengthen responsibility towards a more intensified inspection. School inspection, 
dating back to the 1860s, is not a new phenomenon in Sweden. However, the function, intensity and scope have varied over time (Lindgren et al. 2012). In the first wave of extensive school reforms during the 1990s inspections of schools was marginalized, due to reforms focusing on decentralization and profession-based governance. After a shift in government in 2006 a new structure for inspections was introduced based on three main areas: (i) knowledge, norms and values, (ii) leadership, and (iii) quality. The model for inspection draw extra attention to what was called attainment/goal fulfilment and leadership, but they also introduced the individual rights of pupils as a separate area to be inspected. In total the new agency summarized four areas to be assessed: (i) attainment/goal fulfilment and results, (ii) educational leadership and development, (iii) learning environment, and finally (iv) individual student rights (Lindgren et al. 2012). This period of inspection relied on an ideology of school improvement accomplished through more control-directed inspections. An overall pattern of change can be identified concerning the state using inspection as a mode of governance in a system of both public and independent schools. There is also a shift in focus from inspection of soft areas, like norms and values, to hard areas, like knowledge and attainment (Lindgren et al. 2012). This shift is tightly interconnected with both inspection techniques in use and a growing reliance on evidence. When state inspection is focused more on these aspects of education, so is research and practice.

\section{Case II: Curriculum Leadership in a Changing Educational Landscape}

In order to describe and analyze curriculum leadership in the changing educational landscape we will take our point of departure in national demands on local systematic quality work. This enables us to illustrate how the national curriculum is linking leaders on different levels in the Swedish educational system. This includes taking into account both the international embeddedness of the local school and the assessment culture permeating and made up by actors at different levels. The overall aim is to elaborate educational leadership within a curriculum theory tradition.

\section{National Demands on Cooperation}

According to the national law every school owner and all schools have to (Education Act 2010, chapter $4 \S \S 2-8$ ) systematically and continuously plan, monitor and develop the work to fulfil the goals in the curricula. The Swedish State School Inspectorate are responsible for the monitoring (Education Act 2010, chapter $26 \S \S$ 19-23). It is mandatory to document the monitoring of the curriculum and to involve the pedagogical staff, pupils and parents. To make this happen the law has an 
implicit assumption, or even request, on leading and leadership. Depending on how curriculum and curriculum work is defined the linking of owners, principals, teachers and parents can be understood as part of the curriculum, and as such be included in discussions of local curriculum work. As such, leading can be considered as an important aspect of curriculum processes.

Since the local systematic quality work is supposed to relate to the classroom work and students learning, visible through results, a question to ask is how this turns out in practice. In the following we focus on educational leaders above teachers and we use findings from a nationwide research project completed in the years 2009-2015. The project included surveys and interviews with superintendents, chairs and members of educational boards in municipalities and principals and preschool leaders in both independent and municipality own schools.

\section{Leading and Communication in Different Local Contexts}

The Swedish governing system includes three major levels of responsibility. The law and school curriculum is formulated at the national level, by politicians and administrators in ministries and national agencies. The responsibility to enact these laws and regulations is given to the boards of local school owners - the municipalities or independent schools. The municipalities have politically elected boards, while the independent schools have their own boards. This is in fact the independent part, since all schools follow the same regulations and curricula, are public financed and inspected by the same authorities. As a consequence, all schools and owners have to perform local systematic quality work. In reality municipality- and independent school owners have different prerequisites and different interpretation of the existing frames. There are also differences between schools connected to the same owner as well as between different school owners.

On a general level the results from the project illuminates a variety of interlinked curriculum processes (Nihlfors and Johansson 2013; Nihlfors et al. 2015; Skott et al. 2015a). The investigated leaders are all rather well-educated, think they have a rather broad scope of action, appreciate their work but they do not rely on other leaders competences on different levels. A main exception is the relationship between the chair of the board and the superintendent in municipalities (Skott 2014a). One explanation can be that they have to trust each other to get power over the political agenda. School leaders (principals and pre-school leaders) very seldom meet politicians to discuss e.g. the result of their systematic quality work and it is also rare that these type of documents ends up in board meeting protocols. Comparatively, it is more common that reports from the State School Inspectorate are on the agenda. However, the structure of some independent schools with internal boards, consisting of staff or parents changes the communication structure. In these schools there are somewhat different kinds of challenges for leading. For instance, a principal can be chair of the board and superintendent at the same time. An added complexity is that the board, which can be seen as the top in a local chain of leading, 
can consist of active teachers. Furthermore, the individuals at these leading positions meet regularly, often on a daily basis. (Skott 2013) In what ways the introduction of independent schools change leading structures on a local level have to be further studied.

\section{Multilevel Structures of Leading in Municipalities}

The lack of knowledge pointed to in the previous section becomes visible when elected members of the municipality school boards express that students' results have enhanced during the last years when it is in fact the opposite. Some explanations why this is the case is found in statements from board members about e.g. PISA results and school performances. On an explanatory level data are often on an aggregated level that makes it difficult to handle for unremunerated politicians having civilian jobs. They have to rely on superintendents' information and they are not fully content with this situation. Also, principals and pre-school leaders express uncertainty on how the superintendents present their school situation for the board.

The school leaders (principals and pre-school leaders) prerequisites for leading are affected by levels above them, by different types of so called middle leaders or superintendents' offices. The more middle layers existing, the more seldom you can find direct communication between school leaders and politicians, this also goes for superintendents. The lack of communication also affects comprehension between different groups. One reason for this is diversity in both culture and language (Nihlfors et al. 2016).

The National Agency for Education is supposed to be a link between the national level and the school owners. This link is however complex since the Agency more often communicates directly with school leaders and teachers, supporting them with general advices. This is strengthened by the fact that all newly appointed principals, according to law, must attend a national program for principals (a university based education), where the educational content is focused around the law and how to enact it (Skott and Törnsén forthcoming). It is not necessarily so that all principals relate themselves as being links in a local chain of governance. This experience is further strengthened through the inspection of single schools. The law also recognizes the principals and the pre-school leaders as responsible for schools inner work. Consequently, the national level "bypasses" the school owner level, making the multilevel leadership of the local school more complex than it has to be. In some questions the different levels are supposed to cooperate, in others the local level are autonomous, even though the results of the lowest levels (and thereby the processes leading up to them) are to be seen as the common denominator.

The findings of the project also indicate that contemporary emphasis on results, and not least PISA results, has together with the national organization of education resulted in feelings of insecurity and perceived lack of courage to act as an educational leader. Some leaders wait for, and even look forward to, the report from the State Inspectorate that gives them a legitimacy to act. However, there is a rather big 
difference between national and local school leaders' interpretation of expectations and confidence. Comparatively, national leaders, even if they have a negative rhetoric, can be looked upon as an ally (Nihlfors and Johansson 2013).

Municipalities' role as school owner is often unclear and sometimes unknown for single school leaders (Skott et al. 2015a, b). At the same time a new actor, the independent schools, are intertwined with municipalities school organizations. There are for one thing no local state authorities handling the transfers of financial means to independent schools. This is done through the municipalities own organizations, where a sum is calculated to follow each child in the municipality as a guarantee for the parents in choosing schools'. This means that the municipalities' middle leaders are not only to be linked to the municipality school leaders, but also to the ones in independent schools. The superintendent often has to balance the numbers of municipality schools in relation to existing and planned independent ones. Communication between leaders on different levels across the educational landscape is necessary. Particularly, this communication is seen between pre-school leaders. While primary and secondary schools are given permissions and are inspected by the State inspectorate, the pre-schools apply for permissions and are inspected by the municipalities. The municipalities' own pre-schools are however inspected by the State inspectorate, on a group level. This means that the same position in a municipality administration is given a double function, where the law encourages a more direct communication between the municipality school office and the independent pre-schools, than it does regarding the municipality pre-schools.

Taken together this raises questions about how local school machineries can be understood in the new horizontal landscapes, how leadership could be enacted to enhance learning, in schools and at different school levels. At the front is local curriculum-making and issues of winner and losers in the curriculum power game.

\section{Curriculum and Leadership Research: Concluding Remarks}

Within the Swedish curriculum theory tradition there has been a focus on different curriculum practices (policy, practice, research), educational message systems (curriculum, pedagogy, evaluation), arenas (formulation, realization, mediation), and curriculum makers (politicians, bureaucrats, school owners, educational leaders, teachers, students and their parents). In addition, researchers have paid attention to different spaces (international, regional, national, local) and times (formation, manifestation, reformation) in relation to the selection, ordering and manifestation of knowledge, norms and values as well as the management and organization of teachers, students and school activities.

Curriculum, codes, context, frames and arenas are empirically based key concepts. Over time, analyses have contributed to elaborations of the concepts and their meaning in relation to data from pre-school to higher education and through analyses of the changes of society as well as educational governance and leadership. The 
aim of curriculum research has been and still is to develop knowledge of the cultural and social reproduction of society. In addition, both curriculum and leadership research has contributed to our knowledge about the reproduction of the school as an institution and an organization.

The history of Swedish curriculum and educational leadership research is inseparable on knowledge and power. They both developed in relation to each other and in tandem with the central welfare state. Knowledge produced was part of that overall program on equity and efficient meritocratic distribution into different educational tracks. Hence, the central state was both the initiator and the receiver of research results. From this position Swedish curriculum research came to develop from investigating quantifiable factors to also include qualitative dimensions and meaning-making processes. Included were perspectives from a range of disciplines, such as education, didactics, philosophy, sociology, history, economics, psychology and linguistics.

In this concluding section we will discuss the changing Swedish educational landscape and based on our two cases elaborate on some of the concepts of the curriculum theoretical tradition. First, we address the context of education in the twenty-first century. Then, we suggest that the framing of education and the codification of educational knowledge, norms and values are best described in terms of a comparative curriculum code. Finally, we single out some issues on how curriculum and leadership research can be further integrated and developed.

\section{The Context of Education in the New Millennium}

While the nation state still is the hub of educational systems the state and its institutions are nowadays explicitly and firmly embedded in the global arena. International organizations like EU and OECD emphasize the importance of education to the knowledge society and the possibility for nations and regions to compete on a global market. The employability and mobility of students and workers are stressed. Standardization and formalization are reached both through regulations and consensus building activities. Mostly the latter, since education on the primary and secondary level are formally regarded as a national matter. However, soft governance tools, like the open method of coordination in EU have contributed to the introduction of both policy and programs on the national and local level. A number of indicators, measurements, reviews, inspections, ranking lists and associations for cooperation have been developed to keep track of member states involvement, rank and progression. At the global level education is framed foremost as an economic value. The economic return of education for regions, nations and individuals is prominent in the political debate.

At the same time globally education for all and nationally a school for all points to the value of inclusion and equality of opportunity. Here, we can also identify programs of sustainable development, especially related to the UN, promoting education for sustainable development (ESD). In comparison to neo-conservative and 
neo-liberal trends in education ESD has had less of an impact on the educational system and its governance. However, there has been a growth of Swedish research producing knowledge about teaching and learning regarding the intersection between ecology, economy, ethics and social justice within the larger framework of ESD (cf. Öhman 2011).

Earlier a school for all in Sweden primarily meant a comprehensive school system without streaming and tracking. With the establishment of the independent school system it takes on another meaning. Rather than one school for all children in a neighborhood, a school for each and every one is put forward, indicating a shift from the social to the individual level. Here education as a commodity with prospect of bringing back expected, specific, predetermined and measurable returns comes to the fore.

The rationale of educational reform in Sweden used to be grounded in centralism, universality, social engineering and consensus. This was followed by reforms based on ideas of decentralization, professionalization and democracy, which lately has shifted to a rationale built on recentralization, management, quality and evaluation. These different rationales relate to power and control over education in different ways. As a consequence, questions of how the system is governed, curriculum goals established and content selected and evaluated are in turn variously answered. Even though earlier rationales are not totally abandoned there are clear alterations in emphasis and thus the relations between policy, practice and research is changed as well. The evidence-based movement and the establishment and closing down of various national agencies are illustrative examples.

In sum, the context of education in the new millennium is very different from the world in which the Swedish curriculum tradition was formed and manifested. While the key-concepts still are viable, their specific meaning require further elaboration to be relevant in relation to this new context.

\section{The Changing Educational System of Governance, Framing and Steering}

Within the new context of education there have been changes of governance on both vertical and horizontal axes related to arenas of formulation, realization and mediation. The educational landscape of governance is today multifaceted and complex. We identified a shift from input and processes towards output. With reference to Hopmann (2003) there are several findings indicating that Sweden has left the German Didaktik tradition of process control in favor of the performativity product control model manifested in the Anglo-Saxon world. The former is characterized by its licensing model with professional self-control and self-evaluation and the latter focus foremost on outcomes and efficiency.

The changes of governance are accompanied by moves within all the frames of educational governance. The Educational Act and curricula of education still stress 
different democratic values the school has to safeguard, employ and promote as well as a broad concept of knowledge. It is, however, evident that the message systems of curricula and pedagogy are less important than the system of evaluation and the overall culture of assessment. The allocation of resources is primarily an issue for the boards of the municipalities and the independent schools. Although, the establishment of a school market together with the voucher system has made the schools more vulnerable. A damaged reputation may in fact in its most drastic form come to the closure of a school. Lately, there has also been a judicalisation of education, with an increasing number of conflicts settled in court, a possibility to fine schools and a growing amount of general guidelines. The complexity also relate to the interplay between different levels within and without the nation and the three level of analysis taken into account in curriculum research; the societal/ideological level, the curriculum level and the teaching and classroom level (Lundgren 1989).

Given our two cases, findings related to the assessment culture are closely aligned to the overall changes of the educational landscape and the message system of education. Findings of the educational leadership projects stress a need to use more specific terms to address the new complex situation in which leadership is exercised. We put forward the steering system including steering, leading and leadership as fruitful concepts to explore how educational leadership can be analyzed focusing both expectations and feed-back in the system (cf. Wahlström et al. 2010). As a consequence more nuanced and specified empirical findings have been reported showing ruptures in expected ways of leading and leadership. Often school owners were by-passed when deviations were reported and rectified. Dialogue between school owners and the national level has during the last decades been rare. It is "a tangled web of couplings" in educational organizations (Rowan 2002). What can be described as loose and tight couplings exist simultaneously and even on the same level in a system of control (Nihlfors et al. 2014).

Curriculum defines what counts as valid knowledge. Analyses of steering, leading and leadership and how these three elements of the steering system are linked in empirical cases may supplement our present knowledge about educational leadership and its prerequisites. Embedded in the steering system is local curriculum-making (Paulsen and Moos 2014). In the long run we may also be able to develop knowledge on how to develop cultures where values can be challenged and the role of educators can be strengthened also in relation to the political agenda (cf. City et al. 2011).

\section{The Comparative Curriculum Code}

What are the implications of the new educational context, the framing of education policy, practice and research and the steering system for the codification of educational knowledge, norms and values? While we argue that it is reasonable to introduce a new code, we do not state that the civic curriculum code with its patriarchal, scientific-rational and democratic conceptions is outdated. However, there are major 
shifts including curriculum practices, message systems, levels, arenas and number of curriculum-makers engaged, so we find it relevant to suggest, what we call the comparative curriculum code.

Like all curriculum codes of modern societies this one is anchored in a meritocratic ideal. Life chances of individuals cannot be legitimized by referring to ancestry or social rank, differences have to be conceived as fair. The meritocratic ideal has emerged as an important symbol of fair distribution of both educational and vocational chances relative to student achievement, as a combination of intelligence/ ability and effort/performance. Today achievements, grades, exams and entrances to further education or working life are established through comparison with specific criteria or in relation to performances of other individuals.

The meritocratic ideal is employed both on individuals, schools, and primary organizers of schools, nations and regions. While the basic legitimation in a meritocracy is student achievement, it is not necessarily prescribed in content, form or how to evaluate and by whom. The ideal of meritocracy may as such be open for curricula aiming for liberal education (Bildung) or schooling, the latter pointing to a more restricted educational program.

Even though, comparisons has a long history in the Swedish educational system, especially in relation to assessment and evaluation it is the way things are played out in the present that puts comparisons on the front stage - no matter what position or function an actor uphold, or where (s)he is active and with what in the educational system.

There are several factors contributing to a situation where comparisons have become the hub of the system: legitimacy problem related to the efficiency of the welfare state and the Swedish school performance; the competitive character ascribed to the global knowledge society; the character and purpose of international organizations; the marketization of Swedish educational landscape; the evidencebased movement; an empirical turn in research; technological developments enabling collection, selection, analyses and dissemination of a large amount of data and findings; a need to reduce complexity in a challenged system and; changes in the distribution of authority, responsibility and accountability. As shown above the assessment culture in total also contributes with its techniques to document, evaluate, rank and mediate performances and results. Important is also to recognize the shortcomings in the system, single schools and failures of individuals with different backgrounds.

Comparisons based primarily on data from student performances have strong implications for standardization and formalization of education. Quantifications, rankings and clear-cut concrete and evaluable objectives, standards and curricula have become popular political means for school leadership and improvement. To help professionals orientate themselves they are provided with shortcuts to knowledge of the educational system through research reviews over areas identified as important for practitioners. Here a number of different knowledge brokers have appeared.

How are curricula, pedagogy and evaluation conceived of within the comparative code? First of all, with comparisons as the guiding principle for codifying the selection, transfer and evaluation of knowledge, the message system of evaluation is 
primary. Curricula end up at the backstage accompanied by a risk for regarding knowledge and values as separate entities and reducing knowledge to prescribed and measurable knowledge. Also the system of pedagogy and transfer is affected, were diagnoses, keeping track and documentation have become more prominent aspect of the transfer process over the last years. Key-elements of the comparison code are legitimacy, standardization and formalization, also three of the main concept in comparative education today again holding a strong position in the field of educational research, policy and practice.

\section{Looking into the Future: Research to Come}

It is crucial that we uphold the Swedish curriculum tradition, i.e. continue to theorize on empirical grounds and closely follow changes in the educational system and develop new critical research question. An overall aim has to be to further elaborate on the key-concepts of the classical era and adding new ones when necessary. It is also important to continue to keep asking questions of the reproduction of the society, the culture and the school and its relation to changes in the production. In this chapter, we have also illustrated how curriculum and leadership research are historically linked. Here, we have put forward some concepts to address the increased complexity of the governance system. But we need also to strengthen how different way of forming the steering system interplay with key curriculum questions. Conclusively, leadership research have to a large extent studied school development on a municipality- and organizational level asking questions on how to manage and guide school development. In contrast, curriculum research have studied school development from a reform- and governmental perspective more asking questions on how to steer educational development through curricula and evaluation. We suggest that these traditions need to be united in order to further develop both traditions in less normative, and more, critical ways, to answer crucial educational questions in a changing educational landscape in glonacal times (Marginson and Rhoades 2001).

\section{References}

Arfwedson, G. (1985). School codes and teachers'work: Three studies on teacher work contexts. Stockholm: Liber Förlag/Gleerups.

Arfwedson, G., \& Lundman, L. (1984). Skolpersonal och skolkoder: Om arbetsplatser i förändring. Stockholm: Liber Utbildningsförlaget.

Ärlestig, H., Johansson, O., \& Nihlfors, E. (2016). Swedish leadership research - An important but neglected area. In H. Ärlestig, O. Johansson, \& C. Day (Eds.), Recent research on principals and their work - Cross cultural perspectives. Dordrecht: Springer.

Arnman, G., \& Jönsson, I. (1983). Segregation och svensk skola: en studie av utbildning, klass och boende. Lund: Arkiv Förlag.

Beach, D. (1995). Making sense of the problems of change: An ethnographic study of a teacher education reform. Gothenburg: Acta Universitatis Gothoburgensis. 
Berg, G. (1986). Att utveckla skolans sociala arbetsmiljö: ett fortbildningsmaterial för skolans personal. Lund: Studentlitteratur.

Berg, G., \& Wallin, E. (1986). Utbildningsreform, implementering och aktörsberedskap. Slutrapport från SIAU-projektet (Pedagogisk forskning i Uppsala, 68). Uppsala: Uppsala Universitet, Pedagogiska institutionen.

Bergh, A. (2010). Vad gör kvalitet med utbildning? Om kvalitetsbegreppets skilda innebörder och dess konsekvenser för utbildning. Örebro: Örebro universitet.

Berner, B., Callewaert, S., \& Silberbrandt, H. (1977). Uddannelse og bevidsthed. Kopenhagen: Cop.

Bernstein, B. (1975). Class, code and control. Volume 3. Towards a theory of educational transmissions. London: Routledge \& Kegan Paul.

Bernstein, B., \& Lundgren, U. P. (1983). Makt, kontroll och pedagogik: studier av den kulturella reproduktionen. Stockholm: Liber Förlag.

Broady, D. (1990). Sociologi och epistemologi: om Pierre Bourdieus författarskap och den historiska epistemologin. Stockholm: HLS Förlag.

Callewaert, S., \& Nilsson, B.-A. (1979). Skolklassen som socialt system. Samhället, skolan och skolans inre arbete. Lund: Lunds bok och tidskrifts AB.

Callewaert, S., \& Nilsson, B.-A. (1980). Skolklassen som socialt system. Lektionsanalyser I och II. Lund: Lunds bok och tidskrifts AB.

City, E. A., Elmore, R. F., Fiarman, S. E., \& Teitel, L. (2011). Instructional rounds in education. A network approach to improving teaching and learning. Cambridge: Harvard Education Press.

Dahllöf, U. (1967). Skoldifferentiering och undervisningsförlopp: komparativa mål- och processanalyser av skolsystem 1. Gothenburg: Göteborg studies in Educational Sciences.

Dahllöf, U. (1971). Ability grouping, content validity, and curriculum process analysis. New York: Teachers College Press.

Education Act. (2010). Skollagen (2010:800): med Lagen om införande av skollagen (2010:801). Stockholm: Norstedts juridik.

Ekholm, M. (1976). Social development in school: Summary and excerpts. Gothenburg: Reports from the Institute of Education. Diss. Gothenburg: University of Gothenburg.

Englund, T. (1986). Curriculum as a political problem: Changing educational conceptions, with special reference to citizenship education. Lund: Studentlitteratur.

Englund, T. (1995). På väg mot undervisning som det ordnade samtalet? In G. Berg, T. Englund, \& S. Lindblad (Eds.), Kunskap, organisation, demokrati. Lund: Studentlitteratur.

Esping-Andersen, G. (1996). Welfare states in transition: National adaptations in global economies. London: SAGE.

Forsberg, E. (2006). International tests, national assessment cultures and reform history. Research project. Stockholm: The Swedish research council.

Forsberg, E. (2011). Curriculum theory revisited: Curriculum as content, pedagogy and evaluation. Saarbrücken: LAP, Lambert Academic publishing.

Forsberg, E. (2015). Homework support as shadow education. Stockholm: The Swedish Research Council.

Forsberg, E., \& Lundahl, C. (2006). Kunskapsbedömningar som styrmedia. Utbildning \& Demokrati, 15(3), 7-29.

Forsberg, E., \& Lundgren, U. P. (2004/2009). Sweden: A welfare state in transition. Change and tradition in global education reform. In I. C. Rotberg (Ed.), Change and tradition in global education reform. Oxford: Scarecrow Education. Addendum.

Forsberg, E., \& Pettersson, D. (2014). European educational transfer and curriculum displacement: The Swedish case. In A. Nordin \& D. Sundberg (Eds.), Transnational policy-flows in European education: Conceptualizing and governing knowledge. Oxford studies in comparative education. East Greenwich: Symposium Books.

Forsberg, E., \& Román, H. (2014). The art of borrowing in Swedish assessment policies. In A. Nordin \& D. Sundberg (Eds.), Transnational policy-flows in European education: Conceptualizing and governing knowledge. Oxford studies in comparative education. East Greenwich: Symposium Books. 
Forsberg, E., \& Wallin, E. (2006). Skolans kontrollregim - ett kontraproduktivt system för styrning? Stockholm: HLS Förlag.

Forsberg, E., Hortlund, T., Malmberg, K. (2016, forthcoming). The assessment culture of school leadership. Nordic Studies in Education, 2016.

Glass, T. E., Björk, L., \& Brunner, C. C. (2000). The study of the American school superintendency. Arlington: American Association of School Administrators.

Gustafsson, C. (1977). Classroom interaction. A study of pedagogical roles in the teaching process. Akademisk avhandling. Stockholm: Stockholm Institute of Education. Department of Educational Research.

Härnqvist, K. (1992). Utbildningsreformer och social selection. In I. R. Åberg (Ed.), Social bakgrund, utbildning, livschanser. Stockholm: Carlssons.

Held, D. (1997). Demokratimodeller: från klassisk demokrati till demokratisk autonomi (2., rev. och utvidgade uppl.). Göteborg: Daidalos.

Hopmann, S. T. (2003). On the evaluation of curriculum reforms. Journal of Curriculum Studies, 35(4), 459-478.

Husén, T., \& Boalt, G. (1968). Educational research and educational change: The case of Sweden. Stockholm: Almqvist \& Wiksell.

Jarl, M. (2012). Skolan och det kommunala huvudmannaskapet. Malmö: Gleerups.

Johnsson Harrie, A. (2009). Staten och läromedlen. En studie av den svenska statliga förhandsgranskningen av läromedel 1938-1991. Linköping: Linköping Studies in Pedagogic Practices No. 10. Linköping University.

Kallos, D., \& Lundgren, U. P. (1979). Curriculum as a pedagogical problem. Lund: Liber Läromedel/Gleerup.

Kowalski, T., McCord, R., Petersen, G. J., Young, I. P., Ellerson, N. M. (2010). American school superintendent: 2010 decennial study. Rowmans \& Littlefied Education/AASA.

Lindblad, S., \& Popkewitz, T. S. (2001). Education governance and social integration and exclusion: Studies in the powers of reason and the reasons of power. A report from the EGSIE project. Uppsala Reports of Education 39, Department of Education, Uppsala: Uppsala University.

Lindblad, S., \& Wallin, E. (1993). On transition of power, democracy and education in Sweden. Journal of Curriculum Studies, 25(1), 77-78.

Lindblad, S., Pettersson, D., \& Popkewitz, T. S. (2015). International comparisons of school results: A systematic review of research on large scale assessments in education. Stockholm: Vetenskapsrådet.

Lindensjö, B., \& Lundgren, U. P. (1986). Politisk styrning och utbildningsreformer. Stockholm: Liber Utbildningsförlag.

Lindensjö, B., \& Lundgren, U. P. (2000). Utbildningsreformer och politisk styrning. Stockholm: HLS Förlag.

Lindgren, J., Hult, A., Segerholm, C., \& Rönnberg, L. (2012). Mediating school inspection - Key dimensions and key words in agency text production 2003-2010. Education Inquiry, 3(4), 569-590.

Linné, A. (1998). Moralen, barnet eller vetenskapen? En studie a tradition och förändring i lärarutbildningen (p. 6). Stockholm: HLS Förlag, Studies in Educational Sciences.

Linné, A. (2015). Curriculum theory and didactics - Towards a theoretical rethinking. Nordic Journal of Studies in Educational Policy, 1(1), 31-39.

Lundahl, C. (2006). Viljan att veta vad andra vet. Kunskapsbedömning i tidigmodern, modern och senmodern skola. Uppsala och Stockholm: Uppsala universitet \& Arbetslivsinstitutet.

Lundahl, L., Erixon Arreman, I., Lundström, U., \& Rönnberg, L. (2010). Setting things right? Swedish upper secondary school reform in a 40-year perspective. European Journal of Education, 45(1), 49-62.

Lundahl, L., Erixon Arreman, I., Holm, A.-S., \& Lundström, U. (2014). Gymnasiet som marknad. Umeå: Boréa.

Lundgren, U. P. (1972). Frame factors and the teaching process (Göteborg studies in educational science 8). Stockholm: Almqvist \& Wiksell. 
Lundgren, U. P. (1977). Model analysis of pedagogical processes (Studies in curriculum theory and cultural reproduction 2). Stockholm: CWK Gleerup.

Lundgren, U. P. (1983). Between hope and happening: Text and context in curriculum. Victoria: Deakin University Press.

Lundgren, U. P. (1989). Att organisera omvärlden. Stockholm: Utbildningsförlaget.

Marginson, S., \& Rhoades, G. (2001). Conceptualising global relations at the glonacal levels. Paper presented at the annual international forum of the Conference of the Association for the Study of Higher Education, Richmond, VA, November 15-18.

Marklund, S. (1985). Skolsverige 1950-1975: 4. Differentieringsfrågan. Stockholm: Skolöverstyrelsen \& Liber utbildningsförlag.

Marklund, C. (2008). Bridging politics and science: The concept of social engineering in Sweden and the USA, Circa 1890-1950. Department of History and Civilization, European University Institute.

Marton, F. (1981). Phenomenography - describing conceptions of the world around us. Instructional Science, 10, 177-200.

Moos, L., \& Paulsen, J. M. (Eds.). (2014). School boards in the governance process (p. 1). Dordrecht: Springer Educational Governance Research.

Moos, L., Nihlfors, E., \& Paulsen, J. M. (Eds.). (2016). Nordic superintendents: Agents in a broken chain. Dordrecht: Springer.

Nihlfors, E. (2003). Skolchefen $i$ skolans styrning och ledning (p. 102). Uppsala: Uppsala University Studies in Education.

Nihlfors, E., \& Johansson, O. (2013). Rektor - en stark länk $i$ styrningen av skolan. Stockholm: SNS Förlag.

Nihlfors, E., Paulsen, J. M., Skedsmo, G., Moos, L., Pulkkinen, S., \& Kanervio, P. (2014). Role and influence of school boards on improving educational quality. In L. Moss \& J. M. Paulsen (Eds.), School boards in the governance process. Dordrecht: Springer.

Nihlfors, E., Jervik Steen, L., \& Johansson, O. (2015). Förskolechefen - en viktig länk i utbildningskedjan. Malmö: Gleerups.

Nihlfors, E., Høyer, H. C., Kofod, K., \& Risku, U. (2016). Competence and understanding in the governance chain. In L. Moos, E. Nihlfors, \& J. M. Paulsen (Eds.), Nordic superintendents: Agents in a broken chain. Dordrecht: Springer.

Nir, A. E. (Ed.). (2014). The educational superintendent; between trust and regulation. An international perspective. New York: Nova Science Publishers.

Nordin, A. (2012). Kunskapens politik: en studie av kunskapsdiskurser i svensk och europeisk utbildningspolicy. Växjö: Växjö University Press.

Nytell, H. (2006). Från kvalitetsidé till kvalitetsregim. Om statlig styrning av skolan. Acta Universitatis Upsaliensis (Uppsala Studies in Education no. 114). Uppsala: Uppsala University.

Öhman, J. (2011). Theme: New Swedish environmental and sustainablility education research. Utbildning \& Demokrati, 20(1), 3-12.

Östman, L. (1995). Socialisation och mening: No-utbildning som politiskt och miljömoraliskt problem. Uppsala: Uppsala University.

Paulsen, J. M., \& Moos, L. (2014). Globalisation and Europeanisation of Nordic governance. In L. Moss \& J. M. Paulsen (Eds.), School boards in the governance process. Dordrecht: Springer.

Pettersson, D. (2008). Internationell kunskapsbedömning som inslag i nationell styrning av skolan (Uppsala Studies in Education No 120). Uppsala: Acta Universitatis Upsaliensis.

Pettersson, D. (2014). Three narratives: National interpretations of PISA. Knowledge Cultures, 2(4), 172-191.

Pierre, J. (Ed.). (2007). Skolan som politisk organisation. Malmö: Gleerup.

Robinson, V., Hohepa, M., \& Lloyd, C. (2009). School leadership and student outcomes: Identifying what works and why. Best evidence synthesis literation. Wellington: Ministry of Education.

Rowan, B. (2002). Rationality and reality in organizational management: Using the coupling metaphor to understand educational (and other) organizations - A concluding comment. Journal of Educational Administration, 40(6), 604. 
Säfström, C. A. (1994). Makt och mening: förutsättningar för en innehållsfokuserad pedagogisk forskning. Uppsala: Uppsala University.

Seashore Louis, K., Leithwood, K., Wahlstrom, K. L., \& Anderson, S. E. (2010). Learning from leadership project - Investigating the links to improved student learning. Minnesota: The Wallace Foundation.

Selander, S. (1992). Forskning om utbildning: en antologi. Stockholm: Symposium.

Skott, P. (2009). Läroplan i rörelse. The Interplay Between National Educational Policy and Local Practice: A study of curriculum processes (p. 102). Uppsala: Uppsala University Studies in Education.

Skott, P. (2013). Att leda inom ramen för olika huvudmannaskap. In O. Johansson \& L. Svedberg (Eds.), Att leda mot skolans mål. Malmö: Gleerup.

Skott, P. (2014). School boards and superintendents doing local curriculum work: Conflict or successful cooperation? International Journal of Educational Management, 28(7), 842-855.

Skott, P., \& Törnsén, M. (forthcoming). Effekter av rektorsutbildning.

Skott, P., Karlsson, S., \& Nihlfors, E. (2015a). Att styra och leda skola - ett lokalt perspektiv. Uppsala: Uppsala University EDU.

Skott, P., Pettersson, D., \& Bergh, A. (2015b). I mötet mellan politik och pedagogik - 70 år av skol(forms)reformer. In S. Lindblad \& L. Lundahl (Eds.), Utbildning: Makt och politik. Lund: Studentlitteratur.

SOU. (1990:44). Maktutredningen. Demokrati och makt i Sverige: Maktutredningens huvudrapport. Stockholm: Allmänna förlaget.

Steiner-Khamsi, G. (2004). The global politics of educational borrowing and lending. New York: Teachers College. Columbia University.

Svedberg, L. (Ed.). (2014). Rektorn, skolchefen och resultaten: mellan profession och politik. Malmö: Gleerup.

Townsend, T., \& MacBeath, J. (Eds.). (2011). International handbook of leadership for learning (p. 25). Dordrecht: Springer International Handbook of Education.

Vislie, L., Popkewitz, T., \& Bjerg, J. (1997). Pedagogy and educational research in Sweden - history and politics. In K. E. Rosengren \& B. Öhngren (Eds.), An evaluation of Swedish research in education. Uppsala: Swedish Council for Research in the Humanities and Social Sciences.

Wahlstrom, K., Seashore Louis, K., Leithwood, K., \& Andersen, S. E. (2010). Learning from leadership project - investigating the links to improve student learning. Executive summary of research findings. Minnesota: The Wallace Foundation.

Wallin, E. (2000). Skola 2000. Nu!: en helhetssyn på pedagogik, arbetsorganisation och fysisk miljö. Stockholm: Rådet för arbetslivsforskning.

Open Access This chapter is licensed under the terms of the Creative Commons Attribution 4.0 International License (http://creativecommons.org/licenses/by/4.0/), which permits use, sharing, adaptation, distribution and reproduction in any medium or format, as long as you give appropriate credit to the original author(s) and the source, provide a link to the Creative Commons license and indicate if changes were made.

The images or other third party material in this chapter are included in the chapter's Creative Commons license, unless indicated otherwise in a credit line to the material. If material is not included in the chapter's Creative Commons license and your intended use is not permitted by statutory regulation or exceeds the permitted use, you will need to obtain permission directly from the copyright holder.

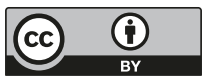




\title{
Chapter 12 \\ Rethinking Authority in Educational Leadership
}

\author{
William F. Pinar
}

\begin{abstract}
Our first experiences with authority, Luxon reminds, are profoundly personal. As children, we experience authority in relationships of varying degrees and orders of intimacy, i.e. parents and other caretakers, teachers, physicians, religious leaders, among others. Invoking elements of Freud and Foucault, I attempt to rethink authority in educational leadership. Freud offers a system supporting the negotiated reconstruction of one's interpretive architecture that is at once personal and professional. His version of psychoanalysis privileged the relationship, not the roles, of analyst and patient, ritualizing subjective and social reconstruction through ongoing and often complicated conversation that questioned the very terms that communicated lived experience. Like psychoanalysis, teaching is a collaborative if structured dialogical encounter across asymmetries of authority. Like Freud's psychoanalysis, Foucault's truth-teller (parrhesiastes) links individual projects of self-formation with collective practices (Luxon). Like psychoanalytic free association, parrhesia is candid conversation that does not coincide with structures of power. Whereas Freud developed interpretative acumen so as to support - even synthesize - the psyches of individuals in the distress of disintegration, Foucault cultivates its potential to intervene in an over-stabilized and socially submerged self. The link between Freud and Foucault lies in their insight that self-formation can result from encounters with authority, under certain specific mutually constituted conditions, even as these encounters simultaneously renegotiate and rewrite the terms of authority that initiate them. Can such conceptions of subjective and social reconstruction contribute to our understanding of how educators can be subordinated subjects and yet nonetheless become authorial agents of educational leadership?
\end{abstract}

\footnotetext{
W.F. Pinar $(\bowtie)$

Department of Curriculum \& Pedagogy, Faculty of Education, University of British Columbia, Vancouver, BC, Canada

e-mail: william.pinar@ubc.ca
} 


\section{Introduction}

"What is it to lead?" (Ted Aoki 2005)

Educational leadership involves (even as it cannot be reduced to) the exercise of authority (often institutionally conferred) to enlist faculty and students in realizing educational objectives, often institutionally conceived and now almost everywhere quantified. This profound depersonalization of education does not eradicate the personal character of curriculum conceived as complicated conversation, the leadership of which is enacted not only by policymakers and administrators but by parents, students, and, especially, teachers ${ }^{1}$. Complicating these conjunctions (see Phelan 2015) of curriculum and leadership acknowledges educational processes of recognition, summoning, Bildsamkeit ${ }^{2}$, as Michael Uljens and Rose Ylimaki (2015) underscore. These processes occur within relationships to authority and its exercise institutionally and educationally. Ted Aoki's ${ }^{3}$ insight that one instance of an education leader - the "principal" - once meant principal teacher, reminding us that authority could be exercised personally and pedagogically. So understood, educational leadership becomes the ongoing opportunity to engage colleagues in complicated conversation that renders experience within relationships educational.

That is my motive here: to engage colleagues in a complicated conversation regarding educational leadership by detaching the concept from its exclusively institutional affiliations and associating it with personal relationships rooted in early often imprinting experience, thereby invoking traditions of study and understanding associated with psychoanalysis, ${ }^{4}$ with its emphasis upon infancy, during and after which dependence becomes renegotiated over time into (relative) relationships of reciprocity. The structures of these early relationships often remain (if modified) into adulthood, transferring patterns of relationality from caregivers to colleagues

\footnotetext{
1 "[U]derstanding educational leadership [is] a multi-level project," Michael Uljens and Rose Ylimaki $(2015,2)$ point out.

${ }^{2}$ While in English the term - defined as plasticity or malleability - implies liability to undue influence, Roth $(2014,168)$ opposes the two: "Conformity is the enemy of learning because in order to conform you restrict our capacity for experience; you constrict our plasticity." What Roth is terming plasticity I would characterize as subjective reconstruction, the phrase underscoring one's capacity - often associated with the concept of agency - to remake what others have made. Such movement - from being imprinted by significant others (persons, ideas, events) to subjective and social reconstruction through academic study - implies the psychoanalytic subtext of education.

3 “A principal of a school at one time," Aoki (2005 [1987], 350) reminds, "was understood as the principal teacher, a leading teacher. In this sense, the principal was a specially recognized teacher, but first and foremost, a teacher. How the word 'principal' became detached and turned into a noun is a bit of a mystery. But we can see how the separation was a prelude to the linking of 'principal' to 'administration,' a term au courant in the world of business." Here Aoki was referencing the managerial discourse that continues to influence educational leadership, a discourse that depersonalizes teaching into its organizational functions.

${ }^{4}$ There is a century of psychoanalysis in education; its primary practitioner today is Deborah P. Britzman (see, for instance, 2015). For a U.S. history see Taubman 2011 and Cremin 1961, 207-215.
} 
whose institutional obligations can inadvertently invoke efforts to re-enact (including contest) dependencies from decades earlier. Through the encouragement of candid encounters - parrhesia $a^{5}$ or "frank speech" - both expressivity and communication might become clarified personally as well as professionally, enabling educational leaders to work with teachers and students more pedagogically.

\section{Relationships}

The classroom is a space in which the personal is magnified, not diminished. (Bryant Keith Alexander 2005, 251)

I emphasize relationships because moving curriculum online threatens to destroy ${ }^{6}$ them, not only students' relationships with teachers, parents, and other significant others, but students' relationships to themselves, and to what they study, and how they work. Relationships are forever fragile; they occur over time and allow for the establishment of trust and intimacy through free even fearless speech. They are structured according to circumstances: time, place, and point (pedagogical, professional, erotic) constitute "circumstance" but also does each participant's relationship history (including the history of the specific relationship itself), one's private situations and states of mind, themselves not unrelated to school climate, curriculum content, and teachers' conduct. Relationships are specific to those engaged in them, and they shift in scale and significance according to the specificities of situations and the singularities of those involved. Today there is much emphasis on relationality - Sam Rocha terms it "irreducible" giance, not a concrete reality questioned in our lives. To appreciate the specificity of relationality we might study autobiographically the history of our relationships, with school subjects, ideas, teachers and other educational leaders, and with ourselves. ${ }^{8}$

\footnotetext{
${ }^{5}$ The standard Greek word for freedom of speech (see Pagels 1989, 171 n. 97), parrhesia here emphasizes "frank" or even "fearless speech," a key concept from Michel Foucault's unpublished lectures on ancient ethical practices as these are discussed by political theorist Nancy Luxon $(2013,2)$, who juxtaposes such speech to the candour of psychoanalytic encounter in order to think through conflated issues of authority and relationship, personal and political.

${ }^{6}$ In addition to replacing face-to-face embodied encounters, moving curriculum online destroys relationships by failing to protect students' privacy. There are "widespread lapses in student data protection across the education technology sector," Singer (2015, B7) reports. "Insecure learning sites, apps and messaging services could potentially expose students, many of them under 13 , to hacking, identity theft, cyber-bullying by their peers, or even unwanted contact from strangers," Singer (2015, B7) warns. When paranoia represents a prudent replacement of trust, relationships end.

${ }^{7}$ Not only do "we arrive, at birth, in relationship, covered in blood," Rocha $(2015,100)$ writes, but subjective singularity is always already a multiplicity: "the human person is a public onto herself, from womb to tomb."

${ }^{8}$ Forty years ago Madeleine Grumet and I (2015 [1976]) argued for making curriculum technologically "poor" in order to forefront subjective presence and thus experience from which one could learn, e.g. that could be educational.
} 
Over the twentieth century and not only in the United States "professionalism" seems to have stripped the personal from student-teacher relationships, rendering them almost anonymous, even when cordial. Intimacy is suspect, due less to rare but sensationalized instances of pedophilia than to fears of the corruption of assessment. ${ }^{9}$ Even when stripped of specificity, the relational bond between teacher and student can be emotionally charged, even exploited. ${ }^{10}$ Students too have been stripped of singularity, often no longer conducting themselves as students but as customers or clients, e.g. schooling as shopping. ${ }^{11}$ For teachers and students, anonymity may be requested, even required, but to preclude the formation of relationship - especially when requested or advised - seems, well, unprofessional.

Despite conceptions of professionalism that strip specificity from teacherstudent relationships, it would be easy to assemble anecdotal evidence for the significance of teachers and other educational leaders in students' lives. ${ }^{12}$ There can be imprinting qualities to especially early relationships. ${ }^{13}$ Such imprinting portends - if unpredictably - forms of relationships later. The work of political

\footnotetext{
${ }^{9}$ The fear of teacher bias in grading has been replaced by fears - justified on occasion - that teachers may alter results on standardized tests. Among the most infamous instances of this corruption of assessment occurred in Atlanta. The National Center for Fair and Open Testing, or Fair Test, Fausset (2014, A19) reported, announced that manipulating scores of standardized tests has occurred in "at least" 39 states and Washington, D.C. "Unfortunately, Atlanta is just the tip of a test cheating iceberg," the organization's public education director, Bob Schaeffer, said in a statement (quoted in Fausset 2014, A19). For additional details regarding the scandal in Atlanta see Severson and Blinder 2014, A9; Blinder 2015, April 2, A12.

10 "Manage" is the verb Labaree $(2004,12)$ uses, but the distinction is lost on me, as his specification of using an "effective" and "authentic teaching persona" to "manage" a "complex and demanding emotional relationship" undermines both concepts: "relationship" and "authentic."

${ }^{11}$ On this point I am able to cite Labaree appreciatively. "An even bigger problem with the marketbased economic solution to the organizational problems in American education," Labaree (2000, 121) notes, "is that it is radically antisocial. By making education entirely subject to the demands of the individual consumer, it leaves no one looking out for the public interest in public education."

${ }^{12}$ I am excluding correlational studies of teachers and student test scores, less a matter of relationship than of outright manipulation and misrepresentation. See Pinar $(2013,17)$ for an egregious instance of correlation misrepresented as causality.

${ }^{13}$ Relationships can be imprinting in negative ways of course. Beginning in 2002, two economists - Victor Lavy of the University of Warwick in England and Edith Sand of Tel Aviv University in Israel - studied three groups of Israeli students from sixth grade through high school, concluding that in math and science their teachers "overestimated the boys' abilities and underestimated the girls', and that this had long-term effects on students' attitudes toward the subjects" (Miller 2015, A10). Ignoring the small and very specific sample size, ignoring how such a variable (such as "estimating") could conceivably function independently of other variables (such as home, religion, class, culture, gender), ignoring their confusion of correlation with causality, Lavy assured the reporter that "similar research had been conducted in several European countries and that he expected the results were applicable to the United States" (Miller 2015, A10). I am thinking of "imprinting" in more subtle and individualistic senses, as psychoanalytic practice demonstrates.
} 
theorist Nancy Luxon - focused on Freud and Foucault, on which I rely here suggests as much. ${ }^{14}$

Referencing psychoanalysis - wherein intimacy is encouraged by the authority of the analyst and the dependency of the patient - Luxon $(2013,126)$ is interested how in the "repeated recurrence" of "rupture" and "repair" within the "transference" relationship ${ }^{15}$ "prepares" persons for the complexities of relationships in "other domains of activity." Those "other domains of activity" include public domains, and Luxon is suggesting that, as in psychoanalysis, political life - I add educational life - is structured personally. ${ }^{16}$ "Political theorists," she judges, "missed the turn to 'relationships' among practicing psychoanalysts to orient a selfformation over -determined neither by trauma nor dominant social conventions" (Luxon 2013, 12). ${ }^{17}$

These three categories of formation - relationship, trauma and convention - are intertwined. Within object relations theories - those summarized and extended by ${ }^{14}$ Efforts to link early experience with later forms of public conduct - from political dispositions
to forms of prejudice - have failed to provide definitive empirical evidence. Dewey, for instance,
abandoned his faith that public school classrooms could be laboratories of democracy. ("By the eve
of World War I," Robert Westbrook (1991, 192) reminds, "Dewey was more fully aware that the
democratic reconstruction of American society he envisioned could not take place simply by a
revolution in the classroom, that, indeed, the revolution in the classroom could not take place until
the society's adults had been won over to radical democracy.") Thirty years later imprinting became
focused on prejudice, first on anti-Semitism (after the Holocaust) and soon, in the U.S., on what
was termed racial prejudice. "Attributing prejudice to social learning or mislearning makes it seem
a superficial matter,' Young-Bruehl (1996, 12-13) points out, spread across all cultures in some-
what the same way that perceptual illusion and historical misinformation are. It normalizes preju-
dice. The obvious next step is to conclude that proper education can eliminate prejudice, that
tolerance can be taught. Just say no to prejudice. Just say yes to the historically victimized. Or, as
many social scientists said - 'let them all learn social science! This hope epitomizes the confident
'just fix it!' attitude of many American educators since the 1950s. The attitude has been able to
perpetuate itself because it has dictated the instruments for measuring prejudice - the statistically
analyzable questionnaire and the opinion poll - and for judging the results of educational pro-
grams." Sixty years on, the terminology has changed, the prejudice - not only racial but method-
ological - has shifted in form but not in substance.

15 "In psychoanalysis," Grumet $(1988,126)$ explains, "transference refers to the process wherein the feelings and attitudes attached to persons involved in the traumatic events in the analysand's history are displaced onto the analyst." These feelings and attitudes from a person's past rarely remain there, re-emerging, maybe in modified forms, when interpersonal circumstances (often inadvertently) stimulate them. For some psychoanalytic theorists, all relationships are, in some measure, transference relationships, as the very capacity to bond with another person requires relational experience that begins in infancy. One project of psychoanalysis is to make these relational trajectories conscious. "We repeat," Sarup $(1992,9)$ notes, "sometimes compulsively, what we cannot properly remember."

${ }^{16}$ Freud, Luxon $(2013,128)$ reminds, noted that one's "first experience with authority is a personal, and not obviously political, one." It was Freud who explained how relationships with others - to "family, teachers, perhaps even a nation" - can have lasting "significance" and "obligation" (2013, 128). For a recent review of Freud's significance to education, see Britzman 2011.

${ }^{17}$ While socialization theories have been eclipsed by more specialized determinisms, "relationship" remains undertheorized in education too. For exceptions see Grumet 1988; Dimitriadis 2003; Waghid 2010, Handa 2011. 
Nancy Chodorow ${ }^{18}$ and Jessica Benjamin ${ }^{19}$, for instance - the internalization of those early life relationships becomes refracted through gender and race, two structuring forms of possible "trauma" and decidedly "dominant social conventions" that Luxon references. Structuring yes, but sources too for "subjective and social reconstruction" (Pinar 2012, 207), within relationships, including within oneself as well as with others. "Uniquely," Luxon $(2013,70)$ writes, emphasizing the point, "psychoanalysis privileges the relationship, not the roles, of analyst and patient." Privileging relationship over roles seems prescient for a professionalism to be restructured, in part, by relationality, wherein institutional roles inform but not definitively define relationships, including within the exercise of leadership.

Not only in psychotherapy do such personal relationships of authority and dependency - and their ongoing renegotiation ${ }^{20}$ and repair through complicated conversation - matter. Family life can underline how "dominant social conventions" and even "trauma" can be the beginning, not the end, of the story. How parents and other caregivers, including teachers (including the "principal teachers" upon whom institutional authority has been conferred), bond with children matters to their formation as persons, students, and as citizens. Political and cultural conservatives emphasize "character," but platitudes depersonalize relationships as they overestimate predictability. Character is no template to be installed; it is to be threaded through the specificities of relationship, study, and circumstance, including the affective as well as material conditions that prevail at home, school, and society. ${ }^{21}$ For children character becomes constituted within the accumulation of experience. ${ }^{22}$ Through its reconstruction one can convert private passion into public service. ${ }^{23}$ Luxon (2013, 292) emphasizes this point:

\footnotetext{
18 "Women's mothering in the isolated nuclear family of contemporary capitalist society" Chodorow $(1978,181)$ argued, "prepares men for participation in a male-dominant family, and society, for their participation in the capitalist world of work" $(1978,181)$, a world that exploits the nurturance of women to perpetuate men's dominance. (Grumet [1988] documents this history in her study of women and teaching.) "It is politically and socially important" Chodorow $(1978,214)$ insisted, "to confront this organization of parenting... It can be changed." The organization of professional relationships - specifically the exercise of educational leadership - can likewise be changed, as this chapter suggests.

19 "Owning the other within," Benjamin $(1998,108)$ suggests, "diminishes the threat of the other without so that the stranger outside is no longer identical with the strange within us - not our shadow, not a shadow over us, but a separate other whose own shadow is distinguishable in the light." Anti-racism, hospitality, cosmopolitanism: a series of historically and professionally urgent concerns cannot be reduced to attitudes or virtues, as they are embodied in singular individuals formed through relationships (Pinar 2009).

20 " $[N]$ egotiation is not a bargaining across clearly defined positions," Luxon $(2013,42)$ explains, "but a 'working-through' that proceeds any real change to belief, value, or practice."

${ }^{21}$ See Pinar 2011, 9-12. Anderson (2006, 48) defines Bildung as "the self-reflexive cultivation of character," a definition that shifts, including historically (see Bruford 2009; Løvlie et al. 2003), and cross-culturally (see Horlacher 2016).

${ }^{22}$ Lived embodied experience that is, not virtualized, as while staring at screens: see Pinar 2015a.

${ }^{23}$ Regarding the relationship see Pinar 2009, 153.
} 
The attention to relationships, however, signals that for all that our ethical institutions rely on individual responsibility in different ways, they further contain an expressive dimension - one that touches on courage, generosity, solidarity, among other qualities - inseparable from commitment to public context.

Specifically Luxon $(2013,16)$ points to the "culturally salient figures of psychoanalyst and truth-teller" - I would add teachers and other educational leaders - as the "nodal points" that "bind self- and political governance." These scales of governance are not the opposite ends of a spectrum, but intertwined subjectively, as Foucault notes: "There is no first or final point of resistance to political power other than in the relationship one has to oneself" (quoted in Koopman 2013, 173).

Like many of Foucault's ideas, this one is ancient, reminiscent of MacIntyre's $(2011,11)$ reminder that "Aquinas says that we only learn adequately when we are on the way to becoming self-teachers." ${ }^{24}$ Such a pedagogical mode of self-self relationality reminds us that experience becomes educational only when we manage to learn from it. One studies and learns not necessarily to realize one's potential - at least when that potential is construed only as human capital ${ }^{25}$ - but for the sake of self-formation the process of study itself supports: an openness to alterity that grappling with whom and what one does not know or understand can encourage.

Ethical self-formation ${ }^{26}$ may not be predictably related to specified structures of pedagogical relations, but even the suggestion of a reciprocal relationship resonates with traditions of liberal learning in the U.S., as Michael Roth makes clear. A "liberal" education has been considered "liberating," Roth $(2014,3)$ reminds, because it both requires "freedom to study" and aspires to "freedom through understanding." In that sense, liberal education is also "useful," he suggests, as the "free pursuit of knowledge" encourages the formation of "free citizens" (Roth 2014, 33).

Historically at least, the emphasis upon utility has been less intense in Canada, but similar ideas have been in play, as George Tomkins documents. ${ }^{27}$ "Nobody is capable of free speech," Northrop Frye (2002 [1963], 93) argued, "unless he knows

\footnotetext{
${ }^{24}$ Here MacIntyre is raising the question of the "relationship between character formation, being able to learn from experience, and being open to political and moral argument." The self-self relationship - specifically the capacity for educational experience and the subjective reconstruction that follows - makes every relationship at least a ménage-a-trois.

${ }^{25}$ The almost universal commodification of humanity as human capacity means, as Lewis $(2013,4)$ appreciates, that "self-knowledge and self-study become forms of self-management and self-governance within an overall biotechnological framework concerned with optimization of liferesources." See, too, Phelan $(2015,28-30)$.

${ }^{26}$ For a helpful explication of the concept, see Moghtader 2015.

${ }^{27}$ Practicality was not taken for granted, as Tomkins $(1981,160)$ records: "Thus the issue of curriculum differentiation was joined in the form of policy debates about the relative emphasis to be given to the traditional academic curriculum and a more practical education suited to a new age." That "practical education" was decoded as "American." As a result, "All these trends [occupational and vocational demands on the curriculum] developed more slowly in Canada, and curriculum differentiation occurred at a slower pace," Tomkins $(1981,163)$ notes. Even Sputnik sounded differently in Canada, as Canadian educators reacted in a "similar, albeit characteristically cautious and typically derivative, manner" $(1981,164)$.
} 
how to use language, and such knowledge is not a gift: it has to be learned and worked at." While "free speech is cultivated speech ... cultivating speech is not just a skill," Frye (2002 [1963], 93) emphasized: "You can't cultivate speech, beyond a certain point, unless you have something to say, and the basis of what you have to say is your vision of society." Reciprocity is implied in Frye's pronouncement, relationship between the personal and the public, between self and society. ${ }^{28}$ Frye's (2002 [1963], 95) "subject" was "the educated imagination." Accordingly, he emphasized education as "something that affects the whole person, not bits and pieces of him. It doesn't just train the mind: it's a social and moral development too" (2002 [1963], 95).

Not only in North America but also in North Europe do these definitions circulate (if differently), as Michael Uljens and Rose Ylimaki (2015) reference. ${ }^{29}$ Gert Biesta $(2003,62)$ traces self-formation to southern Europe, to ancient Athens and Rome, defining Bildung as "the cultivation of the inner life, that is, of the human soul, the human mind and the human person; or, to be more precise, the person's humanity." Contrary to twentieth-century progressivism, "content" was key, as it was constitutive of the process. ${ }^{30}$ In the vernacular one might say you are what you know. ${ }^{31}$ Since Herder and Humboldt, Biesta (2003, 62) asserts, "Bildung has always also been self-Bildung." That may be so, but "always" took different forms in different historical eras. ${ }^{32}$ In our time, potential tethered to employability threatens to end such education. In such circumstances what forms can educational leadership take?

\section{Parrhesia}

Simply, parrhesia is frank speech irreducible to power or interest. Nancy Luxon $(2013,133)$

\footnotetext{
${ }^{28}$ Reciprocity includes tension, which can be generative as Aoki noted. On this point (if in a different context) Tomkins $(1974,16)$ quotes Frye: "The tensions between this political sense of unity and the imaginative sense of locality is the essence of whatever the word 'Canadian' means."

${ }^{29}$ For a detailed study of the convergences of North European and North American traditions see Autio 2006.

30 “After all," Biesta $(2003,66)$ comments, "Herbert Spencer's famous question 'which knowledge is of most worth?' suggests that the criterion for decisions about what to include in the curriculum is the quality of knowledge." In Rocha's terms, that "quality" is less epistemological than ontological. In my terms, it is also a matter of relevance, personally and historically, themselves not necessarily separate domains.

${ }^{31}$ In my juxtaposition of Bildung with currere, I emphasize this point, ending by associating selfformation with "becoming historical" $(2011,126)$.

${ }^{32}$ Writing in 1934, Robert Musil (1990, 259) complained that "classicism's ideal of education [e.g., Bildung] was largely replaced by the idea of entertainment, even if it was entertainment with a patina of art."
} 
While a form of truth telling, such speech is not necessarily equivalent to truth, nor is it independent of time, place, and relationship. ${ }^{33}$ While no panacea, parrhesia might provide one passage through the present. For Freud in fin-de-siècle Vienna, Luxon (2013, 133) notes, parrhesia encouraged the cultivation of interpretative skills that might stabilize patients facing psychic "disintegration"; for Foucault almost 100 later in Paris, its "potential" was the contrary: disrupting an "overstabilized self." ${ }^{4}$ The "link," Luxon $(2013,134)$ suggests, is "their insight that selfformation results from a confrontation with authority, under certain conditions, even as this confrontation simultaneously negotiates and rewrites the terms of authority." Parrhesia is communication that could reconstruct the circumstances in which it occurs: complicated conversation in service to subjective and social reconstruction.

The emphasis on what Luxon 2013, 134) characterizes as the "irreducible relationality of parrhesia" enables her to posit that people can be "subordinated subjects" and "yet nonetheless become authorial agents of change." It is within networks of relationality - including relations of subordination - that one, through truth telling (even if only to oneself), participates in subjective and social reconstruction, even through institutional reorganization. ${ }^{35}$

For Luxon $(2013,136)$, the point is that the cultivation of "liberty" occurs within "personal relationship to authority." No doubt she would also acknowledge that anonymous authority depersonalizes; intense or extreme personal authority can crush. One prerequisite of leadership, then, is an institutionally encouraged willingness to work through in relationship the educational situation one faces. For Luxon $(2013,141)$ "risk" - intensified in situations of unequal power - can become articulated as engaging with a specific "authoritative interpretation" rather than resisting "all authority," suggesting how the "broader relations of political hierarchy" could "come to be re-interpreted, challenged, and exploded from within." Those "broader relations" can also be reconstructed, I add, if apparently accepted, through acts of dissimulation and intransigence Luxon does not here allow.

For Foucault, Luxon $(2013,141)$ points out, parrhesia implies both a kind of "speech" and a "set of practices," not mutually excluding categories. For Foucault, Luxon $(2013,142)$ continues, parrhesia "encompasses a broader set of personalized

\footnotetext{
33 "In Foucault's usage," Luxon (2013, 141-142) explains, "parrhesia denotes both a particular category of speech as well as the set of practices that govern its usage. Foucault distinguishes it from other types of speech: from the flattery of a sophist, from the too-free flow of chatter, and from coercive persuasion and rhetoric. Yet parrhesia is also more than verbal utterances; by Foucault's reading it also encompasses a broader set of personalized ethical practices that finish by constructing relationships to oneself, to authority, and to truth.” Truthfulness - rather than persuasion or, say, entertainment - animates parrhesia (Luxon 2013, 142).

${ }^{34}$ Certain academic knowledge - systematized parrhesia - in the service of self-shattering could, I argued, disrupt the white racist self (Pinar 2006, 181).

${ }^{35}$ Despite drawing a sharp distinction between reconstruction and reorganization (Pinar 2011, 87-90), I am acknowledging here that reconstruction can conceivably occur through reorganization, provided the latter enables - rather than substitutes for - the former (as it so often did in the Eight-Year Study).
} 
ethical practices that finish by constructing relationships to oneself, to authority, and to truth." Crucially, she $(2013,142)$ concludes, parrhesia "aims at truthfulness rather than at persuasion or entertainment." The relationship is not only or even primarily about itself, but about the truth of the educational situation in which the relationship is embedded.

Truth is, in part, what in curriculum studies we have characterized (often dismissively) as “content." In Luxon's (2013, 142, emphasis added) reading, Foucault associates the practices of parrhesia with "context" and "manner of speech, rather than in the matter, or content of that speech." Surely content is as least as (if not more) important as context and manner, a point driven home by civil rights the patina of No Child Left Behind.${ }^{36}$ Of course context and manner matter, but so do the facts. ${ }^{37}$ As style and substance, parrhesia is a medium of subjective and social reconstruction that, as Luxon $(2013,155)$ notes, an "obligation one bears to oneself, absent any reinforcement from political context; while parrhesia can occur in a democracy, in a monarchy, or in a dictatorship, it cannot be compelled." Monarchies and dictatorships are surely more restrictive than many - maybe not all, especially in this age of accountability and surveillance - schools, a point of comparison that could discourage teachers and other educational leaders from claiming institutional climate as disabling parrhesia altogether. In authoritarian regimes, intransigence ${ }^{38}$ relocates parrhesia to the private sphere where private plotting replaces public planning.

Luxon's final point in the quoted passage above - that parrhesia cannot be compelled - acknowledges agency. For parrhesia to be experienced subjectively as ethical obligation implies a wedding of relationships. Let's call it a commitment ceremony that becomes public however private its history, invisible its participants and singular its subjective formation. Whoever, wherever, and whatever comprises the present circumstances in which one works, fidelity ${ }^{39}$ to those no longer physical present informs - indeed may structure - one's engagement in the present, including those persons occupying it. Autobiography provides one means to issue invitations, register who is present, what vows are made, and how they might be honoured.

\footnotetext{
${ }^{36}$ While claiming to include all, especially poor, children in the upward mobility schooling in America presumably promises, what NCLB achieved was the reduction of black bodies to their economic potential, realizable only when children complied with an authoritarian regulation of their education through test-taking.

${ }^{37}$ Of course they can be intertwined as well as distinct, as Luxon $(2013,149)$ points out: "Parrhesia stages and so attests to an individual's relation to truth and the political field that enables or constrains this relationship." The great public pedagogue and anti-lynching activist Ida B. Wells is an exemplary instance, as she combined calm (against white audience expectations of "black" in the late nineteenth century) with facts (black men were not raping white women) to persuade the British public to protest the "peculiar" American practice of castrating then killing young black men (Pinar 2015b, 137-151).

${ }^{38}$ See Pinar 2012, 238.

${ }^{39}$ Luxon $(2013,179)$ emphasizes that: "Solitary individuals are not to be taken as starting point; the relations that bind them to one another are."
} 
While one is wedded to others, fidelity is finally personal. The "ethical" obligation of parrhesia," Luxon $(2013,156)$ acknowledges, "draws on the speaker's capacities to bear alone the burden of speaking truthfully." Such subjective coherence $^{40}$ is prerequisite for the struggle - social and subjective - that speaking frankly can entail, "life lived in relation to truth," as Luxon $(2013,164)$ summarizes the matter. It is truth constantly uncovered, critiqued, and reasserted, truth "underwritten by relations of care" (Luxon 2013, 175), care for others and oneself through care for truthfulness. ${ }^{41}$

While relations of care can structure speech within classrooms and with colleagues, including figures of authority, it also inspires engagement with persons no longer present, with ideas past as well as present, and with oneself. Noting that the practices of parrhesia enable us to rethink conceptions of "free speech," "democratic contestation, and "rhetorical persuasion," Luxon $(2013,180)$ points out that "these" are not the practices Foucault invokes. Rather, she $(2013,180)$ continues, Foucault's parrhesia "schools" one to recast "these practices from within." Working from within ${ }^{42}$ means, as Luxon $(2013,159)$ appreciates, that "freedom" is to be "exercised rather than attained." (Or conferred, I might add.) Such exercise is less in the service of getting it right as much as it is, Luxon $(2013,177)$ notes, the "shakiness" accompanying efforts to "orient" and "steady oneself" within relationships with "oneself, to others, and to truth-telling." For parrhesia to inspire "ethical selfgovernance," Luxon $(2013,177)$ continues, its "practices" must contribute to the formation of "coherent subjects," without "objectifying the individual into a "body of knowledge'," or, I might add, a "role-defined" professional. Roles may be contractually specified, but learning and leadership are personal.

\section{Conclusion}

Self-government without authority is a sham, and site-based management programs can be a hoax when it comes to enchanting professionalism. (David Berliner and Bruce Biddle 1996, 339)

Relying on Luxon's linking of Freud and Foucault, I have worked to "rethink" the relationships between "ethical self-governance" and "political governance" as threaded through "personal relationships" (Luxon 2013, 186). The scale, intensity,

\footnotetext{
${ }^{40}$ Luxon $(2013,191)$ prefers a "steadiness," but our point seems the same. "Yet if agency is pried away from any strong sense of self," Luxon $(2013,176)$ cautions, "then the only political engagement possible is resistance from within the field of power." Non-coincidence is cause and consequence of such subjective coherence (see Pinar 2015b, 113-116).

41 'The parrhesiastic promise," Luxon $(2013,177)$ explains, "is that through a relationship to a truth-teller, students of parrhesia develop their own authorial capacities" that "care" for the "self" as well as "others." Regarding the relationship between the two, see Jung-Hoon Jung (2016).

${ }^{42}$ That idea is a constant theme in my professional-personal life: see Pinar 1972.
} 
and intimacy of such relationships alter according to time, place, and circumstance, but in each instance affect is acknowledged, singularity affirmed while privacy is to be protected. Working through the complicated conversation of classrooms saturated as such conversation is with class, culture, and the unconscious ${ }^{43}$ - requires personal enactments of expressivity, parrhesia, tempered by professional discretion and animated by psychological courage.

By situating individuals within relationships, Luxon (2013, 186-187) reminds, Foucault made relationships the domain of "ethical experience," provoking "action" as they provide "structural constancy" supporting "stable ethical norms binding one individual to another." Indeed, she $(2013,187)$ adds the "dynamics" of specific "personal relationships" can "educate individuals to the arts of ruling and being ruled." These - "ruling" and "being ruled" - may seem overstatements in schools in democratic societies, but such words are also unadorned instances of parrhesia, frank speech that, recontextualized within discussion of leadership, spell out the subjugation educators risk when leadership is reduced to administration or management.

In our era of endless collaboration, leadership practiced instrumentally in the service of implementation can become an Orwellian dissimulation of enforcement. Exercising authority transparently, within acknowledged relationships, relationships with histories and characterized by candour, committed to truth telling, enables "principal teachers" to demonstrate leadership as seeking the truth of the present situation. Seeking and articulating what is found affirms the relationships through which ethical governance - of oneself with others - can recast those patterns of professionalism our predecessors have produced and that we can summon the courage to reconstruct.

\section{References}

Alexander, B. K. (2005). Embracing the teachable moment: The black gay body in the classroom as embodied text. In E. Patrick Johnson \& M. G. Henderson (Eds.), Black queer studies: A critical anthology (pp. 249-265). Durham: Duke University Press.

Anderson, A. (2006). The way we argue now: A study in the cultures of theory. Princeton: Princeton University Press.

Aoki, T. (2005 [1987]). Revisiting the notions of leadership and identity. In W. F. Pinar \& R. L. Irwin (Eds.), Curriculum in a new key: The collected works of Ted T. Aoki (349-355). Mahwah: Lawrence Erlbaum.

Autio, T. (2006). Subjectivity, schooling, society: Between and beyond German Didaktik and Anglo-American curriculum studies. Mahwah: Lawrence Erlbaum.

Benjamin, J. (1998). In the shadow of the other. New York: Routledge.

Berliner, D. C., \& Biddle, B. J. (1996). The manufactured crisis: Myths, Fraud and the assault on America's public schools. Cambridge, MA: Perseus.

\footnotetext{
${ }^{43}$ Psychic material - the sphere of the personal - includes sexual content, as Gilbert $(2014, \mathrm{x})$ affirms: "There can be no education without the charge of sexuality; love, curiosity, and aggression fuel our engagements with knowledge."
} 
Biesta, G. (2003). How general can Bildung be? Reflections on the future of a modern educational ideal. In L. Løvlie, K. P. Mortensen, \& S. E. Nordenbo (Eds.), Educating humanity: Bildung in postmodernity (pp. 61-74). Oxford: Blackwell.

Blinder, A. (2015). Atlanta educators are convicted of racketeering. Jury declares 11 of 12 defendants guilty in a case of cheating on standardized tests. New York Times, CLXIV $(56,824)$, A12-A16.

Britzman, D. P. (2011). Freud and education. New York: Routledge.

Britzman, D. P. (2015). A psychoanalyst in the classroom: On the human condition in education. Albany: State University of New York Press.

Bruford, W. H. (2009 [1975]). The German tradition of self-cultivation: "Bildung" from Humboldt to Thomas Mann. Cambridge: Cambridge University Press.

Chodorow, N. J. (1978). The reproduction of mothering. Berkeley: University of California Press.

Cremin, L. A. (1961). The transformation of the school: Progressivism in American education, 1876-1957. New York: Alfred A. Knopf.

Dimitriadis, G. (2003). Friendship, cliques, and gangs: Young black men coming of age in America. New York: Teachers College Press.

Fausset, R. (2014). Atlanta trial opens in school cheating scandal. The New York Times, CLXIV(56,640), A19.

Frye, N. (2002 [1963]). The educated imagination. Toronto: Anansi.

Gilbert, J. (2014). Sexuality in school. The limits of education. Minneapolis: University of Minnesota Press. Please check what page is referred to/replace x. Referred to at p 30.

Grumet, M. R. (1988). Bitter milk. Women and teaching. Amherst: University of Massachusetts Press.

Handa, Y. (2011). What does understanding mean for teachers? Relationship as a metaphor for knowing. New York: Routledge.

Horlacher, R. (2016). The educated subject and the German concept of Bildung. A comparative cultural history. New York: Routledge.

Jung, J.-H. (2016). The concept of care in curriculum studies. New York: Routledge.

Koopman, C. (2013). Genealogy as critique: Foucault and the problems of modernity. Bloomington: Indiana University Press.

Labaree, D. F. (2004). The trouble with Ed schools. New Haven: Yale University Press.

Lewis, T. E. (2013). On study. Giorgio Agamben and educational potentiality. New York: Routledge.

Løvlie, L., Mortensen, K. P., \& Nordenbo, S. E. (Eds.). (2003). Educating humanity: Bildung in postmodernity. Oxford: Blackwell.

Luxon, N. (2013). Crisis of authority. Politics, trust, and truth-telling in Freud and Foucault. Cambridge: Cambridge University Press.

MacIntyre, A. (2011). How Aristotelianism can become revolutionary. Ethics, resistance, and Utopia. In P. Blackledge \& K. Knight (Eds.), Virtue and politics: Alasdair MacIntyre's revolutionary Aristotelianism (pp. 11-19). Notre Dame: University of Notre Dame Press.

Miller, C. C. (2015). How teacher biases can sway girls from math and science. The New York Times, CLXIV(56,770), A10.

Moghtader, B. (2015). Foucault and educational ethics. New York: Palgrave Macmillan.

Musil, R. (1990). Precision and Soul: Essays and Addresses. [Edited and translated by Burton Pike and David S. Luft.] Chicago: University of Chicago Press.

Pagels, E. (1989). Adam, Eve, and the serpent. New York: Vintage.

Phelan, A. (2015). Curriculum theorizing and teacher education: Complicating conjunctions. New York: Routledge.

Pinar, W. F. (2006). Race, religion and a curriculum of reparation. New York: Palgrave Macmillan.

Pinar, W. F. (2009). The worldliness of a Cosmopolitan education: Passionate lives in public service. New York: Routledge.

Pinar, W. F. (2011). The character of curriculum studies. Bildung, Currere, and the recurring question of the subject. New York: Palgrave Macmillan. 
Pinar (2012) What is curriculum theory? (2nd ed.) New York: Routledge.

Pinar, W. F. (2013). Curriculum studies in the United States. Intellectual histories, present circumstances. New York: Palgrave Macmillan.

Pinar, W. F. (2015a). Without experience is teacher development possible? In Z. Hua \& W. F. Pinar (Eds.), Autobiography and teacher development in China: Subjectivity and culture in curriculum studies (pp. 179-192). New York: Palgrave Macmillan.

Pinar, W. F. (2015b). Educational experience as lived: Knowledge, history, alterity. New York: Routledge.

Pinar, W. F., \& Grumet, M. R. (2015 [1976]). Toward a poor curriculum (3rd ed.). Kingston: Educator's International Press.

Pinar, W. F. (1972). Working from within. Educational Leadership, 29 (4), 329-331.

Rocha, S. D. (2015). Folk phenomenology. Education, study, and the human person. Eugene: Pickwick Publications.

Roth, M. S. (2014). Beyond the University. In Why liberal education matters. New Haven: Yale University Press.

Sarup, M. (1992). Jacques Lacan. Toronto: University of Toronto Press.

Severson, K., \& Blinder, A. (2014). Test scandal in Atlanta brings more guilty pleas. The New York Times, CLXIII (56,374), A9.

Singer, N. (2015). Uncovering security flows in sites for schoolchildren. New York Times, CLXIV (56,772), B1-B7.

Taubman, P. M. (2011). Disavowed knowledge. New York: Routledge.

Tomkins, G. (1974). National consciousness, the curriculum, and Canadian studies. In G. Milburn \& J. Herbert (Eds.), National consciousness and the curriculum: The Canadian case (pp. 15-29). Toronto: Institute for Studies in Education, Department of Curriculum.

Tomkins, G. (1981). Foreign influences on curriculum and curriculum policy making in Canada: Some impressions in historical and contemporary perspective. Curriculum Inquiry, 11(2), 157-166.

Uljens, M., \& Ylimaki, R. (2015). Discursive curriculum and leadership theory: A general framework for an international dialogue. A paper presented at the annual meeting of the American Educational Research Association, Chicago.

Waghid, Y. (2010). Toward authentic teaching and learning in post-apartheid South Africa: In defense of freedom, friendship, and democratic citizenship. In W. F. Pinar (Ed.), Curriculum studies in South Africa: Intellectual histories, present circumstances (pp. 201-220). New York: Palgrave Macmillan.

Westbrook, Robert (1991) John Dewey and American Philosophy. Ithaca, NY: Cornell University Press.

Young-Bruehl, E. (1996). The anatomy of prejudices. Cambridge, MA: Harvard University Press.

Open Access This chapter is licensed under the terms of the Creative Commons Attribution 4.0 International License (http://creativecommons.org/licenses/by/4.0/), which permits use, sharing, adaptation, distribution and reproduction in any medium or format, as long as you give appropriate credit to the original author(s) and the source, provide a link to the Creative Commons license and indicate if changes were made.

The images or other third party material in this chapter are included in the chapter's Creative Commons license, unless indicated otherwise in a credit line to the material. If material is not included in the chapters Creative Commons license and your intended use is not permitted by statutory regulation or exceeds the permitted use, you will need to obtain permission directly from the copyright holder.

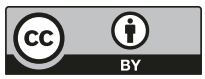




\section{Part V \\ Discursive and Multi-level Perspectives}

This part moves to a discursive and multi-level perspective on leadership and education within and between nation states combining educational leadership and curriculum work. More specifically, we feature Uljens and Rajakaltio's chapter that - considering our theorethical framework based on a non-affirmative understanding of educational influence Dietrich Benner and discursive institutionalism by Vivienn Schmidt. The study reconstructs the discursive dynamics regarding educational leadership as curriculum work at the nation-state level. The National Board of Education (NBE) in Finland operates rather independently between the Ministry of Education and municipal level reflecting a tradition of communicative discourse The study reveals how National Board of Education (NBE) utilises its degrees of freedom in the preparatory discursive processes of curriculum reform in relation to, on the one hand, intentions expressed by the political system and the Ministry of Education and, on the other hand, between NBE and schools and municipalities. Educational leadership as curriculum work on the nation state level mediates between different epistemic fields and value spheres.

Here curriculum work is an interruption in the Other's relation to himself/herself, other persons and the increasingly global world. This recognition-based Hegelian philosophy, updated by Axel Honneth along with modern education theory concepts, a non-affirmative perspective, and discursive institutionalism provide a general frame for understanding how the curriculum works as a pedagogical intervention of influence. The study is an example of our framework where educational leadership and curriculum work in the Finnish curriculum reform recognizes the subject as relationally free. Here influence does not mean support for implementation or development of extant ideas but rather an invitation to dialogue in order to transcend what is given. When curriculum work is seen as an invitational activity approach, general Finnish curriculum reform aims are proposed but translations to practice are not dictated or decided at the national level in advance. Importantly, in this chapter, the authors' position also acknowledges the leader/subject's own agency as a necessary requirement for the ability to transcend what is given. In sum, this chapter illustrates relationships between curriculum making as invitation to professional 
self-activity and professional self-formation in which these relationships may create discursive spaces within and between leadership levels of a nation state.

The other chapter in this part takes a similar discursive perspective and considers educational leadership and curriculum work at the national and transnational levels. More specifically, Sivesind and Wahlstrom (this volume) re-conceptualize school leadership using curriculum theory as well as discursive institutionalism, taking as a point of departure both classical curriculum theory and institutional (societal and programmatic) arenas. Drawing on their own conceptual framework as well as findings from a comparative study of policy documents, Sivesind and Walhstrom explore the meaning of leadership education within and between leadership levels of Norway and Sweden. This chapter provides a well-argued example applying the concept of discursive institutionalism to curriculum theory in leadership at different levels and in different arenas with various coordinations of discourses, ideas, and actors. In conclusion, Sivesind and Wahlstrom argue that educational leadership can contribute to curriculum theorizing by emphasizing the role of leaders as actors in institutions that are embedded in particular but changing social, cultural, and political contexts. In their view, the meaning of leadership is deeply intertwined with ideological discourses on education that have traditionally been the subject of curriculum theorizing.

In combination, the chapters in this part offer important new applications for discursive institutionalism in educational settings with leadership as a multi-level project as well as a closer relationship between education theory, curriculum theorizing, and schools as societal institutions. Moreover, these studies explicitly illustrate the interplay among curriculum policies, societal aims, and educational relations within new forms of governance. In our research program, we bring all of these Parts or elements together toward a theory of educational leadership as curriculum work. 


\title{
Chapter 13 \\ National Curriculum Development as Educational Leadership: A Discursive and Non-affirmative Approach
}

\author{
Michael Uljens and Helena Rajakaltio
}

\begin{abstract}
This chapter reconstructs the making and implementation of the new national curriculum in Finland (2012-2016). This research draws on non-affirmative theory of education and discursive institutionalism. The curriculum making process is perceived as a non-hierarchical educational leadership process where the National Board of Education (NBE) mediates and positions itself concerning (a) aims, (b) contents and (b) methods between transnational policies, national political decision making and policy work, various pressure and expert groups as well as school practice. The data consisted of interviews with three key actors within the Steering Committee of Curriculum Development (SCCD) and document analysis. The results demonstrate a shift towards stronger political steering, which in fact is a deviation from previous, trustbased policy regarding national education administration. In terms of discursive institutionalism the policy culture in Finland framing the curriculum leadership is still coordinative and dialogical, i.e. typical of a political consensus culture with broad governments, providing more autonomy for the educational administration. Second, curricular aims in the New Curriculum from 2016 reflect a movement towards a competence based curriculum, i.e. a more performative educational ideal is supported. The key competencies promoted are now similar to those promoted by the OECD since 2006. Third, a collaborative and development oriented professional culture around teaching methods is strengthened. Learning of the contents should now promote the development of more general key competencies. There are no indications of that the school system in Finland would be leaving a strong subject centered curriculum and evaluation.
\end{abstract}

\footnotetext{
M. Uljens ( $\square)$

Åbo Akademi University, Vaasa, Finland

e-mail: michael.uljens@abo.fi

H. Rajakaltio

Faculty of Education, University of Tampere, Tampere, Finland

e-mail: helena.rajakaltio@staff.uta.fi
} 


\section{Introduction}

This chapter investigates the national curriculum process in Finland (2012-2016). In the first half of the article features of a theoretical framework for curriculum work as educational leadership is outlined. The position draws on non-affirmative theory of education and Bildung as well as discursive institutionalism from political sciences. The second half of the chapter investigates the curriculum making process as a non-hierarchical top-down and bottom-up educational leadership process where the National Board of Education (NBE) mediates between political decision making, pressure groups and school practice. This mediation falls into two parts. The first relation, between National Board of Education and Ministry of Education and Culture, concern the establishment of new Decrees and decisions on allocation of time over school subjects. These decrees create a foundation for the later curriculum making process. The second relation, that between the National board of Education (NBE) and practitioners is based on document analysis. Concerning the curriculum itself the results point out changes concerning aims, contents and methods. The curricular aims in Core Curriculum in Finland 2016 partially reflect a movement towards a competence oriented curriculum. A collaborative and development oriented culture around teaching methods is emphasized. The subject-matter itself is more clearly seen as a vehicle for Bildung purposes.

Questions and Design The aim of this chapter is to investigate the national core curriculum reform (National Board of Education 2014) as a curriculum leadership process at a national level. The whole process is called Curriculum reform 2016. The official curriculum making process is seen as a non-hierarchical top-down and bottom-up educational leadership process where the Finnish National Board of Education (FNBE) mediates between transnational and national political decisionmaking, pressure groups, stakeholders and school practice (Robertson 2006, 2007). The interaction between these levels is considered non-hierarchal as, for example, FNBE, assigned by the Ministry (politics), prepares the ground work for the Decrees to decided upon by the Ministry. As political powers then have decided upon the aims and other questions the curriculum construction process led by FNBE may start. This is, simplified, the shape and form of the non-hierarchical procedure administration prepares for Decrees, Decrees direct the work of the administration, the administration (FNBE) approves the curriculum.

More precisely the two-level design of this analysis is divided between studying, first, the vertical dialogue and process between the FNBE and the Ministry of Education and Government, and, second, vertical dynamic relations between FNBE and stake holders, pressure groups and practitioners. While at the first level, i.e. between FNBE and the Ministry, we study the generative process through which the Decrees are created that later direct the later curriculum constructing process, while the second level tries to catch the dynamics of how FNBE cooperate with the field of practitioners in implementing the curriculum. It is in this sense we see FNBE demonstrating educational leadership as a mediating instance between politics and practice. In this mediating and translating process FNBE is provided with degrees of freedom, a relative independence, to make decisions. 
Third, the curriculum itself is analyzed according to the general part of the curriculum. Finally some development trajectories are described in the Finnish comprehensive school with relevance for the study.

Our three empirical research questions are:

1. How may the recent tradition of revision processes of the national core curriculum in Finland be described?

2. What features may be identified in the discursive dynamics between FNBE and the Ministry of Education (Government) regarding the preparation of Decrees regulating later curriculum construction?

3. How was the curriculum development process designed with respect to cooperation between FNBE, municipalities and schools?

The first question is answered through a reconstruction of recent developments concerning curriculum work in Finland. The answer on this question forms the starting point for the analysis of the second and third research questions.

The data analyzed questions 2 and 3 consist of laws, decrees and other documents regulating the curriculum process. The main data sources are the Government Decree (Government Decree 422/2012) passed in June 2012 and the National core curriculum passed in November 2014 (National Board of Education 2014) which will be implemented in August 2016. In addition we analyze official policy documents, plans, public process descriptions and information produced by the national committee, and interviews with education officials at the national level. Furthermore we have carried out interviews with core officials at the FNBE responsible for leading the curriculum construction process the past 5 years. The interviews were carried out by both of us being present as interviewers at all three occasions. The sessions lasted around $2 \mathrm{~h}$ each, which were transcribed. In this study we utilize understandings that we developed during the interviews.

Given that curriculum development forms a part of a more general process concerning school development a number of significant other parallel decisions concerning school governance are pointed out. Such initiatives may be considered as additional sources of information to be interpreted in order to gain a more coherent picture. These other school governance initiatives have to do with the renewal of the evaluation system, new developmental plans expected to be used by the schools, financial models, and national reform work on principal education. Furthermore in the present curriculum reform several national core curricula were drawn up simultaneously i.e. a national core curriculum for pre-primary education, a core curriculum for general upper secondary education and a core for curriculum basic education in arts, as well as the curricula for preparatory education for immigrants.

\section{Theoretical Framework}

To pursue these aims we will, first, outline features of a theoretical platform for how 'leadership as curriculum development' may be approached. To this end we describe a non-affirmative and discursive educational leadership approach (Uljens 2015; 
Uljens and Ylimaki 2015). This position draws on different but related contributions considered valuable, but which alone are perceived limited for a comprehensive understanding of curriculum reform at the national level. These are non-affirmative theory of education general education (Benner 1991; Uljens 2002), Didaktik (Uljens 1997), research on curriculum leadership (Ylimaki 2011), as well as discursive institutionalism (Schmidt 2008). The framework to be described is related to but not the same as intersubjective and recognition based social philosophy (Honneth 1995) in a critical Bildung tradition (Benner and English 2004). The position assumes individual agency as discursively embedded leadership practice. Educational leadership as professional activity include an interpersonal moral relation, carried out in historically developed societal institutions framed by a policy context, ideologies and occurring within a larger cultural historical tradition (Rajakaltio 2011; Uljens and Ylimaki 2015). An additional framing of the empirical analysis consist of a structural model describing curriculum decentralisation and recentralisation as well as externalisation of evaluation, originally based on a reflective theory of school didactics.

There are many reasons for viewing curriculum work at the nation state level as educational leadership. By turning our attention to 'curriculum work as educational leadership' we expect being able to highlight some of the mechanisms through which the political ideas, initiatives and positions transforms into a 'pedagogical agenda' offered to practitioners. Curriculum is thus both a political, pedagogical and practical challenge. We are interested in how this curricular agenda is initiated, established, adapted, enacted, defended and negotiated on different levels, however, without forgetting to include key actors on the national level. How do those in charge for large scale education reforms act as educational leaders? How do they mediate between political interests, pressure groups, academic research and practitioners' interests? To lead a national reform process is also a huge organizational and practical undertaking. How, and why, is the process, including so many actors, organized as it is?

In demonstrating such a processual and activity oriented focus we connect to research traditions studying the initiation, implementation and institutionalisation of curriculum (Hopmann 2003; Goodlad 1979; Lundgren 1989; Phillips and Hawthorne 1978). Following Erich Weniger's (1975) view curriculum making is a complex practical and political problem, where education as a science can contribute but cannot have or be given the responsibility for the process. As Künzli (2013) points out there is no traditional truth criteria to be applied for evaluating the process, rather "situative and historic appropriateness". Neither is the process predictable or possible to control. In many respects Schwab's (1978) position is reminiscent of Wenigers.

Curriculum making is about construing a platform or frame not only for teaching but also for subsequent leadership of the educational system. We assume that the curriculum may be viewed as a programmatic interruption in the practitioner's way of understanding herself and carrying out one's professional tasks. Here we make use of Foucault's view of politics as an invitation to self-formation while ethics is taken to refer the individual's response, how the individual chooses to relate to herself. An interruption of this kind is an intervention in the Other's relation to herself, 
other persons and the world (Honneth 2003). Such a recognition based Hegelian philosophy provides a general frame for understanding how the curriculum itself, as well the construction process, operates, and is used, as a pedagogical intervention in order to influence. Here influence does not mean implementation of readymade ideas but an invitation to a dialogue. In our view, in doing so educational leadership as curriculum work recognizes the subject as radically free as this makes her able to transcend what is given. But the position also acknowledges the necessity of the subject's own agency as a necessary requirement for transcending a given state. The effect of curriculum development activity is, obviously, also in the hands of the receivers enacting given intentions.

In line with discursive and non-affirmative leadership theory (Uljens and Ylimaki 2015) curriculum making discourse is considered as an invitation to self-activity and self-formation create spaces within and between institutionalized levels. Consequently, also national education leaders' ways, patterns or cultures of inviting practitioners, principals and teachers, in developmental work around the curriculum can be built upon a recognition based view of intersubjectivity and subjectivity in the way Honneth has suggested.

Discursive Institutionalism Not only does a curriculum form a platform for educational leadership practice. Also the very making of the curriculum is a kind of leadership. In curriculum making there is typically a complex interaction occurring between politics and the administration. One result of this process, e.g. law and decrees, form the point of departure for the actual working out the curriculum. In this study we limit ourselves to the process starting when the laws and decrees are accepted. Yet, as a curriculum is a part of a more general ideological and politically informed pedagogical policy agenda (Weniger 1975; Schwab 1978; Apple 1996), 'educational leadership as curriculum making' cannot be disconnected from these politically agreed general aims of education and must be analysed in relation to them, which will be done. In essence we see national authorities working with the making of curriculum as mediating between politics and educational practice. We also make the assumption that how this national educational leadership process of curriculum making is and may be carried out is dependent on the political culture of each country. Although the curriculum is central to both Didaktik and curriculum theory the policy culture of leadership is often not thematized, which is something that discursive educational leadership expands towards.

We argue that analyzing curriculum making as educational leadership may utilize the concepts of 'discourse' and 'ideas', as developed by Schmidt (2008) in discursive institutionalism. Given that "ideas are the substantive contents of discourse", discourse is "the interactive process of conveying ideas" (Schmidt 2008). Discursive institutionalism takes its point of departure in normative and $\operatorname{cog}$ nitive ideas on a philosophy, policy and program level:

Cognitive ideas speak to how ...policies offer solutions to the problems at hand, how ... programs define the problems to be solved and identify the methods by which to solve them, and how both policies and programs mesh with the deeper core of ... principles and norms of relevant scientific disciplines or technical practices. Normative ideas instead 
attach values to political action and serve to legitimate the policies in a program through reference to their appropriateness... Normative ideas speak to how ... policies meet the aspirations and ideals of the general public and how ... programs as well as ... policies resonate with a deeper core of ... principles and norms of public life, whether the newly emerging values of a society or the long-standing ones in the societal repertoire. (Schmidt 2008, 307)

These ideas are considered to manifest themselves in coordinative and communicative discourses. Coordinative discourses mainly occur among policy makers, and communicative discourses occur between policy making and the public. Schmidt points out that different nation states demonstrate different polities or political cultures. Coordinative cultures are frequent in simple or consensus oriented polities and are featured by broad policy preparing procedures and practices widely including different policy actors. Communicative polities in turn typically are frequent in nation states dominated by either left or right wing governments or complex polities. In these last policies political work is more narrowly based, i.e. led by the government parties, typically resulting in a so called communicative culture, i.e. where politicians have to market decisions made, as no broad coalitions necessarily back them up.

First, it is obvious that curriculum making work around both cognitive and normative ideas reflected in the aims and contents of education. We see the meaning of these ideas as evolving due to the discursive processes in relation to given a context at different levels - a philosophical, policy and program level. In this perspective a discursive approach to educational leadership also may reveal how processes and dynamics between actors and levels are related to how these substantive ideas are reconstructed.

Non-affirmative and Discursive Theory of Educational Leadership Despite obvious merits of a politological approach like discursive institutionalism only limited attention is directed to the pedagogical dimension of these discourses. We see a need to overcome this limitation of discursive institutionalism in understanding educational leadership. How may this be done?

In line with non-affirmative leadership theory (Uljens 2015; Uljens and Ylimaki 2015) we make use of some fundamental theoretical categories in non-affirmative education theory (Benner 1991). A first assumption is to adopt a non-hierarchical view of how societal forms of practice are related (Gruber 1979). This means that various forms of societal practices like education, politics, law and economy are not sub- or super-ordinated in relation to each other. For example, on the one hand politicians decide about new laws, on the other politics is regulated by law. Education is politically directed, but in such a way that an educated individual can change future politics. In this sense education is not limited to socialization into given norms but supports the individuals growth into a deliberating subject (Englund 1996) able to transcend what is given.

Given the above we accept the view of curriculum making as a 'complicated conversation' (Pinar 2011) in a procedural and deliberative democracy. Curriculum making is a contingent processes where tradition, political and moral will in addi- 
tion to rational reason operate in relation to self-formation. Regarding the influence of (political) will and (rational) reflection, our assumption is that in a consensus oriented political culture, like Finland, more room is left for rational deliberation in curriculum work and also for teacher autonomy. This gets support from Schmidt (2008) who assumes that simple polities, i.e. consensus cultures, are featured by coordinative rather than a communicative discourse. Consequently, in systems following a stronger political e.g. left-right wing culture we would expect that the role of the administration is more executive and managerial directed by politics, while being less autonomous and balancing between political, academic and practical interests. In many countries also the central administrators are replaced as the result of elections, seldom so in Finland. In comparison a culture of trust in professional deliberation rather than control may partly be explained by this political culture in Finland (Uljens and Nyman 2013).

In our view curriculum may be seen as an invitation to practitioners to reflect on their pedagogical work. A curriculum may be seen as a "summons" to self-reflection and activity (Benner 1991). In summoning it is always assumed that those being summoned have a will of their own. Fundamentally, the idea of influencing somebody by summoning recognizes the individual's transcendental freedom and present empirical condition. The practitioner's self-realization would thus mean that the individual relates to a curriculum as to an "interrupting" summons, an invitational offering. But the process of self-realization is completed only through the indivual's own activity. Here we refer to the concept of Bildsamkeit, initiated by J. G. Fichte and carried further by Hegel, Herbart and Schleiermacher and subsequently by e.g. Dewey, Mead and Vygotsky, in different versions, though the root is the same.

In addition we want to emphasize that educational leadership in the form of curriculum implementation, demonstrate a paradoxical relation to praxis. How? Let us give an example. Although the aims, content or methods proposed in the curriculum may be new the practitioners are treated as if they would understand these new ideas and as if they were capable of transforming their praxis, even if they, by definition, not necessarily are yet able of doing this. The paradox consists in that the practitioners are approached as if they already were able to do what they are expected to become able of doing. Yet, only by being approached in this way, they may transcend their current praxis (Benner 1991; Uljens 2002), i.e. the curriculum is an invitational disruption.

The previously described non-hierarchical relation between societal forms of practice means then that, on the previous grounds, a simple top-down implementation process in launching new curricula is not possible. The validity of the modern version of the pedagogical paradox, i.e. to be recognized as a reflecting and free individual although it is through this very recognizing agency of the Other that one may become a culturally free and reflecting individual, is not limited to the intersubjective relation in a teaching-studying-learning process in the classroom, but is also relevant in describing educational leadership at other levels. We can see that educational leadership on a national level is then not only about managing educational institutions or supporting the growth of professional competence but includes a pedagogical dimension. 
From a discursive curriculum leadership perspective we turn our attention to the normative and cognitive ideas behind these intentional "disturbances" as well as the shapes they take in different political, cultural and administrative systems - as intentions, interpretations and negotiations. The task would thus be to try to grasp the dynamics in a given cultural, historical, political, institutional and societal context. In fact, the very change from an old public administration (OPA) model to a new public management (NPM) model has reminded us how strongly any governance model directly, and mostly indirectly, affect the individual's self-formation and identity (Pinar 2011). In this respect we see soft-governance as a 'politics' inviting or even forcing the subject to new forms of self-formation (Foucault). Utilizing this insight it is also possible to study the intentions of 'normalisation' and creation of cultural coherence by curricular work.

Adopting a non-hierarchical view a view has consequences for how we consider educational administration in a democracy to operate: not only teachers but also education leaders at different levels are both allowed and assumed to make use of degrees freedom given. The system builds upon the previously mentioned paradox. Curriculum making cannot on these grounds be unconditionally sub-ordinate even to those very laws and decrees directing the process of making the curriculum as the curriculum in any western democracy prepares the younger generation to become citizens to participate in changing the very laws.

Deliberative Approaches and Discursive Educational Leadership Theory Given the above focus on recognition of individual and professional agency we see it fruitful also to relate to critical theory of social action inspired by Habermas to help us reflect upon educational leadership in curriculum making. Following a hegelian tradition emphasizing intersubjective legitimation of values and knowledge, Habermas' ideal principles for communication may be used as a reference point in investigating how educational leadership as curriculum making in a democratic society works. This is in coherence with was previously said about discursive institutionalism and educational leadership theory. Communicative action is here considered to refer to a process where participants may act in their own interests but harmonized with interests of others, thus pointed at the centrality of negotiation (Englund 1996). The deliberational aspect also point at that self-formation (Bildung) does not occur without a reference to an Other, on the contrary. We see this kind of communicative action as being about will formation as well as personal and cultural identity, but also about supporting rational reflection in valuing an orientation towards being comprehensible and truthful, sincere (honest), sensitive for others interests and also critical with respect to authorities (Habermas 1987). A consensual political tradition, like the one in Finland, offers, so we believe, more degrees of freedom for such a rational dialogue. A discourse ethical approach thus pay attention to what kinds of procedural communicative dialogues are carried out (Roth 2000). In this study the education officials' work is framed by political legislation but the political decisions do not transform into practices by themselves. Policies are enacted on several levels of the educational system (Ball et al. 2011), and involves moral, political and rational agency (Carleheden 2006). 
Conceptually then, curriculum making as educational leadership is here understood as a mediational process operating between on the one hand values and, on the other, various epistemologies. By epistemologies is referred to that decision making in educational leadership is related to knowledge of e.g. teaching practices, culture, students, law, financial systems, technology, communication, demographics, working life and management. By values is referred to both ethical and political questions. Educational leadership within the administration on the school, municipal, state and transnational level thus partly consist of making use of the degrees of freedom offered in this critical-hermeneutic process. In this process policies are both constructed and enacted.

Educational leadership as curriculum work is also mediational in other respects. Leadership is horizontally distributed over professional actors both within and between institutions (cf. policy borrowing). Educational leadership is also vertically distributed between e.g. transnational level, the state, municipal level and the school level. We can identify a first, second, third and fourth order of educational leadership where the object of what is led varies. Teachers' leading students' study activities is first order leadership. Principals leading teachers' professional teaching activities is second order leadership. Education officials (eg. superintendents) leading principals' leadership activities is third order leadership. While national education authorities leading the previous activities is called fourth order educational leadership. In this study we consciously delimit ourselves to a national level, although we fully accept that transnational interests clearly influence the national curriculum process in many ways (Frontini 2009).

A final argument for viewing curriculum work as educational leadership is that empirical and theoretical curriculum research often, but not entirely, has overseen educational leadership. A similar limitation holds true for the Didaktik tradition. Leadership research in turn has typically not related itself to curriculum making or theory (Uljens and Ylimaki 2015). Thus to consider curriculum work as discursive educational leadership may point at new openings.

\section{Results}

The Policy Culture Regarding the Revision Process of the National Core Curriculum in Finland The curricular reform work in Finland is traditionally carried out as a process of systemic educational leadership from the top of the administration to the single school. The national core curriculum is a national regulation in compliance with which the local curricula are designed. The purpose of the national core curriculum is to support and steer the work in schools and to promote equality and the underlying values of basic education as democracy, cultural diversity as a richness and sustainability as a way of living. Education providers, most commonly municipalities, are fairly autonomous in practicing local educational policy due to their own development strategies and draw up their own local curricula based on the national core curricula. They are responsible for the preparation 
and development of the local curriculum as well for practical teaching arrangements and the quality of its education. Local authorities has the right to choose whether there will be a common local school curriculum or if some schools will set up a curriculum together or if there will be school specific curricula. They determine how much autonomy is passed on to schools (National Board of Education 2012). The relation between state and the municipalities has changed since the 1990s. The municipalities are more self-governing than before (The Local Government Act 365/1995). But because of financial straits some municipalities have cut their resources and a segregation process can be noticed due to various economic and social structures in different municipalities (Nakari and Sjöblom 2009).

A renewal of the National Core Curriculum for basic education in Finland has so far been carried out about every tenth year $(1970,1985,1994,2004)$ although some important amendments in the legislation have been accomplished in the years between. The Basic Act from late 1990s (Basic Education Act 628/1998) still applies. The present national core curriculum issued in 2004 is based on the Act 1998, which stated the single-structure basic education (grades 1-9) by abolishing the traditional (administrative) division between primary and lower secondary schools (National Board of Education 2004). The single-structure school is based on the principle of continuity. The Basic Education Act 1998 pointed out individualization as a pervasive principle, which responds to societal orientation towards neoliberal individualism. According to the Act every pupil has the right to receive tuition corresponding to his/her talents and prerequisites. In the national core curriculum 2004 this individualistic orientation was embedded in the development of the 9-11 years single-structure basic education as the idea of individual learning pathways and devoted attention to learning plans which could be set up for every pupil as it was stated.

The individualistic view - combined with a diagnostic culture in defining special needs - had led to an explosion of enrollments into special student status. In 2010 significant changes were made in the administration guidelines for special education, in the legislation and in the national core curriculum 2004 which affirmed the basic principles: early identification of risks and a three-step-support system for inclusive education. The supplementary to the national core curriculum had a strong emphasis on diversity and equality in all aspects; sex, age, ethnicity and nationality, language, religion, conviction, opinion, health and disability. The changes call for a safe and collaborative school community, which enhances all pupil's well-being, differentiation, cooperation and meaningful learning (National Board of Education 2010). These changes and amendments are embedded in the national core curriculum 2014 (National Board of Education 2014).

The national education policy in Finland is promoting an ideology of inclusion. The change emphasizes recognition of diversity and differences to labeling and diagnosing students and to prevent exclusion by early identification of risks and by offering supportive inclusive practices. The education provider is obliged to ensure that the pupils' right to receive support is implemented in practice. The purpose of the reform is to reinforce the learning support mechanisms for all students. The issue of developing inclusive forms of education has led to increased challenges at 
school level in curriculum development and everyday practices, and teachers struggle to respond to the actual needs of a diverse student population. The need of collaboration among teachers and welfare staff is facing a cultural shift in the traditionally individualistic work culture into a more collaborative culture (Rajakaltio and Mäkinen 2013). The need of a change of school culture as a community was identified in the government decree (Government Decree 422/2012) and is promoted in the national core curriculum 2014.

The principle of neighbourhood school was launched and included in the Basic Education Act 1998 as well. According to this principle every child has got the right to attend a school closest to her/his home. The school may take pupils outside the catchment area if there are vacant places. In fact local authorities (municipalities) have interpreted and modified this principle in various ways and research findings show that parental choice occurs in bigger towns which are facing a segregation process in schools (Varjo and Kalalahti 2011; Seppänen et al. 2015).

The Finnish curriculum tradition has been described as a kind of a hybrid model, a nationally contested mix between Anglo-American curriculum and German Scandinavian Bildung (Autio 2013). The curriculum tradition with Ralph Tyler's Rationale as its icon exemplifies a technical-rational view on curriculum as an organizational framework, which positions the teacher as a technician, whose task is to implement the curriculum, written as a manual. This is the case in many countries where education and curriculum work is based on accountability and standardization. In the Finnish way of mixing the curriculum traditions teachers are positioned as autonomous, intellectual actors in the reform work of the school. Curriculum is seen both as an organizational and intellectual centerpiece of education (Autio 2013). In the Finnish curriculum educational leadership is leadership in both of these fields. It is also possible to identify various positions within the Finnish curriculum leadership tradition over the past 40 years, i.e. during the era of the 9 -year comprehensive school system (1972-).

In Fig. 13.1 major changes during the past 40 years in educational policy, leadership and administration are pointed out. The model is based on a reflective model of Didaktik for schools. The main dimensions in the figure are (a) curriculum work as the vertical axis describing degrees of centralization and decentralization and (b) evaluation of education as the horisontal axis pointing at to what extent evaluation is controlled internally by teachers or externally by other interests. Using these simple distinctions we are able to identify four positions that quite well describe educational policy in Finland concerning curriculum work and evaluation procedures.

First, in Finland the 1972 curriculum is generally considered a product of the heyday of directing schools with laws, inspection and curricula (Position 1: Management by objectives and rules). Here teachers were responsible for evaluating students' learning achievement. The movement from position 1 to position 2 indicate a two step decentralization of curricular work in 1980s and then in the 1990s. From the late 1970s, Finland started to move from a traditional administrationcentred to a qualification-oriented and decentralized way of governing schools. Parallel to decentralization of curriculum work, teachers' vocation was stepwise being professionalized by academiation. Together positions 1 and 2 reflect the edu- 


\section{Positions and Changes in Educational Leadership Policy 1972-2012 in Finland}

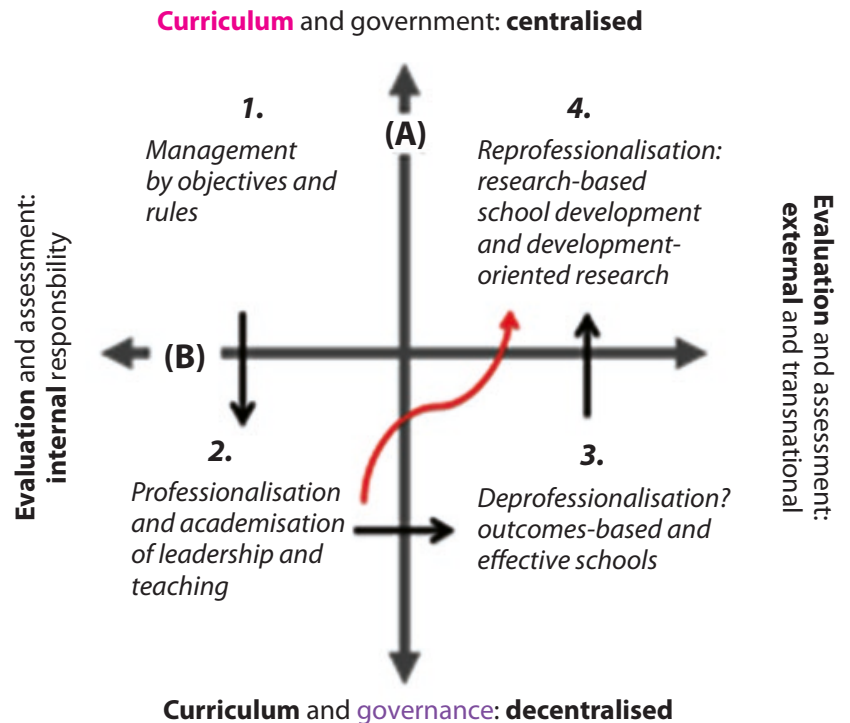

Fig. 13.1 A reconstruction of how educational leadership as curriculum work and evaluation is carried out during different periods

cational policy of the social democratic welfare state. Internationally the educational mentality of the past two decades has to a growing extent reflected a stronger discourse on excellence, efficiency, productivity, competition, internationalization, increased individual freedom and responsibility as well as deregulation in all societal areas. This change is indicated by an arrow from position 2 to position 3 indicating the establishment of regime of performative accountability in public administration. Generally, position 3 demonstrates that evaluation as a tool traditionally used by teachers to control students was turned into a tool for controlling teaching. However, a unique feature of Finnish education policy after 1989 is that a testing culture has to this day not been developed, other than those national exams having existed for over 100 years in upper secondary schools. National authorities have not even developed instruments for following up each and every school's results. Instead survey methods are applied to monitor the state of art in Finnish schools. The final movement, to position 4 , is a stronger recentralization of curriculum meaning that in the 2004 national curriculum a much stronger grip was taken concerning the aims content. We return in the discussion to the ongoing development in Finland.

Curriculum Leadership as a Dynamics Between FNBE and the Ministry According to the design and research questions of this study we first intend to investigate the dynamics between FNBE and the Ministry of Education. 
We aim at a reconstruction of the process leading up the decisions framing the later curriculum construction process. Consequently our study begins by investigating the first step in the national core curriculum process, which is to formulate the Government Decrees that specify the goals of education and the distribution of lesson hours. In principle, these Decrees are expected to reflect the government program and the Ministry's Development Plan for Education and Research (Ministry of Education and Culture 2012a).

The allocation of time to be used for teaching the school subjects is only a seemingly small question. In reality it has been fraught with conflicts. It is a battleground for different stakeholders according to their interests in different subjects. This was also the case in the initial phase of the planning process of the curriculum reform 2016.

What steps and tensions may then be identified in this process? In the spring of 2009 the Ministry of Education appointed a committee on renewal of national aims and distribution of lesson hours (Ministry of Education 2010). The committee consisted of fifteen members who represented political parties and both labor and employers' organizations and the parental union. Both the chairman and the secretary represented the Finnish National Board of Education (FNBE). The Director General of FNBE leading the preparatory committee was Timo Lankinen, representing right-wing party Kansallinen Kokoomus (National Coalition Party-NCP) appointed in 2008 for 5 years. The committee was expected to deliver a report in June 2010.

The central role of FNBE in this process follows existing practices and regulations. The Finnish National Board of Education (FNBE) as the executive body of authority is responsible for designing the national core curriculum, approving it and implementing it as well as other policy aims. The national core curriculum has to be formulated pursuant to the Basic Education Act and Decree (Basic Education Act 628/1998; Basic Education Decree 852/1998) and Government Decree, specifying the goals of education and the distribution of lesson hours among school subjects (Government Decree 422/2012). The Government Decree directs further the overall time allocation by defining the minimum number of lessons allocated to core subjects in basic education. In essence, this national administrative agency (FNBE) was then leading the preparatory work for the later Decrees to be decided upon. This preparatory work resulted in a report (proposition) that demonstrate the conclusions drawn by the committee (Ministry of Education and Culture 2010). Already now it is obvious for the reader how central the role of FNBE was. In fact, the design of this curriculum construction process in Finland demonstrates an institutionalized trust regarding the national administration. However, it should be observed that this tradition was connected to a strong tradition of public civil servants in the state administration. Contrary to most other European countries in Finland central leaders of institutions where not replaced after elections but survived new governments. This tradition was stepwise broken during the past decade. For example the Director General for FNBE was appointed for 5 years at a time.

In the report, or proposition, the committee examined changes in the national and international operational environments that had to be taken account for in the 
renewal of basic education for the future. According to this preparation work some of the transnational trends, as the OECD policy documents discussing key competences, had an influence in shaping the proposition. The proposition argued in favour of several profound developments, which should be taken into consideration in the subsequent curricular planning work. The proposition classified the objectives for citizens' future skills into five groups of competences an individual is expected to need in a future society: (1) Thinking skills, (2) Ways of working and interaction, (3) Crafts and expressive skills, (4) Participation and initiative and (5) Selfawareness and personal responsibility. Further the proposition headed at a significant increase concerning students' individual choices regarding subjects. The proposal argued for a curriculum divided between compulsory and optional subjects. Six multi-disciplinary subject groups were suggested to be mandatory consisting of different subjects. The optionality was located within these multi-disciplinary groups. The multi-disciplinary subject groups were: Language and interaction, Mathematics, Environment, Science and technology, Individual, enterprise and society, Arts and craft as well as Health and personal functionality.

Optionality may be connected to distribution of lesson hours. The committee proposed a considerable increase of lesson hours reserved for optional subjects for all grades. Typically optionality increases the higher up in the school system pupils move. Now the committee proposed increased optionality even for pupils in the lower grades. The group thought there should be 13 weekly optional lesson hours per year in grades 3-6 and 21 weekly optional lesson hours per year in grades 7-9.

Two new subjects were introduced: drama and ethics. By supporting Drama the aim was to strengthen a comprehensive approach to art education in the multidisciplinary group of Arts and crafts. Ethics was seen to reinforce the basic values of the Finnish society within the subject group 'Individual, enterprise and society'.

Foreign language education and second national language studies were diversified and introduced earlier than before. The group proposed also that the minimum amount of annual number of pupil's weekly lessons hours should be increased by $4 \mathrm{~h}$.

The radical proposition included many controversial elements reflected by a lively political debate during the whole process, in public and in the media. The committee could not agree upon the above proposal and no unified view was put forth. Six group members out of 15 made objections to the proposition.

In this preparatory political phase of the curricular work political tensions in the group were clearly visible. The Social Democrats, the Greens and the Center parties as well as both labor and employer organizations, made objections to the proposition for several reasons. One of the main argument against the proposition was related to the costs of the reform. There was a fear of increasing inequality between the municipalities because of their different financial situations. A second objection, also related to equality, was that the substantial addition of lesson hours for optional subjects would not in practice increase the pupils' freedom of choice as pupils' choices are systematically connected to families' social background. Several studies over the years have shown that the students' socio-cultural and economic status significantly influences pupil's school choices (Seppänen et al. 2015). The multidisciplinary subject groups were criticized to abolish the subject-based curriculum, 
e.g. by the teacher union. One of the objections the green party made was that the proposition did not strengthen the education in arts and craft. The center party made an objection to the formulation of the subject group Individual, enterprise and society and suggested a formulation that include Humanity instead of Individual. The social democrats lambasted the expert group's way of working on a too tight schedule with no space for discussions (Ministry of Education and Culture 2010). The chair represented the right-wing party as did the Minister.

In the autumn of 2010 the government refuted the proposition. As previously observed the work was led by a Director General representing a right-wing party appointed for 5 years, obviously making political steering of FNBE easier. The report was put forth despite many objections. In addition there was a change of government due to elections and a new Minister, now representing social democrats instead of the right wing party, was elected. Extensive public and political debate was carried out from the publication of the report until Spring the next year, 2011.

In August 2011 the Ministry of Education and Culture appointed a second expert group with the task of preparing a new foundation for the curriculum work to come. This second committee consisted only of governmental officials from the Ministry who worked out the second proposal behind closed doors. FNBE, that previously and traditionally had a key role in the process was locked out from this process. The new committee took all criticism into account and developed a proposal made public in February 2012 (Ministry of Education and Culture 2012b). This time the proposal was, not unexpected, much more in line with social democratic policies than the first one. The new Government Decree (422/2012) was accepted in June 2012.

What about the result? Comparing the Government Decree (422/2012) there were no significant changes compared to the previous one from 2001, but a greater emphasis was put on school as a community (Government Decree 1435/2001; Uljens and Rajakaltio 2015). The educational principles are fundamentally moral. The Government Decree contains of three sections with several objectives. The value building national goals to be considered in preparing the National Core Curriculum are as follows: Growth as human being and membership in society, Requisite knowledge and skills and Promotion of knowledge and ability, equality and lifelong learning. These goals steer also the preparation of the local curriculum and the work at school. At this level the objectives are rather open and there was a need of an interpretation as the starting point for the curricular reform process. The national goals are briefly summarized as follows.

The section two Growth as human being and membership in society presupposes that basic education should support pupils to become active and ethically responsible citizens, who are promoting sustainable development. Education promotes knowledge and understanding of cultures, ideological, philosophical and religious traditions. The decree highlights respect for human rights, the democratic values of Finnish society, including equity and equality.

The objectives according to section three Requisite knowledge and skills are related to education as laying a foundation on which pupils can build extensive general knowledge and abilities and broaden their world view and of oneself. The emphasis in this section is on the individual pupils' health, welfare and safety and 
competence in taking care of oneself and managing daily life. The objective is furthermore to foster the competencies required in working life and entrepreneurship, e.g. ICT skills. The decree states that the education must be based on scientific knowledge.

The section four Promotion of knowledge and ability, equality and lifelong learning is directing the organization of education and pupil welfare. A new aspect in the present decree is to promote a more collaborative school culture. The whole school community is taken into consideration as a learning environment. It presupposes a more active role of the whole learning community for enhancing learning and growth and welfare. It emphasizes inclusive education in all respects and pupils' involvement and participation. All education must improve the pupils' learning-tolearn skills and capabilities for lifelong learning.

Conclusion The intentions of the decree indicates a shift from a work culture based on individually working teachers' towards a collaborative one. Still, teacher is seen as an autonomous professional who has got the power to choose how to teach but who is invited to reflect on curricular issues in communication with others. Furthermore there is a more profound orientation in fostering societal, sustainable and ethical thinking and activities in preparing pupils for an active citizenship.

These developments are good indicators of educational leadership at the national level as working in relation to political interests. Deviating from a consensual tradition, the committee, led by the Director General at FNBE leading the first committee, obviously was not able to produce a result reflecting a compromise, but a report reflecting the interests of the right wing government for the time being. It appears as if the Ministry of Education and Culture perceived of the FNBE in a new way, instead of a longstanding tradition of a politically more balanced way of working. Ministry now expected this governmental body (FNBE) to produce a politically biased committee report. This shift is interpreted as to represent a new governance culture regarding curriculum making in Finland. Yet, due to the independence of FNBE reflecting a trust from the politicians, we see the Finnish policy discourse regarding the dynamics between politics and governance still representing a coordinative, rather than a communicative, discourse.

How Was the Curriculum Development Process Designed? The third question in this study was about reconstruction of how FNBE was working out the new curriculum, especially with respect to stake holders, pressure groups and practitioners.

The curricular planning work started in the summer of 2012 when the renewed Government Decree (422/2012) was approved by the government in the end of June 2012 as the result of a short-term work.

As described earlier the legislation of the Government Decree governing the national objectives and distribution of lessons hours in the basic education is a starting point for the curricular development work. The Finnish National Board of Education (FNBE) as the executive authority body led and organized the national core curricular work. As a first step in this curricular work on the national level FNBE was leading the process of codifying the legislative guidelines as defined in 
the Governmental Decree into core curriculum outlines. The educational experts at FNBE have some freedom in interpreting the governmental decree but they are loyal to what is prescribed in the decree. This may be seen as a part of the consensual policy in the curricular work.

FNBE has a lot of power in the curricular work process but the role is characterized as a mediating role, which is based on interaction processes between different actors in several communicative spaces. Multidisciplinary working groups, supported by online consultation groups, were outlining the core curriculum. There were altogether 34 groups working in different fields, a steering group and a small group which was coordinating the whole process. The seven members of the coordination group were educational experts at FNBE. All educational experts at FNBE were involved in the process as support groups.

The secretary of the first Decree committee Irmeli Halinen was appointed the head of the core curriculum work. Meanwhile the second government decree was under construction by the second Committee the head of the core curriculum work organized preparatory work for the curriculum planning process at the Finnish Board of Education (FNBE). The educational experts did a thorough preparation work by mapping out current research and evaluation findings both nationally and internationally, educational policy and transnational educational trends in different countries. They studied EU and OECD documents and estimated the changes in the operating environment, analyzed the current state, e.g. national development projects, other legislative changes and development tasks and policy guidelines to be considered in outlining the core curriculum. The officials made acquaintance with development projects and every day experiences of municipalities and schools as well. Several stakeholders and representatives from different organizations were heard and consulted during the preparation process. The educational experts at FNBE were well prepared to take responsibility of the core curriculum work.

In the distribution of lesson hours there are some changes compared to the previous decree (Government Decree 1145/2001). The expert group representing the Ministry made a more conventional proposition on the renewal of the decree than did the earlier representative expert group (Ministry of Education and Culture 2010). According to the optional lesson hours the Ministry expert group took an opposite standpoint by reducing them. There is no change in minimum of lesson hours for the individual pupil, which is still $222 \mathrm{~h}$ as was prescribed in the previous decree. However, there are some changes between the minimum hours of the subjects in different grades. No new subjects are added, but some subjects have more lesson hours and some less. The hours for optional subjects are reduced with $4 \mathrm{~h}$ from 13-9. More lesson hours are devoted to social studies $(+2)$ on an earlier stage, physical education $(+2)$, music and visual arts (both $+1 \mathrm{~h}$ ). The hours in religion is reduced with $1 \mathrm{~h}$. The integrated environmental studies in grades 1-6 include biology, geography, physics, chemistry and health studies. Home economics is integrated as a part of the subject group of Art education. There will be a more varied language program. The decree seems to head at a more participatory, physically active, creative and linguistically enriched school with integrated teaching and 
learning. As a conclusion of the change in distribution of lesson hours the optional of lesson hours in different subjects has declined from 13 to $9 \mathrm{~h}$.

The steering group had an advisory but a key role during the whole process and continued its work until the final version of the national core curriculum was delivered in the end of 2014. The group started its work in August 2012 directly after the government had approved the decree. The members of the steering committee were presenting 16 key representatives from e.g. the teachers' trade union, the Finnish principals' association, Ministry of education and culture, Ministry of social care and health, the association for parents, the delegation for ethnical relations, the institute for health and wellbeing, the confederation of Finnish industries and the Association of Finnish Local and Regional Authorities. The chair, secretary and the presenting official were representing FNBE. The steering group was appointed to settle the principles for the revision work of the core curricula for pre-primary education, the basic education and voluntary additional basic education.

As noted earlier, the inner coordination group had done a thorough preparation work. Three other groups started their work beside the steering group in August 2012. The groups had core tasks in outlining the national guidelines and general principles. One group was working with structures and objectives (e.g. guidelines for integration). Another group was defining the learning concept, learning methods and evaluation, and finally one group was working for a more cultural and multilingual aware school. These groups had some subgroups as well. The steering group's task was to support the working groups and to emphasize an overall societal perspective in the preparation work, to foster the interest and the positive attitude to curriculum work and to keep the group members' partners and organizations informed.

The steering committee approved the curriculum guidelines for planning the curriculum in autumn 2012, which should be taken into consideration in all aspects in all curricular working groups. They were defined as follows:

- Promoting equity and equality in all areas of education

- Strengthening coherence and consistency of basic education, learning continuums

- Supporting pupil's growth and development, welfare and other prerequisites for learning

- Promoting a sustainable future as an objective

- Working with knowledge, taking into account technological change,

- Promoting broad-based multimodal literacy, media, ict that crosses all subjects

- Promoting awareness of languages and cultures, regarding them as richness

- Respecting dependences on international and on global dimensions (Halinen 2013)

According to the general guidelines the school should create better prerequisites for the school's pedagogical work, for meaningful learning and welfare for all pupils (principle of inclusion) and for a sustainable future and a democratic society. The guidelines underlined that the focus should be on deep learning and in creating versatile learning environments (Halinen 2013). 
The process of drawing up the national core curriculum document was designed as a large scale partnership based process buttressed by trust and recognition and built on a broad-based co-operation in dialogue with education experts, researchers, administrators, teachers and various stakeholders, working teams and internet crowdsourcing open to everybody. Crowdsourcing was realized as a new kind of mode for opening the dialogue and engaging more participants in the renewal process of the core curriculum. The website was opened four times during the process: in November 2012 (general guidelines), September 2013 (pre-primary education) and in April 2014 (basic education and voluntary additional). Key stakeholders; education providers were asked to provide their official opinions on the new national core curriculum during the autumn of 2014. NBE's website's comments during the process were collected. According to our informants the comments considered a part of the work in the working groups and were taken into account in the process. Some stakeholders were very active, almost like pressure groups, e.g. representatives for entrepreneurs and for nature associations, which had an influence in formulating the key competencies. Because of numerous arenas and groups the dialogue between different stakeholders and school experts was more intensive than in earlier curriculum work processes. During the preparatory work more than 300 researchers, teacher educators, providers' representatives, teachers, school leaders and other school staff were heard personally. The aim was to encourage also parents and pupils to participate in the process (Halinen et al. 2013). The process could be characterized as communicative discourse (Schmidt 2008).

The national core curriculum includes the objectives and core contents of different subjects, as well as the principles of pupil assessment, and the inclusive oriented support system, pupils' welfare and educational guidance and the principles for a learning community. The Government Decree pointed the way to introduce competences for the first time in the Finnish National Core Curriculum. Also the preparatory work which was made of the educational experts at FNBE was influenced of other EU and OECD countries' educational policy trends, e.g. competence-based curricula. The descriptions of the competences were codified from the government decree and defined in relation to changes in the environment.

The competences are described as broad-based competences referring to knowledge, skills, values, attitudes, capacity and will. In the definitions it is possible to identify an influence of the transnational process of harmonization of educational objectives as competences (Stoer and Magalhaes 2009). Dimensions of broad-based competence as objectives for learning defined in the Finnish national core curriculum are defined as follows (FNBE 2014):

- Thinking and learning to learn

- Cultural competence, interaction and self-expression

- Taking care of oneself and others, managing daily life

- Multiliteracy

- Competence in information and communication technology

- Working life competence and entrepreneurship

- Participation, involvement and building a sustainable future. 
To be put into practice the competencies as aims it presumes cooperation across school subjects and various kinds of working methods. The subject specific groups which began their work in January 2013 had to take into account these seven areas of competence, the general guidelines, the invited experts and website comments. Altogether 25 working groups were preparing the guidelines for subject based curricular parts. The groups were chaired by officials from FNBE. These chairmen were coordinating their job in several meetings. Additionally there were four groups with special tasks related to different educational challenges: small schools, preprimary education, basic education for adults and voluntary additional basic education. The groups worked during meetings and in between through web links. All the groups were free to invite representatives from schools; principals, teachers and education providers and other experts for consultancy. The groups were also supported by online consultation groups. To what extent these online consultation groups had an influence on the groups' work is a question of a separate study. The subject specific groups finished their outlining work in April 2014. There is a significant change in subject syllabi compared to the actual one. The traditionally divided curriculum in a general and subject specific part is integrated through the competence areas, which are interconnected. The competence-based and subjectbased teaching are combined in a new way. The objectives in the subject syllabi include competence goals. The competences will also be assessed as a part of the subject assessment. Moreover, collaborative teaching is enhanced by bringing about multi-disciplinary learning modules. The schools should have at least one learning module per year for the pupils, but otherwise they are free to decide about the learning modules. According to Halinen, Harmanen and Mattila (in press) the learning modules are efficient tools in promoting the transversal competences and pupils' understanding of interconnectivity between different phenomena.

The renewed core curriculum was completed by the end of 2014 and thereafter the reform work has continued as local curriculum development work due to local needs and policies both on a municipal level and at a local school level. The core curriculum consists of the intentions of the educational experts, planners and politicians. These official intentions will meet the reality in schools, principal's and teacher's work. These agencies at school level have a "make or break" role of curricular activities (Kelly 2009). This is the next phase to be studied. The curriculum reform work was completed in spring 2016 and local curricula were approved by 1st of August 2016 in order to introduce the new curricula in the beginning of the autumn term in 2016 for grades 1-6, in August 2017 for grade 7, in 2018 for grade 8 and finally 2019 for grade 9.

FNBE is active in supporting the municipalities and schools in the implementation process to succeed. During the curricular process at national level FNBE offered continuing education in cooperation with the Normal schools at universities. These network programs offered spaces for reflection for school leaders, local authorities and teachers and researchers. Supportive material has also been available for the development work at the website of FNBE. The national core curriculum documents are provided in an electronic and structured form as e-curriculum documents. An "e-library" has been established where all local authorities' curricula will 
be available. This is also way of supporting - and pushing - the curriculum work to be done on a local level. It gives the national administrative authorities an overview in the curriculum reform process throughout the whole country and can also be seen as a tool for control.

FNBE is the executive authority body in the curriculum making process. The Ministry of Education was represented in the steering group. A research group (2,1 $\mathrm{mme}$ ) is financed from the Ministry to do follow up studies of the whole curriculum process (2012-2018), (Pyhältö et al. 2012).

\section{Interpretations and Discussion}

Leadership as Mediation Between the Transnational and Local Level As a starting point we assumed that educational leadership as curriculum work at the national level features mediation between transnational and local level. This can be observed by studying the new key competences accepted in December 2014 and previous EU policies. The objectives in the national decree from 2011 in Finland were developed and reformulated in the curriculum for the comprehensive education in terms of seven key competencies (FNBE 2014):

- Thinking and learning to learn

- Cultural competence, interaction and self-expression

- Taking care of oneself and others, managing daily life

- Multiliteracy

- Competence in information and communication technology

- Working life competence and entrepreneurship

- Participation, involvement and building a sustainable future.

The above key competencies correspond to some degree with those eight keycompetencies furthered by European Union since a decade (Official Journal L 394 of 30.12.2006):

- Communication in the mother tongue

- Communication in foreign languages

- Mathematical competence and basic competences in science and technology

- Digital competence

- Learning to learn

- Social and civic competences

- Sense of initiative and entrepreneurship

- Cultural awareness and expression.

Bildung and Transversal Competencies: - A Combinatory Curriculum Approach Our impression is that curriculum work as discursive educational leadership practice at the national level is about finding a way to create a balance between cultural coherence and room for individual development. In spelling this out, in this curriculum both individual, local, national and global perspectives are visible. 
Generally, the new decree emphasizes stronger than before, sustainable development and global responsibility as an objective. Inclusive education covers the students' well-being safety and equality. Meeting the students individual learning needs are put forth, as well as pupils' empowerment and will formation. These dimensions point at classical Bildung or character formation ideals. Maybe a future weakened welfare state and reorganized labor market is envisioned by the expectation to take care of one self and others and in emphasizing entrepreneurship? Cultural and linguistic interaction and diversity is offered more room and is considered as enrichment. This may be seen as a response to the global increase of cultural diversity within nation states, as well as international communication. A truly plural nation state is visible which can be seen against the hitherto low numbers of immigrants and refugees in Finland. The technological development requiring ICT and multimodal literacy competencies are clearly expressed. Skills for working life and entrepreneurship are pointed out. In our mind this represents a partly new dimension. On the one hand we think we see a curriculum for will formation, identity, recognition, care and responsibility, and on the other, a curriculum for political, cultural and economic citizenship, according to principles of sustainability. Critical thinking is not very visible.

Transversal Competencies and the Subject Matter The curriculum process from 2004 demonstrated a clear recentralization of many aspects related to the curriculum. The change 2004 also reflected a movement towards a more closed curriculum in an epistemological sense, emphasizing subject matter (Vitikka 2009). The current reform does not take this process any further, although the eligibility of lesson hours in different subjects was reduced. Rather, there is a shift in how objectives, contents and methods are conceptualized.

While the curriculum 2004 put the emphasis on contents, the curriculum 2014 emphasizes the general objectives in terms of key competencies. As a result, the role of the subject matter in the teaching process is now expected to change. Now the question is more clearly about to what extent teaching in a school subject supports the learner's development with respect to the key competencies above? Thus the Core curriculum 2014 for basic education does not only demonstrate an orientation towards a more holistic educational approach through an integration of school subjects, in multidisciplinary learning modules by expecting teachers to work together around so call phenomena. In addition, aforementioned cross-curricular or transversal competences are emphasized. Transversal competence "refers to an entity consisting of knowledge, skills, values, attitudes and will. Competence also means an ability to apply knowledge and skills in a given situation (FNBE 2014; OECD 2015). The manner in which the pupils will use their knowledge and skills is influenced by the values and attitudes they have adopted and their willingness to take action" (Halinen et al. in press, p. 140).

Our interpretation is that the 2014 Curriculum partly represents a continuation of a Bildung oriented curriculum in Finland since the beginning of the 1970s. This is evident in the general objectives as expressed in the Government Decree 2012 and by emphasizing personality development in a holistic manner as observed above. 
A new perspective in the current curriculum is the orientation towards key competencies. Compared to the Bildung inspired line of thought the aims expressed in terms of competencies more strongly emphasize pragmatic, instrumental and performative qualifications.

The How-Question: Towards a Collaborative Teaching Culture in Finland If the decentralised curriculum of the 1990s was recentralised in 2004, the philosophy behind the ongoing reform not only has an emphasis on the traditional curriculum questions of what and why of teaching and learning, but also on the how of education on a school level. The how moves the focus towards a more collaborative teaching culture where teachers in different subjects are expected to strive for common aims or competencies. Thus, the school is seen as a learning community with the task of developing the school's overall activity culture, i.e. as a pedagogical community. The teacher's classical freedom of choosing and working with right methods is now completed with viewing the school in its totality. However, the municipal level should not be forgotten here and is in fact included as apart of the local the unit of educational activities. Recent renewal of principals' education supports this change (National Board of Education 2014) and is very coherent with the idea behind the new school development plans launched 2013 (Pitkälä 2013). The aim is to engage school leaders, teachers and school personnel in discussions of how the schools could improve their activities. The municipal development plans may thus be seen as a part of a soft-governance system where the national agency provides the schools with a structure and a unified frame for development work. If resources will be allocated to qualified development plans this will be a strong incitament to take these plans seriously on the municipal and the school level. These plans can be investigated from a discursive institutionalist and systemic perspective where time, social practices, technologies, traditions, relations and position are united (Fairclough 2003).

A key question for the reform work to be successful is how the school communities will cope with the transformation process due to the new reform. The development work at the school level is a big challenge for the school and there is a need of a developed educational leadership and new collaboration. The curriculum reform presupposes that the schools will develop as professional communities. The school leaders together with the municipal education superintendents are in a key position in fostering the development of a professional learning community with spaces for reflection, sharing experiences and knowledge and in order to get enough unanimity in the school community for promoting the reform work in practice.

\section{Non-affirmative Curriculum Leadership}

The Finnish educational policy as a meta-practice of governance on a national level reframes the policy at the municipal level in the field of education. Local providers, usually local authorities are fairly autonomous in practising the educational policy 
within the National Core Curriculum framework. Accordingly the steering group of the National Board of Education points out that there should be space and support for pedagogical development at the local level (Halinen 2013). Decisions on the local curriculum level are, as before, made by local authorities but now expected to be related to municipal educational development strategies. This involves the superintendents in school development at least on a strategic level together with the schools. This also supports the approach outlined in the theoretical frame for this study: the curriculum reform process is not considered a simple implementation process. The process rather reflects an invitational action structure. The general aims are there but how they are to be interpreted and put into practice cannot be dictated at the national level. As there is a space for local interpretations both teachers' and municipalities' autonomy is respected. This is why we call curriculum making as pedagogical leadership at the national level a non-affirmative practice. It is non-affirmative both in the sense that the National Board of Education itself is authorized to decide about the approval of the curriculum and also in the sense that the municipalities are given the ultimate responsibility to evaluate compulsory education and to make own interpretations of the curricular aims.

School reforms and changes in teacher's work are complex social processes that teachers interpret based on their personal understanding and experiences in curriculum development and everyday practices (Rajakaltio 2011). This truly distributed model of responsibilities is the foundation for a more discursive process in curriculum making. According to key-actors in the curriculum construction process trust is of paramount significance: "The key is trust. Teachers trust that the FNBE really listens to their experiences, needs and ideas, and the FNBE trusts that local authorities and teachers do their best in drawing up the local curricula and working according to the common guidelines." (Halinen et al. in press). It should be observed that this trust is not only about the prevailing educational ethos or organizational culture. As noted above, the National Board of Education itself is trusted to make autonomous decisions on the part of political steering and the municipalities have the right and obligation by law to lead, evaluate and develop basic education.

\section{References}

Apple, M. (1996). Cultural politics and education. New York: Teachers College Press.

Autio, T. (2013). The internalization of curriculum research. In W. F. Pinar (Ed.), International handbook of curriculum research (2nd ed.). New York: Routledge.

Ball, S., Maguire, M., \& Brown, A. (2011). How schools do policy: Policy enactments in secondary schools. London: Routledge.

Basic Education Act. 628/1998. Lagen om grundläggande utbildning. http://www.finlex.fi/en/laki/ kaannokset/1998/en19980628.pdf

Basic Education Decree 852/1998. Förordningen om grundläggande utbildning. http://www.finlex. fi/sv/laki/ajantasa/1998/19980852

Benner, D. (1991). Allgemeine Pädagogik. Weinheim: Juventa.

Benner, D., \& English, A. (2004). Critique and negativity: Towards a pluralism of critique in educational practice, theory and research. Journal of Philosophy of Education, 38(3), 409-428. 
Carleheden, M. (2006). Towards democratic foundations: A Habermasian perspective on the politics of education. Journal of Curriculum Studies, 38(5), 521-543.

Carlgren, I. (1995). National curriculum as social compromise or discursive politics? Some reflections on a curriculum-making process. Journal of Curriculum Studies, 27(4), 411-430.

Englund, T. (1996). Deliberative communication: A pragmatist proposal. Journal of Curriculum Studies, 38(5), 499-501.

Fairclough, N. (2003). Analysing discourse. Textual analysis for social research. London: Routledge.

FNBE. (2014). Perusopetuksen opetussuunnitelman perusteet (National Core Curriculum for Basic Education). Helsinki: Opetushallitus.

Frontini, S. (2009). Global influences and national peculiarities in education and training: The Finnish case. In H. B. Holmarsdottir \& M. O'Dowd (Eds.), Nordic voices. Teaching and researching comparative and international education in the Nordic countries. Rotterdam: Sense Publishers.

Goodlad, J. F. (1979). Curriculum inquiry. New York: McGraw-Hill.

Government Decree 1435/2001. The Government Decree on the General National Objectives and Distribution of Lesson Hours in Basic Education (1435/2001). http://www.minedu.fi/ $\mathrm{OPM} /$ ?lang=en

Government Decree 422/2012. Government Decree on the General National Objectives and Distribution of Lesson Hours in Basic Education. http://www.finlex.fi/sv/laki/ alkup/2012/20120422

Gruber, E. (1979). Nicht-hierarchische Verhältnistheorie und pädagogische Praxis. München: Wilhelm Fink Verlag.

Habermas, J. (1987). The theory of communicative action. Lifeworld and system: A critique of functionalist reason. Boston: Beacon Press.

Halinen, I. (2013). Curriculum reform in Finland. http://www.oph.fi/download/151294_ops2016_ curriculum_reform_in_finland.pdf

Halinen, I., Holappa, A.-S., \& Jääskeläinen, L. (2013). Opetussuunnitelmatyö ja yleissivistävän koulutuksen uudistaminen. Kasvatus, 44(2), 187-194.

Halinen, I., Harmanen, M. \& Mattila, P. (in press). Making sense of complexity of the world today: Why Finland is introducing multiliteracy in teaching and learning. CIDREE 2015.

Honneth, A. (1995). The struggle for recognition. The moral grammar of social conflicts. Cambridge: Polity.

Hopmann, S. (2003). On the evaluation of curriculum reforms. Journal of Curriculum Studies, 35(4), 459-478.

Kelly, A. V. (2009). The curriculum. Theory and practice (6th ed.). London: Sage.

Künzli, R. (2013). Memorizing a memory: Schwab's the practical in a German context. Journal of Curriculum Studies, 45(5), 668-683.

Lundgren, U. P. (1989). Att organisera omvärlden. Stockholm: Utbildningsförlaget.

Ministry of Education and Culture. (2010). Basic education 2020 - the national general objectives and distribution of lesson hours. Reports of the Ministry of Education and Culture, Finland, $2010,1$.

Ministry of Education and Culture. (2012a). The education and research plan 2012-2016. A development plan. Reports of the Ministry of Education and Culture, Finland 2012:3. http://www. oph.fi/download/148961_The_Education_and_Research_Development_Plan.pdf

Ministry of Education and Culture. (2012b). Future basic education - National objectives and distribution of lesson hours. Reports of the Ministry of Education and Culture, Finland, 2012, 6.

Nakari, R., \& Sjöblom, S. (2009). Toimiva kunnallinen palveluorganisaatio: Työelämän laadun, asiakastyytyväisyyden ja palvelukustannusten väliset yhteydet strategisen henkilöstöjohtamisen näkökulmasta. Acta 209. Helsinki: Suomen Kuntaliitto.

National Board of Education. (2004). Grunderna för läroplanen för den grundläggande utbildningen 2004. Helsingfors: Utbildningsstyrelsen. 
National Board of Education. (2010). Changes and amendments 50/011/2010 in national core curriculum for basic education.

National Board of Education. (2012). National core curriculum outlines. 13.11. 2012. http://www. oph.fi/english/education_development/current_reforms/curriculum_reform_2016

National Board of Education. (2014). National core curriculum for basic education. Helsinki.

OECD (2015). Education at a Glance 2015. OECD Indicators. http://dx.doi.org/10.1787/ eag-2015-en

Phillips, J. A. \& Hawthorne, R. (1978). Political dimensions of curriculum decision making. Educational Leadership, February, 362-366.

Pinar, W. F. (2011). The character of curriculum studies. Bildung, Currere and the recurring question of the subject. New York: Palgrave MacMillan.

Pitkälä, A. (2013). Millaista kehitystä nyt tarvitaan? (What kind of development do we need now?). http://www.oph.fi/kuntakesu

Pyhältö, K., Soini, T., \& Pietarinen, J. (2012). Do comprehensive school teachers perceive themselves as active agents in school reforms? Journal of Educational Change, 13(1), 95-116.

Rajakaltio, H. (2011). Moninaisuus yhtenäisyydessä. Peruskoulu muutosten ristipaineessa (Diversity in coherence - Comprehensive school in the cross-pressure of change). Acta Universitatis Tamperensis 1686. Tampere: Tampere University Press.

Rajakaltio, H., \& Mäkinen, M. (2013). The Finnish school in cross-pressures of change. In: Proceedings of European Conference of Curriculum Studies. Future directions: Uncertainty and possibilities, 530-536.

Robertson, S. L. (2006). Globalisation, GATS and trading in education services. In J. Kall \& R. Rinne (Eds.), Supranational regimes and national education policies-encountering challenge. Finnish Education Research Association: Helsinki.

Robertson, S. L. (2007). Globalisation, rescaling, national education systems and citizenship regimes. In K. Roth \& N. Burbules (Eds.), Citizenship education in national, transnational and global contexts. Rotterdam: Sense Publishers.

Roth, K. (2000). Democracy, education and citizenship. Stockholm: Stockholm Institute of Education Press.

Schmidt, V. (2008). Discursive institutionalism: The explanatory power of ideas and discourse. Annual Review of Political Science, 11, 303-326.

Schwab, J. J. (1978). The practical: A language for curriculum. In J. J. Schwab (Ed.), Science, curriculum and liberal education (pp. 287-321). Chicago: Chicago University Press.

Seppänen, P., Kalalahti, M., Rinne, R., \& Simola, H. (2015). Lohkoutuva peruskoulu. Perheiden kouluvalinnat, yhteiskuntaluokat ja koulutuspolitiikka. Kasvatusalan tutkimuksia 68. Jyväskylä: Suomen kasvatustieteellinen seura.

Stoer, S. R., \& Magalhaes, A. M. (2009). Education, knowledge and the network society. In R. Dale \& S. Robertson (Eds.), Globalisation and Europeanisation in education (pp. 45-63). Oxford: Symposium Books.

Uljens, M. (1997). School didactics and learning. Hove, East Sussex: Psychology Press.

Uljens, M. (2002). The idea of a universal theory of education - An impossible but necessary project? Journal of Philosophy of Education, 36(3), 353-375.

Uljens, M. (2015). Curriculum work as educational leadership - Paradoxes and theoretical foundations. Nordic Journal of Studies in Educational Policy, 1(1), 22-30. http://www.nordstep.net/ index.php/nstep/article/view/27010.

Uljens, M., \& Nyman, C. (2013). Educational leadership in Finland or building a nation with Bildung. In L. Moos (Ed.), Transnational influences on values and practices in Nordic educational leadership: Is there a Nordic model? (pp. 31-48). Dordrecht: Springer.

Uljens, M. \& Rajakaltio, H. (2015). National curriculum development in Finland as distributed and non-affirmative educational leadership. In: M. Uljens (Ed.). Educational leadership - Theory, research and school development. (pp. 208-234). Report from the Faculty of Education and Welfare Studies, Åbo Akademi, report 38. 
Uljens, M. \& Ylimaki, R. (2015). Towards a discursive and non-affirmative framework for curriculum studies, Didaktik and Educational Leadership. Nordic Journal of Educational Policy. NordSTEP. 2015(1), 30177 -http://dx.doi.org/10.3402/nstep.v1.30177

Varjo, J., \& Kalalahti, M. (2011). Koulumarkkinoiden institutionaalisen tilan rakentuminen. Yhdyskuntasuunnittelu, 49(4), 8-25.

Vitikka, E. (2009). Opetussuunnitelman mallin jäsennys. Sisältö ja pedagogiikka kokonaisuuden rakentajina. Kasvatusalan tutkimuksia 44. Jyväskylä: Suomen Kasvatustieteellinen Seura.

Weniger, E. (1975). Theorie der Bildungsinhalte und des Lehrplans. In E. Weniger (Ed.), Ausgewählte Schriften zur geisteswissenschatligen Pädagogik (pp. 194-294). Weinheim: Beltz.

Ylimaki, R. (2011). Critical curriculum leadership. New York: Routledge.

Open Access This chapter is licensed under the terms of the Creative Commons Attribution 4.0 International License (http://creativecommons.org/licenses/by/4.0/), which permits use, sharing, adaptation, distribution and reproduction in any medium or format, as long as you give appropriate credit to the original author(s) and the source, provide a link to the Creative Commons license and indicate if changes were made.

The images or other third party material in this chapter are included in the chapter's Creative Commons license, unless indicated otherwise in a credit line to the material. If material is not included in the chapter's Creative Commons license and your intended use is not permitted by statutory regulation or exceeds the permitted use, you will need to obtain permission directly from the copyright holder. 


\title{
Chapter 14 \\ Curriculum and Leadership in Transnational Reform Policy: A Discursive-Institutionalist Approach
}

\author{
Kirsten Sivesind and Ninni Wahlström
}

\begin{abstract}
Educational leadership research has in general focused on organizational conditions and expectations for managing and leading activities (Leithwood et al. 1994, pp. 38-61; Spillane and Healey 2010; Møller 2006, pp. 53-69) in parallel curriculum theories have offered insights into substantial societal problems that must be addressed in school and society (Hopmann 1999, pp. 89-105; Westbury 2000, pp. 15-54). This chapter presents a study in which we link curriculum theory both to discursive institutionalism and educational leadership policy and research findings. By including discursive institutionalism (Schmidt 2012) within a framework of curriculum theory, it is possible to distinguish between different forms of discourses and their functions in forming and conveying ideas. Thus, we explore educational leadership policy using a reflexive approach to reforms as intertwined with public discourses and research. A transnational perspective on leadership confirms the applicability of reforms across geographical territories, relating to wider societal and cultural contexts. Following an institutional-discursive approach, we argue that the ways in which social and educational questions become intertwined in actual reforms are dependent on cognitive and normative ideas in the public sphere. Thus, reforms to education leadership are related to coordinative and communicative discourses beyond the individual reform, while solutions to curriculum and leadership problems are anchored in educational policies and practices. Against this background, we argue that a deeper understanding of the meaning of educational leadership discourse and the conditions under which such a discourse is conducted is crucial.
\end{abstract}

\footnotetext{
K. Sivesind $(\bowtie)$

Department of Education, University of Oslo, Oslo, Norway

e-mail: kirsten.sivesind@iped.uio.no

N. Wahlström

Department of Education, Linnaeus University, Växjö, Sweden

e-mail: ninni.wahlstrom@lnu.se
} 


\section{Introduction}

Curriculum theory and leadership research reflect similar issues and problems, albeit based on different theoretical perspectives and origins. While curriculum theory is rooted in theories of education and society, educational leadership research takes as its starting point normative and cognitive models concerning organizations, situations, and persons. Moreover, the scientific orientation makes leadership research a useful recourse for political control across various sites and sectors. Our discourse analysis of 14 policy documents published by the EU (European Union), the OECD (the Organization for Economic Co-operation and Development), and various associated companies, demonstrates that the general and generic orientation of leadership models fits well with the overall ambitions and goals of transnational reform policy. Thus, educational leadership is regarded as an opportunity to improve the efficiency of transnational policy decisions and strategies, not least by reforming the tertiary education of school principals in the member countries. However, our main argument in this chapter is that there is a need to problematize the relationship between school leadership research, reform policy, education, and society. How are policy and leadership rooted in societal discourses? Can leadership models be applied in education without regard for curriculum issues and problems? How can these two fields, curriculum and leadership studies, learn from each other by promoting critical stances towards policy reform discourses?

There are three primary reasons for our suggestion of exploring how curriculum theory might contribute to educational leadership as a research field and as policy. First, the meaning of educational leadership is embedded within the larger context of transnational policy. Second, the meaning of educational leadership is deeply intertwined with ideological discourses on education at large, since this meaning cannot be distinguished from important curriculum matters related to teaching and learning in schools. Third, to fully understand the complexity of educational leadership, there is a need for a conceptual framework that takes ideology, structure, and actors into account. This implies a sensibility for sector-specific dimensions, which in our case relates to educational mandates, curriculum reform and the purposes of education.

Drawing on discursive institutionalism (Schmidt 2008, 2012), the purpose of this chapter is to introduce a framework for the analysis of transnational educational leadership policies that include underlying assumptions about society and education in general. First, we offer a short introduction to the history of the curriculum and leadership research fields in order to demonstrate the overlapping scopes and interests as well as the differences and relationships. We then introduce a discursiveinstitutionalist approach as a framework for the analysis of policy documents concerning educational leadership that addresses transnational problems and perspectives in the current school systems in Europe and beyond. Curriculum theory 
comes into the discussion as both a foundation for understanding how the perceptions of leadership as reflected in our documentation relate to institutional and societal ideas, and how policy- and research-based conceptions of leadership forms are attributable to programmatic ideas and norms within education (as illustrated in Table 14.1). Finally, we make use of the institutional framework to discuss (i) the ways in which the established discourses on educational leadership associated with curriculum problems are linked to the basic notions of community needs, (ii) how the transnational meaning of educational leadership is maintained through a coordinative discourse, and (iii) how this agreed meaning is promoted to the nation states through a communicative discourse.

\section{Curriculum Theory}

The long-standing issues of curriculum and reform have both been subject to extensive research over the years. In the field of curriculum studies, educational reform is above all analyzed according to the origin and institutionalization of public schooling, which has today developed into comprehensive education systems (Hopmann 2003; Lundgren 2003; Westbury 2003). The curriculum as a research field links the study of contemporary problems in education to the tradition of historiography and on to a large range of reflection theories that developed from the early nineteenth century onwards. Moreover, the field of curriculum research has primarily dealt with the history of educational ideas and the study of educational systems and institutions in which legislation and reforms have been a primary topic of study (Doyle 1992; Gundem 1994). However, contemporary researchers add new perspectives to the field by examining governmental changes across and within specific geographical territories and in relation to comparative and international studies that consider the uniqueness of the reforms to a wider societal and cultural context beyond the national (Sivesind and Karseth 2014). A traditional focus of curriculum research has been to examine the topical structure of teaching practices in the context of pedagogy and schooling (Hopmann 2007; Uljens 1997). In curriculum research, the policy and practice of schooling, as well as its programmatic dimensions, have been approached by research that draws on different theories and traditions. First, in the curriculum field, a major presupposition is that the curriculum, whether understood as a curricular framework, teaching material, or a course of study, relates to formalized and institutionalized modes of teaching practices that can be traced back to the pre-Renaissance (i.e., between 1000 and 1250) when schools were licensed by church authorities and not by the state (Hamilton 1989: 12-13). According to this perspective, the relevant ideas and concepts are related to the classical thinkers of Ancient Greece (Hopmann 1999; Lundgren 1979). Since that time, thoughts and 
theoretical distinctions are considered perennial, although they have undergone ground-breaking changes with reference to alterations to the education system and the surrounding society (Hopmann 1999; Schubert 1986; Westbury 2000). For this reason, curriculum theories serve as more than a source for empirical research on institutions, organizations or persons in various settings. Curriculum theories approach practical and moral problems of education, first of all by making use of ideological and societal perspectives to critically reflect upon core conditions and processes in education.

Second, the reconceptual approach became central to curriculum studies during the 1970s in both the American and European contexts. William Pinar stands out as a key researcher in this tradition (Pinar 1978), while Englund (1990) argues that this approach has also been the core idea of curriculum history research in Scandinavia and Europe. The main aim of this perspective has been to establish a critical response to historiography studies and empirical conceptual approaches, which were part of the prevailing tradition of curriculum studies in the Anglo-American field in the 1960s.

In the Scandinavian context, Lundgren (1972) examined several questions that were of political interest during the early 1970s, such as the consequences of dividing pupils into homogeneous versus heterogeneous school classes with respect to the differences in their learning achievements. He later called for increased attention to be paid to the political aspects of education along with a reconceptualizing approach. This position was inspired by British scholars, particularly Basil Bernstein and Michael Young, who both argued for a new direction for the sociology of education from the early 1970s (Bernstein 1971; Young 1971). The conceptual configuration of this "new direction" expresses what occurred during the 1970s as a theoretical shift. Formalized regulations and institutions were no longer viewed as the solution, but rather as the problem. Instead, research on the sociology of education focused on outcomes and the structures that could explain those outcomes. For example, the main goal for Young was to shift the focus from thinking of schooling as being determined by society to instead contributing to the determination of society (Young and Whitty 1977). This shift in perspective highlighted the potential to change society through education research.

The main viewpoint of the new sociologists was that knowledge is produced and reproduced by dominant groups in society and is, therefore, highly ideological and political in character. One major presumption of Bernstein's theory was that the school system favored pupils from the middle class over those from the working class, partly because of its way of structuring the use of language that contributes to a power dynamic. A vertical dimension connecting politics and schooling was introduced, and it conceptualized the curriculum as socially organized knowledge involving different kinds of interests (Young 1971). Later books and articles written by Bernstein (2000/1996) and Young (1998) centered on pedagogy, social discourse, and a recontextualized approach to identity and knowledge.

In recent decades, when curriculum standards entered the arena of educational reforms, they became a focus of attention too (Muller 2000). As a consequence, new research issues emerge within critical research that are informed by educational and 
societal perspectives (Sundberg and Wahlström 2012; Yates 2009; Young 2008). For this reason, curriculum theories serve as more than a source for empirical research on institutions, organizations or persons in various settings. Curriculum theories approach practical and moral problems of education, first of all by making use of ideological perspectives. However, later theories have integrated the formal and societal-discursive perspectives, as well as their materiality, to critically reflect upon core conditions and processes in education. From this point of view, structural reforms possess interpretive dimensions that must be considered according to practicalities, discretion, and sensibleness in policy, research, and practice. Nonetheless, the features of education cannot be comprehended as organizational categories alone, but are instead institutionalized within their societal environment (Menck 2000; Reid 1986, 1999).

At the beginning of the 2000s, a relevant question is how schooling as an institutionalized pursuit is challenged by the views of what counts as core units of analysis, such as competences and outcomes, compared with other kinds of purposes and pursuits, as part of the larger context of society. The interest in scientific approaches to reforming teaching in schools, which was followed by a constructivist approach to research and education, has also called for a stronger focus on the actors and systems and how they contribute to both the reproduction of society and the renewal of the education system. It is within this area, by framing our analysis within an institutional-discursive approach, that we can see an interface between curriculum theory and leadership research.

\section{Leadership Research}

Leadership research examines how various actors are influencing the work of others in order to accomplish certain goals. In school leadership research, the school principal stands out as the key actor within the school organization. For this reason, educational leadership research has traditionally placed emphasis on how the school principal performs a specific role and function within the school as an organization. The responsibility of school principals can be related to different tasks and duties within the schools, as well as to teaching, although the teachers have been considered to control the agency over classroom decisions. In educational leadership research, one strand of research focuses on management as the key concept characterizing what school principals do within their organizations, while another strand of research considers leadership as a broader concept, which includes not only administrative but also relational and human features.

Until recently, educational leadership studies have focused on how leaders conduct leadership through power relations, thereby influencing others within the context of the school organization. Based on theories from the larger field of organization studies, these studies can be broadly sketched by their orientation to traits, contingency, and transformational theories (Lingard et al. 2003). While traits theories 
regard leadership in light of personal dispositions and qualities as dependent on the style and capacity of the person who is leading an organization, contingency theories emphasize how styles and behaviors are adapted to particular situations. The leader is, in this case, expected to coordinate goals and activities, being the prime instigator of the delegation of tasks and responsibilities by regulating other people's mind sets and activities. Transformational leadership theories add the dimensions of vision and vision building to leadership studies. This perspective also integrates ideas regarding how transactions are played out by individuals, assuming that persons act rationally toward each other to achieve certain benefits from their involvement with organizations (Leithwood and Jantzi 2006). A more recent theoretical approach suggests that distributed leadership is a helpful perspective for studying how schools exercise informal power that goes beyond the boundaries of both formal responsibility and the school context. This perspective also invites discussion on what constitutes democratic leadership in light of the discursive and political aspects.

Educational researchers have stressed the importance of situational theories and site-based work. Noteworthy studies utilizing this approach have been conducted by Hallinger and Heck (2010), who argue that instructional leadership is dependent on the mutual influence between students' learning and school capacity, which is shaping and is shaped by the collective leadership that is conceptualized as school-wide actions. These actions involve actors such as principals, teachers, administrators, and others. Based on this approach, educational features, such as constructing a school curriculum, are not separated from school leadership but are essentially a part of it (Hallinger 2010). Further, instructional leadership is seen as encouraging transformation, not merely by normative means but by developing the organization's cognitive capacity to select its purposes and support the development of instructional practices. This approach includes an interest in curriculum matters and school cultures, as well as the ways in which schools are capable of fostering high expectations for learning. Not only first-order change is highlighted, wherein technologies or persons directly regulate the work of students and teachers, but also a second-order phenomenon, whereby the school conditions and the climate require persons to change their actions within the organization (Hallinger 2003, p. 338). Consequently, instructional leadership is thought to exert both a direct and an indirect influence on individuals' actions and activities within the school organization.

In transformational leadership theories, common goals and shared visions serve as a key focus Thus, the shaping of the ideologies of members of an organization is a key issue in these studies (Leithwood 1994). The attention paid to goals is also important for examining transactional leadership as different from, or simply as an aspect of, transformational leadership. To examine how leaders and their followers exchange gratifications in order to achieve the best results, rational choice theories are built into these research approaches (Bass 1997, 1999; Burns 1978). Researchers who adopt this position often refer to empirical evidence to support their arguments concerning how such transactions make an impact on the processes within school organizations as well as their outcomes (Leithwood and Jantzi 2006; Leithwood et al. 1994; Oterkiil and Ertesvåg 2014). One consequence of this research approach 
is the study of particular factors that might explain the actions and priorities of the actors involved, such as the significance of a school's leadership, in terms of teachers' resilience. Day and Gu's (2010) study of effective and trusting relationships within organizations demonstrates that a school's leadership is actually regarded as the most important factor for organizational resilience, which supports transformational and transactional theories (see also Mulford (2003)).

In recent years, within school leadership research, as well as in national and local policy making, a distributive perspective on school leadership has aroused intense interest. A distributive view of leadership recognizes that leading schools can involve multiple individuals in addition to the school principal- "the leader-plus aspect"-and that leading a school is fundamentally concerned with interactions rather than about the actions of individual leaders- "the practice aspect" (Spillane and Healey 2010). According to Harris (2007), this view of distributed leadership rests on an idea of leadership as cognition. Research on distributed leadership is characterized by an interest in opening up new perspectives on the question of who can take part and who can be counted on when studying leadership practices. For example, Woods et al. (2004) understand distributed leadership as an emergent property of a group or network of interacting individuals operating within an unlimited space in which distributed expertise extends the boundaries by going beyond the formal functions of an organization. It has been argued that distributed leadership does not necessarily guarantee democratic processes and practices because it does not explain who is in control of whom and on what premise; therefore, it is suggested that there is a need to integrate sociological theories into discussions about the complexity of organizations and leadership studies (Gronn 2009). In the debate regarding democratic leadership, Lingard et al. (2003) argue that educational leadership must acknowledge complexity, diversity, and equity as salient characteristics in the formal leadership task of every school principal. Based on this perspective, the democratic features are specifically taken into consideration, and they have also been the focus of several studies on educational leadership within the Scandinavian context (Johansson 2001; Moos et al. 2004; Møller 2006). However, the political/democratic aspects of leadership practices cannot be viewed as merely being generated by a single leader and leadership practices in schools. This argument has led to sociological and critical-oriented studies that seek to interpret leadership as an aspect of policy formation related to the democratic problems of society (Gunter 2012; Møller and Skedsmo 2013).

This brief overview of the leadership theories indicates that educational leadership research represents a diverse field that would benefit from a theoretical framework with the ability to simultaneously capture the societal and ideological policy level and the different dimensions of the governance of institutionalized education. From both an instructional and a transformational perspective, and based upon the most recent discussion on distributed perspectives, it becomes clear that a deep understanding of the context of educational reform and policy changes is as important as an awareness of the relevant actions of school leaders, teachers, and others who are involved in school leadership. Moreover, comparing the field of theories in leadership research and transnational policy, we see an urgent need for self-reflection 
on the ways in which leadership definitions are included in the justification of policy. In light of curriculum theory, there seem to be several overlapping policies and practices between the research fields (Uljens and Ylimaki 2015). However, the understanding of educational leadership as it is described in articles and textbooks risks being either too general-that is, there is no distinction between leading a school or leading other social practices (see Young 2008) — or too restricted-that is, each school/district is considered to be its own independent unit.

We thus suggest that the meaning of educational leadership needs to be conceptualized by means of an institutional multilevel analysis, whereby transnational educational policy formations, as well as national governing discourses, curriculum problems, and local school leadership practices, are taken into consideration. In the following section, we turn to the theory of discursive institutionalism to frame our analysis into an integrative approach linking transnational policies on educational leadership to curriculum theories and problems. Our aim is to create a framework through which educational leadership can relate to curriculum theory, including the wider societal/ideological perspectives that are helpful in understanding the discourse of educational leadership. By this, we will not enter into the field of educational practices, but will instead examine the policy level within a transnational policy discourse.

\section{A Theoretical Framework: Discursive Institutionalism and Curriculum Theory}

Discursive institutionalism is a collective term for exploring the content of ideas and the interactive processes that are reflected through discourses in institutional contexts. This fourth version of the new institutionalism approach is focused on both the theorizing of the substantive content of cognitive and normative ideas and the interactive discursive processes and argumentation by which these ideas are produced, conveyed, and potentially lead to collective actions (Schmidt 2012).

Schmidt (2012) argues that agents within institutions possess two forms of abilities: background ideational abilities and foreground discursive abilities. Schmidt (2012) interprets background ideational abilities as a human capacity to understand and structure the environment from know-how concerning how the known world works. Human background ideational abilities allow actors inside and outside institutions to speak and think about institutions in certain ways, thereby contributing to both creating and maintaining institutions through collective discursive actions. Discourses work simultaneously at two levels in institutions. The background ideational abilities represent an everyday level of communication within and about the institution. The foreground discursive abilities, conversely, comprise actions when people distance themselves from the everyday institutional activities and discuss and reflect on the institution at a more general level, which also involves reflection and change from an "outside" perspective. Thus, the foreground discursive abilities 
provide the basis for a coordinative discourse that is characterized by the creation, elaboration, and justification of a certain policy. They also provide the basis for a communicative discourse that takes the ideas and policies outside the institution to "the public" for discussion and deliberation. The public here includes political actors, media, interest groups, and ordinary citizens who can act on and contribute to the discourse by engaging in the discussion (Schmidt 2012).

Discursive institutionalism distinguishes between two types of ideas: cognitive and normative. Cognitive ideas offer solutions to the problems at hand and represent the reasoning for what should be done and why. They include proposals, suggestions, guidelines, and so on that are based on what should be done upon consideration of the circumstances; that is, they express "necessary" actions that are evoked by certain causes and logic. Normative ideas, contrastingly, offer values as the bases for cognitive ideas. They add values to policy actions and policy solutions, and they legitimate policy with reference to normative values and ideologies. If cognitive ideas are legitimized by factual causes, normative ideas are argued for in terms of more general values (Schmidt 2008).

By combining the levels of cognitive and normative ideas with coordinative and communicative discourses, it becomes possible to analyze questions regarding when and where educational discourses on school leadership matter, who is setting the agenda, and to whom the agenda is communicated. When a special meaning that is embedded in a specific material context is translated to another context, it opens it up to a "discursive gap." This gap constitutes a space for the production of discourse through the recontextualization of meanings; that is to say, when a discourse moves away from its original site and into a new arena, a transformation occurs by means of which different elements of meaning within the discourse are selectively appropriated, relocated, refocused, and so on. Although elements of the discourse can be recognized, it is not totally the same discourse anymore, since possibilities for partly new meanings have been created through the transformation from one arena to another. The construction of policy discourses and educational leadership discourses can be understood as processes recontextualizing discourses by moving between different arenas, thereby reinterpreting certain dominant concepts of a discourse (Bernstein 2000/1996).

\section{The Policy Documents: Data and Analysis}

Discursive institutionalism as a research strand helps to classify groups of statements in policy documentation that articulate how actors approach formal and substantive aspects of societal fields as well as their communicative character. In our study, we selected policy documents from the EU and the OECD, which we expected to reflect a transnational discourse on education reform policy beyond the national level. By initially screening a cluster of policy documents that we downloaded from the EU and OECD websites, referred by national authorities in our own countries 
(i.e., Sweden and Norway), we selected a total of 14 documents that articulated meaning concerning the ways in which leadership makes sense as part of reform policy within and across national contexts. Thus, in our examination of the documents, we conducted a qualitative study that sought to unravel their meaning (Coffey 2014, p. 8) as well as the discourses this meaning related to. Discourses were then thought of as "an ensemble of ideas, concepts, and categories through which meaning is given to social and physical phenomena, and which is produced and reproduced through an identifiable set of practices" (Hajer 2003, p. 300).

To avoid a biased selection of documents compared to the existing corpus of policy documents published by the two organizations, we compared the reference lists between the documents and we made use of a snowball method by checking for other documents concerning leadership and leadership research that were mentioned within the papers. In this way, we could trace the ideas and discourses using a forward and backward tracing strategy, utilizing references in one document as pointers to other documents and thereby constructing a text corpus.

The documents were examined with regard to theory and, at the same time, informed by the narrative descriptions that we condensed in order to generate images of what was written about leadership, leadership research, and reform policy. The narrative descriptions were constructed by the use of a technique for identifying themes (Ryan and Bernard 2003). The themes were discovered by reading through the selected documents and clustering expressions in light of the theoretical perspectives framed by an institutional-discursive theory. Dimensions such as spheres, normative and cognitive ideas, and coordinative and communicative discourses guided our reading of the documents and thereby our means of identifying and categorizing themes. By analyzing the themes and the narrative descriptions, as condensed by re-reading the documents, we were also able to point out ways in which leadership and leadership research were conceptualized within the discourses of reform policy for each of the fields and levels included in our study. Thus, we did not apply an evaluative approach to study the documents, but rather an interpretative point of view, for the sake of developing analytical perspectives and theories (Kuckartz 2014). In order to make the theoretical and deductive dimensions transparent, we will present the core concepts and perspectives of Schmidt's (2012) theory. However, our core purpose is not merely to make use of this theory in a deductive way, but with regard to the documents, to conduct an analysis that helps to renew perspectives on curriculum and leadership research.

In terms of discursive institutionalism, Schmidt (2012) distinguishes between three different spheres, although for this study only the first two are relevant. The public philosophy sphere represents a basic sphere of public worldviews and assumptions that underpin the policy solutions, although these assumptions are seldom consciously formulated or even recognized. Instead, they form a background for the sphere of programmatic ideas. The public philosophy sphere generally includes two forms of ideas: normative ideas and cognitive ideas. Normative ideas are concerned with legitimizing policy programs that express the suggested solutions by referring to how they are rooted in a deeper understanding of the world. Cognitive ideas are instead concerned with the interpretation of these background 
assumptions in the public philosophy sphere in order to define the problems to be solved and identify the solutions to be used in the programmatic sphere. The sphere of programmatic ideas consists of a more general program that functions as a common frame of reference for ideas, actions, and solutions that are suggested as possible responses to a problem. This sphere is characterized by its foreground position, which means that ideas and suggestions are debated and discussed on a regular basis.

The spheres of discursive institutionalism are broadly in line with the levels of analysis that are generally suggested within curriculum theory: a societal/ideological arena in which basic assumptions regarding the needs of a society and ideas about education are formed, and a programmatic arena that represents the actual concrete policy documents proposed. A third level of analysis points to the so-called classroom level, where the suggested policy solutions are interpreted and performed in local practice (Deng and Luke 2008; Lundgren 1979). By combining these arenas with the public philosophy sphere and the sphere of programmatic ideas of policy formation, discursive institutionalism contributes to curriculum theory with a more elaborate tool of analysis capable of distinguishing between cognitive and normative ideas, as well as between coordinated and communicative discourses.

In this chapter, the focus of the analysis is transnational educational leadership policy. Therefore, only the first two spheres or arenas are relevant to this study. The analytical interpretive framework combines concepts and meaning from discursive institutionalism and curriculum theory via an analysis of leadership discourses emerging in transnational policy documents. An overview of the combined framework for the present study is presented in Table 14.1 below (see also Wahlström \& Sundberg 2017).

Table 14.1 The framework of analysis and the links between the perspectives in discursive institutionalism, curriculum theory, and leadership discourses

\begin{tabular}{l|l|l|l}
\hline Transnational arenas & $\begin{array}{l}\text { Discursive } \\
\text { institutionalism }\end{array}$ & Curriculum theory & $\begin{array}{l}\text { Leadership } \\
\text { discourses }\end{array}$ \\
\hline The societal/institutional realm & $\begin{array}{l}\text { Public } \\
\text { philosophy }\end{array}$ & $\begin{array}{l}\text { Institutional/societal } \\
\text { ideas }\end{array}$ & $\begin{array}{l}\text { Organizational } \\
\text { theories }\end{array}$ \\
\cline { 2 - 4 } & $\begin{array}{l}\text { Normative and } \\
\text { cognitive ideas } \\
\text { about society } \\
\text { and education }\end{array}$ & $\begin{array}{l}\text { Basic assumptions } \\
\text { concerning society and } \\
\text { education }\end{array}$ & $\begin{array}{l}\text { General ideas of } \\
\text { the need for } \\
\text { leadership in } \\
\text { schools }\end{array}$ \\
\hline The programmatic realm & $\begin{array}{l}\text { Programmatic } \\
\text { ideas }\end{array}$ & $\begin{array}{l}\text { Programmatic ideas } \\
\text { and norms }\end{array}$ & $\begin{array}{l}\text { Programmatic } \\
\text { models }\end{array}$ \\
\cline { 2 - 4 } & $\begin{array}{l}\text { Coordinative } \\
\text { and } \\
\text { communicative } \\
\text { discourses }\end{array}$ & $\begin{array}{l}\text { Common policy } \\
\text { understanding and } \\
\text { recommendations to } \\
\text { reform education }\end{array}$ & $\begin{array}{l}\text { Policy- and } \\
\text { research-based } \\
\text { suggestions for } \\
\text { the type of } \\
\text { leadership } \\
\text { needed }\end{array}$ \\
\hline
\end{tabular}




\section{Societal Ideas and Programmatic Discourses on Educational Leadership in the Transnational Arena}

In this section, we present the results of the analysis in terms of the normative and cognitive ideas in the sphere of public philosophy on education in general and on school leadership in particular. Further, we report on coordinated as well as communicative discourses concerning educational leadership in the field of programmatic ideas.

\section{Public Philosophy: Ideas and Assumptions Concerning Education and Educational Leadership}

Often in policy texts, the references and justifications in the overarching discourse refer to "the rapidly changing world." This is also true for the overall discourse on education and school leadership. At a very general level, the basic assumption can be formulated as in the following example:

Across the globe, the 21 st century is seeing rapid economic and social change. Social and population mobility allied with technological advances and an increased focus on schools to perform mean that students today face very different challenges from their predecessors (Pont et al. 2008b, p. 3).

Together, these changes are assumed to alter the role of schools, as well as the role of school leaders, in fundamental ways. Three distinctive implications of the current challenges for education in relation to leadership can be noticed in the OECD's argumentation: (i) school autonomy, (ii) school accountability, and (iii) school leadership going beyond the individual school. With the increased autonomy of schools comes the increased autonomy of school leaders. Therefore, school autonomy is closely linked to school accountability in terms of student performance, for which school leaders are, in turn, ultimately responsible. The school leaders are also supposed to take on a broader responsibility, including for other schools and the local community in terms of a system-wide school improvement. The aim of this form of school leadership is to develop what the OECD refers to as system leadership. It is linked to a normative idea of a learning organization. Further, system leadership, with its systemic focus, is assumed to have the potential to contribute to system transformation (Pont et al. 2008a). However, system leadership can only exercise influence in relation to the degree to which it is focused on teaching and learning. Thus, a coordinated discourse of system leadership opens up programmatic discourses of personal leadership (as "system thinkers"), of distributed leadership, as well as of instructional leadership. The cognitive idea of the need for leadership of the school itself is never questioned in these transnational policy documents. Instead, the interest is focused on the meaning of education leadership in a rapidly changing world. A cognitive answer to this challenge is that educational 
leaders need to take on a new role as system leaders in a broader understanding of leadership. The cognitive idea is that only if school leaders are both working close to their own school and with other schools and school leaders, caring for the success of both their own and other schools, can they contribute to changing the whole system to be more effective and successful in terms of results.

The belief that it is necessary to change the school system as a whole is related to another basic cognitive idea of the effect of education on economic growth. Within this set of cognitive ideas, human capital is a core concept (Boarini et al. 2012). The way in which the OECD deals with this issue, for example, is to relate students' skills, as measured by the Programme for International Student Assessment (PISA), to economic growth by means of recent economic modeling. The OECD's conclusion is that if each member state boosts its average PISA scores by 25 points over the next 20 years, there will be "an aggregate gain of OECD GDP of USD 115 trillion over the lifetime of the generation born in 2010" (OECD 2010, p. 6). A similar idea was expressed in the Lisbon Strategy by the EU in 2000: "The Union has today set itself a new strategic goal for the next decade: to become the most competitive and dynamic knowledge-based economy in the world"' (European Council 2000 , p. 2, italics in original).

To meet the demands for change, the main normative idea in the transnational arena is that of continuous learning from cradle to grave. In the discourse on lifelong learning, "competence" is a key concept. The identified competences are supposed to express the individual ability that is needed to meet the complex demands created by globalization and modernization in an increasingly interconnected world characterized by diversity. Both the OECD and the EU have formulated frameworks of key competencies, and both systems of competencies are measured by the PISA surveys (European Commission 2007, 2013; Rychen and Salganik 2005). The idea of lifelong learning also implies that school leaders need to develop effective leadership skills for leading learning communities. By implementing the frameworks of key competencies, both the OECD, through the PISA surveys, and the EU (European Council 2000), through the Open Method of Coordination (OMC), have the normative tools for monitoring and evaluating their member states' adaptations of a framework for lifelong learning.

\section{Programmatic Ideas of Education and Leadership in the Transnational Policy Arena}

Programmatic ideas about education and school leadership in the transnational policy arena are analyzed by examining the coordinative and communicative discourses of educational leadership. On the transnational programmatic level, ideas of curriculum are incorporated in a technical- instrumental coordinated discourse. The technical-instrumental curriculum discourse is characterized by the assumption that curricula ought to be designed in accordance with economic and labor market 
related demands. It is in light of this discourse that the emergence of performancebased and standards-based reforms can be understood because of their ability to measure "knowledge outcomes" in a seemingly comparable way (Sundberg and Wahlström 2012).

\section{A Coordinative Discourse of Effective Leadership}

The "Rethinking Education" program, which was launched by the EU in 2012, argued for the importance of member states investing in education and skills training as the most valuable tool for increasing Europe's competitiveness and productivity (European Commission 2012a). It is noted that school leaders spend more than $40 \%$ of their time on management and administrative activities. It is also noted that the number of applications for school leadership posts is often very low in the member states. The European Commission states that educational leadership is second only to teaching among the school-related factors that determine the outcomes of students' learning (European Commission 2012b, p. 43). The quality of school leadership is believed to affect both the motivation of teaching staff and the quality of their teaching. It is argued that the PISA results are higher in countries in which teachers are held accountable to school leaders and to external inspectors through the monitoring of lessons. The school leaders' impact on the ethos of the school is also believed to be significant (European Commission 2012b). In a policy discourse in which efficiency and equity are placed at the foreground of education, school leaders are at the intersection of educational policy and its implications at the school level. The efficiency discourse emphasizes teachers' motivation, skills, and competences as key factors for achieving high quality learning outcomes; in turn, the quality of educational leadership is an essential factor for achieving the goal of good teaching with an ability to inspire all students and make them all want to learn. What is referred to as "effective school leadership" is viewed as crucial for shaping an effective environment for teaching and learning - a learning environment with high aspirations that are supported by appropriate organizational structures and a good school climate. Two factors are highlighted in the efficiency discourse for school leaders: first, it is of key importance to ensure that school leaders are not overburdened with administrative work; and, second, it is important to ensure that school leaders have the capacities and qualities needed to handle an increasing number of very diverse tasks (European Council 2009). The coordinative discourse is consistent with a discourse of transformational leadership research, which expresses the purpose of leadership as motivating followers to work toward common goals. The leaders need to use their charisma and their ability to inspire their staff (Leithwood 1994; Leithwood and Jantzi 2006). In later texts, instructional leadership and managerial leadership have been subsumed within the overall concept of transformational leadership. Transformational leadership has a clear connection to the policy-based and neoliberal concept of New Public Management (Hall et al. 2013), and its representatives argue that it is possible to find correlation between 
transformational leadership and student outcomes (e.g. Sun and Leithwood 2012). Basically, transformational leadership promotes a role for the education leader as a "lonely hero" leading the team toward common goals to improve the school, although advocates of this leadership conception have also tried to go beyond a onesided, individually shaped leadership. In the coordinative discourse there is, however, still a strong normative idea about who can take on a role as principal, what set of central skills he or she needs to have, and that, in the end, the success of the school is dependent on the abilities of its principal.

\section{Communicative Discourses: Implementing Policies}

The policy slogan of getting the right people to be principals has gained lot of media attention in recent years. The motto is also echoed in other policy documents from the OECD (2013) and the private sector (Barber and Mourshed 2007; Mourshed et al. 2010), and it mirrors the individual leadership expressed in the coordinating discourse above. The slogan summarizes a political understanding of leadership against which the communicated leadership discourses on the programmatic arena are reflected.

The McKinsey report from 2007 comprised several simple solutions for school systems that want to reach the top of the results table, including advice concerning educational leadership. It is all about "getting the right people to become school leaders," providing "these people with the right set of skills," and structuring "the roles, expectations and incentives to ensure that its principals focus on instructional leadership, not on school administration" (Barber and Mourshed 2007, p. 30). In this efficiency discourse, the "right" school leader is him- or herself an excellent instructor who spends his or her time coaching the teachers to become better instructors and, as a consequence of this coaching, improves the teachers' ability to increase the students' achievements. The ideal principal is a person who focuses on helping the teachers to learn from each other, a person who is constantly spending time out in the classrooms and halls among the students, and a person who engages in e-mails and administration only after everyone else has left the school for the day. On the other hand, in a follow-up study, the need to support the school leader with a school system that includes adequate administrative staff is emphasized to ensure that the principal can focus on educational leadership. The ideal in this subsequent report is rather the "collective capacity" that is reached when teachers and school leaders work together to examine "what works" and to improve instruction together (Mourshed et al. 2010). In these two examples of the efficiency discourse on educational leadership, the message is addressed directly to the national policy arena. This is a discourse that is pre-eminently interconnected with a standards-based curriculum format because the leadership logic is based on the premise that "good" education leadership manifests itself in improved knowledge results. According to the EU (2012a), EU reforms have streamlined curricula across the EU by introducing standardized tests and infrastructure for literacy, mathematics, and science centers. 
Thus, the ideal of education leadership needs to be interpreted in close relation to the promotion of standards-based curriculum reform at a programmatic transnational level.

Furthermore, the policy text has the character of a decontextualized manual: if you act like this, your national school system will improve regardless of where in the world you are. In the two reports referred to above, a displacement from an individually to a more collective leadership ideal can be discerned. Stated otherwise, in the programmatic arena the communicative discourse is moving from an individual to a distributed leadership discourse, although still remaining within a normative idea of system leadership and learning in organizations as well as within a coordinative discourse of transformational leadership.

Based on references to current research within the field of education leadership, the OECD also communicates a discourse of distributed educational leadership for schools. The assumption is that effective leadership does not need to be exclusively related to one or a few formal positions; rather, it can be distributed across a number of individuals and teams in the school. According to the OECD (Pont et al. 2008a), the "increased responsibilities and accountability of school leadership are creating the need for distribution of leadership, both within schools and across schools." In accordance with this formulation, distributive leadership can be understood as part of the "system leadership" concept, formed in the field of cognitive ideas in the societal/institutional arena. In the same vein, the European Commission (2012b) argues that the core competencies for educational leadership can be supported by policy, for example, the impact of the principal tends to be stronger where the degree of school autonomy is higher. The Commission defines the core competencies as the ability to have vision and to be able to inspire others, to think strategically, to enhance learning environments and learning cultures, to improve the quality of students' learning and their learning outcomes, to manage resources effectively, to have good knowledge of the school system, to have strong communication skills, and to be able to solve problems. While all of those competencies can be said to represent systemic skills, the Commission also believes that successful education leadership requires specific personal characteristics. "School leadership staff are also likely to be most effective if they possess personal attributes such as courage, optimism, resilience, tolerance, emotional intelligence, self-awareness, energy, ambition, commitment and a desire for learning" (European Commission 2012b, p. 46). As school leaders are given greater responsibility for their school's academic achievement, the EU urges their member states to promote distributed leadership as a route to school improvement. A collaborative approach to leadership includes a "greater degree of networking and mutual learning between school leaders at local, national and European level" to promote "self-reflection, encourage further professional development, facilitate mutual support, disseminate policy and practice ... and mitigate some of the effects of between-school competition" (European Commission 2012b, p. 51).

So far, there have been strong links between personal (transformational) leadership and collective (distributed) leadership in the programmatic ideas of the transnational policy arena. There is a clear communicative discourse advocating that 
although a distributed leadership is increasingly favored, a collective leadership still needs to be led by a charismatic leader with a multitude of requested characteristics. At the same time as both individual and collective leadership represent two strong, intertwined conceptions of leadership in education policy documents, there is also a third theme of leadership in the communicative discourse. In 2013, the OECD published an expert report that outlined the concept of "learning leadership" as a distinct new concept of leadership compared with, for example, instructional leadership and leadership for learning. It is emphasized that learning is a basic starting point for educational leadership policy. "As learning is the core business of education, it provides the paramount form and purpose of leadership focused on creating and sustaining environments that are conducive to good learning" (OECD 2013, p. 9). This approach is fully in line with the normative idea of lifelong learning as a public philosophy in the societal arena:

...learning leadership is specifically focused on the design, implementation and sustainability of innovative, powerful learning environments through distributed, connected activity and relationships of a range of formal and informal leaders throughout a learning system (OECD 2013, p. 9).

Learning leadership builds on earlier findings concerning the importance of school autonomy, distributed leadership, and leadership as the path to school improvement. In fact, the latter is specifically emphasized in the claim that leadership is highly influential for learning and outcomes at all levels of the school system. The interest is centered on leadership itself, which means that all those included in a distributed leadership are affected, not only those who hold formal positions. The purpose of a learning leadership is to shape the conditions for "deep learning" to take place, in contrast to a merely instrumental view of learning. It is about creating innovative learning environments adapted to the challenges of the twenty-first century, calling for leaders "to be creative, thinking differently, and taking risks as they push themselves out of their comfort zones and experiment with developing and implementing new designs and encouraging others to do the same without fear of failure" (OECD 2013, p. 23).

Even if the basic analysis is still the same, leadership has an important impact on school results and so that leadership needs to be distributed to many. The requirements have now been increased and the demand of an innovative learning leadership implies that leaders are expected to continuously put themselves at stake. This approach is echoed in a curriculum strategy emphasizing that countries should "foster entrepreneurial skills through new and creative ways of teaching and learning" and focus on "the opportunity of business creation as a career destination" (European Commission 2012a, p. 4). The communicative discourse of both education leadership and curriculum expresses dissatisfaction with a school that is perceived to be too traditional and poorly adapted to the current requirements of the global labor market. Though the innovative approach is highlighted and it characterizes the discourse by introducing a partly new way of talking about leadership, the original purpose of the improvement of students' outcomes is still intact. In this "new" discourse, leadership is spoken of as dominated "by the relatively enclosed world of 
formal schooling"; a learning leadership can, however, "open horizons" and explore what leadership means in "complex environments that mix different players, settings, and styles" (OECD 2013, p. 14).

The concept of leadership that emphasizes "to lead" as well as to distribute a collective leadership is related to the modernization of the public sector in terms of the New Public Management (NPM). During the same period, distributed leadership has also aroused a considerable interest within educational leadership research. Gunter et al. (2013, p. 559) use the term "functional" to designate approaches that focus on "removing dysfunctions from the system, particularly in globalizing economy where governments have adapted modernizing accountability processes." The term "normative" expresses a desire to change and improve practice. A functionalnormative perspective of distributed leadership thus represents research that provides findings that form the basis for specifically designed national leadership reforms with the purpose of promoting good practice, or the so-called evidencebased policy initiatives. The research orientation is related to the research fields of school improvement and school effectiveness, with international organizations such as the OECD and the EU acting as mediators. The research within this field claims that the principal cannot lead the school alone; instead, the principal needs to share the leadership with others. It is argued that research makes it possible to identify the best way in terms of how and to whom the leadership should be distributed, which leads to practical advice concerning the leadership factors affecting the school and its outcomes in a positive direction (Gunter et al. 2013).

\section{Educational Reform, School Improvement, and Leadership}

In this study, we have linked curriculum theory to discursive institutionalism and to educational leadership policy and research. Curriculum theory is helpful for illustrating how policy formations are recontextualized in different transnational and national arenas. We demonstrate how different actors, both governmental organizations and private multinational companies, form coordinated horizontal discourses of what it means to be a "good" leader. By including discursive institutionalism within a framework of curriculum theory, it becomes possible to distinguish between different forms of discourses and their different functions in forming and conveying ideas (Schmidt 2012). Coordinative discourses are nourished by ideas available in the public philosophy of a society through the elusive phenomenon of the "public spirit." Such a strong normative vision of the overarching international arena is the idea of lifelong learning and the need for all citizens to learn throughout their lives.

A dominant cognitive idea on the societal international level concerning educational leadership is that schools need to have principals and that those principals have the ability to affect the outcomes of the school. The transnational perspective on leadership confirms the presence of reforms as travelling across geographical territories relating to wider societal and cultural contexts (Steiner-Khamsi 2013). The basic supposition is that when society changes rapidly in terms of its commu- 
nication and migration patterns, the principal also needs to go beyond his/her own school and exercise leadership in a wider system in order to change and transform the school in a more powerful way (Pont et al. 2008b).

These normative and cognitive societal ideas about lifelong learning and system leadership are reflected in the transnational discourse coordinated between different international actors on a programmatic level. There is a common understanding of educational leadership as being exerted by an individual person capable of leading followers toward common goals for an improved school. The focus on improved results requires a common transnational policy of a standards-based curriculum with predetermined goals and standardized national and international tests (Sundberg and Wahlström 2012). From this coordinated vision, there has been a gradual displacement towards a distributed leadership involving both formal and informal leaders of the school (Leithwood and Jantzi 2006; Lingard et al. 2003; Spillane and Healey 2010); however, the image of the impact of the leader from a transformational leadership concept is still very tangible within a distributive understanding of leadership in policy documents.

A collective view of leadership develops to be the dominant coordinative discourse, and from this common base of distributed leadership, three different communicative discourses of functional-normative beliefs (Gunter et al. 2013) about leadership can be distinguished. There is a strong discourse of getting "the right person" to be a leader linked to a transformational leadership (Barber and Mourshed 2007). However, there is also strong advocating for a distributed leadership, not least from the OECD (Pont et al. 2008a, b). More recently, as if neither the transformational nor the distributed leadership has been able to offer enough force to seriously challenge and improve the school system, a learning leadership has been introduced (OECD 2013). The latter has close connotations to innovation and "thinking outside the box," manifested through the language used within this orientation of leadership. Learning leadership is not a new form of leadership, but rather it is a variation of distributed leadership developed into network formations and experimentalism to provoke the system from within. The suggested solution for improving learning and knowledge results in schools is to keep the focus exclusively on learning, leaving other institutional commitments aside. Learning leadership can be said to illustrate a well-known truth within curriculum theory, namely that it is not easy to get reforms to deliver the expected results (Karabel and Halsey 1977; Lundgren 2006). In educational leadership policy and in much of the research on leadership, the expectations of the results of school reforms and school improvements are placed on the shoulders of principals as the central actors in schools (Barber and Mourshed 2007; Mourshed et al. 2010; OECD 2013; Pont et al. 2008a, b).

By relating leadership research to communicative transnational discourses of educational leadership policy, it seems clear that certain international research and transnational policy initiatives can be viewed as symbiotic. In particular, the functional-normative orientation of distributive leadership is developed in close connection to the policy needs of evidence-informed recommendations concerning factors expected to contribute to improved educational outcomes. However, as this 
chapter has shown, both societal and educational issues are crucial in reform policy, as well as the formal, substantive, and discursive dimensions. Following an institutional-discursive approach, we will argue that the ways in which social and educational questions become intertwined in actual reforms are dependent on cognitive and normative ideas in the public sphere. Thus, reforms to education leadership are related to coordinative and communicative discourses beyond the individual reform, while solutions to curriculum and leadership problems are anchored in educational policies and practices.

Against this background, we argue that a deeper understanding of the meaning of educational leadership discourse and the conditions under which such a discourse is conducted is crucial. While educational leadership research has so far focused on the organizational conditions and expectations for managing and leading activities, curriculum theories have offered insights into societal and educational problems to be dealt with in school and in society. We suggest taking both fields into consideration in future policies and practices; however, not without a reflexivity around how reform and research are intertwined. It is with this ambition in mind that we have approached curriculum and leadership from a discursive-institutionalist perspective.

\section{References}

Barber, M., \& Mourshed, M. (2007). How the world's best-performing school systems come ut on ttop. New York: McKinsey \& Company.

Bass, B. M. (1997). Does the transactional-transformational leadership paradigm transcend organizational and national boundaries? American Psychologist, 52(2), 130-139.

Bass, B. M. (1999). Two decades of research and development in transformational leadership. European Journal of Work and Organizational Psychology, 8(1), 9-32.

Bernstein, B. (1971). On the classification and framing of educational knowledge. In M. F. D. Young (Ed.), Knowledge and control: New directions for the sociology of education (pp. 47-69). London: Collier MacMillan Publishers.

Bernstein, B. (2000/1996). Pedagogy, symbolic control and identity: Theory, research, critique (rev. ed.). Lanham: Rowman \& Littlefield.

Boarini, R, d'Ercole, M. M., \& Liu, G. (2012). Approaches to measuring the stock of human capital: A review of country practices. http:/www.oecd-ilibrary.org/economics/ approaches-to-measuring-the-stock-of-human-capital_5k8zlm5bc3ns-en

Burns, J. (1978). Leadership. NY: Harper \& Row.

Coffey, A. (2014). Analysing documents. The SAGE handbook of qualitative data analysis. SAGE Publications Ltd. In U. Flick (Ed.), The Sage handbook of qualitative data analysis (pp. 367380). London: SAGE Publications Ltd..

Day, C., \& Gu, Q. (2010). The new lives of teachers. London: Routledge.

Deng, Z., \& Luke, A. (2008). Subject matter: Defining and theorizing shcool subjects. In F. Michael Connelly, M. F. He, \& J. A. Phillion (Eds.), The Sage handbook of curriculum and instruction (pp. 66-87). Los Angeles: Sage Publications.

Doyle, W. (1992). Curriculum and pedagogy. In P. W. Jackson (Ed.), Handbook of research on curriculum (pp. 497-516). New York: Macmillan Publishing Company.

Englund, T. (1990). Curriculum history reconsidered. Scandinavian Journal of Educational Research, 34(2), 91-102. 
European Commission. (2007). Key competences for lifelong learning-European reference framework. (Vol. 25). Luxembourg: Office for Official Publications of the European Communities.

European Commission. (2012a). Rethinking education: Investing in skills for better socioeconomic outcomes. Communication from the Comission to the European Parliamanet, the Council, the European Economic and Social Commitee and the Commitee of Regions, COM. 669 final. Strasbourg: European Comission.

European Commission. (2012b). Supporing the teaching professions for better learning outcomes. Accompanying the document "Rethinking education: Investing in skills for better socio-economic outcomes" Comission staff working document. SWD. 374 final. Strasbourg: European Comission.

European Commission. (2013). PISA 2012: EU performance and first inferences regarding education and training policies in Europe. Brussels: Directorate- general for Education and Culture.

European Council. (2000). Presidency conclusions: European Council, Lisbon, 23 and 24 March 2000. http://www.europarl.europa.eu/summits/lis1_en.htm

European Council. (2009). Council conclusions of 26 November 2009 on the Professional Development of Teachers and School Leaders. Official Journal 2009/C 302/04. Brussels: European Council.

Gronn, P. (2009). Leadership configurations. Leadership, 5(3), 381-394. doi:10.1177/1742715009337770.

Gundem, B. B. (1994). Curriculum: The history of. In T. Husén \& T. Neville Postlethwaite (Eds.), The international encyclopedia of education (Vol. 2, pp. 1301-1308). New York: Pergamon Press.

Gunter, H. M. (2012). Leadership and the reform of education. Bristol: The Policy Press.

Gunter, H., Hall, D., \& Bragg, J. (2013). Distributed leadership: A study in knowledge production. Educational Management Administration \& Leadership, 41(5), 555-580. doi:10.1177/1741143213488586.

Hajer, M. A. (2003). Coalitions, practices, and meaning in environmental politics: From acid rain to BCE. In D. R. Howarth \& J. Torfing (Eds.), Discourse theory in European politics (pp. 297 314). London: Palgrave Macmillan.

Hall, D., Gunter, H., \& Bragg, J. (2013). Leadership, new public management and the re-modelling and regulation of teacher identities. International Journal of Leadership in Education, 16(2), 173-190.

Hallinger, P. (2003). Leading educational change: Reflections on the practice of instructional and transformational leadership. Cambridge Journal of Education, 33(3), 329-352. doi:10.1080/0 305764032000122005.

Hallinger, P. (2010). Developing instructional leadership. In B. Daavis \& M. Brundrett (Eds.), Developing successful leadership (Vol. 11, pp. 61-76). Dordrecht: Springer.

Hallinger, P., \& Heck, R. H. (2010). Collaborative leadership and school improvement: Understanding the impact on school capacity and student learning. School Leadership \& Management, 30(2), 95-110. doi:10.1080/13632431003663214.

Hamilton, D. (1989). Towards a theory of schooling. London: The Falmer Press.

Harris,A. (2007).Distributed leadership:Conceptual confusion andempirical reticence.International Journal of Leadership in Education, 10(3), 315-325. doi:10.1080/13603120701257313.

Hopmann, S. T. (1999). The curriculum as a standard of public education. In Studies in philosophy and education (Vol. 18, pp. 89-105). Dordrecht: Kluwer Academic Publishers.

Hopmann, S. T. (2003). On the evaluation of curriculum reforms. Journal of Curriculum Studies, 35(4), 459-478.

Hopmann, S. T. (2007). Restrained teaching: The common core of Didaktik. European Educational Research Journal, 6(2), 109-124.

Johansson, O. (2001). Swedish school leadership in transition: In search of a democratic, learning and communicative leadership? Pedagogy Culture \& Society, 9(3), 387-406. doi:10.1080/14681360100200122. 
Karabel, J., \& Halsey, A. H. (1977). Power and ideology in education. New York: Oxford University Press.

Kuckartz, U. (2014). Three basic methods of qualitative text analysis. QSAGE Publications Ltd. In U. Kuckartz (Ed.), Quatlitative text analysis: A guide to methods, practice \& using software (pp. 65-121). London: SAGE Publications Ltd..

Leithwood, K. (1994). Leadership for school restructuring. Educational Administration Quarterly, 30(4), 498-518.

Leithwood, K., \& Jantzi, D. (2006). Transformational school leadership for large-scale reform: Effects on students, teachers, and their classroom practices. School Effectiveness and School Improvement, 17(2), 201-227. doi:10.1080/09243450600565829.

Leithwood, K., Menzies, T., \& Jantzi, D. (1994). Earning teachers' commitment to curriculum reform. Peabody Journal of Education, 69(4), 38-61. doi:10.2307/1492691.

Lingard, B., Hayes, D., \& Mills, M. (2003). Leading learning: Making hope practical in schools: Making hope practical in schools. Maidenhead: McGraw-Hill Education (UK).

Lundgren, U. P. (1972). Frame factors and the teaching process. A contribution to curriculum theory and theory on teaching. Stockholm: Almqvist og Wiksell.

Lundgren, U. P. (1979). Att organisera omvärlden. En introduktion till läroplansteori. Vällingby: Liber Förlag.

Lundgren, U. P. (2003). The political governing (Governance) of education and evaluation. In P. Haug, \& T. A. Schwandt (Eds.), Evaluating educational reforms: Scandinavian reforms (pp. 99-110). Information Age Publishing.

Lundgren, Ulf P. (2006). Political governing and curriculum change - From active to reactive curriculum reforms. The need for a reorientation of curriculum theory. Studies in Educational Policy and Educational Philosophy, 1, 1-12. Utdanningspolitiska Institutet http://www.upi. artisan.se/docs/Doc262.pdf

Menck, P. (2000). Looking into classrooms: Papers on Didactics. Stamford: Ablex Publishing Corporation.

Møller, J. (2006). Democratic schooling in Norway: Implications for leadership in practice. Leadership and Policy in Schools, 5, 53-69.

Møller, J., \& Skedsmo, G. (2013). Modernising education: New public management reform in the Norwegian education system. Journal of Educational Administration and History, 45(4), 336-353. doi:10.1080/00220620.2013.822353.

Moos, L., Möller, J., \& Johansson, O. (2004). A Scandinavian perspective on educational leadership. The Educational Forum, 68(3), 200-210. doi:10.1080/00131720408984632.

Mourshed, M., Chijioke, C., \& Barber, M. (2010). How the world's most improved school systems keep getting better. New York: McKinsey London.

Mulford, B. (2003). School leaders: Challenging roles and impact on teacher and school effectivenes. Paris: Comissioned Paper by the Educationand Training Policy division, OECD, for the Activity "Attracting, Developing and Retaining Effective Teachers".

Muller, J. (2000). Reclaiming knowledge: Social theory, curriculum, and education policy (Knowledge, identity, and school life series 8). London: Routledge.

OECD. (2010). The high cost of low educational performance: The long-run economic impact of improving PISA outcomes. The High Cost of Low Educational Performance. http://www.oecd. org/pisa/44417824.pdf

OECD. (2013). Leadership for 21 st century learning. Paris: OECD (Organisation For Economic, Co-Operation Development) Publishing.

Oterkiil, C., \& Ertesvåg, S. K. (2014). Development of a measurement for transformational and transactional leadership in schools taking on a school-based intervention. Educational Management Administration \& Leadership, 42 (4 suppl), 5-27. doi:10.1177/1741143214523011.

Pinar, W. F. (1978). The reconceptualization of curriculum studies. Journal of Curriculum Studies, 10(3), 205-214. 
Pont, Beatriz, Nusche, Deborah, \& Hopkins, David. (2008a). Improving school leadership, volume 1 case studies on system leadership: Case studies on system leadership, (Vol. 2). OECD Publishing.

Pont, B., Nusche, D., \& Moorman, H. (2008b). Improving school leadership: Volume 2, Case studies on system leadership. Paris: Organisation for Economic Co-operation and Development.

Reid, W. (1986). Curriculum theory and curriculum change: What can we learn from history? Journal of Curriculum Studies, 18(2), 159-166.

Reid, W. A. (1999). Curriculum as institution and practice. Essays in the deliberative tradition. London: Lawrence Erlbaum Associates, Publishers.

Ryan, G. W., \& Bernard, H. R. (2003). Techniques to identify themes. Field Methods, 15(1), 85-109. doi:10.1177/1525822x02239569.

Rychen, DS, \& Salganik, LH. (2005). The definition and selection of key competencies: Executive summary (Vol. 1).

Schmidt, V. A. (2008). Discursive institutionalism: The explanatory power of ideas and discourse. Annual Review of Political Science, 11(1), 303-326. doi:10.1146/annurev. polisci.11.060606.135342.

Schmidt, V. A. (2012). Scope, dynamics, and philosophical underpinnings. In F. Fischer \& J. Forester (Eds.), The argumentative turn revisited: Public policy as communicative practice (p. 85). Durham: Duke University Press.

Schubert, W. H. (1986). Curriculum. perspective, paradigm, and possibility. New York: Macmillan Publishing Company.

Sivesind, K., \& Karseth, B. (2014). Curriculum theory and research in Norway: Traditions, trends, and topics. International handbook of curriculum research (pp. 362-375). Lawrence Erlbaum Associates.

Spillane, J. P., \& Healey, K. (2010). Conceptualizing school leadership and management from a distributed perspective: An exploration of some study operations and measures. The Elementary School Journal, 111(2), 253-281.

Steiner-Khamsi, G. (2013). What is wrong with the 'What-Went-Right' approach in educational policy? European Educational Research Journal, 12(1).

Sun, J., \& Leithwood, K. (2012). Transformational school leadership effects on student achievement. Leadership and Policy in Schools, 11(4), 418-451.

Sundberg, D., \& Wahlström, N. (2012). Standards-based curricula in a denationalised conception of education: The case of Sweden. European Education Research Journal, 11(3).

Uljens, M. (1997). School didactics and learning: A school didactic model framing an analysis of pedagogical implications of learning theory. Hove: Psychology Press.

Uljens, M., \& Ylimaki, R. (2015). Towards a discursive and non-affirmative framework for curriculum studies, Didaktik and educational leadership. Nordic Journal of Studies in Educational Policy, 2015(3), 30177. doi:10.3402/nstep.v1.30177.

Westbury, I. (2000). Teaching as a reflective practice: What might Didaktik teach curriculum? In I. Westbury, S. Hopmann, \& K. Riquarts (Eds.), Teaching as a reflectice practice. The German Didaktik Tradition (pp. 15-54). London: Lawrence Erlbaum Associates, Publishers.

Westbury, I. (2003). Evaluating a national curriculum reform. In P. Haug \& T. A. Schwandt (Eds.), Evaluating educational reforms: Scandinavian perspectives. Greenwich: Information Age Publishing.

Woods, P. A., Bennett, N., Harvey, J. A., \& Wise, C. (2004). Variabilities and dualities in distributed leadership findings from a systematic literature review. Educational Management Administration \& Leadership, 32(4), 439-457.

Yates, L. (2009). From curriculum to pedagogy and back again: Knowledge, the person and the changing world. Pedagogy, Culture \& Society, 17(1), 17-28. doi:10.1080/14681360902742837.

Young, M. F. D. (Ed.). (1971). Knowledge and control: New directions for the sociology of education. London: Collier Macmillan Publishers.

Young, M. F. D. (1998). The Curriculum of the future: From the "New sociology of education" to $a$ "Critical theory of learning". London: Falmer Press. 
Young, M. F. D. (2008). From constructivism to realism in the sociology of the curriculum. Review of Research in Education, 32(2008), 1-28.

Young, M. F. D., \& Whitty, G. (Eds.). (1977). Society, state and schooling. Readings on the possibilities for radical education. London: The Falmer Press.

Wahlström, N., \& Sundberg, D. (2017). Discursive institutionalism: Towards a framework for analysing the relation between policy and curriculum. Journal of Education Policy, 1-21. http:// dx.doi.org/10.1080/02680939.2017.1344879

Open Access This chapter is licensed under the terms of the Creative Commons Attribution 4.0 International License (http://creativecommons.org/licenses/by/4.0/), which permits use, sharing, adaptation, distribution and reproduction in any medium or format, as long as you give appropriate credit to the original author(s) and the source, provide a link to the Creative Commons license and indicate if changes were made.

The images or other third party material in this chapter are included in the chapter's Creative Commons license, unless indicated otherwise in a credit line to the material. If material is not included in the chapter's Creative Commons license and your intended use is not permitted by statutory regulation or exceeds the permitted use, you will need to obtain permission directly from the copyright holder.

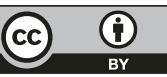




\section{Part VI \\ Conclusions and Implications}




\title{
Chapter 15 \\ Curriculum Theory, Didaktik, and Educational Leadership: Reflections on the Foundations of the Research Program
}

\author{
Rose M. Ylimaki and Michael Uljens
}

\begin{abstract}
This chapter provides concluding reflections and next steps in a research program bridging curriculum theory/Didaktik and educational leadership studies. The bridging utilizes non-affirmative education theory as the theoretical ground. To begin, we present a retrospective discussion of the project. We then relate the approach to the contributions included in this volume, especially focusing on the normativity of education theories, and pointing at how non-affirmative education theory corresponds to deliberation oriented democratic-hermeneutic initiatives. Non-affirmative education theory identifies both leadership, teaching and curriculum work as critical deliberation based professional activities driven by subjects, individual agency in historically developed cultural and societal institutions framed by policies. Non-affirmative educational leadership practices are expected to take a critical stand regarding given policies, and other expectations, yet mindful of that education in democratic societies, typically following a Bildung tradition aim at individuals making up of their own minds and learning that practices, also moral and political ones, may and can be changed. The approach applied in this volume, i.e. to point at the roots of modern European education theory not only helps us to better see connections between this Bildung tradition, Deweyan pragmatism and deliberative democracy but is also used as a point of departure to continue towards comparative research on how educational leadership work is carried out in the intersection between curriculum as a policy document and leadership practices at different levels as discursive practices.
\end{abstract}

\footnotetext{
R.M. Ylimaki $(\bowtie)$

University of South Carolina, Columbia, SC, USA

e-mail:YLIMAKIR@mailbox.sc.edu
}

M. Uljens

Åbo Akademi University, Vaasa, Finland

e-mail: michael.uljens@abo.fi 
Since their inceptions as academic fields, curriculum theory/Didaktik and educational leadership have developed as disparate fields with different traditions and theoretical logics (Uljens and Ylimaki 2015; Ylimaki and Uljens 2017). Curriculum theory has taken many shapes as theory of education and Bildung, theory of governance of institutionalized education, as a cultural critique, or education as a criticalideological force in societal transformation. Educational leadership literature, has evolved from an organizational theory and management foundation to an empirically driven field, developing leadership models or forms grounded in organizational theory and, more recently, institutional theory or critical theories as well as previous empirical studies. Regardless of grounding, the tendency is to approach schooling on an individual, interactive and a practitioner level, offering leadership approaches or forms that contribute to the school (organizational) issues, social dynamics between education professionals and being directed by "best practices" (instructional and otherwise) or social justice where curriculum or aims and content of education have been distant. As we argued in Part I, we see that both fields have demonstrated more or less blind spots to the interactional level between societal aims and social interactions in schools.

While curriculum/Didaktik primarily focuses on curriculum theorizing, all of which focus on broader questions of societal aims, values and ideals translated into content and methods, educational leadership scholars have produced approaches and forms (e.g. instructional leadership, transformational, transformative) primarily from an empirical base. The same holds true both within the Nordic (European) and US traditions. In Part I, we also argued that scholars in both fields have produced important literature but through approaches with inherent normativity problems, thereby grounding their work in social reproduction or social transformation perspectives on the relations between schools and society. For example, normativity problems occur when current and future ideals are clearly identified, perhaps leaving insufficient room to consider the future as an open question. In our view, such practices are problematic for education in a democratic society; however, as Carolyn Shields (2011) also reminds us in her Foreword, education is not value neutral or norm free.

Hence, in Part I, in framing this project, we support a less normative, nonaffirmative approach and the use of modern education theory concepts. The term 'non-affirmative education theory', indeed modern education theory and its core concepts, is unfamiliar to many curriculum and leadership scholars outside the German-Nordic frame of reference, including North America and many other countries worldwide. This volume may be the first in which a non-affirmative approach is proposed for scholars in both fields and in both contexts. In our view, a nonaffirmative approach has the potential to decenter instrumentalism that has, as Autio powerfully argues, dominated educational scholarship and practice, including curriculum and leadership.

Going forward, regardless of whether curriculum theorizing/Didaktik and leadership studies are treated coherently or not, both of these fields have developed in relation to perspectives on education, curriculum, governance, and leadership at the nation state level. As we and other contributors have argued throughout this volume, we must now consider curriculum and leadership at the transnational level as well 
as within nation states with both similar and particular historical and cultural contexts. With this volume, we lay a foundation for a coherent agenda that brings curriculum theory/Didaktik and leadership studies closer together in new research fields amidst what we have termed globopolitanism.

More specifically, our agenda built upon an assumption that three dilemmas are crucial for any theory of education, curriculum, or educational leadership thereof. First, we asked how a theory specifies the relation between education and other societal practices including e.g. culture, economy, politics), and here we argued for a non-hierarchical relation. This question has traditionally been the focus of curriculum studies, considering how curricula specify what education should be aiming at and what cultural contents should be selected in order to reach these aims. We argued that curriculum work to answer these questions is inherently leadership. Second, we asked how we explain human interaction and educational influence, questions that have pointed at methods in curriculum studies. While we recognize that the term influence has been widely utilized from different traditions and empirically driven logics (e.g. a control paradigm in curriculum theorizing and numerous leadership studies grounded in transactional and transformational leadership approaches) but the term has not been theorized as we do here with education theory. Rather, we argue that in answering the second question regarding the nature of individual interactions or relations in any theory of education, there is a necessary element of pedagogical influence, invitation or provocation in the Bildung process. As noted in part one, theories of (general) education are aimed at addressing both a theory of Bildung (aims and contents) and a theory of teaching (educational influence) in a systematic manner. Theory of Bildung traditionally includes reflection on the aims of education and how selected cultural contents may support reaching these aims, while theory of education explicates those educative interactions involved in treating the contents for reaching given aims.

It should, thus, be observed that exchanging the term 'influence' to another one does not do away with the core problem at hand. The paradox of modern education is that it seems we have to accept the individual as being indeterminate, i.e. free, in order for learning to occur and for education to be a meaningful activity. On the other hand, it seems we have to accept that we can reach cultural freedom only by living among others - "man becomes man only among men" (Kant, Fichte). That is, only by experiences, activity and reflection that we learn from other humans we become a part of the culture sharing language, practices and norms. Without these we would not be culturally free (Benner 1991; Uljens 2002). The modern heritage in education theory reminds us that this process of pedagogically supported growing into a culture can occur in ways that allow me to become aware of what kind of culture I seem to be growing into. Education also can help the individual transcend the very culture into which she or he has grown into. This is not problematic. The dilemma comes here: if the individual really is assumed free in this radical sense as being indeterminate, then it appears that it is exactly this and nothing else that, first, makes education possible, but also, second, that might allow external influences to mold the individual. On the other, if the individual is free in this radical sense, then it seems that what experiences are paid attention to is in the hands of the individual 
herself and her way of relating to the world, others and herself. Suddenly, in this light it appears as if education would be a completely impossible task. Modern education theory tried to locate a position between unlimited possibility to influence the learner and the the impossibility to influence the learner, given that determinist assumptions about the world and the human was abandoned.

Or, we could always try to communicate with others and convey things, but it would definitely be beyond our control to lead the individual to predetermined aims, as is done in reproductive, socialization oriented pedagogy or in transformative pedagogy striving towards making ideals real for the future by educational means. With modernity our western tradition left the idea of man being determined by birth, religion or social class. We also left a teleological view of development. The future became radically open. Human beings turned from being only law abiding to law establishing, political subjects accepting to live by the laws we make ourselves. In the same vein, we became historical: if the future is open the way we live, we cannot avoid making history. Faced with this dramatic shift from a teleological view of man and world, founding fathers of education as a science and theory tried conceptually to find a room for pedagogical action that did not perceive of itself an almighty power to form indetermined objects according to his/her own ideas about the future, and which, on the other hand did not fall into despair of being unable to support others growth. The theoretical concept of non-affirmativity in pedagogy refers to an invitational activity is relational in its core: only to the extent that the learner accepts the invitation, she can, through own activity, engage in a self-transcending activity. Fichte called this relation activity Bildsamkeit on the learner's side. On the educator's side, he used the concept of summoning to self-activity.

One of the cornerstones of modern pedagogy is the notion that autonomy (Mündigkeit) is the highest objective of education - discerning thought and action as regards issues of both knowledge and values. According to Herbart, moral freedom means following the reflected will, not acting conventionally from impulse or emotion. Consequently, education consists in the summoning of the Other to reflect over, for instance, the reasonableness of one's own will in relation to others and to the interests of others. Educating the will is then about the cultivation of discernment with the help of reason. Here we have argued that we need to (re)theorize educational leadership from a root of existing education theory, drawing explicitly on the German tradition including particularly Benner's (1991) reconstruction. This tradition is also present in John Dewey's philosophy that has a Hegelian root. As we argued in detail earlier, Hegel draws on Kant and Fichte's critique of Kant that opened the way for Hegel on which Dewey builds his philosophy. Further, John Dewey developed his philosophy in relation to a core tradition of western citizenship, identity, and democracy 100 years ago in a nation with a relatively new U.S. democracy from classical theorists who developed ideas 100 years even prior. Interestingly, education theory in its classical tradition and applications with Dewey and others have survived over time. At the same time, we see the increasing relevance of interculturality and multiculturalism to our non-affirmative education theoretical framing in what we have termed an era of globopolitanism increasingly characterized by populism and neo-nationalist discourses. Of course we also recog- 
nize that Kant Fichte and others were writing about cosmopolitanism, but we are now living in a time with a paradoxical neo-nationalist discourse along with globopolitanism. Despite globopolitanism and multiculturalism, we see the need to retain core theoretical positioning on what it means to become a human cultural being (etnos, identity) and citizen (demos).

We also recognize that non-affirmative education theory in the foreground of our theoretical framing was constructed in relation to education at the nation state level (Uljens 2016). National curriculum making and curriculum theory are challenged by globally growing political, economic and technological interdependencies, transnational homogenization and aggregation processes. In addition, increasing pluralisms within nation states present new topics to be solved. We have argued that although the world never before has had the shape and form it we experience today, these issues are, in principle not new from an education theory perspective. The modern, or classic, approach early on identified dilemmas connected to a reproduction- and transformation oriented curricula, as well as dilemmas emanating from descriptive-technological and normative theory. A contribution we have from modern education is a concept explaining how e.g. socialization (becoming a subject sharing things with others) and personalization (developing an individual unique identity) may be considered as integrated rather than excluding processes.

The nation-states face new dilemmas both in their external relations and internal conditions. 'Globalization' has brought cosmopolitanism back on the agenda after about 200 years of constructing independent, legal states (Rechtsstaat) based on some concept of collective nationhood, often invented around language, formal equity of citizens and history (Lewellen 2002). What is needed today is a renewed and extended discussion on cosmopolitanism and the modern, Hegelian educational heritage (e.g. Brincat 2009; Moland 2011). As a topic cosmopolitanism has reoccurred many times in European history, but always in new constellations and with new motives. We know that in their reaction against the aristocratic society both Kant and Herbart proposed cosmopolitanism as an ideal. "Das Weltbeste", (Kant 1915), the best for the world, rather than private or national interests, was to be the aim of education.

We recognize and support a deliberative notion of curriculum (Englund 2006) and theorizing curriculum as complicated conversation (Pinar 2012) with explicit attention to leadership amidst the contemporary situation. The third question in our framework again considers how relations between schools and society are explained and how educational influence is explained both within nation states and at transnational levels. Although the global perspective has been present as an educational ideal and in policy borrowing/lending for centuries, the scale and quality of how policy-curriculum work and educational leadership are connected with transnational developments in globopolitanism has changed. The question is how far the modern heritage can take us in this respect? Modernism, however, includes both a tradition of cosmopolitan thinking as represented by Kant, while educational theory in the form we know it today is mainly Hegelian acknowledging the empirical Other as a condition for developing subjectivity. We also must recognize that we have been here before. Historically, we have been at a point where modern nation states 
developed from cosmopolitanism, and education had a crucial role in building societal cohesion. Thus, again we also look to modern education theory (Benner 1991) and its core concepts (recognition, summoning to self-activity, Bildsamkeit), asking to what extent these modern concepts are still relevant for contemporary challenges. We argue that modern education theory and its core concepts are still relevant; however, we have not yet fully examined how these core education concepts apply to curriculum work-leadership at transnational levels. We also look to discursive institutionalism to explain leadership interactions within and between levels; however, we recognize that discursive institutionalism does not have an education language. Further, we acknowledge that discursive institutionalism provides us a language to understand policy discourse but it does not explicitly consider internal organizational dimensions found to be relevant for leadership in schools, districts, and perhaps transnational organizations. Finally, it is important to acknowledge that we are presenting a grand narrative in a certain way with our overarching theoretical framing. At the same time, in a spirit of a non-affirmative perspective, we also acknowledge that the future is an open question and our framing will be subject to counter arguments in relation to future changes. To that point, we defend the position presented here as we take it to an empirical phase. Next steps feature an empirical phase grounded in our theoretical framing and hermeneutic-phenomenological methodology.

We purposely opened Part II with an examination of the macro level influences on educational leadership and curriculum/Didaktik. As Lejf Moos clearly demonstrates with his detailed description of empirical realities in Europe, new transnationally related movements focused on governance and evaluation/curriculum policies bring curriculum/Didaktik and leadership studies closer together on a global agenda. Closely related, Gert Biesta's analysis of leadership demonstrates how neoliberalism and related externalized evaluation policies have instrumentalized administrative/leadership of teaching and learning on a global scale, shifting for example, discourse from head teachers to lead learners. In so doing, these chapters lay the groundwork to understand how Curriculum Theory-Educational Leadership and Didaktik-Educational Leadership connect in a global perspective as well as nation state perspective. Paraskeva continues this argument, proposing a critical, non-abyssal position that respects epistemological diversity, demonstrates vertical as well as horizontal cosmopolitanism, and aligns with a non-affirmative position outlined in Part I.

Part III continues with an examination of the history and implications of dialogues occurring in the Curriculum Meets Didaktik project of the 1990s. While the Curriculum Meets Didaktik project did not explicitly consider the role of leadership, that project is a model for comparative dialogues and inspired projects that help us further consider how to bridge curriculum and leadership today. From a US perspective, Doyle describes how the Curriculum Theory Meets Didaktik project inspired empirical studies of the relations among content, pedagogical processes, and the practicalities of classroom-level curriculum. He argues that the present situation of current curriculum evaluation policies and governances changes makes leadership increasingly relevant to curriculum studies, and in his chapter, he considers how curriculum and leadership may be considered closer together. From a 
European perspective, Knapp and Hopmann also illustrate how the Curriculum Meets Didaktik project inspired many empirical projects, including most recently, their own recent study of school leadership as gap management in Austria. Autio then traces the history of ideas and its emphasis on instrumentalist thinking and rationality, and then argues powerfully for a reconsideration of this thinking in the contemporary situation, one that more clearly connects curriculum and leadership. In his final part of his chapter, Autio moves beyond western epistemologies, and poses a view of educational leadership from a base of Chinese wisdom traditions. Further, Autio reminds us that when we consider leadership in relation to a culture other than our own, we must clearly understand our own traditions and history of ideas.

The disproportion of chapters in the remainder of this volume then illustrate, through varying traditions and perspectives, an empirical reality of how, from a North American and European perspective respectively, we came to a place where curriculum theory/Didaktik and leadership are coming together on global and nation state agendas. We began with Bogotch and colleagues' description of powerful historical examples when leadership and curriculum have come together to promote aims of multicultural education, democracy and social justice in the US. Huber and colleagues also consider leadership connections with curriculum but focus on tensions between accountability policy pressures and long-standing tradition in Didaktik and Bildung. Castner and colleagues continue the focus on tensions between ideological arguments on the right and left, arguing for a new conceptualization of teacher as leader grounded in a Reconceptualist curriculum tradition. We appreciate the focus on theorizing teacher leadership from a curriculum base as this chapter opens a complicated conversation regarding leadership in pedagogical relations. William F. Pinar, the leader of the Reconceptualist movement in North America and elsewhere, then brings his expertise in curriculum theorizing, Bildung, and parrhesia ('frank speech') to posit a new lens for leadership authority. Here we again see important connections between Pinar's perspectives on intersubjectivity and the core concepts from modern education theory (Benner 1991) that are foundational for understanding leadership relations in our framework. Moving back to the Nordic countries with some contrast, Forsberg and colleagues anchor their consideration of curriculum theory and leadership in classical curriculum perspectives (codes), arguing for a new comparative code to explain the contemporary global situation bringing leadership and curriculum together. The final chapters also feature a multi-level perspective on leadership, using discursive institutionalism along with curriculum theory/Didaktik and leadership research. Here Uljens and Rajakaltio's empirical study reconstructs the discursive dynamics regarding educational leadership as curriculum work at the nation-state level. In much the same vein, Sivesind and Wahlstrom take their point of departure from both classical curriculum theory and institutional (societal and programmatic) arenas, reconceptualizing school leadership using curriculum theory as well as discursive institutionalism.

Going forward, in conclusion, we offer two interrelated propositions. Our first proposition is methodological. The non-affirmative position is coherent with a 
hermeneutics as epistemology. We have also pointed out the connection between hermeneutics and theory of Bildung. Practitioners and policymakers at all levels of the education system are mediating between, as well as contributing to, various epistemic practices and value discourses. To what extent do different policy practices frame this work? How to practitioners contribute to the existing network of professionals acting? Here we argue that a comparative international dialogue regarding curriculum and leadership requires new comparative inquiry approaches and methods as well as a further consideration of globopolitanism. One reason is that obvious differences may be observed both within and between regions (NorthAmerica and the Nordic countries/Europe) and increasingly between these regions and non-Western regions, especially in terms of leadership and evaluation policies and practices, despite increasing similarities in curriculum and output-centered evaluation policies supported by transnational organizations. In our view, comparative inquiry must involve reflexive relations that both differentiate globalizing policy and the subject at all levels (classrooms, schools, districts/municipalities, states or regions, nation states, and transnational levels), with some variation between the nation state curriculum/evaluation policy and leadership preparation level and the transnational policy level (Uljens et al. 2016).

At school and district/municipality levels, we then ask:

What notions of curriculum are promoted by transnational organizations, nations, and states, municipalities, regions and schools and how do they mediate among these and their students?

What kind of school leadership, teacher's professionality and school development are promoted and practised at the different levels within the state and as cooperations between regions and states? How may these school development initiatives be supported by research?

At the nation state level (and states as well as regions, districts, municipalities within these), we have curricula (e.g. aims, content, methods) as well as policies. Implications for a comparative inquiry project include the following questions:

How do nation states initiate and develop curriculum changes or reforms and how do they mediate and position themselves between transnational and local realities within the nation-state? If appropriate, we also ask how do states within nation states initiate, develop, and mediate curriculum changes or reforms? How are various nation states, with their differing cultural and historical traditions, responding to global, common changes and dilemmas? What are the conceptions of citizens' Bildung, competencies, skills, and learning of each nation state and how are these promoted through policies and the process of education?

How does the dynamics within the nation-state look like, regarding the dynamics between policy making, governance research and practitioners?

At the transnational level, we do not have curricula; rather, we have transnational curriculum and evaluation policies that could be compared. We ask:

What curriculum and evaluation policies and practices are promoted by transnational organizations as members of continuously evolving configurations of nation state systems? 
Here curriculum may be considered as a culture emerging in relations within and between new forms of governance.

At all levels, we acknowledge that categories are discursive, dynamic, and evolving within and between nation states.

Our second proposition is that domestically oriented theorists, researchers, and practitioners now considering curriculum work and educational leadership grounded in our framework could enlarge their repertory by further looking into theoretical developments regarding the issues we have opened up for, including empirical and theoretical studies regarding to what extent educational leadership and school development practices may be understood as affirmative vs non affirmative practices. Cosmopolitanism, both in terms of theoretical ideals and empirical realities now manifest within and between nations is another central topic. We can look to theoretical ideals of cosmopolitanism (Kant) and existing approaches to explain the empirical reality, such as policy borrowing and lending inspired by systems theories (Luhmann), world systems theory (Wallerstein) and those who have considered their approaches in policy borrowing and lending (e.g. Steiner-Khamsi, Waldow). As noted in Part I as well as other chapters (Uljens and Rajakaltio Chap. 13, Sivesind and Wahlstrom Chap. 14), we also see the relevance of organizational theory for schools and transnational organizations along with discursive institutionalism as a mediational approach to analysis of how ideas move within and between levels in different polities. Our approach considers these perspectives and offers an alternative for future comparative inquiry.

At the same time, we recognize and appreciate the cultural/contextual challenges involved in comparative inquiry or even dialogue. We also recognize the challenges involved when scholars move outside of their traditional fields of study with longstanding theoretical logics and meanings associated with particular terminology, such as influence. Going beyond the earlier important Curriculum Meets Didaktik project, in this project bridging curriculum theory/Didaktik and educational leadership studies, scholars must not only engage in cross-national dialogue and transnational study but also must engage in study across fields and disciplines. That said, we invite readers from curriculum/Didaktik and educational leadership to continue this dialogue as this project is ongoing.

As a final note, we appreciate the perspectives, questions, and critiques from those who wrote commentaries - namely, William F. Pinar, Carolyn Shields, and Tomas Englund. They all inspired and challenged our thinking in this volume and for future scholarship. This is how we grow as scholars and as learning subjects. It is our greatest hope that the kind of cross-field/disciplinary and cross-national dialogue initiated here will be inspirational for others, building upon our new coherent theoretical agenda of education, bridging curriculum theory/Didaktik and leadership amidst globopolitanism and beyond. 


\section{References}

Benner, D. (1991). Allegemeine Paedagogik. Eine systematisch-problemgeschichtliche Einfuhrung in die Grundstruktur pädagogischen Denkens and Handelns. Weinheim: Juventa.

Brincat, S. (2009). Hegel's gesture to radical cosmopolitanism. Journal of Critical Globalisation Studies, 1, 47-65.

Englund, T. (2006). Deliberative communication: A pragmatist proposal. Journal of Curriculum Studies, 38(5), 503-520.

Kant, I. (1915). Avhandlingar om fred och rätt. Stockholm: Albert Bonnier.

Lewellen, T. C. (2002). Anthropology of globalization, the: Cultural anthropology enters the 21st century. London: Bergin \& Garvey.

Moland, L. L. (2011). Hegel on political identity. Patriotism, nationality, cosmopolitanism. Evanston: Northwestern University Press.

Pinar, W. (2012). What is curriculum theory? (2nd ed.). New York: Routledge.

Shields, C. M. (2011). Transformative leadership: An introduction. Counterpoints, 409, 1-17.

Uljens, M. (2002). The idea of a universal theory of education - An impossible but necessary project? Journal of Philosophy of Education, 36(3), 353-375.

Uljens, M. (2016). Non-affirmative curriculum theory in a cosmopolitan era? Revista Tempos $e$ Espaços em Educação, Sergipe, Brasil, (Time and Space in Education), 9(18), 121-132. http:// www.seer.ufs.br/index.php/revtee/article/view/4970.

Uljens, M., \& Ylimaki, R. (2015). Towards a discursive and non-affirmative framework for curriculum studies, Didaktik and educational leadership. Nordic Journal of Studies in Educational Policy, 1(3). http://nordstep.net/index.php/nstep/article/view/30177

Uljens, M., Sundqvist, R., \& Smeds-Nylund, A.-S. (2016). Educational leadership for sustained multi-level school development in Finland - A non-affirmative approach. Nordic Studies in Education, 36(2), 103-124.

Ylimaki, R., \& Uljens, M. (2017). Theorizing educational leadership studies, curriculum, and Didaktik. Leadership and Policy in Schools, 17(2).

Open Access This chapter is licensed under the terms of the Creative Commons Attribution 4.0 International License (http://creativecommons.org/licenses/by/4.0/), which permits use, sharing, adaptation, distribution and reproduction in any medium or format, as long as you give appropriate credit to the original author(s) and the source, provide a link to the Creative Commons license and indicate if changes were made.

The images or other third party material in this chapter are included in the chapter's Creative Commons license, unless indicated otherwise in a credit line to the material. If material is not included in the chapter's Creative Commons license and your intended use is not permitted by statutory regulation or exceeds the permitted use, you will need to obtain permission directly from the copyright holder.

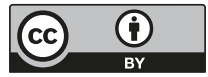

